Center for Transportation Analysis

Energy and Transportation Science Division

\title{
TRANSPORTATION ENERGY DATA BOOK: EDITION 29
}

\author{
Stacy C. Davis \\ Susan W. Diegel
}

Oak Ridge National Laboratory

Robert G. Boundy

Roltek, Inc.

July 2010

Transportation Energy Data Book: Edition 29 can be found on line at:

cta.ornl.gov/data

\author{
Prepared for the \\ Vehicle Technologies Program \\ Office of Energy Efficiency and Renewable Energy \\ U.S. Department of Energy
}

Prepared by the Oak Ridge National Laboratory

Oak Ridge, Tennessee 37831-6073

Managed by UT-BATTELLE, LLC

for the

U.S. DEPARTMENT OF ENERGY

under Contract No. DE-AC05-00OR22725 


\section{DOCUMENT AVAILABILITY}

Reports produced after January 1, 1996, are generally available free via the U.S. Department of Energy (DOE) Information Bridge:

Web site: http://www.osti.gov/bridge

Reports produced before January 1, 1996, may be purchased by members of the public from the following source:

National Technical Information Service

5285 Port Royal Road

Springfield, VA 22161

Telephone: 703-605-6000 (1-800-553-6847)

TDD: 703-487-4639

Fax: 703-605-6900

E-mail: info@ntis.fedworld.gov

Web site: http://www.ntis.gov/support/ordernowabout.htm

Reports are available to DOE employees, DOE contractors, Energy Technology Data Exchange (ETDE) representatives, and International Nuclear Information System (INIS) representatives from the following source:

Office of Scientific and Technical Information

P.O. Box 62

Oak Ridge, TN 37831

Telephone: $865-576-8401$

Fax: 865-576-5728

E-mail: reports@adonis.osti.gov

Web site: http://www.osti.gov/contact.html

This report was prepared as an account of work sponsored by an agency of the United States Government. Neither the United States government nor any agency thereof, nor any of their employees, makes any warranty, express or implied, or assumes any legal liability or responsibility for the accuracy, completeness, or usefulness of any information, apparatus, product, or process disclosed, or represents that its use would not infringe privately owned rights. Reference herein to any specific commercial product, process, or service by trade name, trademark, manufacturer, or otherwise, does not necessarily constitute or imply its endorsement, recommendation, or favoring by the United States Government or any agency thereof. The views and opinions of authors expressed herein do not necessarily state or reflect those of the United States Government or any agency thereof. 
Users of the Transportation Energy Data Book are encouraged to comment on errors, omissions, emphases, and organization of this report to one of the persons listed below. Requests for additional complementary copies of this report, additional data, or information on an existing table should be referred to Ms. Stacy Davis, Oak Ridge National Laboratory.

\author{
Stacy C. Davis \\ Oak Ridge National Laboratory \\ National Transportation Research Center \\ 2360 Cherahala Boulevard \\ Knoxville, Tennessee 37932 \\ Telephone: (865) 946-1256 \\ FAX: (865) 946-1314 \\ E-mail: DAVISSC@ornl.gov \\ Web Site Location: cta.ornl.gov/data \\ Philip D. Patterson \\ Vehicle Technologies Program \\ Energy Efficiency and Renewable Energy \\ Department of Energy, EE-2G \\ Forrestal Building, Room \\ 1000 Independence Avenue, S.W. \\ Washington, D.C. 20585 \\ Telephone: (202) 586-9121 \\ FAX: (202) 586-1637 \\ E-mail: PHILIP.PATTERSON@ee.doe.gov \\ Web Site Location: www.eere.energy.gov \\ Jacob W. Ward \\ Vehicle Technologies Program \\ Energy Efficiency and Renewable Energy \\ Department of Energy, EE-2G \\ Forrestal Building \\ 1000 Independence Avenue, S.W. \\ Washington, D.C. 20585 \\ Telephone: (202) 586-7606 \\ FAX: (202) 586-1600 \\ E-mail: JACOB.WARD@ee.doe.gov \\ Web Site Location: www.eere.energy.gov
}

Spreadsheets of the tables in the

Transportation Energy Data Book can be found on the web at:

cta.ornl.gov/data 
Find useful data and information in other U.S.

Department of Energy, Office of Energy Efficiency and Renewable Energy Data Books.

Biomass Energy Data Book:

cta.ornl.gov/bedb

Buildings Energy Data Book:

www.btscoredatabook.net

Hydrogen Data Book:

hydrogen.pnl.gov/cocoon/morf/hydrogen/article/103

Power Technologies Energy Data Book:

www.nrel.gov/analysis/power_databook 


\section{TABLE OF CONTENTS}

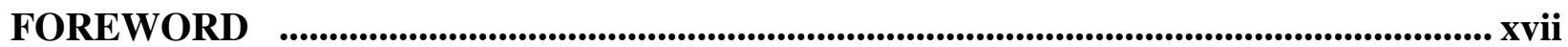

ACKNOWLEDGMENTS .......................................................................................................... xix

ABSTRACT

INTRODUCTION........................................................................................................................ xxiii

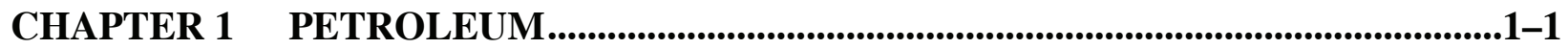

Table 1.1 World Fossil Fuel Potential ………............................................................

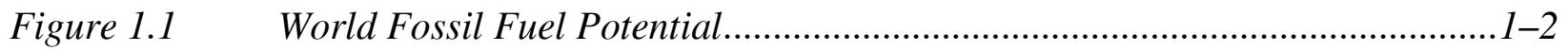

Table 1.2 World Crude Oil Production, 1960-2009 .........................................................1-3

Table 1.3 World Petroleum Production, 1973-2009 ………………………................1-4

Table 1.4 World Petroleum Consumption, 1960-2009 ……….....................................1-5

Figure 1.2 World Oil Reserves, Production and Consumption, 2008................................1-6

Table 1.5 World Oil Reserves, Production and Consumption, 2008 …….........................1-6

Figure 1.3 World Natural Gas Reserves, Production and Consumption, 2008..................1-7

Table 1.6 World Natural Gas Reserves, Production and Consumption, 2008...................1-7

Table 1.7 U.S. Petroleum Imports by World Region of Origin, 1960-2009 .....................1-8

Figure 1.4 Oil Price and Economic Growth, 1970-2009.............................................1-9

Figure 1.5 Costs of Oil Dependence to the U.S. Economy, 1970-2009..........................1-10

Table 1.8 Summary of Military Expenditures for Defending Oil Supplies from the Middle East ...............................................................................................1-11

Figure 1.6 Refinery Gross Output by World Region, 1999 and 2009.............................1-12

Table 1.9 U.S. Refinery Input of Crude Oil and Petroleum Products, 1987-2008.........1-13

Table 1.10 Refinery Yield of Petroleum Products from a Barrel of Crude Oil,

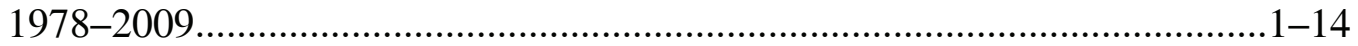

Table 1.11 United States Petroleum Production, Imports and Exports, 1950-2009 ........1-15 
Table 1.12 Petroleum Production and Consumption and Some Important Percent Shares, 1950-2009 $1-16$

Figure 1.7 United States Petroleum Production and Consumption - All Sectors, 1973-2035..... $1-17$

Figure $1.8 \quad$ United States Petroleum Production, Transportation and Consumption, 1970-2035...... $1-18$

Table 1.13 Consumption of Petroleum by End-Use Sector, 1973-2009 $1-19$

Table 1.14 Highway Transportation Petroleum Consumption by Mode, 1970-2008 ......1-20

Table 1.15 Nonhighway Transportation Petroleum Consumption by Mode, 1970-2008 1-21

Table 1.16 Transportation Petroleum Use by Mode, 2007-2008 $1-22$

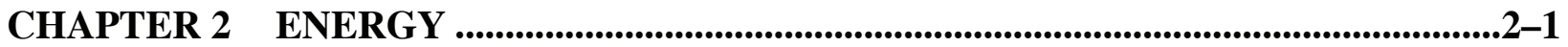

Figure 2.1 World Consumption of Primary Energy, 2007 ...........................................2-2

Table 2.1 U. S. Consumption of Total Energy by End-Use Sector, 1973-2009 .............2-3

Table 2.2 Distribution of Energy Consumption by Source, 1973 and 2009..................2-4

Table 2.3 Alternative Fuel and Oxygenate Consumption, 2003-2008 _........................2-5

Table $2.4 \quad$ Ethanol Consumption, 1995-2008.........................................................

Table 2.5 Domestic Consumption of Transportation Energy by Mode and

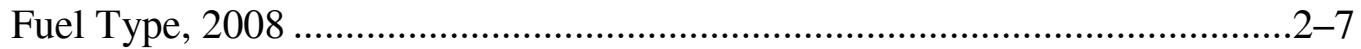

Table 2.6 Transportation Energy Use by Mode, 2007-2008 _.....................................2-8

Table 2.7 Highway Transportation Energy Consumption by Mode, 1970-2008 ............2-9

Table 2.8 Nonhighway Transportation Energy Consumption by Mode, 1970-2008 .....2-10

Table 2.9 Off-highway Transportation-related Fuel Consumption from the NonRoad

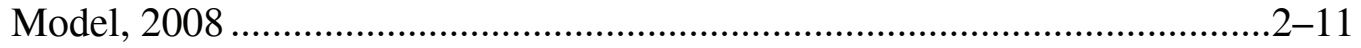

Table 2.10 Fuel Consumption from Lawn and Garden Equipment, 2008 ....................2-12

Table 2.11 Highway Usage of Gasoline and Special Fuels, 1973-2008 ......................2-13

Table 2.12 Passenger Travel and Energy Use, 2008.................................................2-14

Table 2.13 Energy Intensities of Highway Passenger Modes, 1970-2008....................2-15 
Table 2.14 Energy Intensities of Nonhighway Passenger Modes, 1970-2008. $.2-16$

Figure 2.2 Energy Intensity of Light Rail Transit Systems, 2008................................2-17

Figure 2.3 Energy Intensity of Heavy Rail Systems, 2008.......................................2-18

Figure 2.4 Energy Intensity of Commuter Rail Systems, 2008....................................2-18

Table 2.15 Intercity Freight Movement and Energy Use in the United States, 2007 and 2008 $.2-19$

Table 2.16 Energy Intensities of Freight Modes, 1970-2008 .....................................2-20

\section{CHAPTER 3 ALL HIGHWAY VEHICLES AND CHARACTERISTICS ....................3-1}

Table 3.1 Car Registrations for Selected Countries, 1960-2008 ..................................3-2

Table 3.2 Truck and Bus Registrations for Selected Countries, 1960-2008 ..................3-3

Table $3.3 \quad$ U.S. Cars and Trucks in Use, 1970-2008 ..................................................

Figure $3.1 \quad$ Vehicles per Thousand People: U.S. (Over Time) Compared to Other Countries (in 1998 and 2008) ........................................................................6

Table 3.4 Vehicles per Thousand People in Other Countries, 1998 and 2008 ................3-8

Table 3.5 Vehicles per Thousand People in the United States, 1990-2008 ...................3-9

Table 3.6 Shares of Highway Vehicle-Miles Traveled by Vehicle Type,

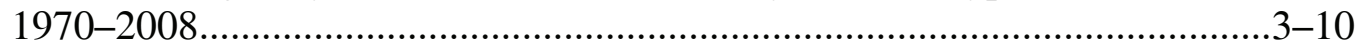

Table 3.7 Cars in Operation and Vehicle Travel by Age, 1970 and 2001 _...................3-11

Table $3.8 \quad$ Trucks in Operation and Vehicle Travel by Age, 1970 and 2001 .................3-12

Table $3.9 \quad$ U.S. Average Vehicle Age, 1995-2009....................................................13

Table 3.10 New Retail Vehicle Sales, 1970-2009.........................................................14

Table 3.11 Car Scrappage and Survival Rates, 1970, 1980 and 1990 Model Years ........3-15

Table 3.12 Light Truck Scrappage and Survival Rates ............................................

Table 3.13 Car and Light Truck Survivability Rates and Lifetime Miles ........................

CHAPTER 4 LIGHT VEHICLES AND CHARACTERISTICS ..................................4-1

Table 4.1 Summary Statistics for Cars, 1970-2008..................................................2 
Table 4.2 Summary Statistics for Two-Axle, Four-Tire Trucks, 1970-2008.................4-3

Table 4.3 Summary Statistics on Class 1, Class 2a, and Class 2b Light Trucks .............4-4

Table 4.4 Sales Estimates of Class 1, Class 2a, and Class 2b Light Trucks, 1989-1999.

Table 4.5 New Retail Car Sales in the United States, 1970-2009 $4-5$

Table 4.6 New Retail Sales of Trucks 10,000 Pounds GVW and Less in the United States, 1970-2009.

Table 4.7 Period Sales, Market Shares, and Sales-Weighted Fuel Economies of New Domestic and Import Cars, Selected Model Years 1975-2009.

Table 4.8 Definition of Wagons in Model Year 2009 $4-8$

Table 4.9 Period Sales, Market Shares, and Sales-Weighted Fuel Economies of New Domestic and Import Light Trucks, Model Years 1975-2009....

Table 4.10 Light Vehicle Market Shares by Size Class, Model Years 1975-2009 $.4-10$

Figure 4.1 Light Vehicle Market Shares, Model Years 1975-2009. $4-11$

Table 4.11 Sales-Weighted Engine Size of New Domestic and Import Cars by Size Class, Model Years 1975-2009 $4-12$

Table 4.12 Sales-Weighted Engine Size of New Domestic and Import Light Trucks by Size Class, Model Years 1975-2009 $.4-13$

Table 4.13 Sales-Weighted Curb Weight of New Domestic and Import Cars by Size Class, Model Years 1975-2009 $.4-14$

Table 4.14 Sales-Weighted Interior Space of New Domestic and Import Cars by Size Class, Model Years 1977-2009 $.4-15$

Table 4.15 Average Material Consumption for a Light Vehicle, Model Years 1995, 2000, and 2008 .

Table 4.16 New Light Vehicle Dealerships and Sales, 1970-2008. .4-17

Table 4.17 Conventional Refueling Stations, 1993-2008 $.4-18$

Table 4.18 Reformed and Unreformed Light Truck Corporate Average Fuel Economy Standards, MY 2008-2011 ..... .4-19

Table 4.19 Fuel Economy and Carbon Dioxide Emissions Standards, MY 2012-2016. 
Table 4.20 Fuel Economy and Carbon Dioxide Targets for Model Year 2016

Table 4.21 Car Corporate Average Fuel Economy (CAFE) Standards versus Sales-Weighted Fuel Economy Estimates, 1978-2010....

Table 4.22 Light Truck Corporate Average Fuel Economy (CAFE) Standards versus Sales-Weighted Fuel Economy Estimates, 1978-2010 . .4-23

Table 4.23 Corporate Average Fuel Economy (CAFE) Fines Collected, 1983-2008.....4-24

Table 4.24 The Gas Guzzler Tax on New Cars .........................................................4-25

Table 4.25 Tax Receipts from the Sale of Gas Guzzlers, 1980-2008 ...........................4-26

Table 4.26 Fuel Economy by Speed, PSAT Model Results ........................................4-27

Table 4.27 Fuel Economy by Speed, 1973, 1984, and 1997 Studies............................4-28

Figure 4.2 Fuel Economy by Speed, 1973, 1984, and 1997 Studies ............................4-29

Table 4.28 Vehicle Specifications for Vehicles Tested in the 1997 Study.....................4-30

Table 4.29 Steady Speed Fuel Economy for Vehicles Tested in the 1997 Study............4-31

Table 4.30 Driving Cycle Attributes...................................................................4-32

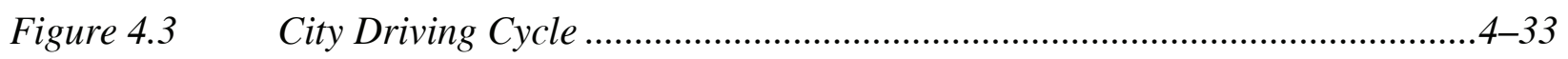

Figure $4.4 \quad$ Highway Driving Cycle .........................................................................3

Figure 4.5 Air Conditioning (SC03) Driving Cycle ...................................................4-34

Figure 4.6 Cold Temperature (Cold FTP) Driving Cycle ............................................4-34

Figure 4.7 High Speed (USO6) Driving Cycle ...........................................................35

Figure $4.8 \quad$ New York City Driving Cycle .............................................................4-36

Figure 4.9 Representative Number Five Driving Cycle ............................................4-36

Table 4.31 Projected Fuel Economies from U.S., European, and Japanese Driving Cycles4-37

Table 4.32 Comparison of U.S., European, and Japanese Driving Cycles ......................4-38

Table 4.33 Summary Statistics on Demand Response Vehicles, 1994-2008.................4-39 
CHAPTER 5 HEAVY VEHICLES AND CHARACTERISTICS........................................5-1

Table 5.1 Summary Statistics for Heavy Single-Unit Trucks, 1970-2008.....................5-2

Table 5.2 Summary Statistics for Combination Trucks, 1970-2008 ….........................5-3

Table 5.3 New Retail Truck Sales by Gross Vehicle Weight, 1970-2009.....................5-4

Table 5.4 Truck Statistics by Gross Vehicle Weight Class, 2002 ...............................5-6

Table 5.5 Truck Harmonic Mean Fuel Economy by Size Class, 1992, 1997,

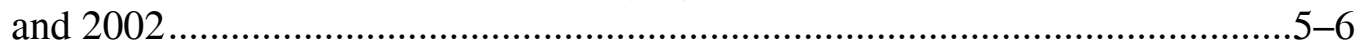

Table $5.6 \quad$ Truck Statistics by Size, 2002 ..............................................................

Table 5.7 Percentage of Trucks by Size Ranked by Major Use, 2002 .........................5-8

Table 5.8 Percentage of Trucks by Fleet Size and Primary Fueling Facility, 2002.........5-9

Table 5.9 Share of Trucks by Major Use and Primary Fueling Facility, 2002 ..............5-10

Figure 5.1 Distribution of Trucks over 26,000 lbs. Less than Two Years Old by Vehicle-Miles Traveled ..........................................................................5-11

Figure 5.2 Share of Heavy Trucks with Selected Electronic Features, 2002 .................5-12

Table 5.10 Effect of Terrain on Class 8 Truck Fuel Economy ......................................5-14

Table 5.11 Fuel Economy for Class 8 Trucks as Function of Speed and Tractor-Trailer

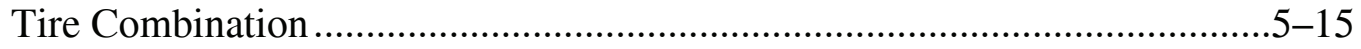

Figure 5.3 Class 8 Truck Fuel Economy as a Function of Speed and Tractor-Trailer Tire Combination and Percentage of Total Distance Traveled as a Function of Speed .....................................................................................5-16

Figure 5.4 Class 8 Truck Percent of Total Fuel Consumed as a Function of Speed and Tractor-Trailer Tire Combination ........................................................5-17

Table 5.12 Class 8 Truck Weight by Component........................................................5-18

Table 5.13 Gross Vehicle Weight vs. Empty Vehicle Weight......................................5-19

Figure 5.5 Distribution of Five-Axle Tractor-Trailers by On-Road Vehicle

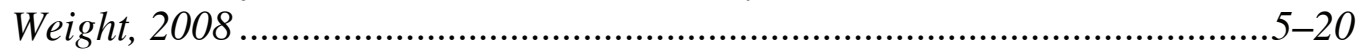

Table 5.14 Growth of Freight in the United States: Comparison of the 2007, 2002 and 1997 Commodity Flow Surveys........................................................5-22 
Table 5.15 Growth of Freight Miles in the United States: Comparison of the 2007, 2002 and 1997 Commodity Flow Surveys

Table 5.16 Summary Statistics on Transit Buses and Trolleybuses, 1994-2008

\section{CHAPTER 6 ALTERNATIVE FUEL AND ADVANCED TECHNOLOGY} VEHICLES AND CHARACTERISTICS 6-1

Table 6.1 Estimates of Alternative Fuel Vehicles in Use, 1995-2008 $.6-3$

Table 6.2 Alternative Fuel Vehicles Available by Manufacturer, Model Year 2009 _......6-4

Table 6.3 Hybrid Electric Vehicles Available by Manufacturer, Model Year 2009 ........6-5

Table 6.4 Number of Alternative Refuel Sites by State and Fuel Type, 2010...............6-6

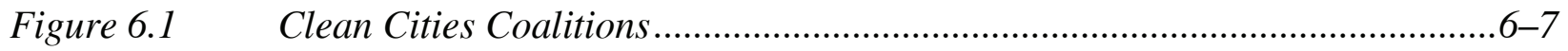

Table 6.5 Properties of Conventional and Alternative Fuels ......................................6-9

CHAPTER 7 FLEET VEHICLES AND CHARACTERISTICS.......................................7-1

Figure 7.1 Fleet Vehicles in Service as of January 1, 2009 ..........................................7-2

Table 7.1 New Light Fleet Vehicle Registrations by Vehicle Type, Model Year 2008 ..................................................................................................

Table 7.2 Average Length of Time Business Fleet Vehicles are in Service, 2008..........7-3

Table 7.3 Average Annual Vehicle-Miles of Travel for Business Fleet Vehicles, 2008

Figure 7.2 Average Miles per Domestic Federal Vehicle by Vehicle Type, 2009.............7-4

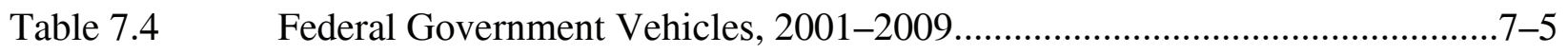

Table 7.5 Federal Fleet Vehicle Acquisitions by Fuel Type, FY 2002- 2009 _...............7-6

Table 7.6 Fuel Consumed by Federal Government Fleets, FY 2000-2009...................7-6

Table $7.7 \quad$ Federal Government Vehicles by Agency, FY 2009 ..................................7-7

CHAPTER 8 HOUSEHOLD VEHICLES AND CHARACTERISTICS..........................8-1

Table 8.1 Population and Vehicle Profile, 1950-2008 ............................................. 8 -2

Table 8.2 Vehicles and Vehicle-Miles per Capita, 1950-2008 ..................................8-3 
Table 8.3 Average Annual Expenditures of Households by Income, 2008 $.8-4$

Table 8.4 Household Vehicle Ownership, 1960-2000 Census $.8-5$

Table 8.5 Demographic Statistics from the 1969, 1977, 1983, 1990, 1995 NPTS and 2001, 2009 NHTS .8-6

Table 8.6 Average Annual Vehicle-Miles, Vehicle Trips and Trip Length per Household 1969, 1977, 1983, 1990, 1995 NPTS and 2001, 2009 NHTS $8-7$

Table 8.7 Average Number of Vehicles and Vehicle Travel per Household, 1990 NPTS and 2001 and 2009 NHTS. $.8-8$

Table 8.8 Trip Statistics by Trip Purpose, 2001 and 2009 NHTS 8-9

Figure 8.1 Average Vehicle Occupancy by Vehicle Type, 1995 NPTS and 2009 NHTS. $8-10$

Figure 8.2 Average Vehicle Occupancy by Trip Purpose, 1977 NPTS and 2009 NHTS... $8-11$

Table 8.9 Average Annual Miles per Household Vehicle by Vehicle Age $.8-12$

Table 8.10 Self-Reported vs. Odometer Average Annual Miles, 1995 NPTS and 2001 NHTS $.8-13$

Figure 8.3 Share of Vehicle Trips by Trip Distance, 2009 NHTS................................8-14

Figure 8.4 Share of Vehicle Trips to Work by Trip Distance, 2009 NHTS ....................8-14

Table 8.11 Share of Vehicles by Annual Miles of Travel and Vehicle Age, 2009 NHTS $.8-15$

Table 8.12 Household Vehicle Trips, 2009 NHTS ..................................................8-16

Figure 8.5 Average Daily Miles Driven (per Driver), 2009 NHTS..............................8-16

Table 8.13 Daily Vehicle Miles of Travel (per Vehicle) by Number of Vehicles in the Household, 2009 NHTS. $.8-17$

Table 8.14 Daily and Annual Vehicle Miles of Travel and Average Age for Each Vehicle in a Household, 2009 NHTS . $.8-17$

Figure 8.6 Daily Vehicle Miles of Travel for Each Vehicle in a Household, 2009 NHTS. $.8-18$

Figure 8.7 Annual Vehicle Miles of Travel for Each Vehicle in a Household, 2009 NHTS. $8-18$ 
Table 8.15 Means of Transportation to Work, 1980, 1990 and 2000 Census

Table 8.16 Characteristics of U.S. Daily per Vehicle Driving vs. Dwelling Unit Type and Density $.8-20$

Table 8.17 Housing Unit Characteristics, 2007. $.8-20$

Table 8.18 Workers by Commute Time, 1990 and 2000 Census $.8-21$

Table 8.19 Bicycle Sales, 1981-2008. $.8-22$

Figure $8.8 \quad$ Walk and Bike Trips by Trip Purpose, 2009 NHTS. $.8-23$

Table 8.20 Long-Distance Trip Characteristics, 2001 NHTS $.8-25$

CHAPTER 9 NONHIGHWAY MODES 9-1

Table 9.1 Nonhighway Energy Use Shares, 1970-2008 $9-2$

Table 9.2 Summary Statistics for U.S. Domestic and International Certificated Route Air Carriers (Combined Totals), 1970-2008 .....................................9-3

Table 9.3 Summary Statistics for General Aviation, 1970-2008 $.9-4$

Table 9.4 Tonnage Statistics for Domestic and International Waterborne Commerce, 1970-2008 $.9-5$

Table 9.5 Summary Statistics for Domestic Waterborne Commerce, 1970-2008 ..........9-6

Table 9.6 Recreational Boat Energy Use, 1970-2008 …...........................................9-7

Table 9.7 Class I Railroad Freight Systems in the United States Ranked by Revenue Ton-Miles, 2008 ....................................................................9-8

Table 9.8 Summary Statistics for Class I Freight Railroads, 1970-2008 .....................9-9

Table 9.9 Intermodal Rail Traffic, 1965-2008 .........................................................10

Table 9.10 Summary Statistics for the National Railroad Passenger Corporation (Amtrak), 1971-2008 ..........................................................................

Table 9.11 Summary Statistics for Commuter Rail Operations, 1984-2008.................9-12

Table 9.12 Summary Statistics for Rail Transit Operations, 1970-2008 _.....................9-13

CHAPTER 10 TRANSPORTATION AND THE ECONOMY ........................................10-1

Figure 10.1 Transportation Services Index, January 1990-January 2010......................10-2 
Table 10.1 Gasoline Prices for Selected Countries, 1990-2008....................................10-3

Table 10.2 Diesel Fuel Prices for Selected Countries, 1998-2008................................10-4

Figure 10.2 Gasoline Prices for Selected Countries, 1990 and 2008............................10-5

Figure 10.3 Diesel Prices for Selected Countries, 1990 and 2008 ................................10-6

Table 10.3 Prices for a Barrel of Crude Oil and a Gallon of Gasoline, 1978-2009 .........10-7

Table 10.4 Retail Prices for Motor Fuel, 1978-2009 …...........................................10-8

Table 10.5 Refiner Sales Prices for Propane and No. 2 Diesel, 1978-2009...................10-9

Table 10.6 Refiner Sales Prices for Aviation Gasoline and Jet Fuel, 1978-2009 ..........10-10

Table $10.7 \quad$ State Tax Exemptions for Gasohol, 2008 ............................................10-11

Table $10.8 \quad$ Federal Excise Taxes on Motor Fuels, 2008 ............................................10-11

Table $10.9 \quad$ Federal and State Alternative Fuel Incentives, 2010 ................................10-12

Table 10.10 Federal and State Advanced Technology Incentives, 2010 ......................10-13

Table 10.11 Average Price of a New Car, 1906-2008 …...........................................10-14

Table 10.12 Average Price of a New Car (Domestic and Import), 1970-2008...............10-15

Table 10.13 Car Operating Cost per Mile, 1985-2009..............................................10-16

Table 10.14 Fixed Car Operating Costs per Year, 1975-2009....................................10-17

Table 10.15 Personal Consumption Expenditures, 1970-2009 ....................................10-18

Table 10.16 Consumer Price Indices, 1970-2009 …..............................................10-18

Table 10.17 Transportation-related Employment, 1999 and 2009 ..............................10-19

Table $10.18 \quad$ U.S. Employment for Motor Vehicles and Motor Vehicle Parts Manufacturing, 1990-2009 ..................................................................10-20

\section{CHAPTER 11 GREENHOUSE GAS EMISSIONS ......................................................11-1}

Table 11.1 World Carbon Dioxide Emissions, 1990 and 2006 ...................................11-2

Table 11.2 Numerical Estimates of Global Warming Potentials Compared with Carbon Dioxide. 
Table $11.3 \quad$ U.S. Emissions of Greenhouse Gases, based on Global Warming Potential, 1990-2008 $11-4$

Table 11.4 Total U.S. Greenhouse Emissions by End-Use Sector, 2008 $11-5$

Table 11.5 U.S. Carbon Emissions from Fossil Energy Consumption by End-Use Sector, 1990-2008 $11-6$

Table 11.6 U.S. Carbon Emissions from Energy Use in the Transportation Sector, 1990-2008 $11-7$

Table 11.7 Transportation Greenhouse Gas Emissions by Mode, 1990 and 2008 $11-8$

Figure 11.1 GREET Model $11-9$

Figure 11.2 GREET Model Feedstocks and Fuels $11-10$

Table 11.8 Sales-Weighted Annual Carbon Footprint of New Domestic and Import Cars by Size Class, Model Years 1975-2009. $11-12$

Table 11.9 Sales-Weighted Annual Carbon Footprint of New Domestic and Import Light Trucks by Size Class, Model Years 1975-2009 $.11-13$

Table 11.10 Average Annual Carbon Footprint by Vehicle Classification, 1975 and 2009 $11-14$

Table 11.11 Carbon Dioxide Emissions from a Gallon of Fuel.....................................11-15

CHAPTER 12 CRITERIA AIR POLLUTANTS $.12-1$

Table 12.1 Total National Emissions of the Criteria Air Pollutants by Sector, 2008 ....... $12-2$

Table 12.2 Total National Emissions of Carbon Monoxide, 1970-2008 $12-3$

Table 12.3 Emissions of Carbon Monoxide from Highway Vehicles, 1970-2005 ..... $12-4$

Table 12.4 Total National Emissions of Nitrogen Oxides, 1970-2008 $12-5$

Table 12.5 Emissions of Nitrogen Oxides from Highway Vehicles, 1970-2005............12-6

Table 12.6 Total National Emissions of Volatile Organic Compounds, 1970-2008 .......12-7

Table 12.7 Emissions of Volatile Organic Compounds from Highway Vehicles, 1970-200512-8

Table 12.8 Total National Emissions of Particulate Matter (PM-10), 1970-2008 $.12-9$

Table 12.9 Emissions of Particulate Matter (PM-10) from Highway Vehicles, 1970-200512-10 
Table 12.10 Total National Emissions of Particulate Matter (PM-2.5), 1990-2008 $.12-11$

Table 12.11 Emissions of Particulate Matter (PM-2.5) from Highway Vehicles, 1990-2005

Table 12.12 U.S. Tier 2 Emission Standards for Cars and Light Trucks..........

Table 12.13 Light Vehicle Exhaust Emission Standards in Effect in 2009 when U.S. Tier 2 Standards are Final.

Table 12.14 California Cars and Light Trucks Emission Certification Standards. $12-15$

APPENDIX A. SOURCES \& METHODOLOGIES . A-1

APPENDIX B. CONVERSIONS.. B-1

APPENDIX C. MAPS. C-1

GLOSSARY G-1

INDEX. I-1 


\section{FOREWORD}

Welcome to this 29th edition of the Transportation Energy Data Book. Twenty-one of these editions have been produced by Stacy Davis. DOE is grateful for the dedication and the skill she has brought to this effort.

We would like to bring to your attention some of the data that are new in this edition:

\section{Figure 2.4: Commuter Rail Transit Energy Intensity}

Data for an additional 10 systems has been added. These data show the variation in commuter rail energy intensity across 17 metropolitan areas in the U.S.

Table 2.9: Off-highway Transportation-related Fuel Consumption from the NonRoad Model, 2008

These data for off-highway vehicles all come from a single EPA source now.

Table 3.9: U.S. Average Vehicle Age, 1995-2009

This replaces a table that had median vehicle ages which Polk no longer makes available.

Table 3.10: New Retail Vehicle Sales, 1970-2008

These summary data were never shown before.

Tables 3.11 and 3.12: Car Scrappage and Survival Rates and Light Truck Scrappage and Survival Rates

These data have been added to this edition.

Table 4.19: Fuel Economy and Carbon Dioxide Emissions Standards, MY 2012-2016

This table shows that the combined 2016 standard is $34.1 \mathrm{MPG}$, not the $35.5 \mathrm{MPG}$ often quoted in the press.

Table 4.20: Fuel Economy and Carbon Dioxide Targets for Model Year 2016 New CAFE standards

Table 7.7: Federal Government Vehicles by Agency, FY 2009

GSA owned over 150,000 vehicles in 1994, but owned only 1,221 vehicles in 2009.

We hope you find value in this data book. We welcome suggestions on how to improve it.

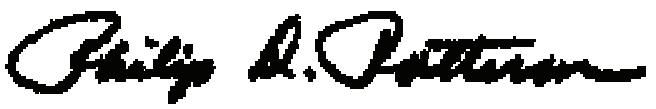


xviii

TRANSPORTATION ENERGY DATA BOOK: EDITION 29—2010 


\section{ACKNOWLEDGMENTS}

The authors would like to express their gratitude to the many individuals who assisted in the preparation of this document. First, we would like to thank Phil Patterson, Jacob Ward, and the Vehicle Technologies Program staff for their continued support of the Transportation Energy Data Book project. We would also like to thank Jamie Payne for the cover design and Patricia $\mathrm{Hu}$ for her guidance and mentoring. Finally, this book would not have been possible without the dedication of Debbie Bain, who masterfully prepared the manuscript. 


\begin{abstract}
The Transportation Energy Data Book: Edition 29 is a statistical compendium prepared and published by Oak Ridge National Laboratory (ORNL) under contract with the U.S. Department of Energy, Office of Energy Efficiency and Renewable Energy, Vehicle Technologies Program. Designed for use as a desk-top reference, the Data Book represents an assembly and display of statistics and information that characterize transportation activity, and presents data on other factors that influence transportation energy use. The purpose of this document is to present relevant statistical data in the form of tables and graphs. The latest edition of the Data Book is available to a larger audience via the Internet (cta.ornl.gov/data).

This edition of the Data Book has 12 chapters which focus on various aspects of the transportation industry. Chapter 1 focuses on petroleum; Chapter 2 - energy; Chapter 3 highway vehicles; Chapter 4 - light vehicles; Chapter 5 - heavy vehicles; Chapter 6 - alternative fuel vehicles; Chapter 7 - fleet vehicles; Chapter 8 - household vehicles; Chapter 9 nonhighway modes; Chapter 10 - transportation and the economy; Chapter 11 - greenhouse gas emissions; and Chapter 12 - criteria pollutant emissions. The sources used represent the latest available data. There are also three appendices which include detailed source information for some tables, measures of conversion, and the definition of Census divisions and regions. A glossary of terms and a title index are also included for the reader's convenience.
\end{abstract}




\section{INTRODUCTION}

In January 1976, the Transportation Energy Conservation (TEC) Division of the Energy Research and Development Administration contracted with Oak Ridge National Laboratory (ORNL) to prepare a Transportation Energy Conservation Data Book to be used by TEC staff in their evaluation of current and proposed conservation strategies. The major purposes of the Data Book were to draw together, under one cover, transportation data from diverse sources, to resolve data conflicts and inconsistencies, and to produce a comprehensive document. The first edition of the TEC Data Book was published in October 1976. With the passage of the Department of Energy (DOE) Organization Act, the work being conducted by the former Transportation Energy Conservation Division fell under the purview of the DOE's Office of Transportation Programs.

Policymakers and analysts need to be well-informed about activity in the transportation sector. The organization and scope of the data book reflect the need for different kinds of information. For this reason, Edition 29 updates much of the same type of data that is found in previous editions.

In any attempt to compile a comprehensive set of statistics on transportation activity, numerous instances of inadequacies and inaccuracies in the basic data are encountered. Where such problems occur, estimates are developed by ORNL. To minimize the misuse of these statistics, an appendix (Appendix A) is included to document the estimation procedures. The attempt is to provide sufficient information for the conscientious user to evaluate the estimates and to form their own opinions as to their utility. Clearly, the accuracy of the estimates cannot exceed the accuracy of the primary data, an accuracy which in most instances is unknown. In cases where data accuracy is known or substantial errors are strongly suspected in the data, the reader is alerted. In all cases it should be recognized that the estimates are not precise.

The majority of the statistics contained in the data book are taken directly from published sources, although these data may be reformatted for presentation by ORNL. Consequently, neither ORNL nor DOE endorses the validity of these data. 
xxiv

TRANSPORTATION ENERGY DATA BOOK: EDITION 29—2010 


\section{Chapter 1 \\ Petroleum}

Summary Statistics from Tables/Figures in this Chapter

\begin{tabular}{|c|c|c|c|}
\hline \multicolumn{4}{|l|}{ Source } \\
\hline Table 1.3 & World Petroleum Production, 2009 (million barrels per day) ${ }^{\mathrm{a}}$ & & 80.25 \\
\hline \multirow{5}{*}{ Table 1.4} & U.S. Production (million barrels per day) & & 7.20 \\
\hline & U.S. Share & & $9.0 \%$ \\
\hline & World Petroleum Consumption, 2009 (million barrels per day) & & 84.04 \\
\hline & U.S. Consumption (million barrels per day) & & 18.69 \\
\hline & U.S. Share & & $22.2 \%$ \\
\hline \multirow[t]{6}{*}{ Figure 1.6} & Average Refinery Yield, 2009 & $\begin{array}{l}\text { OECD } \\
\text { Europe }\end{array}$ & $\begin{array}{l}\text { North } \\
\text { America }\end{array}$ \\
\hline & Gasoline & $20.0 \%$ & $43.0 \%$ \\
\hline & Diesel oil & $39.0 \%$ & $25.0 \%$ \\
\hline & Residual fuel & $13.0 \%$ & $6.0 \%$ \\
\hline & Kerosene & $7.0 \%$ & $8.0 \%$ \\
\hline & Other & $21.0 \%$ & $18.0 \%$ \\
\hline Table 1.12 & $\begin{array}{l}\text { U.S. transportation petroleum use as a percent of U.S. petroleum } \\
\text { production, } 2009\end{array}$ & & $179.4 \%$ \\
\hline Table 1.12 & Net imports as a percentage of U.S. petroleum consumption, 2009 & & $51.9 \%$ \\
\hline Table 1.13 & Transportation share of U.S. petroleum consumption, 2009 & & $71.4 \%$ \\
\hline Table 1.16 & Highway share of transportation petroleum consumption, 2008 & & $84.9 \%$ \\
\hline Table 1.16 & Light vehicle share of transportation petroleum consumption, 2008 & & $66.0 \%$ \\
\hline
\end{tabular}

In this document, petroleum is defined as crude oil (including lease condensate) and natural gas plant liquids.

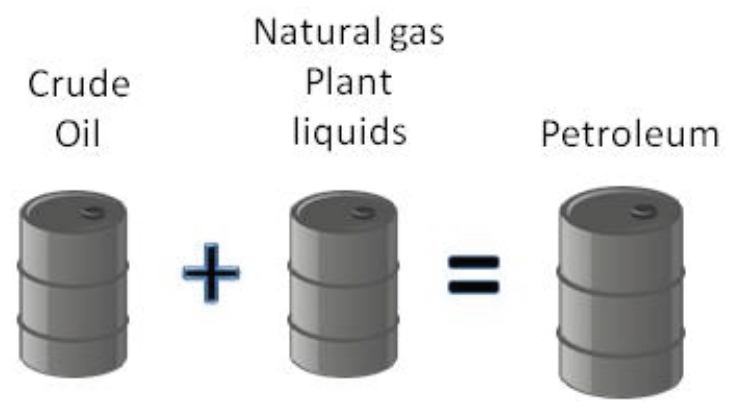

${ }^{\text {a }}$ Because other liquids and processing gain are not included, the world total is smaller than world petroleum consumption. 
Although the world has consumed about $40 \%$ of estimated conventional oil resources, the total fossil fuel potential is huge. Methane hydrates-a potential source of natural gas-are included in the "additional occurrences" of unconventional natural gas, and constitute the largest resource.

Table 1.1

World Fossil Fuel Potential

(gigatonnes of carbon)

\begin{tabular}{lccrr}
\hline & $\begin{array}{c}\text { Consumption } \\
(1860-1998)\end{array}$ & Reserves & Resources & $\begin{array}{c}\text { Additional } \\
\text { occurrences }\end{array}$ \\
\hline Oil $\quad r$ & 120 & 121 & 0 \\
$\quad$ Conventional & 97 & 102 & 305 & 914 \\
$\quad$ Unconventional & 6 & & & \\
Natural Gas & & 83 & 170 & 0 \\
$\quad$ Conventional & 36 & 144 & 364 & 14,176 \\
$\quad$ Unconventional & 1 & 533 & 4,618 & a \\
Coal & 155 & & & \\
\hline
\end{tabular}

\section{Source:}

Rogner, H.H., World Energy Assessment: Energy and the Challenge of Sustainability, Part II, Chapter 5, 2000, p. 149.

Figure 1.1 World Fossil Fuel Potential

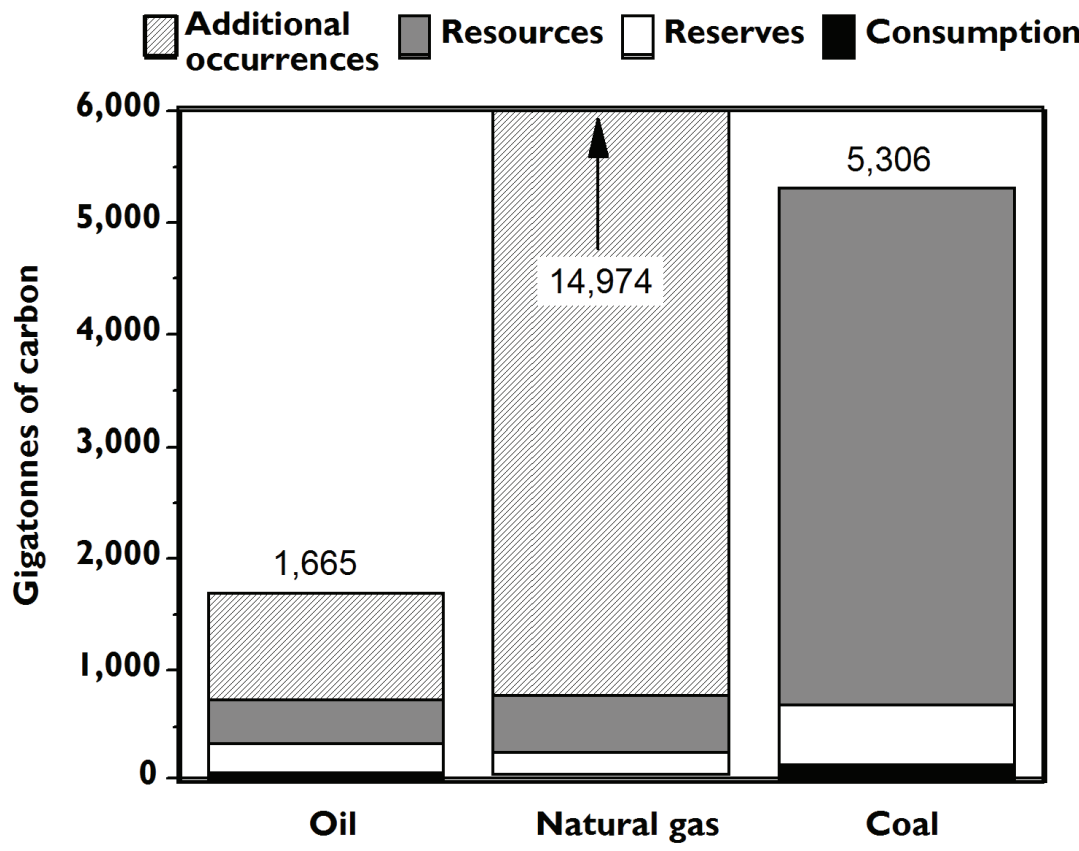

Source:

See Table 1.1

${ }^{a}$ Data are not available. 
In 2009, the Organization of Petroleum Exporting Countries (OPEC) accounted for more than $40 \%$ of world oil production. Responding to low oil prices in early 2000, Mexico, Norway, Russia, and Oman joined OPEC in cutting production. This group of oil countries, referred to here as OPEC+, account for almost $65 \%$ of world oil production.

Table 1.2

World Crude Oil Production, 1960-2009 (million barrels per day)

\begin{tabular}{|c|c|c|c|c|c|c|c|c|c|c|}
\hline Year & $\begin{array}{l}\text { United } \\
\text { States }\end{array}$ & $\begin{array}{l}\text { U.S. } \\
\text { share }\end{array}$ & $\begin{array}{c}\text { Total } \\
\text { OPEC }^{\mathrm{b}}\end{array}$ & $\begin{array}{c}\text { OPEC } \\
\text { share }\end{array}$ & $\begin{array}{c}\text { OPEC } \\
+^{c} \\
\end{array}$ & $\begin{array}{c}\text { OPEC } \\
+{ }^{\mathrm{c}} \text { share } \\
\end{array}$ & $\begin{array}{c}\text { Total } \\
\text { non- } \\
\text { OPEC } \\
\end{array}$ & $\begin{array}{l}\text { Persian } \\
\text { Gulf } \\
\text { nations }^{\mathrm{d}}\end{array}$ & $\begin{array}{l}\text { Persian } \\
\text { Gulf }^{\mathrm{d}} \\
\text { share }\end{array}$ & World \\
\hline 1960 & 7.04 & $33.5 \%$ & 8.70 & $41.4 \%$ & 12.25 & $58.3 \%$ & 12.29 & 5.27 & $25.1 \%$ & 20.99 \\
\hline 1965 & 7.80 & $25.7 \%$ & 14.35 & $47.3 \%$ & 19.83 & $65.4 \%$ & 15.98 & 8.37 & $27.6 \%$ & 30.33 \\
\hline 1970 & 9.64 & $21.0 \%$ & 23.30 & $50.8 \%$ & 31.12 & $67.8 \%$ & 22.59 & 13.39 & $29.2 \%$ & 45.89 \\
\hline 1975 & 8.38 & $15.9 \%$ & 26.79 & $50.3 \%$ & 37.55 & $71.1 \%$ & 27.04 & 18.93 & $35.8 \%$ & 52.83 \\
\hline 1980 & 8.60 & $14.4 \%$ & 26.38 & $44.3 \%$ & 40.80 & $68.5 \%$ & 34.18 & 17.96 & $30.2 \%$ & 59.56 \\
\hline 1985 & 8.97 & $16.6 \%$ & 15.37 & $28.5 \%$ & 30.98 & $57.4 \%$ & 38.60 & 9.63 & $17.8 \%$ & 53.97 \\
\hline 1986 & 8.68 & $15.4 \%$ & 18.28 & $32.5 \%$ & 34.05 & $60.6 \%$ & 37.95 & 11.70 & $20.8 \%$ & 56.23 \\
\hline 1987 & 8.35 & $14.7 \%$ & 18.52 & $32.7 \%$ & 34.72 & $61.3 \%$ & 38.15 & 12.10 & $21.4 \%$ & 56.67 \\
\hline 1988 & 8.14 & $13.9 \%$ & 20.32 & $34.6 \%$ & 36.66 & $62.4 \%$ & 38.42 & 13.46 & $22.9 \%$ & 58.74 \\
\hline 1989 & 7.61 & $12.7 \%$ & 22.07 & $36.9 \%$ & 38.50 & $64.3 \%$ & 37.79 & 14.84 & $24.8 \%$ & 59.86 \\
\hline 1990 & 7.36 & $12.2 \%$ & 22.49 & $37.2 \%$ & 38.34 & $63.4 \%$ & 38.00 & 15.28 & $25.3 \%$ & 60.49 \\
\hline 1991 & 7.42 & $12.3 \%$ & 23.27 & $38.6 \%$ & 38.53 & $64.0 \%$ & 36.94 & 14.74 & $24.5 \%$ & 60.21 \\
\hline 1992 & 7.17 & $11.9 \%$ & 24.40 & $40.5 \%$ & 37.67 & $62.6 \%$ & 35.81 & 15.97 & $26.5 \%$ & 60.21 \\
\hline 1993 & 6.85 & $11.4 \%$ & 25.12 & $41.7 \%$ & 37.65 & $62.5 \%$ & 35.12 & 16.71 & $27.7 \%$ & 60.24 \\
\hline 1994 & 6.66 & $10.9 \%$ & 25.51 & $41.8 \%$ & 37.67 & $61.8 \%$ & 35.48 & 16.96 & $27.8 \%$ & 60.99 \\
\hline 1995 & 6.56 & $10.5 \%$ & 25.54 & $40.9 \%$ & 37.77 & $60.5 \%$ & 36.85 & 17.21 & $27.6 \%$ & 62.39 \\
\hline 1996 & 6.47 & $10.1 \%$ & 26.02 & $40.8 \%$ & 38.70 & $60.7 \%$ & 37.73 & 17.37 & $27.2 \%$ & 63.75 \\
\hline 1997 & 6.45 & $9.8 \%$ & 27.29 & $41.5 \%$ & 40.28 & $61.3 \%$ & 38.45 & 18.10 & $27.5 \%$ & 65.74 \\
\hline 1998 & 6.25 & $9.3 \%$ & 28.37 & $42.4 \%$ & 41.21 & $61.5 \%$ & 38.70 & 19.34 & $28.9 \%$ & 66.97 \\
\hline 1999 & 5.88 & $8.9 \%$ & 27.22 & $41.3 \%$ & 40.14 & $60.9 \%$ & 38.70 & 18.67 & $28.3 \%$ & 65.92 \\
\hline 2000 & 5.82 & $8.5 \%$ & 28.98 & $42.3 \%$ & 42.66 & $62.3 \%$ & 39.52 & 19.89 & $29.0 \%$ & 68.50 \\
\hline 2001 & 5.80 & $8.5 \%$ & 28.16 & $41.4 \%$ & 42.35 & $62.2 \%$ & 39.94 & 19.10 & $28.0 \%$ & 68.10 \\
\hline 2002 & 5.75 & $8.6 \%$ & 26.39 & $39.3 \%$ & 41.01 & $61.1 \%$ & 40.77 & 17.79 & $26.5 \%$ & 67.17 \\
\hline 2003 & 5.68 & $8.2 \%$ & 27.98 & $42.3 \%$ & 43.34 & $62.4 \%$ & 41.45 & 19.06 & $27.5 \%$ & 69.43 \\
\hline 2004 & 5.42 & $7.5 \%$ & 30.41 & $42.0 \%$ & 46.30 & $63.9 \%$ & 42.07 & 20.79 & $28.7 \%$ & 72.48 \\
\hline 2005 & 5.18 & $7.0 \%$ & 31.87 & $43.2 \%$ & 47.72 & $64.7 \%$ & 41.85 & 21.60 & $29.3 \%$ & 73.72 \\
\hline 2006 & 5.10 & $6.9 \%$ & 31.69 & $43.2 \%$ & 47.42 & $64.6 \%$ & 41.84 & 21.23 & $28.9 \%$ & 73.44 \\
\hline 2007 & 5.06 & $6.9 \%$ & 31.21 & $42.8 \%$ & 46.70 & $64.0 \%$ & 41.77 & 20.67 & $28.3 \%$ & 72.98 \\
\hline 2008 & 4.96 & $6.7 \%$ & 32.48 & $44.1 \%$ & 47.58 & $64.6 \%$ & 41.21 & 21.91 & $29.7 \%$ & 73.69 \\
\hline 2009 & 5.31 & $7.3 \%$ & 30.65 & $42.4 \%$ & 45.62 & $63.1 \%$ & 41.61 & 20.40 & $28.2 \%$ & 72.26 \\
\hline \multicolumn{11}{|c|}{ Average annual percentage change } \\
\hline 1960-2009 & $-0.6 \%$ & & $2.6 \%$ & & $2.7 \%$ & & $2.5 \%$ & $2.8 \%$ & & $2.6 \%$ \\
\hline 1970-2009 & $-1.6 \%$ & & $0.5 \%$ & & $0.4 \%$ & & $0.7 \%$ & $0.4 \%$ & & $0.7 \%$ \\
\hline 1999-2009 & $-1.0 \%$ & & $1.2 \%$ & & $1.5 \%$ & & $0.7 \%$ & $0.9 \%$ & & $0.9 \%$ \\
\hline
\end{tabular}

\section{Source:}

U.S. Department of Energy, Energy Information Administration, Monthly Energy Review, March 2010, Washington, DC, 2010, Table 11.1a and 11.1b. (Additional resources: www.eia.doe.gov)

\footnotetext{
${ }^{\text {a }}$ Includes lease condensate. Excludes natural gas plant liquids.

${ }^{\mathrm{b}}$ See Glossary for membership.

${ }^{c}$ OPEC+ includes all OPEC nations plus Russia, Mexico, Norway and Oman.

${ }^{\mathrm{d}}$ See Glossary for Persian Gulf Nations.
} 
This table shows petroleum production, which includes both crude oil and natural gas plant liquids. Because other liquids and processing gain are not included, the world total is smaller than world petroleum consumption (Table 1.4). The United States was responsible for $9.0 \%$ of the world's petroleum production in 2009 and $7.3 \%$ of the world's crude oil production (Table 1.2).

Table 1.3

World Petroleum Production, 1973-2009a

(million barrels per day)

\begin{tabular}{|c|c|c|c|c|c|c|c|c|c|}
\hline Year & $\begin{array}{l}\text { United } \\
\text { States }\end{array}$ & $\begin{array}{l}\text { U.S. } \\
\text { share }\end{array}$ & $\begin{array}{c}\text { Total } \\
\text { OPEC }^{b}\end{array}$ & $\begin{array}{c}\text { OPEC } \\
\text { share }\end{array}$ & $\begin{array}{c}\text { Total } \\
\text { non- } \\
\text { OPEC }\end{array}$ & $\begin{array}{c}\text { Non- } \\
\text { OPEC } \\
\text { share }\end{array}$ & $\begin{array}{c}\text { Persian } \\
\text { Gulf } \\
\text { nations }^{c}\end{array}$ & $\begin{array}{c}\text { Persian } \\
\text { Gulf }^{c} \\
\text { share }\end{array}$ & World \\
\hline 1973 & 10.95 & $18.7 \%$ & 29.99 & $51.3 \%$ & 28.48 & $48.7 \%$ & 20.86 & $35.7 \%$ & 58.47 \\
\hline 1974 & 10.44 & $17.8 \%$ & 29.67 & $50.7 \%$ & 28.84 & $49.3 \%$ & 21.51 & $36.8 \%$ & 58.51 \\
\hline 1975 & 10.01 & $18.0 \%$ & 26.16 & $47.0 \%$ & 28.48 & $51.2 \%$ & 19.18 & $34.5 \%$ & 55.62 \\
\hline 1976 & 9.74 & $16.2 \%$ & 29.55 & $49.1 \%$ & 30.66 & $50.9 \%$ & 21.81 & $36.2 \%$ & 60.21 \\
\hline 1977 & 9.86 & $15.7 \%$ & 30.06 & $47.9 \%$ & 32.64 & $52.1 \%$ & 22.06 & $35.2 \%$ & 62.69 \\
\hline 1978 & 10.27 & $16.2 \%$ & 28.70 & $45.4 \%$ & 34.54 & $54.6 \%$ & 21.02 & $33.2 \%$ & 63.24 \\
\hline 1979 & 10.14 & $15.4 \%$ & 29.95 & $45.4 \%$ & 36.01 & $54.6 \%$ & 21.52 & $32.6 \%$ & 65.96 \\
\hline 1980 & 10.17 & $16.1 \%$ & 26.05 & $41.3 \%$ & 35.77 & $56.8 \%$ & 18.50 & $29.3 \%$ & 63.03 \\
\hline 1981 & 10.18 & $17.1 \%$ & 21.95 & $36.8 \%$ & 37.73 & $63.2 \%$ & 15.84 & $26.5 \%$ & 59.68 \\
\hline 1982 & 10.20 & $17.9 \%$ & 18.54 & $32.5 \%$ & 38.55 & $67.5 \%$ & 12.77 & $22.4 \%$ & 57.09 \\
\hline 1983 & 10.25 & $18.0 \%$ & 17.26 & $30.3 \%$ & 39.65 & $69.7 \%$ & 11.63 & $20.4 \%$ & 56.90 \\
\hline 1984 & 10.51 & $18.0 \%$ & 17.29 & $29.6 \%$ & 41.09 & $70.4 \%$ & 11.39 & $19.5 \%$ & 58.38 \\
\hline 1985 & 10.58 & $18.3 \%$ & 16.22 & $28.0 \%$ & 40.90 & $70.6 \%$ & 10.28 & $17.7 \%$ & 57.91 \\
\hline 1986 & 10.23 & $16.9 \%$ & 18.40 & $30.5 \%$ & 41.17 & $68.2 \%$ & 12.40 & $20.5 \%$ & 60.36 \\
\hline 1987 & 9.94 & $16.3 \%$ & 18.69 & $30.7 \%$ & 41.47 & $68.1 \%$ & 12.82 & $21.0 \%$ & 60.92 \\
\hline 1988 & 9.77 & $15.5 \%$ & 20.79 & $32.9 \%$ & 41.86 & $66.3 \%$ & 14.27 & $22.6 \%$ & 63.18 \\
\hline 1989 & 9.16 & $14.2 \%$ & 22.51 & $35.0 \%$ & 41.19 & $64.0 \%$ & 15.69 & $24.4 \%$ & 64.30 \\
\hline 1990 & 8.91 & $13.7 \%$ & 23.70 & $36.4 \%$ & 40.80 & $62.6 \%$ & 16.21 & $24.9 \%$ & 65.13 \\
\hline 1991 & 9.08 & $14.0 \%$ & 23.71 & $36.5 \%$ & 40.53 & $62.4 \%$ & 15.67 & $24.1 \%$ & 65.01 \\
\hline 1992 & 8.87 & $13.7 \%$ & 25.03 & $38.5 \%$ & 39.37 & $60.6 \%$ & 16.97 & $26.1 \%$ & 64.96 \\
\hline 1993 & 8.58 & $13.2 \%$ & 25.82 & $39.6 \%$ & 38.82 & $59.5 \%$ & 17.76 & $27.2 \%$ & 65.23 \\
\hline 1994 & 8.39 & $12.6 \%$ & 26.54 & $39.9 \%$ & 39.30 & $59.0 \%$ & 18.29 & $27.5 \%$ & 66.57 \\
\hline 1995 & 8.32 & $12.2 \%$ & 27.23 & $40.0 \%$ & 40.29 & $59.2 \%$ & 18.57 & $27.3 \%$ & 68.04 \\
\hline 1996 & 8.30 & $11.9 \%$ & 27.71 & $39.9 \%$ & 41.33 & $59.4 \%$ & 18.72 & $26.9 \%$ & 69.53 \\
\hline 1997 & 8.27 & $11.5 \%$ & 29.07 & $40.6 \%$ & 42.12 & $58.8 \%$ & 19.52 & $27.2 \%$ & 71.66 \\
\hline 1998 & 8.01 & $11.0 \%$ & 30.21 & $41.4 \%$ & 42.41 & $58.1 \%$ & 20.83 & $28.5 \%$ & 73.03 \\
\hline 1999 & 7.73 & $10.7 \%$ & 29.13 & $40.4 \%$ & 43.03 & $59.6 \%$ & 20.16 & $27.9 \%$ & 72.17 \\
\hline 2000 & 7.79 & $10.4 \%$ & 30.89 & $41.2 \%$ & 44.07 & $58.8 \%$ & 21.45 & $28.6 \%$ & 74.96 \\
\hline 2001 & 7.67 & $10.2 \%$ & 30.03 & $40.1 \%$ & 44.84 & $59.9 \%$ & 20.82 & $27.8 \%$ & 74.86 \\
\hline 2002 & 7.63 & $10.3 \%$ & 28.27 & $38.2 \%$ & 45.76 & $61.8 \%$ & 19.59 & $26.5 \%$ & 74.03 \\
\hline 2003 & 7.40 & $9.7 \%$ & 29.70 & $38.8 \%$ & 46.87 & $61.2 \%$ & 21.04 & $27.5 \%$ & 76.57 \\
\hline 2004 & 7.23 & $9.1 \%$ & 32.22 & $40.3 \%$ & 47.64 & $59.7 \%$ & 22.89 & $28.7 \%$ & 79.86 \\
\hline 2005 & 6.90 & $8.5 \%$ & 33.59 & $41.3 \%$ & 47.76 & $58.7 \%$ & 23.78 & $29.2 \%$ & 81.35 \\
\hline 2006 & 6.84 & $8.4 \%$ & 33.33 & $41.0 \%$ & 47.88 & $59.0 \%$ & 23.52 & $29.0 \%$ & 81.21 \\
\hline 2007 & 6.85 & $8.5 \%$ & 32.99 & $40.8 \%$ & 47.91 & $59.2 \%$ & 22.99 & $28.4 \%$ & 80.91 \\
\hline 2008 & 6.73 & $8.3 \%$ & 34.27 & $42.0 \%$ & 47.30 & $58.0 \%$ & 24.25 & $29.7 \%$ & 81.57 \\
\hline 2009 & 7.20 & $9.0 \%$ & 32.53 & $40.5 \%$ & 47.71 & $59.5 \%$ & 22.77 & $28.4 \%$ & 80.25 \\
\hline \multicolumn{10}{|c|}{ Average annual percentage change } \\
\hline 1973-2009 & $-1.2 \%$ & & $0.2 \%$ & & $1.4 \%$ & & $0.2 \%$ & & $0.9 \%$ \\
\hline 1999-2009 & $-0.7 \%$ & & $1.1 \%$ & & $1.0 \%$ & & $1.2 \%$ & & $1.1 \%$ \\
\hline
\end{tabular}

Source:

U.S. Department of Energy, Energy Information Administration, International Petroleum Monthly, March 2010, Tables 4.1c, 4.1d and 4.3. (Additional resources: www.eia.doe.gov)

${ }^{\text {a }}$ Includes natural gas plant liquids, crude oil and lease condensate. Does not account for all inputs or refinery processing gain.

${ }^{\mathrm{b}}$ Organization of Petroleum Exporting Countries. See Glossary for membership.

${ }^{\mathrm{c}}$ See Glossary for Persian Gulf Nations. 
The United States has accounted for almost one-quarter of the world's petroleum consumption for the last two decades, but in 2009 accounted for only 22.2\%. World petroleum consumption decreased in 2008 and 2009, though non-OECD consumption increased.

Table 1.4

World Petroleum Consumption, 1960-2009 (millions barrels per day)

\begin{tabular}{|c|c|c|c|c|c|}
\hline Year & United States & U.S. share & Total OECD ${ }^{\mathrm{a}}$ & Total non-OECD & World \\
\hline 1960 & 9.80 & $45.9 \%$ & 15.78 & 5.56 & 21.34 \\
\hline 1965 & 11.51 & $37.0 \%$ & 22.81 & 8.33 & 31.14 \\
\hline 1970 & 14.70 & $31.4 \%$ & 34.69 & 12.12 & 46.81 \\
\hline 1975 & 16.32 & $29.0 \%$ & 39.14 & 17.06 & 56.20 \\
\hline 1976 & 17.46 & $29.3 \%$ & 41.72 & 17.95 & 59.67 \\
\hline 1977 & 18.43 & $29.8 \%$ & 42.78 & 19.05 & 61.83 \\
\hline 1978 & 18.85 & $29.4 \%$ & 43.98 & 20.18 & 64.16 \\
\hline 1979 & 18.51 & $28.4 \%$ & 44.39 & 20.84 & 65.22 \\
\hline 1980 & 17.06 & $27.0 \%$ & 41.76 & 21.35 & 63.11 \\
\hline 1981 & 16.06 & $26.4 \%$ & 39.49 & 21.45 & 60.94 \\
\hline 1982 & 15.30 & $25.7 \%$ & 37.77 & 21.78 & 59.54 \\
\hline 1983 & 15.23 & $25.9 \%$ & 36.91 & 21.87 & 58.78 \\
\hline 1984 & 15.73 & $26.3 \%$ & 37.69 & 22.12 & 59.82 \\
\hline 1985 & 15.73 & $26.2 \%$ & 37.48 & 22.60 & 60.09 \\
\hline 1986 & 16.28 & $26.3 \%$ & 38.60 & 23.21 & 61.81 \\
\hline 1987 & 16.67 & $26.4 \%$ & 39.34 & 23.75 & 63.10 \\
\hline 1988 & 17.28 & $26.6 \%$ & 40.65 & 24.31 & 64.97 \\
\hline 1989 & 17.33 & $26.2 \%$ & 41.33 & 24.75 & 66.08 \\
\hline 1990 & 16.99 & $25.5 \%$ & 41.61 & 25.07 & 66.68 \\
\hline 1991 & 16.71 & $24.8 \%$ & 42.00 & 25.28 & 67.28 \\
\hline 1992 & 17.03 & $25.2 \%$ & 42.95 & 24.52 & 67.46 \\
\hline 1993 & 17.24 & $25.5 \%$ & 43.30 & 24.30 & 67.60 \\
\hline 1994 & 17.72 & $25.7 \%$ & 44.44 & 24.43 & 68.86 \\
\hline 1995 & 17.73 & $25.3 \%$ & 44.90 & 25.17 & 70.07 \\
\hline 1996 & 18.31 & $25.6 \%$ & 45.98 & 25.65 & 71.63 \\
\hline 1997 & 18.62 & $25.4 \%$ & 46.78 & 26.66 & 73.43 \\
\hline 1998 & 18.92 & $25.5 \%$ & 46.94 & 27.13 & 74.07 \\
\hline 1999 & 19.52 & $25.8 \%$ & 47.87 & 27.89 & 75.76 \\
\hline 2000 & 19.70 & $25.7 \%$ & 47.93 & 28.82 & 76.74 \\
\hline 2001 & 19.65 & $25.4 \%$ & 47.98 & 29.49 & 77.47 \\
\hline 2002 & 19.76 & $25.3 \%$ & 47.94 & 30.18 & 78.12 \\
\hline 2003 & 20.03 & $25.1 \%$ & 48.64 & 31.04 & 79.68 \\
\hline 2004 & 20.73 & $25.1 \%$ & 49.43 & 33.02 & 82.46 \\
\hline 2005 & 20.80 & $24.8 \%$ & 49.80 & 34.24 & 84.04 \\
\hline 2006 & 20.69 & $24.3 \%$ & 49.49 & 35.71 & 85.20 \\
\hline 2007 & 20.68 & $24.0 \%$ & 49.16 & 36.98 & 86.14 \\
\hline 2008 & 19.50 & $22.7 \%$ & 47.55 & 38.20 & 85.75 \\
\hline 2009 & 18.69 & $22.2 \%$ & 45.38 & 38.66 & 84.04 \\
\hline \multicolumn{6}{|c|}{ Average annual percentage change } \\
\hline 1960-2009 & $1.3 \%$ & & $2.2 \%$ & $4.0 \%$ & $2.8 \%$ \\
\hline 1970-2009 & $0.6 \%$ & & $0.7 \%$ & $3.0 \%$ & $1.5 \%$ \\
\hline 1999-2009 & $-0.4 \%$ & & $-0.5 \%$ & $3.3 \%$ & $1.0 \%$ \\
\hline
\end{tabular}

\section{Source:}

U.S. Department of Energy, Energy Information Administration, International Petroleum Monthly, April 2010. (Additional resources: www.eia.doe.gov)

${ }^{\text {a }}$ Organization for Economic Cooperation and Development. See Glossary for membership. 
Figure 1.2. World Oil Reserves, Production and Consumption, 2008

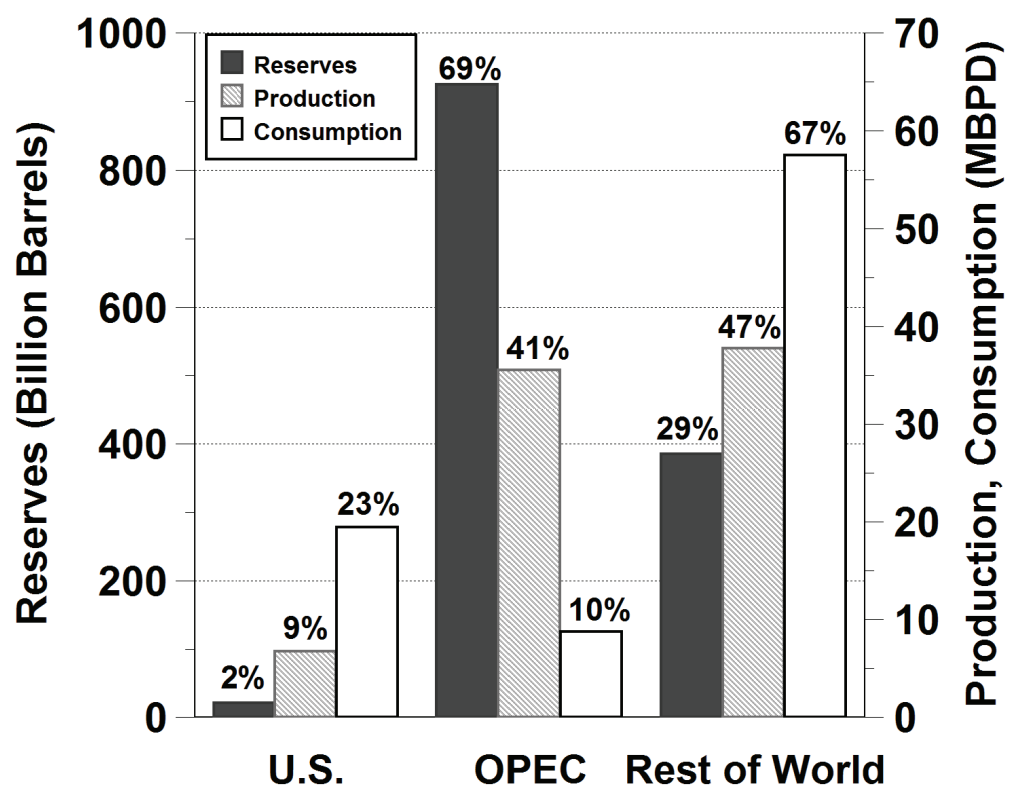

Table 1.5

World Oil Reserves, Production and Consumption, 2008

\begin{tabular}{lcccccc}
\hline & $\begin{array}{c}\text { Crude oil } \\
\text { reserves } \\
\text { (billion } \\
\text { barrels) }\end{array}$ & $\begin{array}{c}\text { Reserve } \\
\text { share }\end{array}$ & $\begin{array}{c}\text { Petroleum } \\
\text { production } \\
\text { (million } \\
\text { barrels per } \\
\text { day }\end{array}$ & $\begin{array}{c}\text { Production } \\
\text { share }\end{array}$ & $\begin{array}{c}\text { Petroleum } \\
\text { consumption } \\
\text { (million } \\
\text { barrels per } \\
\text { day) }\end{array}$ & $\begin{array}{c}\text { Consumption } \\
\text { share }\end{array}$ \\
\hline United States & 21.3 & $2 \%$ & 6.8 & $9 \%$ & 19.5 & $23 \%$ \\
OPEC & 925 & $69 \%$ & 35.6 & $41 \%$ & 8.8 & $10 \%$ \\
Rest of world & 385.8 & $29 \%$ & 37.8 & $47 \%$ & 57.5 & $67 \%$ \\
\hline
\end{tabular}

Sources:

Reserves - Energy Information Administration, International Energy Statistics, April 2010.

Production - Energy Information Administration, International Petroleum Monthly, March 2010, Tables 1.1c, 1.1d, 1.3 , and 1.7 .

Consumption - Energy Information Administration, International Energy Statistics, April 2010, and International Petroleum Monthly, March 2010, Table 1.7. (Additional resources: www.eia.doe.gov)

Note: Total consumption is higher than total production due to refinery gains including alcohol and liquid products produced from coal and other sources. OPEC countries include Venezuela, Iran, Iraq, Kuwait, Qatar, Saudi Arabia, United Arab Emirates, Algeria, Libya, Nigeria, Indonesia, Gabon, and Ecuador. 
Figure 1.3. World Natural Gas Reserves, Production and Consumption, 2008

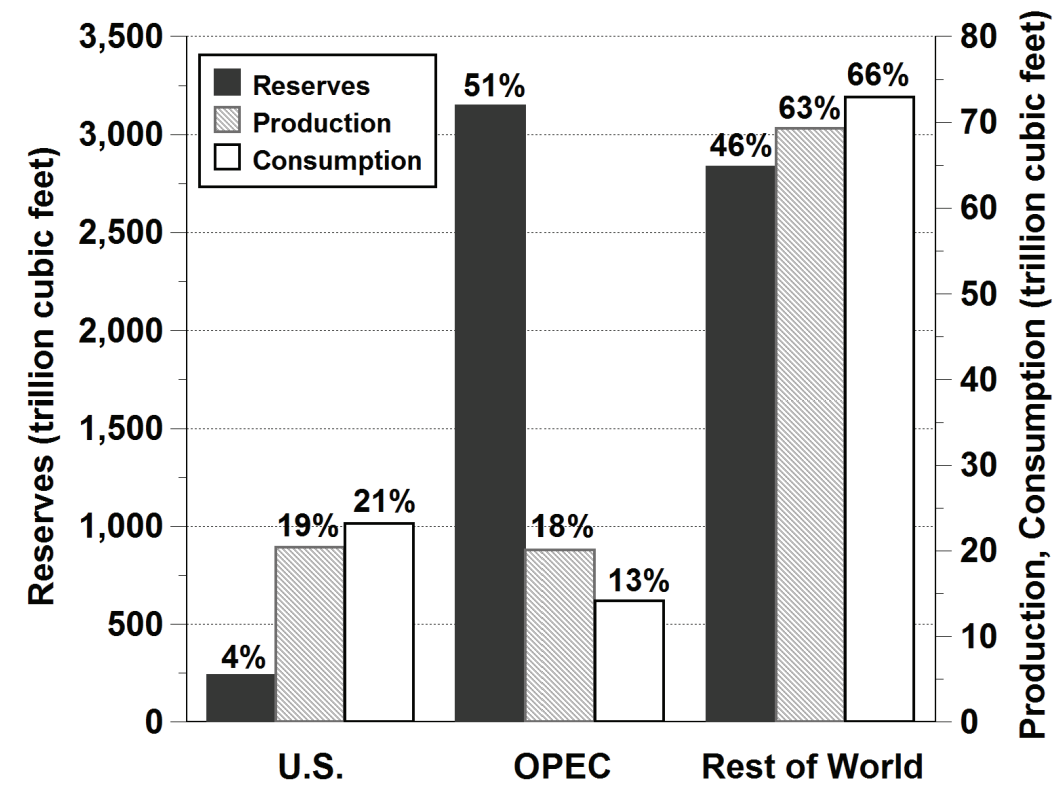

Table 1.6

World Natural Gas Reserves, Production and Consumption, 2008 (trillion cubic feet)

\begin{tabular}{lrrrrrr}
\hline & $\begin{array}{c}\text { Natural } \\
\text { gas } \\
\text { reserves }\end{array}$ & $\begin{array}{c}\text { Reserve } \\
\text { share }\end{array}$ & $\begin{array}{c}\text { Natural gas } \\
\text { production }\end{array}$ & $\begin{array}{c}\text { Production } \\
\text { share }\end{array}$ & $\begin{array}{c}\text { Natural gas } \\
\text { consumption }\end{array}$ & $\begin{array}{c}\text { Consumption } \\
\text { share }\end{array}$ \\
\hline U.S. & 237.7 & $4 \%$ & 20.4 & $19 \%$ & 23.2 & $21 \%$ \\
OPEC & $3,143.4$ & $51 \%$ & 20.1 & $18 \%$ & 14.1 & $13 \%$ \\
Rest of world & $2,831.4$ & $46 \%$ & 69.3 & $63 \%$ & 72.9 & $66 \%$ \\
\hline
\end{tabular}

Source:

Energy Information Administration, International Energy Statistics, 2010. (Additional resources: www.eia.doe.gov)

Note: Reserves as of April 2010. Production data are dry gas production. 
The share of petroleum imported to the United States can be calculated using total imports or net imports. Net imports, which is the preferred data, rose to 50\% of U.S. petroleum consumption for the first time in 1998, while total imports reached 50\% for the first time in 1993. OPEC share of net imports has been below $50 \%$ since 1993.

Table 1.7

U.S. Petroleum Imports by World Region of Origin, 1960-2009 (million barrels per day)

\begin{tabular}{|c|c|c|c|c|c|c|c|}
\hline Year & $\begin{array}{l}\text { Net OPEC } \\
\text { imports }\end{array}$ & $\begin{array}{l}\text { Net OPEC } \\
\text { share }\end{array}$ & $\begin{array}{l}\text { Net Persian } \\
\text { Gulf nation } \\
\text { imports }\end{array}$ & $\begin{array}{l}\text { Net Persian } \\
\text { Gulf share }\end{array}$ & $\begin{array}{c}\text { Net } \\
\text { imports }\end{array}$ & $\begin{array}{l}\text { Net imports as a } \\
\text { share of U.S. } \\
\text { consumption }\end{array}$ & $\begin{array}{c}\text { Total } \\
\text { imports }\end{array}$ \\
\hline 1960 & 1.31 & $81.3 \%$ & c & c & 1.61 & c & 1.82 \\
\hline 1965 & 1.48 & $64.7 \%$ & c & c & 2.28 & c & 2.47 \\
\hline 1970 & 1.34 & $42.5 \%$ & $\mathrm{c}^{-}$ & & 3.16 & & 3.42 \\
\hline 1975 & 3.60 & $61.6 \%$ & 1.17 & $19.2 \%$ & 5.85 & $35.8 \%$ & 6.06 \\
\hline 1980 & 4.30 & $62.2 \%$ & $1.52^{\circ}$ & $22.0 \%$ & 6.36 & $37.3 \%$ & 6.91 \\
\hline 1981 & 3.32 & $55.4 \%$ & 1.22 & $20.3 \%$ & 5.40 & $33.6 \%$ & 6.00 \\
\hline 1982 & 2.15 & $42.0 \%$ & 0.70 & $13.7 \%$ & 4.30 & $28.1 \%$ & 5.11 \\
\hline 1983 & 1.86 & $36.9 \%$ & 0.44 & $8.7 \%$ & 4.31 & $28.2 \%$ & 5.05 \\
\hline 1984 & 2.05 & $37.7 \%$ & 0.51 & $9.4 \%$ & 4.72 & $29.9 \%$ & 5.44 \\
\hline 1985 & 1.83 & $36.1 \%$ & 0.31 & $6.1 \%$ & 4.29 & $27.3 \%$ & 5.07 \\
\hline 1986 & 2.84 & $45.6 \%$ & 0.91 & $14.6 \%$ & 5.44 & $33.4 \%$ & 6.22 \\
\hline 1987 & 3.06 & $45.8 \%$ & 1.08 & $16.2 \%$ & 5.91 & $35.4 \%$ & 6.68 \\
\hline 1988 & 3.52 & $47.6 \%$ & 1.54 & $20.8 \%$ & 6.59 & $38.0 \%$ & 7.40 \\
\hline 1989 & 4.14 & $51.4 \%$ & 1.86 & $23.1 \%$ & 7.20 & $41.3 \%$ & 8.06 \\
\hline 1990 & 4.30 & $53.6 \%$ & 1.97 & $24.6 \%$ & 7.16 & $42.2 \%$ & 8.02 \\
\hline 1991 & 4.09 & $53.7 \%$ & 1.84 & $24.1 \%$ & 6.63 & $38.9 \%$ & 7.63 \\
\hline 1992 & 4.09 & $51.9 \%$ & 1.78 & $22.6 \%$ & 6.94 & $40.9 \%$ & 7.89 \\
\hline 1993 & 4.27 & $49.6 \%$ & 1.78 & $20.6 \%$ & 7.62 & $44.9 \%$ & 8.62 \\
\hline 1994 & 4.25 & $47.2 \%$ & 1.73 & $19.2 \%$ & 8.05 & $45.7 \%$ & 9.00 \\
\hline 1995 & 4.00 & $45.3 \%$ & 1.57 & $17.8 \%$ & 7.89 & $44.5 \%$ & 8.84 \\
\hline 1996 & 4.21 & $44.4 \%$ & 1.60 & $16.9 \%$ & 8.50 & $46.4 \%$ & 9.48 \\
\hline 1997 & 4.57 & $45.0 \%$ & 1.76 & $17.3 \%$ & 9.16 & $49.2 \%$ & 10.16 \\
\hline 1998 & 4.91 & $45.8 \%$ & 2.14 & $19.9 \%$ & 9.76 & $51.6 \%$ & 10.71 \\
\hline 1999 & 4.95 & $45.6 \%$ & 2.46 & $22.7 \%$ & 9.91 & $50.8 \%$ & 10.85 \\
\hline 2000 & 5.20 & $45.4 \%$ & 2.49 & $21.7 \%$ & 10.42 & $52.9 \%$ & 11.46 \\
\hline 2001 & 5.53 & $46.6 \%$ & 2.76 & $23.3 \%$ & 10.90 & $55.5 \%$ & 11.87 \\
\hline 2002 & 4.61 & $39.9 \%$ & 2.27 & $19.7 \%$ & 10.55 & $53.4 \%$ & 11.53 \\
\hline 2003 & 5.16 & $42.1 \%$ & 2.50 & $20.4 \%$ & 11.24 & $56.1 \%$ & 12.26 \\
\hline 2004 & 5.70 & $43.4 \%$ & 2.49 & $19.0 \%$ & 12.10 & $58.4 \%$ & 13.15 \\
\hline 2005 & 5.59 & $40.7 \%$ & 2.33 & $17.0 \%$ & 12.55 & $60.3 \%$ & 13.71 \\
\hline 2006 & 5.52 & $40.2 \%$ & 2.21 & $16.1 \%$ & 12.39 & $59.9 \%$ & 13.71 \\
\hline 2007 & 5.98 & $44.4 \%$ & 2.16 & $16.1 \%$ & 12.04 & $58.2 \%$ & 13.47 \\
\hline 2008 & 5.95 & $46.1 \%$ & 2.37 & $18.4 \%$ & 11.11 & $57.0 \%$ & 12.92 \\
\hline 2009 & 4.79 & $40.8 \%$ & 1.70 & $14.5 \%$ & 9.70 & $51.9 \%$ & 11.72 \\
\hline \multicolumn{8}{|c|}{ Average annual percentage change } \\
\hline 1960-2009 & $2.7 \%$ & & $\mathrm{c}$ & & $3.7 \%$ & & $3.9 \%$ \\
\hline 1970-2009 & $3.3 \%$ & & c & & $2.9 \%$ & & $3.2 \%$ \\
\hline 1999-2009 & $-0.3 \%$ & & $-3.6 \%$ & & $-0.2 \%$ & & $0.8 \%$ \\
\hline
\end{tabular}

\section{Source:}

U.S. Department of Energy, Energy Information Administration, Monthly Energy Review, Washington, DC, March 2010, Table 3.3a.

${ }^{\text {a }}$ Organization of Petroleum Exporting Countries. See Glossary for membership.

${ }^{\mathrm{b}}$ See Glossary for Persian Gulf Nations.

${ }^{\mathrm{c}}$ Data are not available. 
Major oil price shocks have disrupted world energy markets five times in the past 30 years (1973-74, 1979-80, 1990-91, 1999-2000, 2008). Most of the oil price shocks were followed by an economic recession in the United States.

Figure 1.4. Oil Price and Economic Growth, 1970-2009

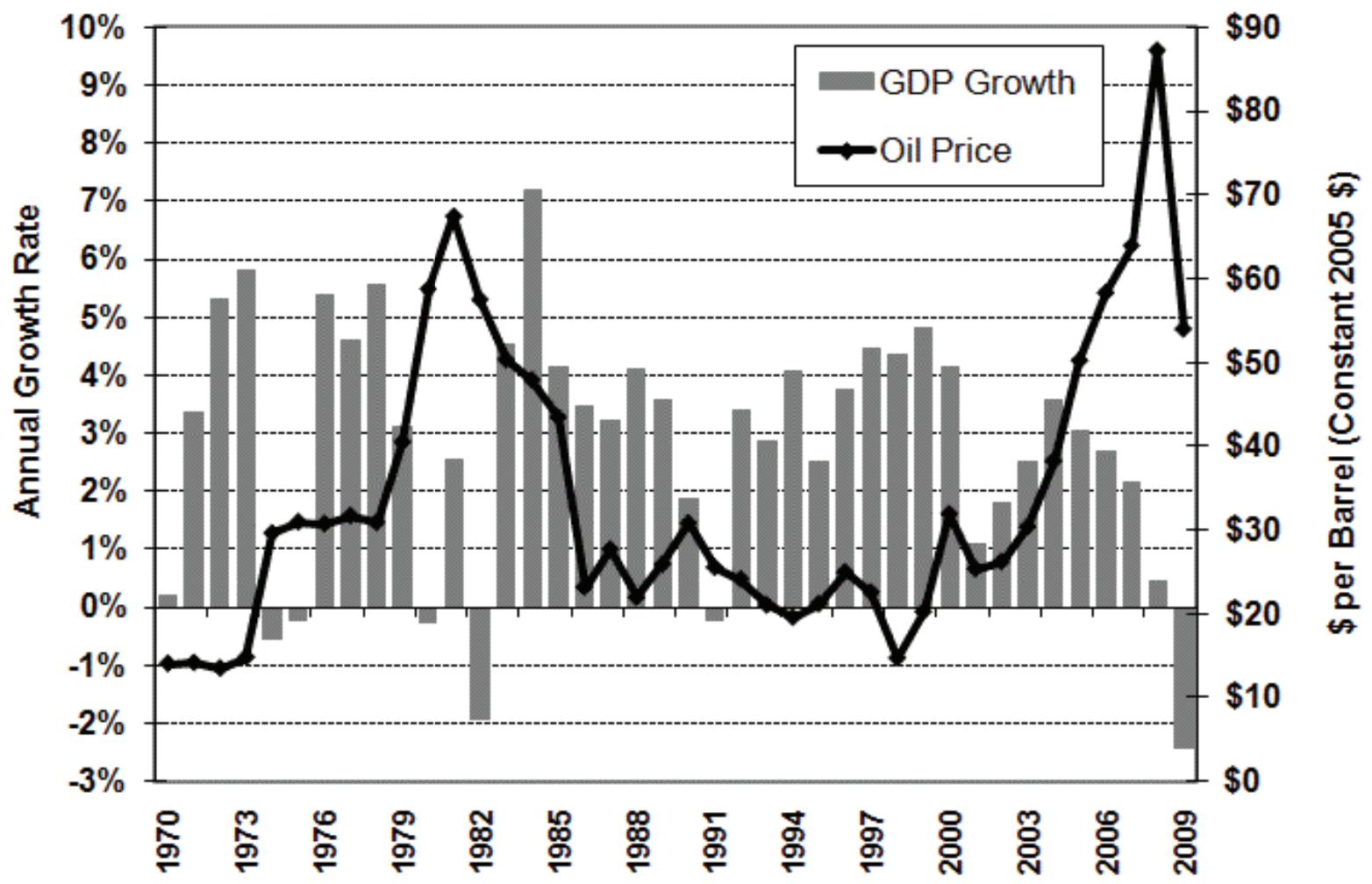

Source:

Greene, D.L. and N. I. Tishchishyna, Costs of Oil Dependence: A 2000 Update, Oak Ridge National Laboratory, ORNL/TM-2000/152, Oak Ridge, TN, 2000, and data updates, 2010. (Additional resources: wwwcta.ornl.gov/publications) 
The United States has long recognized the problem of oil dependence and the economic problems that arise from it. According to Oak Ridge National Laboratory (ORNL) researchers Greene and Hopson, oil dependence is a combination of four factors: (1) a noncompetitive world oil market strongly influenced by the OPEC cartel, (2) high levels of U.S. imports, (3) the importance of oil to the U.S. economy, and (4) the lack of economical and readily available substitutes for oil. ORNL developed a model to estimate the historical cost of oil dependence and analyze the potential effectiveness of policies on likely future costs. The most recent study using this model shows that the U.S. economy suffered the greatest losses in 2008 when wealth transfer and GDP losses (combined) amounted to approximately half a trillion dollars. However, when comparing oil dependence to the size of the economy, the year 1980 is the highest. Oil dependence costs were almost 4.5\% of GDP in 1980, but were under $3.5 \%$ in 2008.

Figure 1.5. Costs of Oil Dependence to the U.S. Economy, 1970-2009

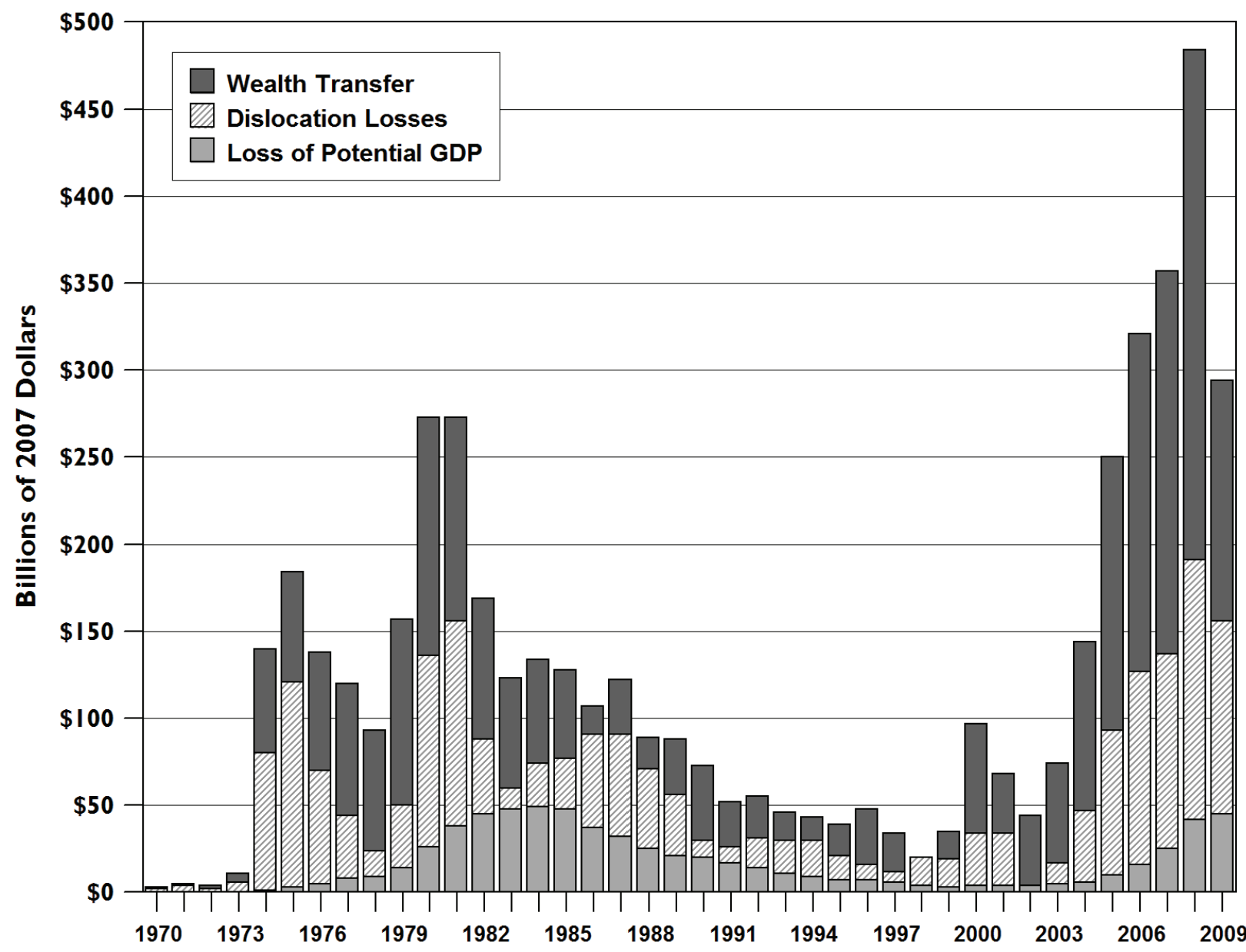

Source:

Greene, David L., and Janet L. Hopson, "The Costs of Oil Dependence 2009," Oak Ridge National Laboratory Memorandum, 2010.

Notes:

Wealth Transfer is the product of total U.S. oil imports and the difference between the actual market price of oil (influenced by market power) and what the price would have been in a competitive market.

Dislocation Losses are temporary reductions in GDP as a result of oil price shocks.

Loss of Potential Gross Domestic Product (GDP) results because a basic resource used by the economy to produce output has become more expensive. As a consequence, with the same endowment of labor, capital, and other resources, our economy cannot produce quite as much as it could have at a lower oil price. 
Estimates of military expenditures for defending oil supplies in the Middle East range from $\$ 6$ to $\$ 60$ billion per year. This wide range in estimates reflects the difficulty in assigning a precise figure to the military cost of defending the U.S. interests in the Middle East. The two main reasons for the difficulty are 1) the Department of Defense does not divide the budget into regional defense sectors and 2) it is difficult to determine how much of the cost is attributable to defending Persian Gulf oil.

Table 1.8

Summary of Military Expenditures for Defending Oil Supplies from the Middle East

\begin{tabular}{lcc}
\hline \multicolumn{1}{c}{ Source } & $\begin{array}{c}\text { Original estimates } \\
\text { (billion dollars) }\end{array}$ & $\begin{array}{c}\text { Year of original estimate } \\
\text { (billion dollars) }\end{array}$ \\
\hline General Accounting Office [1] & $\$ 33$ & 1990 \\
Congressional Research Service [2] & $\$ 6.4$ & 1990 \\
Greene and Leiby [3] & $\$ 14.3$ & 1990 \\
Kaufmann and Steinbruner [4] & $\$ 64.5$ & 1990 \\
Ravenal [5] & $\$ 50$ & 1992 \\
Delucchi and Murphy ${ }^{\mathrm{a}}[6]$ & $\$ 20-40$ & 1996 \\
National Defense Council Foundation [7] & $\$ 49.1$ & 2003 \\
Delucchi and Murphy ${ }^{\mathrm{a}}[8]$ & $\$ 47-98$ & 2004 \\
\hline
\end{tabular}

[1] U.S. General Accounting Offices, Southwest Asia: Cost of Protecting U.S. Interests, GAO/NSIAD-91-250, Washington, DC, August 1991.

[2] Congressional Research Service, The External Costs of Oil Used in Transportation, prepared for the U.S. Alternative Fuels Council, Washington, DC, June 1992

[3] Greene, D.L., and P. Leiby, The Social Costs to the U.S. of Monopolization of the World Oil Market, 19721991, ORNL-6744, Oak Ridge National Laboratory, Oak Ridge, TN, March 1993.

[4] Kaufmann, W.W., and J.D. Steinbruner, Decisions for Defense: Prospects for a New Order, The Brookings Institution, Washington, DC, 1991.

[5] Ravenal, E.C., Designing Defense for a New World Order: The Military Budget in 1992 and Beyond, Cato Institute, Washington, DC, 1991.

[6] Delucchi, M.A., and J. Murphy, U.S. Military Expenditures to Protect the Use of Persian-Gulf Oil for Motor Vehicles, UCD-ITS-RR-96-3 (15), University of California, Davis, California, April 1996.

[7] Copulas, Milton R., America's Achilles Heel -- The Hidden Costs of Imported Oil, National Defense Council Foundation, Washington, DC, October 2003.

[8] Delucchi, M.A. and J. Murphy, "U.S. Military Expenditures to Protect the Use of Persian-Gulf Oil for Motor Vehicles," Energy Policy, April 2008.

\footnotetext{
${ }^{a}$ Annual cost to defend all U.S. interests in the Persian Gulf.
} 
Other parts of the world refine crude oil to produce more diesel fuel and less gasoline than does North America. The OECD Europe countries produce the lowest share of gasoline in 2009.

Figure 1.6. Refinery Gross Output by World Region, 1999 and 2009

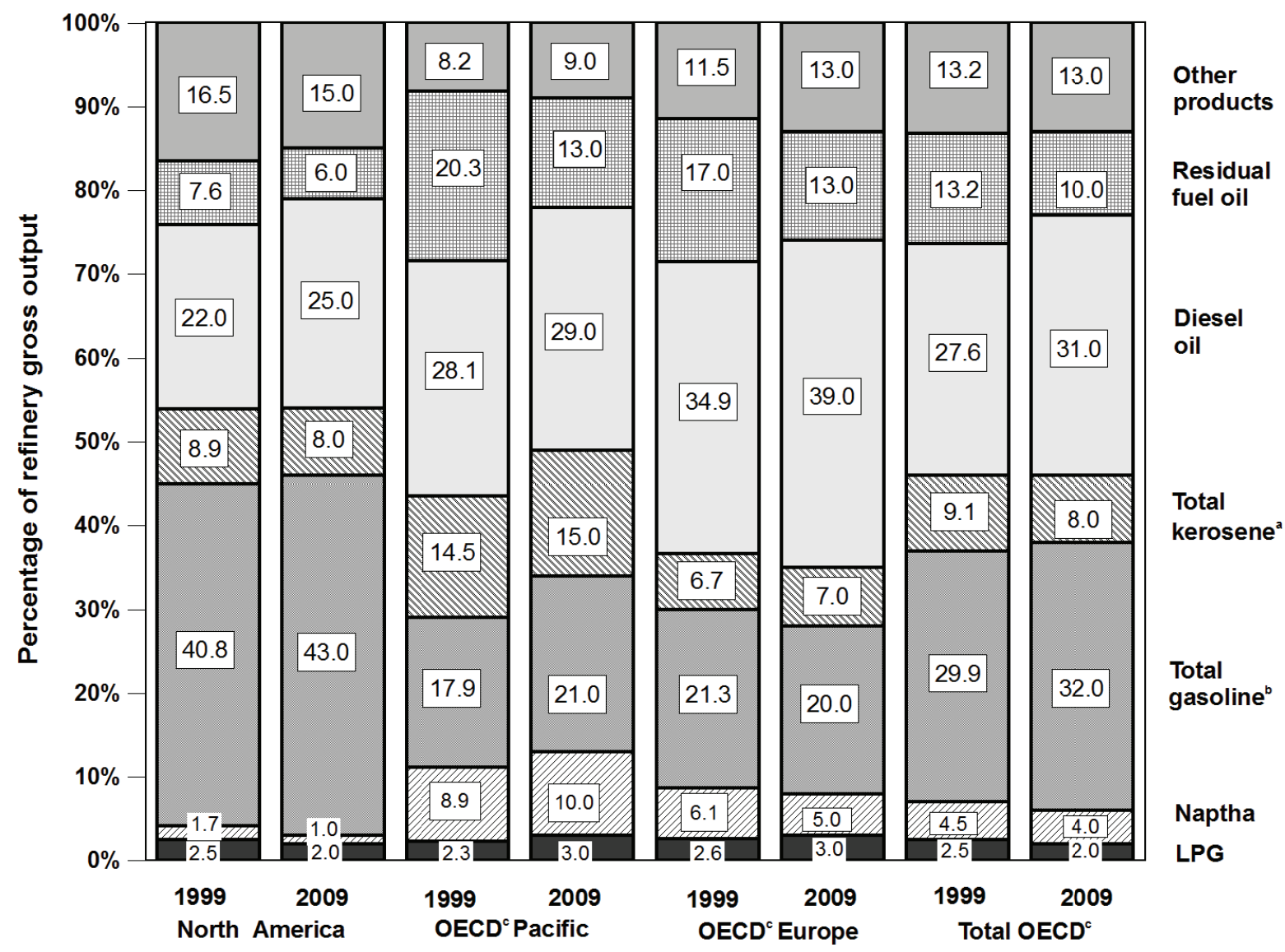

Source:

International Energy Agency, Monthly Oil Survey, January 2010.(Additional resources: www.iea.org)

${ }^{\mathrm{a}}$ Includes jet kerosene and other kerosene.

${ }^{\mathrm{b}}$ Includes motor gasoline, jet gasoline, and aviation gasoline.

${ }^{\mathrm{c}}$ Organization for Economic Cooperation and Development. See Glossary for membership. 
Oxygenate refinery input increased significantly in 1995, most certainly due to the Clean Air Act Amendments of 1990 which mandated the sale of reformulated gasoline in certain areas beginning in January 1995. The use of MTBE has declined in recent years due to many states banning the additive. The other hydrocarbons and liquids category includes unfinished oils, motor gasoline blending components and aviation gasoline blending components. In 2005 the gasoline blending components rose significantly.

Table 1.9

U.S. Refinery Input of Crude Oil and Petroleum Products, 1987-2008 (thousand barrels)

\begin{tabular}{|c|c|c|c|c|c|c|c|}
\hline \multirow[b]{2}{*}{ Year } & \multirow[b]{2}{*}{ Crude oil } & \multirow[b]{2}{*}{$\begin{array}{l}\text { Natural gas } \\
\text { liquids }\end{array}$} & \multicolumn{3}{|c|}{ Oxygenates } & \multirow{2}{*}{$\begin{array}{c}\text { Other } \\
\text { hydrocarbons } \\
\text { and liquids }\end{array}$} & \multirow[b]{2}{*}{$\begin{array}{l}\text { Total input to } \\
\text { refineries }\end{array}$} \\
\hline & & & $\begin{array}{c}\text { Fuel } \\
\text { ethanol }\end{array}$ & MTBE $^{\mathrm{a}}$ & $\begin{array}{c}\text { Other } \\
\text { oxygenates }\end{array}$ & & \\
\hline 1987 & $4,691,783$ & 280,889 & c & c & d & 132,720 & $5,105,392$ \\
\hline 1988 & $4,848,175$ & 304,566 & c & c & d & 105,645 & $5,258,386$ \\
\hline 1989 & $4,891,381$ & 182,109 & c & c & d & 223,797 & $5,297,287$ \\
\hline 1990 & $4,894,379$ & 170,589 & c & c & d & 260,108 & $5,325,076$ \\
\hline 1991 & $4,855,016$ & 172,306 & c & c & d & 280,265 & $5,307,587$ \\
\hline 1992 & $4,908,603$ & 171,701 & c & c & d & 272,676 & $5,352,980$ \\
\hline 1993 & $4,968,641$ & 179,213 & 3,351 & 49,393 & 1,866 & 280,074 & $5,482,538$ \\
\hline 1994 & $5,061,111$ & 169,868 & 3,620 & 52,937 & 1,918 & 193,808 & $5,483,262$ \\
\hline 1995 & $5,100,317$ & 172,026 & 9,055 & 79,396 & 4,122 & 190,411 & $5,555,327$ \\
\hline 1996 & $5,195,265$ & 164,552 & 11,156 & 79,407 & 3,570 & 214,282 & $5,668,232$ \\
\hline 1997 & $5,351,466$ & 151,769 & 11,803 & 86,240 & 4,246 & 201,268 & $5,806,792$ \\
\hline 1998 & $5,434,383$ & 146,921 & 11,722 & 89,362 & 4,038 & 206,135 & $5,892,561$ \\
\hline 1999 & $5,403,450$ & 135,756 & 13,735 & 94,784 & 4,147 & 225,779 & $5,877,651$ \\
\hline 2000 & $5,514,395$ & 138,921 & 15,268 & 90,288 & 4,005 & 201,135 & $5,964,012$ \\
\hline 2001 & $5,521,637$ & 156,479 & 16,929 & 87,116 & 4,544 & 192,632 & $5,979,337$ \\
\hline 2002 & $5,455,530$ & 155,429 & 26,320 & 90,291 & 2,338 & 224,567 & $5,955,475$ \\
\hline 2003 & $5,585,875$ & 152,763 & 55,626 & 67,592 & 1,937 & 163,459 & $6,027,252$ \\
\hline 2004 & $5,663,861$ & 154,356 & 74,095 & 47,600 & 940 & 194,203 & $6,135,055$ \\
\hline 2005 & $5,555,332$ & 161,037 & 84,088 & 39,751 & 612 & 295,064 & $6,135,884$ \\
\hline 2006 & $5,563,354$ & 182,924 & 117,198 & 11,580 & 57 & 322,989 & $6,198,102$ \\
\hline 2007 & $5,532,097$ & 184,383 & 136,603 & 1,610 & 0 & 349,807 & $6,204,500$ \\
\hline 2008 & $5,361,287$ & 177,559 & 190,084 & 480 & 0 & 548,843 & $6,277,893$ \\
\hline \multicolumn{8}{|c|}{ Average annual percentage change } \\
\hline $1987-2008$ & $0.6 \%$ & $-2.2 \%$ & 80 & 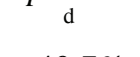 & & $7.0 \%$ & $1.0 \%$ \\
\hline 1998-2008 & $-0.1 \%$ & $1.9 \%$ & $32.1 \%$ & $-40.7 \%$ & $-100.0 \%$ & $10.3 \%$ & $0.6 \%$ \\
\hline
\end{tabular}

Source:

U.S. Department of Energy, Energy Information Administration, Petroleum Supply Annual 2008, Vol. 1, June 2009, Table 15, and annual. (Additional resources: www.eia.doe.gov)

${ }^{\text {a }}$ Methyl tertiary butyl ether (MTBE).

${ }^{\mathrm{b}}$ Includes methanol and other oxygenates.

c Reported in "Other" category in this year.

${ }^{\mathrm{d}}$ Data are not available. 
When crude oil and other hydrocarbons are processed into products that are, on average, less dense than the input, a processing volume gain occurs. Due to this gain, the product yield from a barrel of crude oil is more than 100\%. The processing volume gain has been growing over the years.

Table 1.10

Refinery Yield of Petroleum Products from a Barrel of Crude Oil, 1978-2009 (percentage)

\begin{tabular}{rcccccc}
\hline Year & $\begin{array}{c}\text { Motor } \\
\text { gasoline }\end{array}$ & $\begin{array}{c}\text { Distillate } \\
\text { fuel oil }\end{array}$ & $\begin{array}{c}\text { Jet fuel } \\
\text { petroleum gas }\end{array}$ & Other $^{\mathrm{a}}$ & Total $^{\mathrm{b}}$ \\
\hline 1978 & 44.1 & 21.4 & 6.6 & 2.3 & 29.6 & 104.0 \\
1979 & 43.0 & 21.5 & 6.9 & 2.3 & 30.3 & 104.0 \\
1980 & 44.5 & 19.7 & 7.4 & 2.4 & 30.0 & 104.0 \\
1981 & 44.8 & 20.5 & 7.6 & 2.4 & 28.7 & 104.0 \\
1982 & 46.4 & 21.5 & 8.1 & 2.2 & 26.2 & 104.4 \\
1983 & 47.6 & 20.5 & 8.5 & 2.7 & 24.8 & 104.1 \\
1984 & 46.7 & 21.5 & 9.1 & 2.9 & 24.2 & 104.4 \\
1985 & 45.6 & 21.6 & 9.6 & 3.1 & 24.6 & 104.5 \\
1986 & 45.7 & 21.2 & 9.8 & 3.2 & 24.8 & 104.7 \\
1987 & 46.4 & 20.5 & 10.0 & 3.4 & 24.5 & 104.8 \\
1988 & 46.0 & 20.8 & 10.0 & 3.6 & 24.4 & 104.8 \\
1989 & 45.7 & 20.8 & 10.1 & 4.0 & 24.2 & 104.8 \\
1990 & 45.6 & 20.9 & 10.7 & 3.6 & 24.1 & 104.9 \\
1991 & 45.7 & 21.3 & 10.3 & 3.8 & 24.1 & 105.2 \\
1992 & 46.0 & 21.2 & 9.9 & 4.3 & 24.0 & 105.4 \\
1993 & 46.1 & 21.9 & 10.0 & 4.1 & 23.3 & 105.4 \\
1994 & 45.5 & 22.3 & 10.1 & 4.2 & 23.2 & 105.3 \\
1995 & 46.4 & 21.8 & 9.7 & 4.5 & 22.9 & 105.3 \\
1996 & 45.7 & 22.7 & 10.4 & 4.5 & 22.4 & 105.7 \\
1997 & 45.7 & 22.5 & 10.3 & 4.6 & 22.5 & 105.6 \\
1998 & 46.2 & 22.3 & 10.4 & 4.4 & 22.5 & 105.8 \\
1999 & 46.5 & 22.3 & 10.2 & 4.5 & 22.3 & 105.8 \\
2000 & 46.2 & 23.1 & 10.3 & 4.5 & 22.0 & 106.1 \\
2001 & 46.2 & 23.8 & 9.8 & 4.3 & 21.7 & 105.8 \\
2002 & 47.3 & 23.2 & 9.8 & 4.3 & 21.5 & 106.1 \\
2003 & 46.9 & 23.7 & 9.5 & 4.2 & 22.1 & 106.4 \\
2004 & 46.8 & 23.9 & 9.7 & 4.0 & 22.2 & 106.6 \\
2005 & 46.2 & 25.0 & 9.8 & 3.6 & 21.6 & 106.2 \\
2006 & 45.8 & 25.4 & 9.3 & 3.9 & 21.7 & 106.1 \\
2007 & 45.5 & 26.1 & 9.1 & 4.1 & 21.5 & 106.3 \\
2008 & 44.3 & 27.8 & 9.7 & 4.1 & 20.6 & 106.5 \\
2009 & 46.1 & 26.9 & 9.3 & 4.1 & 20.1 & 106.5 \\
\hline & & & & &
\end{tabular}

Source:

Department of Energy, Energy Information Administration, Petroleum Supply Navigator, Vol.1, April 2010. (Additional resources: www.eia.doe.gov)

${ }^{a}$ Includes aviation gasoline $(0.1 \%)$, kerosene $(0.1 \%)$, residual fuel oil $(4.0 \%)$, naphtha and other oils for petrochemical feedstock use $(1.0 \%)$, other oils for petrochemical feedstock use $(1.0 \%)$, special naphthas $(0.2 \%)$, lubricants $(1.0 \%)$, waxes $(0.1 \%)$, petroleum coke $(5.3 \%)$ asphalt and road oil (2.4\%), still gas (4.3\%), and miscellaneous products $(0.5 \%)$.

${ }^{b}$ Products sum greater than $100 \%$ due to processing gain. The processing gain for years 1978 to 1980 is assumed to be 4 percent. 
Most of the petroleum imported by the United States is in the form of crude oil. The United States does export small amounts of petroleum, mainly refined petroleum products which go to Canada and Mexico.

Table 1.11

United States Petroleum Production, Imports and Exports, 1950-2009 (million barrels per day)

\begin{tabular}{|c|c|c|c|c|c|c|c|c|c|}
\hline & \multicolumn{3}{|c|}{ Domestic Production } & \multicolumn{3}{|c|}{ Net Imports } & \multicolumn{3}{|c|}{ Exports } \\
\hline & $\begin{array}{c}\text { Crude } \\
\text { oil }\end{array}$ & $\begin{array}{l}\text { Natural } \\
\text { gas } \\
\text { plant } \\
\text { liquids }\end{array}$ & Total $^{\mathrm{a}}$ & $\begin{array}{c}\text { Crude } \\
\text { oil }\end{array}$ & $\begin{array}{c}\text { Petroleum } \\
\text { products }\end{array}$ & Total & $\begin{array}{c}\text { Crude } \\
\text { oil }\end{array}$ & $\begin{array}{c}\text { Petroleum } \\
\text { products }\end{array}$ & Total \\
\hline 1950 & 5.41 & 0.50 & 5.91 & 0.49 & 0.36 & 0.85 & 0.10 & 0.21 & 0.31 \\
\hline 1955 & 6.81 & 0.77 & 7.58 & 0.78 & 0.47 & 1.25 & 0.03 & 0.34 & 0.37 \\
\hline 1960 & 7.05 & 0.93 & 7.98 & 1.02 & 0.80 & 1.82 & 0.01 & 0.19 & 0.20 \\
\hline 1965 & 7.80 & 1.21 & 9.01 & 1.24 & 1.23 & 2.47 & 0.00 & 0.18 & 0.19 \\
\hline 1970 & 9.64 & 1.66 & 11.30 & 1.32 & 2.10 & 3.42 & 0.01 & 0.25 & 0.26 \\
\hline 1975 & 8.38 & 1.63 & 10.01 & 4.11 & 1.95 & 6.06 & 0.01 & 0.20 & 0.21 \\
\hline 1980 & 8.60 & $1.57^{-}$ & 10.17 & 5.26 & 1.65 & 6.91 & 0.29 & 0.26 & 0.54 \\
\hline 1981 & 8.57 & 1.61 & 10.18 & 4.40 & 1.60 & 6.00 & 0.23 & 0.37 & 0.60 \\
\hline 1982 & 8.65 & 1.55 & 10.20 & 3.49 & 1.63 & 5.11 & 0.24 & 0.58 & 0.82 \\
\hline 1983 & 8.69 & 1.56 & 10.25 & 3.33 & 1.72 & 5.05 & 0.16 & 0.58 & 0.74 \\
\hline 1984 & 8.90 & 1.63 & 10.53 & 3.43 & 2.01 & 5.44 & 0.18 & 0.54 & 0.72 \\
\hline 1985 & 8.97 & 1.61 & 10.58 & 3.20 & 1.87 & 5.07 & 0.20 & 0.58 & 0.78 \\
\hline 1986 & 8.68 & 1.55 & 10.23 & 4.18 & 2.05 & 6.22 & 0.15 & 0.63 & 0.79 \\
\hline 1987 & 8.35 & 1.60 & 9.95 & 4.67 & 2.00 & 6.68 & 0.15 & 0.61 & 0.76 \\
\hline 1988 & 8.16 & 1.63 & 9.97 & 5.11 & 2.30 & 7.40 & 0.16 & 0.66 & 0.82 \\
\hline 1989 & 7.61 & 1.55 & 9.16 & 5.84 & 2.22 & 8.06 & 0.14 & 0.72 & 0.86 \\
\hline 1990 & 7.36 & 1.56 & 8.91 & 5.89 & 2.12 & 8.02 & 0.11 & 0.75 & 0.86 \\
\hline 1991 & 7.42 & 1.66 & 9.08 & 5.78 & 1.84 & 7.63 & 0.12 & 0.89 & 1.00 \\
\hline 1992 & 7.18 & 1.70 & 8.88 & 6.08 & 1.81 & 7.89 & 0.09 & 0.86 & 0.95 \\
\hline 1993 & 6.85 & 1.74 & 8.59 & 6.79 & 1.83 & 8.62 & 0.10 & 0.90 & 1.00 \\
\hline 1994 & 6.66 & 1.73 & 8.39 & 7.06 & 1.93 & 9.00 & 0.10 & 0.84 & 0.94 \\
\hline 1995 & 6.56 & 1.76 & 8.32 & 7.23 & 1.61 & 8.84 & 0.10 & 0.86 & 0.95 \\
\hline 1996 & 6.47 & 1.83 & 8.30 & 7.51 & 1.97 & 9.48 & 0.11 & 0.87 & 0.98 \\
\hline 1997 & 6.45 & 1.82 & 8.27 & 8.23 & 1.94 & 10.16 & 0.11 & 0.90 & 1.00 \\
\hline 1998 & 6.25 & 1.76 & 8.01 & 8.71 & 2.00 & 10.71 & 0.11 & 0.84 & 0.95 \\
\hline 1999 & 5.88 & 1.85 & 7.73 & 8.73 & 2.12 & 10.85 & 0.12 & 0.82 & 0.94 \\
\hline 2000 & 5.82 & 1.91 & 7.73 & 9.07 & 2.39 & 11.46 & 0.05 & 0.99 & 1.04 \\
\hline 2001 & 5.80 & 1.87 & 7.67 & 9.33 & 2.54 & 11.87 & 0.02 & 0.95 & 0.97 \\
\hline 2002 & 5.75 & 1.88 & 7.63 & 9.14 & 2.39 & 11.53 & 0.01 & 0.98 & 0.98 \\
\hline 2003 & 5.68 & 1.72 & 7.40 & 9.67 & 2.60 & 12.26 & 0.01 & 1.01 & 1.03 \\
\hline 2004 & 5.42 & 1.81 & 7.23 & 10.09 & 3.06 & 13.15 & 0.03 & 1.02 & 1.05 \\
\hline 2005 & 5.18 & 1.72 & 6.90 & 10.13 & 3.59 & 13.71 & 0.03 & 1.13 & 1.17 \\
\hline 2006 & 5.10 & 1.74 & 6.84 & 10.12 & 3.59 & 13.71 & 0.03 & 1.29 & 1.32 \\
\hline 2007 & 5.06 & 1.78 & 6.85 & 10.03 & 3.42 & 13.47 & 0.03 & 1.41 & 1.43 \\
\hline 2008 & 4.99 & 1.78 & 6.73 & 9.78 & 3.13 & 12.92 & 0.03 & 1.77 & 1.80 \\
\hline 2009 & 5.31 & 1.89 & 7.20 & 9.06 & 2.67 & 11.73 & 0.04 & 1.98 & 2.03 \\
\hline \multicolumn{10}{|c|}{ Average annual percentage change } \\
\hline 1950-2009 & $0.0 \%$ & $2.7 \%$ & $0.4 \%$ & $6.0 \%$ & $5.1 \%$ & $5.4 \%$ & $-1.8 \%$ & $4.6 \%$ & $3.8 \%$ \\
\hline 1970-2009 & $-2.0 \%$ & $0.4 \%$ & $-1.5 \%$ & $6.6 \%$ & $0.8 \%$ & $4.2 \%$ & $4.7 \%$ & $7.1 \%$ & $7.1 \%$ \\
\hline 1999-2009 & $-1.0 \%$ & $0.2 \%$ & $-0.7 \%$ & $0.4 \%$ & $2.3 \%$ & $0.8 \%$ & $-10.4 \%$ & $9.2 \%$ & $8.0 \%$ \\
\hline
\end{tabular}

Source:

U.S. Department of Energy, Energy Information Administration, Monthly Energy Review, March 2010, Tables 3.1 and $3.3 b$.

${ }^{a}$ Total domestic production includes crude oil, natural gas plant liquids and small amounts of other liquids. 
The U.S. share of the world's petroleum consumption is approximately one-quarter. The United States relies heavily on imported petroleum. Imports accounted for nearly 52\% of U.S. petroleum consumption in 2009.

Table 1.12

Petroleum Production and Consumption and Some Important Percent Shares, 1950-2009

\begin{tabular}{|c|c|c|c|c|c|c|c|c|}
\hline & $\begin{array}{l}\text { Domestic } \\
\text { petroleum } \\
\text { production }^{\mathrm{a}}\end{array}$ & $\begin{array}{c}\text { Net } \\
\text { petroleum } \\
\text { imports }\end{array}$ & $\begin{array}{l}\text { Transportation } \\
\text { petroleum } \\
\text { consumption }\end{array}$ & $\begin{array}{c}\text { U.S. } \\
\text { petroleum } \\
\text { consumption }\end{array}$ & $\begin{array}{c}\text { World } \\
\text { petroleum } \\
\text { consumption }\end{array}$ & \multirow{2}{*}{$\begin{array}{c}\text { Net imports } \\
\text { as a share of } \\
\text { U.S. } \\
\text { consumption } \\
8.4 \%\end{array}$} & $\begin{array}{l}\text { U.S. petroleum } \\
\text { consumption as } \\
\text { a share of world } \\
\text { consumption }\end{array}$ & \multirow{2}{*}{$\begin{array}{l}\text { Transportation } \\
\text { petroleum use as } \\
\text { a share of } \\
\text { domestic } \\
\text { production }\end{array}$} \\
\hline & \multicolumn{5}{|c|}{ (million barrels per day) } & \multicolumn{2}{|c|}{ consumption consumption } & \\
\hline 1950 & 5.91 & 0.55 & 3.36 & 6.46 & $\mathrm{~b}$ & & - & $56.8 \%$ \\
\hline 1955 & 7.58 & 0.88 & 4.46 & 8.46 & $\mathrm{~b}$ & $10.4 \%$ & b & $58.8 \%$ \\
\hline 1960 & 7.99 & 1.62 & $5.15^{-}$ & $9.82^{-}$ & 21.34 & $16.5 \%$ & $46.0 \%$ & $64.5 \%$ \\
\hline 1965 & 9.01 & 2.28 & 6.04 & 11.51 & 31.14 & $19.8 \%$ & $37.0 \%$ & $67.0 \%$ \\
\hline 1970 & 11.30 & 3.16 & $7.78^{\circ}$ & $14.70^{-}$ & 46.81 & $21.5 \%$ & $31.4 \%$ & $68.9 \%$ \\
\hline 1975 & 10.01 & 5.85 & 8.95. & 16.32 & 56.20 & $35.8 \%$ & $29.0 \%$ & $89.4 \%$ \\
\hline 1980 & 10.17 & 6.36 & 9.57 & 17.06 & 63.11 & $37.3 \%$ & $27.0 \%$ & $94.1 \%$ \\
\hline 1981 & 10.18 & 5.40 & 9.49 & 16.06 & 60.94 & $33.6 \%$ & $26.3 \%$ & $93.2 \%$ \\
\hline 1982 & 10.20 & 4.30 & 9.31 & 15.30 & 59.54 & $28.1 \%$ & $25.7 \%$ & $91.2 \%$ \\
\hline 1983 & 10.25 & 4.31 & 9.41 & 15.23 & 58.78 & $28.3 \%$ & $25.9 \%$ & $91.8 \%$ \\
\hline 1984 & 10.51 & 4.72 & 9.71 & 15.73 & 59.82 & $30.0 \%$ & $26.3 \%$ & $92.4 \%$ \\
\hline 1985 & 10.58 & 4.29 & 9.84 & 15.73 & 60.08 & $27.3 \%$ & $26.2 \%$ & $93.0 \%$ \\
\hline 1986 & 10.23 & 5.44 & 10.19 & 16.28 & 61.81 & $33.4 \%$ & $26.3 \%$ & $99.6 \%$ \\
\hline 1987 & 9.94 & 5.91 & 10.50 & 16.67 & 63.10 & $35.5 \%$ & $26.4 \%$ & $105.7 \%$ \\
\hline 1988 & 9.76 & 6.59 & 10.88 & 17.28 & 64.97 & $38.1 \%$ & $26.6 \%$ & $111.4 \%$ \\
\hline 1989 & 9.16 & 7.20 & 10.94 & 17.33 & 66.08 & $41.6 \%$ & $26.2 \%$ & $119.4 \%$ \\
\hline 1990 & 8.91 & 7.16 & 10.89 & 16.99 & 66.63 & $42.2 \%$ & $25.5 \%$ & $122.2 \%$ \\
\hline 1991 & 9.08 & 6.63 & 10.76 & 16.71 & 67.22 & $39.6 \%$ & $24.9 \%$ & $118.5 \%$ \\
\hline 1992 & 8.87 & 6.94 & 10.91 & 17.03 & 67.39 & $40.8 \%$ & $25.3 \%$ & $123.0 \%$ \\
\hline 1993 & 8.58 & 7.62 & 11.12 & 17.24 & 67.51 & $44.2 \%$ & $25.5 \%$ & $129.7 \%$ \\
\hline 1994 & 8.39 & 8.05 & 11.13 & 17.72 & 68.78 & $45.5 \%$ & $25.8 \%$ & $132.6 \%$ \\
\hline 1995 & 8.32 & 7.89 & 11.61 & 17.73 & 69.99 & $44.5 \%$ & $25.3 \%$ & $139.5 \%$ \\
\hline 1996 & 8.30 & 8.50 & 11.91 & 18.31 & 71.54 & $46.4 \%$ & $25.6 \%$ & $143.5 \%$ \\
\hline 1997 & 8.27 & 9.16 & 12.05 & 18.62 & 73.43 & $49.2 \%$ & $25.4 \%$ & $145.7 \%$ \\
\hline 1998 & 8.01 & 9.76 & 12.36 & 18.92 & 74.07 & $51.6 \%$ & $25.5 \%$ & $154.3 \%$ \\
\hline 1999 & 7.73 & 9.91 & 12.70 & 19.52 & 75.58 & $50.8 \%$ & $25.8 \%$ & $164.3 \%$ \\
\hline 2000 & 7.73 & 10.42 & 12.98 & 19.70 & 76.74 & $52.9 \%$ & $25.7 \%$ & $167.9 \%$ \\
\hline 2001 & 7.67 & 10.90 & 12.86 & 19.65 & 77.44 & $55.5 \%$ & $25.4 \%$ & $167.7 \%$ \\
\hline 2002 & 7.63 & 10.55 & 13.12 & 19.76 & 78.12 & $53.4 \%$ & $25.3 \%$ & $172.0 \%$ \\
\hline 2003 & 7.40 & 11.24 & 13.20 & 20.03 & 79.68 & $56.1 \%$ & $25.1 \%$ & $178.4 \%$ \\
\hline 2004 & 7.23 & 12.10 & 13.61 & 20.73 & 82.46 & $58.4 \%$ & $25.1 \%$ & $188.2 \%$ \\
\hline 2005 & 6.90 & 12.55 & 13.79 & 20.80 & 84.04 & $60.3 \%$ & $24.8 \%$ & $200.0 \%$ \\
\hline 2006 & 6.84 & 12.39 & 13.95 & 20.69 & 85.20 & $59.9 \%$ & $24.3 \%$ & $203.9 \%$ \\
\hline 2007 & 6.85 & 12.04 & 14.00 & 20.68 & 86.14 & $58.2 \%$ & $24.0 \%$ & $204.5 \%$ \\
\hline 2008 & 6.73 & 11.11 & 13.36 & 19.50 & 85.75 & $57.0 \%$ & $22.7 \%$ & $198.4 \%$ \\
\hline 2009 & 7.20 & 9.70 & 12.91 & 18.69 & 84.04 & $51.9 \%$ & $22.2 \%$ & $179.4 \%$ \\
\hline & & & Average ar & nual percenta & ge change & & & \\
\hline 1950-2009 & $0.3 \%$ & $5.0 \%$ & $2.3 \%$ & $1.8 \%$ & b & & & \\
\hline 1970-2009 & $-1.1 \%$ & $2.9 \%$ & $1.3 \%$ & $0.6 \%$ & $1.5 \%$ & & & \\
\hline 1999-2009 & $-0.7 \%$ & $-0.2 \%$ & $0.2 \%$ & $-0.4 \%$ & $1.1 \%$ & & & \\
\hline
\end{tabular}

Sources:

U.S. Department of Energy, Energy Information Administration, Monthly Energy Review, March 2010, Tables 2.5, 3.1, and A3. (Pre-1973 data from the Annual Energy Review). World petroleum consumption - U.S. Department of Energy, Energy Information Administration, International Petroleum Monthly, March 2010, Table 1.7 and annual. (Additional resources: www.eia.doe.gov)

\footnotetext{
${ }^{\text {a }}$ Total domestic production includes crude oil, natural gas plant liquids and small amounts of other liquids.

${ }^{\mathrm{b}}$ Data are not available.
} 
Before 1989 the U.S. produced enough petroleum to meet the needs of the transportation sector, but was still short of meeting the petroleum needs of all the sectors, including industrial, residential and commercial, and electric utilities. In 1973 the gap between what the U.S. produced and what was consumed was 5.6 million barrels per day. By 2035, the gap is expected to be at least 10.8 million barrels per day if all sources of petroleum are included or 13.9 million barrels per day if only conventional petroleum sources are used.

Figure 1.7. United States Petroleum Production and Consumption - All Sectors, 1973-2035

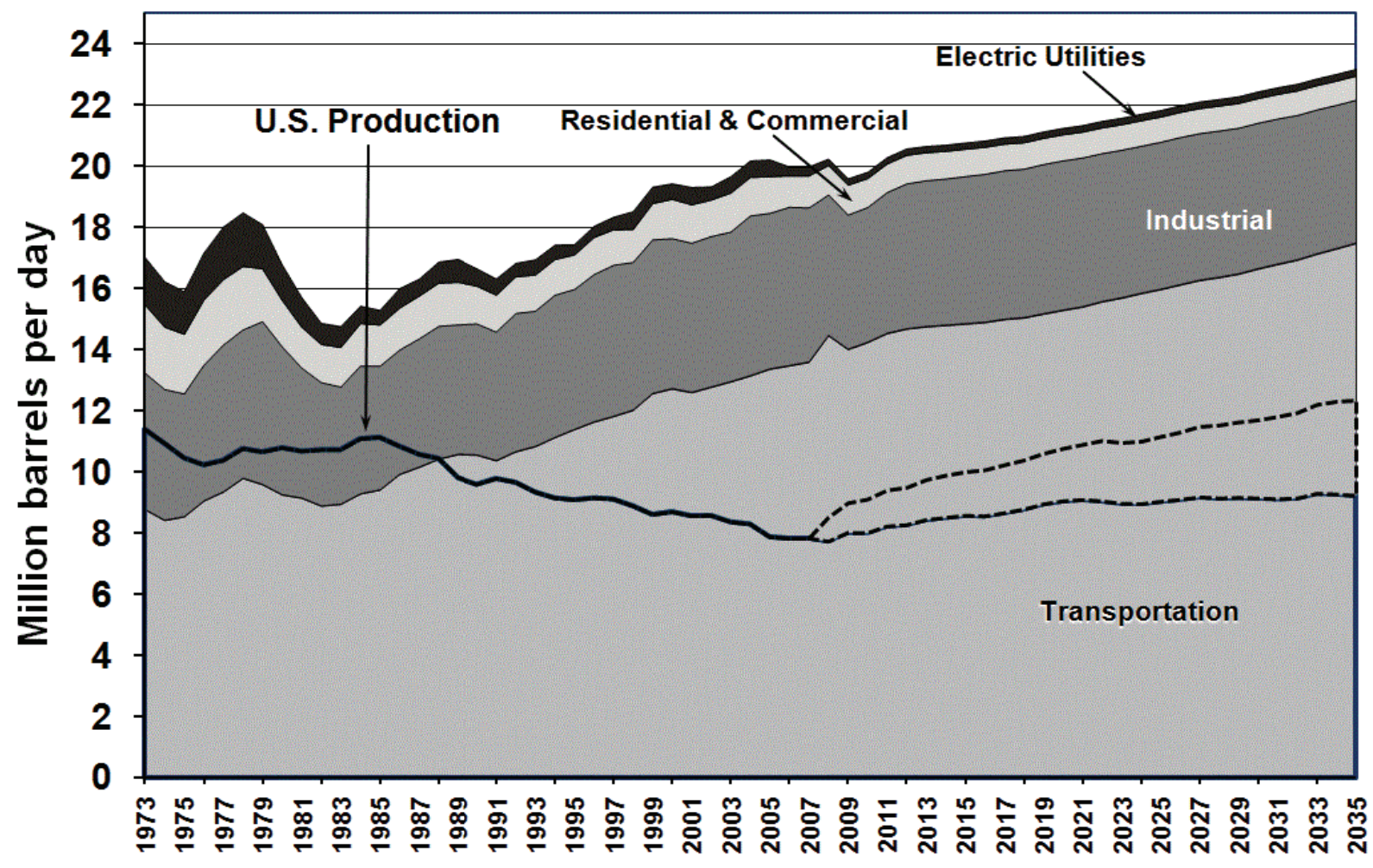

\section{Source:}

See Tables 1.12 and 2.6. Projections are from the Energy Information Administration, Annual Energy Outlook 2010, March 2010.

Notes: The U.S. Production has two lines after 2005. The solid line is conventional sources of petroleum, including crude oil, natural gas plant liquids, and refinery gains. The dashed line adds in other non-petroleum sources, including ethanol, biomass, liquids from coal, other blending components, other hydrocarbons, and ethers.

The sharp increase in values between 2007 and 2008 are caused by the data change from historical to projected values. 
In 1989 the transportation sector petroleum consumption surpassed U.S. petroleum production for the first time, creating a gap that must be met with imports of petroleum. By the year 2035, transportation petroleum consumption is expected to grow to more than 17 million barrels per day; at that time, the gap between U.S. production and transportation consumption will be about 5 million barrels per day (when including the nonpetroleum sources).

Figure 1.8. United States Petroleum Production, Transportation and Consumption, 1970-2035

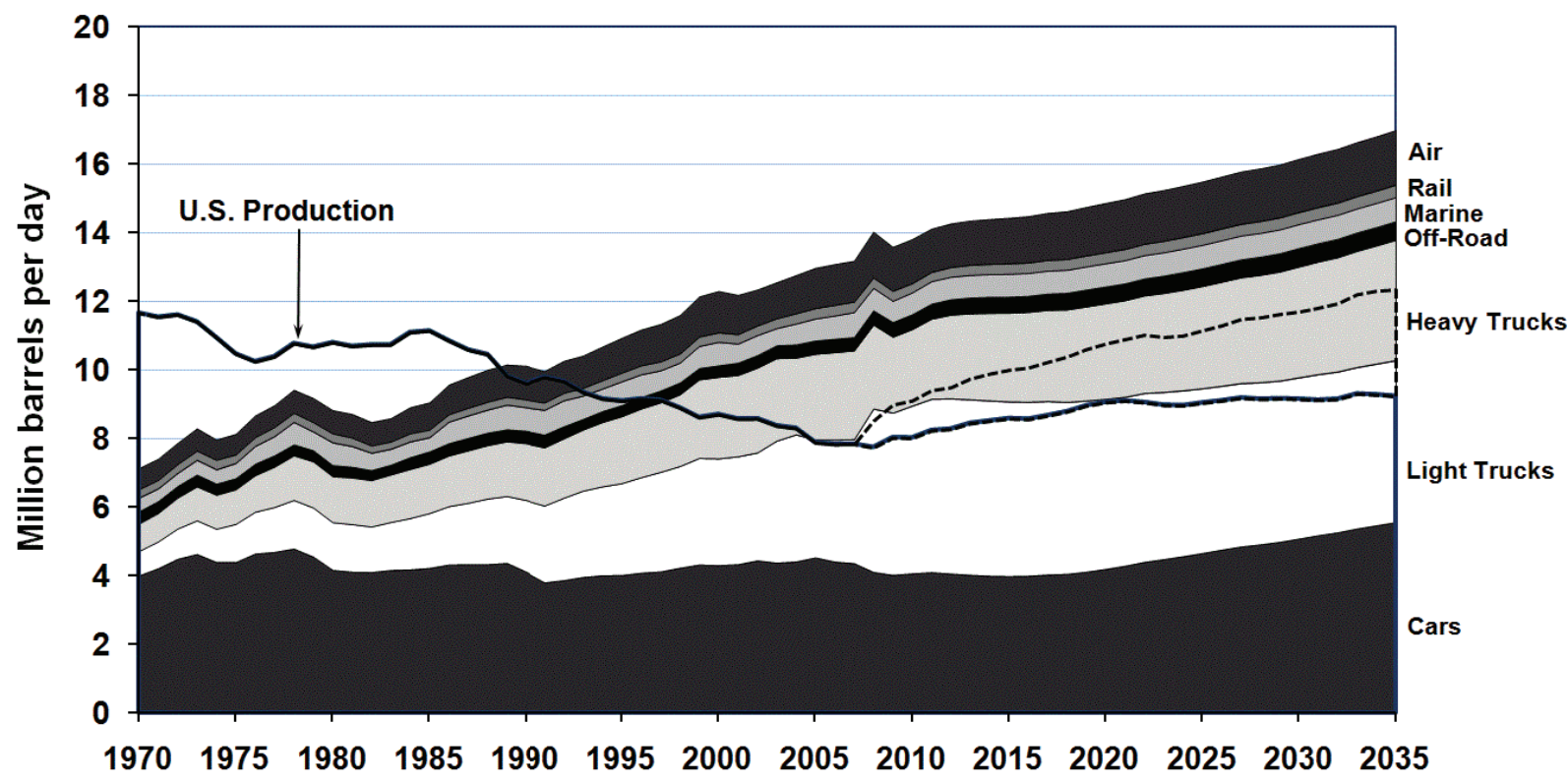

\section{Source:}

See Tables 1.12 and 2.6. Projections are from the Energy Information Administration, Annual Energy Outlook 2010, March 2010.

Notes: The U.S. Production has two lines after 2005. The solid line is conventional sources of petroleum, including crude oil, natural gas plant liquids, and refinery gains. The dashed line adds in other non-petroleum sources, including ethanol, biomass, liquids from coal, other blending components, other hydrocarbons, and ethers.

The sharp increase in values between 2007 and 2008 are caused by the data change from historical to projected values. 
Transportation accounted for more than $70 \%$ of the U.S. petroleum use in 2009. Total petroleum consumption reached more than 20 million barrels per day from 2004 to 2007, but has decreased for the last two years.

Table 1.13

Consumption of Petroleum by End-Use Sector, 1973-2009 (million barrels per day)

\begin{tabular}{|c|c|c|c|c|c|c|c|}
\hline Year & Transportation & Percentage & Residential & Commercial & Industrial & $\begin{array}{l}\text { Electric } \\
\text { utilities }\end{array}$ & Total \\
\hline 1973 & 9.05 & $52.3 \%$ & 1.49 & 0.75 & 4.48 & 1.54 & 17.31 \\
\hline 1974 & 8.84 & $53.1 \%$ & 1.36 & 0.68 & 4.30 & 1.48 & 16.65 \\
\hline 1975 & 8.95 & $54.8 \%$ & 1.32 & 0.63 & 4.04 & 1.39 & 16.32 \\
\hline 1976 & 9.40 & $53.7 \%$ & 1.43 & 0.70 & 4.46 & 1.52 & 17.51 \\
\hline 1977 & 9.76 & $53.0 \%$ & 1.42 & 0.72 & 4.82 & 1.71 & 18.43 \\
\hline 1978 & 10.16 & $53.9 \%$ & 1.38 & 0.69 & 4.87 & 1.75 & 18.85 \\
\hline 1979 & 10.01 & $54.0 \%$ & 1.09 & 0.63 & 5.34 & 1.44 & 18.51 \\
\hline 1980 & 9.57 & $56.0 \%$ & 0.91 & 0.61 & 4.86 & 1.15 & 17.10 \\
\hline 1981 & 9.49 & $59.1 \%$ & 0.81 & 0.52 & 4.27 & 0.96 & 16.06 \\
\hline 1982 & 9.31 & $60.9 \%$ & 0.76 & 0.48 & 4.06 & 0.69 & 15.30 \\
\hline 1983 & 9.41 & $61.8 \%$ & 0.74 & 0.55 & 3.85 & 0.68 & 15.23 \\
\hline 1984 & 9.62 & $61.0 \%$ & 0.78 & 0.61 & 4.21 & 0.56 & 15.77 \\
\hline 1985 & 9.84 & $62.6 \%$ & 0.84 & 0.50 & 4.07 & 0.48 & 15.72 \\
\hline 1986 & 10.19 & $62.6 \%$ & 0.82 & 0.54 & 4.09 & 0.64 & 16.28 \\
\hline 1987 & 10.50 & $63.0 \%$ & 0.87 & 0.53 & 4.21 & 0.55 & 16.67 \\
\hline 1988 & 10.88 & $62.7 \%$ & 0.90 & 0.52 & 4.36 & 0.69 & 17.34 \\
\hline 1989 & 10.94 & $62.8 \%$ & 0.90 & 0.49 & 4.33 & 0.75 & 17.41 \\
\hline 1990 & 10.89 & $64.7 \%$ & 0.77 & 0.47 & 4.15 & 0.57 & 16.84 \\
\hline 1991 & 10.76 & $63.2 \%$ & 0.77 & 0.44 & 4.53 & 0.53 & 17.03 \\
\hline 1992 & 10.91 & $64.2 \%$ & 0.78 & 0.42 & 4.45 & 0.44 & 17.00 \\
\hline 1993 & 11.12 & $63.8 \%$ & 0.80 & 0.38 & 4.64 & 0.50 & 17.44 \\
\hline 1994 & 11.13 & $64.2 \%$ & 0.78 & 0.39 & 4.57 & 0.47 & 17.33 \\
\hline 1995 & 11.61 & $64.9 \%$ & 0.77 & 0.36 & 4.83 & 0.33 & 17.90 \\
\hline 1996 & 11.91 & $64.6 \%$ & 0.84 & 0.37 & 4.96 & 0.36 & 18.44 \\
\hline 1997 & 12.05 & $65.2 \%$ & 0.81 & 0.35 & 4.86 & 0.41 & 18.47 \\
\hline 1998 & 12.36 & $65.5 \%$ & 0.74 & 0.33 & 4.84 & 0.58 & 18.86 \\
\hline 1999 & 12.70 & $65.3 \%$ & 0.85 & 0.34 & 5.03 & 0.53 & 19.46 \\
\hline 2000 & 12.98 & $65.9 \%$ & 0.90 & 0.38 & 4.92 & 0.51 & 19.68 \\
\hline 2001 & 12.86 & $65.7 \%$ & 0.88 & 0.38 & 4.89 & 0.56 & 19.57 \\
\hline 2002 & 13.12 & $66.7 \%$ & 0.85 & 0.35 & 4.93 & 0.43 & 19.67 \\
\hline 2003 & 13.20 & $66.3 \%$ & 0.89 & 0.39 & 4.90 & 0.53 & 19.91 \\
\hline 2004 & 13.61 & $66.0 \%$ & 0.88 & 0.38 & 5.23 & 0.54 & 20.64 \\
\hline 2005 & 13.79 & $66.8 \%$ & 0.83 & 0.36 & 5.10 & 0.55 & 20.63 \\
\hline 2006 & 13.95 & $68.2 \%$ & 0.71 & 0.32 & 5.19 & 0.29 & 20.45 \\
\hline 2007 & 14.00 & $68.7 \%$ & 0.73 & 0.31 & 5.05 & 0.29 & 20.39 \\
\hline 2008 & 13.36 & $69.8 \%$ & 0.67 & 0.29 & 4.60 & 0.21 & 19.13 \\
\hline 2009 & 12.91 & $71.4 \%$ & 0.69 & 0.29 & 4.02 & 0.17 & 18.08 \\
\hline \multicolumn{8}{|c|}{ Average annual percentage change } \\
\hline 1973-2009 & $1.0 \%$ & & $-2.1 \%$ & $-2.6 \%$ & $-0.3 \%$ & $-5.9 \%$ & $0.1 \%$ \\
\hline 1999-2009 & $0.2 \%$ & & $-2.1 \%$ & $-1.6 \%$ & $-2.2 \%$ & $-10.4 \%$ & $-0.8 \%$ \\
\hline
\end{tabular}

\section{Source:}

U.S. Department of Energy, Energy Information Administration, Monthly Energy Review, March 2010, Tables 2.22.6. Converted to million barrels per day using Table A3. (Additional resources: www.eia.doe.gov) 
The highway sector used over 13 million barrels of petroleum per day in 2008, mostly in light vehicles. Light trucks include pick-ups, minivans, sport-utility vehicles, and vans. See Table 2.7 for highway energy use in trillion Btu.

Table 1.14

Highway Transportation Petroleum Consumption by Mode, 1970-2008 ${ }^{\text {a }}$ (thousand barrels per day)

\begin{tabular}{|c|c|c|c|c|c|c|c|c|}
\hline Year & Autos & $\begin{array}{l}\text { Light } \\
\text { trucks }\end{array}$ & $\begin{array}{c}\text { Light } \\
\text { vehicles } \\
\text { subtotal }\end{array}$ & $\begin{array}{l}\text { Motor- } \\
\text { cycles }\end{array}$ & Buses & $\begin{array}{l}\text { Heavy } \\
\text { trucks }\end{array}$ & $\begin{array}{l}\text { Highway } \\
\text { subtotal }\end{array}$ & $\begin{array}{c}\text { Total } \\
\text { transportation }\end{array}$ \\
\hline 1970 & 4,424 & 803 & 5,227 & 4 & 62 & 738 & 6,031 & 7,333 \\
\hline 1975 & 4,836 & 1,245 & 6,081 & 7 & 58 & $952^{\circ}$ & 7,099 & 8,472 \\
\hline 1976 & 5,107 & 1,359 & 6,466 & 8 & 63 & 1,005 & 7,542 & 8,969 \\
\hline 1977 & 5,157 & 1,460 & 6,617 & 8 & 65 & 1,114 & 7,805 & 9,314 \\
\hline 1978 & 5,261 & 1,576 & 6,837 & 9 & 66 & 1,247 & 8,160 & 9,793 \\
\hline 1979 & 4,996 & 1,595 & 6,591 & 11 & 68 & 1,299 & 7,969 & 9,725 \\
\hline 1980 & 4,565 & 1,552 & 6,117 & 13 & 68 & 1,302 & 7,500 & 9,118 \\
\hline 1981 & 4,508 & 1,546 & 6,054 & 14 & 69 & 1,329 & 7,466 & 9,175 \\
\hline 1982 & 4,509 & 1,481 & 5,989 & 13 & 71 & 1,330 & 7,403 & 8,944 \\
\hline 1983 & 4,587 & 1,562 & 6,149 & 11 & 72 & 1,354 & 7,586 & 9,077 \\
\hline 1984 & 4,609 & 1,670 & 6,280 & 11 & 69 & 1,398 & 7,758 & 9,364 \\
\hline 1985 & 4,665 & 1,785 & 6,450 & 12 & 72 & 1,396 & 7,930 & 9,536 \\
\hline 1986 & 4,773 & 1,897 & 6,670 & 12 & 76 & 1,426 & 8,184 & 9,896 \\
\hline 1987 & 4,782 & 1,996 & 6,778 & 12 & 77 & 1,469 & 8,336 & 10,111 \\
\hline 1988 & 4,784 & 2,130 & 6,914 & 13 & 80 & 1,495 & 8,503 & 10,343 \\
\hline 1989 & 4,821 & 2,170 & 6,992 & 14 & 79 & 1,534 & 8,618 & 10,505 \\
\hline 1990 & 4,538 & 2,323 & 6,861 & 12 & 78 & 1,597 & 8,549 & 10,440 \\
\hline 1991 & 4,196 & 2,493 & 6,688 & 12 & 83 & 1,630 & 8,413 & 10,257 \\
\hline 1992 & 4,268 & 2,670 & 6,938 & 12 & 87 & 1,660 & 8,698 & 10,595 \\
\hline 1993 & 4,374 & 2,795 & 7,169 & 13 & 86 & 1,711 & 8,979 & 10,820 \\
\hline 1994 & 4,428 & 2,878 & 7,305 & 13 & 86 & 1,806 & 9,211 & 11,089 \\
\hline 1995 & 4,440 & 2,975 & 7,415 & 13 & 87 & 1,881 & 9,396 & 11,346 \\
\hline 1996 & 4,515 & 3,089 & 7,604 & 13 & 88 & 1,931 & 9,636 & 11,601 \\
\hline 1997 & 4,559 & 3,222 & 7,781 & 13 & 91 & 1,949 & 9,834 & 11,776 \\
\hline 1998 & 4,677 & 3,292 & 7,969 & 13 & 93 & 2,012 & 10,086 & 12,060 \\
\hline 1999 & 4,780 & 3,448 & 8,228 & 14 & 96 & 2,212 & 10,550 & 12,639 \\
\hline 2000 & 4,766 & 3,453 & 8,219 & 14 & 98 & 2,298 & 10,630 & 12,791 \\
\hline 2001 & 4,798 & 3,491 & 8,290 & 13 & 93 & 2,295 & 10,690 & 12,671 \\
\hline 2002 & 4,923 & 3,602 & 8,525 & 12 & 91 & 2,401 & 11,029 & 12,938 \\
\hline 2003 & 4,866 & 3,963 & 8,829 & 12 & 90 & 2,334 & 11,265 & 13,107 \\
\hline 2004 & 4,919 & 4,137 & 9,055 & 13 & 92 & 2,162 & 11,323 & 13,343 \\
\hline 2005 & 5,050 & 3,840 & 8,890 & 12 & 93 & 2,426 & 11,422 & 13,536 \\
\hline 2006 & 4,893 & 3,959 & 8,852 & 14 & 94 & 2,476 & 11,436 & 13,604 \\
\hline 2007 & 4,852 & 4,034 & 8,885 & 16 & 92 & 2,517 & 11,511 & 13,717 \\
\hline 2008 & 4,664 & 3,992 & 8,656 & 17 & 95 & 2,394 & 11,162 & 13,149 \\
\hline \multicolumn{9}{|c|}{ Average annual percentage change } \\
\hline 1970-2008 & $0.1 \%$ & $4.3 \%$ & $1.3 \%$ & $3.9 \%$ & $1.1 \%$ & $3.1 \%$ & $1.6 \%$ & $1.5 \%$ \\
\hline 1998-2008 & $0.0 \%$ & $1.9 \%$ & $0.8 \%$ & $2.7 \%$ & $0.2 \%$ & $1.8 \%$ & $1.0 \%$ & $0.9 \%$ \\
\hline
\end{tabular}

Source:

See Appendix A for Highway Energy Use.

${ }^{a}$ Each gallon of petroleum product was assumed to equal one gallon of crude oil. The oil used to produce electricity is also estimated. See Appendix A, p. 18 for details.

${ }^{b}$ Total transportation figures do not include military and off-highway energy use and may not include all possible uses of fuel for transportation (e.g., snowmobiles). 
Although about $20 \%$ of transportation energy use is for nonhighway modes, only $16 \%$ of transportation petroleum use is for nonhighway. This is because some nonhighway modes, such as pipelines and transit rail, use electricity. An estimate for the petroleum used to make electricity is included in the data. See Table 2.8 for nonhighway transportation energy use in trillion Btu.

Table 1.15

Nonhighway Transportation Petroleum Consumption by Mode, 1970-2008 ${ }^{\text {a }}$ (thousand barrels per day)

\begin{tabular}{|c|c|c|c|c|c|c|}
\hline Year & Air & Water & Pipeline & Rail & $\begin{array}{c}\text { Nonhighway } \\
\text { subtotal }\end{array}$ & $\begin{array}{c}\text { Total } \\
\text { transportation }\end{array}$ \\
\hline 1970 & 625 & 381 & 43 & 253 & 1,302 & 7,333 \\
\hline 1975 & 651 & 423 & 50 & 249 & 1,373 & 8,472 \\
\hline 1980 & 697 & 625 & 35 & 262 & 1,618 & 9,118 \\
\hline 1981 & 706 & 722 & 29 & 253 & 1,709 & 9,175 \\
\hline 1982 & 701 & 604 & 21 & 214 & 1,541 & 8,944 \\
\hline 1983 & 699 & 561 & 19 & 212 & 1,490 & 9,077 \\
\hline 1984 & 781 & 577 & 16 & 232 & 1,606 & 9,364 \\
\hline 1985 & 814 & 564 & 13 & 216 & 1,606 & 9,536 \\
\hline 1986 & 884 & 601 & 17 & 210 & 1,712 & 9,896 \\
\hline 1987 & 920 & 626 & 15 & 213 & 1,775 & 10,111 \\
\hline 1988 & 958 & 644 & 18 & 220 & 1,840 & 10,343 \\
\hline 1989 & 960 & 688 & 18 & 221 & 1,887 & 10,505 \\
\hline 1990 & 1,006 & 655 & 14 & 216 & 1,891 & 10,440 \\
\hline 1991 & 940 & 690 & 12 & 202 & 1,845 & 10,257 \\
\hline 1992 & 954 & 724 & 10 & 208 & 1,897 & 10,595 \\
\hline 1993 & 961 & 653 & 11 & 215 & 1,841 & 10,820 \\
\hline 1994 & 1,002 & 635 & 11 & 230 & 1,878 & 11,089 \\
\hline 1995 & 1,036 & 668 & 7 & 239 & 1,950 & 11,346 \\
\hline 1996 & 1,068 & 644 & 8 & 245 & 1,965 & 11,601 \\
\hline 1997 & 1,114 & 574 & 9 & 246 & 1,942 & 11,776 \\
\hline 1998 & 1,148 & 566 & 12 & 248 & 1,974 & 12,060 \\
\hline 1999 & 1,196 & 625 & 11 & 257 & 2,089 & 12,639 \\
\hline 2000 & 1,234 & 662 & 10 & 256 & 2,162 & 12,791 \\
\hline 2001 & 1,167 & 546 & 11 & 257 & 1,982 & 12,671 \\
\hline 2002 & 1,071 & 572 & 8 & 257 & 1,909 & 12,938 \\
\hline 2003 & 1,073 & 496 & 10 & 263 & 1,843 & 13,107 \\
\hline 2004 & 1,136 & 596 & 10 & 278 & 2,021 & 13,343 \\
\hline 2005 & 1,199 & 625 & 10 & 281 & 2,115 & 13,536 \\
\hline 2006 & 1,216 & 661 & 5 & 286 & 2,168 & 13,604 \\
\hline 2007 & 1,215 & 709 & 5 & 277 & 2,206 & 13,717 \\
\hline 2008 & 1,159 & 560 & 4 & 265 & 1,988 & 13,149 \\
\hline \multicolumn{7}{|c|}{ Average annual percentage change } \\
\hline 1970-2008 & $1.6 \%$ & $1.0 \%$ & $-6.1 \%$ & $0.1 \%$ & $1.1 \%$ & $1.5 \%$ \\
\hline 1998-2008 & $0.1 \%$ & $-0.1 \%$ & $-10.4 \%$ & $0.7 \%$ & $0.1 \%$ & $0.9 \%$ \\
\hline
\end{tabular}

Source:

See Appendix A for Nonhighway Energy Use.

${ }^{a}$ Each gallon of petroleum product was assumed to equal one gallon of crude oil. The oil used to produce electricity is also estimated. See Appendix A, p. 18 for details.

${ }^{b}$ Total transportation figures do not include military and off-highway energy use and may not include all possible uses of fuel for transportation (e.g., snowmobiles). 
Highway vehicles were responsible for over $84 \%$ of all transportation petroleum use in 2008. See Table 2.6 for transportation energy use in trillion Btu.

Table 1.16

Transportation Petroleum Use by Mode, 2007-2008

\begin{tabular}{|c|c|c|c|c|}
\hline & \multicolumn{2}{|c|}{ Thousand barrels per day } & \multicolumn{2}{|c|}{ Percentage of total } \\
\hline & 2007 & 2008 & 2007 & 2008 \\
\hline HIGHWAY & $11,510.8$ & $11,161.6$ & $83.9 \%$ & $84.9 \%$ \\
\hline Light vehicles & $8,901.2$ & $8,672.7$ & $64.9 \%$ & $66.0 \%$ \\
\hline Cars & $4,851.7$ & $4,663.9$ & $35.4 \%$ & $35.5 \%$ \\
\hline Light trucks $^{\mathrm{b}}$ & $4,033.7$ & $3,992.1$ & $29.4 \%$ & $30.4 \%$ \\
\hline Motorcycles & 15.8 & 16.7 & $0.1 \%$ & $0.1 \%$ \\
\hline Buses & 92.4 & 94.8 & $0.7 \%$ & $0.7 \%$ \\
\hline Transit & 43.8 & 45.2 & $0.3 \%$ & $0.3 \%$ \\
\hline Intercity & 14.0 & 14.3 & $0.1 \%$ & $0.1 \%$ \\
\hline School & 34.6 & 35.3 & $0.3 \%$ & $0.3 \%$ \\
\hline Medium/heavy trucks & $2,517.2$ & $2,394.2$ & $18.4 \%$ & $18.2 \%$ \\
\hline NONHIGHWAY & $2,205.7$ & $1,987.8$ & $16.1 \%$ & $15.1 \%$ \\
\hline Air & $1,214.9$ & $1,158.6$ & $8.9 \%$ & $8.8 \%$ \\
\hline General aviation & 120.0 & 130.5 & $0.9 \%$ & $1.0 \%$ \\
\hline Domestic air carriers & 892.5 & 826.3 & $6.5 \%$ & $6.3 \%$ \\
\hline International air & 202.4 & 201.8 & $1.5 \%$ & $1.5 \%$ \\
\hline Water & 708.7 & 560.0 & $5.2 \%$ & $4.3 \%$ \\
\hline Freight & 582.7 & 434.1 & $4.2 \%$ & $3.3 \%$ \\
\hline Recreational & 126.0 & 125.8 & $0.9 \%$ & $1.0 \%$ \\
\hline Pipeline & 5.3 & 3.8 & $0.0 \%$ & $0.0 \%$ \\
\hline Rail & 276.9 & 265.5 & $2.0 \%$ & $2.0 \%$ \\
\hline Freight (Class I) & 266.6 & 255.1 & $1.9 \%$ & $1.9 \%$ \\
\hline Passenger & 10.3 & 10.3 & $0.1 \%$ & $0.1 \%$ \\
\hline Transit & 1.0 & 0.7 & $0.0 \%$ & $0.0 \%$ \\
\hline Commuter & 5.3 & 5.4 & $0.0 \%$ & $0.0 \%$ \\
\hline Intercity & 4.0 & 4.1 & $0.0 \%$ & $0.0 \%$ \\
\hline HWY \& NONHWY TOTAL ${ }^{c}$ & $13,716.5$ & $13,149.5$ & $100.0 \%$ & $100.0 \%$ \\
\hline Off-Highway & 961.3 & 980.5 & & \\
\hline
\end{tabular}

Source:

See Appendix A for Energy Use Sources.

${ }^{a}$ Each gallon of petroleum product was assumed to equal one gallon of crude oil. The oil used to produce electricity is also estimated. See Appendix A, p. 18 for details.

${ }^{\mathrm{b}}$ Two-axle, four-tire trucks.

${ }^{c}$ Civilian consumption only. Totals may not include all possible uses of fuels for transportation (e.g., snowmobiles). 


\section{Chapter 2 \\ Energy}

Summary Statistics from Tables in this Chapter

\section{Source}

Table 2.1 Transportation share of U.S. energy consumption, 2009

$27.1 \%$

Table 2.2 Petroleum share of transportation energy consumption,

$95.8 \%$ 2009

Table 2.3 Alternative fuel and oxygenate consumption, 2008

$\begin{array}{lrr} & \begin{array}{r}\text { (thousand gasoline } \\ \text { equivalent gallons) }\end{array} & \text { (share) } \\ \text { Ethanol in gasohol } & 6,442,781 & 89.5 \% \\ \text { MTBE } & 0 & 0.0 \% \\ \text { Liquified petroleum gas } & 147,784 & 2.7 \% \\ \text { Compressed natural gas } & 189,358 & 3.5 \% \\ \text { E85 } & 62,464 & 1.1 \% \\ \text { Liquified natural gas } & 25,554 & 0.5 \% \\ \text { Electricity } & 5,050 & 0.1 \%\end{array}$

Table 2.6 Transportation energy use by mode, 2008

(trillion Btu) (share)

Cars

$8,831 \quad 32.9 \%$

Light trucks

$7,572 \quad 28.2 \%$

Medium/heavy trucks

$5,022 \quad 18.7 \%$

Buses

$200 \quad 0.7 \%$

Total Highway

$21,656 \quad 80.7 \%$

Air

$2,393 \quad 8.9 \%$

Water

$1,227 \quad 4.6 \%$

Pipeline

$911 \quad 3.4 \%$

Rail

$634 \quad 2.4 \%$


Petroleum accounted for $36 \%$ of the world's energy use in 2007. Though petroleum is the dominant energy source for both OECD countries and non-OECD countries, the non-OECD countries rely on coal, natural gas, and hydroelectric power more than OECD countries do.

Figure 2.1. World Consumption of Primary Energy, 2007

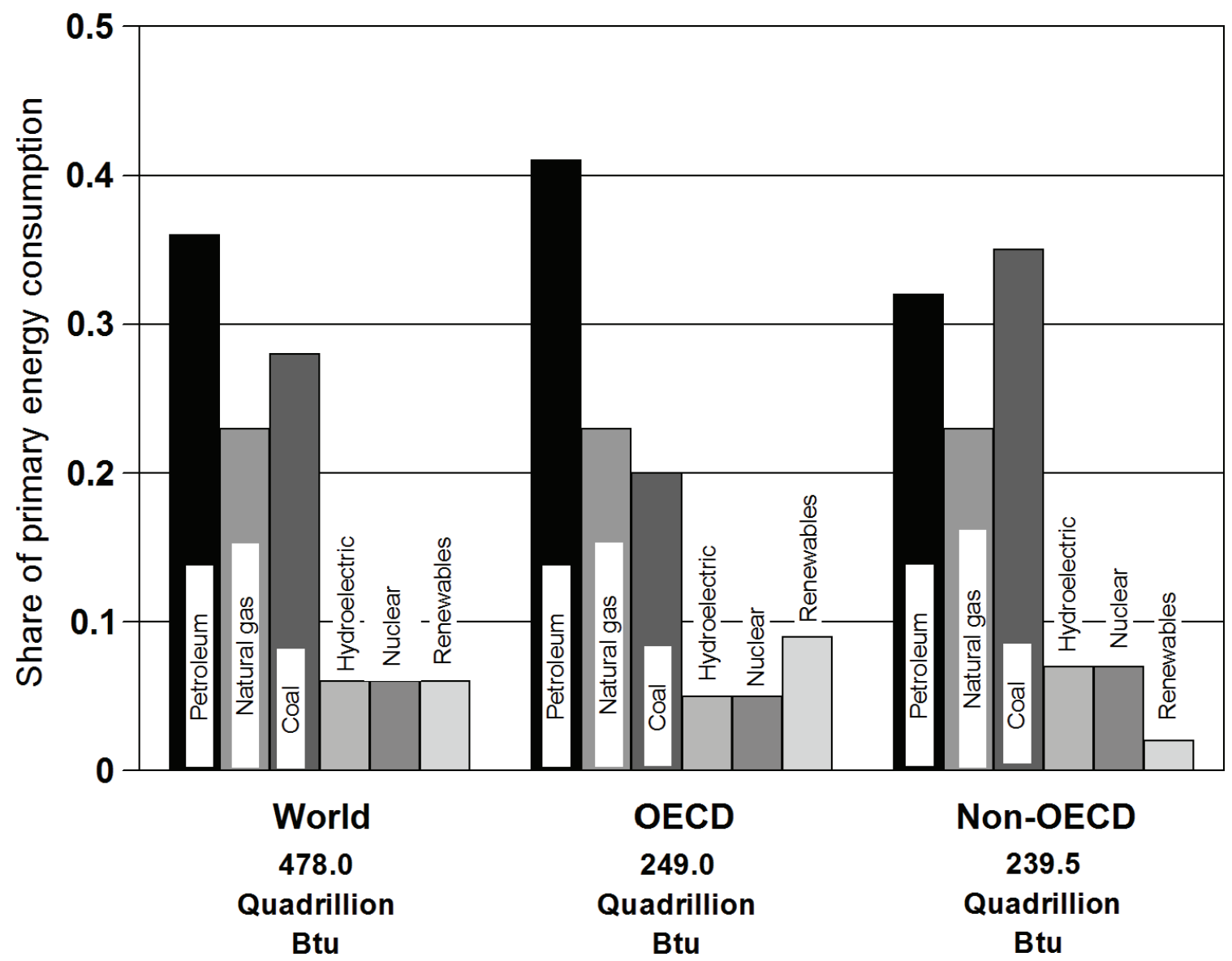

Source:

U.S. Department of Energy, Energy Information Administration, International Energy Statistics Database, May 2010. (Additional resources: www.eia.doe.gov) 
Total energy use was 99.6 quads in 2009 with transportation using 27.1\%. The Energy Information Administration includes renewable energy in each sector.

Table 2.1

\section{U. S. Consumption of Total Energy by End-Use Sector, 1973-2009 (quadrillion Btu)}

\begin{tabular}{|c|c|c|c|c|c|c|}
\hline Year & Transportation & $\begin{array}{c}\text { Percentage } \\
\text { transportation of } \\
\text { total }\end{array}$ & Industrial & Commercial & Residential & Total $^{\mathrm{a}}$ \\
\hline 1973 & 18.6 & $24.6 \%$ & 32.7 & 9.5 & 14.9 & 75.7 \\
\hline 1974 & 18.1 & $24.5 \%$ & 31.8 & 9.4 & 14.7 & 74.0 \\
\hline 1975 & 18.2 & $25.3 \%$ & 29.4 & 9.5 & 14.8 & 72.0 \\
\hline 1976 & 19.1 & $25.1 \%$ & 31.4 & 10.0 & 15.4 & 76.0 \\
\hline 1977 & 19.8 & $25.4 \%$ & 32.3 & 10.2 & 15.7 & 78.0 \\
\hline 1978 & 20.6 & $25.8 \%$ & 32.7 & 10.5 & 16.2 & 80.0 \\
\hline 1979 & 20.5 & $25.3 \%$ & 34.0 & 10.6 & 15.8 & 80.9 \\
\hline 1980 & 19.7 & $25.2 \%$ & 32.1 & 10.6 & 15.8 & 78.1 \\
\hline 1981 & 19.5 & $25.6 \%$ & 30.8 & 10.6 & 15.4 & 76.3 \\
\hline 1982 & 19.1 & $26.1 \%$ & 27.7 & 10.9 & 15.6 & 73.3 \\
\hline 1983 & 19.2 & $26.2 \%$ & 27.5 & 11.0 & 15.5 & 73.1 \\
\hline 1984 & 19.9 & $25.9 \%$ & 29.6 & 11.5 & 15.8 & 76.7 \\
\hline 1985 & 20.1 & $26.3 \%$ & 28.9 & 11.5 & 16.1 & 76.5 \\
\hline 1986 & 20.9 & $27.2 \%$ & 28.4 & 11.5 & 15.9 & 76.8 \\
\hline 1987 & 21.5 & $27.2 \%$ & 29.5 & 12.0 & 16.2 & 79.2 \\
\hline 1988 & 21.4 & $25.8 \%$ & 30.8 & 12.6 & 17.1 & 82.8 \\
\hline 1989 & 22.6 & $26.6 \%$ & 31.4 & 13.2 & 17.8 & 85.0 \\
\hline 1990 & 22.4 & $26.5 \%$ & 31.9 & 13.4 & 17.0 & 84.7 \\
\hline 1991 & 22.2 & $26.2 \%$ & 31.5 & 13.5 & 17.1 & 84.6 \\
\hline 1992 & 22.5 & $26.2 \%$ & 32.7 & 13.4 & 17.4 & 86.0 \\
\hline 1993 & 22.9 & $26.1 \%$ & 36.7 & 13.8 & 18.3 & 87.6 \\
\hline 1994 & 23.5 & $26.3 \%$ & 33.6 & 14.1 & 18.1 & 89.3 \\
\hline 1995 & 23.8 & $26.2 \%$ & 34.0 & 14.7 & 18.5 & 91.2 \\
\hline 1996 & 24.4 & $25.9 \%$ & 35.0 & 15.2 & 19.5 & 94.2 \\
\hline 1997 & 24.7 & $26.1 \%$ & 35.3 & 15.7 & 19.0 & 94.8 \\
\hline 1998 & 25.3 & $26.8 \%$ & 34.9 & 16.0 & 19.0 & 95.2 \\
\hline 1999 & 25.9 & $26.8 \%$ & 34.9 & 16.4 & 19.6 & 96.8 \\
\hline 2000 & 26.5 & $26.8 \%$ & 34.8 & 17.2 & 20.4 & 99.0 \\
\hline 2001 & 26.3 & $27.3 \%$ & 32.8 & 17.2 & 20.1 & 96.3 \\
\hline 2002 & 26.8 & $27.4 \%$ & 32.8 & 17.4 & 20.8 & 97.9 \\
\hline 2003 & 27.0 & $27.5 \%$ & 32.6 & 17.4 & 21.1 & 98.2 \\
\hline 2004 & 27.9 & $27.8 \%$ & 33.6 & 17.7 & 21.1 & 100.4 \\
\hline 2005 & 28.4 & $28.2 \%$ & 32.5 & 17.9 & 21.7 & 100.5 \\
\hline 2006 & 28.8 & $28.9 \%$ & 32.5 & 17.8 & 20.7 & 99.9 \\
\hline 2007 & 29.1 & $28.7 \%$ & 32.6 & 18.3 & 21.6 & 101.6 \\
\hline 2008 & 29.0 & $29.2 \%$ & 31.4 & 18.4 & 21.6 & 99.6 \\
\hline 2009 & 27.0 & $27.1 \%$ & 28.2 & 18.1 & 21.2 & 99.6 \\
\hline \multicolumn{7}{|c|}{ Average annual percentage change } \\
\hline 1973-2009 & $1.0 \%$ & & $-0.4 \%$ & $1.8 \%$ & $1.0 \%$ & $0.8 \%$ \\
\hline 1999-2009 & $0.4 \%$ & & $-2.1 \%$ & $1.0 \%$ & $0.8 \%$ & $0.3 \%$ \\
\hline
\end{tabular}

\section{Source:}

U.S. Department of Energy, Energy Information Administration, Monthly Energy Review, April 2010, Washington, DC, Table 2.1. (Additional resources: www.eia.doe.gov)

\footnotetext{
${ }^{\text {a }}$ Electrical energy losses have been distributed among the sectors.
} 
In transportation, the alcohol fuels blended into gasoline to make gasohol (10\% ethanol or less) are counted under "renewables" and are not in with petroleum. The petroleum category, however, still contains other blending agents, such as MTBE, that are not actually petroleum, but are not broken out into a separate category.

Table 2.2

Distribution of Energy Consumption by Source, 1973 and 2009 (percentage)

\begin{tabular}{|c|c|c|c|c|c|c|}
\hline \multirow{2}{*}{$\begin{array}{l}\text { Energy } \\
\text { source }\end{array}$} & \multicolumn{2}{|c|}{ Transportation } & \multicolumn{2}{|c|}{ Residential } & \multicolumn{2}{|c|}{ Commercial } \\
\hline & 1973 & 2009 & 1973 & 2009 & 1973 & 2009 \\
\hline Petroleum $^{\mathrm{a}}$ & 95.8 & 93.8 & 18.9 & 5.5 & 16.5 & 3.1 \\
\hline Natural gas ${ }^{\mathrm{b}}$ & 4.0 & 2.5 & 33.3 & 22.9 & 27.9 & 17.6 \\
\hline Coal & 0.0 & 0.0 & 0.6 & 0.0 & 1.7 & 0.3 \\
\hline Renewable & 0.0 & 3.4 & 2.4 & 2.8 & 0.1 & 0.7 \\
\hline Nuclear & 0.0 & 0.0 & 0.0 & 0.0 & 0.0 & 0.0 \\
\hline Electricity $^{\mathrm{c}}$ & 0.2 & 0.3 & 44.7 & 68.7 & 53.9 & 78.3 \\
\hline Total & 100.00 & 100.00 & 100.00 & 100.00 & 100.00 & 100.00 \\
\hline
\end{tabular}

\begin{tabular}{lrrrrr} 
Energy & \multicolumn{2}{c}{ Industrial } & & \multicolumn{2}{c}{ Electric utilities } \\
\cline { 2 - 3 } \cline { 5 - 6 } source $^{1973}$ & 2009 & & 1973 & \multicolumn{2}{c}{2009} \\
\hline Petroleum $^{\mathrm{a}}$ & 27.9 & 26.8 & & 17.8 & 1.0 \\
Natural gas $^{\mathrm{b}}$ & 31.8 & 27.1 & & 19.0 & 18.3 \\
Coal & 12.4 & 5.0 & & 43.9 & 48.0 \\
Renewable & 3.7 & 7.3 & & 14.6 & 10.7 \\
Nuclear $_{\text {Electricity }}^{\mathrm{c}}$ & 0.0 & 0.0 & & 4.6 & 21.6 \\
Total & 24.2 & 33.8 & & 0.0 & 0.3 \\
\hline
\end{tabular}

\section{Source:}

U.S. Department of Energy, Energy Information Administration, Monthly Energy Review, March 2010, Washington, DC, Tables 2.2, 2.3, 2.4, 2.5, and 2.6. (Additional resources: www.eia.doe.gov)

Note: Numbers may not add due to rounding.

\footnotetext{
${ }^{\text {a }}$ In transportation, the petroleum category contains some blending agents which are not petroleum.

${ }^{\mathrm{b}}$ Includes supplemental gaseous fuels. Transportation sector includes pipeline fuel and natural gas vehicle use.

${ }^{\mathrm{c}}$ Includes electrical system energy losses.
} 
Oxygenates are blended with gasoline to be used in conventional vehicles. The amount of oxygenate use dwarfs the alternative fuel use. Gasoline-equivalent gallons are used in this table to allow comparisons of different fuel types.

Table 2.3

Alternative Fuel and Oxygenate Consumption, 2003-2008 (thousand gasoline-equivalent gallons)

\begin{tabular}{lrrrrrr}
\hline & 2003 & 2004 & 2005 & 2006 & 2007 & 2008 \\
\hline Alternative fuel & & & & & & \\
Liquified petroleum gas & 224,697 & 211,883 & 188,171 & 173,130 & 152,360 & 147,784 \\
Compressed natural gas & 133,222 & 158,903 & 166,878 & 172,011 & 178,585 & 189,358 \\
Liquified natural gas $^{\text {E85 }}$ & 13,503 & 20,888 & 22,409 & 23,474 & 24,594 & 25,554 \\
Electricity $^{\mathrm{b}}$ & 26,376 & 31,581 & 38,074 & 44,041 & 54,091 & 62,464 \\
Hydrogen $_{\text {Biodiesel }}$ & 5,141 & 5,269 & 5,219 & 5,104 & 5,037 & 5,050 \\
Other & 2 & 8 & 25 & 41 & 66 & 117 \\
Subtotal & 18,220 & 28,244 & 91,649 & 260,606 & 367,764 & 324,329 \\
\hline Oxygenates $_{\text {MTBE }}$ & 0 & 0 & 2 & 2 & 2 & 2 \\
$\quad$ & $\mathbf{4 2 1 , 1 6 1}$ & $\mathbf{4 5 6 , 7 6 6}$ & $\mathbf{5 1 2 , 4 2 7}$ & $\mathbf{6 7 8 , 4 0 9}$ & $\mathbf{7 8 2 , 4 7 9}$ & $\mathbf{7 5 4 , 6 5 8}$ \\
\hline Ethanol in gasohol & & & & & & \\
\hline Total & $2,368,400$ & $1,877,300$ & $1,654,500$ & 435,000 & 0 & 0 \\
\hline
\end{tabular}

\section{Source:}

U.S. Department of Energy, Energy Information Administration, Alternatives to Traditional Transportation Fuels, 2008, Washington, DC, April 2010, Web site www.eia.doe.gov/cneaf/alternate/page/atftables/afv-atf2008.pdf. (Additional resources: www.eia.doe.gov)

${ }^{\mathrm{a}}$ Consumption includes gasoline portion of the mixture.

${ }^{\mathrm{b}}$ Vehicle consumption only; does not include power plant inputs.

${ }^{c}$ Methyl Tertiary Butyl Ether. This category includes a very small amount of other ethers, primarily Tertiary Amyl Methyl Ether (TAME) and Ethyl Tertiary Butyl Ether (ETBE). 
Ethanol is used as an oxygenate, blended with gasoline to be used as gasohol in conventional vehicles. The amount of ethanol used in gasohol dwarfs the amount used in E85. Production of E95 ended in 2000.

Table 2.4

Ethanol Consumption, 1995-2008 (thousand gallons)

\begin{tabular}{lcccc}
\hline & \multicolumn{2}{c}{ Ethanol blends } & & \\
\cline { 2 - 3 } & E85 & E95 & Ethanol in gasohol & Total \\
\hline 1995 & 166 & 970 & 934,615 & 935,751 \\
2000 & 10,530 & 12 & $1,114,313$ & $1,124,855$ \\
2001 & 12,756 & 0 & $1,173,323$ & $1,186,079$ \\
2002 & 15,513 & 0 & $1,450,721$ & $1,466,234$ \\
2003 & 26,376 & 0 & $1,919,572$ & $1,945,948$ \\
2004 & 31,581 & 0 & $2,414,167$ & $2,445,748$ \\
2005 & 38,074 & 0 & $2,756,663$ & $2,794,737$ \\
2006 & 44,041 & 0 & $3,729,168$ & $3,773,209$ \\
2007 & 54,091 & 0 & $4,694,304$ & $4,748,395$ \\
2008 & 62,464 & 0 & $6,442,781$ & $6,505,245$ \\
\hline 2008 Percentage & $1.0 \%$ & $0.0 \%$ & $99.0 \%$ & $0.0 \%$ \\
\hline
\end{tabular}

Source:

U.S. Department of Energy, Energy Information Administration, Alternatives to Traditional Transportation Fuels, 2008, Washington, DC, April 2010, Web site:

http://www.eia.doe.gov/cneaf/alternate/page/atftables/afv-atf2008.pdf, Table C1. (Additional resources: www.eia.doe.gov)

Note: Gallons of E85, E95 and Ethanol in gasohol, do not include the gasoline portion of the blended fuel. 
As data about alternative fuel use become available, an attempt is made to incorporate them into this table. Sometimes assumptions must be made in order to use the data. Please see Appendix A for a description of the methodology used to develop these data. See Table 1.16 for transportation petroleum use in thousand barrels per day.

Table 2.5

Domestic Consumption of Transportation Energy by Mode and Fuel Type, 2008 (trillion Btu)

\begin{tabular}{|c|c|c|c|c|c|c|c|c|}
\hline & Gasoline & $\begin{array}{c}\text { Diesel } \\
\text { fuel }\end{array}$ & $\begin{array}{l}\text { Liquified } \\
\text { petroleum } \\
\text { gas }\end{array}$ & Jet fuel & $\begin{array}{l}\text { Residual } \\
\text { fuel oil }\end{array}$ & $\begin{array}{c}\text { Natural } \\
\text { gas }\end{array}$ & Electricity & Total \\
\hline HIGHWAY & $16,513.8$ & $5,060.8$ & 61.5 & & & 20.4 & 0.6 & $21,657.1$ \\
\hline$\overline{\text { Light vehicles }}$ & $16,001.9$ & 389.1 & 44.7 & & & 0.0 & 0.0 & $16,435.7$ \\
\hline Cars & $8,781.8$ & 49.6 & & & & & & $8,831.4$ \\
\hline Light trucks ${ }^{\mathrm{b}}$ & $7,188.0$ & 339.5 & 44.7 & & & & & $7,572.2$ \\
\hline Motorcycles & 32.0 & & & & & & & 32.0 \\
\hline Buses & 7.2 & 171.5 & 0.0 & & & 20.4 & 0.6 & 199.8 \\
\hline Transit & 0.5 & 73.7 & 0.0 & & & 20.4 & 0.6 & 95.3 \\
\hline Intercity & & 30.3 & & & & & & 30.3 \\
\hline School & 6.8 & 67.5 & & & & & & 74.3 \\
\hline Medium/heavy trucks & 504.7 & $4,500.2$ & 16.8 & & & & & $5,021.6$ \\
\hline NONHIGHWAY & 237.7 & 794.3 & 0.0 & $2,355.6$ & 795.8 & 668.0 & 314.0 & $5,165.4$ \\
\hline Air & 37.7 & 0.0 & $\mathbf{0 . 0}$ & $2,355.6$ & 0.0 & $\mathbf{0 . 0}$ & 0.0 & $2,393.3$ \\
\hline General aviation & 37.7 & & & 228.0 & & & & 265.7 \\
\hline Domestic air carriers & & & & $1,710.0$ & & & & $1,710.0$ \\
\hline International air carriers ${ }^{c}$ & & & & 417.6 & & & & 417.6 \\
\hline Water & 200.0 & 231.5 & & & 795.8 & & & $1,227.2$ \\
\hline Freight & & 185.8 & & & 795.8 & & & 981.6 \\
\hline Recreational & 200.0 & 45.7 & & & & & & 245.7 \\
\hline Pipeline & $\mathbf{0 . 0}$ & 0.0 & 0.0 & 0.0 & 0.0 & 668.0 & 242.5 & 910.5 \\
\hline Rail & 0.0 & 562.9 & 0.0 & 0.0 & 0.0 & 0.0 & 71.5 & 634.4 \\
\hline Freight (Class I) & & 542.5 & & & & & & 542.5 \\
\hline Passenger & & 20.4 & & & & & 71.5 & 91.9 \\
\hline Transit & & 0.0 & & & & & 47.8 & 47.8 \\
\hline Commuter & & 11.6 & & & & & 17.8 & 29.3 \\
\hline Intercity & & 8.8 & & & & & 6.0 & 14.8 \\
\hline $\begin{array}{l}\text { TOTAL HWY \& } \\
\text { NONHWY }\end{array}$ & $16,751.5$ & $5,855.2$ & 61.5 & $2,355.6$ & 795.8 & 688.5 & 314.6 & 26,822.6 \\
\hline
\end{tabular}

Source:

See Appendix A for Energy Use Sources.

${ }^{a}$ Civilian consumption only. Totals may not include all possible uses of fuels for transportation (e.g., snowmobiles).

${ }^{\mathrm{b}}$ Two-axle, four-tire trucks.

${ }^{\mathrm{c}}$ One half of fuel used by domestic carriers in international operation. 
Highway vehicles were responsible for $80.7 \%$ of all transportation energy use in 2008 . See Table 1.16 for transportation energy use in thousand barrels per day.

Table 2.6

Transportation Energy Use by Mode, 2007-2008 ${ }^{a}$

\begin{tabular}{|c|c|c|c|c|}
\hline & \multicolumn{2}{|c|}{ Trillion Btu } & \multicolumn{2}{|c|}{ Percentage of total based on Btus } \\
\hline & 2007 & 2008 & 2007 & 2008 \\
\hline HIGHWAY & $22,405.0$ & $21,657.1$ & $80.0 \%$ & $80.7 \%$ \\
\hline Light vehicles & $16,930.4$ & $16,435.7$ & $60.4 \%$ & $61.3 \%$ \\
\hline Cars & $9,221.0$ & $8,831.4$ & $32.9 \%$ & $32.9 \%$ \\
\hline Light trucks ${ }^{\mathrm{b}}$ & $7,679.2$ & $7,572.2$ & $27.4 \%$ & $28.2 \%$ \\
\hline Motorcycles & 30.3 & 32.0 & $0.1 \%$ & $0.1 \%$ \\
\hline Buses & 194.9 & 199.8 & $0.7 \%$ & $0.7 \%$ \\
\hline Transit & 92.3 & 95.3 & $0.3 \%$ & $0.4 \%$ \\
\hline Intercity & 29.7 & 30.3 & $0.1 \%$ & $0.1 \%$ \\
\hline School & 72.9 & 74.3 & $0.3 \%$ & $0.3 \%$ \\
\hline Medium/heavy trucks & $5,279.6$ & $5,021.6$ & $18.8 \%$ & $18.7 \%$ \\
\hline NONHIGHWAY & $5,608.0$ & $5,165.4$ & $20.0 \%$ & $19.3 \%$ \\
\hline Air & $2,509.5$ & $2,393.3$ & $9.0 \%$ & $8.9 \%$ \\
\hline General aviation & 243.6 & 265.7 & $0.9 \%$ & $1.0 \%$ \\
\hline Domestic air carriers & $1,847.0$ & $1,710.0$ & $6.6 \%$ & $6.4 \%$ \\
\hline International air & 418.8 & 417.6 & $1.5 \%$ & $1.6 \%$ \\
\hline Water & $1,559.3$ & $1,227.2$ & $5.6 \%$ & $4.6 \%$ \\
\hline Freight & $1,313.5$ & 981.6 & $4.7 \%$ & $3.7 \%$ \\
\hline Recreational & 245.8 & 245.7 & $0.9 \%$ & $0.9 \%$ \\
\hline Pipeline & 881.8 & 910.5 & $3.1 \%$ & $3.4 \%$ \\
\hline Rail & 657.5 & 634.4 & $2.3 \%$ & $2.4 \%$ \\
\hline Freight (Class I) & 566.9 & 542.5 & $2.0 \%$ & $2.0 \%$ \\
\hline Passenger & 90.5 & 91.9 & $0.3 \%$ & $0.3 \%$ \\
\hline Transit & 46.6 & 47.8 & $0.2 \%$ & $0.2 \%$ \\
\hline Commuter & 29.4 & 29.3 & $0.1 \%$ & $0.1 \%$ \\
\hline Intercity & 14.5 & 14.8 & $0.1 \%$ & $0.1 \%$ \\
\hline HWY \& NONHWY TOTAL & $28,013.0$ & $26,822.6$ & $100.0 \%$ & $100.0 \%$ \\
\hline Off-Highway & $1,918.0$ & $1,957.9$ & & \\
\hline
\end{tabular}

Source:

See Appendix A for Energy Use Sources.

a Civilian consumption only. Totals may not include all possible uses of fuels for transportation (e.g., snowmobiles).

${ }^{\mathrm{b}}$ Two-axle, four-tire trucks. 
The highway sector is by far the largest part of transportation energy use. Light truck energy use has increased at the greatest rate, due to their increased use as personal passenger vehicles. Light trucks include pick-ups, minivans, sport-utility vehicles, and vans. See Table 1.14 for highway petroleum use in thousand barrels per day.

Table 2.7

Highway Transportation Energy Consumption by Mode, 1970-2008 (trillion Btu)

\begin{tabular}{|c|c|c|c|c|c|c|c|c|}
\hline Year & Autos & $\begin{array}{l}\text { Light } \\
\text { trucks }\end{array}$ & $\begin{array}{c}\text { Light } \\
\text { vehicles } \\
\text { subtotal }\end{array}$ & $\begin{array}{l}\text { Motor- } \\
\text { cycles }\end{array}$ & Buses & $\begin{array}{l}\text { Heavy } \\
\text { trucks }\end{array}$ & $\begin{array}{l}\text { Highway } \\
\text { subtotal }\end{array}$ & $\begin{array}{c}\text { Total } \\
\text { transportation }\end{array}$ \\
\hline 1970 & 8,479 & 1,539 & 10,018 & 7 & 129 & 1,553 & 11,707 & 15,395 \\
\hline 1975 & 9,298 & 2,384 & 11,682 & 14 & 124 & 2,003 & 13,823 & 17,424 \\
\hline 1976 & 9,826 & 2,602 & 12,428 & 15 & 134 & 2,114 & 14,691 & 18,491 \\
\hline 1977 & 9,928 & 2,797 & 12,725 & 16 & 137 & 2,344 & 15,222 & 19,126 \\
\hline 1978 & 10,134 & 3,020 & 13,154 & 18 & 141 & 2,607 & 15,920 & 20,097 \\
\hline 1979 & 9,629 & 3,056 & 12,685 & 22 & 144 & 2,697 & 15,548 & 19,652 \\
\hline 1980 & 8,800 & 2,975 & 11,775 & 26 & 143 & 2,686 & 14,630 & 18,940 \\
\hline 1981 & 8,693 & 2,963 & 11,656 & 27 & 145 & 2,724 & 14,552 & 18,741 \\
\hline 1982 & 8,673 & 2,837 & 11,510 & 25 & 151 & 2,707 & 14,393 & 18,237 \\
\hline 1983 & 8,802 & 2,990 & 11,792 & 22 & 152 & 2,770 & 14,736 & 18,368 \\
\hline 1984 & 8,837 & 3,197 & 12,034 & 22 & 146 & 2,873 & 15,075 & 18,962 \\
\hline 1985 & 8,932 & 3,413 & 12,345 & 23 & 153 & 2,883 & 15,404 & 19,205 \\
\hline 1986 & 9,138 & 3,629 & 12,767 & 23 & 160 & 2,958 & 15,908 & 20,276 \\
\hline 1987 & 9,157 & 3,819 & 12,976 & 24 & 164 & 3,061 & 16,225 & 20,771 \\
\hline 1988 & 9,158 & 4,078 & 13,236 & 25 & 169 & 3,118 & 16,548 & 21,327 \\
\hline 1989 & 9,232 & 4,156 & 13,388 & 26 & 169 & 3,199 & 16,782 & 21,685 \\
\hline 1990 & 8,688 & 4,451 & 13,139 & 24 & 167 & 3,334 & 16,664 & 21,613 \\
\hline 1991 & 8,029 & 4,774 & 12,803 & 23 & 177 & 3,402 & 16,405 & 21,205 \\
\hline 1992 & 8,169 & 5,117 & 13,286 & 24 & 184 & 3,468 & 16,962 & 21,866 \\
\hline 1993 & 8,368 & 5,356 & 13,724 & 25 & 183 & 3,577 & 17,509 & 22,322 \\
\hline 1994 & 8,470 & 5,515 & 13,985 & 26 & 183 & 3,778 & 17,972 & 22,926 \\
\hline 1995 & 8,489 & 5,695 & 14,184 & 25 & 184 & 3,937 & 18,330 & 23,465 \\
\hline 1996 & 8,634 & 5,917 & 14,551 & 24 & 186 & 4,045 & 18,806 & 23,973 \\
\hline 1997 & 8,710 & 6,168 & 14,878 & 25 & 192 & 4,086 & 19,181 & 24,327 \\
\hline 1998 & 8,936 & 6,304 & 15,240 & 26 & 196 & 4,218 & 19,680 & 24,757 \\
\hline 1999 & 9,134 & 6,602 & 15,736 & 26 & 203 & 4,638 & 20,603 & 25,948 \\
\hline 2000 & 9,100 & 6,607 & 15,707 & 26 & 209 & 4,819 & 20,761 & 26,268 \\
\hline 2001 & 9,161 & 6,678 & 15,839 & 24 & 196 & 4,813 & 20,872 & 25,958 \\
\hline 2002 & 9,391 & 6,883 & 16,274 & 24 & 192 & 5,035 & 21,525 & 26,521 \\
\hline 2003 & 9,255 & 7,551 & 16,806 & 24 & 190 & 4,895 & 21,915 & 26,672 \\
\hline 2004 & 9,331 & 7,861 & 17,192 & 25 & 194 & 4,535 & 21,946 & 27,065 \\
\hline 2005 & 9,579 & 7,296 & 16,875 & 24 & 196 & 5,088 & 22,183 & 27,526 \\
\hline 2006 & 9,316 & 7,550 & 16,866 & 28 & 199 & 5,193 & 22,286 & 27,759 \\
\hline 2007 & 9,221 & 7,679 & 16,900 & 30 & 195 & 5,280 & 22,405 & 28,013 \\
\hline 2008 & 8,831 & 7,572 & 16,404 & 32 & 200 & 5,022 & 21,657 & 26,823 \\
\hline \multicolumn{9}{|c|}{ Average annual percentage change } \\
\hline 1970-2008 & $0.1 \%$ & $4.3 \%$ & $1.3 \%$ & $4.1 \%$ & $1.1 \%$ & $3.1 \%$ & $1.6 \%$ & $1.5 \%$ \\
\hline $1998-2008$ & $-0.1 \%$ & $1.9 \%$ & $0.7 \%$ & $2.1 \%$ & $0.2 \%$ & $1.8 \%$ & $1.0 \%$ & $0.8 \%$ \\
\hline
\end{tabular}

Source:

See Appendix A for Highway Energy Use.

Note: Totals may not add due to rounding.

${ }^{\text {a }}$ Total transportation figures do not include military and off-highway energy use and may not include all possible uses of fuel for transportation (e.g., snowmobiles). These data have been revised due to a new data series for recreational boats. 
About $20 \%$ of transportation energy use is for nonhighway modes. Air travel accounts for over $45 \%$ of nonhighway energy use. See Table 1.15 for nonhighway petroleum use in thousand barrels per day.

Table 2.8

Nonhighway Transportation Energy Consumption by Mode, 1970-2008 (trillion Btu)

\begin{tabular}{|c|c|c|c|c|c|c|}
\hline Year & Air & Water & Pipeline & Rail & $\begin{array}{c}\text { Nonhighway } \\
\text { subtotal }\end{array}$ & $\begin{array}{c}\text { Total } \\
\text { transportation }^{\mathrm{a}}\end{array}$ \\
\hline 1970 & 1,307 & 836 & 990 & 555 & 3,688 & 15,395 \\
\hline 1975 & 1,274 & 927 & 840 & 559 & 3,601 & 17,424 \\
\hline 1976 & 1,333 & 1,083 & 803 & 581 & 3,800 & 18,491 \\
\hline 1977 & 1,350 & 1,177 & 786 & 591 & 3,904 & 19,126 \\
\hline 1978 & 1,423 & 1,382 & 784 & 588 & 4,177 & 20,097 \\
\hline 1979 & 1,488 & 1,149 & 860 & 607 & 4,104 & 19,652 \\
\hline 1980 & 1,434 & 1,393 & 896 & 588 & 4,310 & 18,940 \\
\hline 1981 & 1,453 & 1,270 & 904 & 561 & 4,189 & 18,741 \\
\hline 1982 & 1,445 & 1,063 & 855 & 481 & 3,844 & 18,237 \\
\hline 1983 & 1,440 & 974 & 740 & 478 & 3,632 & 18,368 \\
\hline 1984 & 1,609 & 964 & 782 & 532 & 3,887 & 18,962 \\
\hline 1985 & 1,677 & 871 & 755 & 498 & 3,801 & 19,205 \\
\hline 1986 & 1,823 & 1,323 & 735 & 487 & 4,368 & 20,276 \\
\hline 1987 & 1,899 & 1,378 & 772 & 498 & 4,546 & 20,771 \\
\hline 1988 & 1,978 & 1,417 & 874 & 511 & 4,779 & 21,327 \\
\hline 1989 & 1,981 & 1,516 & 890 & 515 & 4,903 & 21,685 \\
\hline 1990 & 2,077 & 1,442 & 923 & 506 & 4,949 & 21,613 \\
\hline 1991 & 1,939 & 1,523 & 860 & 478 & 4,800 & 21,205 \\
\hline 1992 & 1,970 & 1,599 & 846 & 490 & 4,904 & 21,866 \\
\hline 1993 & 1,986 & 1,437 & 885 & 505 & 4,813 & 22,322 \\
\hline 1994 & 2,070 & 1,394 & 951 & 539 & 4,954 & 22,926 \\
\hline 1995 & 2,141 & 1,468 & 967 & 559 & 5,135 & 23,465 \\
\hline 1996 & 2,206 & 1,411 & 979 & 572 & 5,167 & 23,973 \\
\hline 1997 & 2,300 & 1,250 & 1,022 & 574 & 5,146 & 24,327 \\
\hline 1998 & 2,371 & 1,232 & 897 & 578 & 5,077 & 24,757 \\
\hline 1999 & 2,471 & 1,367 & 908 & 599 & 5,345 & 25,948 \\
\hline 2000 & 2,549 & 1,454 & 904 & 601 & 5,507 & 26,268 \\
\hline 2001 & 2,411 & 1,186 & 886 & 603 & 5,086 & 25,958 \\
\hline 2002 & 2,213 & 1,247 & 931 & 605 & 4,996 & 26,521 \\
\hline 2003 & 2,217 & 1,074 & 850 & 617 & 4,757 & 26,672 \\
\hline 2004 & 2,348 & 1,299 & 822 & 650 & 5,119 & 27,065 \\
\hline 2005 & 2,477 & 1,368 & 842 & 657 & 5,343 & 27,526 \\
\hline 2006 & 2,511 & 1,450 & 842 & 670 & 5,473 & 27,759 \\
\hline 2007 & 2,509 & 1,559 & 882 & 657 & 5,608 & 28,013 \\
\hline 2008 & 2,393 & 1,227 & 911 & 634 & 5,165 & 26,823 \\
\hline \multicolumn{7}{|c|}{ Average annual percentage change } \\
\hline 1970-2008 & $1.6 \%$ & $1.0 \%$ & $-0.2 \%$ & $0.4 \%$ & $0.9 \%$ & $1.5 \%$ \\
\hline $1998-2008$ & $0.1 \%$ & $0.0 \%$ & $0.2 \%$ & $1.0 \%$ & $0.0 \%$ & $1.0 \%$ \\
\hline
\end{tabular}

Source:

See Appendix A for Nonhighway Energy Use.

Note: Totals may not add due to rounding.

${ }^{a}$ Total transportation figures do not include military and off-highway energy use and may not include all possible uses of fuel for transportation (e.g., snowmobiles). 
The Environmental Protection Agency's NONROAD2008a model estimates fuel use for different types of equipment and off-highway vehicles. Most of these vehicles/equipment use diesel fuel. Recreational equipment, such as offhighway motorcycles, snowmobiles, and ATVs, are mainly fueled by gasoline.

Table 2.9

Off-highway Transportation-related Fuel Consumption from the NonRoad Model, 2008 (trillion Btus)

\begin{tabular}{lrrrrr}
\hline & Gasoline & Diesel & LPG & CNG & Total \\
\hline $\begin{array}{l}\text { Agricultural Equipment } \\
\quad \text { Tractors, mowers, combines, balers, and otherfarm }\end{array}$ & 8.3 & 531.8 & 0.0 & 0.0 & 540.1 \\
$\quad \begin{array}{l}\text { equipment which has utility in its movement. } \\
\text { Airport Ground Equipment }\end{array}$ & 0.3 & 13.2 & 0.2 & $\mathrm{a}$ & 13.7 \\
$\begin{array}{l}\text { Construction and Mining Equipment } \\
\quad \text { Pavers, rollers, drill rigs, graders, backhoes, } \\
\text { excavators, cranes, mining equipment }\end{array}$ & 11.5 & 849.3 & 1.8 & $\mathrm{a}$ & 862.6 \\
$\begin{array}{l}\text { Industrial Equipment } \\
\quad \text { Forklifts, terminal tractors, sweeper/scrubbers }\end{array}$ & 14.2 & 120.6 & 196.7 & 18.4 & 349.8 \\
$\begin{array}{l}\text { Logging Equipment } \\
\quad \text { Feller/buncher/skidder }\end{array}$ & 1.6 & 23.7 & $\mathrm{a}$ & $\mathrm{a}$ & 25.3 \\
$\begin{array}{l}\text { Railroad Maintenance Equipment } \\
\text { Recreational Equipment } \\
\quad \text { Off-road motorcycles, snowmobiles, all-terrain }\end{array}$ & 0.2 & 3.2 & 0.0 & $\mathrm{a}$ & 3.4 \\
$\quad$ vehicles, golf carts, specialty vehicles & 161.2 & 1.8 & 0.1 & $\mathrm{a}$ & 163.1 \\
\hline Total & 197.3 & $1,543.4$ & 198.9 & 18.4 & $1,958.0$ \\
\hline
\end{tabular}

Source:

Environmental Protection Agency, NONROAD2008a model, www.epa.go/otaq/nonrdmdl.htm.

${ }^{a}$ There is no equipment listed for this fuel type. 
Mowing equipment consumes nearly half of all the fuel used by lawn and garden equipment. The gasoline used in lawn and garden equipment is $1.8 \%$ of total gasoline use.

Table 2.10

Fuel Consumption from Lawn and Garden Equipment, 2008 (million gallons)

\begin{tabular}{|c|c|c|c|c|c|}
\hline Equipment & Classification & Gasoline & Diesel & LPG & $\begin{array}{c}\text { Total fuel } \\
\text { consumption }\end{array}$ \\
\hline \multicolumn{6}{|l|}{ Mowing Equipment } \\
\hline Front mowers & Commercial & 19.37 & 102.62 & 0.00 & 121.99 \\
\hline Lawn \& garden tractors & Commercial & 221.36 & 21.18 & 0.00 & 242.55 \\
\hline Lawn \& garden tractors & Residential & 527.79 & 0.00 & 0.00 & 527.79 \\
\hline Lawn mowers & Commercial & 149.57 & 0.00 & 0.00 & 149.57 \\
\hline Lawn mowers & Residential & 201.78 & 0.00 & 0.00 & 201.78 \\
\hline Rear engine riding mowers & Commercial & 16.28 & 0.00 & 0.00 & 16.28 \\
\hline Rear engine riding mowers & Residential & 39.25 & 0.00 & 0.00 & 39.25 \\
\hline Total & & $1,175.41$ & 123.80 & 0.00 & $1,299.22$ \\
\hline \multicolumn{6}{|l|}{ Soil and Turf Equipment } \\
\hline Commercial turf equipment $\mathrm{a}^{\mathrm{a}}$ & Commercial & 713.64 & 16.46 & 0.00 & 730.10 \\
\hline Rotary tillers $<6 \mathrm{HP}$ & Commercial & 83.66 & 0.00 & 0.00 & 83.66 \\
\hline Rotary tillers $<6 \mathrm{HP}$ & Residential & 18.43 & 0.00 & 0.00 & 18.43 \\
\hline Total & & 815.72 & 16.46 & 0.00 & 832.18 \\
\hline \multicolumn{6}{|l|}{ Wood Cutting Equipment } \\
\hline Chain saws $<6 \mathrm{HP}$ & Commercial & 71.03 & 0.00 & 0.00 & 71.03 \\
\hline Chain saws $<6 \mathrm{HP}$ & Residential & 17.69 & 0.00 & 0.00 & 17.69 \\
\hline Chippers/stump grinders & Commercial & 38.02 & 139.65 & 19.00 & 196.75 \\
\hline Shredders $<6 \mathrm{HP}$ & Commercial & 8.96 & 0.00 & 0.00 & 8.96 \\
\hline Total & & 135.70 & 139.65 & 19.00 & 294.34 \\
\hline \multicolumn{6}{|l|}{ Blowers and Vacuums } \\
\hline Leafblowers/vacuums & Commercial & 198.58 & 0.01 & 0.00 & 198.60 \\
\hline Leafblowers/vacuums & Residential & 17.95 & 0.00 & 0.00 & 17.95 \\
\hline Snowblowers & Commercial & 33.56 & 1.75 & 0.00 & 35.38 \\
\hline Snowblowers & Residential & 17.77 & 0.00 & 0.00 & 17.77 \\
\hline Total & & 267.85 & 1.83 & 0.00 & 269.69 \\
\hline \multicolumn{6}{|l|}{ Trimming Equipment } \\
\hline Trimmers/edgers/brush cutter & Commercial & 60.41 & 0.00 & 0.00 & 60.41 \\
\hline Trimmers/edgers/brush cutter & Residential & 25.66 & 0.00 & 0.00 & 25.66 \\
\hline Other lawn \& garden equipment ${ }^{\mathrm{b}}$ & Commercial & 23.00 & 0.39 & 0.00 & 23.39 \\
\hline Other lawn \& garden equipment $\mathrm{t}^{\mathrm{b}}$ & Residential & 19.28 & 0.00 & 0.00 & 19.28 \\
\hline Total & & 128.34 & 0.39 & 0.00 & 128.73 \\
\hline Total All Equipment & & $2,523.02$ & 282.14 & 19.00 & $2,824.16$ \\
\hline
\end{tabular}

Source:

U.S. Environmental Protection Agency, NONROAD2008a Model, www.epa.gov/otaq/nonrdmdl.htm.

${ }^{a}$ Includes equipment such as aerators, dethatchers, sod cutters, hydro-seeders, turf utility vehicles, golf course greens mowers, and sand trap groomers.

${ }^{\mathrm{b}}$ Includes equipment not otherwise classified such as augers, sickle-bar mowers, and wood splitters. 
The Federal Highway Administration (FHWA) cautions that data from 1993 on may not be directly comparable to earlier years. Some states have improved reporting procedures in recent years, and the estimation procedures were revised in 1994. The definition of gasohol was expanded in 1993 to include blends that are less than 10\%. Now, the FHWA does not publish separate estimates of gasohol or ethanol used in gasohol. See Table 2.3 for details on oxygenate usage.

Table 2.11

Highway Usage of Gasoline and Special Fuels, 1973-2008 (billion gallons)

\begin{tabular}{|c|c|c|c|c|c|c|c|}
\hline Year & Gasoline & Gasohol & $\begin{array}{l}\text { Ethanol used } \\
\text { in gasohol }^{\mathrm{a}}\end{array}$ & $\begin{array}{c}\text { Total gasoline } \\
\text { and gasohol }\end{array}$ & Diesel $^{\mathrm{b}}$ & $\begin{array}{c}\text { Percent } \\
\text { diesel }\end{array}$ & $\begin{array}{l}\text { Total highway } \\
\text { fuel use }\end{array}$ \\
\hline 1973 & $\mathrm{c}$ & $\mathrm{c}$ & $\mathrm{c}$ & 100.6 & 9.8 & $8.9 \%$ & 110.5 \\
\hline 1975 & c & c & c & 99.4 & 9.6 & $8.8 \%$ & 109.0 \\
\hline 1980 & 100.7 & 0.5 & 0.0 & 101.2 & 13.8 & $12.0 \%$ & 115.0 \\
\hline 1981 & 98.9 & 0.7 & 0.1 & 99.6 & 14.9 & $13.0 \%$ & 114.5 \\
\hline 1982 & 96.2 & 2.3 & 0.2 & 98.5 & 14.9 & $13.1 \%$ & 113.4 \\
\hline 1983 & 95.9 & 4.3 & 0.4 & 100.1 & 16.0 & $13.8 \%$ & 116.1 \\
\hline 1984 & 96.0 & 5.4 & 0.5 & 101.4 & 17.3 & $14.6 \%$ & 118.7 \\
\hline 1985 & 95.6 & 8.0 & 0.8 & 103.6 & 17.8 & $14.6 \%$ & 121.3 \\
\hline 1986 & 98.6 & 8.1 & 0.8 & 106.8 & 18.4 & $14.7 \%$ & 125.2 \\
\hline 1987 & 101.8 & 6.9 & 0.8 & 108.7 & 19.0 & $14.9 \%$ & 127.7 \\
\hline 1988 & 101.7 & 8.1 & 0.8 & 109.8 & 20.1 & $15.5 \%$ & 129.9 \\
\hline 1989 & 103.7 & 6.9 & 0.7 & 110.6 & 21.2 & $16.1 \%$ & 131.9 \\
\hline 1990 & 102.6 & 7.5 & 0.8 & 110.2 & 21.4 & $16.3 \%$ & 131.6 \\
\hline 1991 & 99.3 & 8.6 & 0.9 & 107.9 & 20.7 & $16.1 \%$ & 128.6 \\
\hline 1992 & 102.1 & 8.8 & 0.9 & 111.0 & 22.0 & $16.5 \%$ & 132.9 \\
\hline 1993 & 103.4 & 10.3 & 1.0 & 113.7 & 23.5 & $17.1 \%$ & 137.2 \\
\hline 1994 & 104.0 & 11.0 & 1.0 & 115.0 & 25.1 & $17.9 \%$ & 140.1 \\
\hline 1995 & 104.0 & 13.1 & 1.2 & 117.1 & 26.2 & $18.3 \%$ & 143.3 \\
\hline 1996 & 107.4 & 12.1 & 1.1 & 119.5 & 27.2 & $18.5 \%$ & 146.7 \\
\hline 1997 & 106.2 & 14.7 & 1.3 & 120.9 & 29.4 & $19.6 \%$ & 150.3 \\
\hline 1998 & 110.7 & 14.0 & 1.3 & 124.7 & 30.2 & $19.5 \%$ & 154.9 \\
\hline 1999 & 114.6 & 14.2 & 1.3 & 128.7 & 31.9 & $19.9 \%$ & 160.7 \\
\hline 2000 & 112.6 & 16.3 & 1.5 & 128.9 & 33.4 & $20.6 \%$ & 162.3 \\
\hline 2001 & 112.3 & 17.4 & 1.5 & 129.7 & 33.4 & $20.5 \%$ & 163.1 \\
\hline 2002 & 112.0 & 21.0 & 2.1 & 133.0 & 34.8 & $20.7 \%$ & 167.8 \\
\hline 2003 & 101.5 & 32.5 & 2.7 & 134.1 & 35.5 & $20.9 \%$ & 169.6 \\
\hline 2004 & 92.4 & 44.0 & 3.7 & 136.5 & 37.4 & $21.5 \%$ & 173.9 \\
\hline 2005 & d & d & $\mathrm{d}$ & 135.2 & 39.1 & $22.4 \%$ & 174.3 \\
\hline 2006 & d & d & d & 134.8 & 40.1 & $22.9 \%$ & 174.9 \\
\hline 2007 & d & d & d & 135.4 & 40.7 & $23.1 \%$ & 176.1 \\
\hline 2008 & d & d & d & 132.2 & 38.6 & $22.6 \%$ & 170.8 \\
\hline \multicolumn{8}{|c|}{ Average annual percentage change } \\
\hline 1973-2008 & d & d & $\mathrm{d}_{\mathrm{d}}$ & $0.8 \%$ & $4.0 \%$ & & $1.3 \%$ \\
\hline $1998-2008$ & d & d & d & $0.6 \%$ & $2.5 \%$ & & $1.0 \%$ \\
\hline
\end{tabular}

\section{Source:}

U.S. Department of Transportation, Federal Highway Administration, Highway Statistics 2008, Washington, DC, 2009, Table MF-21 and annual. (Additional resources: www.fhwa.dot.gov)

${ }^{\text {a }}$ Estimated for 1980-92 and 2002 as 10\% of gasohol consumption.

${ }^{\mathrm{b}}$ Consists primarily of diesel fuel, with small quantities of liquefied petroleum gas.

${ }^{c}$ Data for gasoline and gasohol cannot be separated in this year.

${ }^{\mathrm{d}}$ Gasohol data is no longer published by the Federal Highway Administration. 
Great care should be taken when comparing modal energy intensity data among modes. Because of the inherent differences among the transportation modes in the nature of services, routes available, and many additional factors, it is not possible to obtain truly comparable national energy intensities among modes. These values are averages, and there is a great deal of variability even within a mode.

Table 2.12

Passenger Travel and Energy Use, 2008

\begin{tabular}{|c|c|c|c|c|c|c|c|}
\hline & \multirow[b]{2}{*}{$\begin{array}{c}\text { Number of } \\
\text { vehicles } \\
\text { (thousands) }\end{array}$} & \multirow[b]{2}{*}{$\begin{array}{l}\text { Vehicle- } \\
\text { miles } \\
\text { (millions) }\end{array}$} & \multirow[b]{2}{*}{$\begin{array}{l}\text { Passenger- } \\
\text { miles } \\
\text { (millions) }\end{array}$} & \multirow[b]{2}{*}{$\begin{array}{c}\text { Load factor } \\
\text { (persons/ } \\
\text { vehicle) }\end{array}$} & \multicolumn{2}{|c|}{ Energy intensities } & \multirow[b]{2}{*}{$\begin{array}{l}\text { Energy use } \\
\text { (trillion Btu) }\end{array}$} \\
\hline & & & & & $\begin{array}{c}\text { (Btu per } \\
\text { vehicle- } \\
\text { mile) }\end{array}$ & $\begin{array}{c}\text { (Btu per } \\
\text { passenger- } \\
\text { mile) }\end{array}$ & \\
\hline Cars & $137,080.0$ & $1,615,850$ & $2,569,202$ & 1.59 & 5,465 & 3,437 & $\mathbf{8 , 8 3 1 . 4}$ \\
\hline Personal trucks & $89,079.8$ & 926,657 & $1,705,049$ & 1.84 & 6,699 & 3,641 & $6,207.6$ \\
\hline Motorcycles & $7,742.9$ & 14,484 & 17,091 & 1.18 & 2,212 & 1,875 & 32.0 \\
\hline Demand response ${ }^{\mathrm{a}}$ & 65.8 & 1,495 & 1,412 & 0.9 & 16,509 & 17,482 & 24.7 \\
\hline Buses & b & b & b & b & b & b & 199.9 \\
\hline Transit & 67.1 & 2,388 & 21,918 & 9.2 & 39,906 & 4,348 & 95.3 \\
\hline Intercity $^{\mathrm{c}}$ & b & $\mathrm{b}$ & $\mathrm{b}$ & b & $\mathrm{b}$ & $\mathrm{b}$ & 30.3 \\
\hline School $^{\mathrm{c}}$ & 683.7 & $\mathrm{~b}$ & $\mathrm{~b}$ & b & $\mathrm{b}$ & b & 74.3 \\
\hline Air & b & b & b & b & b & $\mathbf{b}$ & $1,975.7$ \\
\hline Certificated route ${ }^{\mathrm{d}}$ & $\mathrm{b}$ & 5,871 & 570,922 & 97.2 & 291,246 & 2,995 & $1,710.0$ \\
\hline General aviation & 228.7 & $\mathrm{~b}$ & $\mathrm{~b}$ & b & $\mathrm{b}$ & $\mathrm{b}$ & 265.7 \\
\hline Recreational boats & $13,188.5$ & b & b & b & b & b & 245.7 \\
\hline Rail & 20.2 & 1,345 & 36,169 & 26.9 & 68,345 & 2,541 & 91.9 \\
\hline Intercity (Amtrak) & 0.3 & 272 & 6,179 & 22.7 & 54,514 & 2,398 & 14.8 \\
\hline Transit & 13.3 & 763 & 18,941 & 24.8 & 62,601 & 2,521 & 47.8 \\
\hline Commuter & 6.6 & 310 & 11,049 & 35.6 & 94,587 & 2,656 & 29.3 \\
\hline
\end{tabular}

Source:

See Appendix A for Passenger Travel and Energy Use.

${ }^{\text {a }}$ Includes passenger cars, vans, and small buses operating in response to calls from passengers to the transit operator who dispatches the vehicles.

${ }^{\mathrm{b}}$ Data are not available.

${ }^{c}$ Energy use is estimated.

${ }^{\mathrm{d}}$ Only domestic service and domestic energy use are shown on this table. (Previous editions included half of international energy.) These energy intensities may be inflated because all energy use is attributed to passengerscargo energy use is not taken into account. 
Great care should be taken when comparing modal energy intensity data among modes. Because of the inherent differences among the transportation modes in the nature of services, routes available, and many additional factors, it is not possible to obtain truly comparable national energy intensities among modes. These values are averages, and there is a great deal of variability even within a mode.

Table 2.13

Energy Intensities of Highway Passenger Modes, 1970-2008

\begin{tabular}{|c|c|c|c|c|c|}
\hline \multirow[b]{2}{*}{ Year } & \multicolumn{2}{|c|}{ Automobiles } & \multirow{2}{*}{$\begin{array}{l}\text { Light truck }^{\mathrm{a}} \\
\text { (Btu per } \\
\text { vehicle-mile) }\end{array}$} & \multicolumn{2}{|c|}{ Transit Buses ${ }^{b}$} \\
\hline & $\begin{array}{c}\text { (Btu per } \\
\text { vehicle-mile) }\end{array}$ & $\begin{array}{c}\text { (Btu per } \\
\text { passenger-mile) }\end{array}$ & & $\begin{array}{c}\text { (Btu per } \\
\text { vehicle-mile) }\end{array}$ & $\begin{array}{c}\text { (Btu per } \\
\text { passenger-mile) }\end{array}$ \\
\hline 1970 & 9,250 & 4,868 & 12,480 & 31,796 & 2,472 \\
\hline 1975 & 8,993 & 4,733 & 11,879 & 33,748 & 2,814 \\
\hline 1976 & 9,113 & 4,796 & 11,524 & 34,598 & 2,896 \\
\hline 1977 & 8,950 & 4,710 & 11,160 & 35,120 & 2,889 \\
\hline 1978 & 8,839 & 4,693 & 10,807 & 36,603 & 2,883 \\
\hline 1979 & 8,647 & 4,632 & 10,468 & 36,597 & 2,795 \\
\hline 1980 & 7,916 & 4,279 & 10,224 & 36,553 & 2,813 \\
\hline 1981 & 7,670 & 4,184 & 9,997 & 37,745 & 3,027 \\
\hline 1982 & 7,465 & 4,109 & 9,268 & 38,766 & 3,237 \\
\hline 1983 & 7,365 & 4,092 & 9,124 & 37,962 & 3,177 \\
\hline 1984 & 7,202 & 4,066 & 8,931 & 38,705 & 3,307 \\
\hline 1985 & 7,164 & 4,110 & 8,730 & 38,876 & 3,423 \\
\hline 1986 & 7,194 & 4,197 & 8,560 & 37,889 & 3,545 \\
\hline 1987 & 6,959 & 4,128 & 8,359 & 36,247 & 3,594 \\
\hline 1988 & 6,683 & 4,033 & 8,119 & 36,673 & 3,706 \\
\hline 1989 & 6,589 & 4,046 & 7,746 & 36,754 & 3,732 \\
\hline 1990 & 6,169 & 3,856 & 7,746 & 37,374 & 3,794 \\
\hline 1991 & 5,912 & 3,695 & 7,351 & 37,732 & 3,877 \\
\hline 1992 & 5,956 & 3,723 & 7,239 & 40,243 & 4,310 \\
\hline 1993 & 6,087 & 3,804 & 7,182 & 39,043 & 4,262 \\
\hline 1994 & 6,024 & 3,765 & 7,212 & 37,259 & 4,262 \\
\hline 1995 & 5,902 & 3,689 & 7,208 & 37,251 & 4,307 \\
\hline 1996 & 5,874 & 3,683 & 7,247 & 37,452 & 4,340 \\
\hline 1997 & 5,797 & 3,646 & 7,251 & 38,861 & 4,434 \\
\hline 1998 & 5,767 & 3,638 & 7,260 & 41,296 & 4,399 \\
\hline 1999 & 5,821 & 3,684 & 7,327 & 40,578 & 4,344 \\
\hline 2000 & 5,687 & 3,611 & 7,158 & 41,695 & 4,531 \\
\hline 2001 & 5,626 & 3,583 & 7,080 & 38,535 & 4,146 \\
\hline 2002 & 5,662 & 3,607 & 7,125 & 37,548 & 4,133 \\
\hline 2003 & 5,535 & 3,525 & 7,673 & 37,096 & 4,213 \\
\hline 2004 & 5,489 & 3,496 & 7,653 & 37,855 & 4,364 \\
\hline 2005 & 5,607 & 3,571 & 7,009 & 37,430 & 4,250 \\
\hline 2006 & 5,511 & 3,510 & 6,974 & 39,568 & 4,316 \\
\hline 2007 & 5,513 & 3,512 & 6,904 & 39,931 & 4,372 \\
\hline 2008 & 5,465 & 3,437 & 6,830 & 39,906 & 4,348 \\
\hline \multicolumn{6}{|c|}{ Average annual percentage change } \\
\hline 1970-2008 & $-1.4 \%$ & $-0.9 \%$ & $-1.6 \%$ & $0.6 \%$ & $1.5 \%$ \\
\hline $1998-2008$ & $-0.5 \%$ & $-0.6 \%$ & $-0.6 \%$ & $-0.4 \%$ & $-0.2 \%$ \\
\hline
\end{tabular}

\section{Source:}

See Appendix A for Highway Passenger Mode Energy Intensities.

${ }^{a}$ All two-axle, four-tire trucks.

${ }^{\mathrm{b}}$ Series not continuous between 1983 and 1984 because of a change in data source by the American Public Transportation Association (APTA). 
Great care should be taken when comparing modal energy intensity data among modes. Because of the inherent differences between the transportation modes in the nature of services, routes available, and many additional factors, it is not possible to obtain truly comparable national energy intensities among modes.

Table 2.14

Energy Intensities of Nonhighway Passenger Modes, 1970-2008

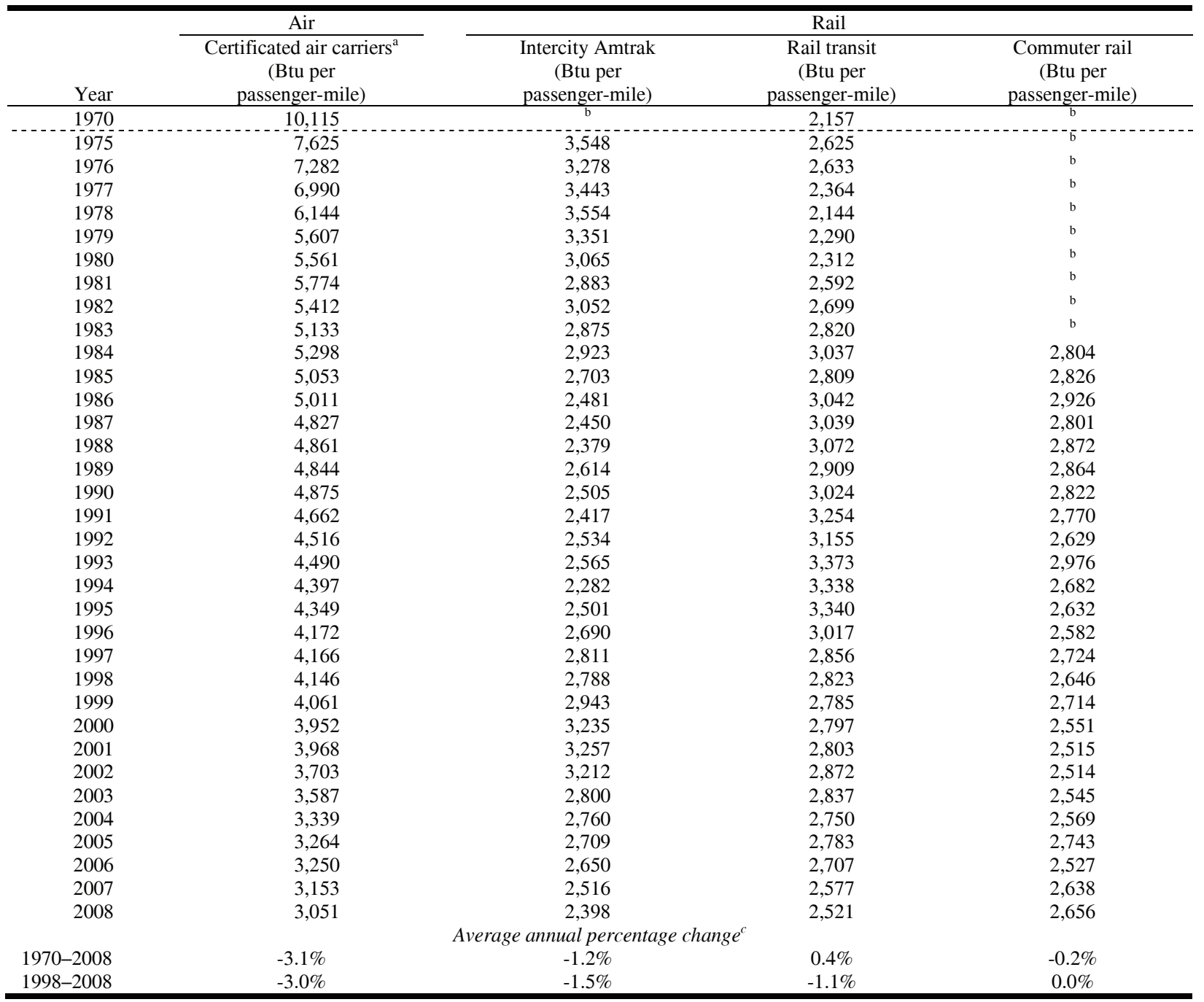

\section{Source:}

See Appendix A for Nonhighway Passenger Mode Energy Intensities.

${ }^{a}$ These data differ from the data on Table 2.12 because they include half of international services. These energy intensities may be inflated because all energy use is attributed to passengers-cargo energy use is not taken into account.

${ }^{\mathrm{b}}$ Data are not available.

${ }^{c}$ Average Annual Percent calculated to latest year possible. 
The energy intensity of light rail systems, measured in btu per passenger-mile varies greatly. The weighted average of all light rail systems in 2008 is 6,436 btu/passenger-mile.

\section{Figure 2.2. Energy Intensity of Light Rail Transit Systems, 2008}

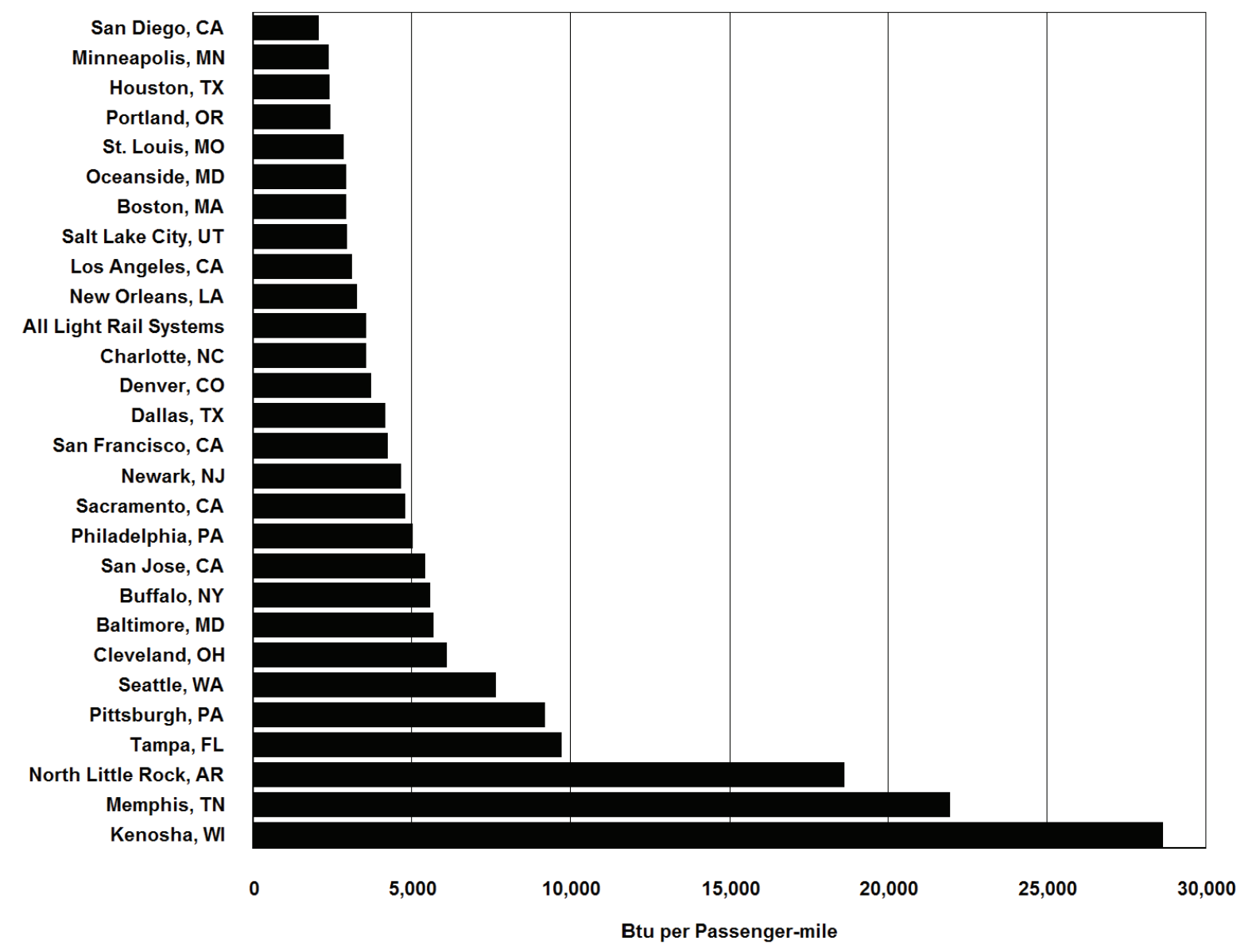

Source:

U.S. Department of Transportation, National Transit Database, May 2010. (Additional resources: http://204.68.195.57/ntdprogram/data.htm) 
Figure 2.3. Energy Intensity of Heavy Rail Systems, 2008

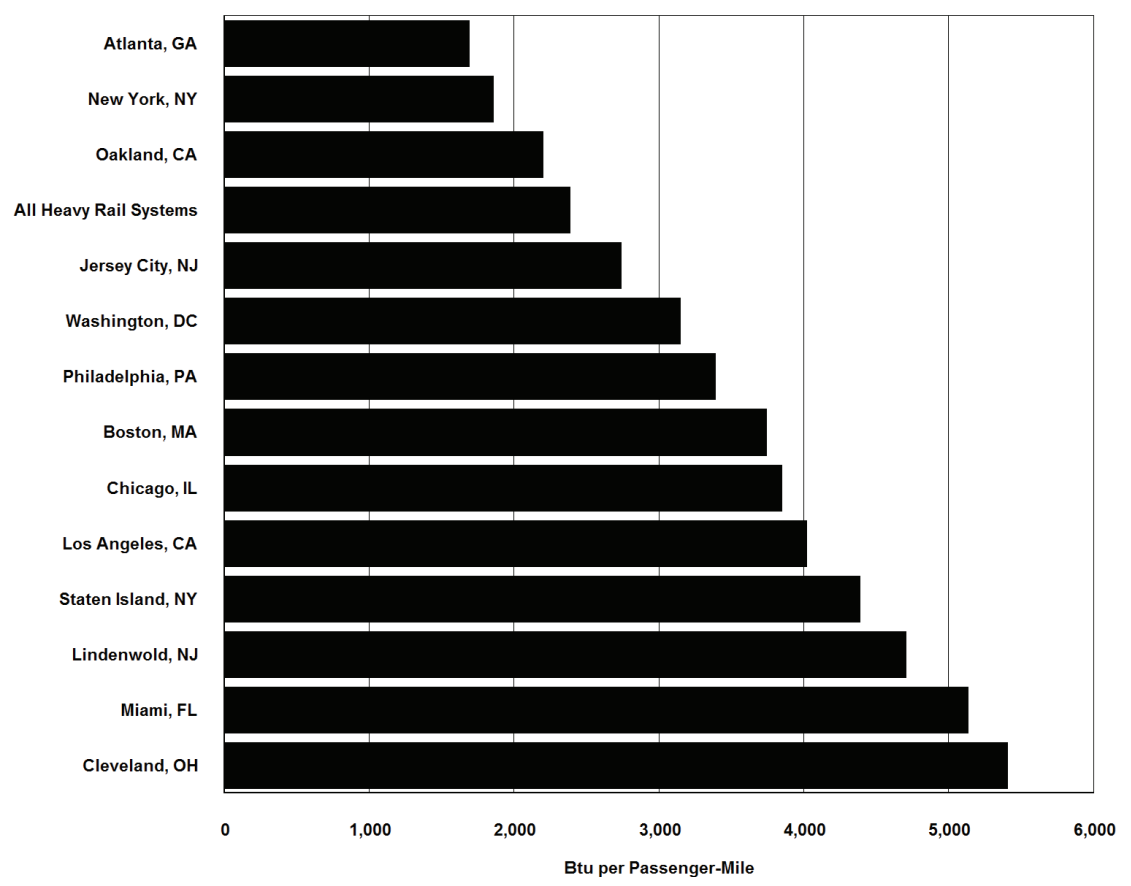

Source:

U.S. Department of Transportation, National Transit Database, May 2010. (Additional resources: www.ntdprogram.gov)

Figure 2.4. Energy Intensity of Commuter Rail Systems, 2008

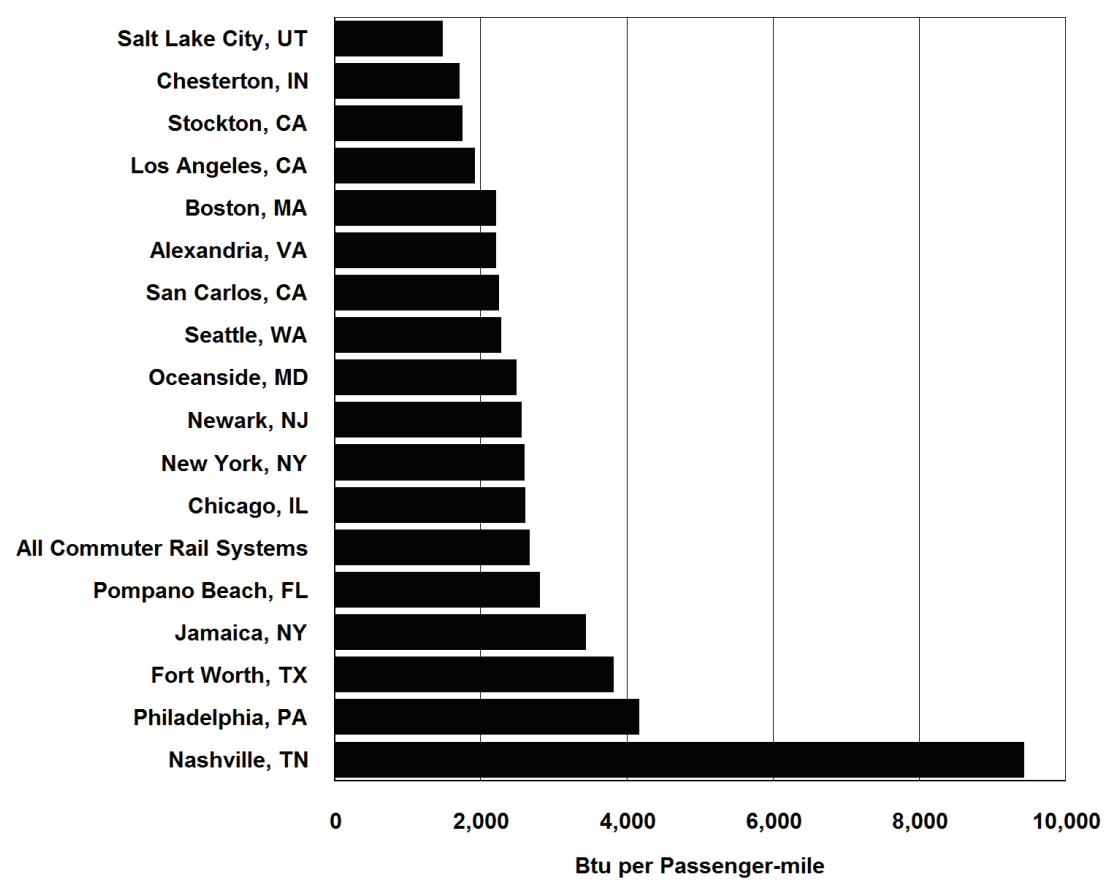

Source:

U.S. Department of Transportation, National Transit Database, May 2010. (Additional resources: www.ntdprogram.gov) 
Great care should be taken when comparing modal energy intensity data among modes. Because of the inherent differences between the transportation modes in the nature of services, routes available, and many additional factors, it is not possible to obtain truly comparable national energy intensities among modes.

Table 2.15

Intercity Freight Movement and Energy Use in the United States, 2007 and 2008

\begin{tabular}{lrrrrr}
\hline & \multicolumn{2}{c}{ Waterborne commerce } & & \multicolumn{2}{c}{ Class I railroads } \\
\cline { 2 - 3 } \cline { 5 - 6 } & 2007 & 2008 & & 2007 & 2008 \\
\hline Number of vehicles (thousands) & 41 & 40 & & $24^{\mathrm{a}}$ & $24^{\mathrm{a}}$ \\
Ton-miles (billions) & 553 & 521 & & 1,771 & 1,777 \\
Tons shipped (millions) & 1,016 & 952 & & 1,940 & 1,934 \\
Average length of haul (miles) & 544 & 547 & & 913 & 919 \\
Energy intensity (Btu/ton-mile) & 590 & 590 & & 320 & 305 \\
Energy use (trillion Btu) & 326 & 218 & & 567 & 542 \\
\hline
\end{tabular}

Source:

See Appendix A for Freight Movement and Energy Use.

\footnotetext{
${ }^{a}$ Number of locomotives.
} 
Great care should be taken when comparing modal energy intensity data among modes. Because of the inherent differences between the transportation modes in the nature of services, routes available, and many additional factors, it is not possible to obtain truly comparable national energy intensities among modes.

Table 2.16

Energy Intensities of Freight Modes, 1970-2008

\begin{tabular}{|c|c|c|c|c|}
\hline \multirow[b]{2}{*}{ Year } & \multirow{2}{*}{$\begin{array}{l}\text { Heavy single-unit and } \\
\text { combination trucks } \\
\text { (Btu per vehicle-mile) }\end{array}$} & \multicolumn{2}{|c|}{ Class I freight railroad } & \multirow{2}{*}{$\begin{array}{c}\text { Domestic waterborne } \\
\text { Commerce } \\
\text { (Btu per ton-mile) }\end{array}$} \\
\hline & & (Btu per freight car-mile) & (Btu per ton-mile) & \\
\hline 1970 & 24,960 & 17,669 & 691 & 545 \\
\hline 1971 & 24,485 & 18,171 & 717 & 506 \\
\hline 1972 & 24,668 & 18,291 & 714 & 522 \\
\hline 1973 & 24,777 & 18,468 & 677 & 576 \\
\hline 1974 & 24,784 & 18,852 & 681 & 483 \\
\hline 1975 & 24,631 & 18,739 & 687 & 549 \\
\hline 1976 & 24,567 & 18,938 & 680 & 468 \\
\hline 1977 & 24,669 & 19,226 & 669 & 458 \\
\hline 1978 & 24,655 & 18,928 & 641 & 383 \\
\hline 1979 & 24,746 & 19,188 & 618 & 436 \\
\hline 1980 & 24,758 & 18,742 & 597 & 358 \\
\hline 1981 & 25,059 & 18,629 & 572 & 360 \\
\hline 1982 & 24,297 & 18,404 & 553 & 310 \\
\hline 1983 & 23,853 & 17,864 & 525 & 286 \\
\hline 1984 & 23,585 & 17,795 & 510 & 346 \\
\hline 1985 & 23,343 & 17,500 & 497 & 446 \\
\hline 1986 & 23,352 & 17,265 & 486 & 463 \\
\hline 1987 & 22,923 & 16,790 & 456 & 414 \\
\hline 1988 & 22,596 & 16,758 & 443 & 373 \\
\hline 1989 & 22,411 & 16,894 & 437 & 423 \\
\hline 1990 & 22,795 & 16,619 & 420 & 387 \\
\hline 1991 & 22,749 & 15,835 & 391 & 386 \\
\hline 1992 & 22,609 & 16,043 & 393 & 398 \\
\hline 1993 & 22,373 & 16,056 & 389 & 389 \\
\hline 1994 & 22,193 & 16,340 & 388 & 369 \\
\hline 1995 & 22,097 & 15,992 & 372 & 374 \\
\hline 1996 & 22,109 & 15,747 & 368 & 412 \\
\hline 1997 & 21,340 & 15,784 & 370 & 415 \\
\hline 1998 & 21,516 & 15,372 & 365 & 435 \\
\hline 1999 & 22,884 & 15,363 & 363 & 457 \\
\hline 2000 & 23,449 & 14,917 & 352 & 473 \\
\hline 2001 & 23,024 & 15,108 & 346 & 460 \\
\hline 2002 & 23,462 & 15,003 & 345 & 470 \\
\hline 2003 & 22,461 & 15,016 & 344 & 418 \\
\hline 2004 & 20,540 & 15,274 & 341 & 510 \\
\hline 2005 & 22,866 & 15,152 & 337 & 515 \\
\hline 2006 & 23,340 & 14,990 & 330 & 571 \\
\hline 2007 & 23,252 & 14,846 & 320 & 590 \\
\hline 2008 & 22,077 & 14,573 & 305 & 418 \\
\hline \multicolumn{5}{|c|}{ Average annual percentage change } \\
\hline 1970-2008 & $-0.3 \%$ & $-0.5 \%$ & $-2.1 \%$ & $-0.7 \%$ \\
\hline $1998-2008$ & $0.3 \%$ & $-0.5 \%$ & $-1.8 \%$ & $-0.4 \%$ \\
\hline
\end{tabular}

Source:

See Appendix A for Freight Mode Energy Intensities. 


\section{Chapter 3 \\ All Highway Vehicles and Characteristics}

Summary Statistics from Tables in this Chapter

\begin{tabular}{rlr}
\hline Source & & \\
\hline Table 3.1 & U.S. share of world car registrations, 2008 & $20.3 \%$ \\
Table 3.2 & U.S. share of world truck \& bus registrations, 2008 & $41.8 \%$ \\
Table 3.3 & Number of U.S. cars, 2008 (thousands) & 135,882 \\
Table 3.3 & Number of U.S. trucks, 2008 (thousands) & 114,357 \\
Table 3.6 & Vehicle miles traveled, 2008 (million miles) & $2,973,509$ \\
& Cars & $53.2 \%$ \\
& Two-axle, four-tire trucks & $36.5 \%$ \\
& Combination trucks & $4.7 \%$ \\
Table 3.9 & Other single-unit trucks & $2.8 \%$ \\
& Motorcycles & $0.5 \%$ \\
& Buses & $0.2 \%$ \\
& Cars (years) & 10.6 \\
& Light trucks (years) & 9.6 \\
& All trucks (years) & 10.2 \\
\hline
\end{tabular}


The 1997 data in this series were never published. Use caution comparing historical data because of disconnects in data series, such as China in 1998. Also, the United States is unique in how many light trucks (SUVs, minivans, pickups) are used for personal travel. Those light trucks are not included on this table. The U.S. share of world cars has been declining since 1998. The growth in the World total comes mainly from developing countries, like China and India.

Table 3.1

Car Registrations for Selected Countries, 1960-2008 (thousands)

\begin{tabular}{|c|c|c|c|c|c|c|c|c|c|c|}
\hline Country & 1960 & 1970 & 1980 & 1990 & 2000 & 2005 & 2006 & 2007 & 2008 & $\begin{array}{c}\text { Average } \\
\text { Annual } \\
\text { Percentage } \\
\text { Change } \\
\text { 1990-2008 }\end{array}$ \\
\hline Brazil & a & a & a & 12,127 & 15,393 & 18,370 & 19,446 & 20.430 & 21884 & $3.3 \%$ \\
\hline Canada $^{\mathrm{b}}$ & 4,104 & 6,602 & 10,256 & 12,622 & 16,832 & 18,124 & 18,739 & 19,199 & 19,613 & $2.5 \%$ \\
\hline China & a & a & 351 & 1,897 & 3,750 & 8,900 & 11,000 & 13,758 & 18,270 & $13.4 \%$ \\
\hline France & 4,950 & 11,860 & 18,440 & 23,550 & 28,060 & 30,100 & 30,500 & 30,550 & 30,850 & $1.5 \%$ \\
\hline India & 4,856 & 14,376 & 23,236 & 35,512 & 43,772 & 46,090 & 41,020 & 41,184 & 41,321 & $0.8 \%$ \\
\hline Germany $^{\mathrm{c}}$ & $\mathrm{a}$ & $\mathrm{a}$ & $\mathrm{a}$ & 2,300 & 5,150 & 7,654 & 8,100 & 8,595 & 9,400 & $8.1 \%$ \\
\hline Japan & 457 & 8,779 & 23,660 & 34,924 & 52,437 & 57,091 & 57,521 & 57,624 & 57,865 & $2.8 \%$ \\
\hline $\begin{array}{l}\text { Russia } \\
\text { United }\end{array}$ & a & a & a & $\mathrm{a}$ & 20,353 & 25,285 & 26,800 & 28,300 & 32,021 & $\mathrm{a}$ \\
\hline Kingdom & 5,650 & 11,802 & 15,438 & 22,528 & 27,185 & 30,652 & 30,995 & 31,225 & 31,167 & $1.8 \%$ \\
\hline $\begin{array}{l}\text { United States } \\
\text { U.S. Percentage } \\
\text { of World }\end{array}$ & $62.7 \%$ & 89,244 & 121,601 & 143,550 & 127,721 & 132,909 & 135,047 & 135,222 & 135,882 & $-0.3 \%$ \\
\hline World total & 98,305 & 193,479 & 320,390 & 444,900 & 548,558 & 617,914 & 630,043 & 645,837 & 667,736 & $2.3 \%$ \\
\hline
\end{tabular}

\section{Source:}

Ward's Communications, Ward's World Motor Vehicle Data, 2009 Edition, Southfield, MI, 2009, pp. 257-260 and annual. (Additional resources: www.wardsauto.com)

${ }^{\mathrm{a}}$ Data are not available.

${ }^{\mathrm{b}}$ Data from 2000 and later are not comparable to prior data. Canada reclassified autos and trucks prior to 2000.

${ }^{\mathrm{c}}$ Data for 1990 and prior include West Germany only. Kraftwagen are included with automobiles. 
The United States totals include SUVs, minivans, and light trucks, many of which are used for personal travel.

Table 3.2

Truck and Bus Registrations for Selected Countries, 1960-2008 (thousands)

\begin{tabular}{|c|c|c|c|c|c|c|c|c|}
\hline Country & 1960 & 1970 & 1980 & 1990 & 2000 & 2005 & 2008 & $\begin{array}{c}\text { Average } \\
\text { Annual } \\
\text { Percentage } \\
\text { Change } \\
\text { 1990-2008 }\end{array}$ \\
\hline Brazil & a & $\mathrm{a}$ & $\mathrm{a}$ & 936 & 3,917 & 4,653 & 5,597 & $10.4 \%$ \\
\hline Canada $^{\mathrm{b}}$ & 1,056 & 1,481 & 2,955 & 3,931 & 739 & 786 & 907 & $-7.8 \%$ \\
\hline China & a & a & 1,480 & 4,314 & 9,650 & 21,750 & 28,750 & $0.7 \%$ \\
\hline France & 1,650 & 1,850 & 2,550 & 4,910 & 5,733 & 6,198 & 6,362 & $1.4 \%$ \\
\hline India & a & a & a & 2,050 & 2,390 & 4,145 & 5,610 & $5.8 \%$ \\
\hline Germany $^{c}$ & 786 & 1,228 & 1,617 & 2,764 & 3,534 & 3,133 & 2,859 & $0.2 \%$ \\
\hline Japan & 896 & 8,803 & 14,197 & 22,773 & 20,211 & 16,734 & 16,127 & $-1.9 \%$ \\
\hline Russia & a & a & a & 7,200 & 5,041 & 5,705 & 6,243 & $-0.8 \%$ \\
\hline United Kingdom & 1,534 & 1,769 & 1,920 & 3,774 & 3,361 & 3,943 & 4,450 & $0.9 \%$ \\
\hline United States & 12,186 & 19,175 & 34,195 & 45,106 & 85,579 & 104,788 & 114,357 & $5.3 \%$ \\
\hline $\begin{array}{l}\text { U.S. Percentage of } \\
\text { World }\end{array}$ & $42.6 \%$ & $36.2 \%$ & $37.7 \%$ & $32.7 \%$ & $42.1 \%$ & $42.6 \%$ & $41.8 \%$ & \\
\hline World total & 28,583 & 52,899 & 90,592 & 138,082 & 203,272 & 245,798 & 273,894 & $13.4 \%$ \\
\hline
\end{tabular}

\section{Source:}

Ward's Communications, Ward's World Motor Vehicle Data, 2009 Edition, Southfield, MI, 2009, pp. 257-260 and annual. (Additional resources: www.wardsauto.com)

${ }^{a}$ Data are not available.

${ }^{\mathrm{b}}$ Data from 2000 and later are not comparable to prior data. Canada reclassified autos and trucks prior to 2000 .

${ }^{\mathrm{c}}$ Data for 1990 and prior include West Germany only. Kraftwagen are included with automobiles. 


\section{VEHICLES IN USE}

Both the Federal Highway Administration (FHWA) and The Polk Company report figures on the car and truck population each year. The two estimates, however, differ by as much as $11.2 \%$ (1981). The differences can be attributed to several factors:

- The FHWA data include all vehicles which have been registered at any time throughout the calendar year. Therefore, the data include vehicles which were retired during the year and may double count vehicles which have been registered in different states or the same states to different owners. The Polk Company data include only those vehicles which are registered on July 1 of the given year.

- The classification of mini-vans, station wagons on truck chassis, and utility vehicles as cars or trucks causes important differences in the two estimates. The Polk Company data included passenger vans in the car count until 1980; since 1980 all vans have been counted as trucks. Recently, the Federal Highway Administration adjusted their definition of cars and trucks. Starting in 1993, some minivans and sport utility vehicles that were previously included with cars were included with trucks. This change produced a dramatic change in the individual percentage differences of cars and trucks. The difference in total vehicles has been less than 5\% each year since 1990 and does not appear to be significantly affected by the FHWA reclassifications.

- The FHWA data include all non-military Federal vehicles, while The Polk Company data include only those Federal vehicles which are registered within a state. Federal vehicles are not required to have State registrations, and, according to the General Services Administration, most Federal Vehicles are not registered.

According to The Polk Company statistics, the number of cars in use in the United States declined from 1991 to 1992. This is the first decline in vehicle stock since the figures were first reported in 1924. However, the data should be viewed with caution. A redesign of Polk's approach in 1992 allowed a national check for duplicate registrations, which was not possible in earlier years. Polk estimates that, due to processing limitations, its vehicle population counts may have been inflated by as much as $1 \frac{1}{2}$ percent. Assuming that percentage is correct, the number of cars in use would have declined from 1991 to 1992 under the previous Polk method. The growing popularity of light trucks being used as passenger vehicles could also have had an impact on these figures. 
In the early 1980's, researchers had to make a conscience choice of which data series to use, since they differed by as much as 11\%. In 2008 the two sources differed by about 1\%. The FHWA series shows a slight decrease in the number of trucks from 2007 to 2008, while The Polk Company data show a slight increase.

Table 3.3

U.S. Cars and Trucks in Use, 1970-2008 (thousands)

\begin{tabular}{|c|c|c|c|c|c|c|c|c|c|}
\hline \multirow[b]{2}{*}{ Year } & \multicolumn{3}{|c|}{ Automobiles } & \multicolumn{3}{|c|}{ Trucks } & \multicolumn{3}{|c|}{ Total } \\
\hline & FHWA & $\begin{array}{l}\text { The Polk } \\
\text { Company }\end{array}$ & $\begin{array}{l}\text { Percentage } \\
\text { difference }\end{array}$ & FHWA & $\begin{array}{l}\text { The Polk } \\
\text { Company }\end{array}$ & $\begin{array}{l}\text { Percentage } \\
\text { difference }\end{array}$ & FHWA & $\begin{array}{l}\text { The Polk } \\
\text { Company }\end{array}$ & $\begin{array}{l}\text { Percentage } \\
\text { difference }\end{array}$ \\
\hline 1970 & 89,243 & 80,448 & $10.9 \%$ & 18,797 & 17,688 & $6.3 \%$ & 108,040 & 98,136 & $10.1 \%$ \\
\hline 1975 & 106,706 & 95,241 & $12.0 \%$ & 25,781 & 24,813 & $3.9 \%$ & 132,487 & 120,054 & $10.4 \%$ \\
\hline 1976 & 110,189 & 97,818 & $12.6 \%$ & 27,876 & 26,560 & $5.0 \%$ & 138,065 & 124,378 & $11.0 \%$ \\
\hline 1977 & 112,288 & 99,904 & $12.4 \%$ & 29,314 & 28,222 & $3.9 \%$ & 141,602 & 128,126 & $10.5 \%$ \\
\hline 1978 & 116,573 & 102,957 & $13.2 \%$ & 31,336 & 30,565 & $2.5 \%$ & 147,909 & 133,522 & $10.8 \%$ \\
\hline 1979 & 118,429 & 104,677 & $13.1 \%$ & 32,914 & 32,583 & $1.0 \%$ & 151,343 & 137,260 & $10.3 \%$ \\
\hline 1980 & 121,601 & 104,564 & $16.3 \%$ & 33,667 & 35,268 & $-4.5 \%$ & 155,267 & 139,832 & $11.0 \%$ \\
\hline 1981 & 123,098 & 105,839 & $16.3 \%$ & 34,644 & 36,069 & $-4.0 \%$ & 157,743 & 141,908 & $11.2 \%$ \\
\hline 1982 & 123,702 & 106,867 & $15.8 \%$ & 35,382 & 36,987 & $-4.3 \%$ & 159,084 & 143,854 & $10.6 \%$ \\
\hline 1983 & 126,444 & 108,961 & $16.0 \%$ & 36,723 & 38,143 & $-3.7 \%$ & 163,166 & 147,104 & $10.9 \%$ \\
\hline 1984 & 128,158 & 112,019 & $14.4 \%$ & 37,507 & 40,143 & $-6.6 \%$ & 165,665 & 152,162 & $8.9 \%$ \\
\hline 1985 & 127,885 & 114,662 & $11.5 \%$ & 43,210 & 42,387 & $1.9 \%$ & 171,095 & 157,049 & $8.9 \%$ \\
\hline 1986 & 130,004 & 117,268 & $10.9 \%$ & 45,103 & 44,826 & $0.6 \%$ & 175,106 & 162,094 & $8.0 \%$ \\
\hline 1987 & 131,482 & 119,849 & $9.7 \%$ & 46,826 & 47,344 & $-1.1 \%$ & 178,308 & 167,193 & $6.6 \%$ \\
\hline 1988 & 133,836 & 121,519 & $10.1 \%$ & 49,941 & 50,221 & $-0.6 \%$ & 183,777 & 171,740 & $7.0 \%$ \\
\hline 1989 & 134,559 & 122,758 & $9.6 \%$ & 52,172 & 53,202 & $-1.9 \%$ & 186,731 & 175,960 & $6.1 \%$ \\
\hline 1990 & 133,700 & 123,276 & $8.5 \%$ & 54,470 & 56,023 & $-2.8 \%$ & 188,171 & 179,299 & $4.9 \%$ \\
\hline 1991 & 128,300 & 123,268 & $4.1 \%$ & 59,206 & 58,179 & $1.8 \%$ & 187,505 & 181,447 & $3.3 \%$ \\
\hline 1992 & 126,581 & 120,347 & $5.2 \%$ & 63,136 & 61,172 & $3.2 \%$ & 189,717 & 181,519 & $4.5 \%$ \\
\hline 1993 & 127,327 & 121,055 & $5.2 \%$ & 66,082 & 65,260 & $1.3 \%$ & 193,409 & 186,315 & $3.8 \%$ \\
\hline 1994 & 127,883 & 121,997 & $4.8 \%$ & 69,491 & 66,717 & $4.2 \%$ & 197,375 & 188,714 & $4.6 \%$ \\
\hline 1995 & 128,387 & 123,242 & $4.2 \%$ & 72,458 & 70,199 & $3.2 \%$ & 200,845 & 193,441 & $3.8 \%$ \\
\hline 1996 & 129,728 & 124,613 & $4.1 \%$ & 75,940 & 73,681 & $3.1 \%$ & 205,669 & 198,294 & $3.7 \%$ \\
\hline 1997 & 129,749 & 124,673 & $4.1 \%$ & 77,307 & 76,398 & $1.2 \%$ & 207,056 & 201,071 & $3.0 \%$ \\
\hline 1998 & 131,839 & 125,966 & $4.7 \%$ & 79,062 & 79,077 & $0.0 \%$ & 210,901 & 205,043 & $2.9 \%$ \\
\hline 1999 & 132,432 & 126,869 & $4.4 \%$ & 83,148 & 82,640 & $0.6 \%$ & 215,580 & 209,509 & $2.9 \%$ \\
\hline 2000 & 133,621 & 127,721 & $4.6 \%$ & 87,108 & 85,579 & $1.8 \%$ & 220,729 & 213,300 & $3.5 \%$ \\
\hline 2001 & 137,633 & 128,714 & $6.9 \%$ & 92,045 & 87,969 & $4.6 \%$ & 229,678 & 216,683 & $6.0 \%$ \\
\hline 2002 & 135,921 & 129,907 & $4.6 \%$ & 92,939 & 91,120 & $2.0 \%$ & 228,860 & 221,027 & $3.5 \%$ \\
\hline 2003 & 135,670 & 131,072 & $3.5 \%$ & 94,944 & 94,810 & $0.1 \%$ & 230,614 & 225,882 & $2.1 \%$ \\
\hline 2004 & 136,431 & 132,469 & $3.0 \%$ & 100,016 & 99,698 & $0.3 \%$ & 236,447 & 232,167 & $1.8 \%$ \\
\hline 2005 & 136,568 & 132,909 & $2.8 \%$ & 103,819 & 105,475 & $-1.6 \%$ & 240,387 & 238,384 & $0.8 \%$ \\
\hline 2006 & 135,400 & 135,047 & $0.3 \%$ & 107,944 & 109,596 & $-1.5 \%$ & 243,344 & 244,643 & $-0.5 \%$ \\
\hline 2007 & 135,933 & 135,222 & $0.5 \%$ & 110,498 & 113,479 & $-2.6 \%$ & 246,431 & 248,701 & $-0.9 \%$ \\
\hline 2008 & 137,080 & 135,882 & $0.9 \%$ & 110,242 & 114,357 & $-3.6 \%$ & 247,322 & 250,239 & $-1.2 \%$ \\
\hline
\end{tabular}

Source:

FHWA - U.S. Department of Transportation, Federal Highway Administration, Highway Statistics 2008, Washington, DC, 2009, Table VM-1 and annual. (Additional resources: www.fhwa.dot.gov)

Polk - The Polk Company, Detroit, Michigan. FURTHER REPRODUCTION PROHIBITED. (Additional resources: www.polk.com) 
The graphs below show the number of motor vehicles per thousand people for various countries. The data for the United States are displayed in the line which goes from 1900 to 2008. The points labeled on that line show data for the other countries/regions around the world and how their vehicles per thousand people compare to the United States at two different points in time, 1998 and 2008. For instance, the graph shows that in 1998, Western Europe's vehicles per thousand people was about where the United States was in 1968, but by 2008 it is about where the United States was in 1972. The lower part of the graph (1900-1940) is shown enlarged on the facing page.

Figure 3.1. Vehicles per Thousand People: U.S. (Over Time) Compared to Other Countries (in 1998 and 2008)

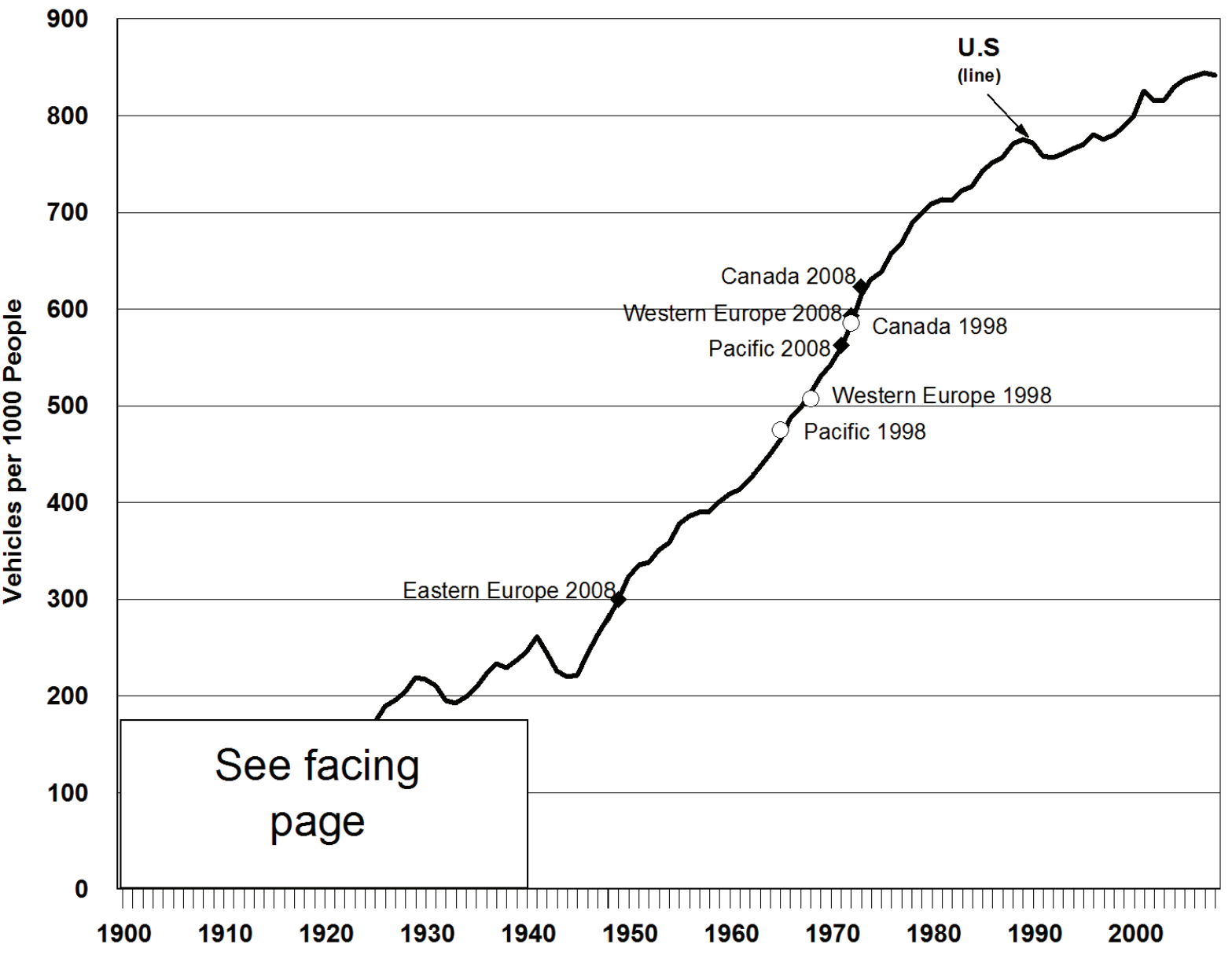




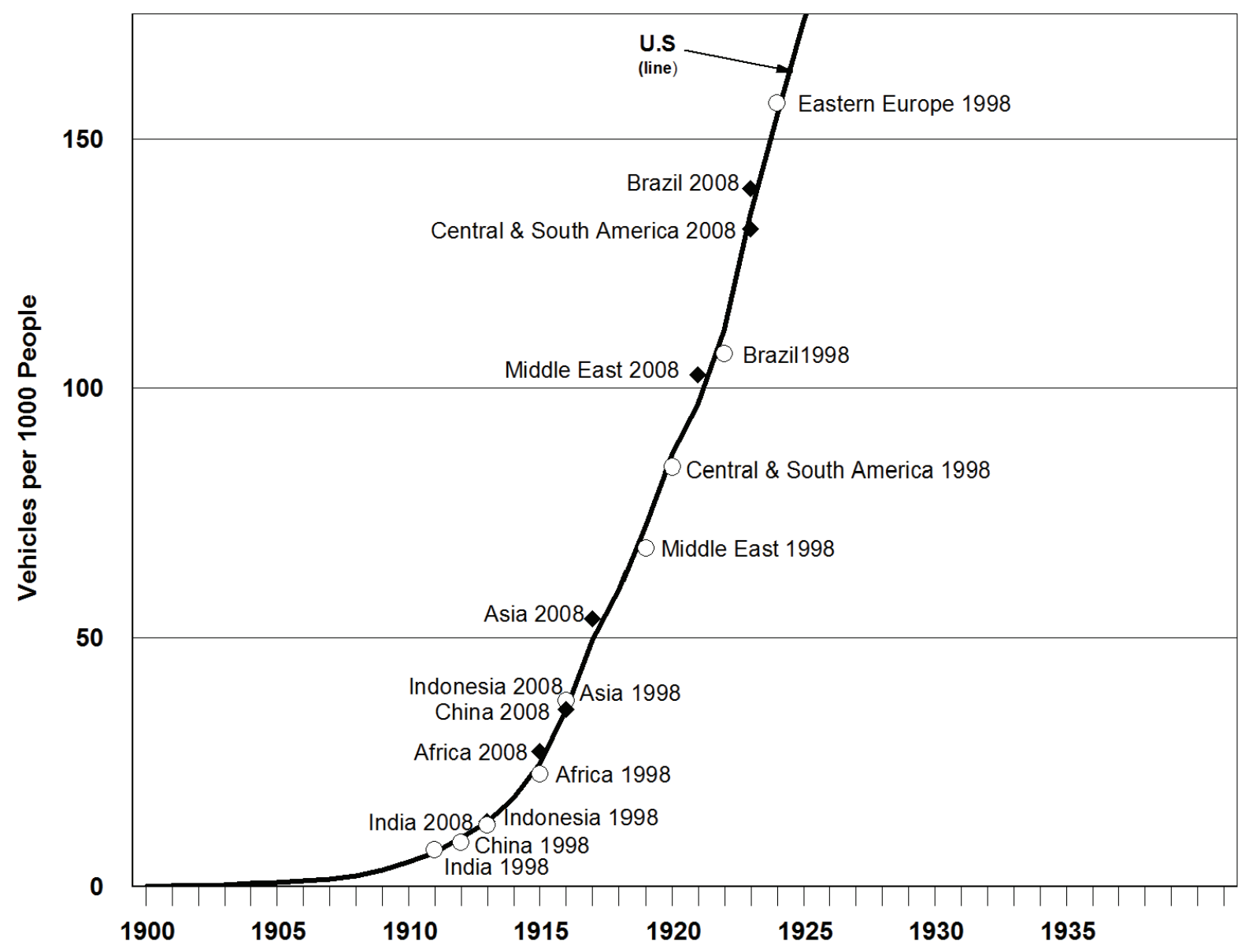

Source:

See Tables 3.4 and 3.5. 
Though some countries are listed separately in this table, those countries are also included in the regional total.

For instance, China is listed separately, but is also included in the Asia, Far East region.

Table 3.4

Vehicles per Thousand People in Other Countries, 1998 and 2008

\begin{tabular}{lcc}
\hline & \multicolumn{2}{c}{ Vehicles per 1,000 people } \\
\cline { 2 - 3 } Country/Region & 1998 & 2008 \\
\hline Africa & 22.8 & 27.2 \\
Asia, Far East & 50.4 & 135.8 \\
Asia, Middle East & 68.0 & 102.7 \\
Brazil & 106.9 & 140.0 \\
Canada & 585.5 & 623.0 \\
Central \& South America & 48.5 & 127.9 \\
China & 9.0 & 35.7 \\
Europe, East & 157.2 & 300.0 \\
Europe, West & 507.2 & 593.2 \\
India & 7.6 & 13.2 \\
Indonesia & 12.5 & 34.7 \\
Pacific & 475.1 & 563.1 \\
\hline
\end{tabular}

\section{Sources:}

Population - (2008) U.S. Census Bureau, Population Division, International Data Base (IDB) World, February 19, 2010. (Additional resources: http://www.census.gov/ipc/www/idb/)

Vehicles - (2008) U.S.: U.S. Department of Transportation, Federal Highway Administration, Highway Statistics 2008, Washington, DC, 2009. All others: Ward's Communications, Ward's Motor Vehicle Data 2009, pp. 257-260. (Additional resources: www.fhwa.dot.gov, www.wardsauto.com) 
The number of vehicles per thousand people in the United States has grown significantly from 1900 to 2008. From 2007 to 2008, however, the number decreased for the first time since 1997.

Table 3.5

Vehicles per Thousand People in the United States, 1990-2008

\begin{tabular}{|c|c|c|c|c|c|c|c|c|c|}
\hline Year & $\begin{array}{c}\text { U.S. } \\
\text { vehicles } \\
\text { per } 1,000 \\
\text { people }\end{array}$ & Year & $\begin{array}{c}\text { U.S. } \\
\text { vehicles } \\
\text { per } 1,000 \\
\text { people }\end{array}$ & Year & $\begin{array}{c}\text { U.S. } \\
\text { vehicles } \\
\text { per } 1,000 \\
\text { people }\end{array}$ & Year & $\begin{array}{c}\text { U.S. } \\
\text { vehicles } \\
\text { per } 1,000 \\
\text { people }\end{array}$ & Year & $\begin{array}{c}\text { U.S. } \\
\text { vehicles } \\
\text { per } 1,000 \\
\text { people }\end{array}$ \\
\hline 1900 & 0.11 & 1922 & 111.53 & 1944 & 220.23 & 1966 & 486.89 & 1988 & 771.27 \\
\hline 1901 & 0.19 & 1923 & 134.90 & 1945 & 221.80 & 1967 & 497.50 & 1989 & 775.35 \\
\hline 1902 & 0.29 & 1924 & 154.35 & 1946 & 243.11 & 1968 & 513.12 & 1990 & 771.82 \\
\hline 1903 & 0.41 & 1925 & 173.26 & 1947 & 262.56 & 1969 & 529.97 & 1991 & 758.66 \\
\hline 1904 & 0.67 & 1926 & 189.10 & 1948 & 280.20 & 1970 & 542.51 & 1992 & 756.84 \\
\hline 1905 & 0.94 & 1927 & 195.77 & 1949 & 299.56 & 1971 & 560.19 & 1993 & 760.95 \\
\hline 1906 & 1.27 & 1928 & 204.87 & 1950 & 322.86 & 1972 & 583.89 & 1994 & 766.04 \\
\hline 1907 & 1.65 & 1929 & 219.31 & 1951 & 335.19 & 1973 & 613.59 & 1995 & 770.18 \\
\hline 1908 & 2.24 & 1930 & 217.34 & 1952 & 338.06 & 1974 & 630.80 & 1996 & 780.37 \\
\hline 1909 & 3.45 & 1931 & 210.37 & 1953 & 350.95 & 1975 & 638.56 & 1997 & 775.27 \\
\hline 1910 & 5.07 & 1932 & 195.38 & 1954 & 358.87 & 1976 & 658.04 & 1998 & 780.46 \\
\hline 1911 & 6.81 & 1933 & 192.38 & 1955 & 377.80 & 1977 & 667.57 & 1999 & 789.35 \\
\hline 1912 & 9.90 & 1934 & 199.90 & 1956 & 385.71 & 1978 & 688.65 & 2000 & 800.30 \\
\hline 1913 & 12.94 & 1935 & 208.61 & 1957 & 390.30 & 1979 & 698.90 & 2001 & 825.61 \\
\hline 1914 & 17.79 & 1936 & 222.62 & 1958 & 390.53 & 1980 & 709.14 & 2002 & 815.44 \\
\hline 1915 & 24.77 & 1937 & 233.33 & 1959 & 401.25 & 1981 & 713.66 & 2003 & 815.82 \\
\hline 1916 & 35.48 & 1938 & 229.65 & 1960 & 408.80 & 1982 & 712.34 & 2004 & 829.69 \\
\hline 1917 & 49.57 & 1939 & 236.93 & 1961 & 413.53 & 1983 & 722.70 & 2005 & 837.12 \\
\hline 1918 & 59.69 & 1940 & 245.63 & 1962 & 424.31 & 1984 & 726.59 & 2006 & 840.74 \\
\hline 1919 & 72.50 & 1941 & 261.57 & 1963 & 436.99 & 1985 & 742.80 & 2007 & 844.38 \\
\hline 1920 & 86.78 & 1942 & 244.73 & 1964 & 449.81 & 1986 & 751.71 & 2008 & 841.67 \\
\hline 1921 & 96.68 & 1943 & 225.89 & 1965 & 465.03 & 1987 & 756.97 & & \\
\hline
\end{tabular}

\section{Sources:}

Population - (2008) U.S. Census Bureau, Population Division, International Data Base (IDB) World, February 19, 2010. (Additional resources: http://www.census.gov/ipc/www/idb/)

Vehicles - (2008) U.S.: U.S. Department of Transportation, Federal Highway Administration, Highway Statistics 2008, Washington, DC, 2009. All others: Ward's Communications, Ward's Motor Vehicle Data 2009, pp. 257-260. (Additional resources: www.fhwa.dot.gov, www.wardsauto.com) 
Total vehicle-miles traveled decreased slightly from 2007 to 2008. It is the first decline since 1980. The trend of using two-axle, four-tire trucks, such as pickups, vans, and sport-utility vehicles, for personal travel is evident in these data; two-axle, four-tire trucks account for 26.2\% more travel in 2008 than in 1970, and cars account for $28.3 \%$ less travel in that time period.

Table 3.6

Shares of Highway Vehicle-Miles Traveled by Vehicle Type, 1970-2008

\begin{tabular}{|c|c|c|c|c|c|c|c|}
\hline Year & Automobiles & Motorcycles & $\begin{array}{l}\text { Two- } \\
\text { axle,four- } \\
\text { tire trucks }\end{array}$ & $\begin{array}{c}\text { Other } \\
\text { single-unit } \\
\text { trucks }\end{array}$ & $\begin{array}{c}\text { Combination } \\
\text { trucks }\end{array}$ & Buses & $\begin{array}{c}\text { Total } \\
\text { vehicle-miles } \\
\text { traveled } \\
\text { (million miles) }\end{array}$ \\
\hline 1970 & $82.6 \%$ & $0.3 \%$ & $11.1 \%$ & $2.4 \%$ & $3.2 \%$ & $0.4 \%$ & $1,109,724$ \\
\hline 1971 & $82.0 \%$ & $0.3 \%$ & $11.7 \%$ & $2.5 \%$ & $3.2 \%$ & $0.4 \%$ & $1,178,811$ \\
\hline 1972 & $81.1 \%$ & $0.3 \%$ & $12.4 \%$ & $2.5 \%$ & $3.2 \%$ & $0.4 \%$ & $1,259,786$ \\
\hline 1973 & $79.7 \%$ & $0.4 \%$ & $13.5 \%$ & $2.6 \%$ & $3.5 \%$ & $0.4 \%$ & $1,313,110$ \\
\hline 1974 & $78.7 \%$ & $0.4 \%$ & $14.3 \%$ & $2.6 \%$ & $3.6 \%$ & $0.4 \%$ & $1,280,544$ \\
\hline 1975 & $77.9 \%$ & $0.4 \%$ & $15.1 \%$ & $2.6 \%$ & $3.5 \%$ & $0.5 \%$ & $1,327,664$ \\
\hline 1976 & $76.9 \%$ & $0.4 \%$ & $16.1 \%$ & $2.6 \%$ & $3.5 \%$ & $0.4 \%$ & $1,402,380$ \\
\hline 1977 & $75.6 \%$ & $0.4 \%$ & $17.1 \%$ & $2.7 \%$ & $3.8 \%$ & $0.4 \%$ & $1,467,027$ \\
\hline 1978 & $74.2 \%$ & $0.5 \%$ & $18.1 \%$ & $2.8 \%$ & $4.1 \%$ & $0.4 \%$ & $1,544,704$ \\
\hline 1979 & $72.8 \%$ & $0.6 \%$ & $19.1 \%$ & $2.7 \%$ & $4.4 \%$ & $0.4 \%$ & $1,529,133$ \\
\hline 1980 & $72.8 \%$ & $0.7 \%$ & $19.0 \%$ & $2.6 \%$ & $4.5 \%$ & $0.4 \%$ & $1,527,295$ \\
\hline 1981 & $72.9 \%$ & $0.7 \%$ & $19.1 \%$ & $2.5 \%$ & $4.4 \%$ & $0.4 \%$ & $1,555,308$ \\
\hline 1982 & $72.8 \%$ & $0.6 \%$ & $19.2 \%$ & $2.5 \%$ & $4.4 \%$ & $0.4 \%$ & $1,595,010$ \\
\hline 1983 & $72.3 \%$ & $0.5 \%$ & $19.8 \%$ & $2.6 \%$ & $4.5 \%$ & $0.3 \%$ & $1,652,788$ \\
\hline 1984 & $71.3 \%$ & $0.5 \%$ & $20.8 \%$ & $2.6 \%$ & $4.5 \%$ & $0.3 \%$ & $1,720,269$ \\
\hline 1985 & $70.2 \%$ & $0.5 \%$ & $22.0 \%$ & $2.6 \%$ & $4.4 \%$ & $0.3 \%$ & $1,774,826$ \\
\hline 1986 & $69.2 \%$ & $0.5 \%$ & $23.1 \%$ & $2.5 \%$ & $4.4 \%$ & $0.3 \%$ & $1,834,872$ \\
\hline 1987 & $68.5 \%$ & $0.5 \%$ & $23.8 \%$ & $2.5 \%$ & $4.5 \%$ & $0.3 \%$ & $1,921,204$ \\
\hline 1988 & $67.6 \%$ & $0.5 \%$ & $24.8 \%$ & $2.4 \%$ & $4.4 \%$ & $0.3 \%$ & $2,025,962$ \\
\hline 1989 & $66.8 \%$ & $0.5 \%$ & $25.6 \%$ & $2.4 \%$ & $4.4 \%$ & $0.3 \%$ & $2,096,487$ \\
\hline 1990 & $65.7 \%$ & $0.4 \%$ & $26.8 \%$ & $2.4 \%$ & $4.4 \%$ & $0.3 \%$ & $2,144,362$ \\
\hline 1991 & $62.5 \%$ & $0.4 \%$ & $29.9 \%$ & $2.4 \%$ & $4.4 \%$ & $0.3 \%$ & $2,172,050$ \\
\hline 1992 & $61.0 \%$ & $0.4 \%$ & $31.5 \%$ & $2.4 \%$ & $4.4 \%$ & $0.3 \%$ & $2,247,151$ \\
\hline 1993 & $59.9 \%$ & $0.4 \%$ & $32.5 \%$ & $2.5 \%$ & $4.5 \%$ & $0.3 \%$ & $2,296,378$ \\
\hline 1994 & $59.6 \%$ & $0.4 \%$ & $32.4 \%$ & $2.6 \%$ & $4.6 \%$ & $0.3 \%$ & $2,357,588$ \\
\hline 1995 & $59.4 \%$ & $0.4 \%$ & $32.6 \%$ & $2.6 \%$ & $4.8 \%$ & $0.3 \%$ & $2,422,696$ \\
\hline 1996 & $59.1 \%$ & $0.4 \%$ & $32.8 \%$ & $2.6 \%$ & $4.8 \%$ & $0.3 \%$ & $2,485,848$ \\
\hline 1997 & $58.7 \%$ & $0.4 \%$ & $33.2 \%$ & $2.6 \%$ & $4.9 \%$ & $0.3 \%$ & $2,561,695$ \\
\hline 1998 & $58.9 \%$ & $0.4 \%$ & $33.0 \%$ & $2.6 \%$ & $4.9 \%$ & $0.3 \%$ & $2,631,522$ \\
\hline 1999 & $58.3 \%$ & $0.4 \%$ & $33.5 \%$ & $2.6 \%$ & $4.9 \%$ & $0.3 \%$ & $2,691,056$ \\
\hline 2000 & $58.3 \%$ & $0.4 \%$ & $33.6 \%$ & $2.6 \%$ & $4.9 \%$ & $0.3 \%$ & $2,746,925$ \\
\hline 2001 & $58.2 \%$ & $0.3 \%$ & $33.8 \%$ & $2.6 \%$ & $4.9 \%$ & $0.3 \%$ & $2,790,372$ \\
\hline 2002 & $58.1 \%$ & $0.3 \%$ & $33.8 \%$ & $2.7 \%$ & $4.9 \%$ & $0.2 \%$ & $2,855,508$ \\
\hline 2003 & $57.8 \%$ & $0.3 \%$ & $34.0 \%$ & $2.7 \%$ & $4.8 \%$ & $0.2 \%$ & $2,890,450$ \\
\hline 2004 & $57.3 \%$ & $0.3 \%$ & $34.6 \%$ & $2.6 \%$ & $4.8 \%$ & $0.2 \%$ & $2,964,788$ \\
\hline 2005 & $57.1 \%$ & $0.3 \%$ & $34.8 \%$ & $2.6 \%$ & $4.8 \%$ & $0.2 \%$ & $2,989,430$ \\
\hline 2006 & $56.1 \%$ & $0.4 \%$ & $35.9 \%$ & $2.7 \%$ & $4.7 \%$ & $0.2 \%$ & $3,014,369$ \\
\hline 2007 & $55.2 \%$ & $0.4 \%$ & $36.7 \%$ & $2.7 \%$ & $4.8 \%$ & $0.2 \%$ & $3,032,399$ \\
\hline 2008 & $53.2 \%$ & $0.5 \%$ & $36.5 \%$ & $2.8 \%$ & $4.7 \%$ & $0.2 \%$ & $2,973,509$ \\
\hline \multicolumn{8}{|c|}{ Average annual percentage change } \\
\hline 1970-2008 & & & & & & & $2.8 \%$ \\
\hline 1998-2008 & & & & & & & $1.7 \%$ \\
\hline
\end{tabular}

Source:

U.S. Department of Transportation, Federal Highway Administration, Highway Statistics 2008, Washington, DC, 2009, Table VM-1 and annual. (Additional resources: www.fhwa.dot.gov) 
Due to data restrictions, the 2001 data are the latest than can be published.

Table 3.7

Cars in Operation and Vehicle Travel by Age, 1970 and 2001

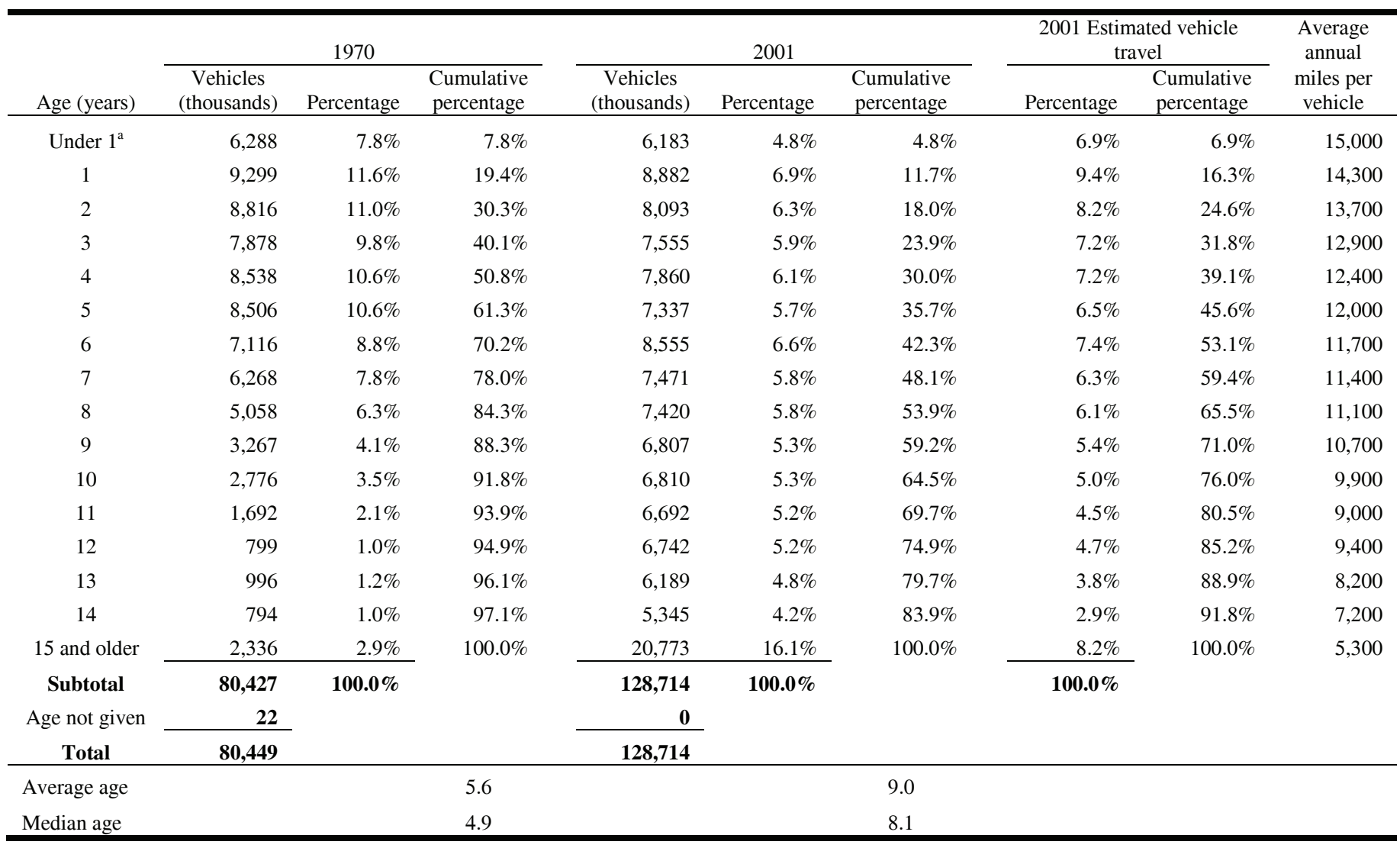

\section{Source:}

The Polk Company, Detroit, MI. FURTHER REPRODUCTION PROHIBITED.

Vehicle travel - Average annual miles per auto by age were multiplied by the number of vehicles in operation by age to estimate the vehicle travel. Average annual miles per auto by age - generated by ORNL from the National Household Travel Survey Web site: nhts.ornl.gov. (Additional resources: www.polk.com, nhts.ornl.gov)

a Includes cars from model year 2002 and 2001 which were sold prior to July 1, 2002, and similarly, model years 1971 and 1970 sold prior to July $1,1970$. 
Due to data restrictions, the 2001 data are the latest than can be published.

Table 3.8

Trucks in Operation and Vehicle Travel by Age, 1970 and 2001

\begin{tabular}{|c|c|c|c|c|c|c|c|c|c|}
\hline Age (years) & \multicolumn{3}{|c|}{1970} & \multicolumn{3}{|c|}{2001} & \multicolumn{2}{|c|}{$\begin{array}{c}2001 \text { Estimated vehicle } \\
\text { travel }\end{array}$} & $\begin{array}{l}\text { Average } \\
\text { annual } \\
\text { miles per } \\
\text { vehicle }\end{array}$ \\
\hline 1 & 1,881 & $10.6 \%$ & $17.8 \%$ & 7,958 & $9.0 \%$ & $16.1 \%$ & $12.0 \%$ & $20.6 \%$ & 19,200 \\
\hline 2 & 1,536 & $8.7 \%$ & $26.5 \%$ & 7,522 & $8.6 \%$ & $24.7 \%$ & $11.7 \%$ & $32.3 \%$ & 19,800 \\
\hline 3 & 1,428 & $8.1 \%$ & $34.6 \%$ & 6,398 & $7.3 \%$ & $31.9 \%$ & $9.0 \%$ & $41.3 \%$ & 17,900 \\
\hline 5 & 1,339 & $7.6 \%$ & $50.5 \%$ & 5,122 & $5.8 \%$ & $44.7 \%$ & $6.8 \%$ & $56.6 \%$ & 17,000 \\
\hline 6 & 1,154 & $6.5 \%$ & $57.1 \%$ & 5,574 & $6.3 \%$ & $51.0 \%$ & $6.8 \%$ & $63.4 \%$ & 15,600 \\
\hline 7 & 975 & $5.5 \%$ & $62.6 \%$ & 5,042 & $5.7 \%$ & $56.8 \%$ & $6.1 \%$ & $69.5 \%$ & 15,400 \\
\hline 8 & 826 & $4.7 \%$ & $67.3 \%$ & 4,148 & $4.7 \%$ & $61.5 \%$ & $4.9 \%$ & $74.4 \%$ & 15,100 \\
\hline 9 & 621 & $3.5 \%$ & $70.8 \%$ & 3,395 & $3.9 \%$ & $65.3 \%$ & $3.5 \%$ & $77.9 \%$ & 13,200 \\
\hline 13 & 417 & $2.4 \%$ & $82.3 \%$ & 3,112 & $3.5 \%$ & $79.8 \%$ & $2.3 \%$ & $89.1 \%$ & 9,200 \\
\hline 14 & 414 & $2.3 \%$ & $84.7 \%$ & 2,544 & $2.9 \%$ & $82.7 \%$ & $1.8 \%$ & $89.0 \%$ & 9,200 \\
\hline 15 and older & 2,710 & $15.3 \%$ & $100.0 \%$ & 15,227 & $17.3 \%$ & $100.0 \%$ & $11.0 \%$ & $100.0 \%$ & 9,200 \\
\hline Subtotal & 17,670 & $100.0 \%$ & & 87,969 & $100.0 \%$ & & $100.0 \%$ & & \\
\hline Age not given & 15 & & & 0 & & & & & \\
\hline Total & 17,685 & & & 87,969 & & & & & \\
\hline Average age & & 7.3 & & & 7.9 & & & & \\
\hline Median age & & 5.9 & & & 6.8 & & & & \\
\hline
\end{tabular}

Source:

The Polk Company, Detroit, MI. FURTHER REPRODUCTION PROHIBITED.

Vehicle travel-The average annual vehicle-miles per truck by age were multiplied by the number of trucks in operation by age to estimate the vehicle travel. Average annual miles per truck by age were generated by ORNL from the 1997 Truck Inventory and Use Survey public use tape provided by U.S. Department of Commerce, Bureau of the Census, Washington, DC, 2000. (Additional resources: www.polk.com, www.census.gov)

${ }^{\text {a }}$ Includes trucks from model year 2002 and 2001 which were sold prior to July 1, 2002, and similarly, model years 1971 and 1970 sold prior to July 1, 1970. 
Table 3.9

U.S. Average Vehicle Age, 1995-2009

\begin{tabular}{cccc}
\hline & Passenger Cars & Light Trucks & All Light Vehicles \\
\hline 1995 & 8.4 & 8.3 & 8.4 \\
1996 & 8.5 & 8.3 & 8.5 \\
1997 & 8.7 & 8.5 & 8.6 \\
1998 & 8.9 & 8.5 & 8.8 \\
1999 & 9.1 & 8.5 & 8.8 \\
2000 & 9.1 & 8.4 & 8.9 \\
2001 & 9.3 & 8.4 & 8.9 \\
2002 & 9.4 & 8.4 & 9.0 \\
2003 & 9.6 & 8.5 & 9.1 \\
2004 & 9.8 & 8.6 & 9.4 \\
2005 & 10.1 & 8.7 & 9.5 \\
2006 & 10.3 & 8.9 & 9.7 \\
2007 & 10.4 & 9.0 & 9.8 \\
2008 & 10.6 & 9.3 & 10.0 \\
2009 & 10.6 & 9.6 & 10.2 \\
\hline
\end{tabular}

Source:

The Polk Company, Detroit, MI. FURTHER REPRODUCTION PROHIBITED. (Additional resources: www.polk.com) 
Table 3.10

New Retail Vehicle Sales, 1970-2009

\begin{tabular}{|c|c|c|c|c|c|}
\hline $\begin{array}{c}\text { Calendar } \\
\text { Year }\end{array}$ & Cars & $\begin{array}{l}\text { Light } \\
\text { Trucks }\end{array}$ & $\begin{array}{c}\text { Subtotal } \\
\text { Light Vehicles }\end{array}$ & $\begin{array}{l}\text { Heavy } \\
\text { Trucks }\end{array}$ & $\begin{array}{c}\text { Total } \\
\text { Vehicle } \\
\text { Sales }\end{array}$ \\
\hline 1970 & 8,399 & 1,463 & 9,862 & 334 & 10,196 \\
\hline 1971 & 10,242 & 1,757 & 11,999 & 340 & 12,339 \\
\hline 1972 & 10,941 & 2,239 & 13,180 & 438 & 13,618 \\
\hline 1973 & 11,424 & 2,745 & 14,169 & 497 & 14,666 \\
\hline 1974 & 8,853 & 2,338 & 11,191 & 424 & 11,615 \\
\hline 1975 & 8,624 & 2,281 & 10,905 & 298 & 11,203 \\
\hline 1976 & 10,110 & 2,956 & 13,066 & 324 & 13,390 \\
\hline 1977 & 11,183 & 3,430 & 14,613 & 376 & 14,989 \\
\hline 1978 & 11,314 & 3,808 & 15,122 & 441 & 15,563 \\
\hline 1979 & 10,673 & 3,311 & 13,984 & 391 & 14,375 \\
\hline 1980 & 8,949 & 2,440 & 11,389 & 271 & 11,660 \\
\hline 1981 & 8,489 & 2,189 & 10,678 & 226 & 10,904 \\
\hline 1982 & 7,956 & 2,470 & 10,426 & 184 & 10,610 \\
\hline 1983 & 9,148 & 2,984 & 12,132 & 189 & 12,321 \\
\hline 1984 & 10,324 & 3,863 & 14,187 & 282 & 14,469 \\
\hline 1985 & 10,979 & 4,458 & 15,437 & 295 & 15,732 \\
\hline 1986 & 11,404 & 4,594 & 15,998 & 277 & 16,275 \\
\hline 1987 & 10,192 & 4,610 & 14,802 & 302 & 15,104 \\
\hline 1988 & 10,547 & 4,800 & 15,347 & 348 & 15,695 \\
\hline 1989 & 9,779 & 4,610 & 14,389 & 330 & 14,719 \\
\hline 1990 & 9,303 & 4,548 & 13,851 & 297 & 14,148 \\
\hline 1991 & 8,189 & 4,123 & 12,312 & 242 & 12,554 \\
\hline 1992 & 8,213 & 4,629 & 12,842 & 276 & 13,118 \\
\hline 1993 & 8,518 & 5,351 & 13,869 & 330 & 14,199 \\
\hline 1994 & 8,991 & 6,033 & 15,024 & 387 & 15,411 \\
\hline 1995 & 8,635 & 6,053 & 14,688 & 428 & 15,116 \\
\hline 1996 & 8,526 & 6,519 & 15,045 & 411 & 15,456 \\
\hline 1997 & 8,272 & 6,797 & 15,069 & 430 & 15,499 \\
\hline 1998 & 8,142 & 7,299 & 15,441 & 526 & 15,967 \\
\hline 1999 & 8,698 & 8,073 & 16,771 & 641 & 17,412 \\
\hline 2000 & 8,847 & 8,387 & 17,234 & 579 & 17,813 \\
\hline 2001 & 8,423 & 8,700 & 17,123 & 452 & 17,575 \\
\hline 2002 & 8,103 & 8,713 & 16,816 & 402 & 17,218 \\
\hline 2003 & 7,610 & 8,938 & 16,548 & 420 & 16,968 \\
\hline 2004 & 7,545 & 9,361 & 16,906 & 538 & 17,444 \\
\hline 2005 & 7,720 & 9,281 & 17,001 & 664 & 17,665 \\
\hline 2006 & 7,821 & 8,684 & 16,505 & 694 & 17,199 \\
\hline 2007 & 7,618 & 8,471 & 16,089 & 537 & 16,626 \\
\hline 2008 & 6,813 & 6,381 & 13,194 & 432 & 13,626 \\
\hline 2009 & 5,456 & 4,945 & 10,401 & 312 & 10,713 \\
\hline \multicolumn{6}{|c|}{ Average annual percentage change } \\
\hline 1970-2009 & $-1.1 \%$ & $3.2 \%$ & $0.1 \%$ & $-0.2 \%$ & $0.1 \%$ \\
\hline $1999-2009$ & $-4.6 \%$ & $-4.8 \%$ & $-4.7 \%$ & $-6.9 \%$ & $-4.7 \%$ \\
\hline
\end{tabular}

\section{Source:}

Domestic and import data - 1970-97: American Automobile Manufacturers Association, Motor Vehicle Facts and Figures, 1998, Detroit, MI, 1998, p. 15 and annual. 1997 data from Economic Indicators, $4^{\text {th }}$ Quarter 1997. 1998-2008: Ward's Communication, Ward's Automotive Yearbook, Detroit, MI, 2009, p. 109.2009 : Ward's Communications, www.wardsauto.com. 
Using current registration data and a scrappage model by Greenspan and Cohen, 1996 paper: http://www.federalreserve.gov/pubs/feds/1996/199640/199640pap.pdf], ORNL calculated new automobile scrappage rates. The expected median lifetime for a 1990 model year automobile is 16.9 years. These data are fitted model values which assume constant economic conditions.

Table 3.11

Car Scrappage and Survival Rates 1970, 1980 and 1990 Model Years

\begin{tabular}{|c|c|c|c|c|c|c|}
\hline \multirow{2}{*}{$\begin{array}{l}\text { Vehicle } \\
\text { age }^{\mathrm{a}} \\
\text { (years) }\end{array}$} & \multicolumn{2}{|c|}{1970 Model Year } & \multicolumn{2}{|c|}{1980 Model Year } & \multicolumn{2}{|c|}{1990 Model Year } \\
\hline & $\begin{array}{c}\text { Survival } \\
\text { rate }^{\mathrm{b}}\end{array}$ & $\begin{array}{l}\text { Scrappage } \\
\text { rate }^{\text {c }}\end{array}$ & $\begin{array}{c}\text { Survival } \\
\text { rate }^{\mathrm{b}}\end{array}$ & $\begin{array}{c}\text { Scrappage } \\
\text { rate }^{\text {c }}\end{array}$ & $\begin{array}{c}\text { Survival } \\
\text { rate }^{\mathrm{b}}\end{array}$ & $\begin{array}{c}\text { Scrappage } \\
\text { rate }^{c}\end{array}$ \\
\hline 4 & 99.0 & 1.0 & 100.0 & 0.0 & 100.0 & 0.0 \\
\hline 5 & 94.1 & 5.0 & 96.3 & 3.7 & 100.0 & 0.0 \\
\hline 6 & 88.4 & 6.1 & 91.3 & 5.1 & 99.4 & 0.6 \\
\hline 7 & 82.0 & 7.2 & 85.7 & 6.1 & 96.3 & 3.2 \\
\hline 8 & 75.2 & 8.3 & 79.7 & 7.1 & 92.7 & 3.7 \\
\hline 9 & 68.1 & 9.5 & 73.3 & 8.1 & 88.7 & 4.3 \\
\hline 10 & 60.9 & 10.6 & 66.6 & 9.0 & 84.4 & 4.9 \\
\hline 11 & 53.8 & 11.7 & 60.0 & 10.0 & 79.8 & 5.5 \\
\hline 12 & 46.9 & 12.8 & 53.3 & 11.0 & 75.0 & 6.1 \\
\hline 13 & 40.3 & 14.0 & 46.9 & 12.0 & 70.0 & 6.7 \\
\hline 14 & 34.2 & 15.1 & 40.8 & 13.0 & 64.9 & 7.3 \\
\hline 15 & 28.7 & 16.2 & 35.1 & 14.0 & 59.7 & 7.9 \\
\hline 16 & 23.7 & 17.4 & 29.8 & 15.0 & 54.6 & 8.6 \\
\hline 17 & 19.3 & 18.5 & 25.0 & 16.1 & 49.5 & 9.3 \\
\hline 18 & 15.5 & 19.6 & 20.8 & 17.1 & 44.6 & 9.9 \\
\hline 19 & 12.3 & 20.8 & 17.0 & 18.1 & 39.9 & 10.6 \\
\hline 20 & 9.6 & 21.9 & 13.8 & 19.1 & 35.4 & 11.3 \\
\hline 21 & 7.4 & 23.0 & 11.0 & 20.1 & 31.1 & 12.0 \\
\hline 22 & 5.6 & 24.2 & 8.7 & 21.2 & 27.2 & 12.7 \\
\hline 23 & 4.2 & 25.3 & 6.7 & 22.2 & 23.5 & 13.5 \\
\hline 24 & 3.1 & 26.4 & 5.2 & 23.2 & 20.2 & 14.2 \\
\hline 25 & 2.2 & 27.5 & 3.9 & 24.2 & 17.1 & 15.0 \\
\hline 26 & 1.6 & 28.6 & 2.9 & 25.3 & 14.5 & 15.7 \\
\hline 27 & 1.1 & 29.7 & 2.2 & 26.3 & 12.1 & 16.5 \\
\hline 28 & 0.8 & 30.8 & 1.6 & 27.3 & 10.0 & 17.2 \\
\hline 29 & 0.5 & 31.9 & 1.1 & 28.4 & 8.2 & 18.0 \\
\hline 30 & 0.4 & 33.0 & 0.8 & 29.4 & 6.6 & 18.8 \\
\hline $\begin{array}{l}\text { Median } \\
\text { lifetime }\end{array}$ & \multicolumn{2}{|c|}{11.5 years } & \multicolumn{2}{|c|}{12.5 years } & \multicolumn{2}{|c|}{16.9 years } \\
\hline
\end{tabular}

Source:

Schmoyer, Richard L., unpublished study on scrappage rates, Oak Ridge National Laboratory, Oak Ridge, TN, 2001.

\footnotetext{
${ }^{\text {a }}$ It was assumed that scrappage for vehicles less than 4 years old is 0 .

${ }^{b}$ The percentage of automobiles which will be in use at the end of the year.

${ }^{c}$ The percentage of automobiles which will be retired from use during the year.
} 
Using current registration data and a scrappage model by Greenspan and Cohen 11996 paper: http://www.federalreserve.gov/pubs/feds/1996/199640/199640pap.pdf], ORNL calculated new light truck scrappage rates. The expected median lifetime for a 1990 model year light truck is 15.5 years. These data are fitted model values which assume constant economic conditions.

Table 3.12

Light Truck ${ }^{\text {a }}$ Scrappage and Survival Rates

\begin{tabular}{|c|c|c|c|c|c|c|}
\hline \multirow{2}{*}{$\begin{array}{l}\text { Vehicle } \\
\text { age }^{\mathrm{b}} \\
\text { (years) }\end{array}$} & \multicolumn{2}{|c|}{1970 model year } & \multicolumn{2}{|c|}{1980 model year } & \multicolumn{2}{|c|}{1990 model year } \\
\hline & $\begin{array}{l}\text { Survival } \\
\text { rate }^{\mathrm{b}}\end{array}$ & $\begin{array}{l}\text { Scrappage } \\
\text { rate }^{\text {c }}\end{array}$ & $\begin{array}{c}\text { Survival } \\
\text { rate }^{\mathrm{b}}\end{array}$ & $\begin{array}{l}\text { Scrappage } \\
\text { rate }^{\mathrm{d}}\end{array}$ & $\begin{array}{c}\text { Survival } \\
\text { rate }^{\mathrm{b}}\end{array}$ & $\begin{array}{l}\text { Scrappage } \\
\text { rate }^{\mathrm{c}}\end{array}$ \\
\hline & 99.7 & 0.3 & 99.1 & 0.9 & 99.3 & 0.7 \\
\hline & 97.5 & 2.2 & 96.6 & 2.5 & 96.9 & 2.4 \\
\hline & 94.9 & 2.7 & 93.7 & 3.1 & 94.1 & 3.0 \\
\hline & 91.8 & 3.2 & 90.2 & 3.7 & 90.7 & 3.6 \\
\hline & 88.3 & 3.8 & 86.3 & 4.3 & 86.9 & 4.2 \\
\hline & 84.4 & 4.4 & 82.0 & 5.0 & 82.7 & 4.8 \\
\hline 1 & 80.2 & 5.0 & 77.3 & 5.7 & 78.2 & 5.5 \\
\hline 1 & 75.7 & 5.6 & 72.4 & 6.4 & 73.4 & 6.1 \\
\hline 1 & 70.9 & 6.3 & 67.3 & 7.1 & 68.4 & 6.8 \\
\hline 1 & 66.0 & 6.9 & 62.1 & 7.8 & 63.3 & 7.5 \\
\hline 1 & 61.0 & 7.6 & 56.8 & 8.5 & 58.0 & 8.2 \\
\hline 1 & 55.9 & 8.3 & 51.5 & 9.3 & 52.8 & 9.0 \\
\hline 1 & 50.8 & 9.0 & 46.3 & 10.1 & 47.7 & 9.7 \\
\hline 1 & 45.9 & 9.8 & 41.3 & 10.8 & 42.7 & 10.5 \\
\hline 1 & 41.1 & 10.5 & 36.5 & 11.6 & 37.9 & 11.3 \\
\hline 1 & 36.4 & 11.3 & 32.0 & 12.4 & 33.3 & 12.1 \\
\hline 2 & 32.1 & 12.0 & 27.7 & 13.3 & 29.0 & 12.9 \\
\hline 2 & 28.0 & 12.8 & 23.8 & 14.1 & 25.0 & 13.7 \\
\hline 2 & 24.2 & 13.6 & 20.3 & 14.9 & 21.4 & 14.5 \\
\hline 2 & 20.7 & 14.4 & 17.1 & 15.8 & 18.1 & 15.4 \\
\hline 2 & 17.5 & 15.2 & 14.2 & 16.7 & 15.2 & 16.2 \\
\hline 2 & 14.7 & 16.1 & 11.7 & 17.5 & 12.6 & 17.1 \\
\hline 2 & 12.2 & 16.9 & 9.6 & 18.4 & 10.3 & 18.0 \\
\hline 2 & 10.1 & 17.8 & 7.7 & 19.3 & 8.4 & 18.8 \\
\hline 2 & 8.2 & 18.6 & 6.2 & 20.2 & 6.7 & 19.7 \\
\hline 2 & 6.6 & 19.5 & 4.9 & 21.1 & 5.3 & 20.6 \\
\hline 3 & 5.2 & 20.4 & 3.8 & 22.1 & 4.2 & 21.5 \\
\hline $\begin{array}{l}\text { Median } \\
\text { lifetime }\end{array}$ & & ears & 15. & rears & & ears \\
\hline
\end{tabular}

Source:

Schmoyer, Richard L., unpublished study on scrappage rates, Oak Ridge National Laboratory, Oak Ridge, TN, 2001.

${ }^{\text {a }}$ Light trucks are trucks less than 10,000 lbs. gross vehicle weight.

${ }^{\mathrm{b}}$ It was assumed that scrappage for vehicles less than 4 years old is 0 .

${ }^{\mathrm{c}}$ The percentage of light trucks which will be retired from use during the year.

${ }^{d}$ The percentage of light trucks which will be in use at the end of the year. 
A typical car will travel 152,137 miles in a lifetime, while a typical light truck will travel 179,954 miles.

Table 3.13

Car and Light Truck Survivability Rates and Lifetime Miles

\begin{tabular}{|c|c|c|}
\hline $\begin{array}{c}\text { Vehicle age } \\
\text { (Years) }\end{array}$ & $\begin{array}{c}\text { Car } \\
\text { Survivability } \\
\text { rate }\end{array}$ & $\begin{array}{c}\text { Light truck } \\
\text { survivability } \\
\text { rate }\end{array}$ \\
\hline 1 & 0.9900 & 0.9741 \\
\hline 2 & 0.9831 & 0.9603 \\
\hline 3 & 0.9731 & 0.9420 \\
\hline 4 & 0.9593 & 0.9190 \\
\hline 5 & 0.9413 & 0.8913 \\
\hline 6 & 0.9188 & 0.8590 \\
\hline 7 & 0.8918 & 0.8226 \\
\hline 8 & 0.8604 & 0.7827 \\
\hline 9 & 0.8252 & 0.7401 \\
\hline 10 & 0.7866 & 0.6956 \\
\hline 11 & 0.7170 & 0.6501 \\
\hline 12 & 0.6125 & 0.6040 \\
\hline 13 & 0.5094 & 0.5517 \\
\hline 14 & 0.4142 & 0.5009 \\
\hline 15 & 0.3308 & 0.4522 \\
\hline 16 & 0.2604 & 0.4062 \\
\hline 17 & 0.2028 & 0.3633 \\
\hline 18 & 0.1565 & 0.3236 \\
\hline 19 & 0.1200 & 0.2873 \\
\hline 20 & 0.0916 & 0.2542 \\
\hline 21 & 0.0696 & 0.2244 \\
\hline 22 & 0.0527 & 0.1975 \\
\hline 23 & 0.0399 & 0.1735 \\
\hline 24 & 0.0301 & 0.1522 \\
\hline 25 & 0.0227 & 0.1332 \\
\hline 26 & a & 0.1165 \\
\hline 27 & a & 0.1017 \\
\hline 28 & $\mathrm{a}$ & 0.0887 \\
\hline 29 & $\mathrm{a}$ & 0.0773 \\
\hline 30 & $\mathrm{a}$ & 0.0673 \\
\hline 31 & a & 0.0586 \\
\hline 32 & $\mathrm{a}$ & 0.0509 \\
\hline 33 & a & 0.0443 \\
\hline 34 & $\mathrm{a}$ & 0.0385 \\
\hline 35 & $\mathrm{a}$ & 0.0334 \\
\hline 36 & $\mathrm{a}$ & 0.0290 \\
\hline Lifetime miles & 152,137 & 179,954 \\
\hline
\end{tabular}

\section{Source:}

U.S. Department of Transportation, National Highway Traffic Safety Administration, Vehicle Survivability and Travel Mileage Schedules, January 2006.

Note: Registration data from 1977 to 2002 were used in developing these estimates. In this analysis, vehicle age was cut off when the estimated survival rate reached approximately a two percent threshold; for cars, this was 25 years, and for trucks it was 36 years.

${ }^{\mathrm{a}}$ Data are not available. 


\section{Chapter 4 \\ Light Vehicles and Characteristics}

Summary Statistics from Tables in this Chapter

Source

Table 4.1 Cars, 2008

Registrations (thousands)

137,080

Vehicle miles (million miles)

$1,615,850$

Fuel economy (miles per gallon)

22.6

Table 4.2 Two-axle, four-tire trucks, 2008

Registrations (thousands)

101,235

Vehicle miles (million miles)

$1,108,603$

Fuel economy (miles per gallon)

18.1

Table 4.6 Light truck share of total light vehicle sales

1970 calendar year $\quad 14.8 \%$

2009 calendar year $\quad 47.5 \%$

Table 4.7 Car sales, 2009 sales period (thousands) $\quad 8,200$

Small 3,040

Midsize 2,606

Large $\quad 1,875$

Table $4.9 \quad$ Light truck sales, 2009 model year (thousands) $\quad 7,797$

Midsize pickup 326

Large pickup $\quad 1,616$

Midsize van 696

Large van 29

Small SUV 191

Midsize SUV 2,463

Large SUV 2,476

Tables 4.21 Corporate average fuel economy (mpg)

$\begin{array}{llr}\text { and } 4.22 \quad \text { Car standard, MY } 2010 & 27.5\end{array}$

Car fuel economy, MY 2010

Light truck standard, MY 2010 (unreformed) 23.5

Light truck fuel economy, MY $2010 \quad 24.9$

Table $4.27 \quad$ Average fuel economy loss from 55 to $70 \mathrm{mph} \quad 17.1 \%$ 
Though car registrations grew slightly from 2007 to 2008, the vehicle travel and fuel use declined. The data in this table from 1985-on DO NOT include minivans, pickups, or sport utility vehicles.

Table 4.1

Summary Statistics for Cars, 1970-2008

\begin{tabular}{|c|c|c|c|c|}
\hline Year & $\begin{array}{c}\text { Registrations }^{\mathrm{a}} \\
\text { (thousands) }\end{array}$ & $\begin{array}{l}\text { Vehicle travel } \\
\text { (million miles) }\end{array}$ & $\begin{array}{c}\text { Fuel use } \\
\text { (million gallons) }\end{array}$ & $\begin{array}{l}\text { Fuel economy } \\
\text { (miles per gallon) }\end{array}$ \\
\hline 1970 & 89,244 & 916,700 & 67,820 & 13.5 \\
\hline 1975 & 106,706 & $1,033,950$ & 74,140 & 13.9 \\
\hline 1980 & 121,601 & $1,111,596$ & 69,981 & 15.9 \\
\hline 1981 & 123,098 & $1,133,332$ & 69,112 & 16.4 \\
\hline 1982 & 123,702 & $1,161,713$ & 69,116 & 16.8 \\
\hline 1983 & 126,444 & $1,195,054$ & 70,322 & 17.0 \\
\hline 1984 & 128,158 & $1,227,043$ & 70,663 & 17.4 \\
\hline $1985^{c}$ & 127,885 & $1,246,798$ & 71,518 & 17.4 \\
\hline 1986 & 130,004 & $1,270,167$ & 73,174 & 17.4 \\
\hline 1987 & 131,482 & $1,315,982$ & 73,308 & 18.0 \\
\hline 1988 & 133,836 & $1,370,271$ & 73,345 & 18.7 \\
\hline 1989 & 134,559 & $1,401,221$ & 73,913 & 19.0 \\
\hline 1990 & 133,700 & $1,408,266$ & 69,568 & 20.2 \\
\hline 1991 & 128,300 & $1,358,185$ & 64,318 & 21.1 \\
\hline 1992 & 126,581 & $1,371,569$ & 65,436 & 21.0 \\
\hline 1993 & 127,327 & $1,374,709$ & 67,047 & 20.5 \\
\hline 1994 & 127,883 & $1,406,089$ & 67,874 & 20.7 \\
\hline 1995 & 128,387 & $1,438,294$ & 68,072 & 21.1 \\
\hline 1996 & 129,728 & $1,469,854$ & 69,221 & 21.2 \\
\hline 1997 & 129,749 & $1,502,556$ & 69,892 & 21.5 \\
\hline 1998 & 131,839 & $1,549,577$ & 71,695 & 21.6 \\
\hline 1999 & 132,432 & $1,569,100$ & 73,283 & 21.4 \\
\hline 2000 & 133,621 & $1,600,287$ & 73,065 & 21.9 \\
\hline 2001 & 137,633 & $1,628,332$ & 73,559 & 22.1 \\
\hline 2002 & 135,921 & $1,658,474$ & 75,471 & 22.0 \\
\hline 2003 & 135,670 & $1,672,079$ & 74,590 & 22.2 \\
\hline 2004 & 136,431 & $1,699,890$ & 75,402 & 22.5 \\
\hline 2005 & 136,568 & $1,708,421$ & 77,418 & 22.1 \\
\hline 2006 & 135,400 & $1,690,534$ & 75,009 & 22.5 \\
\hline 2007 & 135,933 & $1,672,467$ & 74,377 & 22.5 \\
\hline 2008 & 137,080 & $1,615,850$ & 71,497 & 22.6 \\
\hline \multicolumn{5}{|c|}{ Average annual percentage change } \\
\hline 1970-2008 & $1.1 \%$ & $1.5 \%$ & $0.1 \%$ & $1.4 \%$ \\
\hline $1998-2008$ & $0.4 \%$ & $0.4 \%$ & $0.0 \%$ & $0.5 \%$ \\
\hline
\end{tabular}

\section{Source:}

U.S. Department of Transportation, Federal Highway Administration, Highway Statistics 2008, Washington, DC, 2009, Table VM-1 and annual. (Additional resources: www.fhwa.dot.gov)

${ }^{a}$ This number differs from R.L. Polk's estimates of "number of cars in use.” See Table 3.3.

${ }^{\mathrm{b}}$ Fuel economy for car population.

${ }^{\mathrm{c}}$ Beginning in this year the data were revised to exclude minivans, pickups and sport utility vehicles which may have been previously included. 
The Federal Highway Administration released revised historical data back to 1985 which better reflected two-axle, four-tire trucks. The definition of this category includes vans, pickup trucks, and sport utility vehicles. The number of two-axle, four-tire trucks registered in the United States decreased from 2007 to 2008, as did vehicle travel and fuel use.

Table 4.2

Summary Statistics for Two-Axle, Four-Tire Trucks, 1970-2008

\begin{tabular}{|c|c|c|c|c|}
\hline Year & $\begin{array}{c}\text { Registrations } \\
\text { (thousands) }\end{array}$ & $\begin{array}{c}\text { Vehicle travel } \\
\text { (million miles) }\end{array}$ & $\begin{array}{c}\text { Fuel use } \\
\text { (million gallons) }\end{array}$ & $\begin{array}{c}\text { Fuel economy } \\
\text { (miles per gallon) }\end{array}$ \\
\hline 1970 & 14,211 & 123,286 & 12,313 & 10.0 \\
\hline 1975 & 20,418 & 200,700 & 19,081 & 10.5 \\
\hline 1976 & 22,301 & 225,834 & 20,828 & 10.8 \\
\hline 1977 & 23,624 & 250,591 & 22,383 & 11.2 \\
\hline 1978 & 25,476 & 279,414 & 24,162 & 11.6 \\
\hline 1979 & 27,022 & 291,905 & 24,445 & 11.9 \\
\hline 1980 & 27,876 & 290,935 & 23,796 & 12.2 \\
\hline 1981 & 28,928 & 296,343 & 23,697 & 12.5 \\
\hline 1982 & 29,792 & 306,141 & 22,702 & 13.5 \\
\hline 1983 & 31,214 & 327,643 & 23,945 & 13.7 \\
\hline 1984 & 32,106 & 358,006 & 25,604 & 14.0 \\
\hline $1985^{\mathrm{a}}$ & 37,214 & 390,961 & 27,363 & 14.3 \\
\hline 1986 & 39,382 & 423,915 & 29,074 & 14.6 \\
\hline 1987 & 41,107 & 456,870 & 30,598 & 14.9 \\
\hline 1988 & 43,805 & 502,207 & 32,653 & 15.4 \\
\hline 1989 & 45,945 & 536,475 & 33,271 & 16.1 \\
\hline 1990 & 48,275 & 574,571 & 35,611 & 16.1 \\
\hline 1991 & 53,033 & 649,394 & 38,217 & 17.0 \\
\hline 1992 & 57,091 & 706,863 & 40,929 & 17.3 \\
\hline 1993 & 59,994 & 745,750 & 42,851 & 17.4 \\
\hline 1994 & 62,904 & 764,634 & 44,112 & 17.3 \\
\hline 1995 & 65,738 & 790,029 & 45,605 & 17.3 \\
\hline 1996 & 69,134 & 816,540 & 47,354 & 17.2 \\
\hline 1997 & 70,224 & 850,739 & 49,389 & 17.2 \\
\hline 1998 & 71,330 & 868,275 & 50,462 & 17.2 \\
\hline 1999 & 75,356 & 901,022 & 52,859 & 17.0 \\
\hline 2000 & 79,085 & 923,059 & 52,939 & 17.4 \\
\hline 2001 & 84,188 & 943,207 & 53,522 & 17.6 \\
\hline 2002 & 85,011 & 966,034 & 55,220 & 17.5 \\
\hline 2003 & 87,187 & 984,094 & 60,758 & 16.2 \\
\hline 2004 & 91,845 & $1,027,164$ & 63,417 & 16.2 \\
\hline 2005 & 95,337 & $1,041,051$ & 58,869 & 17.7 \\
\hline 2006 & 99,125 & $1,082,490$ & 60,685 & 17.8 \\
\hline 2007 & 101,470 & $1,112,271$ & 61,836 & 18.0 \\
\hline 2008 & 101,235 & $1,108,603$ & 61,199 & 18.1 \\
\hline \multicolumn{5}{|c|}{ Average annual percentage change } \\
\hline 1970-2008 & $5.3 \%$ & $6.0 \%$ & $4.3 \%$ & $1.6 \%$ \\
\hline $1998-2008$ & $3.6 \%$ & $2.5 \%$ & $1.9 \%$ & $0.5 \%$ \\
\hline
\end{tabular}

Source:

U.S. Department of Transportation, Federal Highway Administration, Highway Statistics 2008, Washington, DC, 2009, Table VM-1 and annual. (Additional resources: www.fhwa.dot.gov)

${ }^{a}$ Beginning in this year the data were revised to include all vans (including mini-vans), pickups and sport utility vehicles. 
Because data on Class $2 b$ trucks are scarce, the U.S. DOE funded a study to investigate available sources of data. In the final report, four methodologies are described to estimate the sales of Class $2 b$ trucks. Until another study is funded, the 1999 data are the latest available.

Table 4.3

Summary Statistics on Class 1, Class 2a, and Class 2b Light Trucks

\begin{tabular}{lccccccc}
\hline & $\begin{array}{c}\text { CY 1999 } \\
\text { truck sales } \\
\text { (millions) }\end{array}$ & $\begin{array}{c}\text { MY 2000 } \\
\text { truck } \\
\text { population } \\
\text { (millions) }\end{array}$ & $\begin{array}{c}\text { Percent } \\
\text { diesel trucks } \\
\text { in } \\
\text { population }\end{array}$ & $\begin{array}{c}\text { Average } \\
\text { age } \\
\text { (years) }\end{array}$ & $\begin{array}{c}\text { Estimated } \\
\text { annual } \\
\text { miles }^{\mathrm{a}} \\
\text { (billions) }\end{array}$ & $\begin{array}{c}\text { Estimated } \\
\text { fuel use } \\
\text { (billion }^{\mathrm{a}} \\
\text { gallons) }\end{array}$ & $\begin{array}{c}\text { Estimated fuel } \\
\text { economy } \\
\text { (miles per } \\
\text { gallon) }\end{array}$ \\
\hline Class 1 & 5.7 & 49.7 & $0.3 \%$ & 7.3 & 672.7 & 37.4 & 18.0 \\
Class 2a & 1.8 & 19.2 & $2.5 \%$ & 7.4 & 251.9 & 18 & 14.0 \\
Class 2b & 0.5 & 5.8 & $24.0 \%$ & 8.6 & 76.7 & 5.5 & 13.9 \\
\hline
\end{tabular}

Source:

Davis, S.C. and L.F. Truett, Investigation of Class $2 b$ Trucks (Vehicles of 8,500 to 10,000 lbs GVWR), ORNL/TM2002/49, March 2002, Table 16.

Note: CY - calendar year. MY - model year.

Table 4.4

Sales Estimates of Class 1, Class 2a, and Class 2b Light Trucks, 1989-1999

\begin{tabular}{ccccc}
\hline & \multicolumn{4}{c}{ Sales estimates (thousands) } \\
\cline { 2 - 5 } Calendar Year & Class 1 & $\begin{array}{c}\text { Class 2a } \\
(6,000 \text { lbs and under })\end{array}$ & $\begin{array}{c}\text { Class 2b } \\
(8,501-10,000 \mathrm{lbs})\end{array}$ & Total \\
\hline 1989 & 3,313 & $91800 \mathrm{lbs})$ & 379 & 4,610 \\
1990 & 3,451 & 829 & 268 & 4,548 \\
1991 & 3,246 & 670 & 206 & 4,122 \\
1992 & 3,608 & 827 & 194 & 4,629 \\
1993 & 4,119 & 975 & 257 & 5,351 \\
1994 & 4,527 & 1,241 & 265 & 6,033 \\
1995 & 4,422 & 1,304 & 327 & 6,053 \\
1996 & 4,829 & 1,356 & 334 & 6,519 \\
1997 & 5,085 & 1,315 & 397 & 6,797 \\
1998 & 5,263 & 1,694 & 342 & 7,299 \\
1999 & 5,707 & 1,845 & 521 & 8,073 \\
$1989-1999$ & & & $37.5 \%$ & $75.1 \%$ \\
\hline
\end{tabular}

\section{Source:}

Davis, S.C. and L.F. Truett, Investigation of Class $2 b$ Trucks (Vehicles of 8,500 to 10,000 lbs GVWR), ORNL/TM2002/49, March 2002, Table 1.

Note: These data were calculated using Methodology 4 from the report.

${ }^{\text {a }}$ Estimates derived using 2000 population data and 1997 usage data. See source for details. 
Car sales in 2009 dropped below 6 million. In 1980, the Big 3 (Chrysler, Ford and General Motors) held 73.8\% of the market; by 2009, that had dropped to $31.8 \%$.

Table 4.5

New Retail Car Sales in the United States, 1970-2009

\begin{tabular}{|c|c|c|c|c|c|c|}
\hline $\begin{array}{c}\text { Calendar } \\
\text { year }\end{array}$ & Domestic $^{\mathrm{a}}$ & $\frac{\text { Import }^{\mathrm{b}}}{\text { (thousands) }^{2}}$ & Total & $\begin{array}{l}\text { Percentage } \\
\text { imports }\end{array}$ & $\begin{array}{c}\text { Percentage } \\
\text { Big } 3 \\
\text { Sales }^{c}\end{array}$ & $\begin{array}{c}\text { Percentage } \\
\text { diesel }\end{array}$ \\
\hline 1970 & 7,119 & 1,280 & 8,399 & $15.2 \%$ & $\mathrm{~d}$ & $\mathrm{~d}$ \\
\hline 1975 & 7,053 & 1,571 & 8,624 & $18.2 \%$ & d & $0.31 \%$ \\
\hline 1980 & 6,580 & 2,369 & 8,949 & $26.5 \%$ & $73.8 \%$ & $4.31 \%$ \\
\hline 1985 & 8,205 & 2,775 & 10,979 & $25.3 \%$ & $72.9 \%$ & $0.82 \%$ \\
\hline 1986 & 8,215 & 3,189 & 11,404 & $28.0 \%$ & $70.9 \%$ & $0.37 \%$ \\
\hline 1987 & 7,085 & 3,107 & 10,192 & $30.5 \%$ & $67.6 \%$ & $0.16 \%$ \\
\hline 1988 & 7,543 & 3,004 & 10,547 & $28.5 \%$ & $69.3 \%$ & $0.02 \%$ \\
\hline 1989 & 7,098 & 2,680 & 9,779 & $27.4 \%$ & $67.9 \%$ & $0.13 \%$ \\
\hline 1990 & 6,919 & 2,384 & 9,303 & $25.6 \%$ & $65.7 \%$ & $0.08 \%$ \\
\hline 1991 & 6,162 & 2,028 & 8,189 & $24.8 \%$ & $64.2 \%$ & $0.10 \%$ \\
\hline 1992 & 6,286 & 1,927 & 8,213 & $23.5 \%$ & $65.8 \%$ & $0.06 \%$ \\
\hline 1993 & 6,742 & 1,776 & 8,518 & $20.8 \%$ & $67.3 \%$ & $0.03 \%$ \\
\hline 1994 & 7,255 & 1,735 & 8,991 & $19.3 \%$ & $65.9 \%$ & $0.04 \%$ \\
\hline 1995 & 7,129 & 1,506 & 8,635 & $17.4 \%$ & $65.3 \%$ & $0.04 \%$ \\
\hline 1996 & 7,255 & 1,271 & 8,526 & $14.9 \%$ & $64.1 \%$ & $0.10 \%$ \\
\hline 1997 & 6,917 & 1,355 & 8,272 & $16.4 \%$ & $62.2 \%$ & $0.09 \%$ \\
\hline 1998 & 6,762 & 1,380 & 8,142 & $16.9 \%$ & $59.7 \%$ & $0.13 \%$ \\
\hline 1999 & 6,979 & 1,719 & 8,698 & $19.8 \%$ & $58.3 \%$ & $0.16 \%$ \\
\hline 2000 & 6,831 & 2,016 & 8,847 & $22.8 \%$ & $55.0 \%$ & $0.26 \%$ \\
\hline 2001 & 6,325 & 2,098 & 8,423 & $24.9 \%$ & $51.4 \%$ & $0.18 \%$ \\
\hline 2002 & 5,878 & 2,226 & 8,103 & $27.5 \%$ & $48.4 \%$ & $0.39 \%$ \\
\hline 2003 & 5,527 & 2,083 & 7,610 & $27.4 \%$ & $47.1 \%$ & $0.51 \%$ \\
\hline 2004 & 5,396 & 2,149 & 7,545 & $28.5 \%$ & $44.9 \%$ & $0.40 \%$ \\
\hline 2005 & 5,533 & 2,187 & 7,720 & $28.3 \%$ & $43.1 \%$ & $0.63 \%$ \\
\hline 2006 & 5,476 & 2,345 & 7,821 & $30.0 \%$ & $41.5 \%$ & $0.82 \%$ \\
\hline 2007 & 5,253 & 2,365 & 7,618 & $31.0 \%$ & $38.0 \%$ & $0.11 \%$ \\
\hline 2008 & 4,535 & 2,278 & 6,813 & $33.4 \%$ & $34.9 \%$ & $0.11 \%$ \\
\hline 2009 & 3,619 & 1,837 & 5,456 & $33.7 \%$ & $31.8 \%$ & $2.09 \%$ \\
\hline \multicolumn{7}{|c|}{ Average annual percentage change } \\
\hline 1970-2009 & $-1.7 \%$ & $0.9 \%$ & $-1.1 \%$ & & & \\
\hline 1999-2009 & $-6.4 \%$ & $0.7 \%$ & $-4.6 \%$ & & & \\
\hline
\end{tabular}

\section{Source:}

Domestic and import data - 1970-97: American Automobile Manufacturers Association, Motor Vehicle Facts and Figures 1998, Detroit, MI, 1998, p. 15, and annual. 1997 data from Economic Indicators, 4th Quarter 1997. 1998-2008: Ward's Communication, Ward's Automotive Yearbook, Detroit, MI, 2009, p. 249. 2009: Ward's Communications, www.wardsauto.com.

Diesel data - Ward's Communications, Ward's Automotive Yearbook, Detroit, MI, 2009, p. 31.

Transplant data - Oak Ridge National Laboratory, Light Vehicle MPG and Market Shares Data System, Oak Ridge,

TN, 2004. (Additional resources: www.aama.com, www.wardsauto.com)

${ }^{a}$ North American built.

${ }^{\mathrm{b}}$ Does not include import tourist deliveries.

${ }^{c}$ Big 3 includes Chrysler, Ford and General Motors. Beginning in 1998, Ford includes Jaguar and Volvo. GM Includes Saab.

${ }^{\mathrm{d}}$ Data are not available. 
Light trucks, which include pick-ups, minivans, sport-utility vehicles, and other trucks less than 10,000 pounds gross vehicle weight $(G V W)$, accounted for more than half of light vehicle sales from 2001 to 2007.

Table 4.6

New Retail Sales of Trucks 10,000 Pounds GVW and Less in the United States, 1970-2009

\begin{tabular}{|c|c|c|c|c|c|c|}
\hline \multirow[b]{2}{*}{$\begin{array}{c}\text { Calendar } \\
\text { year }\end{array}$} & \multirow[b]{2}{*}{$\begin{array}{l}\text { Light truck } \\
\text { sales }^{\mathrm{a}} \\
\text { (thousands) }\end{array}$} & \multicolumn{5}{|c|}{ Percentages } \\
\hline & & Import $^{\mathrm{b}}$ & $\begin{array}{l}\text { Big } 3 \\
\text { Sales }^{\text {c }}\end{array}$ & Diesel $^{\mathrm{d}}$ & $\begin{array}{l}\text { Light trucks of } \\
\text { light-duty } \\
\text { vehicle sales }\end{array}$ & $\begin{array}{c}\text { Light trucks } \\
\text { of total } \\
\text { truck sales }\end{array}$ \\
\hline 1970 & 1,463 & $4.5 \%$ & & $\mathrm{f}$ & $14.8 \%$ & $80.4 \%$ \\
\hline 1975 & 2,281 & $10.0 \%$ & & $\mathrm{f}$ & $20.9 \%$ & $87.9 \%$ \\
\hline 1980 & 2,440 & $19.7 \%$ & & $3.6 \%$ & $21.4 \%$ & $88.9 \%$ \\
\hline 1981 & 2,189 & $20.3 \%$ & & $3.1 \%$ & $20.4 \%$ & $89.8 \%$ \\
\hline 1982 & 2,470 & $16.5 \%$ & & $8.5 \%$ & $23.6 \%$ & $92.8 \%$ \\
\hline 1983 & 2,984 & $15.6 \%$ & & $6.7 \%$ & $24.5 \%$ & $93.6 \%$ \\
\hline 1984 & 3,863 & $15.7 \%$ & $78.8 \%$ & $4.8 \%$ & $27.1 \%$ & $93.0 \%$ \\
\hline 1985 & 4,458 & $17.2 \%$ & $78.2 \%$ & $3.8 \%$ & $28.8 \%$ & $93.6 \%$ \\
\hline 1986 & 4,594 & $20.1 \%$ & $76.9 \%$ & $3.7 \%$ & $28.6 \%$ & $94.3 \%$ \\
\hline 1987 & 4,610 & $17.9 \%$ & $78.3 \%$ & $2.3 \%$ & $31.0 \%$ & $93.9 \%$ \\
\hline 1988 & 4,800 & $12.6 \%$ & $81.6 \%$ & $2.3 \%$ & $31.1 \%$ & $93.2 \%$ \\
\hline 1989 & 4,610 & $10.9 \%$ & $81.9 \%$ & $2.9 \%$ & $31.8 \%$ & $93.3 \%$ \\
\hline 1990 & 4,548 & $13.2 \%$ & $80.9 \%$ & $3.1 \%$ & $32.8 \%$ & $93.9 \%$ \\
\hline 1991 & 4,123 & $12.8 \%$ & $79.4 \%$ & $3.2 \%$ & $33.5 \%$ & $94.5 \%$ \\
\hline 1992 & 4,629 & $8.6 \%$ & $83.1 \%$ & $3.3 \%$ & $36.0 \%$ & $94.4 \%$ \\
\hline 1993 & 5,351 & $6.8 \%$ & $83.4 \%$ & $3.7 \%$ & $38.6 \%$ & $94.2 \%$ \\
\hline 1994 & 6,033 & $6.5 \%$ & $82.9 \%$ & $3.9 \%$ & $40.2 \%$ & $94.0 \%$ \\
\hline 1995 & 6,053 & $6.5 \%$ & $83.4 \%$ & $4.1 \%$ & $41.2 \%$ & $93.4 \%$ \\
\hline 1996 & 6,519 & $6.6 \%$ & $83.8 \%$ & $3.7 \%$ & $43.3 \%$ & $94.1 \%$ \\
\hline 1997 & 6,797 & $8.4 \%$ & $81.9 \%$ & $4.8 \%$ & $46.6 \%$ & $94.1 \%$ \\
\hline 1998 & 7,299 & $8.9 \%$ & $80.5 \%$ & $1.7 \%$ & $47.3 \%$ & $93.3 \%$ \\
\hline 1999 & 8,073 & $9.5 \%$ & $78.0 \%$ & $5.9 \%$ & $48.1 \%$ & $92.6 \%$ \\
\hline 2000 & 8,387 & $9.9 \%$ & $76.1 \%$ & $4.8 \%$ & $48.7 \%$ & $93.9 \%$ \\
\hline 2001 & 8,700 & $11.3 \%$ & $75.3 \%$ & $5.3 \%$ & $50.8 \%$ & $96.1 \%$ \\
\hline 2002 & 8,713 & $12.2 \%$ & $74.7 \%$ & $4.9 \%$ & $51.8 \%$ & $96.4 \%$ \\
\hline 2003 & 8,938 & $13.5 \%$ & $72.4 \%$ & $4.3 \%$ & $54.0 \%$ & $95.5 \%$ \\
\hline 2004 & 9,361 & $13.1 \%$ & $70.1 \%$ & $5.5 \%$ & $55.4 \%$ & $95.5 \%$ \\
\hline 2005 & 9,281 & $13.2 \% \mathrm{f}$ & $68.2 \%$ & $3.7 \%$ & $54.7 \%$ & $94.9 \%$ \\
\hline 2006 & 8,684 & $15.6 \%$ & $64.1 \%$ & $3.7 \%$ & $52.6 \%$ & $94.1 \%$ \\
\hline 2007 & 8,471 & $16.4 \%$ & $65.0 \%$ & $3.9 \%$ & $52.6 \%$ & $95.8 \%$ \\
\hline 2008 & 6,381 & $17.2 \%$ & $62.1 \%$ & $4.1 \%$ & $48.4 \%$ & $95.5 \%$ \\
\hline 2009 & 4,945 & $17.9 \%$ & $59.1 \%$ & $4.8 \%$ & $47.5 \%$ & $96.1 \%$ \\
\hline \multicolumn{7}{|c|}{ Average annual percentage change } \\
\hline 1970-2009 & $3.2 \%$ & & & & & \\
\hline 1999-2009 & $-4.8 \%$ & & & & & \\
\hline
\end{tabular}

\section{Source:}

Ward's Communications, Ward's Automotive Yearbook, Detroit, MI, 2009, and updates at www.wardsauto.com. (Additional resources: www.wardsauto.com)

${ }^{\text {a }}$ Includes all trucks of 10,000 pounds gross vehicle weight and less sold in the United States.

${ }^{\mathrm{b}}$ Excluding transplants.

${ }^{c}$ Big 3 includes Chrysler, Ford and General Motors. Beginning in 1998, Ford includes Land Rover and Volvo light trucks and GM includes Saab. Trucks include light, medium and heavy trucks.

${ }^{\mathrm{d}}$ Based on model year factory installations.

${ }^{\mathrm{e}}$ Light-duty vehicles include cars and light trucks.

${ }^{\mathrm{f}}$ Indicates less than 1 percent. 
The sales-weighted fuel economy of cars increased dramatically from 1975 (15.4 mpg) to 1990 (26.2 mpg), but has

risen only about $3.1 \mathrm{mpg}$ since then.

Table 4.7

Period Sales, Market Shares, and Sales-Weighted Fuel Economies

of New Domestic and Import Cars, Selected Model Years 1975-2009 ${ }^{a}$

(thousands)

\begin{tabular}{|c|c|c|c|c|c|c|c|c|}
\hline & \multicolumn{7}{|c|}{ Sales Period } & \multirow[b]{2}{*}{2009} \\
\hline & 1975 & 1980 & 1985 & 1990 & 1995 & 2000 & 2005 & \\
\hline \multicolumn{9}{|l|}{ CARS } \\
\hline \multicolumn{9}{|l|}{ Small } \\
\hline Total sales, units & 4,088 & 4,825 & 5,519 & 4,999 & 5,190 & 4,266 & 3,185 & 3,040 \\
\hline Market share, \% & $49.6 \%$ & $51.1 \%$ & $51.1 \%$ & $56.8 \%$ & $55.2 \%$ & $46.7 \%$ & $39.7 \%$ & $37.1 \%$ \\
\hline $\begin{array}{l}\text { Fuel economy, mpg } \\
\text { Midsize }\end{array}$ & 18.3 & 26.1 & 29.8 & 29.8 & 30.7 & 30.3 & 31.1 & 32.6 \\
\hline Total sales, units & 1,631 & 2,987 & 2,777 & 2,342 & 2,515 & 2,894 & 2,886 & 2,606 \\
\hline Market share, \% & $19.8 \%$ & $31.6 \%$ & $25.7 \%$ & $26.6 \%$ & $26.8 \%$ & $31.7 \%$ & $36.0 \%$ & $31.8 \%$ \\
\hline $\begin{array}{l}\text { Fuel economy, mpg } \\
\text { Large }\end{array}$ & 13.6 & 21.6 & 24.9 & 26.2 & 26.1 & 27.0 & 29.8 & 31.7 \\
\hline Total sales, units & 1,555 & 963 & 1,512 & 1,092 & 1,306 & 1,665 & 1,234 & 1,875 \\
\hline Market share, \% & $18.9 \%$ & $10.2 \%$ & $14.0 \%$ & $12.4 \%$ & $13.9 \%$ & $18.2 \%$ & $15.4 \%$ & $22.9 \%$ \\
\hline Fuel economy, mpg & 13.1 & 19.1 & 22.3 & 23.7 & 24.5 & 25.6 & 26.4 & 27.5 \\
\hline \multicolumn{9}{|l|}{ WAGONS } \\
\hline \multicolumn{9}{|l|}{ Small } \\
\hline Total sales, units & 477 & 310 & 496 & 160 & 198 & 68 & 365 & 549 \\
\hline Market share, \% & $5.8 \%$ & $3.3 \%$ & $4.6 \%$ & $1.8 \%$ & $2.1 \%$ & $0.7 \%$ & $4.5 \%$ & $6.7 \%$ \\
\hline \multicolumn{9}{|l|}{ Midsize } \\
\hline Total sales, units & 289 & 257 & 341 & 184 & 176 & 234 & 238 & 122 \\
\hline Market share, \% & $3.5 \%$ & $2.7 \%$ & $3.2 \%$ & $2.1 \%$ & $1.9 \%$ & $2.6 \%$ & $3.0 \%$ & $1.5 \%$ \\
\hline $\begin{array}{l}\text { Fuel economy, mpg } \\
\text { Large }\end{array}$ & 13.2 & 21.1 & 25.2 & 25.3 & 26.6 & 27.3 & 26.0 & 27.7 \\
\hline Total sales, units & 197 & 102 & 145 & 31 & 10 & 0 & 118 & 77 \\
\hline Market share, \% & $2.4 \%$ & $1.1 \%$ & $1.3 \%$ & $0.4 \%$ & $0.1 \%$ & $0.0 \%$ & $1.5 \%$ & $0.1 \%$ \\
\hline Fuel economy, mpg & 11.9 & 19.1 & 20.9 & 22.7 & 22.8 & & 22.2 & 21.8 \\
\hline \multicolumn{9}{|l|}{ TOTAL } \\
\hline Total sales, units & 8,237 & 9,443 & 10,791 & 8,810 & 9,396 & 9,128 & 8,027 & 8,200 \\
\hline Market share, \% & $100.0 \%$ & $100.0 \%$ & $100.0 \%$ & $100.0 \%$ & $100.0 \%$ & $100.0 \%$ & $100.0 \%$ & $100.0 \%$ \\
\hline Fuel economy, mpg & 15.8 & 23.5 & 27.0 & 27.8 & 28.3 & 28.2 & 29.5 & 30.9 \\
\hline
\end{tabular}

Source:

U.S. Environmental Protection Agency, Light-Duty Automotive Technology, Carbon Dioxide Emissions, and Fuel Economy Trends: 1975 Through 2009, November 2009. (Additional resources: www.epa.gov/otaq/fetrends.htm)

\footnotetext{
${ }^{a}$ The fuel economy data on this table are EPA laboratory test values.

${ }^{\mathrm{b}}$ No vehicles in this category were sold in this model year.
} 
The term "wagon" conjures up images of the station wagons from the 1960's. However, most of the cars that are now classified as wagons have little in common with those station wagons. The wagons below make up the category "wagon" on Tables 4.7 through 4.14.

Table 4.8

Definition of Wagons in Model Year 2009

\begin{tabular}{l}
\multicolumn{1}{c}{ Small Wagon } \\
\hline Audi A3 \\
Audi A3 Quattro \\
Audi A4 Avant Quattro \\
BMW 328I Sport \\
BMW 328XI Sport Xdriv \\
Chrysler PT Cruiser \\
General Motors HHR FWD \\
General Motors HHR Panel FWD \\
General Motors Vibe \\
Honda Fit \\
Hyundai Elantra Touring \\
Mitsubishi Lancer Sportback \\
Nissan Cube \\
Saab 9-3 Aero Sportcombi AWD \\
Saab 9-3 Sportcombi \\
Subaru Impreza WGN-Outback SPT AWD \\
Suzuki SX4 \\
Suzuki SX4 AWD \\
Toyota Corolla Matrix \\
Toyota Scion XB \\
Volkswagen Jetta Sportwagen \\
Volvo V50 AWD \\
Volvo V50 FWD \\
\hline \\
Audi A6 Avant Quattro \\
BMW 535XI Sport Xdriv \\
Daimler AG E350 4MATIC \\
Daimler AG E63 AMG \\
Kia Rondo \\
Mazda Mazda 5 \\
Saab 9-5 Sportcombi \\
Subaru Outback AWD \\
Volkswagen Passat \\
Volvo V70 FWD \\
Volvo XC 70 AWD \\
\hline Daimler AG R350 Bluetec \\
Daimler AG R350 4MATIC \\
\hline \\
\hline
\end{tabular}

Source:

U.S. Environmental Protection Agency, Light-Duty Automotive Technology, Carbon Dioxide Emissions, and Fuel Economy Trends: 1975 Through 2009, November 2009. (Additional resources: www.epa.gov/otaq/fetrends.htm) 
Sales of light trucks in 2009 are almost four times that of 1975. Similar to the car trend, the sales-weighted fuel economy of light trucks increased substantially during the late '70's and '80's, but has increased slowly since then.

Table 4.9

Period Sales, Market Shares, and Sales-Weighted Fuel Economies ${ }^{\mathrm{a}}$ of New Domestic and Import Light Trucks, Model Years 1975-2009

(thousands)

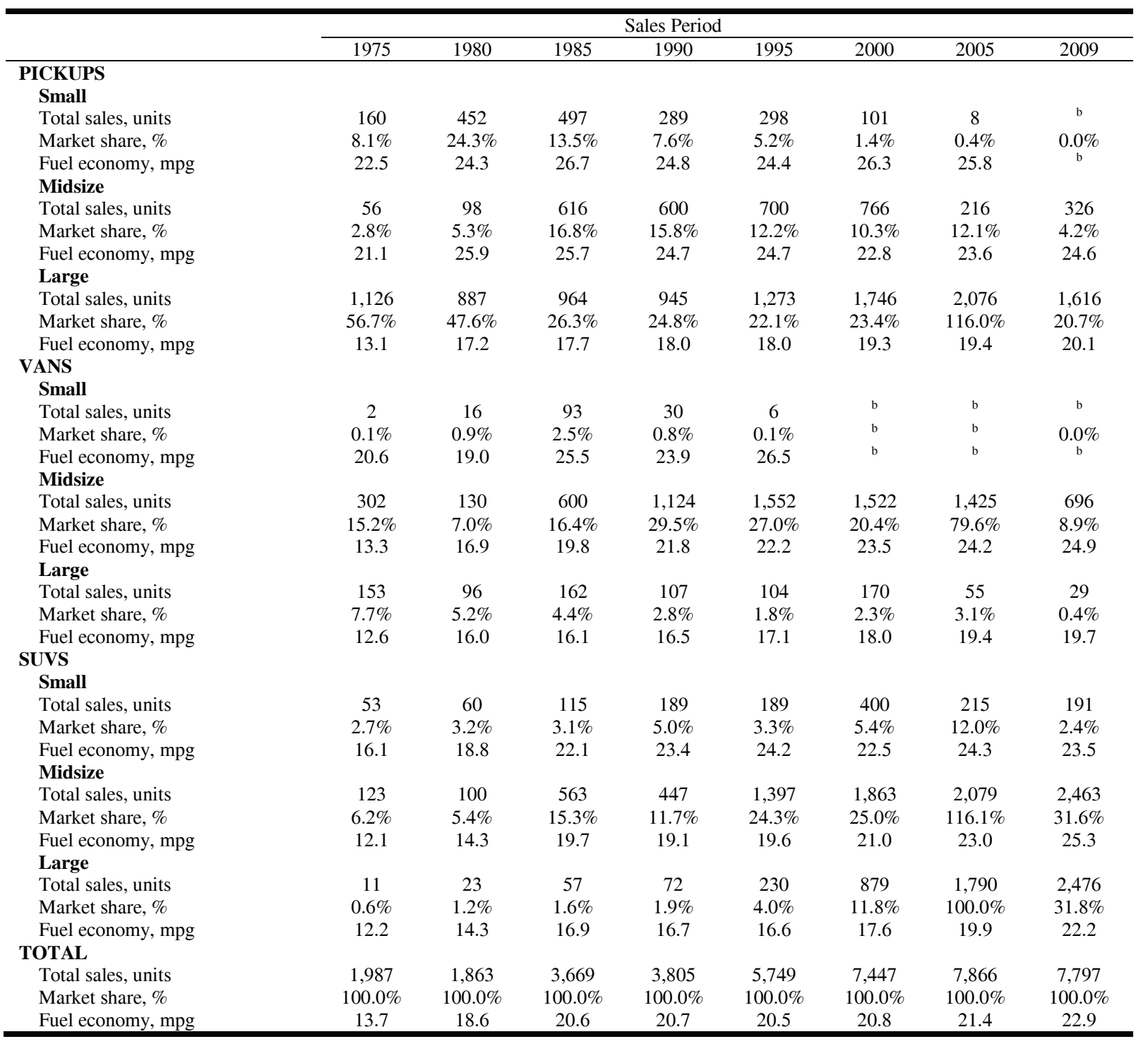

\section{Source:}

U.S. Environmental Protection Agency, Light-Duty Automotive Technology, Carbon Dioxide Emissions, and Fuel Economy Trends: 1975 Through 2009, November 2009. (Additional resources: www.epa.gov/otaq/fetrends.htm)

Note: Includes light trucks of $8,500 \mathrm{lbs}$. or less.

${ }^{a}$ The fuel economy data on this table are EPA laboratory test values.

${ }^{\mathrm{b}}$ No vehicles in this category were sold in this model year. 
Back in 1975 only 19\% of new light vehicle sales were light trucks. Because of the boom in sales of minivans, sport utility vehicles, and pick-up trucks, today almost half of light vehicle sales are light trucks.

Table 4.10

Light Vehicle Market Shares by Size Class, Model Years 1975-2009

\begin{tabular}{|c|c|c|c|c|c|c|c|c|}
\hline & \multicolumn{8}{|c|}{ Model Year } \\
\hline & 1975 & 1980 & 1985 & 1990 & 1995 & 2000 & 2005 & 2009 \\
\hline Small car & $40.0 \%$ & $42.7 \%$ & $38.2 \%$ & $39.6 \%$ & $34.3 \%$ & $25.7 \%$ & $20.0 \%$ & $19.0 \%$ \\
\hline Midsize car & $16.0 \%$ & $26.4 \%$ & $19.2 \%$ & $18.6 \%$ & $16.6 \%$ & $17.5 \%$ & $18.2 \%$ & $16.3 \%$ \\
\hline Large car & $15.2 \%$ & $8.5 \%$ & $10.5 \%$ & $8.7 \%$ & $8.6 \%$ & $10.0 \%$ & $7.8 \%$ & $11.7 \%$ \\
\hline Small wagon & $4.7 \%$ & $2.7 \%$ & $3.4 \%$ & $1.3 \%$ & $1.3 \%$ & $0.4 \%$ & $2.3 \%$ & $3.4 \%$ \\
\hline Midsize wagon & $2.8 \%$ & $2.3 \%$ & $2.4 \%$ & $1.5 \%$ & $1.2 \%$ & $1.4 \%$ & $1.5 \%$ & $0.8 \%$ \\
\hline Large wagon & $1.9 \%$ & $0.9 \%$ & $1.0 \%$ & $0.2 \%$ & $0.1 \%$ & $0.0 \%$ & $0.7 \%$ & $0.0 \%$ \\
\hline Small pickup & $1.6 \%$ & $4.0 \%$ & $3.4 \%$ & $2.3 \%$ & $2.0 \%$ & $0.6 \%$ & $0.1 \%$ & $0.0 \%$ \\
\hline Midsize pickup & $0.5 \%$ & $0.9 \%$ & $4.3 \%$ & $4.8 \%$ & $4.6 \%$ & $4.6 \%$ & $1.4 \%$ & $2.0 \%$ \\
\hline Large pickup & $11.0 \%$ & $7.8 \%$ & $6.7 \%$ & $7.5 \%$ & $8.4 \%$ & $10.5 \%$ & $13.1 \%$ & $10.1 \%$ \\
\hline Small van & $0.0 \%$ & $0.1 \%$ & $0.6 \%$ & $0.2 \%$ & $0.0 \%$ & $0.0 \%$ & $0.0 \%$ & $0.0 \%$ \\
\hline Midsize van & $3.0 \%$ & $1.1 \%$ & $4.1 \%$ & $8.9 \%$ & $10.2 \%$ & $9.2 \%$ & $9.0 \%$ & $4.4 \%$ \\
\hline Large van & $1.5 \%$ & $0.8 \%$ & $1.1 \%$ & $0.9 \%$ & $0.7 \%$ & $1.0 \%$ & $0.3 \%$ & $0.2 \%$ \\
\hline Small SUV & $0.5 \%$ & $0.5 \%$ & $0.8 \%$ & $1.5 \%$ & $1.3 \%$ & $2.4 \%$ & $1.4 \%$ & $1.2 \%$ \\
\hline Midsize SUV & $1.2 \%$ & $1.0 \%$ & $3.9 \%$ & $3.5 \%$ & $9.2 \%$ & $11.2 \%$ & $13.1 \%$ & $15.4 \%$ \\
\hline Large SUV & $0.1 \%$ & $0.2 \%$ & $0.4 \%$ & $0.6 \%$ & $1.5 \%$ & $5.3 \%$ & $11.3 \%$ & $15.5 \%$ \\
\hline \multirow{2}{*}{$\begin{array}{l}\text { Total light vehicles sold } \\
\text { (thousands) }\end{array}$} & & & & & & & & \\
\hline & 10,224 & 11,306 & 14,460 & 12,615 & 15,145 & 16,575 & 15,893 & 15,997 \\
\hline Cars & $80.6 \%$ & $83.5 \%$ & $74.6 \%$ & $69.8 \%$ & $62.0 \%$ & $55.1 \%$ & $50.5 \%$ & $51.3 \%$ \\
\hline Light trucks & $19.4 \%$ & $16.5 \%$ & $25.4 \%$ & $30.2 \%$ & $38.0 \%$ & $44.9 \%$ & $49.5 \%$ & $48.7 \%$ \\
\hline
\end{tabular}

Source:

U.S. Environmental Protection Agency, Light-Duty Automotive Technology, Carbon Dioxide Emissions, and Fuel Economy Trends: 1975 Through 2009, November 2009. (Additional resources: www.epa.gov/otaq/fetrends.htm)

Note: Includes light trucks of $8,500 \mathrm{lbs}$. or less. 
Light trucks were gaining market share from the early 1980s until 2004, mainly due to increases in the market share of sport utility vehicles (SUVs) and pickup trucks.

Figure 4.1. Light Vehicle Market Shares, Model Years 1975-2009

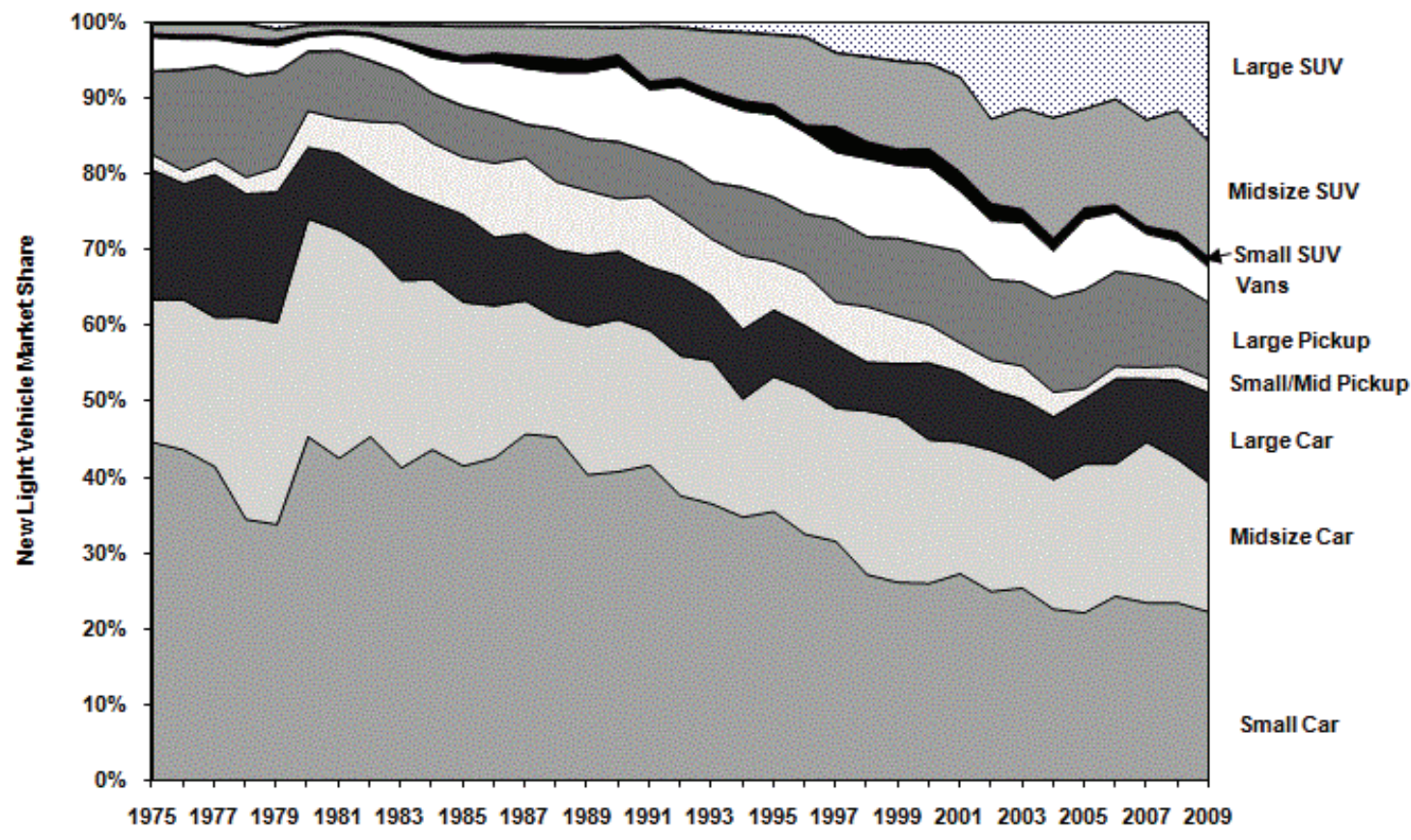

Source:

See Table 4.10 
The midsize and large cars and wagons sales-weighted engine sizes have decreased at an average of about $2 \%$ per year since 1975 .

Table 4.11

Sales-Weighted Engine Size of New Domestic and Import Cars by Size Class, Model Years 1975-2009 $\left(\right.$ liters ${ }^{\mathrm{a}}$ )

\begin{tabular}{|c|c|c|c|c|c|c|}
\hline \multirow[b]{2}{*}{ Model Year } & \multicolumn{3}{|c|}{ Cars } & \multicolumn{3}{|c|}{ Wagons } \\
\hline & Small & Midsize & Large & Small & Midsize & Large \\
\hline 1975 & 3.67 & 5.78 & 6.70 & 2.10 & 5.92 & 6.72 \\
\hline 1976 & 3.70 & 5.62 & 6.72 & 2.23 & 5.16 & 6.82 \\
\hline 1977 & 3.67 & 5.44 & 6.00 & 2.20 & 4.87 & 5.98 \\
\hline 1978 & 2.90 & 4.79 & 5.85 & 2.20 & 4.23 & 5.80 \\
\hline 1979 & 2.72 & 4.46 & 5.56 & 2.02 & 4.08 & 5.46 \\
\hline 1980 & 2.25 & 3.74 & 5.15 & 1.85 & 3.74 & 5.29 \\
\hline 1981 & 2.11 & 3.61 & 4.98 & 1.77 & 3.16 & 5.11 \\
\hline 1982 & 2.15 & 3.46 & 4.79 & 1.79 & 3.36 & 5.01 \\
\hline 1983 & 2.25 & 3.47 & 4.79 & 1.72 & 3.28 & 5.03 \\
\hline 1984 & 2.29 & 3.44 & 4.82 & 1.75 & 2.82 & 5.00 \\
\hline 1985 & 2.26 & 3.36 & 4.57 & 1.74 & 2.79 & 5.00 \\
\hline 1986 & 2.25 & 3.18 & 4.26 & 1.85 & 2.65 & 4.98 \\
\hline 1987 & 2.20 & 3.08 & 4.24 & 1.90 & 2.84 & 4.98 \\
\hline 1988 & 2.18 & 3.00 & 4.29 & 1.85 & 2.80 & 4.98 \\
\hline 1989 & 2.15 & 2.97 & 4.28 & 1.84 & 2.88 & 4.98 \\
\hline 1990 & 2.15 & 3.06 & 4.23 & 1.97 & 2.97 & 4.98 \\
\hline 1991 & 2.15 & 3.13 & 4.33 & 1.97 & 2.97 & 4.98 \\
\hline 1992 & 2.20 & 3.13 & 4.29 & 2.00 & 3.08 & 5.54 \\
\hline 1993 & 2.18 & 3.15 & 4.20 & 1.93 & 3.08 & 5.57 \\
\hline 1994 & 2.25 & 3.10 & 4.06 & 1.98 & 2.95 & 5.74 \\
\hline 1995 & 2.25 & 3.10 & 4.06 & 1.93 & 2.74 & 5.74 \\
\hline 1996 & 2.23 & 2.97 & 4.10 & 2.00 & 2.64 & 5.74 \\
\hline 1997 & 2.18 & 3.02 & 3.97 & 2.03 & 2.62 & $\mathrm{~b}$ \\
\hline 1998 & 2.25 & 2.90 & 3.93 & 2.03 & 2.54 & $\mathrm{~b}$ \\
\hline 1999 & 2.31 & 2.87 & 3.85 & 2.05 & 2.57 & $\mathrm{~b}$ \\
\hline 2000 & 2.28 & 2.85 & 3.62 & 2.08 & 2.51 & b \\
\hline 2001 & 2.29 & 2.87 & 3.62 & 2.38 & 2.54 & $\mathrm{~b}$ \\
\hline 2002 & 2.31 & 2.90 & 3.57 & 2.38 & 2.49 & $\mathrm{~b}$ \\
\hline 2003 & 2.36 & 2.85 & 3.67 & 2.08 & 2.47 & $\mathrm{~b}$ \\
\hline 2004 & 2.39 & 2.85 & 3.69 & 2.06 & 2.59 & 3.52 \\
\hline 2005 & 2.36 & 2.75 & 3.69 & 2.00 & 3.00 & 3.56 \\
\hline 2006 & 2.47 & 2.77 & 3.75 & 2.08 & 2.98 & 3.59 \\
\hline 2007 & 2.39 & 2.70 & 3.75 & 2.08 & 2.64 & 3.88 \\
\hline 2008 & 2.43 & 2.67 & 3.49 & 2.13 & 2.67 & 3.72 \\
\hline 2009 & 2.41 & 2.69 & 3.51 & 2.13 & 2.59 & 3.43 \\
\hline \multicolumn{7}{|c|}{ Average annual percentage change } \\
\hline 1975-2009 & $-1.2 \%$ & $-2.2 \%$ & $-1.9 \%$ & $0.0 \%$ & $-2.4 \%$ & $-2.0 \%$ \\
\hline 1999-2009 & $0.4 \%$ & $-0.6 \%$ & $-0.9 \%$ & $0.4 \%$ & $0.1 \%$ & $-3.9 \%^{\mathrm{c}}$ \\
\hline
\end{tabular}

Source:

U.S. Environmental Protection Agency, Light-Duty Automotive Technology, Carbon Dioxide Emissions, and Fuel Economy Trends: 1975 Through 2009, November 2009. (Additional resources: www.epa.gov/otaq/fetrends.htm)

${ }^{\mathrm{a}} 1$ liter $=61.02$ cubic inches.

${ }^{\mathrm{b}}$ No vehicles in this category were sold in this model year.

c 1996-2009. 
The engine size of large sport utility vehicles (SUVs) declined an average of $2.5 \%$ per year from 1999 to 2009 , while the size of a small SUV engine increased by $2.6 \%$.

Table 4.12

Sales-Weighted Engine Size of New Domestic and Import Light Trucks by Size Class, Model Years 1975-2009 $\left(\right.$ liters $^{\mathrm{a}}$ )

\begin{tabular}{|c|c|c|c|c|c|c|c|c|c|}
\hline \multirow[b]{2}{*}{ Model Year } & \multicolumn{3}{|c|}{ Pickups } & \multicolumn{3}{|c|}{ Vans } & \multicolumn{3}{|c|}{ SUVs } \\
\hline & Small & Midsize & Large & Small & Midsize & Large & Small & Midsize & Large \\
\hline 1975 & 1.93 & 1.79 & 5.62 & 1.93 & 5.08 & 5.47 & 4.47 & 5.72 & 5.97 \\
\hline 1976 & 1.95 & 1.79 & 5.64 & 1.97 & 5.20 & 5.49 & 4.47 & 5.80 & 6.11 \\
\hline 1977 & 1.97 & 2.03 & 5.69 & 1.97 & 5.34 & 5.62 & 4.49 & 5.72 & 6.08 \\
\hline 1978 & 1.95 & 2.03 & 5.56 & 1.97 & 5.36 & 5.49 & 4.51 & 5.87 & 6.11 \\
\hline 1979 & 1.97 & 2.15 & 5.41 & 1.97 & 5.24 & 5.51 & 4.28 & 5.64 & 6.15 \\
\hline 1980 & 2.00 & 2.18 & 5.00 & 1.97 & 4.72 & 5.16 & 3.72 & 5.31 & 5.57 \\
\hline 1982 & 2.25 & 2.49 & 4.90 & 1.82 & 4.65 & 5.15 & 3.39 & 5.24 & 5.64 \\
\hline 1983 & 2.33 & 2.39 & 4.95 & 1.93 & 4.82 & 5.15 & 3.44 & 4.10 & 5.82 \\
\hline 1984 & 2.33 & 2.43 & 4.93 & 1.97 & 4.06 & 5.15 & 3.05 & 3.70 & 5.75 \\
\hline 1985 & 2.34 & 2.52 & 5.00 & 1.98 & 3.82 & 5.11 & 2.74 & 3.47 & 5.74 \\
\hline 1986 & 2.38 & 2.41 & 4.88 & 2.15 & 3.67 & 5.01 & 2.74 & 3.34 & 5.74 \\
\hline 1987 & 2.41 & 2.61 & 5.06 & 2.20 & 3.70 & 5.06 & 2.64 & 3.54 & 5.74 \\
\hline 1988 & 2.43 & 2.70 & 5.21 & 2.20 & 3.65 & 5.06 & 2.57 & 3.83 & 5.75 \\
\hline 1989 & 2.51 & 2.90 & 5.21 & 2.13 & 3.57 & 5.06 & 2.80 & 4.16 & 5.75 \\
\hline 1991 & 2.49 & 3.11 & 5.16 & 2.03 & 3.51 & 5.11 & 2.38 & 3.87 & 5.38 \\
\hline 1992 & 2.49 & 3.20 & 5.11 & 2.11 & 3.57 & 5.16 & 2.39 & 3.82 & 5.42 \\
\hline 1993 & 2.41 & 3.24 & 4.97 & 1.98 & 3.46 & 5.16 & 2.46 & 3.97 & 5.65 \\
\hline 1994 & 2.47 & 3.23 & 5.18 & 2.21 & 3.59 & 5.21 & 2.28 & 3.90 & 5.62 \\
\hline 1995 & 2.57 & 3.11 & 5.18 & 2.20 & 3.70 & 5.15 & 2.26 & 3.88 & 5.69 \\
\hline 1996 & 2.61 & 3.06 & 5.16 & 2.33 & 3.46 & 5.33 & 1.75 & 4.08 & 5.64 \\
\hline 1997 & 2.39 & 3.20 & 4.97 & b & 3.44 & 4.92 & 2.98 & 3.85 & 5.38 \\
\hline 1998 & 2.62 & 3.13 & 5.05 & b & 3.43 & 4.87 & 2.65 & 3.87 & 5.13 \\
\hline 1999 & 2.84 & 3.28 & 5.13 & b & 3.49 & 4.87 & 2.57 & 3.74 & 5.29 \\
\hline 2000 & 2.43 & 3.15 & 4.74 & b & 3.41 & 4.85 & 2.80 & 3.75 & 5.11 \\
\hline 2001 & 2.41 & 3.39 & 4.79 & b & 3.38 & 4.97 & 2.51 & 3.51 & 4.64 \\
\hline 2002 & 2.90 & 3.70 & 4.82 & b & 3.44 & 4.80 & 2.56 & 3.34 & 4.54 \\
\hline 2003 & 2.92 & 3.21 & 4.82 & b & 3.47 & 4.74 & 2.64 & 3.36 & 4.72 \\
\hline 2004 & 3.02 & 3.59 & 4.93 & b & 3.51 & 4.79 & 2.97 & 3.51 & 4.74 \\
\hline 2005 & 2.46 & 3.15 & 4.82 & b & 3.49 & 4.72 & 2.92 & 3.34 & 4.46 \\
\hline 2006 & 2.46 & 3.23 & 4.75 & b & 3.47 & 4.64 & 3.28 & 3.34 & 4.24 \\
\hline 2009 & b & 3.38 & 5.01 & b & 3.61 & 4.83 & 3.31 & 3.18 & 4.10 \\
\hline \multicolumn{10}{|c|}{ Average annual percentage change } \\
\hline 1975-2009 & c & $1.9 \%$ & $-0.3 \%$ & c & $-1.0 \%$ & $-0.4 \%$ & $-0.9 \%$ & $-1.7 \%$ & $-1.1 \%$ \\
\hline 1999-2009 & $\mathrm{c}$ & $0.3 \%$ & $-0.2 \%$ & $\mathrm{c}$ & $0.3 \%$ & $-0.1 \%$ & $2.6 \%$ & $-1.6 \%$ & $-2.5 \%$ \\
\hline
\end{tabular}

Source:

U.S. Environmental Protection Agency, Light-Duty Automotive Technology, Carbon Dioxide Emissions, and Fuel Economy Trends: 1975 Through 2009, November 2009. (Additional resources: www.epa.gov/otaq/fetrends.htm)

Note: Includes light trucks of 8,500 lbs. or less.

\footnotetext{
${ }^{\mathrm{a}} 1$ liter $=61.02$ cubic inches.

${ }^{\mathrm{b}}$ No vehicles in this category were sold in this model year.

${ }^{\mathrm{c}}$ Data are not available.
} 
Table 4.13

Sales-Weighted Curb Weight of New Domestic and Import Cars by Size Class, Model Years 1975-2009 (pounds)

\begin{tabular}{|c|c|c|c|c|c|c|}
\hline \multirow[b]{2}{*}{ Model Year } & \multicolumn{3}{|c|}{ Cars } & \multicolumn{3}{|c|}{ Wagons } \\
\hline & Small & Midsize & Large & Small & Midsize & Large \\
\hline 1975 & 3,440 & 4,630 & 5,142 & 2,833 & 4,791 & 5,453 \\
\hline 1976 & 3,474 & 4,558 & 5,156 & 2,902 & 4,555 & 5,444 \\
\hline 1977 & 3,486 & 4,473 & 4,482 & 2,801 & 4,410 & 4,713 \\
\hline 1978 & 3,029 & 3,820 & 4,394 & 2,805 & 3,836 & 4,664 \\
\hline 1979 & 2,936 & 3,710 & 4,210 & 2,711 & 3,758 & 4,466 \\
\hline 1980 & 2,717 & 3,362 & 4,130 & 2,591 & 3,534 & 4,423 \\
\hline 1981 & 2,648 & 3,346 & 4,108 & 2,531 & 3,285 & 4,394 \\
\hline 1982 & 2,684 & 3,321 & 4,034 & 2,580 & 3,384 & 4,396 \\
\hline 1983 & 2,734 & 3,316 & 4,041 & 2,565 & 3,348 & 4,379 \\
\hline 1984 & 2,776 & 3,318 & 4,022 & 2,620 & 3,298 & 4,371 \\
\hline 1985 & 2,771 & 3,318 & 3,841 & 2,579 & 3,356 & 4,354 \\
\hline 1986 & 2,791 & 3,241 & 3,719 & 2,647 & 3,355 & 4,381 \\
\hline 1987 & 2,803 & 3,247 & 3,696 & 2,795 & 3,434 & 4,348 \\
\hline 1988 & 2,818 & 3,293 & 3,730 & 2,757 & 3,378 & 4,349 \\
\hline 1989 & 2,841 & 3,314 & 3,721 & 2,766 & 3,436 & 4,334 \\
\hline 1990 & 2,897 & 3,450 & 3,799 & 3,026 & 3,498 & 4,337 \\
\hline 1991 & 2,885 & 3,412 & 3,893 & 3,005 & 3,506 & 4,402 \\
\hline 1992 & 2,921 & 3,515 & 3,872 & 3,076 & 3,503 & 4,500 \\
\hline 1993 & 2,903 & 3,515 & 3,831 & 2,882 & 3,498 & 4,500 \\
\hline 1994 & 2,965 & 3,529 & 3,858 & 2,908 & 3,532 & 4,500 \\
\hline 1995 & 2,988 & 3,546 & 3,830 & 2,859 & 3,482 & 4,500 \\
\hline 1996 & 2,977 & 3,527 & 3,894 & 2,952 & 3,661 & 4,500 \\
\hline 1997 & 2,977 & 3,551 & 3,821 & 2,901 & 3,666 & a \\
\hline 1998 & 3,013 & 3,534 & 3,784 & 2,874 & 3,668 & $\mathrm{a}$ \\
\hline 1999 & 3,085 & 3,540 & 3,854 & 2,923 & 3,691 & a \\
\hline 2000 & 3,079 & 3,550 & 3,782 & 3,107 & 3,572 & a \\
\hline 2001 & 3,101 & 3,566 & 3,774 & 3,470 & 3,775 & a \\
\hline 2002 & 3,125 & 3,549 & 3,767 & 3,504 & 3,731 & a \\
\hline 2003 & 3,179 & 3,567 & 3,841 & 3,262 & 3,745 & a \\
\hline 2004 & 3,192 & 3,577 & 3,858 & 3,235 & 3,860 & 4,769 \\
\hline 2005 & 3,163 & 3,544 & 3,933 & 3,160 & 3,839 & 4,791 \\
\hline 2006 & 3,255 & 3,568 & 4,014 & 3,255 & 3,827 & 4,806 \\
\hline 2007 & 3,238 & 3,581 & 4,026 & 3,264 & 3,727 & 4,785 \\
\hline 2008 & 3,284 & 3,564 & 3,966 & 3,300 & 3,808 & 5,017 \\
\hline 2009 & 3,267 & 3,575 & 3,942 & 3,337 & 3,761 & 5,500 \\
\hline \multicolumn{7}{|c|}{ Average annual percentage change } \\
\hline 1975-2009 & $-0.2 \%$ & $-0.8 \%$ & $-0.8 \%$ & $0.5 \%$ & $-0.7 \%$ & $0.0 \%$ \\
\hline 1999-2009 & $0.6 \%$ & $0.1 \%$ & $0.2 \%$ & $1.3 \%$ & $0.2 \%$ & $1.6 \%{ }^{\mathrm{b}}-\mathrm{r}-\mathrm{r}$ \\
\hline
\end{tabular}

Source:

U.S. Environmental Protection Agency, Light-Duty Automotive Technology, Carbon Dioxide Emissions, and Fuel Economy Trends: 1975 Through 2009, November 2009. (Additional resources: www.epa.gov/otaq/fetrends.htm)

${ }^{\text {a }}$ Data are not available.

b 1996-2009. 
The interior space of new small and midsize cars in 2009 was about the same as in the late 1980's; large cars, however, had smaller interior space.

Table 4.14

Sales-Weighted Interior Space of New Domestic and Import Cars by Size Class, Model Years 1977-2009

(cubic feet)

\begin{tabular}{|c|c|c|c|c|c|c|}
\hline \multirow[b]{2}{*}{ Model Year } & \multicolumn{3}{|c|}{ Cars } & \multicolumn{3}{|c|}{ Wagons } \\
\hline & Small & Midsize & Large & Small & Midsize & Large \\
\hline 1977 & 95.4 & 112.9 & 128.1 & 108.0 & 143.6 & 163.1 \\
\hline 1978 & 90.9 & 113.0 & 128.5 & 108.0 & 140.0 & 162.4 \\
\hline 1979 & 89.2 & 113.1 & 130.0 & 105.1 & 139.7 & 162.5 \\
\hline 1980 & 90.0 & 113.2 & 130.9 & 108.2 & 139.7 & 161.5 \\
\hline 1981 & 91.6 & 113.9 & 131.0 & 110.6 & 136.2 & 161.4 \\
\hline 1982 & 92.2 & 113.9 & 131.0 & 112.2 & 136.1 & 161.3 \\
\hline 1983 & 95.1 & 113.8 & 131.3 & 108.2 & 136.2 & 161.6 \\
\hline 1984 & 95.2 & 113.7 & 130.9 & 116.5 & 135.9 & 161.7 \\
\hline 1985 & 95.8 & 113.6 & 129.3 & 117.7 & 134.8 & 161.7 \\
\hline 1986 & 96.7 & 113.8 & 127.4 & 118.4 & 137.8 & 161.4 \\
\hline 1987 & 96.9 & 113.7 & 127.0 & 120.0 & 140.2 & 161.8 \\
\hline 1988 & 98.5 & 113.4 & 128.1 & 118.7 & 139.4 & 161.7 \\
\hline 1989 & 98.3 & 113.6 & 127.4 & 118.6 & 139.9 & 161.8 \\
\hline 1990 & 97.6 & 113.7 & 126.7 & 122.2 & 141.6 & 161.6 \\
\hline 1991 & 97.6 & 113.5 & 129.0 & 123.3 & 142.3 & 169.1 \\
\hline 1992 & 97.9 & 113.9 & 129.6 & 123.7 & 142.6 & 170.3 \\
\hline 1993 & 98.3 & 113.9 & 128.9 & 123.0 & 137.7 & 169.3 \\
\hline 1994 & 98.7 & 113.5 & 128.3 & 122.9 & 137.4 & 169.2 \\
\hline 1995 & 99.6 & 114.3 & 127.9 & 122.1 & 135.9 & 169.3 \\
\hline 1996 & 99.9 & 114.1 & 128.1 & 118.0 & 136.9 & 170.2 \\
\hline 1997 & 99.2 & 114.5 & 127.4 & 119.5 & 136.5 & a \\
\hline 1998 & 98.8 & 114.0 & 127.4 & 116.9 & 135.3 & a \\
\hline 1999 & 98.9 & 114.0 & 127.0 & 117.9 & 136.4 & a \\
\hline 2000 & 99.4 & 113.6 & 124.9 & 119.7 & 134.0 & a \\
\hline 2001 & 99.2 & 113.7 & 124.8 & 119.6 & 133.6 & a \\
\hline 2002 & 98.9 & 114.8 & 124.0 & 118.2 & 133.6 & a \\
\hline 2003 & 98.6 & 114.6 & 124.8 & 115.2 & 133.5 & a \\
\hline 2004 & 99.0 & 114.0 & 124.7 & 117.5 & 135.0 & 165.0 \\
\hline 2005 & 99.1 & 114.5 & 125.0 & 115.9 & 133.3 & 165.0 \\
\hline 2006 & 98.8 & 114.0 & 124.7 & 118.4 & 135.6 & 164.4 \\
\hline 2007 & 99.3 & 113.8 & 123.8 & 112.0 & 135.4 & 159.2 \\
\hline 2008 & 98.3 & 113.3 & 123.2 & 115.0 & 132.2 & 160.1 \\
\hline 2009 & 98.5 & 113.5 & 123.5 & 114.4 & 131.1 & 161.7 \\
\hline \multicolumn{7}{|c|}{ Average annual percentage change } \\
\hline 1977-2009 & $0.1 \%$ & $0.0 \%$ & $-0.1 \%$ & $0.2 \%$ & $-0.3 \%$ & $0.0 \%$ \\
\hline 1999-2009 & $0.0 \%$ & $0.0 \%$ & $-0.3 \%$ & $-0.3 \%$ & $-0.4 \%$ & $-0.4 \%^{\mathrm{b}}$ \\
\hline
\end{tabular}

Source:

U.S. Environmental Protection Agency, Light-Duty Automotive Technology, Carbon Dioxide Emissions, and Fuel Economy Trends: 1975 Through 2009, November 2009. (Additional resources: www.epa.gov/otaq/fetrends.htm)

\footnotetext{
${ }^{a}$ No vehicles in this category were sold in this model year.

b 1996-2009.
} 
The average light vehicle in 2008 contained more than 2,000 pounds of steel, most of it conventional steel. High and medium strength steel, however, made up more than 10\% of the vehicle. The use of aluminum grew from 1995 to 2008, while the use of iron castings declined.

Table 4.15

Average Material Consumption for a Domestic Light Vehicle, Model Years 1995, 2000, and 2008

\begin{tabular}{|c|c|c|c|c|c|c|}
\hline \multirow[b]{2}{*}{ Material } & \multicolumn{2}{|c|}{1995} & \multicolumn{2}{|c|}{2000} & \multicolumn{2}{|c|}{2008} \\
\hline & Pounds & Percentage & Pounds & Percentage & Pounds & Percentage \\
\hline Regular steel & $1,630.0$ & $44.1 \%$ & $1,655.0$ & $42.4 \%$ & $1,629.0$ & $40.0 \%$ \\
\hline High and medium strength steel & 324.0 & $8.8 \%$ & 408.0 & $10.5 \%$ & 523.0 & $12.9 \%$ \\
\hline Stainless steel & 51.0 & $1.4 \%$ & 62.0 & $1.6 \%$ & 75.0 & $1.8 \%$ \\
\hline Other steels & 46.0 & $1.2 \%$ & 26.0 & $0.7 \%$ & 34.0 & $0.8 \%$ \\
\hline Iron castings & 466.0 & $12.6 \%$ & 432.0 & $11.1 \%$ & 301.0 & $7.4 \%$ \\
\hline Aluminum & 231.0 & $6.3 \%$ & 268.0 & $6.9 \%$ & 315.0 & $7.7 \%$ \\
\hline Magnesium castings & 4.0 & $0.1 \%$ & 8.0 & $0.2 \%$ & 11.0 & $0.3 \%$ \\
\hline Copper and brass & 50.0 & $1.4 \%$ & 52.0 & $1.3 \%$ & 64.0 & $1.6 \%$ \\
\hline Lead & 33.0 & $0.9 \%$ & 36.0 & $0.9 \%$ & 45.0 & $1.1 \%$ \\
\hline Zinc castings & 19.0 & $0.5 \%$ & 13.0 & $0.3 \%$ & 10.0 & $0.2 \%$ \\
\hline Powder metal parts & 29.0 & $0.8 \%$ & 36.0 & $0.9 \%$ & 43.0 & $1.1 \%$ \\
\hline Other metals & 4.0 & $0.1 \%$ & 4.0 & $0.1 \%$ & 5.0 & $0.1 \%$ \\
\hline Plastics and plastic composites & 240.0 & $6.5 \%$ & 286.0 & $7.3 \%$ & 343.0 & $8.4 \%$ \\
\hline Rubber & 149.0 & $4.0 \%$ & 166.0 & $4.3 \%$ & 185.0 & $4.5 \%$ \\
\hline Coatings & 23.0 & $0.6 \%$ & 25.0 & $0.6 \%$ & 28.0 & $0.7 \%$ \\
\hline Textiles & 42.0 & $1.10 \%$ & 44.0 & $1.10 \%$ & 48.0 & $1.2 \%$ \\
\hline Fluids and lubricants & 192.0 & $5.20 \%$ & 207.0 & $5.30 \%$ & 214.0 & $5.3 \%$ \\
\hline Glass & 97.0 & $2.60 \%$ & 103.0 & $2.60 \%$ & 106.0 & $2.6 \%$ \\
\hline Other materials & 64.0 & $1.70 \%$ & 71.0 & $1.80 \%$ & 91.0 & $2.2 \%$ \\
\hline Total & $3,694.0$ & $100.0 \%$ & $3,902.0$ & $100.0 \%$ & $4,070.0$ & $100.0 \%$ \\
\hline
\end{tabular}

Source:

Ward's Communications, Ward's Motor Vehicle Facts and Figures, 2009, (and updates, 2010), Detroit, MI, 2009, p. 63. 
The number of franchised dealerships which sell new light-duty vehicles (cars and light trucks) has declined over $30 \%$ since 1970, though new vehicle sales have increased. The average number of vehicles sold per dealer in 2008 was 635 vehicles per dealer - almost double the 1970 number.

Table 4.16

New Light Vehicle Dealerships and Sales, 1970-2008

\begin{tabular}{|c|c|c|c|}
\hline Calendar year & $\begin{array}{l}\text { Number of franchised new } \\
\text { light vehicle dealerships }{ }^{\mathrm{a}}\end{array}$ & $\begin{array}{l}\text { New light vehicle sales } \\
\text { (thousands) }\end{array}$ & $\begin{array}{c}\text { Light vehicle sales per } \\
\text { dealer }\end{array}$ \\
\hline 1970 & 30,800 & 9,862 & 320 \\
\hline 1975 & 29,600 & 10,905 & 368 \\
\hline 1976 & 29,300 & 13,066 & 446 \\
\hline 1977 & 29,100 & 14,613 & 502 \\
\hline 1978 & 29,000 & 15,122 & 521 \\
\hline 1979 & 28,500 & 13,984 & 491 \\
\hline 1980 & 27,900 & 11,389 & 408 \\
\hline 1981 & 26,350 & 10,678 & 405 \\
\hline 1982 & 25,700 & 10,426 & 406 \\
\hline 1983 & 24,725 & 12,132 & 491 \\
\hline 1984 & 24,725 & 14,187 & 574 \\
\hline 1985 & 24,725 & 15,437 & 624 \\
\hline 1986 & 24,825 & 15,998 & 644 \\
\hline 1987 & 25,150 & 14,802 & 589 \\
\hline 1988 & 25,025 & 15,347 & 613 \\
\hline 1989 & 25,000 & 14,389 & 576 \\
\hline 1990 & 24,825 & 13,851 & 558 \\
\hline 1991 & 24,200 & 12,312 & 509 \\
\hline 1992 & 23,500 & 12,842 & 546 \\
\hline 1993 & 22,950 & 13,869 & 604 \\
\hline 1994 & 22,850 & 15,024 & 658 \\
\hline 1995 & 22,800 & 14,688 & 644 \\
\hline 1996 & 22,750 & 15,046 & 661 \\
\hline 1997 & 22,700 & 15,069 & 664 \\
\hline 1998 & 22,600 & 15,441 & 683 \\
\hline 1999 & 22,400 & 16,771 & 748 \\
\hline 2000 & 22,250 & 17,234 & 774 \\
\hline 2001 & 22,150 & 17,123 & 773 \\
\hline 2002 & 21,800 & 16,816 & 771 \\
\hline 2003 & 21,725 & 16,548 & 762 \\
\hline 2004 & 21,650 & 16,867 & 779 \\
\hline 2005 & 21,640 & 16,948 & 783 \\
\hline 2006 & 21,495 & 16,505 & 768 \\
\hline 2007 & 21,200 & 16,089 & 759 \\
\hline 2008 & 20,770 & 13,194 & 635 \\
\hline \multicolumn{4}{|c|}{ Average annual percentage change } \\
\hline 1970-2008 & $-1.0 \%$ & $0.8 \%$ & $1.8 \%$ \\
\hline $1998-2008$ & $-0.8 \%$ & $-1.6 \%$ & $-0.7 \%$ \\
\hline
\end{tabular}

\section{Source:}

Number of dealers - National Automobile Dealers Association, Automotive Executive Magazine, 2009. (Additional resources: http://www.nada.org/Publications/NADADATA/) Light-duty vehicle sales - See tables 4.5 and 4.6.

\footnotetext{
${ }^{a}$ As of the beginning of the year.
} 
The number of conventional refueling stations is declining while the number of vehicles fueling at those stations continues to rise. In 2008, there were 0.65 fueling stations per thousand vehicles or 1.55 thousand vehicles per station.

Table 4.17

Conventional Refueling Stations, 1993-2008

\begin{tabular}{|c|c|c|c|c|}
\hline & $\begin{array}{c}\text { Number of retail } \\
\text { outlets }\end{array}$ & $\begin{array}{l}\text { Vehicles in } \\
\text { operation } \\
\text { (thousands) }\end{array}$ & $\begin{array}{c}\text { Stations per } \\
\text { thousand vehicles }\end{array}$ & $\begin{array}{l}\text { Thousand } \\
\text { vehicles per } \\
\text { station }\end{array}$ \\
\hline Year & \multicolumn{3}{|c|}{ Conventional fuels } & \\
\hline 1993 & 207,416 & 186,315 & 1.11 & 0.90 \\
\hline 1994 & 202,878 & 188,714 & 1.08 & 0.93 \\
\hline 1995 & 195,455 & 193,441 & 1.01 & 0.99 \\
\hline 1996 & 190,246 & 198,294 & 0.96 & 1.04 \\
\hline 1997 & 187,892 & 201,071 & 0.93 & 1.07 \\
\hline 1998 & 182,596 & 205,043 & 0.89 & 1.12 \\
\hline 1999 & 180,567 & 209,509 & 0.86 & 1.16 \\
\hline 2000 & 175,941 & 213,300 & 0.82 & 1.21 \\
\hline 2001 & 172,169 & 216,683 & 0.79 & 1.26 \\
\hline 2002 & 170,018 & 221,027 & 0.77 & 1.30 \\
\hline 2003 & 167,571 & 225,882 & 0.74 & 1.35 \\
\hline 2004 & 167,346 & 232,167 & 0.72 & 1.39 \\
\hline 2005 & 168,987 & 238,384 & 0.71 & 1.41 \\
\hline 2006 & 167,476 & 244,643 & 0.69 & 1.46 \\
\hline 2007 & 164,292 & 248,701 & 0.66 & 1.51 \\
\hline 2008 & 161,768 & 250,239 & 0.65 & 1.55 \\
\hline
\end{tabular}

\section{Sources:}

Conventional refueling stations: National Petroleum News Survey, 2008.

Conventional vehicles: The Polk Company, Detroit, MI, FURTHER REPRODUCTION PROHIBITED.

Notes: The County Business Patterns (CBP) data published by the Bureau of the Census tells the number of establishments by North American Industry Classification System (NAICS). NAICS is an industry classification system that groups establishments into industries based on the activities in which they are primarily engaged. NAICS 447 represents gasoline stations. However, the CBP gasoline station data differ from the National Petroleum News Survey data by as much as 30\% (117,189 stations in 2005); the CBP may not include every gasoline retail outlet due to the classification of the primary activity of the business.

Alternative Fuel Refueling Stations are listed in Chapter 6. 
In 2006 the National Highway Traffic Safety Administration (NHTSA) established new requirements for the light truck Corporate Average Fuel Economy (CAFE) standards. In the new rule, there are Unreformed CAFE standards for model years (MY) 2008 through 2010 using the same CAFE calculations as in the past, and there are Reformed CAFE standards for those years as well, using a new methodology for the calculation of CAFE. For MY 2008 through 2010, the manufacturers can choose either standard. See Table 4.19 for details on the Reformed CAFE calculation. Another change with the Reformed CAFE standards is that larger passenger vans and sport utility vehicles (8,500-10,000 lbs gross vehicle weight) are included, whereas they are not included in the Unreformed CAFE calculations.

For MY 2011, new standards for cars and light trucks were established on March 30, 2009, using a methodology similar to the Reformed light truck standards. NHTSA is currently researching future standards.

Table 4.18

Reformed and Unreformed Light Truck Corporate Average Fuel Economy Standards, MY 2008-2011

\begin{tabular}{cccccc}
\hline & \multicolumn{2}{c}{$\begin{array}{c}\text { CAFE Standard } \\
\text { (miles per gallon) }\end{array}$} & & \multicolumn{2}{c}{$\begin{array}{c}\text { Estimated Fuel Savings } \\
\text { (billions of gallons) }\end{array}$} \\
\cline { 2 - 3 } \cline { 5 - 6 } Model Year & Unreformed & Reformed & & Unreformed & Reformed \\
\hline & & Light trucks & & \\
\hline 2008 & 22.5 & 22.7 & & 0.6 & 1.9 \\
2009 & 23.1 & 23.4 & & 1.8 & 2.2 \\
2010 & 23.5 & 23.7 & & 2.0 & Not applicable \\
2011 & None & 24.1 & & Not available \\
\hline & & Cars & & \\
\hline 2011 & None & 30.2 & & Not applicable & Not available \\
\hline
\end{tabular}

Source:

Federal Register, vol. 71, No. 66, April 6, 2006.

Federal Register, Vol. 74, No. 59, March 30, 2009. 
The National Highway Traffic Safety Administration and the Environmental Protection Agency issued joint rulemaking in March 2010 to establish a new National Program to regulate fuel economy and greenhouse gas emissions for model year 2012-2016 cars and light trucks.

Table 4.19

Fuel Economy and Carbon Dioxide Emissions Standards, MY 2012-2016

\begin{tabular}{cccc}
\hline Year & Cars & Light Trucks & $\begin{array}{c}\text { Combined Cars and } \\
\text { Light Trucks }\end{array}$ \\
\hline \multicolumn{4}{c}{$\begin{array}{c}\text { Average Required Fuel Economy } \\
\text { (miles per gallon) }\end{array}$} \\
\hline 2012 & 33.3 & 25.4 & 29.7 \\
2013 & 34.2 & 26.0 & 30.5 \\
2014 & 34.9 & 26.6 & 31.3 \\
2015 & 36.2 & 27.5 & 32.6 \\
2016 & 37.8 & 28.8 & 34.1 \\
\hline \multicolumn{5}{c}{ Average Projected Emissions Compliance Levels under } \\
2012 & \multicolumn{4}{c}{ the Footprint-based Carbon Dioxide Standards } \\
2013 & 263 & (grams per mile) & 295 \\
2014 & 256 & 336 & 286 \\
2016 & 247 & 326 & 263 \\
\hline
\end{tabular}

Source:

Federal Register, Vol. 74, No. 186, September 2009. (Additional resources: www.nhtsa.dot.gov/portal/fueleconomy.jsp)

Note: The required fuel economy, along with projections of $\mathrm{CO}_{2}$ emissions, are shown here. 
The target levels for the proposed fuel economy and carbon dioxide emission standards for vehicles manufactured in model years 2012-2016 are assigned based on a vehicle's "footprint." Each footprint has a different target. The vehicle footprint is calculated as:

$$
\text { footprint }=\text { track width } \times \text { wheelbase, }
$$

where

track width = lateral distance between the centerlines of the base tires at ground, and wheelbase = longitudinal distance between the front and rear wheel centerlines.

Table 4.20

Fuel Economy and Carbon Dioxide Targets for Model Year 2016

\begin{tabular}{|c|c|c|c|c|}
\hline Vehicle type & Example models & $\begin{array}{c}\text { Example } \\
\text { model footprint } \\
\text { (square feet) }\end{array}$ & $\begin{array}{c}\mathrm{CO}_{2} \text { emissions } \\
\text { target } \\
\text { (grams per mile) }\end{array}$ & $\begin{array}{l}\text { Fuel economy target } \\
\text { (miles per gallon) }\end{array}$ \\
\hline \multicolumn{5}{|c|}{ Example Passenger Cars } \\
\hline Compact car & Honda Fit & 40 & 214 & 41.4 \\
\hline Midsize car & Ford Fusion & 46 & 237 & 37.3 \\
\hline Fullsize car & Chrysler 300 & 53 & 270 & 32.8 \\
\hline \multicolumn{5}{|c|}{ Example Light-Duty Trucks } \\
\hline Small SUV & 4WD Ford Escape & 44 & 269 & 32.8 \\
\hline Midsize crossover & Nissan Murano & 49 & 289 & 30.6 \\
\hline Minivan & Toyota Sienna & 55 & 313 & 28.2 \\
\hline Large pickup truck & Chevy Silverado & 67 & 358 & 24.7 \\
\hline
\end{tabular}

Source:

Federal Register, Vol. 74, No. 186, September 28, 2009.

Note: Examples use model year 2008 vehicle specifications. 
The Corporate Average Fuel Economy standards were established by the U.S. Energy Policy and Conservation Act of 1975 (PL94-163). These standards must be met at the manufacturer level. Some manufacturers fall short of meeting the standards while others exceed them. Legislation passed in December 2007 will change the CAFE standards beginning in the 2011 model year. The new standards have a target of combined fleet fuel economy of 35 mpg by 2020, for all cars and light trucks.

Table 4.21

Car Corporate Average Fuel Economy (CAFE) Standards versus Sales-Weighted Fuel Economy Estimates, 1978-2010 ${ }^{\mathrm{a}}$ (miles per gallon)

\begin{tabular}{|c|c|c|c|c|c|}
\hline \multirow{3}{*}{$\begin{array}{l}\text { Model } \\
\text { year }^{\mathrm{b}}\end{array}$} & \multicolumn{4}{|c|}{ Cars } & \multirow{3}{*}{$\begin{array}{l}\text { CAFE estimates } \\
\text { Cars and light } \\
\text { trucks combined }\end{array}$} \\
\hline & \multirow{2}{*}{$\begin{array}{c}\text { CAFE } \\
\text { standards }\end{array}$} & \multicolumn{3}{|c|}{ CAFE estimates $^{\mathrm{c}}$} & \\
\hline & & Domestic & Import & Combined & \\
\hline 1978 & 18.0 & 18.7 & 27.3 & 19.9 & 19.9 \\
\hline 1979 & 19.0 & 19.3 & 26.1 & 20.3 & 20.1 \\
\hline 1980 & 20.0 & 22.6 & 29.6 & 24.3 & 23.1 \\
\hline 1981 & 22.0 & 24.2 & 31.5 & 25.9 & 24.6 \\
\hline 1982 & 24.0 & 25.0 & 31.1 & 26.6 & 25.1 \\
\hline 1983 & 26.0 & 24.4 & 32.4 & 26.4 & 24.8 \\
\hline 1984 & 27.0 & 25.5 & 32.0 & 26.9 & 25.0 \\
\hline 1985 & 27.5 & 26.3 & 31.5 & 27.6 & 25.4 \\
\hline 1986 & 26.0 & 26.9 & 31.6 & 28.2 & 25.9 \\
\hline 1987 & 26.0 & 27.0 & 31.2 & 28.5 & 26.2 \\
\hline 1988 & 26.0 & 27.4 & 31.5 & 28.8 & 26.0 \\
\hline 1989 & 26.5 & 27.2 & 30.8 & 28.4 & 25.6 \\
\hline 1990 & 27.5 & 26.9 & 29.9 & 28.0 & 25.4 \\
\hline 1991 & 27.5 & 27.3 & 30.1 & 28.4 & 25.6 \\
\hline 1992 & 27.5 & 27.0 & 29.2 & 27.9 & 25.1 \\
\hline 1993 & 27.5 & 27.8 & 29.6 & 28.4 & 25.2 \\
\hline 1994 & 27.5 & 27.5 & 29.6 & 28.3 & 24.7 \\
\hline 1995 & 27.5 & 27.7 & 30.3 & 28.6 & 24.9 \\
\hline 1996 & 27.5 & 28.1 & 29.6 & 28.5 & 24.9 \\
\hline 1997 & 27.5 & 27.8 & 30.1 & 28.7 & 24.6 \\
\hline 1998 & 27.5 & 28.6 & 29.2 & 28.8 & 24.7 \\
\hline 1999 & 27.5 & 28.0 & 29.0 & 28.3 & 24.5 \\
\hline 2000 & 27.5 & 28.7 & 28.3 & 28.5 & 24.8 \\
\hline 2001 & 27.5 & 28.7 & 29.0 & 28.8 & 24.5 \\
\hline 2002 & 27.5 & 29.1 & 28.8 & 29.0 & 24.7 \\
\hline 2003 & 27.5 & 29.1 & 29.9 & 29.5 & 25.1 \\
\hline 2004 & 27.5 & 29.9 & 28.7 & 29.5 & 24.6 \\
\hline 2005 & 27.5 & 30.5 & 29.9 & 30.3 & 25.4 \\
\hline 2006 & 27.5 & 30.3 & 29.7 & 30.1 & 25.8 \\
\hline 2007 & 27.5 & 30.6 & 32.2 & 31.2 & 26.6 \\
\hline 2008 & 27.5 & 31.2 & 32.1 & 31.6 & 27.1 \\
\hline 2009 & 27.5 & 32.8 & 32.4 & 32.6 & 28.8 \\
\hline 2010 & 27.5 & 32.8 & 35.2 & 33.8 & 29.2 \\
\hline
\end{tabular}

Source:

U.S. Department of Transportation, NHTSA, "Summary of Fuel Economy Performance," Washington, DC, March 2010. (Additional resources: www.nhtsa.dot.gov)

${ }^{a}$ Only vehicles with at least 75 percent domestic content can be counted in the average domestic fuel economy for a manufacturer.

${ }^{\mathrm{b}}$ Model year as determined by the manufacturer on a vehicle by vehicle basis.

${ }^{\mathrm{c}}$ All CAFE calculations are sales-weighted. 
The Corporate Average Fuel Economy standards for light trucks are lower than the car standards. Light trucks include pickups, minivans, sport utility vehicles and vans. New legislation passed in December 2007 will change the CAFE standards beginning in the 2011 model year. The new standards have a target of combined fleet fuel economy of 35 mpg by 2020, for all cars and light trucks.

Table 4.22

Light Truck Corporate Average Fuel Economy (CAFE) Standards versus Sales-Weighted Fuel Economy Estimates, 1978-2010 a (miles per gallon)

\begin{tabular}{|c|c|c|c|c|c|}
\hline \multirow{3}{*}{$\begin{array}{l}\text { Model } \\
\text { year }^{\mathrm{c}}\end{array}$} & \multicolumn{4}{|c|}{ Light trucks ${ }^{b}$} & \multirow{3}{*}{$\begin{array}{c}\text { CAFE estimates } \\
\text { Cars and light } \\
\text { trucks combined }\end{array}$} \\
\hline & \multirow{2}{*}{$\begin{array}{c}\text { CAFE } \\
\text { standards }\end{array}$} & \multicolumn{3}{|c|}{ CAFE estimates $^{\mathrm{d}}$} & \\
\hline & & Domestic & Import & Combined & \\
\hline 1978 & 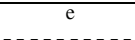 & & & 7 & 19.9 \\
\hline 1980 & $-\mathrm{e}^{-}$ & 16.8 & 24.3 & 18.5 & 23.1 \\
\hline 1985 & 19.5 & 19.6 & 26.5 & 20.7 & 25.4 \\
\hline 1986 & 20.0 & 20.0 & 25.9 & 21.5 & 25.9 \\
\hline 1987 & 20.5 & 20.5 & 25.2 & 21.7 & 26.2 \\
\hline 1988 & 20.5 & 20.6 & 24.6 & 21.3 & 26.0 \\
\hline 1989 & 20.5 & 20.4 & 23.5 & 21.0 & 25.6 \\
\hline 1990 & 20.0 & 20.3 & 23.0 & 20.8 & 25.4 \\
\hline 1991 & 20.2 & 20.9 & 23.0 & 21.3 & 25.6 \\
\hline 1992 & 20.2 & 20.5 & 22.7 & 20.8 & 25.1 \\
\hline 1993 & 20.4 & 20.7 & 22.8 & 21.0 & 25.2 \\
\hline 1994 & 20.5 & 20.5 & 22.1 & 20.8 & 24.7 \\
\hline 1995 & 20.6 & 20.3 & 21.5 & 20.5 & 24.9 \\
\hline 1996 & 20.7 & 20.5 & 22.2 & 20.8 & 24.9 \\
\hline 1997 & 20.7 & 20.1 & 22.1 & 20.6 & 24.6 \\
\hline 1998 & 20.7 & 20.5 & 23.0 & 21.0 & 24.7 \\
\hline 1999 & 20.7 & 20.4 & 22.5 & 20.9 & 24.5 \\
\hline 2000 & 20.7 & 21.1 & 19.7 & 21.3 & 24.8 \\
\hline 2001 & 20.7 & 20.6 & 21.8 & 20.9 & 24.5 \\
\hline 2002 & 20.7 & 20.6 & 21.9 & 21.4 & 24.7 \\
\hline 2003 & 20.7 & 21.8 & 22.4 & 21.8 & 25.1 \\
\hline 2004 & 20.7 & 20.7 & 22.3 & 21.5 & 24.6 \\
\hline 2005 & 21.0 & $\mathrm{f}$ & $\mathrm{f}$ & 22.1 & 25.4 \\
\hline 2006 & 21.6 & f & f & 22.5 & 25.8 \\
\hline 2007 & 22.2 & $\mathrm{f}$ & f & 23.1 & 26.6 \\
\hline 2008 & $22.5^{\mathrm{g}}$ & f & f & 23.6 & 27.1 \\
\hline 2009 & $23.1^{\mathrm{g}}$ & f & f & 24.6 & 28.8 \\
\hline 2010 & $23.5^{\mathrm{g}}$ & f & f & 24.9 & 29.2 \\
\hline
\end{tabular}

Source:

U.S. Department of Transportation, NHTSA, "Summary of Fuel Economy Performance," Washington, DC, March 2010. (Additional resources: www.nhtsa.dot.gov)

${ }^{a}$ Only vehicles with at least $75 \%$ domestic content can be counted in the average domestic fuel economy for a manufacturer.

${ }^{\mathrm{b}}$ Represents two- and four-wheel drive trucks combined. Gross vehicle weight of 0-6,000 pounds for model year 1978-1979 and 0-8,500 pounds for subsequent years.

${ }^{c}$ Model year as determined by the manufacturer on a vehicle by vehicle basis.

${ }^{\mathrm{d}}$ All CAFE calculations are sales-weighted.

${ }^{\mathrm{e}}$ Standards were set for two-wheel drive and four-wheel drive light trucks, but no combined standard was set in this year.

${ }_{\mathrm{f}}^{\mathrm{f}}$ Data are not available.

${ }^{\mathrm{g}}$ Unreformed standards. See Table 4.18 for reformed standards. 
Manufacturers of cars and light trucks whose vehicles do not meet the CAFE standards are fined. Data from the National Highway Traffic Safety Administration show CAFE fine collection dropped under \$25 million in 2002 and 2003; this was due to several factors, including the CAFE credit system, manufacturer mergers, and fines not being paid in the same year they were assessed.

Table 4.23

Corporate Average Fuel Economy (CAFE) Fines Collected, 1983-2008 (thousands)

\begin{tabular}{crc}
\hline Model year & $\begin{array}{c}\text { Current } \\
\text { dollars }\end{array}$ & $\begin{array}{c}\text { 2008 constant } \\
\text { dollars }\end{array}$ \\
\hline 1983 & 58 & 125 \\
1984 & 5,958 & 12,346 \\
1985 & 15,565 & 31,144 \\
1986 & 29,872 & 58,681 \\
1987 & 31,261 & 59,247 \\
1988 & 44,519 & 81,024 \\
1989 & 47,501 & 82,476 \\
1990 & 48,309 & 79,579 \\
1991 & 42,243 & 66,777 \\
1992 & 38,287 & 58,754 \\
1993 & 28,688 & 42,745 \\
1994 & 31,499 & 45,761 \\
1995 & 40,787 & 57,623 \\
1996 & 19,302 & 26,487 \\
1997 & 36,212 & 48,576 \\
1998 & 21,740 & 28,716 \\
1999 & 27,516 & 35,560 \\
2000 & 51,067 & 63,850 \\
2001 & 35,507 & 43,167 \\
2002 & 20,042 & 23,986 \\
2003 & 15,225 & 17,816 \\
2004 & 33,637 & 38,339 \\
2005 & 27,487 & 30,302 \\
2006 & 43,183 & 46,119 \\
2007 & 37,386 & 38,821 \\
2008 & 12,922 & 12,922 \\
\hline
\end{tabular}

Source:

U.S. Department of Transportation, National Highway Traffic Safety Administration, Office of Vehicle Safety Compliance, Washington, DC, February 2010. (Additional resources: www.nhtsa.dot.gov)

${ }^{a}$ These are fines which are actually collected. Fines which are assessed in certain year may not have been collected in that year.

${ }^{\mathrm{b}}$ Adjusted using the Consumer Price Inflation Index. 
Consumers must pay the Gas Guzzler Tax when purchasing a car that has an Environmental Protection Agency (EPA) fuel economy rating (combined city and highway) less than that stipulated in the table below. The Gas Guzzler Tax doubled in 1991 after remaining constant from 1986 to 1990. The tax has not changed since 1991.

This tax does not apply to light trucks such as pickups, minivans, sport utility vehicles, and vans.

Table 4.24

The Gas Guzzler Tax on New Cars (dollars per vehicle)

\begin{tabular}{crrrrrrrr}
\hline $\begin{array}{c}\text { Vehicle fuel } \\
\text { economy (mpg) }\end{array}$ & 1980 & \multicolumn{1}{c}{1981} & \multicolumn{1}{c}{1982} & 1983 & 1984 & 1985 & $1986-90$ & $1991-$ on \\
\hline Over 22.5 & 0 & 0 & 0 & 0 & 0 & 0 & 0 & 0 \\
$22.0-22.5$ & 0 & 0 & 0 & 0 & 0 & 0 & 500 & 1,000 \\
$21.5-22.0$ & 0 & 0 & 0 & 0 & 0 & 0 & 500 & 1,000 \\
$21.0-21.5$ & 0 & 0 & 0 & 0 & 0 & 0 & 650 & 1,300 \\
$20.5-21.0$ & 0 & 0 & 0 & 0 & 0 & 500 & 650 & 1,300 \\
$20.0-20.5$ & 0 & 0 & 0 & 0 & 0 & 500 & 850 & 1,700 \\
$19.5-20.0$ & 0 & 0 & 0 & 0 & 0 & 600 & 850 & 1,700 \\
$19.0-19.5$ & 0 & 0 & 0 & 0 & 450 & 600 & 1,050 & 2,100 \\
$18.5-19.0$ & 0 & 0 & 0 & 350 & 450 & 800 & 1,050 & 2,100 \\
$18.0-18.5$ & 0 & 0 & 200 & 350 & 600 & 800 & 1,300 & 2,600 \\
$17.5-18.0$ & 0 & 0 & 200 & 500 & 600 & 1,000 & 1,300 & 2,600 \\
$17.0-17.5$ & 0 & 0 & 350 & 500 & 750 & 1,000 & 1,500 & 3,000 \\
$16.5-17.0$ & 0 & 200 & 350 & 650 & 750 & 1,200 & 1,500 & 3,000 \\
$16.0-16.5$ & 0 & 200 & 450 & 650 & 950 & 1,200 & 1,850 & 3,700 \\
$15.5-16.0$ & 0 & 350 & 450 & 800 & 950 & 1,500 & 1,850 & 3,700 \\
$15.0-15.5$ & 0 & 350 & 600 & 800 & 1,150 & 1,500 & 2,250 & 4,500 \\
$14.5-15.0$ & 200 & 450 & 600 & 1,000 & 1,150 & 1,800 & 2,250 & 4,500 \\
$14.0-14.5$ & 200 & 450 & 750 & 1,000 & 1,450 & 1,800 & 2,700 & 5,400 \\
$13.5-14.0$ & 300 & 550 & 750 & 1,250 & 1,450 & 2,200 & 2,700 & 5,400 \\
$13.0-13.5$ & 300 & 550 & 950 & 1,250 & 1,750 & 2,200 & 3,200 & 6,400 \\
$12.5-13.0$ & 550 & 650 & 950 & 1,550 & 1,750 & 2,650 & 3,200 & 6,400 \\
Under 12.5 & 550 & 650 & 1,200 & 1,550 & 2,150 & 2,650 & 3,850 & 7,700 \\
\hline
\end{tabular}

Source:

Internal Revenue Service, Form 6197, (Rev. 10-05), "Gas Guzzler Tax." (Additional resources: www.irs.ustreas.gov) 
Consumers continue to demand gas guzzling cars. The IRS collected over $\$ 172$ million in 2008 from those buying cars with combined city/highway fuel economy less than 22.5 miles per gallon. This tax does not apply to light trucks such as pickups, minivans, sport utility vehicles, and vans. It is worthy to note that total revenue from fines paid by consumers to purchase gas-guzzling vehicles greatly exceeds the overall fines paid by manufacturers whose vehicles fail to meet CAFE standards (see Table 4.23).

Table 4.25

Tax Receipts from the Sale of Gas Guzzlers, 1980-2008 (thousands)

\begin{tabular}{|c|c|c|}
\hline Model year & Current dollars & $\begin{array}{c}2008 \\
\text { constant dollars }\end{array}$ \\
\hline 1980 & 740 & 1,934 \\
\hline 1981 & 780 & 1,847 \\
\hline 1982 & 1,720 & 3,838 \\
\hline 1983 & 4,020 & 8,690 \\
\hline 1984 & 8,820 & 18,277 \\
\hline 1985 & 39,790 & 79,618 \\
\hline 1986 & 147,660 & 290,070 \\
\hline 1987 & 145,900 & 276,520 \\
\hline 1988 & 116,780 & 212,537 \\
\hline 1989 & 109,640 & 190,370 \\
\hline 1990 & 103,200 & 170,002 \\
\hline 1991 & 118,400 & 187,165 \\
\hline 1992 & 144,200 & 221,288 \\
\hline 1993 & 111,600 & 166,282 \\
\hline 1994 & 64,100 & 93,124 \\
\hline 1995 & 73,500 & 103,837 \\
\hline 1996 & 52,600 & 72,179 \\
\hline 1997 & 48,200 & 64,658 \\
\hline 1998 & 47,700 & 63,006 \\
\hline 1999 & 68,300 & 88,266 \\
\hline 2000 & 70,800 & 88,522 \\
\hline 2001 & 78,200 & 95,069 \\
\hline 2002 & 79,700 & 95,384 \\
\hline 2003 & 126,700 & 148,255 \\
\hline 2004 & 140,800 & 160,480 \\
\hline 2005 & 163,800 & 180,577 \\
\hline 2006 & 200,200 & 213,808 \\
\hline 2007 & 178,700 & 185,561 \\
\hline 2008 & 172,428 & 172,428 \\
\hline
\end{tabular}

Source:

Ward's Communications, Detroit, MI, 2010. Original data source: Internal Revenue Service.

${ }^{\mathrm{a}}$ Adjusted using the Consumer Price Inflation Index. 
The Powertrain System Analysis Toolkit (PSAT) provides vehicle simulations for a variety of research purposes. It is used by the Department of Energy to evaluate the fuel efficiency potential of advanced powertrain configurations for different driving conditions. Recently, PSAT was used to develop data on the relationship between speed and fuel economy.

Table 4.26

Fuel Economy by Speed, PSAT Model Results

\begin{tabular}{|c|c|c|c|c|c|c|c|c|c|c|}
\hline \multirow[b]{2}{*}{ Speed (mph) } & \multicolumn{3}{|c|}{ Gasoline Conventional } & \multicolumn{3}{|c|}{ Diesel Conventional } & \multicolumn{4}{|c|}{ Hybrid Vehicles } \\
\hline & $\begin{array}{c}\text { Midsize } \\
\text { Car }\end{array}$ & $\begin{array}{l}\text { Small } \\
\text { SUV }\end{array}$ & $\begin{array}{l}\text { Large } \\
\text { SUV }\end{array}$ & $\begin{array}{c}\text { Midsize } \\
\text { Car }\end{array}$ & $\begin{array}{l}\text { Small } \\
\text { SUV }\end{array}$ & $\begin{array}{l}\text { Large } \\
\text { SUV }\end{array}$ & $\begin{array}{c}2000 \\
\text { Insight }^{\mathrm{a}}\end{array}$ & $\begin{array}{l}2004 \\
\text { Prius } \\
\end{array}$ & $\begin{array}{c}2007 \\
\text { Camry }^{\mathrm{a}} \\
\end{array}$ & $\begin{array}{r}2008 \\
\text { Tahoe }^{\mathrm{a}} \\
\end{array}$ \\
\hline 45 & 39.1 & 32.5 & 29.5 & 56.4 & 47.7 & 43.6 & 101.3 & 72.0 & 52.2 & 32.2 \\
\hline 55 & 41.7 & 34.3 & 30.0 & 57.0 & 46.0 & 39.9 & 94.3 & 66.0 & 46.8 & 27.1 \\
\hline 65 & 36.9 & 29.1 & 23.0 & 47.9 & 37.6 & 32.5 & 80.0 & 57.0 & 40.9 & 23.7 \\
\hline 75 & 31.9 & 24.5 & 19.8 & 40.2 & 30.8 & 26.9 & 60.6 & 42.0 & 35.0 & 21.1 \\
\hline \multicolumn{11}{|c|}{ Fuel economy loss } \\
\hline $55-65 \mathrm{mph}$ & $11.5 \%$ & $15.2 \%$ & $23.5 \%$ & $16.0 \%$ & $18.3 \%$ & $18.5 \%$ & $15.2 \%$ & $13.6 \%$ & $12.6 \%$ & $12.4 \%$ \\
\hline $65-75 \mathrm{mph}$ & $13.6 \%$ & $15.8 \%$ & $13.8 \%$ & $16.2 \%$ & $18.1 \%$ & $17.2 \%$ & $24.3 \%$ & $26.3 \%$ & $14.5 \%$ & $11.1 \%$ \\
\hline $55-75 \mathrm{mph}$ & $23.5 \%$ & $28.6 \%$ & $34.0 \%$ & $29.6 \%$ & $33.1 \%$ & $32.6 \%$ & $35.8 \%$ & $36.4 \%$ & $25.3 \%$ & $22.1 \%$ \\
\hline
\end{tabular}

\section{Source:}

Argonne National Laboratory, Powertrain System Analysis Toolkit, July 16, 2009,

www.transportation.anl.gov/modeling_simulation/PSAT/. (Additional resources: www.transportation.anl.gov)

${ }^{a}$ From Argonne National Laboratory Advanced Powertrain Research Facility (Vehicle Test Data). 
The two earlier studies by the Federal Highway Administration (FHWA) indicate maximum fuel efficiency was achieved at speeds of 35 to $40 \mathrm{mph}$. The recent FHWA study indicates greater fuel efficiency at higher speeds. Note that the 1973 study did not include light trucks.

Table 4.27

Fuel Economy by Speed, 1973, 1984, and 1997 Studies (miles per gallon)

\begin{tabular}{cccc}
\hline $\begin{array}{c}\text { Speed } \\
\text { (miles per hour) }\end{array}$ & $\begin{array}{c}1973^{\mathrm{a}} \\
(13 \text { vehicles })\end{array}$ & $\begin{array}{c}1984^{\mathrm{b}} \\
(15 \text { vehicles })\end{array}$ & $\begin{array}{c}1997^{\mathrm{c}} \\
(9 \text { vehicles })\end{array}$ \\
\hline 15 & $\mathrm{~d}$ & 21.1 & 24.4 \\
20 & $\mathrm{~d}$ & 25.5 & 27.9 \\
25 & $\mathrm{~d}$ & 30.0 & 30.5 \\
30 & 21.1 & 31.8 & 31.7 \\
35 & 21.1 & 33.6 & 31.2 \\
40 & 21.1 & 33.6 & 31.0 \\
45 & 20.3 & 33.5 & 31.6 \\
50 & 19.5 & 31.9 & 32.4 \\
55 & 18.5 & 30.3 & 32.4 \\
60 & 17.5 & 27.6 & 31.4 \\
65 & 16.2 & 24.9 & 29.2 \\
70 & 14.9 & 22.5 & 26.8 \\
75 & $\mathrm{~d}$ & 20.0 & 24.8 \\
& Fuel economy loss & & \\
$55-65 \mathrm{mph}$ & $12.4 \%$ & $17.8 \%$ & $9.7 \%$ \\
$65-70 \mathrm{mph}$ & $8.0 \%$ & $9.6 \%$ & $17.1 \%$ \\
$55-70 \mathrm{mph}$ & $19.5 \%$ & $25.7 \%$ & \\
\hline
\end{tabular}

\section{Sources:}

1973- U.S. Department of Transportation, Federal Highway Administration, Office of Highway Planning, The Effect of Speed on Automobile Gasoline Consumption Rates, Washington, DC, October 1973.

1984 - U.S. Department of Transportation, Federal Highway Administration, Fuel Consumption and Emission Values for Traffic Models, Washington, DC, May 1985.

1997 - West, B.H., R.N. McGill, J.W. Hodgson, S.S. Sluder, and D.E. Smith, Development and Verification of Light-Duty Modal Emissions and Fuel Consumption Values for Traffic Models, FHWA Report (in press), Washington, DC, April 1997, and additional project data, April 1998. (Additional resources: www.fhwatsis.com)

\footnotetext{
${ }^{a}$ Model years 1970 and earlier cars.

${ }^{\mathrm{b}}$ Model years 1981-84 cars and light trucks.

${ }^{\mathrm{c}}$ Model years 1988-97 cars and light trucks.

${ }^{\mathrm{d}}$ Data are not available.
} 
Figure 4.2. Fuel Economy by Speed, 1973, 1984, and 1997 Studies

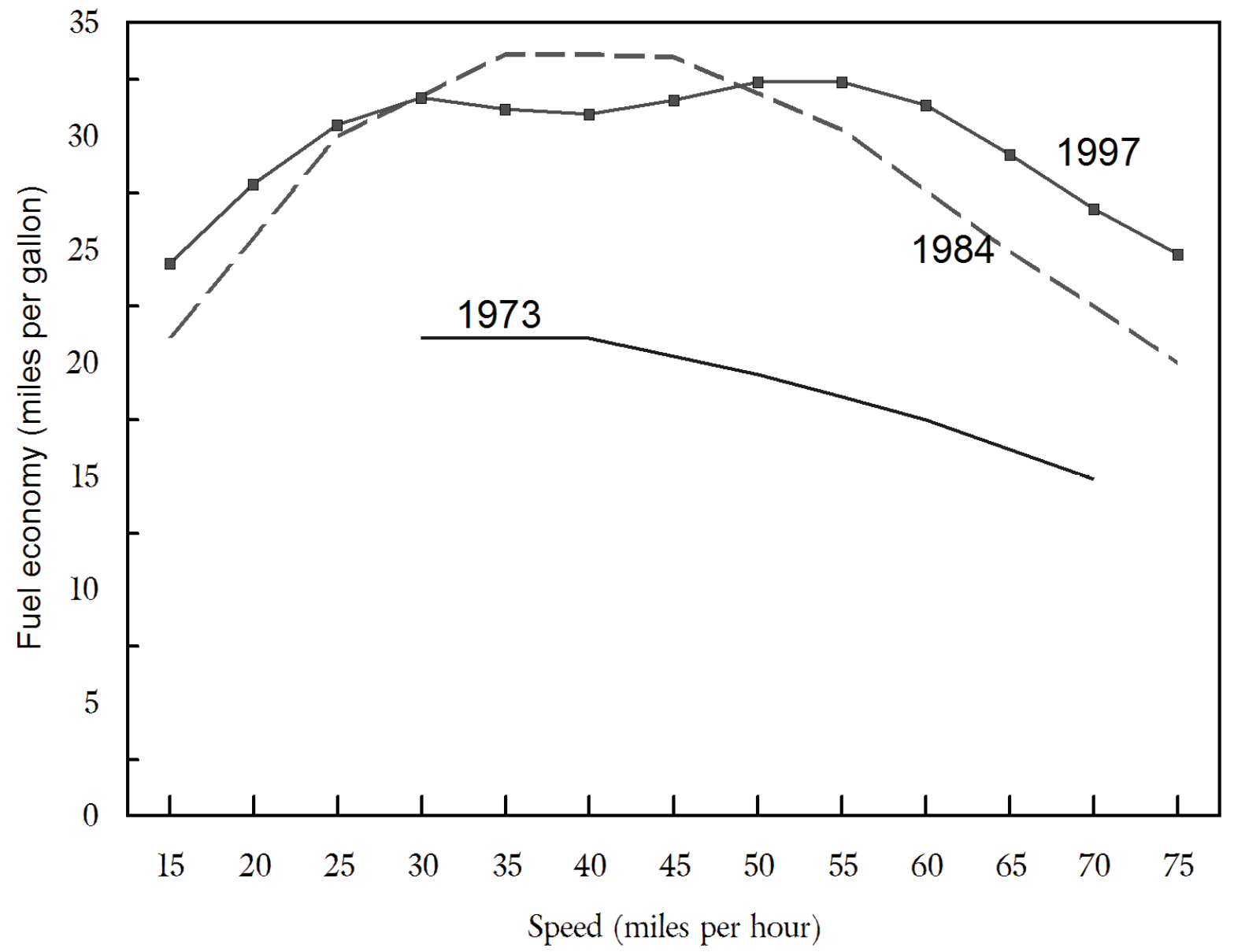

Source:

See Table 4.27. 
Table 4.28

Vehicle Specifications for Vehicles Tested in the 1997 Study

\begin{tabular}{|c|c|c|c|c|c|c|}
\hline \multirow[b]{2}{*}{ Vehicle } & \multirow{2}{*}{$\begin{array}{c}\text { Curb } \\
\text { weight }\end{array}$} & \multirow[b]{2}{*}{ Engine } & \multirow{2}{*}{$\begin{array}{c}\text { Fuel } \\
\text { delivery } \\
\text { system }^{\mathrm{a}}\end{array}$} & \multirow{2}{*}{$\begin{array}{l}\text { Trans- } \\
\text { mission }\end{array}$} & \multicolumn{2}{|c|}{ EPA fuel economy } \\
\hline & & & & & City & Highway \\
\hline 1988 Chevrolet Corsica & 2,665 & 2.8 liter V6 & PFI & M5 & 19 & 29 \\
\hline 1994 Olds Cutlass Supreme & 3,290 & 3.4 liter V6 & PFI & $\mathrm{L} 4$ & 17 & 26 \\
\hline 1994 Oldsmobile 88 & 3,433 & 3.8 literV6 & PFI & L4 & 19 & 29 \\
\hline 1994 Mercury Villager & 4,020 & 3.0 liter V6 & PFI & L4 & 17 & 23 \\
\hline 1995 Geo Prizm & 2,359 & 1.6 liter I-4 & PFI & L3 & 26 & 30 \\
\hline 1994 Jeep Grand Cherokee & 3,820 & 4.0 liter I-6 & PFI & $\mathrm{L} 4$ & 15 & 20 \\
\hline 1994 Chevrolet Pickup & 4,020 & 5.7 liter V8 & TBI & $\mathrm{L} 4$ & 14 & 18 \\
\hline 1993 Subaru Legacy & 2,800 & 2.2 liter $\mathrm{H} 4$ & PFI & $\mathrm{L} 4$ & 22 & 29 \\
\hline 1997 Toyota Celica & 2,395 & 1.8 liter I4 & PFI & L4 & 27 & 34 \\
\hline
\end{tabular}

Source:

West, B.H., R.N. McGill, J.W. Hodgson, S.S. Sluder, and D.E. Smith, Development and Verification of Light-Duty Modal Emissions and Fuel Consumption Values for Traffic Models, Washington, DC, April 1997 and additional project data, April 1998.

${ }^{\mathrm{a}} \mathrm{PFI}=$ port fuel injection. $\mathrm{TBI}=$ throttle- body fuel injection. 
Of the tested vehicles, the 1994 Oldsmobile Olds 88 had the greatest fuel economy loss from 55 mph to 75 mpg. The 1997 Toyota Celica tested fuel economy was slightly better at $65 \mathrm{mph}$ than at $55 \mathrm{mph}$.

Table 4.29

\section{Steady Speed Fuel Economy for Vehicles Tested in the 1997 Study} (miles per gallon)

\begin{tabular}{|c|c|c|c|c|c|c|c|c|c|}
\hline $\begin{array}{l}\text { Speed } \\
(\mathrm{mph})\end{array}$ & $\begin{array}{c}1988 \\
\text { Chevrolet } \\
\text { Corsica } \\
\end{array}$ & $\begin{array}{c}1993 \\
\text { Subaru } \\
\text { Legacy }\end{array}$ & $\begin{array}{c}1994 \\
\text { Oldsmobile } \\
\text { Olds } 88 \\
\end{array}$ & $\begin{array}{c}1994 \\
\text { Oldsmobile } \\
\text { Cutlass } \\
\end{array}$ & $\begin{array}{c}1994 \\
\text { Chevrolet } \\
\text { Pickup } \\
\end{array}$ & $\begin{array}{c}1994 \text { Jeep } \\
\text { Grand } \\
\text { Cherokee }\end{array}$ & $\begin{array}{c}1994 \\
\text { Mercury } \\
\text { Villager }\end{array}$ & $\begin{array}{c}1995 \\
\text { Geo } \\
\text { Prizm } \\
\end{array}$ & $\begin{array}{c}1997 \\
\text { Toyota } \\
\text { Celica } \\
\end{array}$ \\
\hline 5 & 10.0 & 14.5 & 10.5 & 5.1 & 7.9 & 8.2 & 12.3 & 18.1 & 19.1 \\
\hline 10 & 16.8 & 24.7 & 14.9 & 7.9 & 16.0 & 11.2 & 19.0 & 23.1 & 34.1 \\
\hline 15 & 17.7 & 31.9 & 22.2 & 11.4 & 16.3 & 17.5 & 22.4 & 38.9 & 41.7 \\
\hline 20 & 21.7 & 34.4 & 26.3 & 12.5 & 19.9 & 24.7 & 25.8 & 39.4 & 46.0 \\
\hline 25 & 23.9 & 37.4 & 28.3 & 15.6 & 22.7 & 21.8 & 30.8 & 41.7 & 52.6 \\
\hline 30 & 28.7 & 39.7 & 29.0 & 19.0 & 26.3 & 21.6 & 30.3 & 40.0 & 50.8 \\
\hline 35 & 28.6 & 38.0 & 30.9 & 21.2 & 24.3 & 25.0 & 26.1 & 39.1 & 47.6 \\
\hline 40 & 29.2 & 37.0 & 33.2 & 23.0 & 26.7 & 25.5 & 29.0 & 38.9 & 36.2 \\
\hline 45 & 28.8 & 33.7 & 32.4 & 23.0 & 27.3 & 25.4 & 27.8 & 42.3 & 44.1 \\
\hline 50 & 31.2 & 33.7 & 34.2 & 27.3 & 26.3 & 24.8 & 30.1 & 39.1 & 44.8 \\
\hline 55 & 29.1 & 37.7 & 34.6 & 29.1 & 25.1 & 24.0 & 31.7 & 37.7 & 42.5 \\
\hline 60 & 28.2 & 35.9 & 32.5 & 28.2 & 22.6 & 23.2 & 27.3 & 36.7 & 48.4 \\
\hline 65 & 28.7 & 33.4 & 30.0 & 25.0 & 21.8 & 21.3 & 25.3 & 34.1 & 43.5 \\
\hline 70 & 26.1 & 31.0 & 26.7 & 22.9 & 20.1 & 20.0 & 23.9 & 31.7 & 39.2 \\
\hline 75 & 23.7 & 28.8 & 24.0 & 21.6 & 18.1 & 19.1 & 22.4 & 28.3 & 36.8 \\
\hline \multicolumn{10}{|c|}{ Fuel economy loss } \\
\hline $55-65 \mathrm{mph}$ & $1.4 \%$ & $11.4 \%$ & $13.3 \%$ & $14.1 \%$ & $13.1 \%$ & $11.3 \%$ & $20.2 \%$ & $9.5 \%$ & $-2.4 \%$ \\
\hline 65-75 mph & $17.4 \%$ & $13.8 \%$ & $20.0 \%$ & $13.6 \%$ & $17.0 \%$ & $10.3 \%$ & $11.5 \%$ & $17.0 \%$ & $15.4 \%$ \\
\hline 55-75 mph & $18.6 \%$ & $23.6 \%$ & $30.6 \%$ & $25.8 \%$ & $27.9 \%$ & $20.4 \%$ & $29.3 \%$ & $24.9 \%$ & $13.4 \%$ \\
\hline
\end{tabular}

\section{Source:}

B.H. West, R.N. McGill, J.W. Hodgson, S.S. Sluder, D.E. Smith, Development and Verification of Light-Duty Modal Emissions and Fuel Consumption Values for Traffic Models, Washington, DC, April 1997, and additional project data, April 1998. (Additional resources: www.fhwa-tsis.com)

Note: For specifications of the tested vehicles, please see Table 4.27. 
This table shows the new methodology that the Environmental Protection Agency (EPA) used to determine fuel economy ratings for new vehicles beginning in model year 2008. In addition to the Urban Driving Cycle and the Highway Driving cycle, the EPA will also use three additional tests to adjust fuel economy ratings to account for higher speeds, air conditioner use, and colder temperatures. Though the EPA uses a complex combination of these five cycles to determine the fuel economy that will be posted on a new vehicle window sticker, the manufacturer's Corporate Average Fuel Economy is still calculated using only the city and highway driving cycles. To know more about new vehicle fuel economy ratings, visit www.fueleconomy.gov.

\section{Table 4.30}

\section{Driving Cycle Attributes}

\begin{tabular}{|c|c|c|c|c|c|}
\hline & \multicolumn{5}{|c|}{ Test Schedule } \\
\hline & City & Highway & High Speed & $\mathrm{AC}$ & Cold Temp \\
\hline Trip type & $\begin{array}{l}\text { Low speeds in } \\
\text { stop-and-go } \\
\text { urban traffic }\end{array}$ & $\begin{array}{c}\text { Free-flow traffic } \\
\text { at highway } \\
\text { speeds }\end{array}$ & $\begin{array}{l}\text { Higher speeds; harder } \\
\text { acceleration \& } \\
\text { braking }\end{array}$ & $\begin{array}{l}\text { AC use under } \\
\text { hot ambient } \\
\text { conditions }\end{array}$ & $\begin{array}{c}\text { City test } \\
\text { w/colder outside } \\
\text { temperature }\end{array}$ \\
\hline Top speed & $56 \mathrm{mph}$ & $60 \mathrm{mph}$ & $80 \mathrm{mph}$ & $54.8 \mathrm{mph}$ & $56 \mathrm{mph}$ \\
\hline Average speed & $20 \mathrm{mph}$ & $48 \mathrm{mph}$ & $48 \mathrm{mph}$ & $22 \mathrm{mph}$ & $20 \mathrm{mph}$ \\
\hline Max. acceleration & $3.3 \mathrm{mph} / \mathrm{sec}$ & $3.2 \mathrm{mph} / \mathrm{sec}$ & $8.46 \mathrm{mph} / \mathrm{sec}$ & $5.1 \mathrm{mph} / \mathrm{sec}$ & $3.3 \mathrm{mph} / \mathrm{sec}$ \\
\hline Simulated distance & $11 \mathrm{mi}$. & $10 \mathrm{mi}$. & $8 \mathrm{mi}$ & $3.6 \mathrm{mi}$. & $11 \mathrm{mi}$. \\
\hline Time & $31 \mathrm{~min}$. & $12.5 \mathrm{~min}$. & $10 \mathrm{~min}$. & 9.9 min. & $31 \mathrm{~min}$. \\
\hline Stops & 23 & None & 4 & 5 & 23 \\
\hline Idling time & $18 \%$ of time & None & $7 \%$ of time & $19 \%$ of time & $18 \%$ of time \\
\hline Engine startup $^{\mathrm{a}}$ & Cold & Warm & Warm & Warm & Cold \\
\hline Lab temperature & $68-86^{\circ} \mathrm{F}$ & $68-86^{\circ} \mathrm{F}$ & $68-86^{\circ} \mathrm{F}$ & $95^{\circ} \mathrm{F}$ & $20^{\circ} \mathrm{F}$ \\
\hline Vehicle air conditioning & Off & Off & Off & On & Off \\
\hline
\end{tabular}

\section{Source:}

U.S. Department of Energy and U.S. Environmental Protection Agency, Fuel Economy Web site, www.fueleconomy.gov.

\footnotetext{
a A vehicle's engine doesn't reach maximum fuel efficiency until it is warm.
} 
These driving cycles simulate the performance of an engine while driving in the city and on the highway. Once the city cycle is completed, the engine is stopped, and then started again for the 8.5 minute hot start cycle. Three additional cycles also influence new vehicle fuel economy ratings beginning with the 2008 model year.

Figure 4.3. City Driving Cycle

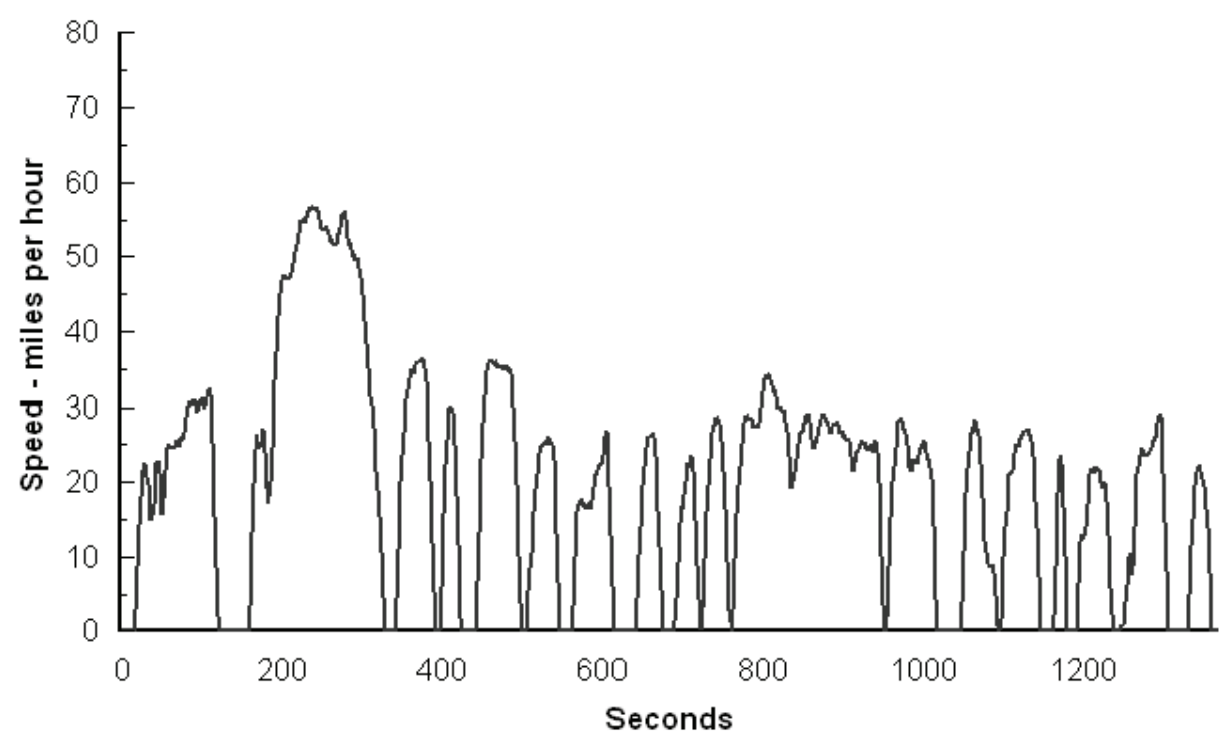

Figure 4.4. Highway Driving Cycle

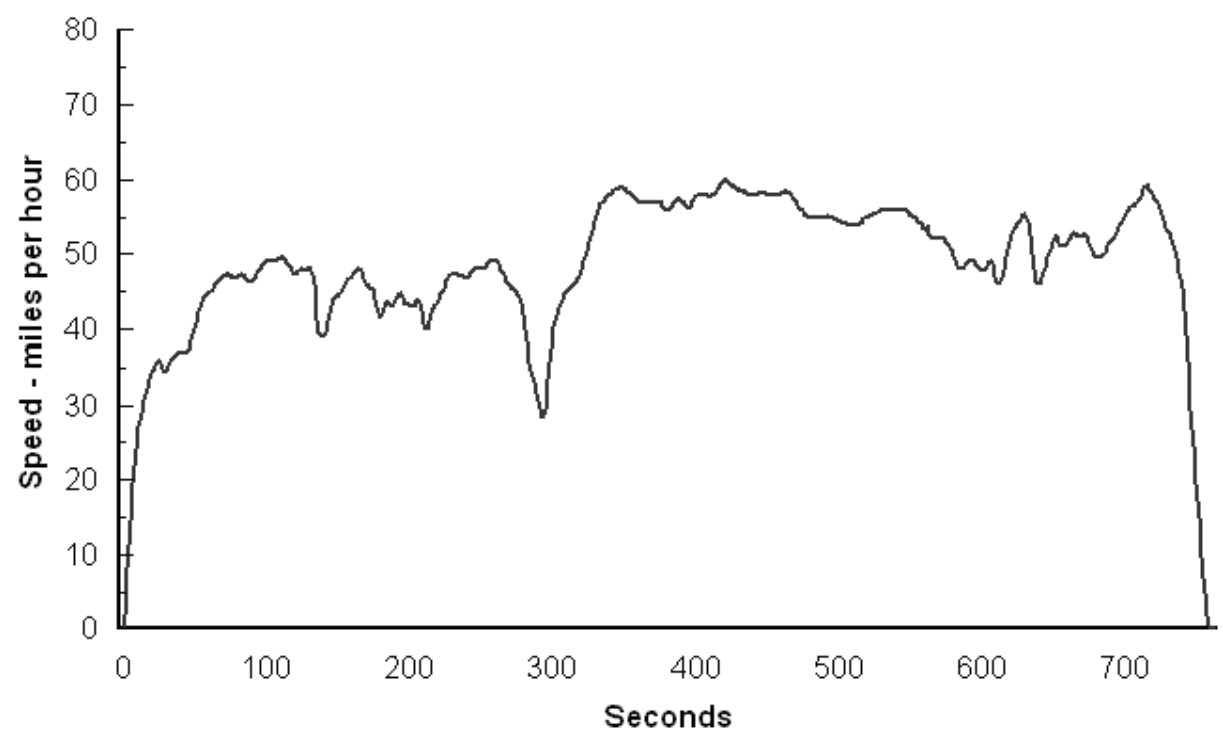

\section{Source:}

Code of Federal Regulations, 40CFR, "Subpart B - Fuel Economy Regulations for 1978 and Later Model Year Automobiles - Test Procedures," July 1, 1988 edition, p. 676. 
Figure 4.5. Air Conditioning (SC03) Driving Cycle

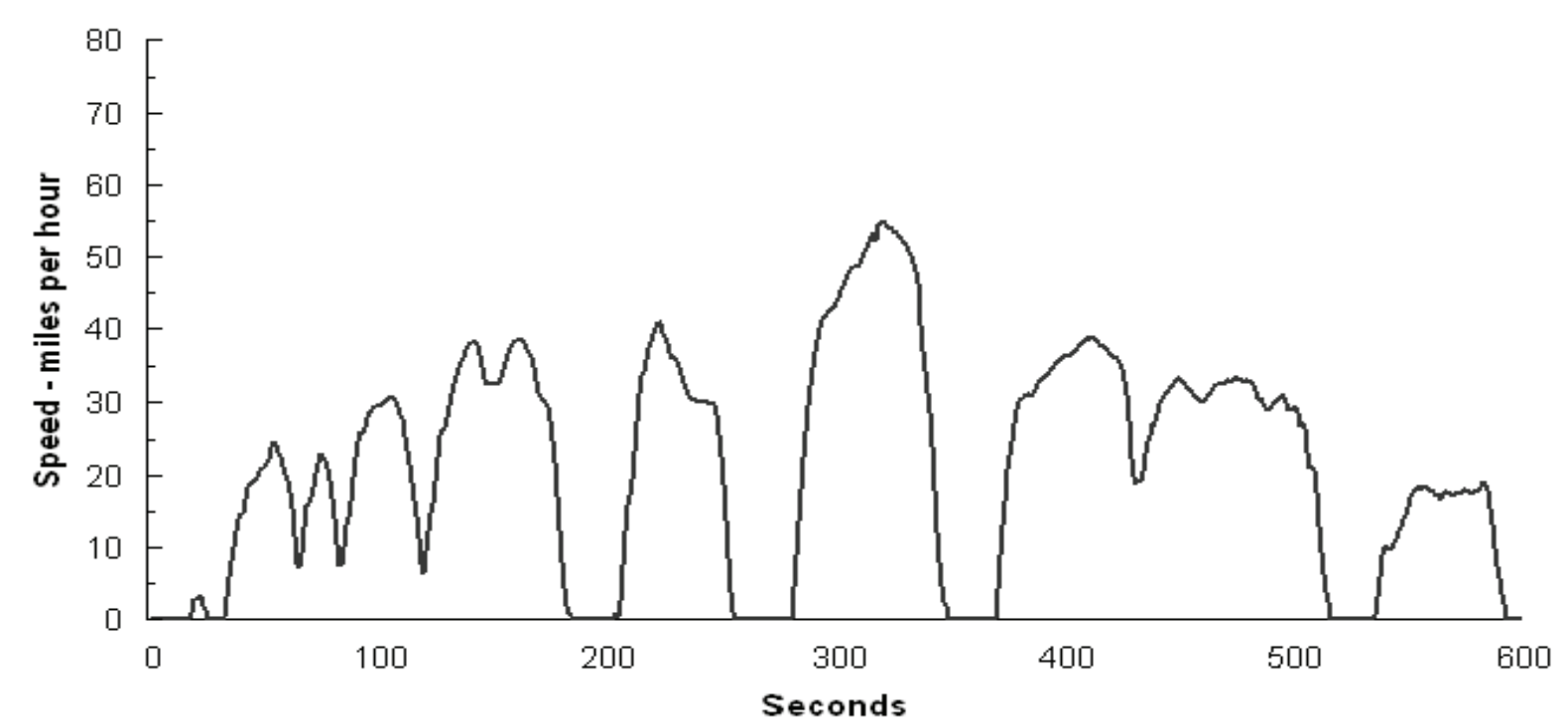

Source:

U.S. Department of Energy and Environmental Protection Agency, Fuel Economy Web site, www.fueleconomy.gov.

Figure 4.6. Cold Temperature (Cold FTP) Driving Cycle

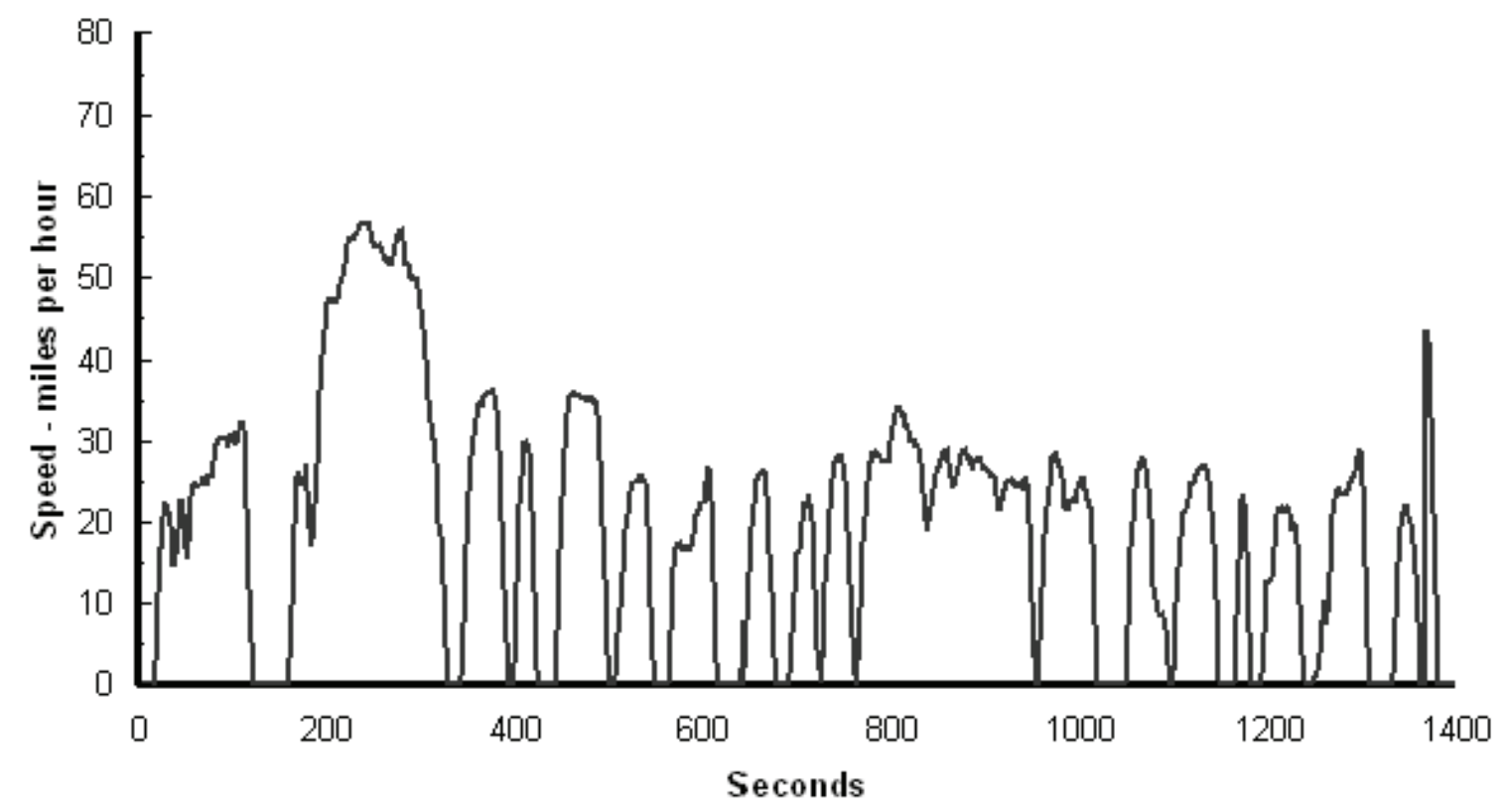

Source:

U.S. Department of Energy and Environmental Protection Agency, Fuel Economy Web site, www.fueleconomy.gov. 
Beginning with the 2008 model year, this cycle influences the new vehicle fuel economy ratings. The USO6 driving cycle was originally developed as a supplement to the Federal Test Procedure. It is a short-duration cycle (600 seconds) which represents hard-acceleration driving.

Figure 4.7. High-Speed (US06) Driving Cycle

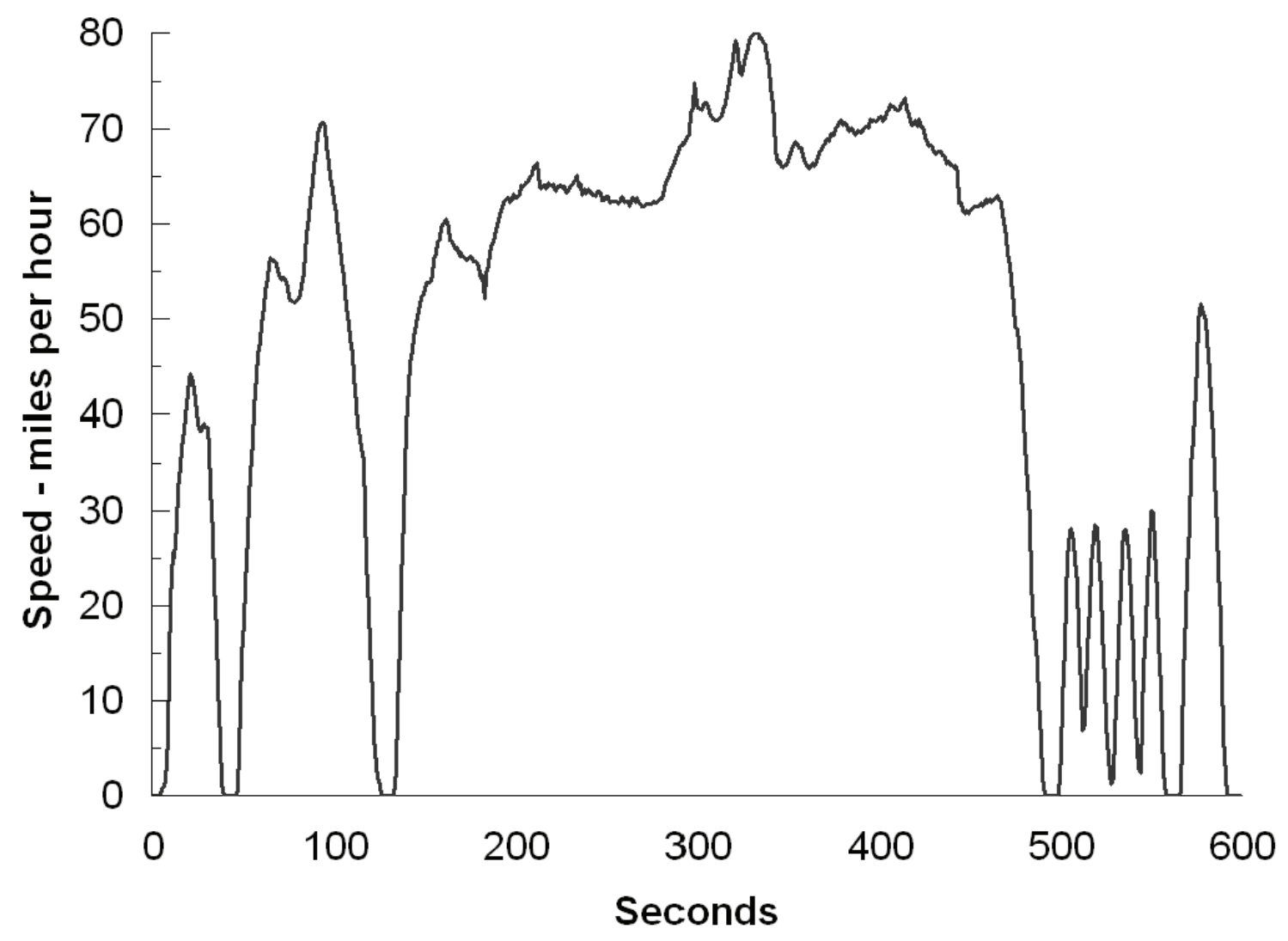

Source:

U.S. Department of Energy and Environmental Protection Agency, Fuel Economy Web site, www.fueleconomy.gov. 
The Environmental Protection Agency also uses other driving cycles to test new vehicles (although these do not affect the fuel economy ratings). The New York Test Cycle was developed in the 1970's in order to simulate driving in downtown congested areas. The Representative Number Five Test Cycle was developed in the 1990's to better represent actual on-road driving by combining modern city and freeway driving.

Figure 4.8. New York City Driving Cycle

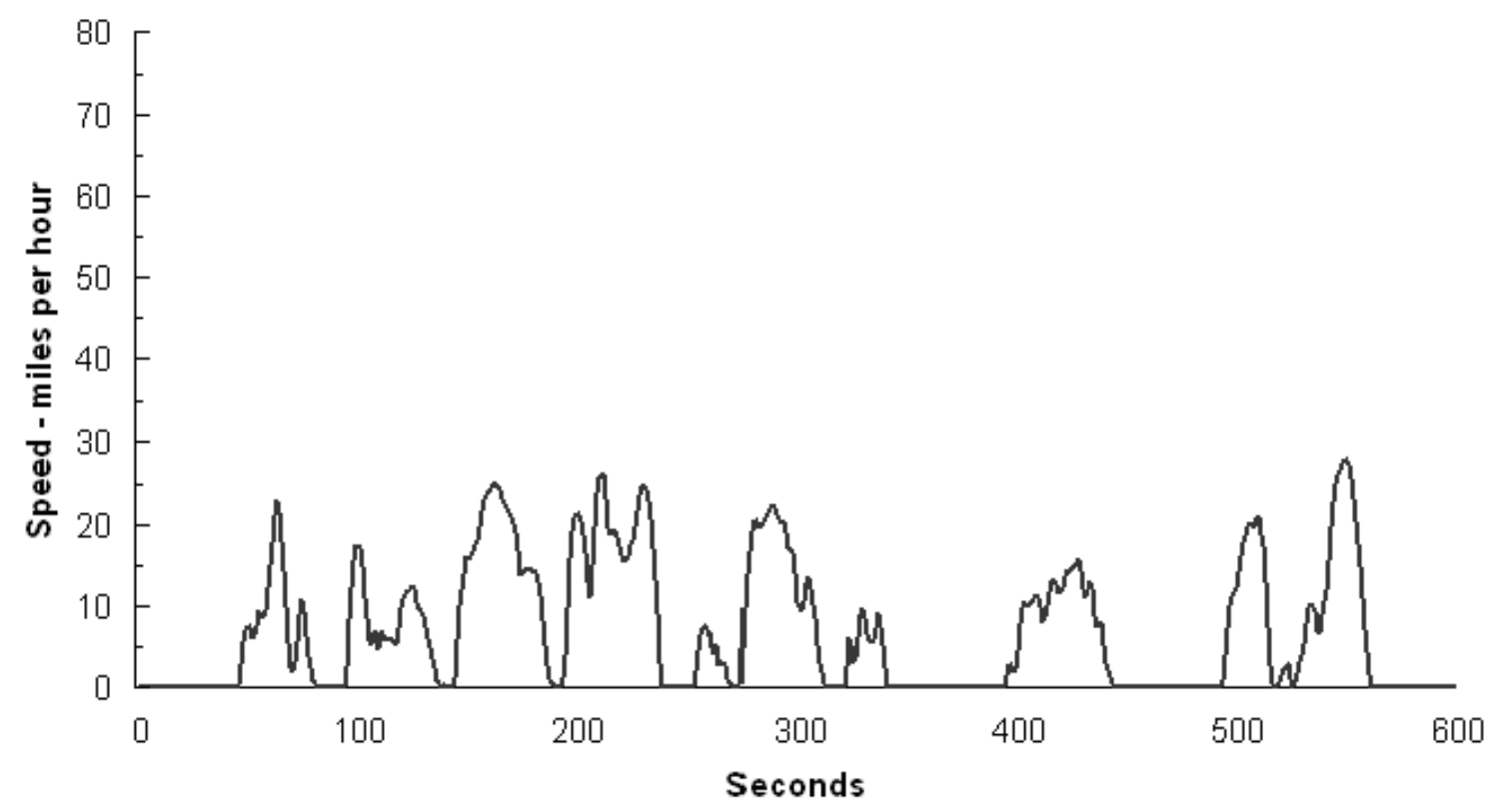

Figure 4.9. Representative Number Five Driving Cycle

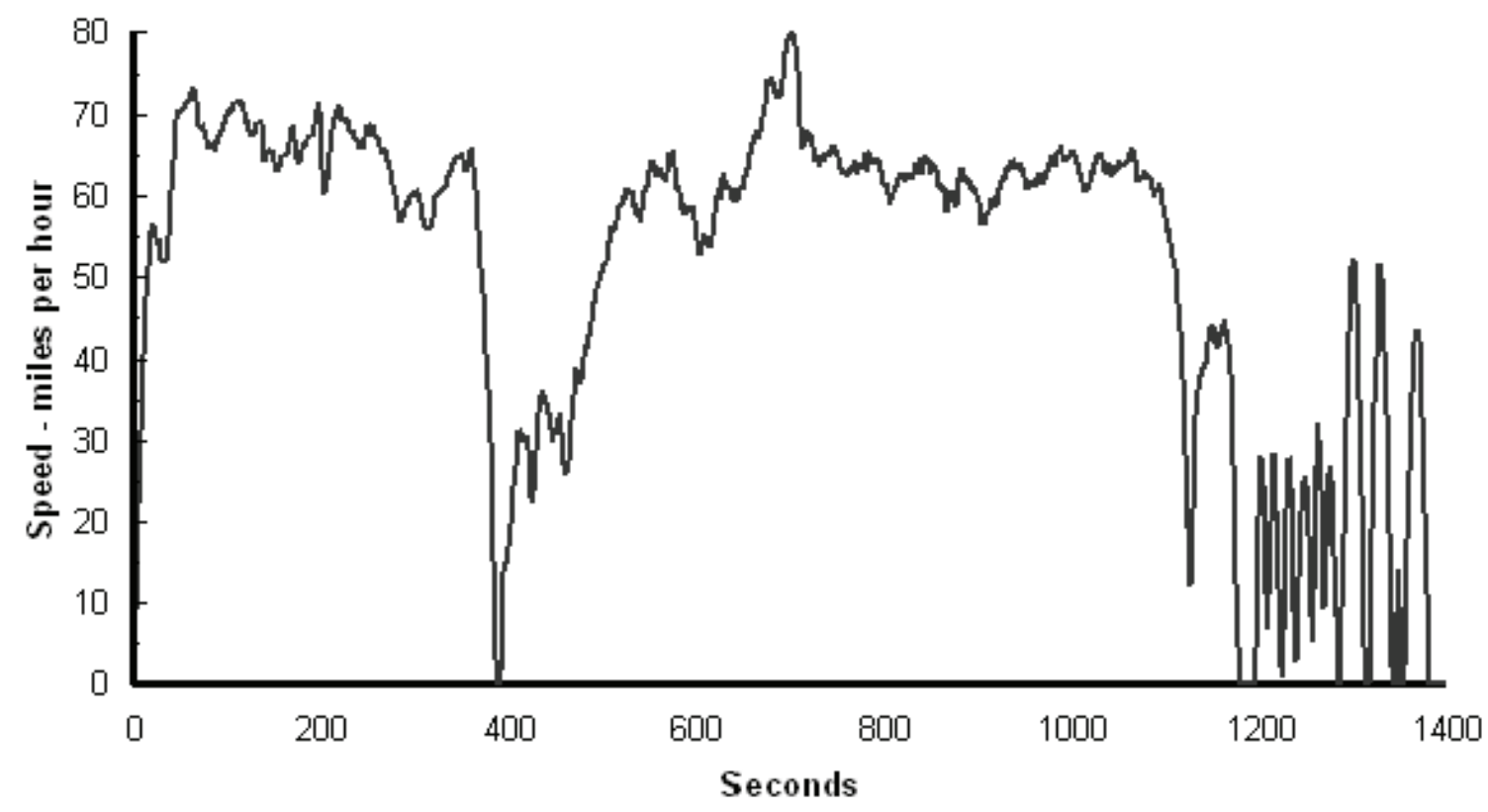

Source:

Data obtained from Michael Wang, Argonne National Laboratory, Argonne, IL, 1997. 
Researchers at Argonne National Laboratory have estimated the fuel economy of a midsize car using driving cycles from different countries. These results illustrate the difference in fuel economy which can be obtained from the same vehicle using different test cycles.

Table 4.31

Projected Fuel Economies from U.S., European, and Japanese Driving Cycles

\begin{tabular}{lc}
\hline Driving Cycle & $\begin{array}{c}\text { Projected fuel economy } \\
\text { for a 1995 composite } \\
\text { midsize vehicle }^{\mathrm{a}}\end{array}$ \\
\hline Japanese 10/15 mode test cycle & $17.5 \mathrm{mpg}$ \\
New European Driving Cycle (NEDC) & $22.0 \mathrm{mpg}$ \\
U.S. EPA city cycle (LA4) & $19.8 \mathrm{mpg}$ \\
U.S. EPA highway cycle & $32.1 \mathrm{mpg}$ \\
U.S. Corporate Average Fuel Economy cycle & $23.9 \mathrm{mpg}$ \\
\hline
\end{tabular}

\section{Source:}

Santini, D., A. Vyas, J. Anderson, and F. An, Estimating Trade-Offs along the Path to the PNGV $3 X$ Goal, presented at the Transportation Research Board 80 ${ }^{\text {th }}$ Annual Meeting, Washington, DC, January 2001.

Note: China and India both use the European Driving Cycle, though India uses a modified version called the Modified Indian Driving Cycle which accounts for lower maximum speeds that better represent driving conditions in India.

a The 1995 composite midsize vehicle is an average of a Chevrolet Lumina, Chrysler Concord, and Ford Taurus. The fuel economies were projected using the National Renewable Energy Laboratory's Advanced Vehicle Simulator (ADVISOR) model. 
When comparing data between countries, one must realize that different countries have different testing cycles to determine fuel economy and emissions. This table compares various statistics on the European, Japanese, and U.S. testing cycles [for fuel economy measurements, the United States uses the formula, $1 /$ fuel economy $=(0.55 /$ city fuel economy + (0.45/highway fuel economy)]. Most vehicles will achieve higher fuel economy on the U.S. test cycle than on the European or Japanese cycles.

Table 4.32

Comparison of U.S., European, and Japanese Driving Cycles

\begin{tabular}{|c|c|c|c|c|c|c|}
\hline & $\begin{array}{c}\text { Time } \\
\text { (seconds) }\end{array}$ & $\begin{array}{l}\text { Percent of } \\
\text { time } \\
\text { stopped } \\
\text { or } \\
\text { decelerating }\end{array}$ & $\begin{array}{l}\text { Distance } \\
\text { (miles) }\end{array}$ & $\begin{array}{l}\text { Average } \\
\text { speed } \\
(\mathrm{mph})\end{array}$ & $\begin{array}{c}\text { Maximum } \\
\text { speed } \\
(\mathrm{mph})\end{array}$ & $\begin{array}{c}\text { Maximum } \\
\text { acceleration } \\
(\mathrm{mph} / \mathrm{s})\end{array}$ \\
\hline $\begin{array}{l}\text { Japanese } 10 / 15 \text { mode } \\
\text { test cycle }\end{array}$ & 631 & 52.3 & 2.6 & 14.8 & 43.5 & 1.8 \\
\hline $\begin{array}{c}\text { New European Driving } \\
\text { Cycle (NEDC) }\end{array}$ & 1,181 & 24.9 & 6.84 & 20.9 & 74.6 & 2.4 \\
\hline $\begin{array}{l}\text { U.S. EPA city cycle } \\
\qquad(\mathrm{LA} 4)^{\mathrm{a}}\end{array}$ & 1,372 & 43.2 & 7.5 & 19.5 & 56.7 & 3.3 \\
\hline U.S. EPA highway cycle & 765 & 9.3 & 17.8 & 48.2 & 59.9 & 3.3 \\
\hline $\begin{array}{c}\text { U.S. Corporate Average } \\
\text { Fuel Economy cycle }\end{array}$ & 2,137 & 27.9 & 10.3 & 29.9 & 59.9 & 3.3 \\
\hline
\end{tabular}

\section{Source:}

Santini, D., A. Vyas, J. Anderson, and F. An, Estimating Trade-Offs along the Path to the PNGV 3X Goal, presented at the Transportation Research Board 80 ${ }^{\text {th }}$ Annual Meeting, Washington, DC, January 2001.

Note: China and India both use the European Driving Cycle, though India uses a modified version called The Modified Indian Driving Cycle which accounts for lower maximum speeds that better represent driving conditions in India.

${ }^{a}$ The actual Federal Procedure (FTP), which is also the test for emissions certification, repeats the first 505 seconds of the Federal Urban Driving Simulation cycle, hot started, after a 10 minute hot soak. Starting with Model Year 2001, the emissions test-but not the fuel economy test-incorporates a supplemental cycle that simulates aggressive urban driving, coupled with an added air conditioning load. 
Demand response (also called paratransit or dial-a-ride) are widely used by transit agencies. In 2007, the data changed substantially due to improved estimation methodologies. Unfortunately, those data are no longer comparable to the rest of the historical series.

Table 4.33

Summary Statistics on Demand Response Vehicles, 1994-2008 ${ }^{\mathrm{a}}$

\begin{tabular}{ccccc}
\hline Year & $\begin{array}{c}\text { Number of active } \\
\text { vehicles }\end{array}$ & $\begin{array}{c}\text { Vehicle-miles } \\
\text { (millions) }\end{array}$ & $\begin{array}{c}\text { Passenger-miles } \\
\text { (millions) }\end{array}$ & $\begin{array}{c}\text { Energy use } \\
\text { (trillion Btu) }\end{array}$ \\
\hline 1994 & 31,090 & 490 & 781 & 9.8 \\
1995 & 31,773 & 538 & 856 & 9.6 \\
1996 & 33,472 & 588 & 958 & 10.2 \\
1997 & 35,657 & 627 & 1,075 & 10.2 \\
1998 & 33,481 & 721 & 1,103 & 10.9 \\
1999 & 36,651 & 784 & 1,258 & 11.2 \\
2000 & 37,957 & 826 & 1,274 & 11.4 \\
2001 & 40,049 & 861 & 1,345 & 11.9 \\
2002 & 40,691 & 879 & 1,336 & 12.3 \\
2003 & 42,578 & 953 & 1,471 & 13.5 \\
2004 & 42,993 & 975 & 1,448 & 14.1 \\
2005 & 48,530 & 1,078 & 1,663 & 15.5 \\
2006 & 51,744 & 1,129 & 1,790 & 16.4 \\
$2007^{\text {a }}$ & 64,865 & 1,471 & 1,502 & 24.7 \\
2008 & 65,799 & 1,495 & 1,412 & 24.7 \\
\hline
\end{tabular}

\section{Source:}

American Public Transportation Association, 2010 Public Transportation Fact Book, Washington, DC, April 2010. (Additional resources: www.apta.com)

Note: See Glossary for detailed definitions of demand response.

a Data are not continuous between 2006 and 2007 due to changes in estimation methodology. See source document for details. 


\section{Chapter 5 \\ Heavy Vehicles and Characteristics}

Summary Statistics from Tables in this Chapter

\section{Source}

Table 5.1 Heavy single-unit trucks, 2008

Registration (thousands)

6,791

Vehicle miles (millions)

83,951

Fuel economy (miles per gallon)

Table 5.2 Combination trucks, 2008

Registration (thousands)

Vehicle miles (millions)

Fuel economy (miles per gallon)

Tables 5.14 Freight Shipments, 2007 Commodity Flow Survey

and 5.15

Value (billion dollars)

11,685

Tons (millions)

12,543

Ton-miles (billions) 
Heavy single-unit trucks include all single-unit trucks which have more than two axles or more than four tires. Most of these trucks would be used for business or for individuals with heavy hauling or towing needs.

Table 5.1

Summary Statistics for Heavy Single-Unit Trucks, 1970-2008

\begin{tabular}{|c|c|c|c|c|}
\hline Year & $\begin{array}{c}\begin{array}{c}\text { Registrations } \\
\text { (thousands) }\end{array} \\
\end{array}$ & $\begin{array}{l}\text { Vehicle travel } \\
\text { (million miles) }\end{array}$ & $\begin{array}{c}\text { Fuel use } \\
\text { (million gallons) }\end{array}$ & $\begin{array}{c}\text { Fuel economy } \\
\text { (miles per gallon) }\end{array}$ \\
\hline 1970 & 3,681 & 27,081 & 3,968 & 6.8 \\
\hline $1975^{\circ}$ & 4,232 & 34,606 & 5,420 & 6.4 \\
\hline 1976 & 4,350 & 36,390 & 5,706 & 6.4 \\
\hline 1977 & 4,450 & 39,339 & 6,268 & 6.3 \\
\hline 1978 & 4,518 & 42,747 & 6,955 & 6.1 \\
\hline 1979 & 4,505 & 42,012 & 7,050 & 6.0 \\
\hline 1980 & 4,374 & 39,813 & 6,923 & 5.8 \\
\hline 1981 & 4,455 & 39,568 & 6,867 & 5.8 \\
\hline 1982 & 4,325 & 40,658 & 6,803 & 6.0 \\
\hline 1983 & 4,204 & 42,546 & 6,965 & 6.1 \\
\hline 1984 & 4,061 & 44,419 & 7,240 & 6.1 \\
\hline 1985 & 4,593 & 45,441 & 7,399 & 6.1 \\
\hline 1986 & 4,313 & 45,637 & 7,386 & 6.2 \\
\hline 1987 & 4,188 & 48,022 & 7,523 & 6.4 \\
\hline 1988 & 4,470 & 49,434 & 7,701 & 6.4 \\
\hline 1989 & 4,519 & 50,870 & 7,779 & 6.5 \\
\hline 1990 & 4,487 & 51,901 & 8,357 & 6.2 \\
\hline 1991 & 4,481 & 52,898 & 8,172 & 6.5 \\
\hline 1992 & 4,370 & 53,874 & 8,237 & 6.5 \\
\hline 1993 & 4,408 & 56,772 & 8,488 & 6.7 \\
\hline 1994 & 4,906 & 61,284 & 9,032 & 6.8 \\
\hline 1995 & 5,024 & 62,705 & 9,216 & 6.8 \\
\hline 1996 & 5,266 & 64,072 & 9,409 & 6.8 \\
\hline 1997 & 5,293 & 66,893 & 9,576 & 7.0 \\
\hline 1998 & 5,414 & 67,894 & 9,741 & 7.0 \\
\hline 1999 & 5,763 & 70,304 & 9,372 & 7.5 \\
\hline 2000 & 5,926 & 70,500 & 9,563 & 7.4 \\
\hline 2001 & 5,704 & 72,448 & 9,667 & 7.5 \\
\hline 2002 & 5,651 & 75,866 & 10,321 & 7.4 \\
\hline 2003 & 5,849 & 77,757 & 8,881 & 8.8 \\
\hline 2004 & 6,161 & 78,441 & 8,959 & 8.8 \\
\hline 2005 & 6,395 & 78,496 & 9,501 & 8.3 \\
\hline 2006 & 6,649 & 80,344 & 9,852 & 8.2 \\
\hline 2007 & 6,807 & 82,014 & 10,044 & 8.2 \\
\hline 2008 & 6,791 & 83,951 & 9,889 & 8.5 \\
\hline \multicolumn{5}{|c|}{ Average annual percentage change } \\
\hline 1970-2008 & $1.6 \%$ & $3.0 \%$ & $2.4 \%$ & $0.6 \%$ \\
\hline 1998-2008 & $2.3 \%$ & $2.1 \%$ & $0.2 \%$ & $2.0 \%$ \\
\hline
\end{tabular}

Source:

U. S. Department of Transportation, Federal Highway Administration, Highway Statistics 2008, Washington, DC, 2009, Table VM1 and annual. (Additional resources: www.fhwa.dot.gov)

Note: Highway Statistics 1999 data were not used. 
Combination trucks include all trucks designed to be used in combination with one or more trailers. The average vehicle travel of these trucks (on a per truck basis) far surpasses the travel of other trucks due to long-haul freight movement.

Table 5.2

Summary Statistics for Combination Trucks, 1970-2008

\begin{tabular}{|c|c|c|c|c|}
\hline Year & $\begin{array}{c}\text { Registrations } \\
\text { (thousands) }\end{array}$ & $\begin{array}{l}\text { Vehicle travel }^{\mathrm{a}} \\
\text { (million miles) }\end{array}$ & $\begin{array}{c}\text { Fuel use } \\
\text { (million gallons) }\end{array}$ & $\begin{array}{c}\text { Fuel economy } \\
\text { (miles per gallon) }\end{array}$ \\
\hline 1970 & 905 & 35,134 & 7,348 & 4.8 \\
\hline 1975 & 1,131 & 46,724 & 9,177 & 5.1 \\
\hline 1980 & 1,417 & 68,678 & 13,037 & 5.3 \\
\hline 1981 & 1,261 & 69,134 & 13,509 & 5.1 \\
\hline 1982 & 1,265 & 70,765 & 13,583 & 5.2 \\
\hline 1983 & 1,304 & 73,586 & 13,796 & 5.3 \\
\hline 1984 & 1,340 & 77,377 & 14,188 & 5.5 \\
\hline 1985 & 1,403 & 78,063 & 14,005 & 5.6 \\
\hline 1986 & 1,408 & 81,038 & 14,475 & 5.6 \\
\hline 1987 & 1,530 & 85,495 & 14,990 & 5.7 \\
\hline 1988 & 1,667 & 88,551 & 15,224 & 5.8 \\
\hline 1989 & 1,707 & 91,879 & 15,733 & 5.8 \\
\hline 1990 & 1,709 & 94,341 & 16,133 & 5.8 \\
\hline 1991 & 1,691 & 96,645 & 16,809 & 5.7 \\
\hline 1992 & 1,675 & 99,510 & 17,216 & 5.8 \\
\hline 1993 & 1,680 & 103,116 & 17,748 & 5.8 \\
\hline 1994 & 1,681 & 108,932 & 18,653 & 5.8 \\
\hline 1995 & 1,696 & 115,451 & 19,777 & 5.8 \\
\hline 1996 & 1,747 & 118,899 & 20,192 & 5.9 \\
\hline 1997 & 1,790 & 124,584 & 20,302 & 6.1 \\
\hline 1998 & 1,831 & 128,159 & 21,100 & 6.1 \\
\hline 1999 & 2,029 & 132,384 & 24,537 & 5.4 \\
\hline 2000 & 2,097 & 135,020 & 25,666 & 5.3 \\
\hline 2001 & 2,154 & 136,584 & 25,512 & 5.4 \\
\hline 2002 & 2,277 & 138,737 & 26,480 & 5.2 \\
\hline 2003 & 1,908 & 140,160 & 23,815 & 5.9 \\
\hline 2004 & 2,010 & 142,370 & 24,191 & 5.9 \\
\hline 2005 & 2,087 & 144,028 & 27,689 & 5.2 \\
\hline 2006 & 2,170 & 142,169 & 28,107 & 5.1 \\
\hline 2007 & 2,221 & 145,046 & 28,545 & 5.1 \\
\hline 2008 & 2,216 & 143,507 & 26,814 & 5.4 \\
\hline \multicolumn{5}{|c|}{ Average annual percentage change } \\
\hline 1970-2008 & $2.4 \%$ & $3.8 \%$ & $3.5 \%$ & $0.3 \%$ \\
\hline 1998-2008 & $1.9 \%$ & $1.1 \%$ & $2.4 \%$ & $-1.2 \%$ \\
\hline
\end{tabular}

Source:

U. S. Department of Transportation, Federal Highway Administration, Highway Statistics 2008, Washington, DC, 2009, Table VM1 and annual. (Additional resources: www.fhwa.dot.gov)

Note: Highway Statistics 1999 data were not used.

a The Federal Highway Administration changed the combination truck travel methodology in 1993. 
Truck sales in 2009 continued to decline in every class. Trucks under 10,000 lbs. continue to dominate truck sales.

Table 5.3

New Retail Truck Sales by Gross Vehicle Weight, 1970-2009 (thousands)

\begin{tabular}{|c|c|c|c|c|c|c|c|c|c|}
\hline $\begin{array}{l}\text { Calendar } \\
\text { year }\end{array}$ & $\begin{array}{c}\text { Class } 1 \\
6,000 \text { lbs. } \\
\text { or less }\end{array}$ & $\begin{array}{c}\text { Class } 2 \\
6,001- \\
10,000 \mathrm{lbs} .\end{array}$ & $\begin{array}{c}\text { Class } 3 \\
10,001- \\
14,000 \mathrm{lbs} .\end{array}$ & $\begin{array}{c}\text { Class } 4 \\
14,001- \\
16,000 \text { lbs. }\end{array}$ & $\begin{array}{c}\text { Class 5 } \\
16,001- \\
19,500 \mathrm{lbs} .\end{array}$ & $\begin{array}{c}\text { Class 6 } \\
19,501- \\
26,000 \mathrm{lbs} .\end{array}$ & $\begin{array}{c}\text { Class } 7 \\
26,001- \\
33,000 \mathrm{lbs} .\end{array}$ & $\begin{array}{c}\text { Class } 8 \\
33,001 \text { lbs. } \\
\text { and over }\end{array}$ & Total \\
\hline \multicolumn{10}{|c|}{ Domestic sales (import data are not available) } \\
\hline $1970^{b}$ & 1,049 & 408 & 6 & 12 & 58 & 133 & 36 & 89 & 1,791 \\
\hline 1975 & 1,101 & 952 & 23 & 1 & 9 & 159 & 23 & 83 & 2,351 \\
\hline 1976 & 1,318 & 1,401 & 43 & $\mathrm{c}^{-}$ & 9 & 153 & 22 & 97 & 3,043 \\
\hline 1977 & 1,306 & 1,803 & 36 & 3 & 5 & 163 & 28 & 141 & 3,485 \\
\hline 1978 & 1,334 & 2,140 & 73 & 6 & 3 & 156 & 41 & 162 & 3,915 \\
\hline 1979 & 1,271 & 1,574 & 15 & 3 & 3 & 146 & 50 & 174 & 3,236 \\
\hline 1980 & 985 & 975 & 4 & c & 2 & 90 & 58 & 117 & 2,231 \\
\hline 1981 & 896 & 850 & 1 & c & 2 & 72 & 51 & 100 & 1,972 \\
\hline 1982 & 1,102 & 961 & 1 & $\mathrm{c}$ & 1 & 44 & 62 & 76 & 2,248 \\
\hline 1983 & 1,314 & 1,207 & c & c & 1 & 47 & 59 & 82 & 2,710 \\
\hline 1984 & 2,031 & 1,224 & 6 & c & 5 & 55 & 78 & 138 & 3,538 \\
\hline 1985 & 2,408 & 1,280 & 11 & c & 5 & 48 & 97 & 134 & 3,983 \\
\hline \multicolumn{10}{|c|}{ Domestic and import sales } \\
\hline 1986 & 3,380 & 1,214 & 12 & c & 6 & 45 & 101 & 113 & 4,870 \\
\hline 1987 & 3,435 & 1,175 & 14 & 2 & 8 & 44 & 103 & 131 & 4,912 \\
\hline 1988 & 3,467 & 1,333 & 14 & 21 & 8 & 54 & 103 & 148 & 5,149 \\
\hline 1989 & 3,313 & 1,297 & 19 & 27 & 7 & 39 & 93 & 145 & 4,942 \\
\hline 1990 & 3,451 & 1,097 & 21 & 27 & 5 & 38 & 85 & 121 & 4,846 \\
\hline 1991 & 3,246 & 876 & 21 & 24 & 3 & 22 & 73 & 99 & 4,365 \\
\hline 1992 & 3,608 & 1,021 & 26 & 26 & 4 & 28 & 73 & 119 & 4,903 \\
\hline 1993 & 4,119 & 1,232 & 27 & 33 & 4 & 27 & 81 & 158 & 5,681 \\
\hline 1994 & 4,527 & 1,506 & 35 & 44 & 4 & 20 & 98 & 186 & 6,421 \\
\hline 1995 & 4,422 & 1,631 & 40 & 53 & 4 & 23 & 107 & 201 & 6,481 \\
\hline 1996 & 4,829 & 1,690 & 52 & 59 & 7 & 19 & 104 & 170 & 6,930 \\
\hline 1997 & 5,085 & 1,712 & 53 & 57 & 9 & 18 & 114 & 179 & 7,226 \\
\hline 1998 & 5,263 & 2,036 & 102 & 43 & 25 & 32 & 115 & 209 & 7,826 \\
\hline 1999 & 5,707 & 2,366 & 122 & 49 & 30 & 48 & 130 & 262 & 8,716 \\
\hline 2000 & 5,965 & 2,421 & 117 & 47 & 29 & 51 & 123 & 212 & 8,965 \\
\hline 2001 & 6,073 & 2,525 & 102 & 52 & 24 & 42 & 92 & 140 & 9,050 \\
\hline 2002 & 6,068 & 2,565 & 80 & 38 & 24 & 45 & 69 & 146 & 9,035 \\
\hline 2003 & 6,267 & 2,671 & 91 & 40 & 29 & 51 & 67 & 142 & 9,357 \\
\hline 2004 & 6,458 & 2,796 & 107 & 47 & 36 & 70 & 75 & 203 & 9,793 \\
\hline 2005 & 6,586 & 2,528 & 167 & 49 & 46 & 60 & 89 & 253 & 9,777 \\
\hline 2006 & 6,136 & 2,438 & 150 & 50 & 49 & 70 & 91 & 284 & 9,268 \\
\hline 2007 & 5,682 & 2,623 & 166 & 51 & 45 & 54 & 70 & 151 & 8,842 \\
\hline 2008 & 4,358 & 1,888 & 135 & 36 & 40 & 39 & 49 & 133 & 6,680 \\
\hline 2009 & 3,528 & 1,306 & 112 & 20 & 24 & 22 & 39 & 95 & 5,145 \\
\hline \multicolumn{10}{|c|}{ Average annual percentage change } \\
\hline 1970-1985 & $5.7 \%$ & $7.9 \%$ & $4.1 \%$ & - & $-15.1 \%$ & $-6.6 \%$ & $6.8 \%$ & $2.8 \%$ & $5.5 \%$ \\
\hline 1986-2009 & $0.2 \%$ & $0.3 \%$ & $10.2 \%$ & - & $6.2 \%$ & $-3.1 \%$ & $-4.1 \%$ & $-0.8 \%$ & $0.2 \%$ \\
\hline 1999-2009 & $-4.7 \%$ & $-5.8 \%$ & $-0.9 \%$ & $-8.6 \%$ & $-2.2 \%$ & $-7.5 \%$ & $-11.3 \%$ & $9.6 \%$ & $-5.1 \%$ \\
\hline
\end{tabular}

\section{Source:}

Ward's Communication's, Motor Vehicle Facts and Figures 2009, Southfield, MI, 2009, p. 26, and annual; 2009:

Ward's Communications, www.wardsauto.com. (Additional resources: www.wardsauto.com)

\footnotetext{
${ }^{\text {a }}$ Sales include domestic-sponsored imports.

${ }^{\mathrm{b}}$ Data for 1970 is based on new truck registrations.

${ }^{\mathrm{c}}$ Data are not available.
} 


\section{Vehicle Inventory and Use Survey}

The Vehicle Inventory and Use Survey (VIUS), which was formerly the Truck Inventory and Use Survey (TIUS), provides data on the physical and operational characteristics of the Nation's truck population. It is based on a probability sample of private and commercial trucks registered (or licensed) in each state. In 1997, the survey was changed to the Vehicle Inventory and Use Survey due to future possibilities of including additional vehicle types. The 2002 VIUS, however, only includes trucks. Copies of the 2002 VIUS report or CD may be obtained by contacting the U.S. Bureau of the Census, Transportation Characteristics Surveys Branch (301) 457-2797. Internet site:

www. census.gov/svsd/www/tiusview.html

Since 1987, the survey has included minivans, vans, station wagons on truck chassis, and sport utility vehicles in addition to the bigger trucks. The 1977 and 1982 surveys did not include those vehicle types. The estimated number of trucks that were within the scope of the 2002 VIUS and registered in the United States as of July 1, 2002, was 85.2 million. These trucks were estimated to have been driven a total of 1,115 billion miles during 2002, an increase of $6.8 \%$ from 1997 . The average annual miles traveled per truck was estimated at 13,100 miles.

In the 2002 VIUS, there are several ways to classify a truck by weight. The survey respondent was asked the average weight of the vehicle or vehicle-trailer combination when carrying a typical payload; the empty weight (truck minus cargo) of the vehicle as it was usually operated; and the maximum gross weight at which the vehicle or vehicle-trailer combination was operated. The Census Bureau also collected information on the Gross Vehicle Weight Class of the vehicles (decoded from the vehicle identification number) and the registered weight of the vehicles from the State registration files. Some of these weights are only provided in categories, while others are exact weights. Since all these weights could be quite different for a single truck, the tabulations by weight can be quite confusing. In the tables presented here, the Gross Vehicle Weight Class was used.

The Census Bureau has discontinued the Vehicle Inventory and Use Survey; it was not conducted in 2007. The 2002 data remain the latest available. 
Table 5.4

Truck Statistics by Gross Vehicle Weight Class, 2002

\begin{tabular}{|c|c|c|c|c|c|c|}
\hline \multicolumn{2}{|c|}{$\begin{array}{c}\text { Manufacturer's gross vehicle } \\
\text { weight class }\end{array}$} & \multirow{2}{*}{$\begin{array}{c}\begin{array}{c}\text { Number of } \\
\text { trucks }\end{array} \\
51,941,389\end{array}$} & \multirow{2}{*}{$\begin{array}{r}\begin{array}{c}\text { Percentage } \\
\text { of trucks }\end{array} \\
61.0 \%\end{array}$} & \multirow{2}{*}{$\begin{array}{c}\text { Average } \\
\text { annual miles } \\
\text { per truck }\end{array}$} & \multirow{2}{*}{$\begin{array}{r}\begin{array}{r}\text { Harmonic } \\
\text { mean fuel } \\
\text { economy }\end{array} \\
17.6\end{array}$} & \multirow{2}{*}{$\begin{array}{r}\begin{array}{r}\text { Percentage } \\
\text { of fuel use }\end{array} \\
42.7 \%\end{array}$} \\
\hline 1) & $6,000 \mathrm{lbs}$ and less & & & & & \\
\hline \multirow[t]{2}{*}{ 2) } & $6,001-10,000 \mathrm{lbs}$ & $28,041,234$ & $32.9 \%$ & 12,684 & 14.3 & $30.5 \%$ \\
\hline & Light truck subtotal & $79,982,623$ & $93.9 \%$ & 12,163 & 16.2 & $73.2 \%$ \\
\hline 3) & $10,001-14,000 \mathrm{lbs}$ & 691,342 & $0.8 \%$ & 14,094 & 10.5 & $1.1 \%$ \\
\hline 4) & $14,001-16,000 \mathrm{lbs}$ & 290,980 & $0.3 \%$ & 15,441 & 8.5 & $0.5 \%$ \\
\hline 5) & $16,001-19,500 \mathrm{lbs}$ & 166,472 & $0.2 \%$ & 11,645 & 7.9 & $0.3 \%$ \\
\hline \multirow[t]{2}{*}{ 6) } & $19,501-26,000 \mathrm{lbs}$ & $1,709,574$ & $2.0 \%$ & 12,671 & 7.0 & $3.2 \%$ \\
\hline & Medium truck subtotal & $2,858,368$ & $3.4 \%$ & 13,237 & 8.0 & $5.2 \%$ \\
\hline 7) & $26,001-33,000 \mathrm{lbs}$ & 179,790 & $0.2 \%$ & 30,708 & 6.4 & $0.9 \%$ \\
\hline \multirow[t]{2}{*}{ 8) } & $33,001 \mathrm{lbs}$ and up & $2,153,996$ & $2.5 \%$ & 45,739 & 5.7 & $20.7 \%$ \\
\hline & Heavy truck subtotal & $2,333,786$ & $2.7 \%$ & 44,581 & 5.8 & $21.6 \%$ \\
\hline \multicolumn{2}{|c|}{ Total } & $85,174,776$ & $100.0 \%$ & 13,088 & 13.5 & $100.0 \%$ \\
\hline
\end{tabular}

Source:

U.S. Department of Commerce, Bureau of the Census, 2002 Vehicle Inventory and Use Survey, Microdata File on CD, 2005. (Additional resources: www.census.gov/svsd/www.tiusview.html)

Table 5.5

Truck Harmonic Mean Fuel Economy by Size Class, 1992, 1997, and 2002 (miles per gallon)

\begin{tabular}{lrrr}
\hline $\begin{array}{c}\text { Manufacturer's gross vehicle } \\
\text { weight class }\end{array}$ & $\begin{array}{c}1992 \\
\text { TIUS }\end{array}$ & 1997 & VIUS \\
\hline 1) 6,000 lbs and less & 17.2 & 17.1 & VIUS \\
2) 6,001-10,000 lbs & 13.0 & 13.6 & 17.6 \\
Light truck subtotal & 15.7 & 15.8 & 14.3 \\
3) 10,000-14,000 lbs & 8.8 & 9.4 & 10.2 \\
4) $14,001-16,000 \mathrm{lbs}$ & 8.8 & 9.3 & 8.5 \\
5) 16,001-19,500 lbs & 7.4 & 8.7 & 7.9 \\
6) 19,501-26,000 lbs & 6.9 & 7.3 & 7.0 \\
Medium truck subtotal & 7.3 & 8.6 & 8.0 \\
7) 26,001-33,000 lbs & 6.5 & 6.4 & 6.4 \\
8) 33,001 lbs and over & 5.5 & 5.7 & 5.7 \\
Large truck subtotal & 5.6 & 6.1 & 5.8 \\
\hline
\end{tabular}

Sources:

Estimates are based on data provided on the following public use files: U.S. Department of Commerce, Bureau of the Census, Census of Transportation, Washington, DC, 1992 Truck Inventory and Use Survey, 1995; 1997 Vehicle Inventory and Use Survey, 2000, and 2002 Vehicle Inventory and Use Survey, 2005. (Additional resources: www.census.gov/svsd/www/tiusview.html)

Note: Based on average fuel economy as reported by respondent. 
As expected, most light trucks travel within 50 miles of their home base and refuel at public stations. About sixty percent of heavy trucks travel over 50 miles from their home base and $36 \%$ of them refuel at central companyowned refueling stations.

Table 5.6

Truck Statistics by Size, 2002

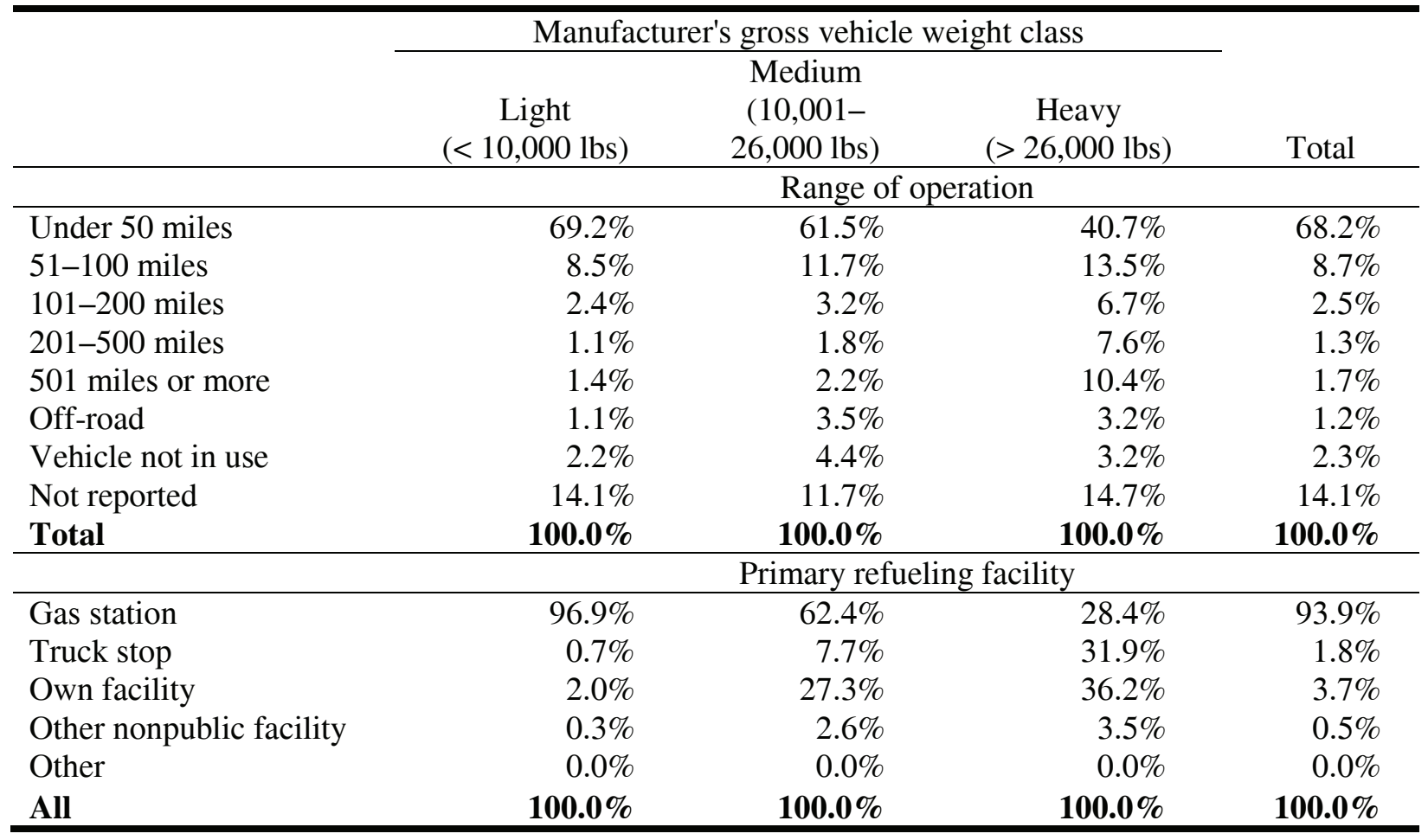

Source:

U.S. Department of Commerce, Bureau of the Census, 2002 Vehicle Inventory and Use Survey, Microdata. File on CD, 2005. (Additional resources: www.census.gov/svsd/www/tiusview.html) 
More medium truck owners listed construction as the truck's major use than any other major use category.

Construction was the second highest major use for light trucks and heavy trucks.

Table 5.7

Percentage of Trucks by Size Ranked by Major Use, 2002

\begin{tabular}{|c|c|c|c|}
\hline Rank & $\begin{array}{c}\text { Light } \\
(<10,000 \mathrm{lbs} \\
\text { average weight })\end{array}$ & $\begin{array}{c}\text { Medium } \\
(10,001-26,000 \mathrm{lbs} \\
\text { average weight) }\end{array}$ & $\begin{array}{c}\text { Heavy } \\
(>26,000 \text { lbs average } \\
\text { weight })\end{array}$ \\
\hline 1 & $\begin{array}{c}\text { Personal } \\
81.5 \%\end{array}$ & $\begin{array}{c}\text { Construction } \\
18.4 \%\end{array}$ & $\begin{array}{c}\text { For hire } \\
30.1 \%\end{array}$ \\
\hline 2 & $\begin{array}{c}\text { Construction } \\
4.6 \%\end{array}$ & $\begin{array}{c}\text { Agriculture } \\
16.2 \%\end{array}$ & $\begin{array}{c}\text { Construction } \\
15.9 \%\end{array}$ \\
\hline 3 & $\begin{array}{c}\text { Other services }^{\mathrm{a}} \\
2.5 \%\end{array}$ & $\begin{array}{c}\text { For hire } \\
9.6 \%\end{array}$ & $\begin{array}{c}\text { Agriculture } \\
12.2 \%\end{array}$ \\
\hline 4 & $\begin{array}{c}\text { Not in use } \\
2.2 \%\end{array}$ & $\begin{array}{l}\text { Retail } \\
7.1 \%\end{array}$ & $\begin{array}{l}\text { Retail } \\
5.4 \%\end{array}$ \\
\hline 5 & $\begin{array}{c}\text { Agriculture } \\
1.9 \%\end{array}$ & $\begin{array}{c}\text { Not in use } \\
6.4 \%\end{array}$ & $\begin{array}{c}\text { Not in use } \\
5.1 \%\end{array}$ \\
\hline 6 & $\begin{array}{c}\text { Retail } \\
1.5 \%\end{array}$ & $\begin{array}{c}\text { Leasing } \\
6.2 \%\end{array}$ & $\begin{array}{c}\text { Waste management } \\
5.0 \%\end{array}$ \\
\hline 7 & $\begin{array}{c}\text { Unknown } \\
1.3 \%\end{array}$ & $\begin{array}{c}\text { Wholesale } \\
5.5 \%\end{array}$ & $\begin{array}{c}\text { Manufacturing } \\
4.9 \%\end{array}$ \\
\hline 8 & $\begin{array}{c}\text { Leasing } \\
0.7 \%\end{array}$ & $\begin{array}{c}\text { Waste management } \\
5.4 \%\end{array}$ & $\begin{array}{c}\text { Wholesale } \\
4.8 \%\end{array}$ \\
\hline 9 & $\begin{array}{c}\text { Manufacturing } \\
0.7 \%\end{array}$ & $\begin{array}{c}\text { Utilities } \\
5.0 \%\end{array}$ & $\begin{array}{c}\text { Leasing } \\
4.6 \%\end{array}$ \\
\hline 10 & $\begin{array}{l}\text { Utilities } \\
0.6 \%\end{array}$ & $\begin{array}{c}\text { Personal } \\
4.8 \%\end{array}$ & $\begin{array}{c}\text { Unknown } \\
3.2 \%\end{array}$ \\
\hline 11 & $\begin{array}{c}\text { Waste management } \\
0.6 \%\end{array}$ & $\begin{array}{c}\text { Unknown } \\
4.4 \%\end{array}$ & $\begin{array}{c}\text { Personal } \\
2.5 \%\end{array}$ \\
\hline 12 & $\begin{array}{c}\text { Wholesale } \\
0.6 \%\end{array}$ & $\begin{array}{c}\text { Manufacturing } \\
3.3 \%\end{array}$ & $\begin{array}{l}\text { Mining } \\
2.4 \%\end{array}$ \\
\hline 13 & $\begin{array}{c}\text { Information services } \\
0.4 \%\end{array}$ & $\begin{array}{c}\text { Other services }^{\mathrm{a}} \\
3.2 \%\end{array}$ & $\begin{array}{c}\text { Other services }^{\mathrm{a}} \\
1.3 \%\end{array}$ \\
\hline 14 & $\begin{array}{c}\text { For hire } \\
0.4 \%\end{array}$ & $\begin{array}{c}\text { Food services } \\
1.6 \%\end{array}$ & $\begin{array}{c}\text { Utilities } \\
1.1 \%\end{array}$ \\
\hline 15 & $\begin{array}{c}\text { Food services } \\
0.3 \%\end{array}$ & $\begin{array}{c}\text { Information services } \\
1.3 \%\end{array}$ & $\begin{array}{c}\text { Food services } \\
1.1 \%\end{array}$ \\
\hline 16 & $\begin{array}{l}\text { Arts } \\
0.2 \%\end{array}$ & $\begin{array}{c}\text { Mining } \\
1.1 \%\end{array}$ & $\begin{array}{l}\text { Arts } \\
0.3 \%\end{array}$ \\
\hline 17 & $\begin{array}{c}\text { Mining } \\
0.1 \%\end{array}$ & $\begin{array}{l}\text { Arts } \\
0.5 \%\end{array}$ & $\begin{array}{c}\text { Information services } \\
0.1 \%\end{array}$ \\
\hline
\end{tabular}

Source:

U.S. Department of Commerce, Bureau of the Census, 2002 Vehicle Inventory and Use Survey, Micro data File on CD, 2005. (Additional resources: www.census.gov/svsd/www/tiusview.html)

${ }^{\mathrm{a}}$ Business and personal services. 
Nearly half of trucks in fleets of 11-20 and 21-50 vehicles use company-owned facilities. Most trucks in smaller

fleets use public gas stations for fueling.

Table 5.8

Percentage of Trucks by Fleet Size and Primary Fueling Facility, 2002

\begin{tabular}{|c|c|c|c|c|c|}
\hline \multirow[b]{2}{*}{ Truck fleet size } & \multicolumn{4}{|c|}{ Primary refueling facility } & \multirow[b]{2}{*}{ Total } \\
\hline & Gas station & Truck stop & Own facility & Other's facility & \\
\hline $1-5$ & $73.8 \%$ & $6.1 \%$ & $18.2 \%$ & $1.9 \%$ & $100.0 \%$ \\
\hline $6-10$ & $55.3 \%$ & $5.7 \%$ & $35.5 \%$ & $3.4 \%$ & $100.0 \%$ \\
\hline $11-20$ & $41.1 \%$ & $5.1 \%$ & $48.9 \%$ & $4.9 \%$ & $100.0 \%$ \\
\hline $21-50$ & $42.9 \%$ & $3.7 \%$ & $49.8 \%$ & $3.6 \%$ & $100.0 \%$ \\
\hline 51 or more & $48.3 \%$ & $6.3 \%$ & $44.4 \%$ & $1.0 \%$ & $100.0 \%$ \\
\hline $\begin{array}{l}\text { Fleets of } 6 \text { or more } \\
\text { vehicles }\end{array}$ & $47.6 \%$ & $5.2 \%$ & $43.9 \%$ & $3.4 \%$ & $100.0 \%$ \\
\hline No fleet & $96.4 \%$ & $1.6 \%$ & $1.7 \%$ & $0.3 \%$ & $100.0 \%$ \\
\hline
\end{tabular}

Source:

U.S. Department of Commerce, Bureau of the Census, 2002 Vehicle Inventory and Use Survey, Microdata File on CD, 2005. (Additional resources: www.census.gov/svsd/www/tiusview.html) 
Most trucks are fueled at gas stations but for-hire or warehousing trucks are more often fueled at truck stops. Mining trucks and vehicle leasing or rental trucks fuel at the companies' own facility more than $30 \%$ of the time.

Table 5.9

Share of Trucks by Major Use and Primary Fueling Facility, 2002

\begin{tabular}{|c|c|c|c|c|c|c|}
\hline Major use & $\begin{array}{c}\text { Gas } \\
\text { station }\end{array}$ & $\begin{array}{c}\text { Truck } \\
\text { stop }\end{array}$ & $\begin{array}{c}\text { Own } \\
\text { facility }\end{array}$ & $\begin{array}{l}\text { Others } \\
\text { facility }\end{array}$ & Other & All \\
\hline Personal & $98.6 \%$ & $0.6 \%$ & $0.7 \%$ & $0.1 \%$ & $0.1 \%$ & $100.0 \%$ \\
\hline Other services & $96.0 \%$ & $1.4 \%$ & $1.6 \%$ & $0.9 \%$ & $0.1 \%$ & $100.0 \%$ \\
\hline All & $93.9 \%$ & $1.8 \%$ & $3.7 \%$ & $0.5 \%$ & $0.0 \%$ & $100.0 \%$ \\
\hline Information services & $92.3 \%$ & $0.4 \%$ & $7.2 \%$ & $0.1 \%$ & $0.0 \%$ & $100.0 \%$ \\
\hline Retail trade & $86.6 \%$ & $3.5 \%$ & $8.6 \%$ & $1.2 \%$ & $0.0 \%$ & $100.0 \%$ \\
\hline Construction & $84.7 \%$ & $3.3 \%$ & $9.8 \%$ & $2.2 \%$ & $0.0 \%$ & $100.0 \%$ \\
\hline Accommodation or food services & $82.4 \%$ & $7.5 \%$ & $8.8 \%$ & $1.3 \%$ & $0.0 \%$ & $100.0 \%$ \\
\hline Manufacturing & $81.5 \%$ & $5.1 \%$ & $11.9 \%$ & $1.5 \%$ & $0.0 \%$ & $100.0 \%$ \\
\hline Arts, entertainment, recreation services & $81.1 \%$ & $4.3 \%$ & $14.2 \%$ & $0.3 \%$ & $0.0 \%$ & $100.0 \%$ \\
\hline Waste mgmt, landscaping, admin/support services & $78.2 \%$ & $3.0 \%$ & $17.1 \%$ & $1.6 \%$ & $0.0 \%$ & $100.0 \%$ \\
\hline Wholesale trade & $76.2 \%$ & $6.6 \%$ & $12.0 \%$ & $5.1 \%$ & $0.0 \%$ & $100.0 \%$ \\
\hline Utilities & $72.6 \%$ & $1.8 \%$ & $24.3 \%$ & $1.3 \%$ & $0.0 \%$ & $100.0 \%$ \\
\hline Agriculture, forestry, fishing, hunting & $62.7 \%$ & $6.7 \%$ & $29.4 \%$ & $1.0 \%$ & $0.1 \%$ & $100.0 \%$ \\
\hline Vehicle leasing or rental & $60.2 \%$ & $1.3 \%$ & $31.8 \%$ & $6.8 \%$ & $0.0 \%$ & $100.0 \%$ \\
\hline Mining & $48.7 \%$ & $8.5 \%$ & $34.3 \%$ & $8.5 \%$ & $0.0 \%$ & $100.0 \%$ \\
\hline For-hire or warehousing & $33.3 \%$ & $38.7 \%$ & $25.8 \%$ & $2.3 \%$ & $0.0 \%$ & $100.0 \%$ \\
\hline Overall & $93.90 \%$ & $1.80 \%$ & $3.70 \%$ & $0.50 \%$ & $0 \%$ & $100.00 \%$ \\
\hline
\end{tabular}

Source:

U.S. Department of Commerce, Bureau of the Census, 2002 Vehicle Inventory and Use Survey, Microdata File on CD, 2005. (Additional resources: www.census.gov/svsd/www/tiusview.html) 
The figure below shows the distribution of annual travel the two types of Class 7 and 8 vehicles-combination units (separate tractor and trailer) and single units (tractor and trailer on a single chassis). This information is for vehicles two years old or less and comes from the 2002 VIUS. Combination trucks, dominated by box-type trailers, display the greatest amount of annual travel of all heavy vehicle types, as is evidenced both by the range of annual use which is up to 250,000 miles per year, and the peaking that occurs in the 100,000 to 140,000-mile segments. Most of the single-unit trucks in the survey travel 40,000 miles per year or less.

Figure 5.1. Distribution of Trucks over 26,000 lbs. Less than Two Years Old by Vehicle-Miles Traveled

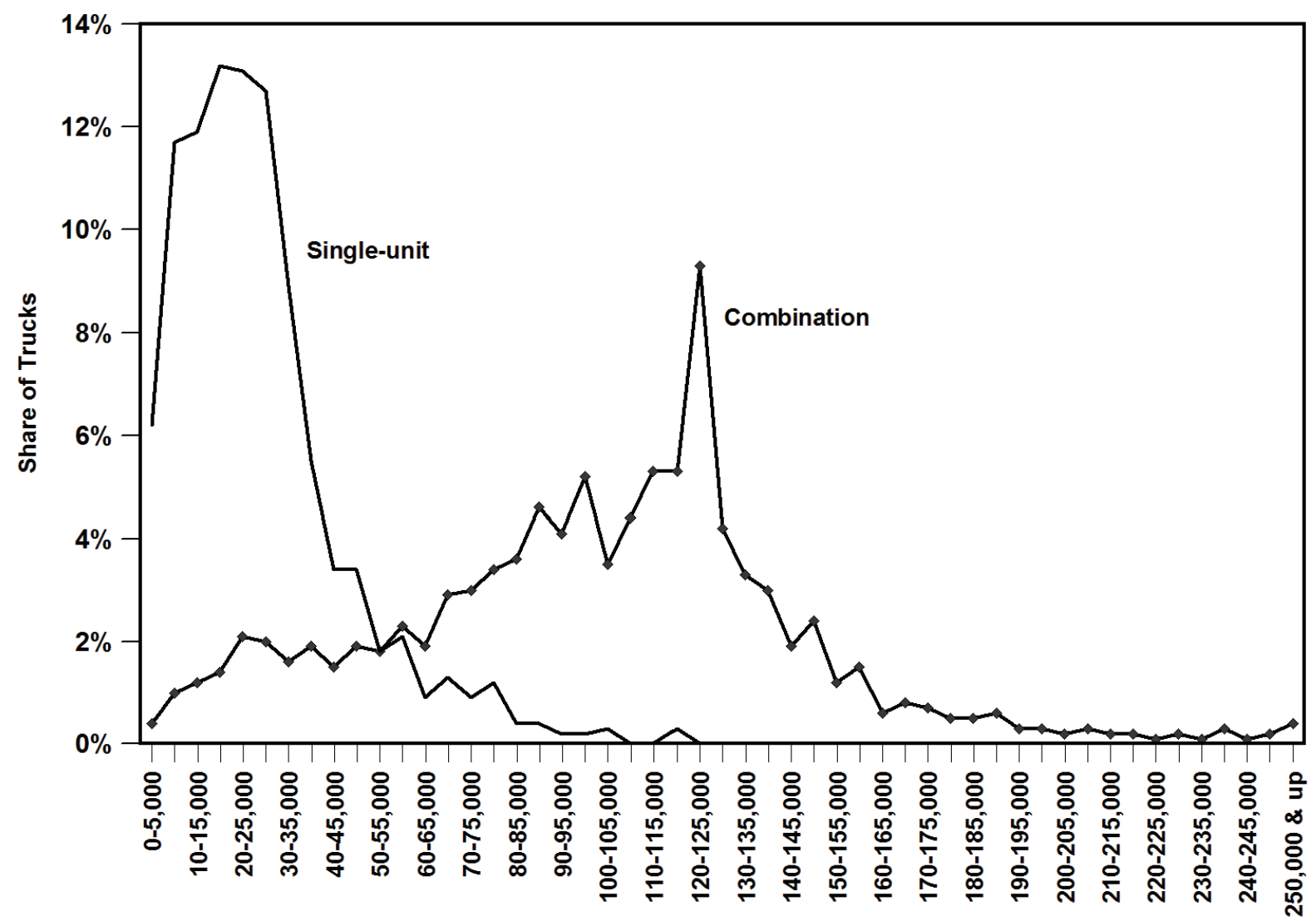

Annual Vehicle-Miles of Travel

\section{Source:}

U.S. Department of Commerce, Bureau of the Census, 2002 Vehicle Inventory and Use Survey, Microdata File on CD, 2005. (Additional resources: www.census.gov/svsd/www/tiusview.html)

Note: Heavy trucks (class $7 \&$ 8) are greater than 26,000 pounds gross vehicle weight based on the manufacturer's rating. 
The latest Vehicle Inventory and Use Survey asked truck owners if the truck had certain features as permanent equipment on the truck. Some of the features asked about were onboard computers, idle-reduction devices, navigational systems, and Internet access. Of the 2.3 million heavy trucks (class 7 \& 8) in the United States, nearly $10 \%$ were equipped with onboard computers that had communication capabilities and another 5\% had onboard computers without communication capabilities. Six percent of heavy trucks were equipped with idle-reducing technology. Navigational systems and Internet access were available in less than one percent of heavy trucks.

Figure 5.2. Share of Heavy Trucks with Selected Electronic Features, 2002

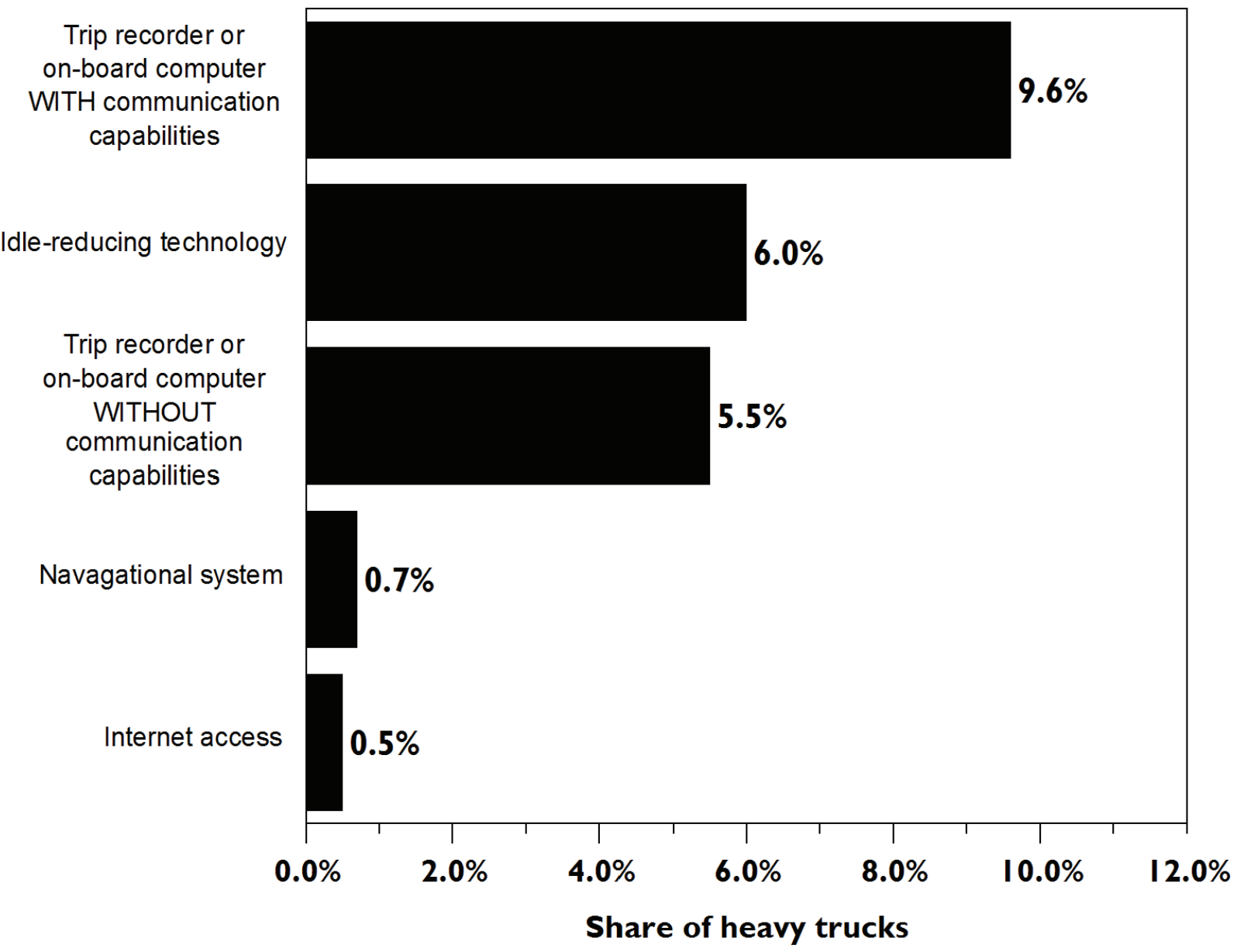

Source:

U.S. Department of Commerce, Bureau of the Census, 2002 Vehicle Inventory and User Survey, Microdata File on CD, 2005.

Note: Heavy trucks (class $7 \& 8$ ) are greater than 26,000 pounds gross vehicle weight based on the manufacturer's rating. 


\section{Fuel Economy Study for Class 8 Trucks}

As part of a long-term study sponsored by the U.S. Department of Energy (DOE) Office of Vehicle Technologies (OVT), the Oak Ridge National Laboratory (ORNL) in conjunction with several industry partners has collected data and information related to heavy-truck operation in real-world highway environments. The primary objective of the project was to collect real-world performance and spatial data for long-haul operations of Class 8 tractor-trailers from a fleet engaged in normal freight operations. Six model year 2005 Class 8 trucks from the selected fleet, which operates within a large area of the country extending from the east coast to Mountain Time Zone and from Canada to the US-Mexican border, were instrumented and 60 channels of data were collected for over a year at a rate of $5 \mathrm{~Hz}$ (or 5 readings per second). Those channels included information such as instantaneous fuel rate, engine speed, gear ratio, vehicle speed, and other information read from the vehicle's databus; weather information (wind speed, precipitation, air temperature, etc.) gathered from an on-board weather station; spatial information (latitude, longitude, altitude) acquired from a GPS (Global Positioning System) device; and instantaneous tractor and trailer weight obtained from devices mounted on the six participating tractors and ten trailers. Three of the six instrumented tractors and five of the ten instrumented trailers were mounted with New Generation Single Wide-Based Tires and the others with regular dual tires. Over the duration of this phase of the project (just over a year) the six tractors traveled nearly 700,000 miles.

To find out more about this project, contact Oscar Franzese, franzeseo@ornl.gov, 865-946-1304. The final report on this project is available on-line at: cta.ornl.gov/cta/Publications/Reports/ORNL_TM_2008-122.pdf. 
The type of terrain a truck is traveling on can cause significant differences in fuel efficiency. This study (see page 5-13 for project description) shows fuel economy on severe upslopes is less than half that on flat terrain. On severe downslopes, the fuel economy was three times higher than on flat terrain.

Table 5.10

Effect of Terrain on Class 8 Truck Fuel Economy

\begin{tabular}{|c|c|c|c|c|c|}
\hline \multirow[b]{2}{*}{ Type of Terrain } & \multicolumn{5}{|c|}{ Average Fuel Efficiency (mpg) } \\
\hline & $\begin{array}{c}\text { Share of Data } \\
\text { Records }\end{array}$ & $\begin{array}{c}\text { All } \\
\text { Trucks }\end{array}$ & $\begin{array}{c}\text { Tractors } \\
\text { with Dual } \\
\text { Tires } \\
\end{array}$ & $\begin{array}{c}\text { Tractors } \\
\text { with Single } \\
\text { (wide) Tires }\end{array}$ & $\begin{array}{c}\text { Difference } \\
\text { between Dual } \\
\text { and Single } \\
\text { Tires (percent) }\end{array}$ \\
\hline Severe upslope $(>4 \%)$ & $0.7 \%$ & 2.90 & 2.86 & 2.94 & $2.91 \%$ \\
\hline Mild upslope (1\% to $4 \%$ ) & $13.2 \%$ & 4.35 & 4.25 & 4.44 & $4.35 \%$ \\
\hline Flat terrain ( $1 \%$ to $1 \%)$ & $72.4 \%$ & 7.33 & 7.08 & 7.58 & $7.13 \%$ \\
\hline Mild downslope (-4\% to $-1 \%)$ & $12.6 \%$ & 15.11 & 14.64 & 15.57 & $6.36 \%$ \\
\hline Severe downslope $(<-4 \%)$ & $1.1 \%$ & 23.5 & 21.82 & 25.3 & $15.97 \%$ \\
\hline
\end{tabular}

Source:

Capps, Gary, Oscar Franzese, Bill Knee, M.B. Lascurain, and Pedro Otaduy. Class-8 Heavy Truck Duty Cycle Project Final Report, ORNL/TM-2008/122, Oak Ridge National Laboratory, Oak Ridge, TN, December 2008. 
Table 5.10 presents a distribution of distance traveled, fuel consumed, and fuel economy by speed and by type of tires for the vehicles participating in the project (see page 5-13 for project description). The speed bins are divided into 5-mile intervals, going from $0+\mathrm{mph}$ (i.e., speed $>0.00 \mathrm{mph}$ ) to $85 \mathrm{mph}$, while the four main columns of Table 5.10 are organized by the type of tires that were mounted on the tractor and trailers. The first row of the table contains information about fuel consumed while the vehicle was idling (i.e., the vehicle was static with the engine on) with the following rows presenting information about the distance traveled, fuel consumed, and fuel economy for each one of the speed intervals. The next-to-the-last row shows the totals for both traveled distances and fuel consumed as well as the overall fuel economy for each tire-combination category. The latter are then used to compute the percentage difference in terms of fuel economy from dual tire tractors and trailers, which is the most common tire setup for large trucks at the present time.

Table 5.11

Fuel Economy for Class 8 Trucks as Function of Speed and Tractor-Trailer Tire Combination

\begin{tabular}{|c|c|c|c|c|c|c|c|c|c|c|c|c|}
\hline \multirow[b]{2}{*}{$\begin{array}{l}\text { Speed } \\
(\mathrm{mph})\end{array}$} & \multicolumn{3}{|c|}{$\begin{array}{c}\text { Dual Tire Tractor - } \\
\text { Dual Tire Trailer }\end{array}$} & \multicolumn{3}{|c|}{$\begin{array}{c}\text { Dual Tire Tractor - } \\
\text { Single (Wide) Tire Trailer }\end{array}$} & \multicolumn{3}{|c|}{$\begin{array}{c}\text { Single (Wide) Tire Tractor - } \\
\text { Dual Tire Trailer }\end{array}$} & \multicolumn{3}{|c|}{$\begin{array}{c}\text { Single (Wide) Tire Tractor - } \\
\text { Single (Wide) Tire Trailer }\end{array}$} \\
\hline & $\begin{array}{c}\text { Distance } \\
\text { Traveled } \\
\text { (miles) }\end{array}$ & $\begin{array}{l}\text { Fuel } \\
\text { Cons. } \\
\text { (gal) }\end{array}$ & $\begin{array}{c}\text { Fuel } \\
\text { Econ. } \\
\text { (MPG) }\end{array}$ & $\begin{array}{c}\text { Distance } \\
\text { Traveled } \\
\text { (miles) }\end{array}$ & $\begin{array}{c}\text { Fuel } \\
\text { Cons. } \\
\text { (gal) }\end{array}$ & $\begin{array}{c}\text { Fuel } \\
\text { Econ. } \\
\text { (MPG) }\end{array}$ & $\begin{array}{c}\text { Distance } \\
\text { Traveled } \\
\text { (miles) }\end{array}$ & $\begin{array}{l}\text { Fuel } \\
\text { Cons. } \\
\text { (gal) }\end{array}$ & $\begin{array}{c}\text { Fuel } \\
\text { Econ. } \\
(\mathrm{MPG})\end{array}$ & $\begin{array}{c}\text { Distance } \\
\text { Traveled } \\
\text { (miles) }\end{array}$ & $\begin{array}{l}\text { Fuel } \\
\text { Cons. } \\
\text { (gal) }\end{array}$ & $\begin{array}{c}\text { Fuel } \\
\text { Econ. } \\
\text { (MPG) }\end{array}$ \\
\hline Idling & N/A & $1,858.5$ & N/A & N/A & 967.9 & N/A & N/A & $1,676.4$ & N/A & N/A & 706.0 & N/A \\
\hline $0+$ to 5 & 281 & 101.8 & 2.76 & 148 & 50.4 & 2.93 & 368.0 & 124.2 & 3.0 & 156 & 52.8 & 2.96 \\
\hline $5+$ to 10 & 674 & 198.8 & 3.39 & 368 & 103.2 & 3.56 & 808.0 & 245.4 & 3.3 & 331 & 98.8 & 3.35 \\
\hline $10+$ to 15 & 723 & 192.0 & 3.77 & 396 & 98.3 & 4.03 & 848.0 & 216.5 & 3.9 & 343 & 87.0 & 3.95 \\
\hline $15+$ to 20 & 744 & 199.1 & 3.73 & 404 & 100.9 & 4.00 & 882.0 & 221.6 & 4.0 & 361 & 90.5 & 3.98 \\
\hline $20+$ to 25 & 938 & 228.4 & 4.11 & 489 & 113.6 & 4.31 & $1,111.0$ & 244.2 & 4.6 & 462 & 101.1 & 4.57 \\
\hline $25+$ to 30 & 1,178 & 266.9 & 4.41 & 609 & 131.5 & 4.63 & $1,420.0$ & 286.9 & 5.0 & 580 & 117.6 & 4.93 \\
\hline $30+$ to 35 & 1,481 & 336.8 & 4.40 & 753 & 154.2 & 4.88 & $1,774.0$ & 341.1 & 5.2 & 708 & 141.1 & 5.02 \\
\hline $35+$ to 40 & 1,917 & 403.5 & 4.75 & 1,000 & 193.6 & 5.17 & $2,284.0$ & 433.6 & 5.3 & 941 & 184.3 & 5.10 \\
\hline $40+$ to 45 & 2,955 & 584.1 & 5.06 & 1,543 & 285.9 & 5.40 & $3,380.0$ & 603.6 & 5.6 & 1,350 & 254.4 & 5.31 \\
\hline $45+$ to 50 & 4,935 & 907.9 & 5.43 & 2,573 & 447.7 & 5.75 & $5,410.0$ & 872.8 & 6.2 & 2,177 & 360.4 & 6.04 \\
\hline $50+$ to 55 & 9,397 & $1,629.8$ & 5.77 & 4,962 & 811.5 & 6.11 & $10,046.0$ & $1,622.7$ & 6.2 & 3,877 & 625.5 & 6.20 \\
\hline $55+$ to 60 & 20,656 & $3,297.2$ & 6.26 & 11,707 & $1,721.9$ & 6.80 & $22,373.0$ & $3,257.8$ & 6.9 & 8,710 & $1,246.9$ & 6.99 \\
\hline $60+$ to 65 & 38,964 & $5,879.6$ & 6.63 & 21,472 & $2,980.8$ & 7.20 & $34,517.0$ & $4,840.0$ & 7.1 & 14,944 & $2,049.4$ & 7.29 \\
\hline \multicolumn{13}{|c|}{ NOT ADJUSTED FOR TERRAIN: See note below. } \\
\hline $65+$ to 70 & 58,304 & $8,313.2$ & 7.01 & 27,931 & $3,652.2$ & 7.65 & $65,063.0$ & $9,256.4$ & 7.0 & 27,144 & $3,880.1$ & 7.00 \\
\hline $70+$ to 75 & 56,378 & $7,483.2$ & 7.53 & 21,751 & $2,745.5$ & 7.92 & $66,882.0$ & $8,435.6$ & 7.9 & 32,887 & $4,056.1$ & 8.11 \\
\hline $75+$ to 85 & 7,849 & 808.2 & 9.71 & 3,610 & 403.2 & 8.95 & $11,513.0$ & 911.1 & 12.6 & 6,817 & 512.2 & 13.31 \\
\hline Total $^{\mathrm{a}}$ & 207,374 & $30,831.0$ & 6.73 & 99,714 & $13,994.0$ & 7.13 & $228,680.0$ & $31,913.0$ & 7.2 & 101,790 & $13,858.0$ & 7.35 \\
\hline $\begin{array}{l}\text { Percent } \\
\text { increase in } \\
\text { fuel } \\
\text { economy } \\
\text { from dual } \\
\text { tire } \\
\text { trac/trail }\end{array}$ & & & $0.00 \%$ & & & $5.93 \%$ & & & $6.53 \%$ & & & $9.20 \%$ \\
\hline
\end{tabular}

Source:

Capps, Gary, Oscar Franzese, Bill Knee, M.B. Lascurain, and Pedro Otaduy. Class-8 Heavy Truck Duty Cycle Project Final Report, ORNL/TM-2008/122, Oak Ridge National Laboratory, Oak Ridge, TN, December 2008.

Note: These data were not adjusted to account for the effects of terrain. The increase in fuel economy for speeds above $70 \mathrm{mph}$ is likely due to the vehicle achieving high speeds while traveling down slope. Therefore, this increase in fuel economy is not expected to be characteristic of all travel at these higher speeds.

a Total Fuel Consumed does not include fuel consumed while idling. 
The fuel economy information presented in Table 5.10 is on the upper limits of today's large-truck fleets and is mostly a result of driver training and the extensive vehicle maintenance (including constant tire pressure) to which the fleet company participating in this project adheres. Nevertheless, the results of this extensive test indicate that there are substantial gains in terms of fuel economy for large trucks when single (wide) tires are used in combination with dual tires or alone (best case). Figure 5.3 shows the information from Table 5.10 in a graphical form (bars) and also displays for each speed bin the percentage of the total distance that is traveled at that speed (line). It is possible to observe that above $80 \%$ of the distance traveled by long-haul Class 8 trucks is done at speeds above $55 \mathrm{mph}$. Therefore, any gains in fuel economies at these speeds derived from a given tire combination would have a very large impact on the overall fuel economy of these type of trucks. Figure 5.3 shows that, except for the $D-S$ combination within the $65+$ to $70 \mathrm{mph}$, the combinations with all single (wide) tires perform better and, therefore, obtain the largest overall fuel economy.

Figure 5.3. Class 8 Truck Fuel Economy as a Function of Speed and Tractor-Trailer Tire Combination and Percentage of Total Distance Traveled as a Function of Speed

NOT ADJUSTED FOR TERRAIN: See note below.

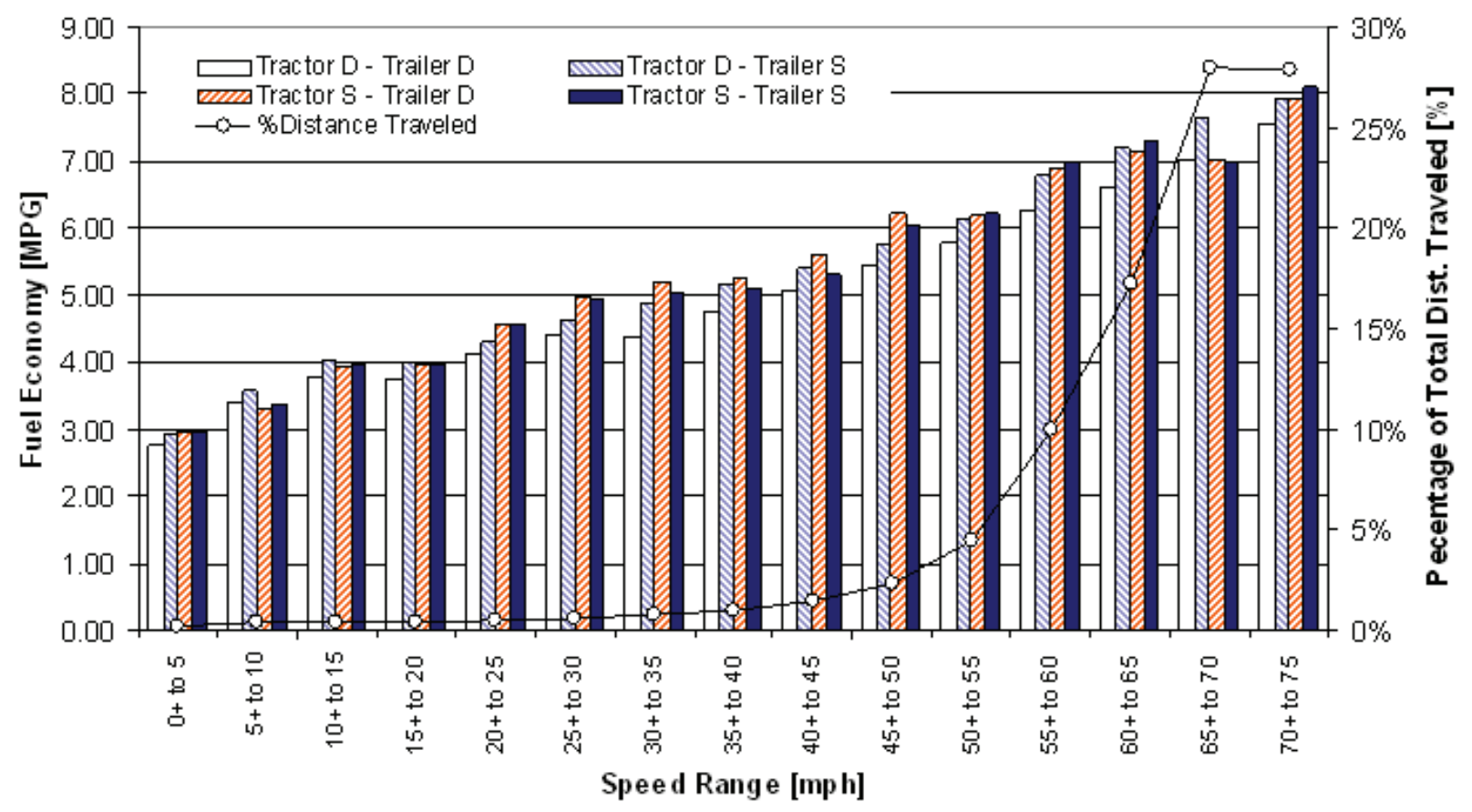

Source:

Capps, Gary, Oscar Franzese, Bill Knee, M.B. Lascurain, and Pedro Otaduy. Class-8 Heavy Truck Duty Cycle Project Final Report, ORNL/TM-2008/122, Oak Ridge National Laboratory, Oak Ridge, TN, December 2008.

Note: $\mathrm{D}=$ Dual tire. $\mathrm{S}=$ Single (wide) tire.

These data were not adjusted to account for the effects of terrain. The increase in fuel economy for speeds above 70 mph is likely due to the vehicle achieving high speeds while traveling down slope. Therefore, this increase in fuel economy is not expected to be characteristic of all travel at these higher speeds. 
This graph presents for each one of the four tire-combination categories the percent of total fuel that is consumed when traveling at different speeds (bars) as well as the average percent of fuel consumed for each speed bin (line). As opposed to Table 5.10, the total fuel consumed on this graph includes the fuel consumed while idling.

Figure 5.4. Class 8 Truck Percent of Total Fuel Consumed as a Function of Speed and Tractor-Trailer Tire Combination

\section{NOT ADJUSTED FOR TERRAIN: See note below.}

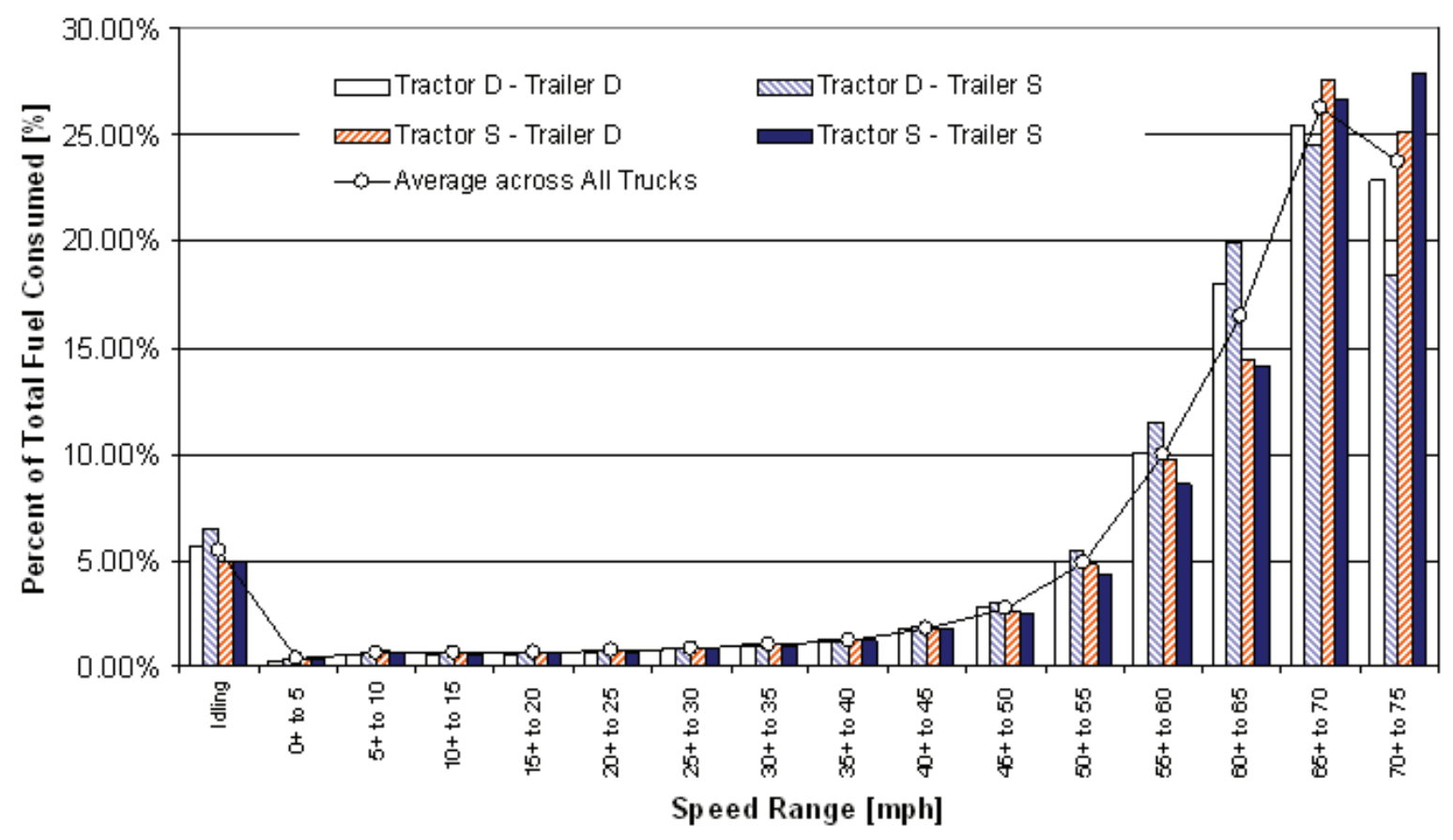

Source:

Capps, Gary, Oscar Franzese, Bill Knee, M.B. Lascurain, and Pedro Otaduy. Class-8 Heavy Truck Duty Cycle Project Final Report, ORNL/TM-2008/122, Oak Ridge National Laboratory, Oak Ridge, TN, December 2008.

Note: $\mathrm{D}=$ Dual tire. $\mathrm{S}=$ Single (wide) tire.

These data were not adjusted to account for the effects of terrain. The increase in fuel economy for speeds above 70 $\mathrm{mph}$ is likely due to the vehicle achieving high speeds while traveling down slope. Therefore, this increase in fuel economy is not expected to be characteristic of all travel at these higher speeds. 
A typical class 8 truck tractor weighs about 17,000 lbs. The powertrain is nearly a quarter of the weight (24\%) while the truck body structure is $19 \%$.

Table 5.12

Class 8 Truck Weight by Component

\begin{tabular}{lrc}
\hline & Pounds & Share of Total \\
\hline Wheels and tires & 1,700 & $10 \%$ \\
Chassis/frame & 2,040 & $12 \%$ \\
Drivetrain and suspension & 2,890 & $17 \%$ \\
Misc. accessories/systems & 3,060 & $18 \%$ \\
Truck body structure & 3,230 & $19 \%$ \\
Powertrain & 4,080 & $24 \%$ \\
\hline Total & 17,000 & $100 \%$ \\
\hline
\end{tabular}

\section{Source:}

National Academy of Sciences, Technologies and Approaches to Reducing the Fuel Consumption of Medium and Heavy-Duty Vehicles, prepublication copy, March 2010, p. 5-42.

\section{Notes:}

- Powertrain includes engine and cooling system, transmission and accessories.

- Truck body structure includes cab-in-white, sleeper unit, hood and fairings, interior and glass.

- Miscellaneous accessories/systems include batteries, fuel system, and exhaust hardware.

- Drivetrain and suspension includes drive axles, steer axle, and suspension system.

- Chassis/frame includes frame rails and crossmembers, fifth wheel and brackets. Wheels and tires include a set of 10 aluminum wheels, plus tires. 
The gross weight of a vehicle $(G V W)$ is the weight of the empty vehicle plus the weight of the maximum payload that the vehicle was designed to carry. In cars and small light trucks, the difference between the empty weight of the vehicle and the $G V W$ is not significantly different (1,000 to 1,500 lbs). The largest trucks and tractor-trailers, however, have a payload capacity share of 200\%, which means they can carry $200 \%$ of their empty weight. The medium-sized trucks (truck classes 3-6) have payload capacity shares between 50\% and 100\%.

Table 5.13

Gross Vehicle Weight vs. Empty Vehicle Weight

\begin{tabular}{|c|c|c|c|c|c|}
\hline Vehicle Description & Truck Class & $\begin{array}{l}\text { Gross Vehicle } \\
\text { Weight Range } \\
\text { (pounds) }\end{array}$ & $\begin{array}{l}\text { Empty Vehicle } \\
\text { Weight Range } \\
\text { (pounds) }\end{array}$ & $\begin{array}{l}\text { Maximum } \\
\text { Payload } \\
\text { Capacity } \\
\text { (pounds) } \\
\end{array}$ & $\begin{array}{c}\text { Payload } \\
\text { Capacity Share } \\
\text { (Percent of } \\
\text { Empty } \\
\text { Weight) } \\
\end{array}$ \\
\hline Cars & & $3,200-6,000$ & $2,400-5,000$ & 1,000 & $20 \%$ \\
\hline $\begin{array}{l}\text { Minivans, Small SUVs, } \\
\text { Small Pick-Ups }\end{array}$ & 1 & $4,000-2,400$ & $3,200-4,500$ & 1,500 & $33 \%$ \\
\hline $\begin{array}{l}\text { Large SUVs, Standard Pick- } \\
\text { Ups }\end{array}$ & $2 \mathrm{a}$ & $6,001-8,500$ & $4,500-6,000$ & 2,500 & $40 \%$ \\
\hline $\begin{array}{l}\text { Large SUVs, Standard Pick- } \\
\text { Ups }\end{array}$ & $2 b$ & $8,501-10,000$ & $5,000-6,300$ & 3,700 & $60 \%$ \\
\hline $\begin{array}{l}\text { Utility Van, Multi- Purpose, } \\
\text { Mini-Bus, Step Van }\end{array}$ & 3 & $10,001-14,000$ & $7,650-8,750$ & 5,250 & $60 \%$ \\
\hline $\begin{array}{l}\text { City Delivery, Parcel } \\
\text { Delivery, Large Walk-in, } \\
\text { Bucket, Landscaping }\end{array}$ & 4 & $14,001-16,000$ & $7,650-8,750$ & 7,250 & $80 \%$ \\
\hline $\begin{array}{l}\text { City Delivery, Parcel } \\
\text { Delivery, Large Walk-in, } \\
\text { Bucket }\end{array}$ & 5 & $16,001-19,500$ & $9,500-10,000$ & 8,700 & $80 \%$ \\
\hline $\begin{array}{l}\text { City Delivery, School Bus, } \\
\text { Large Walk-in, Bucket }\end{array}$ & 6 & $19,501-26,000$ & $11,500-14,500$ & 11,500 & $80 \%$ \\
\hline $\begin{array}{l}\text { City Bus, Furniture, } \\
\text { Refrigerated, Refuse, Fuel } \\
\text { Tanker, Dump, Tow, } \\
\text { Concrete, Fire Engine, } \\
\text { Tractor-Trailer }\end{array}$ & 7 & $26,001-33,000$ & $11,500-14,500$ & 18,500 & $125 \%$ \\
\hline $\begin{array}{l}\text { Refuse, Concrete, Furniture, } \\
\text { City Bus, Tow, Fire Engine } \\
\text { (straight trucks) }\end{array}$ & $8 \mathrm{a}$ & $33,001-80,000$ & $20,000-26,000$ & 54,000 & $200 \%$ \\
\hline $\begin{array}{l}\text { Tractor-Trailer: Van, } \\
\text { Refrigerated, Bulk Tanker, } \\
\text { Flat Bed (combination } \\
\text { trucks) }\end{array}$ & $8 b$ & $33,001-80,000$ & $20,000-26,000$ & 54,000 & $200 \%$ \\
\hline
\end{tabular}

Source:

National Academy of Sciences, Technologies and Approaches to Reducing the Fuel Consumption of Medium and Heavy-Duty Vehicles, prepublication copy, March 2010, pp. 2-2 and 5-42. 
According to weigh-in-motion data collected by fifteen states, the majority of 5-axle tractor-trailers on the road weigh between 33,000 and 73,000 lbs. Eleven percent of the tractor-trailers had weight recorded around 72,800 lbs and $10 \%$ around 68,300 lbs. Another 10\% of tractor-trailers were on the lighter end of the scale - around 37,500

lbs. These data show that only a small percent of trucks on the road are near the maximum roadway gross vehicle weight of 80,000 lbs. Thus, most trucks are filling the trailer space to capacity (cubing-out) before they reach the maximum weight limit (weighing-out).

Figure 5.5. Distribution of Five-Axle Tractor-Trailers by On-Road Vehicle Weight, 2008

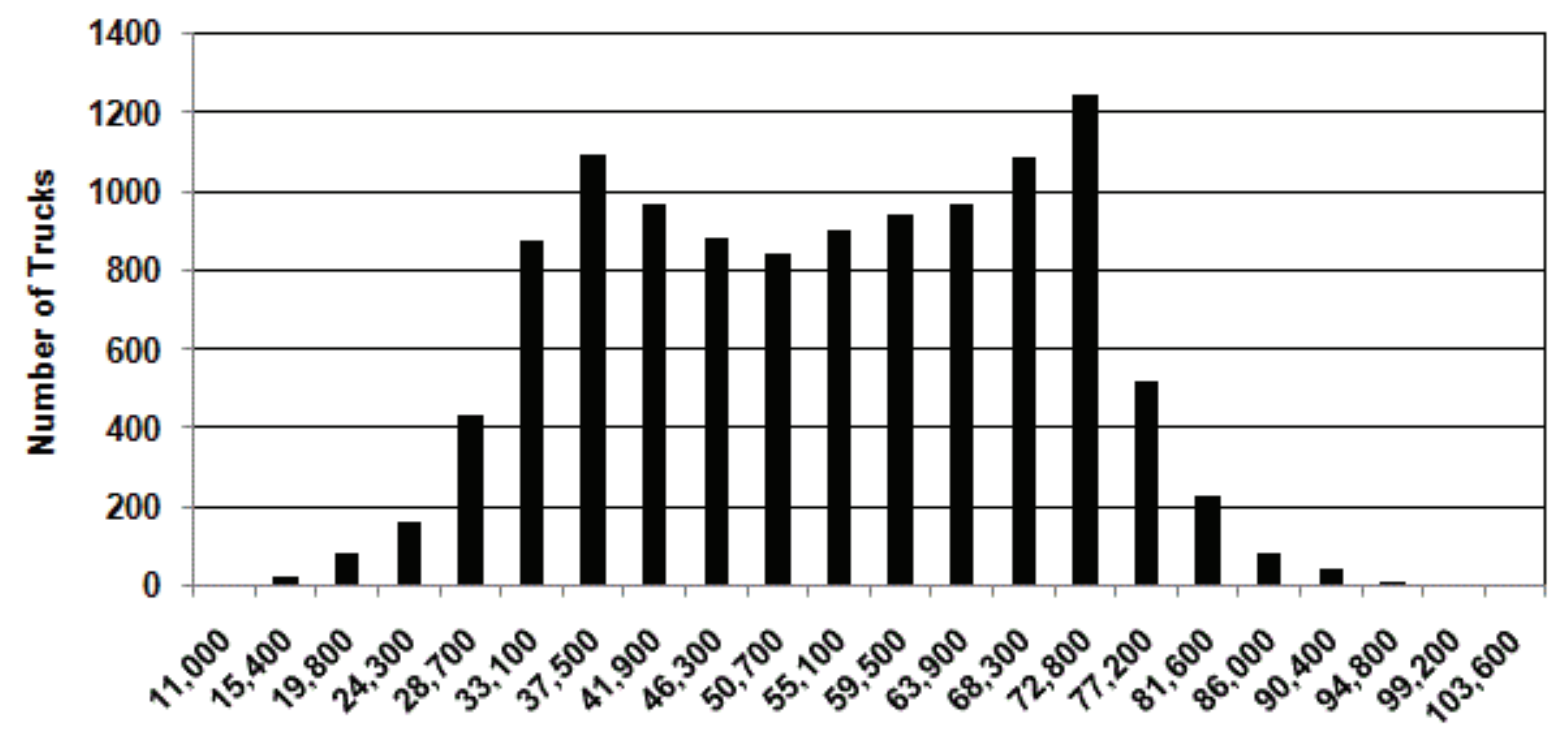

Truck Weight (pounds)

Source:

National Academy of Sciences, Technologies and Approaches to Reducing the Fuel Consumption of Medium and Heavy-Duty Vehicles, prepublication copy, March 2010, p. 5-45. Original source: Federal Highway Administration, Vehicle Travel Information System, 2008.

Note: Data are from these 15 States: California, Connecticut, Florida, Georgia, Hawaii, Iowa, Minnesota, Missouri, Montana, North Carolina, Oregon, Pennsylvania, South Dakota, Texas, and Washington. 


\section{Commodity Flow Survey}

The Commodity Flow Survey (CFS) is designed to provide data on the flow of goods and materials by mode of transport. The 1993, 1997, 2002, and 2007 CFS are a continuation of statistics collected in the Commodity Transportation Survey from 1963 through 1977, and include major improvements in methodology, sample size, and scope. The 2007 CFS covers business establishments with paid employees that are located in the United States and are classified using the North American Industry Classification System (NAICS) in mining, manufacturing, wholesale trade, and select retail trade industries, namely, electronic shopping and mail-order houses. Establishments classified in services, transportation, construction, and most retail industries are excluded from the survey. Farms, fisheries, foreign establishments, and most government-owned establishments are also excluded. ${ }^{\text {a }}$

The 1993, 1997, 2002, and 2007 CFS differ from previous surveys in their greatly expanded coverage of intermodalism (i.e., shipments which travel by at least two different modes, such as rail and truck). Earlier surveys reported only the principal mode. Route distance for each mode for each shipment was imputed using methodologies developed by Oak Ridge National Laboratory. Distance, in turn, was used to compute ton-mileage by mode of transport.

The data can be viewed at: www.bts.gov/publications/commodity_flow_survey.

${ }^{\text {a }}$ Bureau of Transportation Statistics and U.S. Bureau of the Census, 2007 Economic Census, 2007 Commodity Flow Survey, December 2008. 
Industries covered by the 2007 Commodity Flow Survey (CFS) shipped over 12 billion tons of goods worth over \$11 trillion. Compared to the 1997 CFS, the value of shipments is up 1.3\% per year and tons shipped are up $1.6 \%$ per year. By value, intermodal shipments increased 4.7\% per year from 1997 to 2007.

Table 5.14

Growth of Freight in the United States: Comparison of the 2007, 2002 and 1997 Commodity Flow Surveys (Detail may not add to total because of rounding)

\begin{tabular}{|c|c|c|c|c|c|c|c|c|}
\hline \multirow[b]{2}{*}{ Mode of Transportation } & \multicolumn{4}{|c|}{ Value of goods shipped } & \multicolumn{4}{|c|}{ Tons } \\
\hline & $\begin{array}{c}2007 \\
\text { (billions) }\end{array}$ & $\begin{array}{c}2002 \\
\text { (billion } \\
2007 \\
\text { dollars) } \\
\end{array}$ & $\begin{array}{c}1997 \\
\text { (billion } \\
2007 \\
\text { dollars) }\end{array}$ & $\begin{array}{c}\text { Average } \\
\text { annual } \\
\text { percent } \\
\text { change } \\
(1997-2007)\end{array}$ & $\begin{array}{c}2007 \\
\text { (millions) }\end{array}$ & $\begin{array}{c}2002 \\
\text { (millions) }\end{array}$ & $\begin{array}{c}1997 \\
\text { (millions) }\end{array}$ & $\begin{array}{l}\text { Average } \\
\text { annual } \\
\text { percent } \\
\text { change }\end{array}$ \\
\hline All modes & $11,684.9$ & $9,678.0$ & $8,970.5$ & $2.7 \%$ & $12,543.4$ & $11,667.9$ & $11,089.7$ & $1.2 \%$ \\
\hline Single modes & $9,539.0$ & $8,124.6$ & $7,388.8$ & $2.6 \%$ & $11,698.1$ & $11,086.7$ & $10,436.5$ & $1.1 \%$ \\
\hline Truck $^{\mathrm{a}}$ & $8,335.8$ & $7,186.0$ & $6,435.3$ & $2.6 \%$ & $8,778.7$ & $7,842.8$ & $7,700.7$ & $1.3 \%$ \\
\hline For-hire truck & $4,955.7$ & $4,330.2$ & $3,748.0$ & $2.8 \%$ & $4,075.1$ & $3,657.3$ & $3,402.6$ & $1.8 \%$ \\
\hline Private truck & $3,380.1$ & $2,818.3$ & $2,630.8$ & $2.5 \%$ & $4,703.6$ & $4,149.7$ & $4,137.3$ & $1.3 \%$ \\
\hline Rail & 436.4 & 359.5 & 412.9 & $0.6 \%$ & $1,861.3$ & $1,873.9$ & $1,549.8$ & $1.8 \%$ \\
\hline Water & 114.9 & 102.9 & 97.9 & $1.6 \%$ & 403.6 & 681.2 & 563.4 & $-3.3 \%$ \\
\hline Shallow draft & 91.0 & 66.3 & 69.6 & $2.7 \%$ & 343.3 & 458.6 & 414.8 & $-1.9 \%$ \\
\hline Great Lakes & b & 0.9 & 1.9 & $\mathrm{~b}$ & 17.8 & 38.0 & 38.4 & $-7.4 \%$ \\
\hline Deep draft & 23.1 & 35.7 & 26.4 & $-1.3 \%$ & 42.5 & 184.6 & 110.2 & $-9.1 \%$ \\
\hline Air (includes truck and air) & 252.3 & 305.4 & 296.0 & $-1.6 \%$ & 3.6 & 3.8 & 4.5 & $-2.2 \%$ \\
\hline Pipeline $^{b}$ & 399.6 & 172.0 & 146.6 & $10.5 \%$ & 650.9 & 685.0 & 618.2 & $0.5 \%$ \\
\hline Multiple modes & $1,866.7$ & $1,243.8$ & $1,221.9$ & $4.3 \%$ & 573.7 & 216.7 & 216.7 & $10.2 \%$ \\
\hline Parcel, U.S. Postal Service & & & & & & & & \\
\hline or courier & $1,561.9$ & $1,138.5$ & $1,105.7$ & $3.5 \%$ & 33.9 & 25.5 & 23.7 & $3.6 \%$ \\
\hline Truck and rail & 187.2 & 80.6 & 97.8 & $6.7 \%$ & 225.6 & 43.0 & 54.2 & $15.3 \%$ \\
\hline Truck and water & 58.4 & 16.6 & 10.6 & $18.6 \%$ & 145.5 & 23.3 & 33.2 & $15.9 \%$ \\
\hline Rail and water & 13.9 & 3.8 & 2.3 & $19.7 \%$ & 54.9 & 105.1 & 79.3 & $-3.6 \%$ \\
\hline Other multiple modes & 45.3 & 4.4 & 5.6 & $-8.0 \%$ & 113.8 & 19.8 & 26.2 & $15.8 \%$ \\
\hline Other and unknown modes & 279.1 & 309.6 & 359.9 & $-2.5 \%$ & 271.6 & 364.6 & 436.5 & $-4.6 \%$ \\
\hline
\end{tabular}

Source:

U.S. Department of Transportation, Bureau of Transportation Statistics and U.S. Department of Commerce, Bureau of the Census, 2007 Commodity Flow Survey, Table 1a. (Additional resources: www.bts.gov/cfs)

a "Truck" as a single mode includes shipments which went by private truck only, for-hire truck only, or a combination of private truck and for-hire truck.

${ }^{\mathrm{b}}$ Denotes data do not meet publication standards because of high sampling variability or poor response quality.

${ }^{\mathrm{c}} \mathrm{CFS}$ data for pipeline exclude most shipments of crude oil. 
Industries covered by the 2007 Commodity Flow Survey (CFS) accounted for 3.3 trillion ton-miles on the nation's highways, railways, waterways, pipelines, and aviation system. Ton-miles increased an average of $2.7 \%$ per year from 1997 to 2007.

Table 5.15

Growth of Freight Miles in the United States: Comparison of the 2007, 2002 and 1997 Commodity Flow Surveys (Detail may not add to total because of rounding)

\begin{tabular}{|c|c|c|c|c|c|c|c|c|}
\hline \multirow[b]{2}{*}{ Mode of Transportation } & \multicolumn{4}{|c|}{ Ton-miles } & \multicolumn{4}{|c|}{ Average miles per shipment } \\
\hline & $\begin{array}{c}2007 \\
\text { (billions) }\end{array}$ & $\begin{array}{c}2002 \\
\text { (billions) }\end{array}$ & $\begin{array}{c}1997 \\
\text { (billions) }\end{array}$ & $\begin{array}{c}\text { Average } \\
\text { annual } \\
\text { percent } \\
\text { change } \\
(1997-2007)\end{array}$ & 2007 & 2002 & 1997 & $\begin{array}{l}\text { Average } \\
\text { annual } \\
\text { percent } \\
\text { change }\end{array}$ \\
\hline All modes & $3,344.7$ & $3,137.9$ & $2,661.4$ & $2.3 \%$ & 619 & 546 & 472 & $2.7 \%$ \\
\hline Single modes & $2,894.3$ & $2,867.9$ & $2,383.5$ & $2.0 \%$ & 234 & 240 & 184 & $2.4 \%$ \\
\hline Truck $^{\mathrm{a}}$ & $1,342.1$ & $1,255.9$ & $1,023.5$ & $2.7 \%$ & 206 & 173 & 144 & $3.6 \%$ \\
\hline For-hire truck & $1,055.6$ & 959.6 & 741.1 & $3.6 \%$ & 599 & 523 & 485 & $2.1 \%$ \\
\hline Private truck & 286.5 & 291.1 & 268.6 & $0.6 \%$ & 57 & 64 & 53 & $0.7 \%$ \\
\hline Rail & $1,344.0$ & $1,261.6$ & $1,022.5$ & $2.8 \%$ & 728 & 807 & 769 & $-0.5 \%$ \\
\hline Water & 157.3 & 282.7 & 261.7 & $-5.0 \%$ & 520 & 568 & 482 & $0.8 \%$ \\
\hline Shallow draft & 117.5 & 211.5 & 189.3 & $-4.7 \%$ & 144 & 450 & 177 & $-2.0 \%$ \\
\hline Great Lakes & 6.9 & 13.8 & 13.4 & $-6.4 \%$ & 657 & 339 & 204 & $12.4 \%$ \\
\hline Deep draft & 33.0 & 57.4 & 59.0 & $-0.1 \%$ & 923 & 664 & 1,024 & $-1.0 \%$ \\
\hline Air (includes truck and air) & 4.5 & 5.8 & 6.2 & $-3.2 \%$ & 1,304 & 1,919 & 1,380 & $-0.6 \%$ \\
\hline Pipeline ${ }^{\mathrm{b}}$ & c & c & $\mathrm{c}^{0.2}$ & & $\mathrm{c}$ & $\mathrm{c}$ & $\mathrm{c}$ & $\mathrm{c}$ \\
\hline Multiple modes & 416.6 & 225.7 & 204.5 & $7.4 \%$ & 975 & 895 & 813 & $1.8 \%$ \\
\hline Parcel, U.S. Postal Service & & & & & & & & \\
\hline or courier & 28.0 & 19.0 & 18.0 & $4.5 \%$ & 975 & 894 & 813 & $1.8 \%$ \\
\hline Truck and rail & 196.8 & 45.5 & 55.6 & $13.5 \%$ & 1,007 & 1,413 & 1,347 & $-2.9 \%$ \\
\hline Truck and water & 98.4 & 32.4 & 34.8 & $11.0 \%$ & 1,429 & 1,950 & 1,265 & $1.2 \%$ \\
\hline Rail and water & 47.1 & 115.0 & 77.6 & $-4.9 \%$ & 1,928 & 957 & 1,092 & $5.8 \%$ \\
\hline Other multiple modes & 46.4 & 13.8 & 18.6 & $0.1 \%$ & 1,182 & $\mathrm{c}$ & $\mathrm{c}$ & \\
\hline Other and unknown modes & 33.8 & 44.2 & 73.4 & $-7.5 \%$ & 116 & 130 & 122 & $-0.5 \%$ \\
\hline
\end{tabular}

Source:

U.S. Department of Transportation, Bureau of Transportation Statistics and U.S. Department of Commerce, Bureau of the Census, 2007 Commodity Flow Survey, Table 1a. (Additional resources: www.bts.gov/cfs)

a "Truck" as a single mode includes shipments which went by private truck only, for-hire truck only, or a combination of private truck and for-hire truck.

${ }^{\mathrm{b}}$ CFS data for pipeline exclude most shipments of crude oil.

${ }^{c}$ Denotes data do not meet publication standards because of high sampling variability or other reasons. Some unpublished estimates can be derived from other data published in this table. However, figures obtained in this manner are subject to these same limitations. 
In 2007, the data changed substantially due to improved estimation methodologies. Unfortunately, those data are no longer comparable to the rest of the historical series.

Table 5.16

Summary Statistics on Transit Buses and Trolleybuses, 1994-2008

\begin{tabular}{cccccc}
\hline Number of & $\begin{array}{c}\text { Vehicle-miles } \\
\text { (millions) }\end{array}$ & $\begin{array}{c}\text { Passenger- } \\
\text { miles } \\
\text { (millions) }\end{array}$ & Btu/passenger-mile & $\begin{array}{c}\text { Energy use } \\
\text { (trillion Btu) }\end{array}$ \\
\hline 1994 & 68,766 & 2,176 & 19,019 & 4,261 & 81.0 \\
1995 & 67,802 & 2,198 & 19,005 & 4,302 & 81.8 \\
1996 & 72,353 & 2,234 & 19,280 & 4,334 & 83.6 \\
1997 & 73,425 & 2,259 & 19,793 & 4,425 & 87.6 \\
1998 & 72,788 & 2,188 & 20,542 & 4,384 & 90.1 \\
1999 & 74,885 & 2,290 & 21,391 & 4,327 & 92.6 \\
2000 & 75,665 & 2,329 & 21,433 & 4,509 & 96.6 \\
2001 & 76,675 & 2,389 & 22,209 & 4,120 & 91.5 \\
2002 & 76,806 & 2,425 & 22,029 & 4,100 & 90.3 \\
2003 & 78,000 & 2,435 & 21,438 & 4,171 & 89.4 \\
2004 & 81,630 & 2,484 & 21,550 & 4,318 & 93.0 \\
2005 & 82,642 & 2,498 & 21,998 & 4,200 & 92.4 \\
2006 & 83,689 & 2,507 & 22,985 & 4,259 & 97.9 \\
$2007^{\text {a }}$ & 65,808 & 2,314 & 21,132 & 4,315 & 91.2 \\
2008 & 67,096 & 2,388 & 21,918 & 4,290 & 94.0 \\
\hline
\end{tabular}

\section{Source:}

American Public Transportation Association, 2010 Public Transportation Fact Book, Washington, DC, April 2010, Tables 6, 8, 9, and 15. (Additional resources: www.apta.com)

${ }^{\text {a }}$ Data are not continuous between 2006 and 2007 due to changes in estimation methodology. See source document for details. 


\section{Chapter 6 \\ Alternative Fuel and Advanced Technology Vehicles and Characteristics}

Summary Statistics from Tables in this Chapter

Source

Table 6.1 Alternative fuel vehicles in use, 2008

775,664

$L P G$

151,049

CNG

113,973

E85

450,327

Electric

56,901

M85

0

$L N G$

3,101

Table 6.4 Number of alternative fuel refuel sites, 2010

6,497

$L P G$

2,410

CNG

827

Biodiesel

679

Electric

506

Hydrogen

Fuel type abbreviations are used throughout this chapter.

$B 20=20 \%$ biodiesel, $80 \%$ petroleum diesel

$C N G=$ compressed natural gas

E85 $=85 \%$ ethanol, $15 \%$ gasoline

E95 $=95 \%$ ethanol, $5 \%$ gasoline

$\mathrm{H}_{2}=$ hydrogen

$L N G=$ liquified natural gas

$L P G=$ liquified petroleum gas

M85 = $85 \%$ methanol, $15 \%$ gasoline

$M 100=100 \%$ methanol 


\begin{abstract}
Alternative Fuels as alternative fuels:

- methanol, ethanol, and other alcohols,

- blends of $85 \%$ or more of alcohol with gasoline,

- natural gas and liquid fuels domestically produced from natural gas,

- liquefied petroleum gas (propane),

- coal-derived liquid fuels

- hydrogen and electricity

- biodiesel,

- P-series.
\end{abstract}

The Energy Policy Act of 1992 defines alternative fuels and allows the U.S. Department of Energy (DOE) to add to the list of alternative fuels if the fuel is substantially nonpetroleum, yields substantial energy security benefits, and offers substantial environmental benefits. DOE currently recognizes the following

\title{
Alternative Fuels \& Advanced Vehicles Data Center
}

DOE established the Alternative Fuels Data Center (AFDC) in 1991 to support its work aimed at fulfilling the Alternative Motor Fuels Act directives. Since then, the AFDC has expanded its focus to include all advanced transportation fuels, vehicles, and technologies. It has been renamed the Alternative Fuels \& Advanced Vehicles Data Center to reflect this broader scope. The AFDC is operated and managed by the National Renewable Energy Laboratory (NREL) in Golden, Colorado.

The purposes of the AFDC are:

- to gather and analyze information on the fuel consumption, emissions, operation, and durability of alternative fuel vehicles, and

- to provide unbiased, accurate information on alternative fuels and alternative fuel vehicles to government agencies, private industry, research institutions, and other interested organizations.

Much of the AFDC data can be obtained through their Web site: www.eere.energy.gov/afdc. Several tables and graphs in this chapter contain statistics which were generated by the AFDC. Below are some links to specific areas of the AFDC Web site.

Alternative \& Advanced Fuels - www.eere.energy.gov/afdc/fuels/index.html

Alternative Fueling Station Locator - www.eere.energy.gov/afdc/fuels/stations_locator.html

Alternative \& Advanced Vehicles - www.eere.energy.gov/afdc/vehicles/index.html

Fleet Information - www.eere.energy.gov/afdc/fleets/index.html

State \& Federal Incentives \& Laws - www.eere.energy.gov/afdc/incentives_laws.html

Data Analysis \& Trends - www.eere.energy.gov/afdc/data/index.html 
There are over 775,000 alternative fuel vehicles in the United States, not including flex-fuel E85 vehicles which operate mainly on gasoline. The E85 vehicles in this table are those believed to be regularly fueled with E85.

Table 6.1

Estimates of Alternative Fuel Vehicles in Use ${ }^{a}$, 1995-2008

\begin{tabular}{|c|c|c|c|c|c|c|c|c|c|c|}
\hline Year & LPG & $\mathrm{CNG}$ & LNG & M85 & M100 & $\mathrm{E} 85^{\mathrm{b}}$ & E95 & Electricity $^{\mathrm{c}}$ & Hydrogen & Total \\
\hline 1995 & 172,806 & 50,218 & 603 & 18,319 & 386 & 1,527 & 136 & 2,860 & 0 & 246,855 \\
\hline 1996 & 175,585 & 60,144 & 663 & 20,265 & 172 & 4,536 & 361 & 3,280 & 0 & 265,006 \\
\hline 1997 & 175,679 & 68,571 & 813 & 21,040 & 172 & 9,130 & 347 & 4,453 & 0 & 280,205 \\
\hline 1998 & 177,183 & 78,782 & 1,172 & 19,648 & 200 & 12,788 & 14 & 5,243 & 0 & 295,030 \\
\hline 1999 & 178,610 & 91,267 & 1,681 & 18,964 & 198 & 24,604 & 14 & 6,964 & 0 & 322,302 \\
\hline 2000 & 181,994 & 100,750 & 2,090 & 10,426 & 0 & 87,570 & 4 & 11,830 & 0 & 394,664 \\
\hline 2001 & 185,053 & 111,851 & 2,576 & 7,827 & 0 & 100,303 & 0 & 17,847 & 0 & 425,457 \\
\hline 2002 & 187,680 & 120,839 & 2,708 & 5,873 & 0 & 120,951 & 0 & 33,047 & 0 & 471,098 \\
\hline 2003 & 190,369 & 114,406 & 2,640 & 0 & 0 & 179,090 & 0 & 47,485 & 9 & 533,999 \\
\hline 2004 & 182,864 & 118,532 & 2,717 & 0 & 0 & 211,800 & 0 & 49,536 & 43 & 565,492 \\
\hline 2005 & 173,795 & 117,699 & 2,748 & 0 & 0 & 246,363 & 0 & 51,398 & 119 & 592,122 \\
\hline 2006 & 164,846 & 116,131 & 2,798 & 0 & 0 & 297,099 & 0 & 53,526 & 159 & 634,559 \\
\hline 2007 & 158,254 & 114,391 & 2,781 & 0 & 0 & 364,384 & 0 & 55,730 & 223 & 695,763 \\
\hline 2008 & 151,049 & 113,973 & 3,101 & 0 & 0 & 450,327 & 0 & 56,901 & 313 & 775,664 \\
\hline \multicolumn{11}{|c|}{ Average annual percentage change } \\
\hline $1995-2008$ & $-1.0 \%$ & $6.5 \%$ & $13.4 \%$ & $-100.0 \%$ & $-100.0 \%$ & $54.9 \%$ & $-100.0 \%$ & $25.9 \%$ & & $9.2 \%$ \\
\hline
\end{tabular}

Source:

U. S. Department of Energy, Energy Information Administration, Alternatives to Traditional Transportation Fuels, 2008, Washington, DC, April 2010, Table VI. 1995-2006, Annual Energy Review, Table 10.4. Estimated Number of Alternative-Fueled Vehicles in Use and Replacement Fuel Consumption. (Additional resources: www.eia.doe.gov/emeu/aer/renew.html and www.eere.energy.gov/afdc/data/vehicles.html)

${ }^{\text {a }}$ Vehicles in Use represent accumulated acquisitions, less retirements, as of the end of each calendar year. They do not include concept and demonstration vehicles.

${ }^{\mathrm{b}}$ Includes only those E85 vehicles believed to be used as alternative-fuels vehicles (AFVs), primarily fleetoperated vehicles; excludes other vehicles with E85-fueling capability. In 1997, some vehicle manufacturers began including E85-fueling capability in certain model lines of vehicles. For 2007, the Energy Information Administration (EIA) estimates that the number of E85 vehicles that are capable of operating on E85, motor gasoline, or both, is about 7.1 million. Many of these AFVs are sold and used as traditional gasoline-powered vehicles.

${ }^{\mathrm{c}}$ Excludes HEVs. 
Table 6.2

Alternative Fuel Vehicles Available by Manufacturer, Model Year 2009

\begin{tabular}{|c|c|c|c|}
\hline Model & Fuel & Type & Emission Class \\
\hline \multicolumn{4}{|c|}{ Chrysler: 1-800-999-FLEET; www.fleet.chrysler.com } \\
\hline Chrysler Sebring & E85 flex fuel & Sedan & Tier 2 Bin 8 \\
\hline Chrysler Aspen & E85 flex fuel & SUV & LEV II, Tier 2 Bin 5 \\
\hline Chrysler Town \& Country & E85 flex fuel & Minivan & LEV II, Tier 2 Bin 5 \\
\hline Dodge Avenger & E85 flex fuel & Sedan & Tier 2 Bin 8 \\
\hline Dodge Caravan & E85 flex fuel & Minivan & LEV II, Tier 2 Bin 5 \\
\hline Dodge Dakota & E85 flex fuel & Pickup & LEV II, Tier 2 Bin 5 \\
\hline Dodge Durango & E85 flex fuel & SUV & LEV II, Tier 2 Bin 5 \\
\hline Dodge Ram 1500 & E85 flex fuel & Pickup & LEV II, Tier 2 Bin 5 \\
\hline Jeep Commander & E85 flex fuel & SUV & Tier 2 Bin 8 \\
\hline Jeep Grand Cherokee & E85 flex fuel & SUV & Tier 2 Bin 8 \\
\hline \multicolumn{4}{|c|}{ Ford: 1-800-34-FLEET; www.fleet.ford.com; www.fordvehicles.com } \\
\hline Ford Crown Victoria & E85 flex fuel & Sedan & ULEV II, Tier 2 Bin 4 \\
\hline Crown Victoria Police & E85 flex fuel & Sedan & ULEV II, Tier 2 Bin 4 \\
\hline Lincoln Town Car & E85 flex fuel & Sedan & ULEV II, Tier 2 Bin 4 \\
\hline Mercury Grand Marquis & E85 flex fuel & Sedan & ULEV II, Tier 2 Bin 4 \\
\hline Ford Expedition & E85 flex fuel & SUV & LEV II, Tier 2 Bin 5 \\
\hline Ford F-150 & E85 flex fuel & Pickup & LEV II, Tier 2 Bin 5 \\
\hline Ford E-Series E150 Van & E85 flex fuel & Van & Tier 2 Bin 8 \\
\hline Lincoln Navigator & E85 flex fuel & SUV & LEV II, Tier 2 Bin 5 \\
\hline \multicolumn{4}{|c|}{ General Motors Corporation: 1-888-GM-AFT-4U; www.gm.com/vehicles } \\
\hline Chevrolet Impala & E85 flex fuel & Sedan & LEV II, Tier 2 Bin 5 \\
\hline Chevrolet HHR & E85 flex fuel & SUV & LEV II, Tier 2 Bin 5 \\
\hline Chevrolet HHR Panel & E85 flex fuel & LD Van & LEV II, Tier 2 Bin 5 \\
\hline Chevrolet Avalanche 1500 & E85 flex fuel & SUV & LEV II, Tier 2 Bin 5 \\
\hline Chevrolet Tahoe 1500 & E85 flex fuel & SUV & LEV II, Tier 2 Bin 5 \\
\hline GMC Yukon 1500 & E85 flex fuel & SUV & LEV II, Tier 2 Bin 5 \\
\hline Chevrolet Suburban 1500 & E85 flex fuel & SUV & LEV II, Tier 2 Bin 5 \\
\hline GMC Yukon XL & E85 flex fuel & SUV & LEV II, Tier 2 Bin 5 \\
\hline GMC Yukon Denali 1500 & E85 flex fuel & SUV & LEV II, Tier 2 Bin 5 \\
\hline Chevrolet Silverado 1500 & E85 flex fuel & Pickup & LEV II, Tier 2 Bin 5 \\
\hline GMC Sierra 1500 & E85 flex fuel & Pickup & LEV II, Tier 2 Bin 5 \\
\hline Chevrolet Express 1500 & E85 flex fuel & Van & ULEV II, Tier 2 Bin 4 \\
\hline GMC Savana 1500 & E85 flex fuel & Van & ULEV II, Tier 2 Bin 4 \\
\hline Buick Lucerne & E85 flex fuel & Sedan & LEV II, Tier 2 Bin 5 \\
\hline Cadillac Escalade & E85 flex fuel & SUV & LEV II, Tier 2 Bin 5 \\
\hline HUMMER H2 & E85 flex fuel & SUV & LEV II, Tier 2 Bin 5 \\
\hline HUMMER H2 SUT & E85 flex fuel & SUV & LEV II, Tier 2 Bin 5 \\
\hline \multicolumn{4}{|c|}{ Honda: 1-888-CC-HONDA; www.honda.com } \\
\hline Civic GX & CNG Dedicated & Sedan & SULEV II, Tier 2 Bin 2 \\
\hline FCX & Hydrogen fuel cell & Sedan & ZEV, Tier 2 Bin 1 \\
\hline \multicolumn{4}{|c|}{ Mercedes-Benz USA: 1-800-FOR-MERCEDES; www.mbusa.com } \\
\hline C300 Sport & E85 flex fuel & Sedan & LEV II, Tier 2 Bin 5 \\
\hline \multicolumn{4}{|c|}{ Mitsubishi: 1-888-MITSU2007; www.mitsubishicars.com } \\
\hline Raider & E85 flex fuel & Pickup & LEV II, Tier 2 Bin 5 \\
\hline \multicolumn{4}{|c|}{ Nissan: 1-800-NISSAN-1; www.nissanusa.com } \\
\hline Armada 2WD & E85 flex fuel & SUV & LEV II, Tier 2 Bin 5 \\
\hline Titan & E85 flex fuel & Pickup & LEV II, Tier 2 Bin 5 \\
\hline \multicolumn{4}{|c|}{ Toyota: 1-800-331-4331; www.toyota.com } \\
\hline Tundra & E85 flex fuel & Pickup & LEV II, Tier 2 Bin 5 \\
\hline
\end{tabular}

Source:

U.S. Department of Energy, National Alternative Fuels Data Center, Web site, ww.afdc.energy.gov/afdc/pdfs/my2009_afv_atv.pdf, February 2010. (Additional resources: ww.eere.energy.gov/afdc/vehicles/index.html)

Note: $L E V=$ low emission vehicle. ILEV=inherently low emission vehicle. ULEV=ultra low emission vehicle. ZEV=zero emission vehicle. TLEV=transitional low emission vehicle. SULEV=super ultra low emission vehicle. See Chapter 12 for details on emissions. 
Table 6.3

Hybrid Electric Vehicles Available by Manufacturer, Model Year 2009

\begin{tabular}{|c|c|c|c|}
\hline Model & Battery Type $^{\mathrm{a}}$ & Type & Emission Class \\
\hline \multicolumn{4}{|c|}{ Chrysler: 1-800-999-FLEET; www.fleet.chrysler.com } \\
\hline Chrysler Aspen Hybrid & NiMH & SUV & LEV II, Tier 2 Bin 5 \\
\hline Dodge Durango Hybrid & $\mathrm{NiMH}$ & SUV & LEV II, Tier 2 Bin 5 \\
\hline \multicolumn{4}{|c|}{ Ford: 1-800-34-FLEET; www.fleet.ford.com; www.fordvehicles.com } \\
\hline Ford Escape Hybrid & NiMH & SUV & ULEV II, Tier 2 Bin 3 \\
\hline Mercury Mariner Hybrid & $\mathrm{NiMH}$ & SUV & ULEV II, Tier 2 Bin 3 \\
\hline \multicolumn{4}{|c|}{ General Motors: 1-888-GM-AFT-4U; www.gm.com/vehicles } \\
\hline Chevrolet Malibu Hybrid & NiMH & Sedan & LEV II, Tier 2 Bin 5 \\
\hline Saturn Aura Hybrid & NiMH & Sedan & LEV II, Tier 2 Bin 5 \\
\hline Saturn VUE Hybrid & $\mathrm{NiMH}$ & SUV & LEV II, Tier 2 Bin 5 \\
\hline Cadillac Escalade Hybrid & $\mathrm{NiMH}$ & SUV & LEV II, Tier 2 Bin 5 \\
\hline Chevrolet Tahoe 1500 Hybrid & NiMH & SUV & LEV II, Tier 2 Bin 5 \\
\hline GMC Yukon 1500 Hybrid & NiMH & SUV & LEV II, Tier 2 Bin 5 \\
\hline Chevrolet Silverado 1500 Hybrid & $\mathrm{NiMH}$ & Pickup & LEV II, Tier 2 Bin 5 \\
\hline \multicolumn{4}{|c|}{ Honda: 1-888-CC-HONDA: www.honda.com } \\
\hline Civic Hybrid & $\mathrm{NiMH}$ & Sedan & SULEV II,Tier 2 Bin 2 \\
\hline \multicolumn{4}{|c|}{ Mazda: 1-800-222-5500; www.mazdausa.com } \\
\hline Tribute & $\mathrm{NiMH}$ & SUV 2WD & SULEV II, Tier 2 Bin 3 \\
\hline \multicolumn{4}{|c|}{ Nissan: 1-800-NISSAN-1; www.nissanusa.com } \\
\hline Altima Hybrid & NiMH & Sedan & SULEV II \\
\hline \multicolumn{4}{|c|}{ Totota: 1-800-331-4331; www.toyota.com } \\
\hline Prius Hybrid & NiMH & Sedan & SULEV II,Tier 2 Bin 3 \\
\hline Camry Hybrid & $\mathrm{NiMH}$ & Sedan & SULEV II,Tier 2 Bin 3 \\
\hline Highlander Hybrid & $\mathrm{NiMH}$ & SUV & SULEV II,Tier 2 Bin 3 \\
\hline
\end{tabular}

Source:

U.S. Department of Energy, National Alternative Fuels Data Center, Web site, www.afdc.energy.gov/afdc/pdfs/my2009_afv_atv.pdf, February 2010 (Additional resources: www.eere.energy.gov/afdc/progs_vehicles_search.php)

Note: LEV = low emission vehicle; ILEV = inherently low emission vehicle; ULEV = ultra low emission vehicle; $\mathrm{ZEV}=$ zero emission vehicle; TLEV $=$ transitional low emission vehicle; SULEV $=$ super ultra low emission vehicle; AT-PZEV = avanced technology - partial zero emissions vehicle. See Chapter 12 for details on emissions.

${ }^{\mathrm{a}} \mathrm{NiMH}=$ Nickel-Metal Hydride; $\mathrm{PbA}=$ Lead-Acid; Mild hybrid = A vehicle that shuts down the engine when coasting, breaking or stopped while continuing to power accessories. There is however, no electric drivetrain like that found on a full hybrid vehicle. 
This list includes public and private refuel sites; therefore, not all of these sites are available to the public.

Table 6.4

Number of Alternative Refuel Sites by State and Fuel Type, 2010

\begin{tabular}{|c|c|c|c|c|c|c|c|c|c|}
\hline State & $\begin{array}{l}\mathrm{CNG} \\
\text { sites }\end{array}$ & $\begin{array}{l}\text { E85 } \\
\text { site }\end{array}$ & $\begin{array}{l}\text { LPG } \\
\text { sites }\end{array}$ & $\begin{array}{l}\text { Electric } \\
\text { sites }\end{array}$ & $\begin{array}{l}\text { Biodiesel } \\
\text { sites }\end{array}$ & $\begin{array}{l}\text { Hydrogen } \\
\text { sites }\end{array}$ & $\begin{array}{c}\text { LNG } \\
\text { sites }\end{array}$ & $\begin{array}{l}\text { LPG } \\
\text { sites }\end{array}$ & Total \\
\hline Alabama & 5 & 16 & 158 & 0 & 10 & 0 & 0 & 158 & 189 \\
\hline Alaska & 1 & 0 & 9 & 0 & 1 & 0 & 0 & 9 & 11 \\
\hline Arizona & 40 & 26 & 54 & 6 & 14 & 1 & 5 & 54 & 146 \\
\hline Arkansas & 5 & 10 & 41 & 0 & 7 & 0 & 0 & 41 & 63 \\
\hline California & 201 & 45 & 188 & 413 & 40 & 22 & 27 & 188 & 936 \\
\hline Colorado & 23 & 80 & 46 & 0 & 19 & 1 & 0 & 46 & 169 \\
\hline Connecticut & 14 & 4 & 12 & 0 & 1 & 2 & 0 & 12 & 33 \\
\hline Delaware & 1 & 1 & 2 & 0 & 3 & 0 & 0 & 2 & 7 \\
\hline Dist. of Columbia & 2 & 3 & 0 & 0 & 1 & 1 & 0 & 0 & 7 \\
\hline Florida & 15 & 30 & 57 & 5 & 17 & 0 & 0 & 57 & 124 \\
\hline Georgia & 16 & 37 & 41 & 1 & 30 & 0 & 0 & 41 & 125 \\
\hline Hawaii & 0 & 0 & 3 & 6 & 5 & 1 & 0 & 3 & 15 \\
\hline Idaho & 7 & 5 & 25 & 5 & 8 & 0 & 1 & 25 & 51 \\
\hline Illinois & 25 & 204 & 73 & 7 & 5 & 1 & 0 & 73 & 315 \\
\hline Indiana & 8 & 109 & 43 & 0 & 7 & 0 & 0 & 43 & 167 \\
\hline Iowa & 0 & 124 & 26 & 4 & 3 & 0 & 0 & 26 & 157 \\
\hline Kansas & 3 & 30 & 45 & 0 & 6 & 0 & 0 & 45 & 84 \\
\hline Kentucky & 0 & 15 & 25 & 0 & 2 & 0 & 0 & 25 & 42 \\
\hline Louisiana & 6 & 5 & 14 & 0 & 1 & 0 & 0 & 14 & 26 \\
\hline Maine & 1 & 0 & 9 & 0 & 4 & 0 & 0 & 9 & 14 \\
\hline Maryland & 5 & 14 & 18 & 0 & 9 & 0 & 0 & 18 & 46 \\
\hline Massachusetts & 21 & 2 & 22 & 3 & 6 & 1 & 0 & 22 & 55 \\
\hline Michigan & 13 & 95 & 68 & 0 & 16 & 6 & 0 & 68 & 198 \\
\hline Minnesota & 1 & 351 & 27 & 2 & 0 & 0 & 0 & 27 & 381 \\
\hline Mississippi & 2 & 4 & 35 & 0 & 7 & 0 & 0 & 35 & 48 \\
\hline Missouri & 10 & 98 & 69 & 0 & 5 & 1 & 0 & 69 & 183 \\
\hline Montana & 3 & 1 & 43 & 0 & 6 & 0 & 0 & 43 & 53 \\
\hline Nebraska & 1 & 48 & 18 & 0 & 4 & 0 & 0 & 18 & 71 \\
\hline Nevada & 11 & 14 & 29 & 0 & 13 & 2 & 0 & 29 & 69 \\
\hline New Hampshire & 3 & 0 & 9 & 2 & 11 & 0 & 0 & 9 & 25 \\
\hline New Jersey & 19 & 2 & 10 & 2 & 4 & 0 & 0 & 10 & 37 \\
\hline New Mexico & 8 & 10 & 50 & 0 & 8 & 0 & 0 & 50 & 76 \\
\hline New York & 99 & 38 & 22 & 2 & 13 & 9 & 0 & 22 & 183 \\
\hline North Carolina & 16 & 19 & 53 & 3 & 139 & 0 & 0 & 53 & 230 \\
\hline North Dakota & 2 & 30 & 23 & 0 & 3 & 1 & 0 & 23 & 59 \\
\hline Ohio & 9 & 63 & 68 & 3 & 21 & 1 & 0 & 68 & 165 \\
\hline Oklahoma & 49 & 11 & 58 & 0 & 6 & 0 & 0 & 58 & 124 \\
\hline Oregon & 12 & 8 & 30 & 23 & 25 & 0 & 0 & 30 & 98 \\
\hline Pennsylvania & 23 & 26 & 62 & 0 & 12 & 2 & 0 & 62 & 125 \\
\hline Rhode Island & 5 & 0 & 5 & 0 & 2 & 0 & 0 & 5 & 12 \\
\hline South Carolina & 3 & 87 & 20 & 0 & 30 & 2 & 0 & 20 & 142 \\
\hline South Dakota & 0 & 78 & 17 & 0 & 2 & 0 & 0 & 17 & 97 \\
\hline Tennessee & 4 & 29 & 42 & 2 & 41 & 0 & 0 & 42 & 118 \\
\hline Texas & 23 & 42 & 535 & 6 & 22 & 0 & 5 & 535 & 633 \\
\hline Utah & 59 & 5 & 24 & 0 & 5 & 0 & 0 & 24 & 93 \\
\hline Vermont & 2 & 0 & 4 & 2 & 2 & 1 & 0 & 4 & 11 \\
\hline Virginia & 12 & 9 & 38 & 3 & 18 & 1 & 0 & 38 & 81 \\
\hline Washington & 15 & 16 & 64 & 4 & 48 & 0 & 0 & 64 & 147 \\
\hline West Virginia & 0 & 3 & 8 & 0 & 1 & 1 & 0 & 8 & 13 \\
\hline Wisconsin & 17 & 127 & 45 & 2 & 1 & 0 & 0 & 45 & 192 \\
\hline Wyoming & 7 & 6 & 23 & 0 & 15 & 0 & 0 & 23 & 51 \\
\hline Totals by Fuel: & 827 & 1,980 & 2,410 & 506 & 679 & 57 & 38 & 2,410 & 6,497 \\
\hline
\end{tabular}

\section{Source:}

U.S. Department of Energy, Alternative Fuels Data Center Web site,

www.eere.energy.gov/afdc/infrastructure/station_counts.html, February 2010. 
Clean Cities is a locally-based government/industry partnership, coordinated by the U.S. Department of Energy to expand the use of alternatives to gasoline and diesel fuel. By combining the decision-making with voluntary action by partners, the "grass-roots" approach of Clean Cities departs from traditional "top-down" Federal programs.

\section{Figure 6.1. Clean Cities Coalitions}

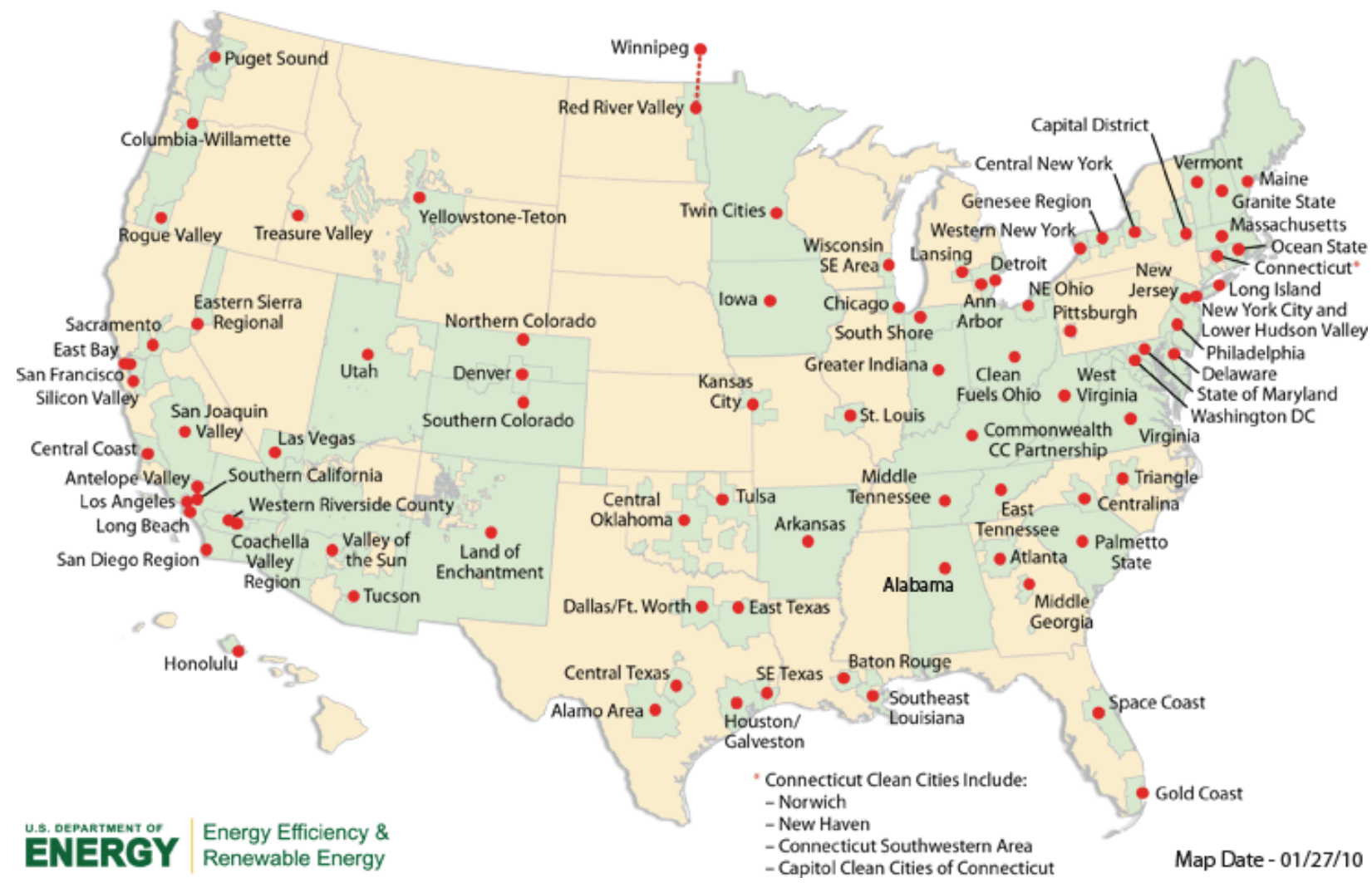

\section{Source:}

U.S. Department of Energy, Alternative Fuel Data Center, February 2010. (Additional resources: www.eere.energy.gov/cleancities/progs/coalition_locations.php) 


\section{Vehicle Technologies Program}

\section{www.eere.energy.gov/vehiclesandfuels}

The Vehicle Technologies Program is administered by the Department of Energy's Office of Energy Efficiency and Renewable Energy. The mission of this program is to develop more energy efficient and environmentally friendly highway transportation technologies that enable America to use less petroleum. The long-term aim is to develop "leap frog" technologies that will provide Americans with greater freedom of mobility and energy security, with lower costs and lower impacts on the environment. For additional information about the Vehicle Technologies Program, visit the Web site listed above.

\section{Hydrogen Analysis Resource Center}

\section{hydrogen.pnl.gov/}

The Hydrogen Analysis Resource Center was developed to provide reliable data and information for hydrogen-related analytical activities. The Center's Web site includes:

- Hydrogen Data Book - contains a wide range of factual information on hydrogen and fuel cells. hydrogen.pnl.gov/cocoon/morf/hydrogen/article/103

- Related Sites - provides links to other sites with data relevant to hydrogen and fuel cell analysis.

- Guidelines and Assumptions for DOE Hydrogen Program Analysis - contains guidelines for conducting analysis (under development) and assumptions.

- Calculator Tools - provides tools to perform conversions of hydrogen and other calculations relevant to hydrogen and fuel cells.

- Analysis Tools - provides links to models and other tools relevant to hydrogen and fuel cells, such as H2A, GREET, PSAT, VISION, MOVES, and other transportation and energy models. 
Table 6.5

Properties of Conventional and Alternative Fuels

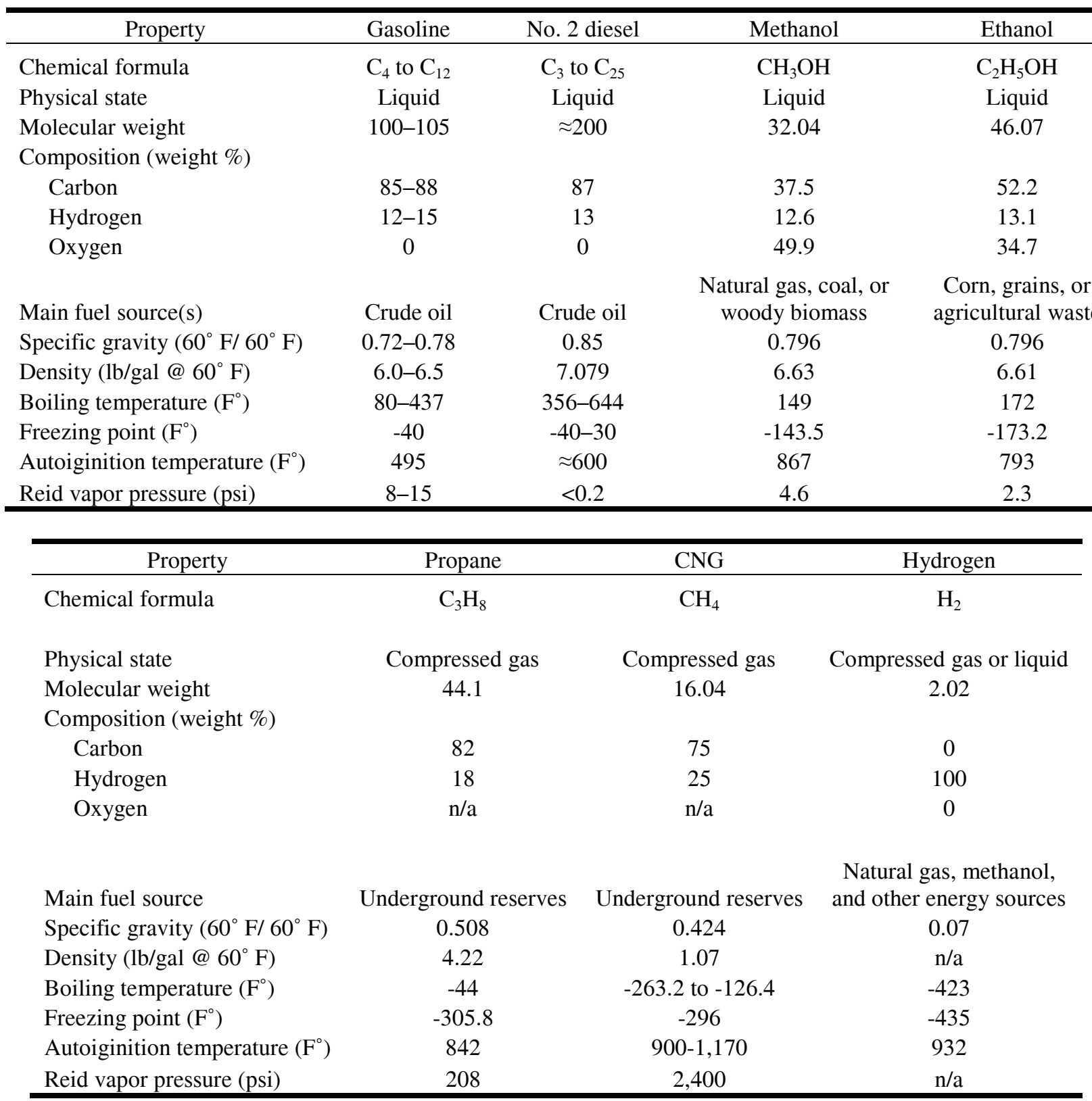

Source:

Alternative Fuels Data Center, "Properties of Fuel," www.eere.energy.gov/afdc/pdfs/fueltable.pdf and "Fuel Comparison," www.eere.energy.gov/afdc/fuels/properties.html, February 2009.

Note: $\mathrm{n} / \mathrm{a}=$ not applicable. 


\section{Chapter 7 \\ Fleet Vehicles and Characteristics}

Summary Statistics from Tables in this Chapter

\begin{tabular}{|c|c|c|}
\hline Source & & \\
\hline Figure 7.1 & Fleet cars, 2008 & $3,986,000$ \\
\hline Figure 7.1 & Fleet trucks $\leq 19,500 \mathrm{lbs} . \mathrm{GVW}, 2008$ & $4,365,000$ \\
\hline \multirow[t]{4}{*}{ Table 7.3} & Average annual miles per business fleet vehicle & \\
\hline & Light trucks & 28,020 \\
\hline & $S U V s$ & 22,968 \\
\hline & Intermediate cars & 23,412 \\
\hline \multirow[t]{8}{*}{ Figure 7.2} & $\begin{array}{l}\text { Average annual miles per Federal Government } \\
\text { fleet vehicle, } 2009\end{array}$ & \\
\hline & Sedans \& station wagons & 11,283 \\
\hline & Buses & 9,799 \\
\hline & $S U V S$ & 9,535 \\
\hline & Heavy trucks & 7,735 \\
\hline & Medium trucks & 7,196 \\
\hline & Light trucks & 5,802 \\
\hline & Ambulances & 5,556 \\
\hline \multirow[t]{6}{*}{ Table 7.4} & Federal government vehicles, FY 2009 & 651,703 \\
\hline & Light trucks $(<8,500 \mathrm{lbs} . G V W)$ & 280,735 \\
\hline & Cars and other passenger vehicles & 239,814 \\
\hline & Medium trucks $(8,500-26,000$ lbs. GVW) & 89,052 \\
\hline & Heavy trucks (>26,000 lbs. GVW) & 32,629 \\
\hline & Buses and ambulances & 9,473 \\
\hline
\end{tabular}


Vehicles in fleets of 15 or more are counted as fleet vehicles, as well as vehicles in fleets where five or more vehicles are purchased annually. Historical data on fleets are not available due to definitional changes of what constitutes a fleet.

Figure 7.1. Fleet Vehicles in Service as of January 1, 2009

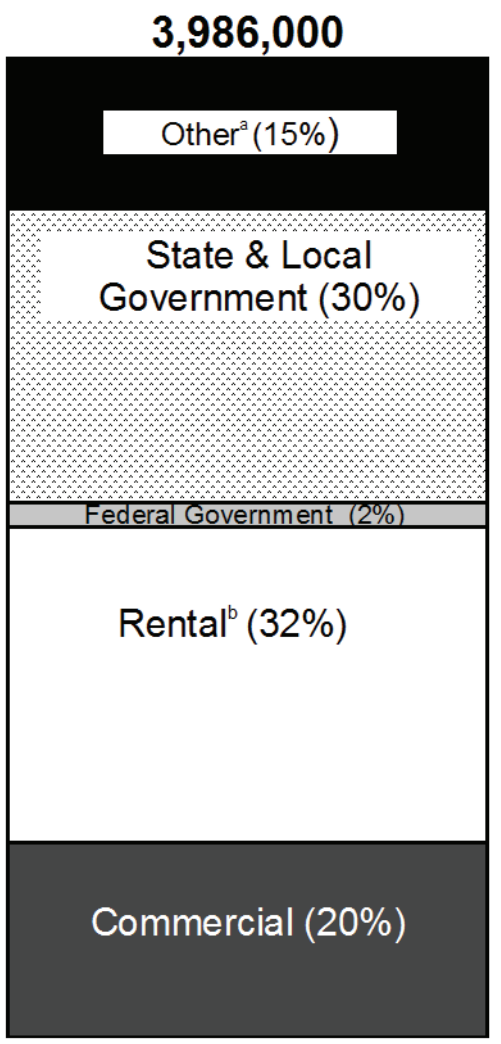

\section{Cars $^{\circ}$}

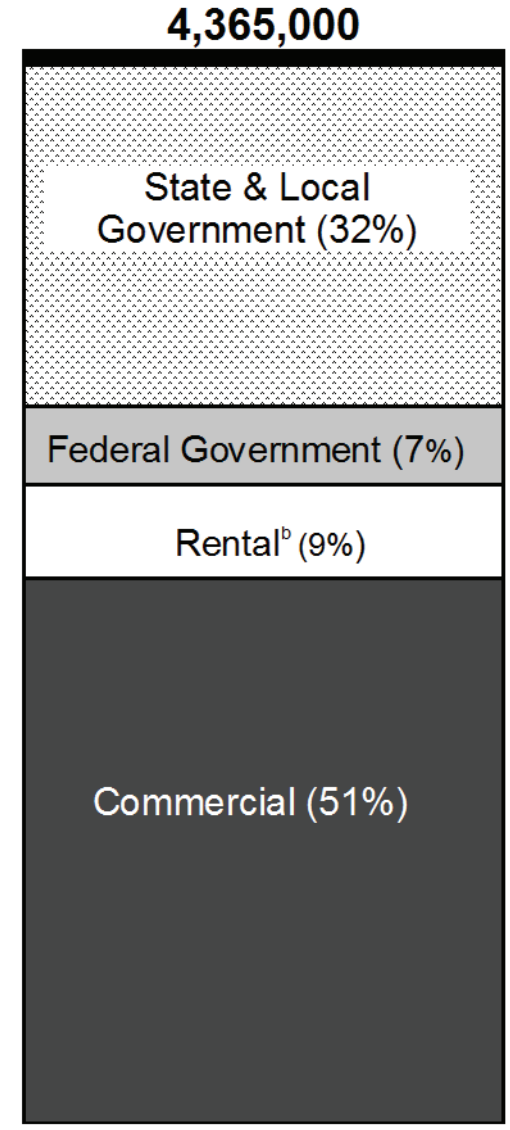

Trucks $<19,50$ I Ibs. gross vehicle weight ${ }^{\circ}$

Source:

Bobit Publishing Company, Automotive Fleet Research Department, Automotive Fleet Factbook 2009, Redondo Beach, CA, 2010. (Additional resources: www.fleet-central.com)

${ }^{a}$ Taxi category includes vans.

${ }^{\mathrm{b}}$ Rental category includes vans and sports utility vehicles under cars, not trucks.

${ }^{\mathrm{c}}$ Fleets of 15 or more in operation or 5 or more fleet vehicles purchased annually. 
Rental companies made the largest light fleet vehicle registrations in 2008 buying nearly 2.0 million vehicles, most of them cars (57.9\%). Over 60\% of the commercial fleet vehicles registrations were pickups.

Table 7.1

New Light Fleet Vehicle Registrations by Vehicle Type, Model Year 2008

\begin{tabular}{|c|c|c|c|c|}
\hline & Commercial & Rental & Government & Total \\
\hline Cars & $27.3 \%$ & $57.9 \%$ & $38.8 \%$ & $47.3 \%$ \\
\hline Light trucks & $64.7 \%$ & $41.1 \%$ & $53.9 \%$ & $49.1 \%$ \\
\hline Medium trucks & $8.0 \%$ & $1.0 \%$ & $7.3 \%$ & $3.6 \%$ \\
\hline Total & 822,864 & $1,779,805$ & 293,639 & $2,896,308$ \\
\hline
\end{tabular}

Source:

Bobit Publishing Company, Automotive Fleet Factbook 2009, www.automotive-fleet.com/statistics. (Additional resources: www.fleet-central.com)

Table 7.2

Average Length of Time Business Fleet Vehicles are in Service, 2008

\begin{tabular}{lc}
\hline Vehicle type & $\begin{array}{c}\text { Average months } \\
\text { in service }\end{array}$ \\
\hline Compact cars & 36 \\
Intermediate cars & 29 \\
Light trucks & 51 \\
Minivans & 36 \\
Sport utility vehicles & 29 \\
Full-size vans & 56 \\
\hline
\end{tabular}

Source:

Bobit Publishing Company, Automotive Fleet Factbook 2009, www.automotive-fleet.com. (Additional resources: www.fleet-central.com)

Note: Based on data collected from four leading Fleet Management companies.

Table 7.3

Average Annual Vehicle-Miles of Travel

for Business Fleet Vehicles, 2008

\begin{tabular}{lc}
\hline Business fleet vehicles & $\begin{array}{c}\text { Average annual miles of } \\
\text { travel }\end{array}$ \\
\hline Compact cars & 23,148 \\
Intermediate cars & 23,412 \\
Light trucks & 28,020 \\
Minivans & 27,852 \\
SUVs & 22,968 \\
Full-size vans & 25,212 \\
\hline
\end{tabular}

\section{Source:}

Bobit Publishing Company, Automotive Fleet Factbook 2009, www.automotive-fleet.com. (Additional resources: www.fleet-central.com) 
These data, which apply to domestic Federal fleet vehicles, indicate that sedans and station wagons have the highest average annual miles per vehicle, followed closely by buses and sport utility vehicles.

Figure 7.2. Average Miles per Domestic Federal Vehicle by Vehicle Type, 2009

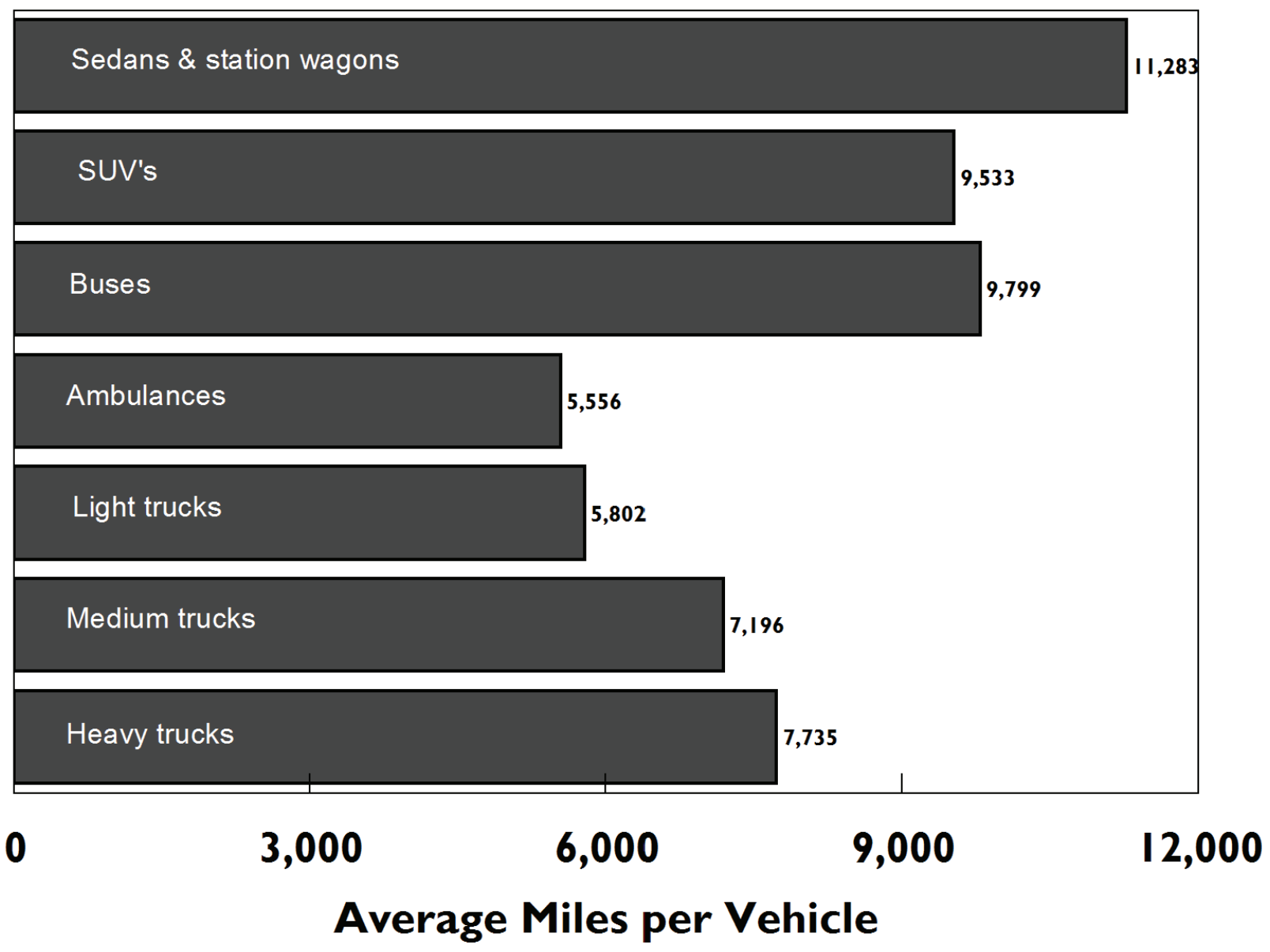

Source:

U.S. General Services Administrations, Federal Vehicle Policy Division, FY 2009 Federal Fleet Report, Washington, DC, January 2010, Table 4-2. (Additional resources: www.gsa.gov) 
The Federal Government vehicle inventory includes more light trucks than passenger vehicles.

Table 7.4

Federal Government Vehicles, 2001-2009

\begin{tabular}{|c|c|c|c|c|c|c|c|c|}
\hline Vehicle Type & 2001 & 2002 & 2004 & 2005 & 2006 & 2007 & 2008 & 2009 \\
\hline \multicolumn{9}{|l|}{ Passenger Vehicles } \\
\hline Subcompact & 5,462 & 4,638 & 4,485 & 2,401 & 2,181 & 1,968 & 3,058 & 5,935 \\
\hline Compact & 60,938 & 57,002 & 55,150 & 58,284 & 56,220 & 48,495 & 41,482 & 36,662 \\
\hline Midsize & 36,921 & 40,779 & 35,012 & 36,656 & 39,762 & 48,622 & 55,157 & 57,284 \\
\hline Large & 11,107 & 11,265 & 16,235 & 15,966 & 11,783 & 11,907 & 10,679 & 10,230 \\
\hline Limousines & 116 & 130 & 227 & 191 & 318 & 217 & 238 & 349 \\
\hline Light Duty Passenger Vans & 56,563 & 61,518 & 42,213 & 42,109 & 41,911 & 43,203 & 43,131 & 41,855 \\
\hline Medium Duty Passenger Vans & 727 & 1,701 & 13,282 & 13,252 & 15,657 & 15,231 & 15,696 & 15,362 \\
\hline Light Duty SUVs & 40,842 & 48,343 & 54,992 & 50,445 & 52,393 & 53,837 & 56,329 & 64,793 \\
\hline Medium Duty SUVs & 0 & 0 & 7,594 & 6,096 & 7,192 & 7,733 & 10,837 & 7,344 \\
\hline Total Passenger Vehicles & 212,676 & 225,376 & 229,190 & 225,400 & 227,417 & 231,213 & 236,607 & 239,814 \\
\hline \multicolumn{9}{|l|}{ Trucks and Other Vehicles } \\
\hline Light Duty $4 \times 2$ & 227,937 & 220,205 & 236,123 & 243,477 & 241,847 & 243,720 & 243,143 & 244,022 \\
\hline Light Duty $4 \times 4$ & 29,975 & 27,108 & 32,121 & 35,417 & 37,019 & 40,115 & 34,962 & 36,713 \\
\hline Medium Duty & 88,993 & 86,949 & 80,474 & 83,747 & 81,721 & 84,414 & 88,509 & 89,052 \\
\hline Heavy Duty & 27,988 & 31,426 & 33,308 & 35,230 & 33,383 & 32,492 & 32,752 & 32,629 \\
\hline Ambulances & 1,819 & 1,710 & 1,405 & 1,580 & 1,601 & 1,982 & 1,474 & 1,433 \\
\hline Buses & 6,726 & 7,313 & 7,530 & 7,837 & 7,752 & 8,297 & 8,044 & 8,040 \\
\hline Total Trucks and Other Vehicles & $\mathbf{3 8 3 , 4 3 8}$ & $\mathbf{3 7 4 , 7 1 1}$ & 390,961 & 407,288 & 403,323 & 411,020 & 408,884 & $\mathbf{4 1 1 , 8 8 9}$ \\
\hline GRAND TOTAL ALL VEHICLES & 596,114 & 600,087 & 620,151 & 632,688 & 630,740 & 642,233 & 645,491 & 651,703 \\
\hline
\end{tabular}

Source:

U.S. General Services Administration, Federal Supply Service, FY 2009 Federal Fleet Report, Washington, DC, 2010, Charts 2-5 and 2-6. (Additional resources: http://www.gsa.gov) 
Table 7.5

Federal Fleet Vehicle Acquisitions

by Fuel Type, FY 2002-2009

\begin{tabular}{lrrrrrrrr}
\hline & \multicolumn{10}{c}{ Acquisitions by Year } \\
\cline { 2 - 9 } Fuel Type & \multicolumn{1}{c}{2002} & \multicolumn{1}{c}{2003} & 2004 & 2005 & 2006 & 2007 & 2008 & \multicolumn{1}{c}{2009} \\
\hline Gasoline & 44,850 & 42,844 & 43,378 & 41,247 & 37,242 & 32,089 & 30,376 & 31,782 \\
Diesel & 8,107 & 5,831 & 5,822 & 6,049 & 6,809 & 5,809 & 5,897 & 4,742 \\
Gasoline hybrid & $\mathrm{a}$ & $\mathrm{a}$ & $\mathrm{a}$ & 222 & 516 & 458 & 531 & 3,959 \\
Diesel hybrid & $\mathrm{b}$ & $\mathrm{b}$ & $\mathrm{b}$ & 1 & 0 & 4 & 0 & 4 \\
CNG & 1,267 & 1,223 & 809 & 188 & 243 & 129 & 123 & 77 \\
E-85 & 8,054 & 19,626 & 13,991 & 16,892 & 18,168 & 26,581 & 27,792 & 27,850 \\
Electric & 7 & 31 & 88 & 13 & 0 & 7 & 6 & 7 \\
LNG & 3 & 0 & 0 & 0 & 0 & 0 & 0 & 0 \\
LPG & 59 & 49 & 26 & 1 & 0 & 4 & 3 & 23 \\
M-85 & 25 & 0 & 0 & 0 & 0 & 0 & 0 & 0 \\
Hydrogen & 0 & 0 & 0 & 0 & 0 & 0 & 1 & 1 \\
\hline Grand Total & 62,372 & 69,604 & 64,114 & 64,613 & 62,978 & 65,081 & 64,729 & 68,445 \\
\hline
\end{tabular}

Source:

U.S. General Services Administrations, Federal Vehicle Policy Division, FY 2009 Federal Fleet Report, Washington, DC, 2010 , Chart 5-4. (Additional resources: www.gsa.gov)

Table 7.6

Fuel Consumed by Federal Government Fleets, FY 2000-2009 (thousand gasoline equivalent gallons)

\begin{tabular}{lrrrrrrrrrr}
\hline & FY00 & \multicolumn{1}{c}{ FY01 } & \multicolumn{1}{c}{ FY02 } & FY03 & FY04 & FY05 & FY06 & FY07 & FY08 & FY09 \\
\hline Gasoline & 284,480 & 281,791 & 281,205 & 296,017 & 284,460 & 300,261 & 288,923 & 293,848 & 292,046 & 301,340 \\
Diesel & 70,181 & 70,761 & 68,487 & 69,109 & 59,199 & 53,363 & 47,489 & 57,700 & 55,617 & 75,540 \\
CNG & 865 & 2,335 & 1,708 & 575 & 1,159 & 1,245 & 807 & 889 & 731 & 499 \\
Electricity & 1 & 35 & 56 & 19 & 3 & 6 & 5 & 5 & 4 & 4 \\
Biodiesel & 569 & 1,315 & 2,252 & 3,753 & 6,470 & 8,052 & 8,334 & 9,483 & 6,976 & 7,379 \\
Methanol/M-85 & 14 & 5 & 4 & 3 & 0 & 0 & 0 & 0 & 0 & 0 \\
LPG & 34 & 102 & 108 & 104 & 126 & 231 & 105 & 322 & 399 & 208 \\
Ethanol/E-85 & 347 & 5,900 & 4,673 & 1,592 & 1,784 & 3,060 & 3,206 & 3,853 & 6,293 & 7,922 \\
LNG & 0 & 52 & 27 & 23 & 91 & 102 & 90 & 95 & 59 & 35 \\
Other & 0 & 0 & 0 & 0 & 0 & 0 & 0 & 0 & 0 \\
\hline Total & 356,491 & 362,296 & 358,520 & 371,195 & 353,292 & 366,320 & 348,959 & 366,195 & 362,125 & 392,927 \\
\hline
\end{tabular}

Source:

U.S. General Services Administrations, Federal Vehicle Policy Division, FY 2009 Federal Fleet Report, Washington, DC, 2010, Chart 5-1. (Additional resources: www.gsa.gov)

${ }^{\mathrm{a}}$ Combined with gasoline.

${ }^{\mathrm{b}}$ Combined with diesel. 
In FY2000, the General Services Administration owned 143,948 vehicles which they leased to other agencies. In FY2009, they owned 1,221 vehicles.

Table 7.7

Federal Government Vehicles by Agency, FY 2009

\begin{tabular}{|c|c|c|c|c|c|c|}
\hline Department or Agency & Cars & Buses & Light trucks ${ }^{\mathrm{a}}$ & $\begin{array}{c}\text { Medium } \\
\text { trucks }^{\mathrm{b}}\end{array}$ & $\begin{array}{l}\text { Heavy } \\
\text { trucks }^{c}\end{array}$ & Total \\
\hline \multicolumn{7}{|l|}{ CIVILIAN } \\
\hline American Battle Monuments Commission & 19 & 0 & 20 & 9 & 1 & 49 \\
\hline Broadcasting Board of Governors & 5 & 10 & 121 & 27 & 19 & 182 \\
\hline Consumer Product Safety Commission & 66 & 0 & 23 & 1 & 0 & 90 \\
\hline Court Services and Offender Supervision Agency & 54 & 0 & 21 & 0 & 0 & 75 \\
\hline Department of Agriculture & 5,488 & 44 & 27,568 & 7,999 & 1,783 & 42,882 \\
\hline Department of Commerce & 350 & 6 & 1,534 & 357 & 51 & 2,298 \\
\hline Department of Education & 30 & 1 & 24 & 0 & 0 & 55 \\
\hline Department of Energy & 840 & 192 & 7,657 & 3,546 & 2,044 & 14,279 \\
\hline Department of Health and Human Services & 1,822 & 8 & 1,935 & 265 & 135 & 4,165 \\
\hline Department of Homeland Security & 13,623 & 470 & 26,891 & 7,367 & 823 & 49,174 \\
\hline Department of Housing and Urban Development & 352 & 0 & 76 & 0 & 0 & 428 \\
\hline Department of Justice & 19,817 & 189 & 17,924 & 1,209 & 842 & 39,981 \\
\hline Department of Labor & 1,249 & 277 & 2,252 & 214 & 33 & 4,025 \\
\hline Department of State & 2,645 & 101 & 6,348 & 2,233 & 438 & 11,765 \\
\hline Department of the Interior & 2,779 & 511 & 16,959 & 9,615 & 3,528 & 33,392 \\
\hline Department of Transportation & 1,624 & 3 & 3,452 & 984 & 101 & 6,164 \\
\hline Department of Treasury & 2,725 & 1 & 1,185 & 10 & 9 & 3,930 \\
\hline Department of Veterans Affairs & 5,024 & 526 & 6,493 & 763 & 653 & 13,459 \\
\hline Environmental Protection Agency & 290 & 7 & 676 & 107 & 29 & 1,109 \\
\hline Equal Employment Opportunity Commission & 62 & 0 & 10 & 0 & 0 & 72 \\
\hline Federal Communications Commission & 8 & 0 & 118 & 0 & 0 & 126 \\
\hline Federal Housing Finance Agency & 2 & 0 & 3 & 0 & 0 & 5 \\
\hline Federal Trade Commission & 1 & 0 & 2 & 1 & 0 & 4 \\
\hline General Services Administration & 659 & 2 & 509 & 45 & 6 & 1,221 \\
\hline Government Printing Office & 6 & 0 & 24 & 0 & 11 & 41 \\
\hline Library of Congress & 8 & 2 & 7 & 2 & 5 & 24 \\
\hline National Aeronautics and Space Administration & 610 & 104 & 1,836 & 801 & 396 & 3,747 \\
\hline National Archives \& Records Administration & 13 & 0 & 44 & 11 & 6 & 74 \\
\hline National Gallery of Art & 1 & 0 & 6 & 3 & 1 & 11 \\
\hline National Labor Relations Board & 39 & 0 & 3 & 0 & 0 & 42 \\
\hline National Science Foundation & 32 & 8 & 174 & 104 & 37 & 355 \\
\hline Nuclear Regulatory Commission & 10 & 0 & 20 & 0 & 5 & 35 \\
\hline Office of Personnel Management & 1,475 & 0 & 108 & 1 & 1 & 1,585 \\
\hline Peace Corps & 25 & 13 & 608 & 0 & 0 & 646 \\
\hline Small Business Administration & 48 & 0 & 29 & 2 & 0 & 79 \\
\hline Smithsonian Institution & 13 & 9 & 329 & 82 & 26 & 459 \\
\hline Social Security Administration & 330 & 7 & 160 & 6 & 29 & 532 \\
\hline Tennessee Valley Authority & 573 & 0 & 1,542 & 671 & 300 & 3,086 \\
\hline U.S. Agency for International Development & 87 & 13 & 658 & 25 & 16 & 799 \\
\hline TOTAL CIVILIAN AGENCIES & 62,804 & 2,504 & 127,349 & 36,460 & 11,328 & $\mathbf{2 4 0 , 4 4 5}$ \\
\hline \multicolumn{7}{|l|}{ MILITARY } \\
\hline Corps of Engineers, Civil Works & 943 & 0 & 4,919 & 1,659 & 656 & 8,177 \\
\hline Defense Agencies & 1,939 & 325 & 2,492 & 562 & 424 & 5,742 \\
\hline Department of Air Force & 4,657 & 1,448 & 17,903 & 17,968 & 5,884 & 47,860 \\
\hline Department of Army & 19,879 & 2,576 & 40,280 & 14,238 & 5,887 & 82,860 \\
\hline Department of Navy & 7,967 & 688 & 18,383 & 6,918 & 2,730 & 36,686 \\
\hline United States Marine Corps & 3,614 & 495 & 5,857 & 2,190 & 1,229 & 13,385 \\
\hline $\begin{array}{r}\text { TOTAL MILITARY AGENCIES } \\
\end{array}$ & 38,999 & $\mathbf{5 , 5 3 2}$ & 89,834 & $\mathbf{4 3 , 5 3 5}$ & 16,810 & $\mathbf{1 9 4 , 7 1 0}$ \\
\hline \multirow[t]{2}{*}{ U.S. POSTAL SERVICE } & 8,657 & 4 & 194,339 & 9,057 & 4,491 & 216,548 \\
\hline & 110,460 & 8,040 & 411,522 & 89,052 & 32,629 & 651,703 \\
\hline
\end{tabular}

Source:

U.S. General Services Administration, Federal Supply Service, FY 2009 Federal Fleet Report, Washington, DC, 2010, Table 1-1. (Additional resources: www.gsa.gov)

${ }^{\mathrm{a}}$ Less than 8,500 pounds GVWR. Includes ambulances.

b $8,501-23,999$ pounds GVWR.

c 24,000 pounds or more GVWR. 


\section{Chapter 8 \\ Household Vehicles and Characteristics}

Summary Statistics from Tables/Figures in this Chapter

\begin{tabular}{|c|c|c|}
\hline Source & & \\
\hline Table 8.2 & Vehicles per capita, 2008 & 0.822 \\
\hline Table 8.3 & Average household transportation expense, 2008 & $17.0 \%$ \\
\hline \multirow[t]{6}{*}{ Table 8.4} & Share of households owning 3 or more vehicles & \\
\hline & 1960 & $2.5 \%$ \\
\hline & 1970 & $5.5 \%$ \\
\hline & 1980 & $17.5 \%$ \\
\hline & 1990 & $17.3 \%$ \\
\hline & 2000 & $18.3 \%$ \\
\hline Table 8.5 & Vehicles per licensed driver, 2009 & 1.03 \\
\hline \multirow[t]{5}{*}{ Figure 8.1} & Average occupancy rates by vehicle type, 2009 & \\
\hline & Pickup Truck & 1.49 \\
\hline & Car & 1.59 \\
\hline & Sports Utility & 1.92 \\
\hline & Van & 2.35 \\
\hline Table 8.9 & Average annual miles per household vehicle, 2009 & 10,100 \\
\hline Table 8.15 & Share of workers who car pooled, 2000 & $11.2 \%$ \\
\hline \multirow[t]{3}{*}{ Table 8.20} & Long-distance trips in the United States, 2001 & \\
\hline & Person-trips & 2,554 million \\
\hline & Person-miles & 1,138 billion \\
\hline
\end{tabular}


The number of vehicles in the United States is growing faster than the population. The growth in vehicle-miles has slowed in recent years. See Table 8.2 for vehicles per capita and vehicle-miles per capita.

Table 8.1

Population and Vehicle Profile, 1950-2008

\begin{tabular}{|c|c|c|c|c|c|c|}
\hline Year & $\begin{array}{l}\text { Resident } \\
\text { population }^{\mathrm{a}} \\
\text { (thousands) }\end{array}$ & $\begin{array}{c}\text { Total } \\
\text { households } \\
\text { (thousands) }\end{array}$ & $\begin{array}{l}\text { Number of } \\
\text { vehicles in } \\
\text { operation } \\
\text { (thousands) }\end{array}$ & $\begin{array}{c}\text { Total vehicle- } \\
\text { miles } \\
\text { (millions) }\end{array}$ & $\begin{array}{c}\text { Number of } \\
\text { licensed drivers } \\
\text { (thousands) }\end{array}$ & $\begin{array}{l}\text { Number of } \\
\text { civilian } \\
\text { employed } \\
\text { persons } \\
\text { (thousands) }\end{array}$ \\
\hline 1950 & 151,868 & 43,554 & 43,501 & 458,246 & 62,194 & 58,920 \\
\hline 1955 & 165,069 & 47,874 & 56,540 & 605,646 & 74,686 & 62,171 \\
\hline 1960 & 179,979 & 52,799 & 67,906 & 718,762 & 87,253 & 65,778 \\
\hline 1965 & 193,526 & 57,436 & 82,066 & 887,812 & 98,502 & 71,088 \\
\hline 1970 & 203,984 & 63,401 & 98,136 & $1,109,724$ & 111,543 & 78,627 \\
\hline 1975 & 215,465 & 71,120 & 120,054 & $1,327,664$ & 129,791 & 85,846 \\
\hline 1980 & 227,225 & 80,776 & 1399831 & $1,527,295$ & 145,295 & 99,303 \\
\hline 1985 & 237,924 & 86,789 & 157,048 & $1,774,826$ & 156,868 & 107,150 \\
\hline 1986 & 240,133 & 88,458 & 162,094 & $1,834,872$ & 159,487 & 109,597 \\
\hline 1987 & 242,804 & 89,479 & 167,193 & $1,921,204$ & 161,975 & 112,440 \\
\hline 1988 & 245,021 & 91,061 & 171,741 & $2,025,962$ & 162,853 & 114,968 \\
\hline 1989 & 247,342 & 92,830 & 175,960 & $2,096,487$ & 165,555 & 117,342 \\
\hline 1990 & 250,132 & 93,347 & 179,299 & $2,144,362$ & 167,015 & 118,793 \\
\hline 1991 & 253,493 & 94,312 & 181,438 & $2,172,050$ & 168,995 & 117,718 \\
\hline 1992 & 256,894 & 95,669 & 181,519 & $2,247,151$ & 173,125 & 118,492 \\
\hline 1993 & 260,255 & 96,391 & 186,315 & $2,296,378$ & 173,149 & 120,259 \\
\hline 1994 & 263,436 & 97,107 & 188,714 & $2,357,588$ & 175,403 & 123,060 \\
\hline 1995 & 266,557 & 98,990 & 193,441 & $2,422,696$ & 176,628 & 124,900 \\
\hline 1996 & 269,667 & 99,627 & 198,294 & $2,485,848$ & 179,539 & 126,708 \\
\hline 1997 & 272,912 & 101,018 & 201,071 & $2,561,695$ & 182,709 & 129,558 \\
\hline 1998 & 276,115 & 102,528 & 205,043 & $2,631,522$ & 184,980 & 131,463 \\
\hline 1999 & 279,295 & 103,874 & 209,509 & $2,691,056$ & 187,170 & 133,488 \\
\hline 2000 & 282,385 & 104,705 & 213,300 & $2,746,925$ & 190,625 & 136,891 \\
\hline 2001 & 285,267 & 108,209 & 216,683 & $2,797,287$ & 191,276 & 136,933 \\
\hline 2002 & 288,028 & 109,297 & 221,027 & $2,855,508$ & 194,296 & 136,485 \\
\hline 2003 & 290,704 & 111,278 & 225,882 & $2,890,450$ & 196,166 & 137,736 \\
\hline 2004 & 293,310 & 112,000 & 231,398 & $2,964,788$ & 198,889 & 139,252 \\
\hline 2005 & 295,994 & 113,343 & 237,697 & $2,989,430$ & 200,549 & 141,730 \\
\hline 2006 & 298,766 & 114,384 & 244,022 & $3,014,371$ & 202,810 & 144,427 \\
\hline 2007 & 301,714 & 116,011 & 248,701 & $3,032,399$ & 205,742 & 146,047 \\
\hline 2008 & 304,483 & 116,783 & 250,239 & $2,973,509$ & 208,321 & 145,362 \\
\hline \multicolumn{7}{|c|}{ Average annual percentage change } \\
\hline 1950-2008 & $1.2 \%$ & $1.7 \%$ & $3.1 \%$ & $3.3 \%$ & $2.1 \%$ & $1.6 \%$ \\
\hline 1998-2008 & $1.0 \%$ & $1.3 \%$ & $2.0 \%$ & $1.2 \%$ & $1.2 \%$ & $1.0 \%$ \\
\hline
\end{tabular}

Sources:

Resident population and civilian employed persons - U.S. Department of Commerce, Bureau of the Census, Statistical Abstract of the United States-2010, Washington, DC, 2010, tables 2, 59, 588, and annual. (Additional resources: www.census.gov)

Vehicles in operation - The Polk Company. FURTHER REPRODUCTION PROHIBITED. (Additional resources: www.polk.com)

Licensed drivers and vehicle-miles - U.S. Department of Transportation, Federal Highway Administration, Highway Statistics 2008, Tables DL-1C and VM-1, and annual. (Additional resources: www.fhwa.dot.gov)

${ }^{\text {a }}$ Estimates as of July 1. Includes Armed Forces in the United States. 
Vehicle-miles per capita reached 10,000 miles in 2004 but have declined since then. There were 1.72 vehicles for every employed civilian in the United States in 2008.

Table 8.2

Vehicles and Vehicle-Miles per Capita, 1950-2008

\begin{tabular}{|c|c|c|c|}
\hline Year & Vehicles per capita & Vehicle-miles per capita & $\begin{array}{c}\text { Vehicles per civilian employed } \\
\text { persons }\end{array}$ \\
\hline 1950 & 0.286 & 3,017 & 0.74 \\
\hline 1955 & 0.343 & 3,669 & 0.91 \\
\hline 1960 & 0.377 & 3,994 & 1.03 \\
\hline 1965 & 0.424 & 4,588 & 1.15 \\
\hline 1970 & 0.481 & 5,440 & 1.25 \\
\hline 1975 & 0.557 & 6,162 & 1.40 \\
\hline $1980^{\circ}$ & 0.615 & 6,722 & 1.41 \\
\hline 1981 & 0.618 & 6,778 & 1.41 \\
\hline 1982 & 0.621 & 6,885 & 1.45 \\
\hline 1983 & 0.629 & 7,069 & 1.46 \\
\hline 1984 & 0.645 & 7,295 & 1.45 \\
\hline 1985 & 0.660 & 7,460 & 1.47 \\
\hline 1986 & 0.675 & 7,641 & 1.48 \\
\hline 1987 & 0.689 & 7,913 & 1.49 \\
\hline 1988 & 0.701 & 8,269 & 1.49 \\
\hline 1989 & 0.711 & 8,476 & 1.50 \\
\hline 1990 & 0.717 & 8,573 & 1.51 \\
\hline 1991 & 0.716 & 8,568 & 1.54 \\
\hline 1992 & 0.707 & 8,747 & 1.53 \\
\hline 1993 & 0.716 & 8,824 & 1.55 \\
\hline 1994 & 0.716 & 8,949 & 1.53 \\
\hline 1995 & 0.726 & 9,089 & 1.55 \\
\hline 1996 & 0.735 & 9,218 & 1.56 \\
\hline 1997 & 0.737 & 9,387 & 1.55 \\
\hline 1998 & 0.743 & 9,531 & 1.56 \\
\hline 1999 & 0.750 & 9,635 & 1.57 \\
\hline 2000 & 0.755 & 9,728 & 1.56 \\
\hline 2001 & 0.760 & 9,808 & 1.58 \\
\hline 2002 & 0.767 & 9,914 & 1.62 \\
\hline 2003 & 0.777 & 9,943 & 1.64 \\
\hline 2004 & 0.789 & 10,108 & 1.66 \\
\hline 2005 & 0.803 & 10,100 & 1.68 \\
\hline 2006 & 0.817 & 10,089 & 1.69 \\
\hline 2007 & 0.824 & 10,051 & 1.70 \\
\hline 2008 & 0.822 & 9,766 & 1.72 \\
\hline \multicolumn{4}{|c|}{ Average annual percentage change } \\
\hline $1950-2008$ & $1.9 \%$ & $2.1 \%$ & $1.5 \%$ \\
\hline $1998-2008$ & $1.1 \%$ & $0.7 \%$ & $0.9 \%$ \\
\hline
\end{tabular}

\section{Sources:}

Resident population and civilian employed persons - U.S. Department of Commerce, Bureau of the Census, Statistical Abstract of the United States-2010, Washington, DC, 2010, tables 2 and 588.

(Additional resources: www.census.gov)

Vehicles in operation - The Polk Company. FURTHER REPRODUCTION PROHIBITED. (Additional resources: www.polk.com)

Vehicle-miles - U.S. Department of Transportation, Federal Highway Administration, Highway Statistics 2008, Table VM-1 and annual. (Additional resources: www.fhwa.dot.gov)

\footnotetext{
${ }^{a}$ Includes all vehicles (light and heavy).
} 
Table 8.3

Average Annual Expenditures of Households by Income, 2008

\begin{tabular}{|c|c|c|c|c|c|}
\hline & \multirow[b]{2}{*}{$\begin{array}{c}\text { All } \\
\text { households }\end{array}$} & \multicolumn{4}{|c|}{ Income before taxes } \\
\hline & & $\begin{array}{r}\text { Less than } \\
\$ 5,000 \\
\end{array}$ & $\begin{array}{r}\$ 5,000- \\
\$ 9,999 \\
\end{array}$ & $\begin{array}{r}\$ 10,000- \\
\$ 14,999 \\
\end{array}$ & $\begin{array}{r}\$ 15,000- \\
\$ 19,999 \\
\end{array}$ \\
\hline \multirow[t]{2}{*}{ Total expenditures } & $\$ 50,486$ & $\$ 23,036$ & $\$ 19,125$ & $\$ 21,120$ & $\$ 25,536$ \\
\hline & \multicolumn{5}{|c|}{ Percentage of total expenditures ${ }^{b}$} \\
\hline Food $^{\mathrm{c}}$ & $12.8 \%$ & $16.8 \%$ & $16.6 \%$ & $15.7 \%$ & $13.9 \%$ \\
\hline Housing & $33.9 \%$ & $39.2 \%$ & $39.9 \%$ & $41.0 \%$ & $39.5 \%$ \\
\hline Apparel and services & $3.6 \%$ & $4.4 \%$ & $4.4 \%$ & $4.7 \%$ & $3.6 \%$ \\
\hline Transportation & $17.0 \%$ & $13.4 \%$ & $15.3 \%$ & $14.1 \%$ & $17.5 \%$ \\
\hline Vehicle purchases (net outlay) & $5.5 \%$ & $1.9 \%$ & $4.2 \%$ & $2.9 \%$ & $5.3 \%$ \\
\hline Gasoline and motor oil & $5.4 \%$ & $5.3 \%$ & $5.7 \%$ & $5.6 \%$ & $5.7 \%$ \\
\hline Other vehicle expenditures & $5.2 \%$ & $4.9 \%$ & $3.9 \%$ & $4.8 \%$ & $5.9 \%$ \\
\hline Public transportation & $1.0 \%$ & $1.3 \%$ & $1.4 \%$ & $0.9 \%$ & $0.6 \%$ \\
\hline Health care & $5.9 \%$ & $6.0 \%$ & $6.3 \%$ & $7.9 \%$ & $8.3 \%$ \\
\hline Entertainment & $5.6 \%$ & $6.0 \%$ & $4.8 \%$ & $4.6 \%$ & $4.6 \%$ \\
\hline Personal Insurance \& pensions & $11.1 \%$ & $1.7 \%$ & $1.6 \%$ & $2.2 \%$ & $3.4 \%$ \\
\hline Others ${ }^{\mathrm{d}}$ & $9.2 \%$ & $11.3 \%$ & $10.1 \%$ & $8.9 \%$ & $8.7 \%$ \\
\hline Households $^{\mathrm{e}}$ (thousands) & 120,770 & 4,463 & 5,340 & 7,883 & 7,625 \\
\hline Percentage of households & $100 \%$ & $3.7 \%$ & $4.4 \%$ & $6.5 \%$ & $6.3 \%$ \\
\hline \multirow[t]{3}{*}{ Average number of vehicles in $\mathrm{HH}$} & 2.0 & 0.9 & 0.8 & 1.0 & 1.2 \\
\hline & \multicolumn{5}{|c|}{ Income before taxes } \\
\hline & $\begin{array}{r}\$ 20,000- \\
\$ 29,999\end{array}$ & $\begin{array}{r}\$ 30,000- \\
\$ 39,999\end{array}$ & $\begin{array}{r}\$ 40,000- \\
\$ 49,999\end{array}$ & $\begin{array}{r}\$ 50,000- \\
\$ 69,999\end{array}$ & $\begin{array}{l}\$ 70,000 \\
\text { and over }\end{array}$ \\
\hline \multirow[t]{2}{*}{ Total expenditures } & $\$ 30,367$ & $\$ 35,778$ & $\$ 40,527$ & $\$ 50,465$ & $\$ 83,700$ \\
\hline & \multicolumn{5}{|c|}{ Percentage of total expenditures ${ }^{b}$} \\
\hline Food $^{\text {c }}$ & $13.9 \%$ & $14.3 \%$ & $13.4 \%$ & $12.7 \%$ & $11.8 \%$ \\
\hline Housing & $37.0 \%$ & $35.1 \%$ & $36.0 \%$ & $33.8 \%$ & $32.0 \%$ \\
\hline Apparel and services & $3.6 \%$ & $3.9 \%$ & $3.1 \%$ & $3.4 \%$ & $3.5 \%$ \\
\hline Transportation & $18.4 \%$ & $18.0 \%$ & $17.8 \%$ & $18.5 \%$ & $16.5 \%$ \\
\hline Vehicle purchases (net outlay) & $5.8 \%$ & $5.8 \%$ & $5.2 \%$ & $6.1 \%$ & $5.5 \%$ \\
\hline Gasoline and motor oil & $6.3 \%$ & $6.5 \%$ & $6.5 \%$ & $6.0 \%$ & $4.7 \%$ \\
\hline Other vehicle expenditures & $5.6 \%$ & $5.0 \%$ & $5.5 \%$ & $5.6 \%$ & $5.0 \%$ \\
\hline Public transportation & $0.7 \%$ & $0.7 \%$ & $0.6 \%$ & $0.8 \%$ & $1.2 \%$ \\
\hline Health care & $7.9 \%$ & $7.5 \%$ & $6.8 \%$ & $6.4 \%$ & $4.9 \%$ \\
\hline Entertainment & $5.4 \%$ & $5.2 \%$ & $5.2 \%$ & $5.8 \%$ & $5.8 \%$ \\
\hline Personal Insurance \& pensions & $5.2 \%$ & $6.7 \%$ & $8.5 \%$ & $10.3 \%$ & $14.6 \%$ \\
\hline Others ${ }^{\mathrm{d}}$ & $7.6 \%$ & $8.3 \%$ & $8.1 \%$ & $8.2 \%$ & $10.0 \%$ \\
\hline Households $^{\mathrm{e}}$ (thousands) & 14,700 & 12,198 & 11,287 & 18,287 & 38,987 \\
\hline Percentage of households & $12.2 \%$ & $10.1 \%$ & $9.3 \%$ & $15.1 \%$ & $32.3 \%$ \\
\hline Average number of vehicles in $\mathrm{HH}$ & 1.5 & 1.7 & 1.9 & 2.2 & 2.7 \\
\hline
\end{tabular}

\section{Source:}

U.S. Department of Labor, Bureau of Labor Statistics, Web site: www.bls.gov/cex/2008/share/income.pdf, October 2009. (Additional resources: www.bls.gov)

${ }^{a}$ Public assistance monies are included in reported income. Data for those reporting income.

${ }^{\mathrm{b}}$ Percentages may not sum to totals due to rounding.

${ }^{\mathrm{c}}$ Includes alcoholic beverages.

d Includes personal care, reading, education, tobacco and smoking supplies, cash contributions, and miscellaneous items.

e The term household refers to a "consumer unit," which is defined differently than households on Table 8.1. 
Household vehicle ownership shows a dramatic increase from 1960 to 1990. In 1960, nearly 79\% of households owned less than two vehicles; by 1990, it declined to 45\%. Census data prior to 1990 indicated that the majority of households owned one vehicle; in 1990 that changed to two vehicles.

Table 8.4

Household Vehicle Ownership, 1960-2000 Census (percentage)

\begin{tabular}{cccccc}
\hline & No & One & Three or & \\
vehicles & vehicle & $\begin{array}{c}\text { Two } \\
\text { vehicles }\end{array}$ & $\begin{array}{c}\text { Total } \\
\text { vehicles }\end{array}$ & \begin{tabular}{c} 
vehicles \\
\hline 1960
\end{tabular} \\
\hline $21.53 \%$ & $56.94 \%$ & $19.00 \%$ & $2.53 \%$ & $54,766,718$ \\
1970 & $17.47 \%$ & $47.71 \%$ & $29.32 \%$ & $5.51 \%$ & $79,002,052$ \\
1980 & $12.92 \%$ & $35.53 \%$ & $34.02 \%$ & $17.52 \%$ & $129,747,911$ \\
1990 & $11.53 \%$ & $33.74 \%$ & $37.35 \%$ & $17.33 \%$ & $152,380,479$ \\
2000 & $9.35 \%$ & $33.79 \%$ & $38.55 \%$ & $18.31 \%$ & $179,417,526$ \\
\hline
\end{tabular}

Source:

U. S. Department of Transportation, Volpe National Transportation Systems Center, Journey-to-Work Trends in the United States and its Major Metropolitan Area, 1960-1990, Cambridge, MA, 1994, p. 2-2.

2000 data - U.S. Bureau of the Census, American Fact Finder, factfinder.census.gov, Table QT-04, August 2001. (Additional resources: www.census.gov)

${ }^{a}$ Estimates using Census Bureau data; these data on the total number of vehicles do not match the figures on Table 8.1. The figures on Table 8.1, from R.L. Polk and Company, are the preferred data. 


\section{National Household Travel Survey Daily Trip Data}

The Department of Transportation (DOT) colleted data on daily trips in 1969, 1977, 1983, 1990 and 1995 via the Nationwide Personal Transportation Survey (NPTS). Data on longer trips were collected in 1977 and 1995 via the American Travel Survey (ATS). For 2001, the DOT combined the collection of long trip and daily trip data into one survey - the 2001 National Travel Household Travel Survey (NHTS).

The NHTS is the nation's inventory of daily and long-distance travel. The survey includes demographic characteristics of households, people, vehicles, and detailed information on daily and longer-distance travel for all purposes by all modes. NHTS survey data are collected from a sample of U.S. households and expanded to provide national estimates of trips and miles by travel mode, trip purpose, and a host of household attributes.

The Nationwide Household Travel Survey was conducted in 2008 but the data have not been released. The 2001 survey data are the latest available at the current time.

The NHTS was designed to continue the NPTS and ATS series, but as with all data surveys, caution should be used when comparing statistics from one survey to another due to changes in terminology, survey procedures, and target population. The 2001 survey collected data on trips of children under 5 years of age, while the previous NPTS did not. Improved methodologies first used in the collection of trip information in the 1995 NPTS make it difficult to compare these data with past NPTS survey data. Thus, the 1990 NPTS trip data have been adjusted to make it comparable with the later surveys.

Table 8.5

Demographic Statistics from the 1969, 1977, 1983, 1990, 1995 NPTS and 2001, 2009 NHTS

\begin{tabular}{lcccccccc}
\hline & & & & & & & & $\begin{array}{c}\text { Percent } \\
\text { change } \\
\end{array}$ \\
& 1969 & 1977 & 1983 & 1990 & 1995 & 2001 & 2009 & $1969-2009$ \\
\hline Persons per household & 3.16 & 2.83 & 2.69 & 2.56 & 2.63 & 2.58 & 2.66 & $-16 \%$ \\
Vehicles per household & 1.16 & 1.59 & 1.68 & 1.77 & 1.78 & 1.89 & 1.92 & $66 \%$ \\
Workers per household & 1.21 & 1.23 & 1.21 & 1.27 & 1.33 & 1.35 & 1.26 & $4 \%$ \\
Licensed drivers per household & 1.65 & 1.69 & 1.72 & 1.75 & 1.78 & 1.77 & 1.87 & $13 \%$ \\
Vehicles per worker & 0.96 & 1.29 & 1.39 & 1.40 & 1.34 & 1.39 & 1.52 & $59 \%$ \\
Vehicles per licensed driver & 0.70 & 0.94 & 0.98 & 1.01 & 1.00 & 1.06 & 1.03 & $47 \%$ \\
Average vehicle trip length (miles) & 8.89 & 8.34 & 7.90 & 8.98 & 9.06 & 9.87 & 10.14 & $14 \%$ \\
\hline
\end{tabular}

Sources:

U.S. Department of Transportation, Federal Highway Administration, 1990 Nationwide Personal Transportation Survey: Summary of Travel Trends, FHWA-PL-92-027, Washington, DC, March 1992, Table 2. Data for 1995, 2001 and 2009 (preliminary) were generated from the Internet site nhts.ornl.gov. (Additional resources: www.fhwa.dot.gov)

Note: Average vehicle trip length for 1990 and 1995 is calculated using only those records with trip mileage information present. The 1969 survey does not include pickups and other light trucks as household vehicles. 
Due to methodology improvements in collecting trip information, the 2001 and 1995 data should be compared only to the 1990 adjusted data. The original 1990 data are comparable to all previous surveys; however, comparisons should always be made with caution because of differing survey methodologies.

Table 8.6

Average Annual Vehicle-Miles, Vehicle Trips and Trip Length per Household 1969, 1977, 1983, 1990, 1995 NPTS and 2001, 2009 NHTS

\begin{tabular}{|c|c|c|}
\hline & ey-to-v & All trips \\
\hline \multicolumn{3}{|c|}{ Average аппиаl vehicle-miles per household } \\
\hline 1969 & 4,183 & 12,423 \\
\hline 1977 & 3,815 & 12,036 \\
\hline 1983 & 3,538 & 11,739 \\
\hline 1990 original & 4,853 & 15,100 \\
\hline 1990 adjusted & 4,853 & 18,161 \\
\hline 1995 & 6,492 & 20,895 \\
\hline 2001 & 5,724 & 21,171 \\
\hline 2009 & 4,325 & 20,251 \\
\hline \multicolumn{3}{|c|}{ Average aпnиal vehicle trips per household } \\
\hline 1969 & 445 & 1,396 \\
\hline 1977 & 423 & 1,442 \\
\hline 1983 & 414 & 1,486 \\
\hline 1990 original & 448 & 1,702 \\
\hline 1990 adjusted & 448 & 2,077 \\
\hline 1995 & 553 & 2,321 \\
\hline 2001 & 479 & 2,171 \\
\hline 2009 & 313 & 2,025 \\
\hline \multicolumn{3}{|c|}{ Average vehicle trip length (miles) } \\
\hline 1969 & 9.4 & 8.9 \\
\hline 1977 & 9.0 & 8.4 \\
\hline 1983 & 8.5 & 7.9 \\
\hline 1990 original & 11.0 & 9.0 \\
\hline 1990 adjusted & 11.0 & 8.9 \\
\hline 1995 & 11.8 & 9.1 \\
\hline 2001 & 12.2 & 9.9 \\
\hline 2009 & 13.9 & 10.1 \\
\hline
\end{tabular}

Sources:

U.S. Department of Transportation, Federal Highway Administration, 1990 Nationwide Personal Transportation Survey: Summary of Travel Trends, FHWA-PL-92-027, Washington, DC, March 1992, Table 7. 1990 adjusted data - Oak Ridge National Laboratory, Oak Ridge, TN, August 1998. 1995 NPTS, 2001, 2009 (preliminary) NHTS data were generated from the Internet site nhts.ornl.gov. (Additional resources: www.fhwa.dot.gov, www-cta.ornl.gov/npts)

${ }^{a}$ It is believed that the methodology changes in the 1995 NPTS did not affect journey-to-work trips; therefore, no adjustment is necessary. 
In 2001 and 2009 vehicle-miles traveled (vmt) for a three-person household is over 28,000 miles. The number of drivers in a household makes a big difference in vmt, as does the presence of children in the household. Households with children have about $75 \%$ more vmt than households without children.

Table 8.7

Average Number of Vehicles and Vehicle Travel per Household, 1990 NPTS and 2001 and 2009 NHTS

\begin{tabular}{|c|c|c|c|c|c|c|}
\hline \multirow{2}{*}{$\begin{array}{l}\text { Number of Licensed } \\
\text { Drivers }\end{array}$} & \multicolumn{3}{|c|}{$\begin{array}{c}\text { Average } \\
\text { number of vehicles } \\
\text { per household }\end{array}$} & \multicolumn{3}{|c|}{$\begin{array}{c}\text { Average } \\
\text { vehicle-miles traveled } \\
\text { per household }\end{array}$} \\
\hline & 1990 & 2001 & 2009 & 1990 & 2001 & 2009 \\
\hline 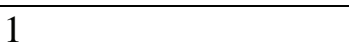 & 1.5 & 1.2 & 1.1 & 15,200 & 9,700 & 11,300 \\
\hline 2 & 2.1 & 2.2 & 2.2 & 22,900 & 25,800 & 23,700 \\
\hline 3 & 2.9 & 3.0 & 3.0 & 29,400 & 37,900 & 36,100 \\
\hline 4 or more & 3.8 & 3.8 & 3.8 & 40,500 & 47,200 & 54,100 \\
\hline \multicolumn{7}{|l|}{ Household size } \\
\hline 1 person & 1.2 & 1.0 & 1.0 & 11,400 & 7,500 & 9,100 \\
\hline 2 persons & 1.9 & 2.0 & 2.0 & 19,300 & 21,200 & 19,100 \\
\hline 3 persons & 2.2 & 2.3 & 2.3 & 23,700 & 28,400 & 28,400 \\
\hline 4 persons & 2.4 & 2.4 & 2.4 & 25,300 & 28,600 & 32,800 \\
\hline 5 persons & 2.4 & 2.4 & 2.5 & 24,900 & 33,200 & 35,500 \\
\hline 6 or more persons & 2.7 & 2.5 & 2.4 & 29,200 & 33,800 & 35,900 \\
\hline \multicolumn{7}{|c|}{ Household urban status } \\
\hline Urban & 1.9 & 1.8 & 1.8 & 19,000 & 19,300 & 20,400 \\
\hline Rural & 2.1 & 2.3 & 2.4 & 22,200 & 28,400 & 27,800 \\
\hline \multicolumn{7}{|c|}{ Household composition } \\
\hline With children & 2.2 & 2.2 & 2.3 & 24,100 & 28,300 & 30,900 \\
\hline Without children & 1.8 & 1.7 & 1.7 & 17,600 & 16,700 & 16,800 \\
\hline All households & 1.8 & 1.9 & 1.9 & 18,300 & 21,200 & 22,200 \\
\hline
\end{tabular}

Source:

Generated from the Department of Transportation, Federal Highway Administration, Nationwide Personal Transportation Survey Public Use Files, Washington, DC, 2000 and the National Household Travel Survey Internet site: nhts.ornl.gov. 2009 data are preliminary. (Additional resources: www-cta.ornl.gov/npts) 
Table 8.8

Trip Statistics by Trip Purpose, 2001 and 2009 NHTS

\begin{tabular}{|c|c|c|c|c|c|c|c|c|}
\hline \multirow[b]{2}{*}{ Trip purpose } & \multicolumn{2}{|c|}{ Share of trips } & \multicolumn{2}{|c|}{$\begin{array}{l}\text { Share of vehicle- } \\
\text { miles traveled }\end{array}$} & \multicolumn{2}{|c|}{$\begin{array}{c}\text { Trip length } \\
\text { (miles) }\end{array}$} & \multicolumn{2}{|c|}{$\begin{array}{c}\text { Trip length } \\
\text { (minutes) }\end{array}$} \\
\hline & 2001 & 2009 & 2001 & 2009 & 2001 & 2009 & 2001 & 2009 \\
\hline To/from work & $22.1 \%$ & $27.1 \%$ & $27.0 \%$ & $27.5 \%$ & 12.1 & 12.7 & 22.3 & 23.4 \\
\hline Work-related business & $4.1 \%$ & $8.4 \%$ & $8.4 \%$ & $6.7 \%$ & 20.3 & 18.0 & 30.9 & 29.3 \\
\hline Shopping & $21.1 \%$ & $16.5 \%$ & $14.5 \%$ & $15.8 \%$ & 6.7 & 6.7 & 14.4 & 14.7 \\
\hline Other family/personal business & $24.7 \%$ & $16.7 \%$ & $18.7 \%$ & $15.7 \%$ & 7.5 & 7.1 & 15.2 & 14.9 \\
\hline School/church & $4.9 \%$ & $3.7 \%$ & $3.7 \%$ & $4.4 \%$ & 7.5 & 8.8 & 15.8 & 17.9 \\
\hline Medical/dental & $2.2 \%$ & $2.2 \%$ & $2.2 \%$ & $2.7 \%$ & 9.9 & 10.2 & 20.7 & 21.1 \\
\hline Vacation & $0.4 \%$ & $1.8 \%$ & $1.8 \%$ & $2.2 \%$ & 47.4 & 32.4 & 59.6 & 42.3 \\
\hline Visit friends/relatives & $6.3 \%$ & $9.1 \%$ & $9.4 \%$ & $10.7 \%$ & 14.9 & 18.3 & 24.4 & 25.8 \\
\hline Other social/recreational & $13.7 \%$ & $13.6 \%$ & $13.2 \%$ & $13.7 \%$ & 9.6 & 8.9 & 18.2 & 17.6 \\
\hline Other & $0.5 \%$ & $0.8 \%$ & $1.0 \%$ & $0.5 \%$ & 18.1 & 18.7 & 31.4 & 28.4 \\
\hline All & $100.0 \%$ & $100.0 \%$ & $100.0 \%$ & $100.0 \%$ & 9.9 & 10.1 & 18.7 & 19.0 \\
\hline
\end{tabular}

Source:

Generated from the National Household Travel Survey Internet site: nhts.ornl.gov. 2009 data are preliminary.

Note: The "All" category for average trip length and duration includes records for which trip purpose was not identified. 
While car occupancy stayed constant from 1995 to 2009, most other vehicle types showed increased occupancy Vans and sport utility vehicles have higher vehicle occupancies than cars.

Figure 8.1. Average Vehicle Occupancy by Vehicle Type, 1995 NPTS and 2009 NHTS

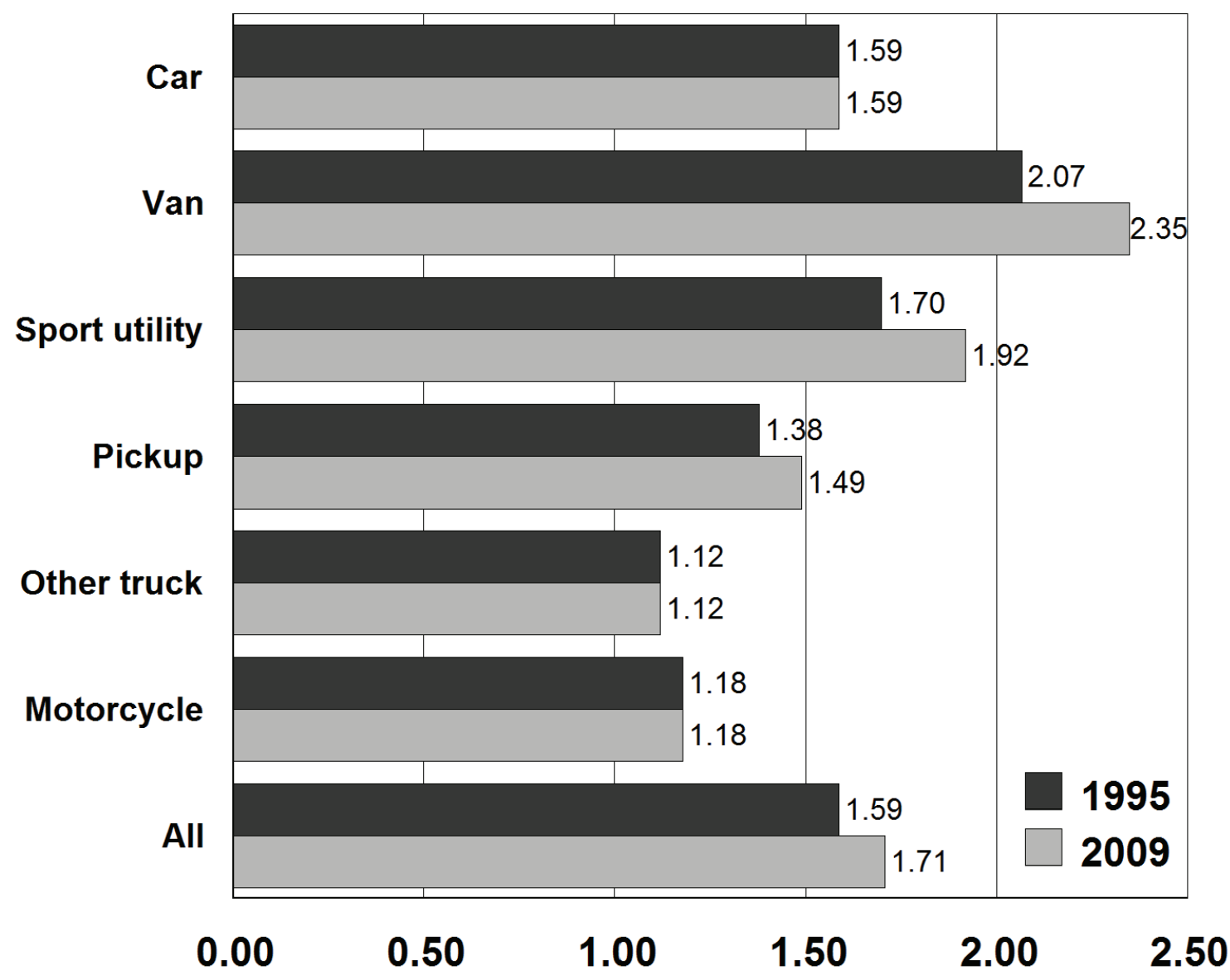

Sources:

U.S. Department of Transportation, Federal Highway Administration, 1995 Nationwide Personal Transportation Survey, Washington, DC, 1997, and 2009 National Household Travel Survey, Washington, DC. 2009 data are preliminary. (Additional resources: www.fhwa.dot.gov, nhts.ornl.gov) 
The average vehicle occupancy, calculated as person-miles per vehicle-mile, is highest for social and recreational purposes. The highest vehicle occupancy levels for all purposes were in 1977. The increase in number of vehicles per household and the decrease in average household size could have contributed to the decline since then.

Figure 8.2. Average Vehicle Occupancy by Trip Purpose 1977 NPTS and 2009 NHTS

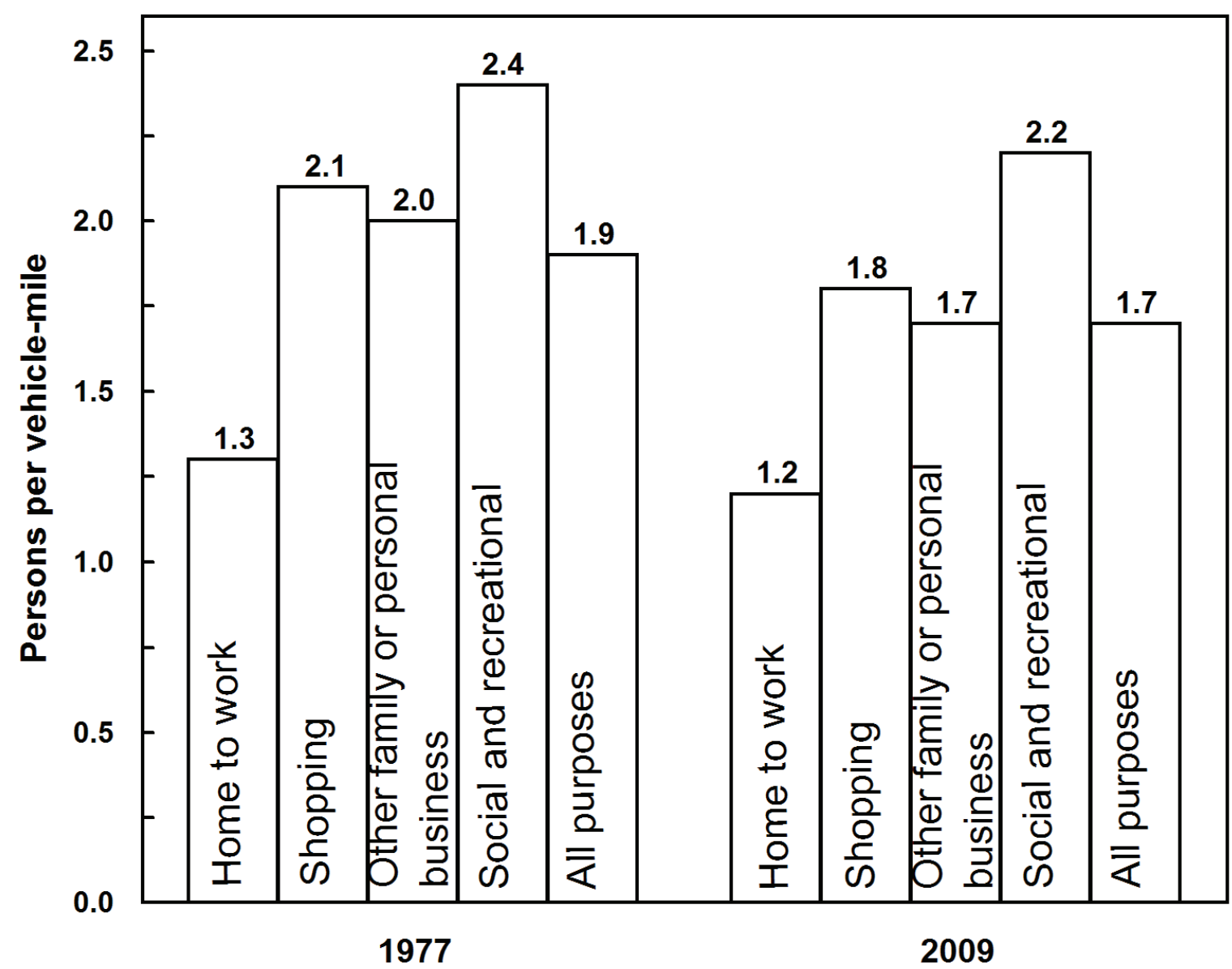

Sources:

U.S. Department of Transportation, Federal Highway Administration, 1990 Nationwide Personal Transportation Survey: Summary of Travel Trends, FHWA-PL-92027, Washington, DC, March 1992, Figure 6. Data from 2009 NHTS were generated from the Internet site nhts.ornl.gov, February 2010. 2009 data are preliminary. (Additional resources: www.fhwa.dot.gov, nhts.ornl.gov) 
The 1990 household survey reports the highest average annual miles per vehicle and the 2009 survey reports the lowest. The low average in 2009 is evidence that the vehicle-miles are growing at a slower rate than the number of vehicles. These data show that younger vehicles are typically driven more miles than older vehicles.

Table 8.9

Average Annual Miles per Household Vehicle by Vehicle Age

\begin{tabular}{cccccc}
\hline $\begin{array}{c}\text { Vehicle age } \\
\text { (years) }\end{array}$ & $\begin{array}{c}1983 \\
\text { self-reported }\end{array}$ & $\begin{array}{c}1990 \\
\text { self-reported }\end{array}$ & $\begin{array}{c}1995 \\
\text { self-reported }\end{array}$ & $\begin{array}{c}2001 \\
\text { self-reported }\end{array}$ & $\begin{array}{c}2009 \\
\text { self-reported }\end{array}$ \\
\hline Under 1 & 8,200 & 19,600 & 15,900 & 15,500 & 12,800 \\
1 & 15,200 & 16,800 & 16,800 & 14,300 & 13,800 \\
2 & 16,800 & 16,600 & 15,500 & 14,000 & 13,500 \\
3 & 14,500 & 14,700 & 14,400 & 13,100 & 12,500 \\
4 & 13,000 & 13,600 & 14,100 & 12,500 & 11,800 \\
5 & 12,100 & 12,900 & 13,500 & 12,000 & 11,700 \\
6 & 11,300 & 13,200 & 13,200 & 11,800 & 11,300 \\
7 & 10,000 & 12,400 & 12,800 & 11,600 & 11,000 \\
8 & 9,800 & 12,600 & 12,200 & 10,900 & 10,300 \\
9 & 9,000 & 11,500 & 12,200 & 10,800 & 9,900 \\
10 and older & 7,300 & 9,200 & 8,900 & 7,400 & 7,300 \\
All household & & & & & \\
vehicles & $\mathbf{1 0 , 4 0 0}$ & $\mathbf{1 2 , 5 0 0}$ & $\mathbf{1 2 , 2 0 0}$ & $\mathbf{1 1 , 1 0 0}$ & $\mathbf{1 0 , 1 0 0}$ \\
\hline
\end{tabular}

Sources:

Nationwide Personal Transportation Study-1983: D. Klinger and J. Richard Kuzmyak, COMSIS Corporation, Personal Travel in the United States, Volume 1: 1983-84 Nationwide Personal Travel Study, prepared for the U.S. Department of Transportation, Washington, DC, August 1986, Table 4-22, p.4-21. 1990: Generated from the 1990 Nationwide Personal Transportation Study Public Use Tape, March 1992. 1995, 2001 and 2009: Generated from the Internet site: nhts.ornl.gov. 2009 data are preliminary. (Additional resources: www.fhwa.dot.gov, www.eia.doe.gov)

Note: Data include all household vehicles, and have been rounded to the nearest hundred. 
Historically, the data from the Nationwide Personal Transportation Survey (NPTS) are based on estimates reported by survey respondents. For the 1995 NPTS and the 2001 National Household Travel Survey (NHTS), odometer data were also collected. The 1995 data indicate that respondents overestimate the number of miles they drive in a year, but the 2001 data do not show that same trend.

Table 8.10

Self-Reported vs. Odometer Average Annual Miles, 1995 NPTS and 2001 NHTS

\begin{tabular}{ccccc}
\hline $\begin{array}{c}\text { Vehicle age } \\
\text { (years) }\end{array}$ & $\begin{array}{c}1995 \\
\text { self-reported }\end{array}$ & $\begin{array}{c}1995 \\
\text { odometer }\end{array}$ & $\begin{array}{c}2001 \\
\text { self-reported }\end{array}$ & $\begin{array}{c}2001 \\
\text { odometer }\end{array}$ \\
\hline Under 1 & 15,900 & 15,600 & 15,500 & 14,500 \\
1 & 16,800 & 14,500 & 14,300 & 14,200 \\
2 & 15,500 & 14,800 & 14,000 & 13,700 \\
3 & 14,400 & 13,800 & 13,100 & 14,100 \\
4 & 14,100 & 12,900 & 12,500 & 13,400 \\
5 & 13,500 & 12,700 & 12,000 & 12,900 \\
6 & 13,200 & 12,400 & 11,800 & 12,400 \\
7 & 12,800 & 11,600 & 11,600 & 12,100 \\
8 & 12,200 & 11,300 & 10,900 & 11,300 \\
9 & 12,200 & 11,200 & 10,800 & 10,500 \\
10 and older & 8,900 & 9,000 & 7,400 & 8,100 \\
All household & & & & \\
vehicles & 12,200 & 11,800 & 11,000 & 11,800 \\
\hline
\end{tabular}

\section{Source:}

Generated from the Internet site: www-cta.ornl.gov/npts and 2001 NHTS public use file.

Note: The 2009 NHTS did not collect similar data. Survey methodology on odometer reading data differs from 1995 to 2001 data. 
Figure 8.3. Share of Vehicle Trips by Trip Distance, 2009 NHTS

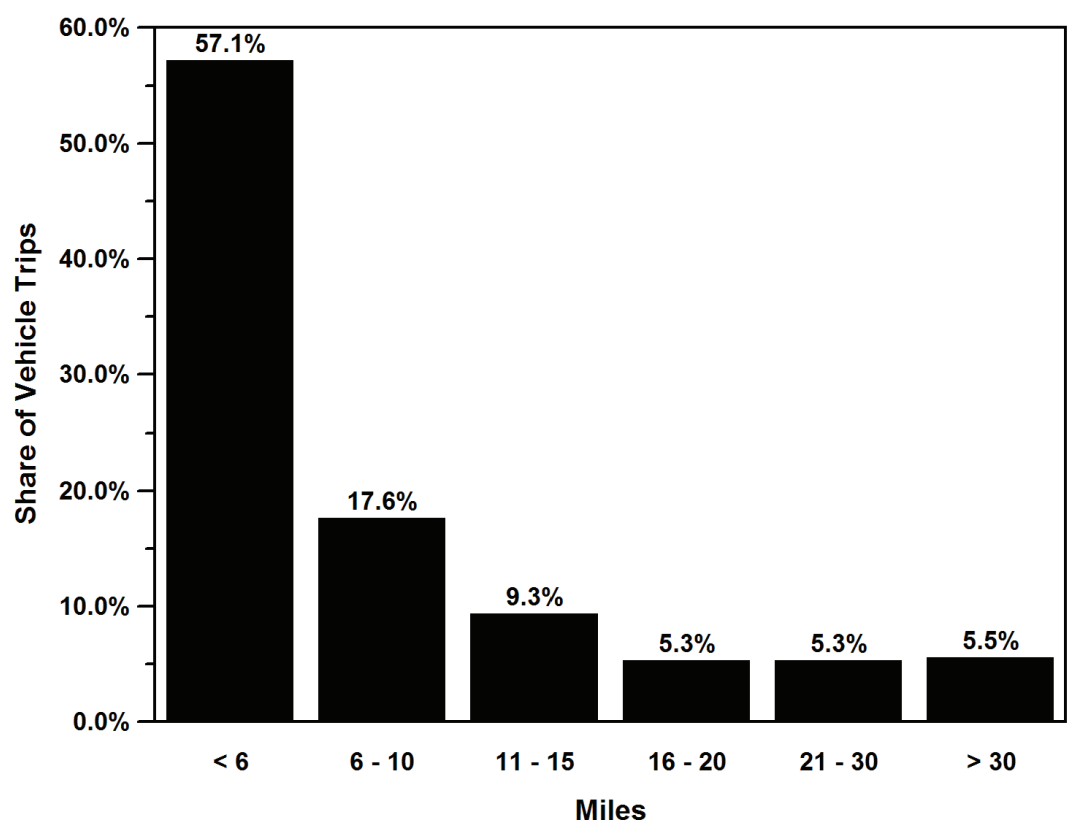

Source:

National Household Travel Survey, https://nhts.ornl.gov. The 2009 data are preliminary.

Figure 8.4. Share of Vehicle Trips to Work by Trip Distance, 2009 NHTS

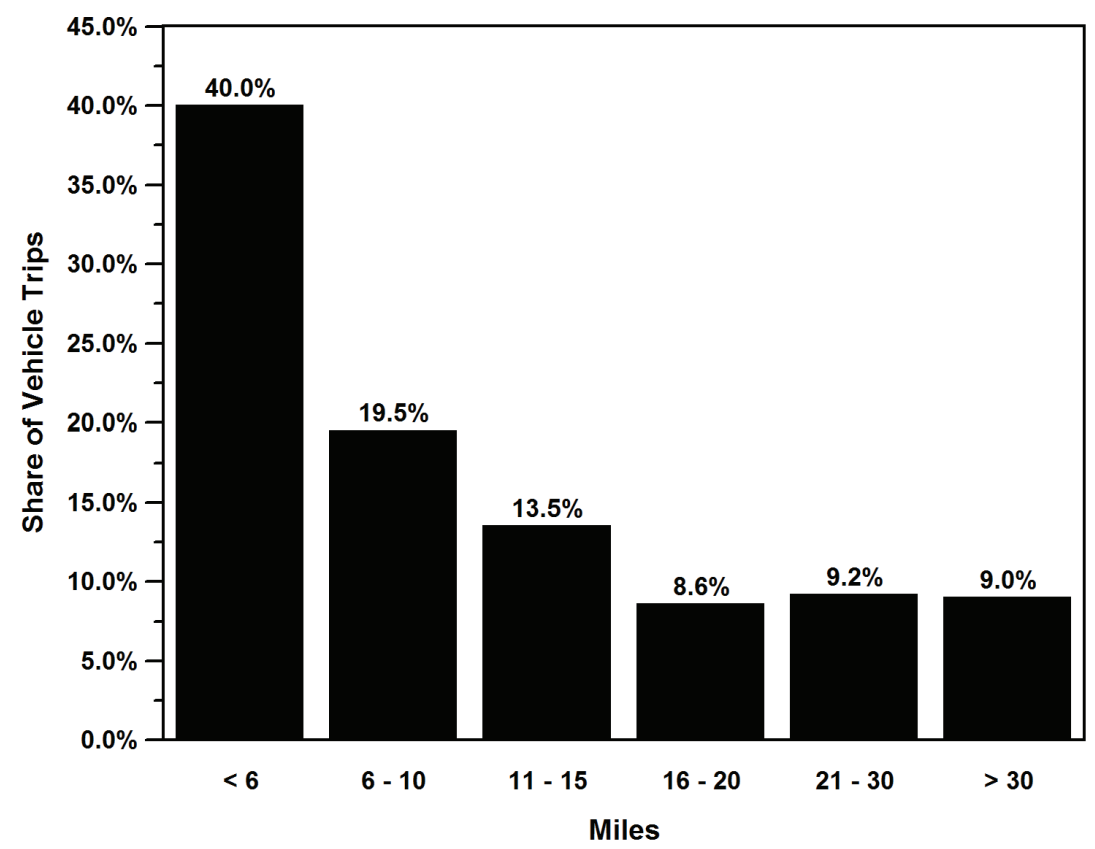

Source:

National Household Travel Survey, https://nhts.ornl.gov. The 2009 data are preliminary. 
Sixteen percent of new vehicles (1 year old and under) travel over 20,000 miles per year. Half of the vehicles over 20 years old travel less than 1,000 miles in a year.

Table 8.11

Share of Vehicles by Annual Miles of Travel and Vehicle Age, 2009 NHTS

\begin{tabular}{|c|c|c|c|c|c|c|c|}
\hline \multirow[b]{2}{*}{$\begin{array}{l}\text { Annual vehicle miles } \\
\text { of travel }\end{array}$} & \multicolumn{7}{|c|}{ Vehicle age (years) } \\
\hline & $\begin{array}{l}1 \text { and } \\
\text { under }\end{array}$ & 2 & 3 & 4 & 5 & 6 & 7 \\
\hline$<1,000$ miles & $2 \%$ & $4 \%$ & $4 \%$ & $4 \%$ & $5 \%$ & $5 \%$ & $5 \%$ \\
\hline $1-2,000$ miles & $2 \%$ & $3 \%$ & $3 \%$ & $3 \%$ & $4 \%$ & $4 \%$ & $4 \%$ \\
\hline 2 - 4,000 miles & $7 \%$ & $8 \%$ & $9 \%$ & $11 \%$ & $10 \%$ & $12 \%$ & $12 \%$ \\
\hline $4-6,000$ miles & $10 \%$ & $9 \%$ & $9 \%$ & $9 \%$ & $9 \%$ & $9 \%$ & $10 \%$ \\
\hline $6-8,000$ miles & $10 \%$ & $11 \%$ & $11 \%$ & $12 \%$ & $12 \%$ & $13 \%$ & $13 \%$ \\
\hline 8 - 10,000 miles & $11 \%$ & $11 \%$ & $11 \%$ & $13 \%$ & $15 \%$ & $14 \%$ & $13 \%$ \\
\hline $10-12,000$ miles & $15 \%$ & $13 \%$ & $12 \%$ & $12 \%$ & $10 \%$ & $12 \%$ & $12 \%$ \\
\hline 12 - 15,000 miles & $14 \%$ & $14 \%$ & $15 \%$ & $14 \%$ & $14 \%$ & $13 \%$ & $13 \%$ \\
\hline 15 - 20,000 miles & $14 \%$ & $12 \%$ & $12 \%$ & $12 \%$ & $12 \%$ & $11 \%$ & $9 \%$ \\
\hline 20 - 30,000 miles & $10 \%$ & $10 \%$ & $9 \%$ & $7 \%$ & $6 \%$ & $6 \%$ & $6 \%$ \\
\hline$>30,000$ miles & $6 \%$ & $5 \%$ & $4 \%$ & $3 \%$ & $3 \%$ & $2 \%$ & $2 \%$ \\
\hline \multirow[t]{3}{*}{ All } & $100 \%$ & $100 \%$ & $100 \%$ & $100 \%$ & $100 \%$ & $100 \%$ & $100 \%$ \\
\hline & \multicolumn{7}{|c|}{ Vehicle age (years) } \\
\hline & 8 & 9 & 10 & $11-15$ & $16-20$ & Over 20 & \\
\hline$<1,000$ miles & $7 \%$ & $7 \%$ & $7 \%$ & $13 \%$ & $21 \%$ & $50 \%$ & \\
\hline $1-2,000$ miles & $5 \%$ & $5 \%$ & $6 \%$ & $7 \%$ & $10 \%$ & $10 \%$ & \\
\hline 2 - 4,000 miles & $14 \%$ & $15 \%$ & $16 \%$ & $18 \%$ & $22 \%$ & $18 \%$ & \\
\hline $4-6,000$ miles & $11 \%$ & $12 \%$ & $12 \%$ & $13 \%$ & $12 \%$ & $7 \%$ & \\
\hline $6-8,000$ miles & $13 \%$ & $13 \%$ & $14 \%$ & $12 \%$ & $9 \%$ & $4 \%$ & \\
\hline 8 - 10,000 miles & $14 \%$ & $14 \%$ & $13 \%$ & $11 \%$ & $8 \%$ & $4 \%$ & \\
\hline 10 - 12,000 miles & $9 \%$ & $9 \%$ & $7 \%$ & $6 \%$ & $5 \%$ & $2 \%$ & \\
\hline 12 - 15,000 miles & $12 \%$ & $10 \%$ & $10 \%$ & $9 \%$ & $6 \%$ & $2 \%$ & \\
\hline 15 - 20,000 miles & $8 \%$ & $8 \%$ & $8 \%$ & $6 \%$ & $4 \%$ & $2 \%$ & \\
\hline 20 - 30,000 miles & $4 \%$ & $4 \%$ & $5 \%$ & $3 \%$ & $2 \%$ & $1 \%$ & \\
\hline$>30,000$ miles & $3 \%$ & $2 \%$ & $2 \%$ & $2 \%$ & $1 \%$ & $1 \%$ & \\
\hline All & $100 \%$ & $100 \%$ & $100 \%$ & $100 \%$ & $100 \%$ & $100 \%$ & \\
\hline
\end{tabular}

\section{Source:}

Generated from the Department of Transportation, Federal Highway Administration, 2009 National Household Travel Survey Internet site: nhts.ornl.gov. 2009 data are preliminary. (Additional resources: nhts.ornl.gov) 
Table 8.12

Household Vehicle Trips, 2009 NHTS

\begin{tabular}{cccc}
\hline & $\begin{array}{c}\text { Number of daily } \\
\text { vehicle trips } \\
\text { (per driver) }\end{array}$ & $\begin{array}{c}\text { Average } \\
\text { vehicle trip } \\
\text { length (miles) }\end{array}$ & $\begin{array}{c}\text { Daily vehicle } \\
\text { miles of travel } \\
\text { (per driver) }\end{array}$ \\
\hline 1990 & 3.3 & 8.9 & 28.5 \\
1995 & 3.6 & 9.1 & 32.1 \\
2001 & 3.4 & 9.9 & 32.7 \\
2009 & 3.0 & 10.1 & 29.7 \\
\hline
\end{tabular}

Source:

U.S. Department of Transportation, Summary of Travel Trends, 2001 Household Travel Survey, December 2004, p. 12. 2009 data from nhts.ornl.gov. 2009 data are preliminary.

Figure 8.5. Average Daily Miles Driven (per Driver), 2009 NHTS

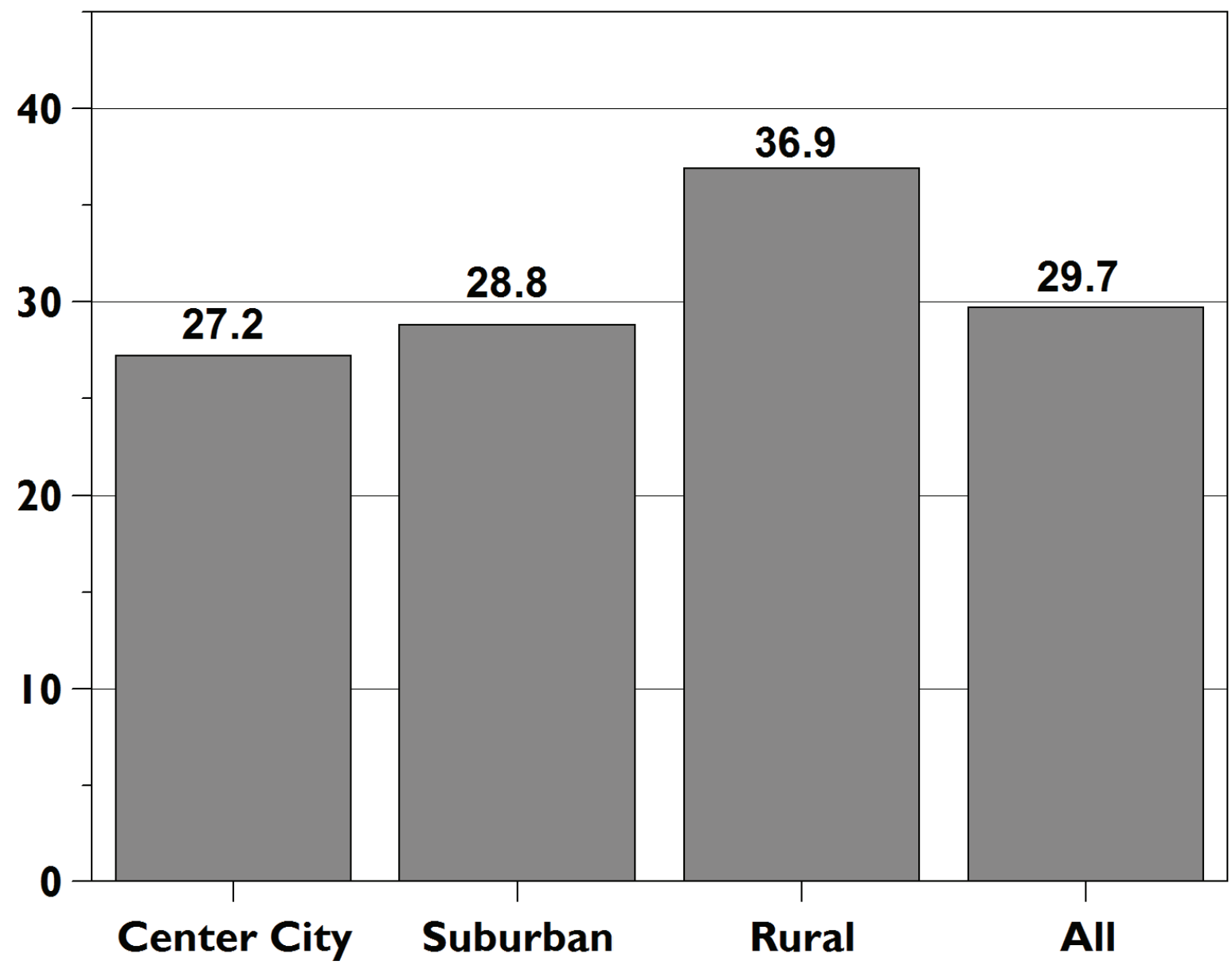

Source:

National Household Travel Survey, nhts.ornl.gov. The 2009 data are preliminary. 
Table 8.13

Daily Vehicle Miles of Travel (per Vehicle) by Number of Vehicles in the Household, 2009 NHTS

\begin{tabular}{ccc}
\hline & \multicolumn{2}{c}{ Daily miles per vehicle } \\
\cline { 2 - 3 } Number of household vehicles & 2001 & 2009 \\
\hline 1 & 25.6 & 25.7 \\
2 & 27.5 & 29.9 \\
3 & 24.2 & 27.2 \\
4 & 23.0 & 26.1 \\
5 & 21.1 & 23.2 \\
More than 5 & 18.4 & 23.8 \\
All & 25.2 & 27.7 \\
\hline
\end{tabular}

Source:

2009 National Household Travel Survey, nhts.ornl.gov. The 2009 data are preliminary.

Table 8.14

Daily and Annual Vehicle Miles of Travel and Average Age for Each Vehicle in a Household, 2009 NHTS

\begin{tabular}{cccc}
\hline $\begin{array}{c}\text { Vehicle number } \\
\text { One-vehicle household }\end{array}$ & $\begin{array}{c}\text { Average } \\
\text { daily miles }\end{array}$ & $\begin{array}{c}\text { Average } \\
\text { annual miles }\end{array}$ & $\begin{array}{c}\text { Average age } \\
\text { (years) }\end{array}$ \\
$\begin{array}{c}\text { Two-vehicle household } \\
1\end{array}$ & 25.7 & 9,400 & 9.0 \\
2 & 39.4 & 14,400 & 7.0 \\
Three-vehicle household & 19.6 & 7,200 & 9.3 \\
1 & 44.7 & 16,300 & 7.0 \\
2 & 24.1 & 8,800 & 8.8 \\
3 & 10.5 & 3,800 & 12.8 \\
Four-vehicle household & & & \\
1 & 50.7 & 18,500 & 7.1 \\
2 & 27.8 & 10,200 & 8.5 \\
3 & 15.4 & 5,600 & 11.6 \\
4 & 6.7 & 2,500 & 15.1 \\
1 & & & \\
2 & 51.3 & 18,700 & 7.2 \\
3 & 29.2 & 10,700 & 9.0 \\
4 & 17.3 & 6,300 & 11.0 \\
5 & 9.7 & 3,500 & 14.1 \\
Five-vehicle household & 4.2 & 1,600 & 18.3 \\
1 & & & \\
2 & 63.6 & 23,200 & 7.6 \\
3 & 32.6 & 11,900 & 9.3 \\
4 & 19.6 & 7,200 & 10.2 \\
5 & 10.7 & 3,900 & 12.2 \\
6 & 5.5 & 2,000 & 16.9 \\
Six-vehicle household & 2.5 & 900 & 20.4 \\
\hline & & &
\end{tabular}

Source:

2009 National Household Travel Survey, nhts.ornl.gov. The 2009 data are preliminary. 
Figure 8.6. Daily Vehicle Miles of Travel for Each Vehicle in a Household, 2009 NHTS

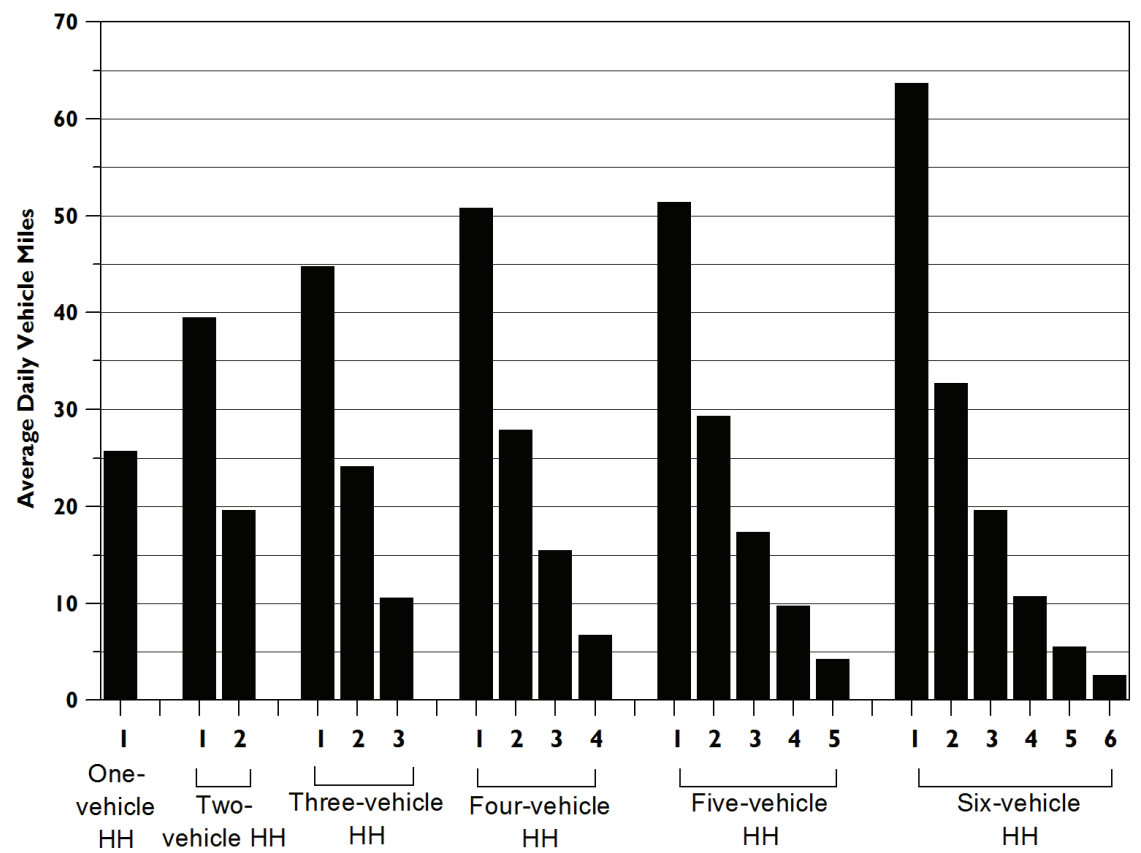

Source:

2009 National Household Travel Survey, nhts.ornl.gov. The 2009 data are preliminary.

Figure 8.7. Annual Vehicle Miles of Travel for Each Vehicle in a Household, 2009 NHTS

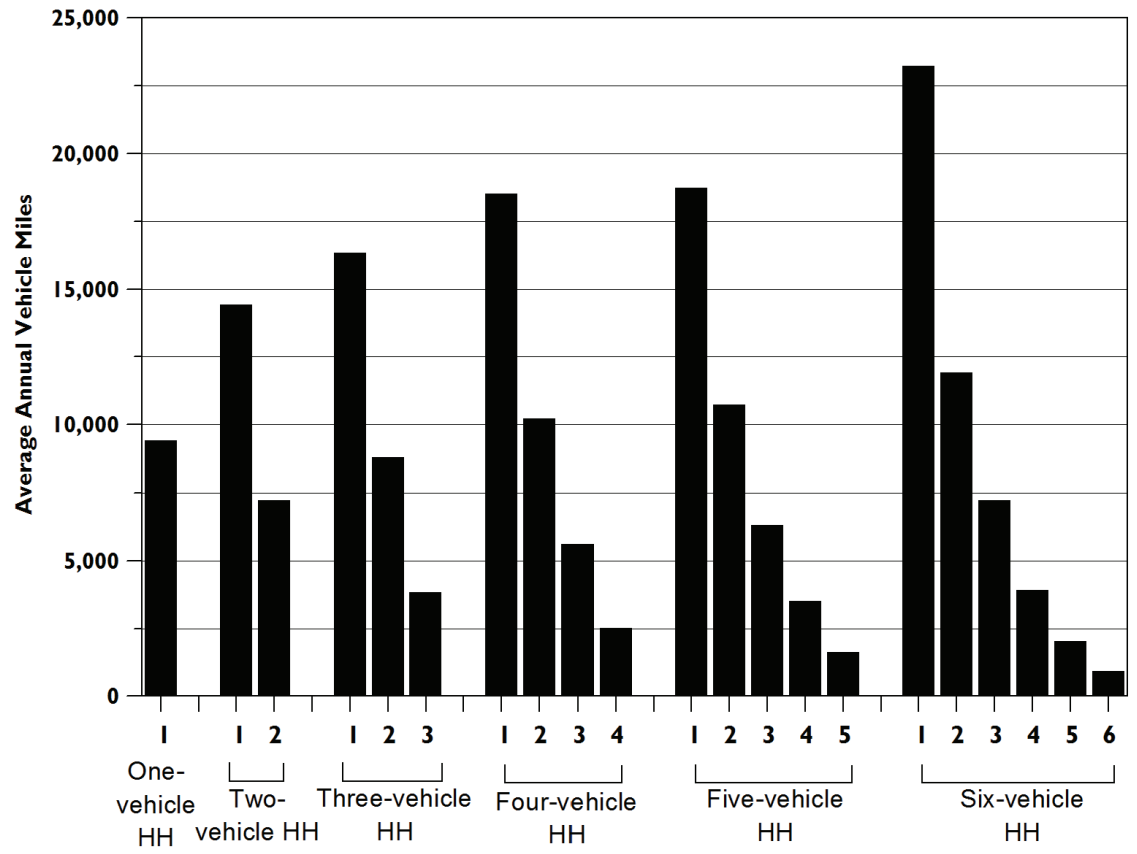

Source:

2009 National Household Travel Survey, nhts.ornl.gov. The 2009 data are preliminary. 
According to the U.S. Census data, the percentage of workers who car pooled has dropped from $19.7 \%$ in 1980 to $11.2 \%$ in 2000. The percent of workers using public transit declined from $6.4 \%$ to $5.3 \%$ in the ten-year period between 1980 and 1990, but stayed relatively the same from 1990 to 2000 (5.2\%). The average travel time increased by 2.6 minutes from 1980 to 2000.

Table 8.15

Means of Transportation to Work, 1980, 1990 and 2000 Census

\begin{tabular}{|c|c|c|c|c|c|c|}
\hline \multirow[b]{2}{*}{ Means of transportation } & \multicolumn{2}{|c|}{1980 Census } & \multicolumn{2}{|c|}{1990 Census } & \multicolumn{2}{|c|}{2000 Census } \\
\hline & $\begin{array}{c}\text { Number of } \\
\text { workers } \\
\text { (thousands) }\end{array}$ & Share & $\begin{array}{c}\text { Number of } \\
\text { workers } \\
\text { (thousands) }\end{array}$ & Share & $\begin{array}{l}\text { Number of } \\
\text { workers } \\
\text { (thousands) }\end{array}$ & Share \\
\hline Private vehicle & 81,258 & $84.1 \%$ & 99,593 & $86.5 \%$ & 111,554 & $87.5 \%$ \\
\hline Drove alone & 62,193 & $64.4 \%$ & 84,215 & $73.2 \%$ & 97,247 & $76.3 \%$ \\
\hline Car pooled & 19,065 & $19.7 \%$ & 15,378 & $13.4 \%$ & 14,307 & $11.2 \%$ \\
\hline Public transportation & 6,175 & $6.4 \%$ & 6,070 & $5.3 \%$ & 6,575 & $5.2 \%$ \\
\hline Bus or trolley bus ${ }^{a}$ & 3,925 & $4.1 \%$ & 3,445 & $3.0 \%$ & 3,572 & $2.8 \%$ \\
\hline Streetcar or trolley car ${ }^{a}$ & $b$ & $b$ & 78 & $0.1 \%$ & 88 & $0.1 \%$ \\
\hline Subway or elevated & 1,529 & $1.6 \%$ & 1,755 & $1.5 \%$ & 1,981 & $1.6 \%$ \\
\hline Railroad & 554 & $0.6 \%$ & 574 & $0.5 \%$ & 696 & $0.5 \%$ \\
\hline Ferryboat & $b$ & $b$ & 37 & $0.0 \%$ & 43 & $0.0 \%$ \\
\hline Taxicab & 167 & $0.2 \%$ & 179 & $0.2 \%$ & 194 & $0.2 \%$ \\
\hline Motorcycle & 419 & $0.4 \%$ & 237 & $0.2 \%$ & 158 & $0.1 \%$ \\
\hline Bicycle & 468 & $0.5 \%$ & 467 & $0.4 \%$ & 563 & $0.4 \%$ \\
\hline Walked only & 5,413 & $5.6 \%$ & 4,489 & $3.9 \%$ & 3,413 & $2.7 \%$ \\
\hline Other means & 703 & $0.7 \%$ & 809 & $0.7 \%$ & 1,099 & $0.9 \%$ \\
\hline Worked at home & 2,180 & $2.3 \%$ & 3,406 & $3.0 \%$ & 4,075 & $3.2 \%$ \\
\hline Total workers & 96,617 & $100.0 \%$ & 115,070 & $100.0 \%$ & 127,437 & $100.0 \%$ \\
\hline Average travel time (minutes) & 21.7 & & 22.4 & & 24.3 & \\
\hline
\end{tabular}

\section{Sources:}

1980-1990 data - Provided by the Journey-to-Work and Migration Statistics Branch, Population Division, U.S. Bureau of the Census

2000 data - U.S. Bureau of the Census, American Fact Finder, factfinder.census.gov, Tables QT-03 and P047, August 2001. (Additional resources: www.census.gov)

\footnotetext{
${ }^{\text {a }}$ This category was "Bus or streetcar" in 1980.

${ }^{\mathrm{b}}$ Data are not available.
} 
Table 8.16

Characteristics of U.S. Daily per Vehicle Driving vs. Dwelling Unit Type and Density

\begin{tabular}{lcccc}
\hline & $\begin{array}{c}\text { Share of } \\
\text { vehicles in } \\
\text { density type }\end{array}$ & $\begin{array}{c}\text { Hours per } \\
\text { vehicle } \\
\text { per day }\end{array}$ & $\begin{array}{c}\text { Average vehicle } \\
\text { speed } \\
\text { (miles/hour) }\end{array}$ & $\begin{array}{c}\text { Miles } \\
\text { per vehicle } \\
\text { per day }\end{array}$ \\
\hline All classes detached single & $79.0 \%$ & 1.24 & 31.4 & 39.0 \\
All classes other & $21.0 \%$ & 1.28 & 29.3 & 37.3 \\
$<1,000 /$ sq. mile detached single & $84.2 \%$ & 1.27 & 34.3 & 43.5 \\
$<1,000 /$ sq. mile all other & $15.8 \%$ & 1.30 & 33.1 & 42.8 \\
1,000-4,000/sq. mile detached single & $80.2 \%$ & 1.21 & 29.3 & 35.5 \\
1,000-4,000/sq. mile all other & $19.8 \%$ & 1.24 & 29.7 & 36.8 \\
4,000-10,000/sq. mile detached single & $72.9 \%$ & 1.19 & 27.1 & 32.3 \\
4,000-10,000/sq. mile all other & $27.1 \%$ & 1.25 & 26.6 & 33.2 \\
10,000-25,000/sq. mile detached single & $46.5 \%$ & 1.31 & 23.3 & 30.6 \\
10,000-25,000/sq. mile all other & $53.5 \%$ & 1.32 & 23.7 & 31.3 \\
$>25,000 /$ sq. mile detached single & $20.5 \%$ & 1.41 & 20.1 & 28.5 \\
$>25,000 /$ sq. mile all other & $79.5 \%$ & 1.40 & 20.8 & 29.1 \\
\hline
\end{tabular}

Source:

Vyas, Anant, Danilo Santini, Michael Duoba, and Mark Alexander, "Plug-In Hybrid Electric Vehicles: How Does One Determine Their Potential for Reducing U.S. Oil Dependence?" Original Data: 2001 National Household Survey.

Note: Data are not currently available in 2009 NHTS to update this table.

Table 8.17

Housing Unit Characteristics, 2007

\begin{tabular}{lcc}
\hline & $\begin{array}{c}\text { Share of occupied } \\
\text { housing units }\end{array}$ & $\begin{array}{c}\text { \% with garage or } \\
\text { carport }\end{array}$ \\
\hline Type of Housing Unit & & \\
New construction (<= 4 years) & $5.2 \%$ & $82.8 \%$ \\
Manufactured/mobile homes $_{\text {With physical problems }}^{\mathrm{a}}$ & $6.3 \%$ & $35.3 \%$ \\
All other & $5.2 \%$ & $38.0 \%$ \\
\hline Geographic Location (Census Region) $^{\text {Northeast }}$ & $83.3 \%$ & $68.2 \%$ \\
Midwest & & \\
South & $18.4 \%$ & $51.1 \%$ \\
West & $22.8 \%$ & $72.8 \%$ \\
\hline Type of Location & $36.7 \%$ & $58.8 \%$ \\
MSA - Central City & $22.0 \%$ & $80.3 \%$ \\
MSA - Suburbs & & \\
Outside MSA & $28.5 \%$ & $55.9 \%$ \\
\hline
\end{tabular}

Source:

U.S. Bureau of the Census, 2007 American Housing Survey, Table 2-7. (Additional information: www.census.gov/prod/2008pubs/h150-07.pdf.)

${ }^{\text {a }}$ Physical problems include problems with plumbing, heating, electric, upkeep, and/or hallways. For detailed definitions of "moderate" and "severe" physical problems, see American Housing Survey for the United States, 2007, pages A-20 and A-21. 
More than half of workers had 15-29 minute commutes in 1990, but that dropped to 35\% by 2000. The share of workers commuting less than 15 minutes increased the most in the ten-year period (14 percentage points), but the share of workers commuting 30 minutes or more also saw small increases.

Table 8.18

Workers by Commute Time, 1990 and 2000 Census

\begin{tabular}{lcc}
\hline \multicolumn{1}{c}{ Commute time } & 1990 & 2000 \\
\hline Less than 15 minutes & $15.9 \%$ & $30.1 \%$ \\
15-29 minutes & $51.6 \%$ & $36.3 \%$ \\
30-39 minutes & $14.7 \%$ & $15.7 \%$ \\
$40-59$ minutes & $9.0 \%$ & $10.7 \%$ \\
60 minutes or more & $5.9 \%$ & $7.3 \%$ \\
\hline Average travel time (minutes) & 22.4 & 24.3 \\
\hline
\end{tabular}

\section{Sources:}

1990 - U. S. Department of Transportation, Volpe National Transportation Systems Center, Journey-to-Work Trends in the United States and its Major Metropolitan Area, 1960-1990, FHWA-PL-94-012, Cambridge, MA, 1994, p. 2-6.

2000 - U.S. Bureau of the Census, American Fact Finder, factfinder.census.gov, Tables QT-03 and P048, August 2001. (Additional resources: www.census.gov) 
Sales of bicycles with wheel sizes of 20 inches and over have grown at an average annual rate of $1.5 \%$ from 1981 to 2008. The largest growth in bicycle sales, however, were bicycles with wheel sizes over 20 inches which grew at an average annual rate of $1.9 \%$ from 1998 to 2008.

Table 8.19

Bicycle Sales, 1981-2008

(millions)

\begin{tabular}{|c|c|c|c|}
\hline & $\begin{array}{c}\text { Wheel } \\
\text { sizes under } \\
20 \text { inches }\end{array}$ & $\begin{array}{l}\text { Wheel sizes } \\
\text { of } 20 \text { inches } \\
\text { and over }\end{array}$ & $\begin{array}{c}\text { All } \\
\text { wheel } \\
\text { sizes }\end{array}$ \\
\hline 1981 & a & 8.9 & $\mathrm{a}$ \\
\hline 1982 & a & 6.8 & a \\
\hline 1983 & a & 9.0 & a \\
\hline 1984 & a & 10.1 & $\mathrm{a}$ \\
\hline 1985 & a & 11.4 & a \\
\hline 1986 & $\mathrm{a}$ & 12.3 & $\mathrm{a}$ \\
\hline 1987 & a & 12.6 & a \\
\hline 1988 & a & 9.9 & a \\
\hline 1989 & a & 10.7 & a \\
\hline 1990 & $\mathrm{a}$ & 10.8 & a \\
\hline 1991 & $\mathrm{a}$ & 11.6 & $\mathrm{a}$ \\
\hline 1992 & 3.7 & 11.6 & 15.3 \\
\hline 1993 & 3.8 & 13.0 & 16.8 \\
\hline 1994 & 4.2 & 12.5 & 16.7 \\
\hline 1995 & 4.1 & 12.0 & 16.1 \\
\hline 1996 & 4.5 & 10.9 & 15.4 \\
\hline 1997 & 4.2 & 11.0 & 15.2 \\
\hline 1998 & 4.7 & 11.1 & 15.8 \\
\hline 1999 & 5.9 & 11.6 & 17.5 \\
\hline 2000 & 9.0 & 11.9 & 20.9 \\
\hline 2001 & 5.4 & 11.3 & 16.7 \\
\hline 2002 & 5.9 & 13.6 & 19.5 \\
\hline 2003 & 5.6 & 12.9 & 18.5 \\
\hline 2004 & 5.3 & 13.0 & 18.3 \\
\hline 2005 & 5.8 & 14.0 & 19.8 \\
\hline 2006 & 5.5 & 12.7 & 18.2 \\
\hline 2007 & 5.4 & 12.8 & 18.2 \\
\hline 2008 & 5.1 & 13.4 & 18.5 \\
\hline \multicolumn{4}{|c|}{ Average annual percentage change } \\
\hline 1981-2008 & a & $1.5 \%$ & $\mathrm{a}$ \\
\hline 1998-2008 & $0.8 \%$ & $1.9 \%$ & $1.6 \%$ \\
\hline
\end{tabular}

\section{Source:}

1981-1996: Bicycle Manufacturers Association. 1997-on: National Bicycle Dealers Association. (Additional resources: www.nbda.com)

\footnotetext{
${ }^{\mathrm{a}}$ Data are not available.
} 
In 2001, 4.5\% of walk trips and 10.9\% of bike trips were to/from work. Forty-six percent of all bike trips were for social/recreational purposes. Fifteen percent of walk trips were shopping trips.

Figure 8.8. Walk and Bike Trips by Trip Purpose, 2009 NHTS

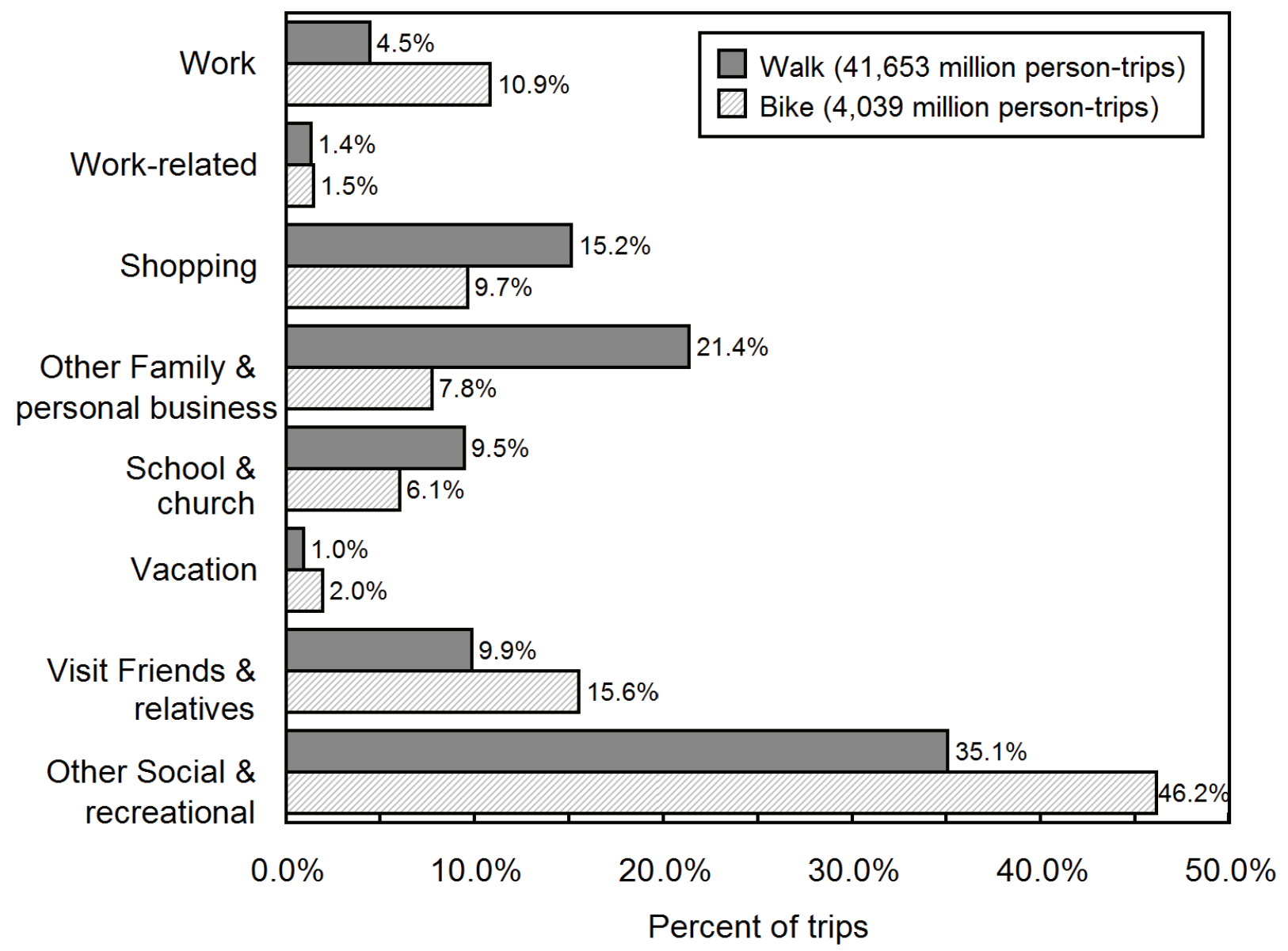

Source:

U.S. Department of Transportation, Federal Highway Administration, 2009 National Household Travel Survey Web site: nhts.ornl.gov. The 2009 data are preliminary. 
In 2009 only data on daily trips were collected in the NHTS. The 2001 data are still the latest available on longdistance trips.

\section{Long Distance Trips - 2001 National Household Travel Survey}

The 2001 National Household Travel Survey (NHTS) collected data on long-distance trips as well as everyday travel. The everyday travel data is a continuation of the Nationwide Personal Transportation Survey (NPTS), while the long-distance travel data is a continuation of the American Travel Survey (ATS) which was collected in 1977 and 1985. The survey collected trip-related data such as mode of transportation, duration, distance and purpose of trip. It also gathered demographic, geographic, and economic data for analysis purposes.

A long-distance trip is defined as a trip of 50 miles or more, one-way. Long-trip data from the 2001 NHTS were released in the summer of 2004. For additional information about the 2001 NHTS data, contact the Bureau of Transportation Statistics at 202-366-3282 or visit the following Internet site: www.bts.gov/programs/national_household_travel_survey. 
Table 8.20

Long-Distance Trip ${ }^{a}$ Characteristics, 2001 NHTS

\begin{tabular}{|c|c|c|c|c|}
\hline \multirow[b]{2}{*}{ Trip characteristic } & \multicolumn{2}{|c|}{ Person trips } & \multicolumn{2}{|c|}{ Person miles } \\
\hline & (thousands) & (percent) & (thousands) & (percent) \\
\hline Total & $2,554,068$ & 100.0 & $1,138,322,697$ & 100.0 \\
\hline \multicolumn{5}{|l|}{ Principal means of transportation: } \\
\hline Personal use vehicles & $2,310,376$ & 90.5 & $735,882,255$ & 64.7 \\
\hline Airplane & 165,039 & 6.5 & $367,888,741$ & 32.3 \\
\hline Commercial airplane & 158,880 & 6.2 & $361,717,015$ & 31.8 \\
\hline Bus $^{\mathrm{b}}$ & 52,962 & 2.1 & $23,747,433$ & 2.1 \\
\hline Intercity bus & 3,456 & 0.1 & $1,765,696$ & 0.2 \\
\hline Charter, tour, or school bus & 45,952 & 1.8 & $21,019,942$ & 1.9 \\
\hline Train & 20,672 & 0.8 & $9,266,373$ & 0.8 \\
\hline \multicolumn{5}{|l|}{ Round trip distance: } \\
\hline 100 to 300 miles & $1,688,358$ & 66.1 & $284,586,370$ & 25.0 \\
\hline 300 to 499 miles & 373,550 & 14.6 & $143,571,597$ & 12.6 \\
\hline 500 to 999 miles & 261,802 & 10.3 & $180,669,482$ & 15.9 \\
\hline 1,000 to 1,999 miles & 125,665 & 4.9 & $178,629,838$ & 15.7 \\
\hline 2,000 miles or more & 104,694 & 4.1 & $350,865,409$ & 30.8 \\
\hline Mean (miles) & 446 & c & c' & $\mathrm{c}$ \\
\hline Median (miles) & 206 & c & c & c \\
\hline \multicolumn{5}{|l|}{ Calendar quarter: } \\
\hline 1st quarter & 566,502 & 22.2 & $246,556,190$ & 21.7 \\
\hline 2nd quarter & 653,310 & 25.6 & $298,154,812$ & 26.2 \\
\hline 3rd quarter & 734,878 & 28.8 & $341,021,290$ & 30.0 \\
\hline 4 th quarter & 599,378 & 23.5 & $252,590,405$ & 22.2 \\
\hline \multicolumn{5}{|l|}{ Main purpose of trip: } \\
\hline Commuting & 329,395 & 12.9 & $65,877,968$ & 5.8 \\
\hline Other business & 405,866 & 15.9 & $242,353,212$ & 21.3 \\
\hline Personal/leisure & $1,406,411$ & 55.1 & $667,471,358$ & 58.7 \\
\hline Personal business & 322,645 & 12.6 & $130,020,982$ & 11.4 \\
\hline Other & 88,230 & 3.5 & $32,031,679$ & 2.8 \\
\hline \multicolumn{5}{|l|}{ Nights away from home: } \\
\hline None & $1,454,847$ & 57.0 & $304,469,524$ & 26.8 \\
\hline 1 to 3 nights & 808,281 & 31.7 & $414,219,147$ & 36.4 \\
\hline 4 to 7 nights & 214,464 & 8.4 & $269,265,597$ & 23.7 \\
\hline 8 or more nights & 76,475 & 3.0 & $150,368,429$ & 13.2 \\
\hline \multicolumn{5}{|l|}{ Destination: } \\
\hline Within Census division & $2,077,810$ & 81.4 & $549,651,116$ & 48.3 \\
\hline Across Census division, within Census & 196,890 & 7.7 & $134,930,113$ & 11.9 \\
\hline Across Census region & 279,367 & 10.9 & $453,741,468$ & 39.9 \\
\hline
\end{tabular}

Source:

U.S. Bureau of Transportation Statistics and the U.S. Federal Highway Administration, 2001 National Household Transportation Survey.

Note: Long-distance trips were not included in the 2009 NHTS.

\footnotetext{
${ }^{\text {a }}$ A long-distance trip is defined as a trip of 50 miles or more, one-way.

${ }^{\mathrm{b}}$ Includes other types of buses.

${ }^{\mathrm{c}}$ Not applicable.
} 
$8-26$

TRANSPORTATION ENERGY DATA BOOK: EDITION 29—2010 


\section{Chapter 9 \\ Nonhighway Modes}

Summary Statistics from Tables in this Chapter

\begin{tabular}{lrr}
\hline \multicolumn{1}{c}{ Source } & & \\
\hline & Passenger-miles & (millions) \\
Table 9.2 & Domestic and international air carrier, 2008 & 823,770 \\
Table 9.10 & Amtrak, 2008 & 6,179 \\
Table 9.11 & Commuter rail, 2008 & 11,049 \\
Table 9.12 & Transit rail, 2008 & 18,941 \\
& Freight ton-miles & (millions) \\
Table 9.5 & Domestic waterborne commerce, 2008 & 521,000 \\
Table 9.8 & Class I railroad, 2008 & (trillion Btus) \\
Table 9.2 & Passenger energy use & $2,542.5$ \\
Table 9.3 & Domestic and international air carrier, 2008 \\
Table 9.6 & General aviation, 2008 & 265.7 \\
Table 9.10 & Recreational boats, 2008 & 245.7 \\
Table 9.11 & Amtrak, 2008 & 14.8 \\
Table 9.12 & Commuter rail, 2008 & 218.0 \\
& Transit rail, 2008 & 542.5 \\
\hline
\end{tabular}


Nonhighway transportation modes accounted for $19.3 \%$ of total transportation energy use in 2008.

Table 9.1

Nonhighway Energy Use Shares, 1970-2008

\begin{tabular}{|c|c|c|c|c|c|c|}
\hline \multirow[b]{2}{*}{ Year } & \multicolumn{6}{|c|}{ Share of transportation energy use } \\
\hline & Air & Water & Pipeline & Rail & $\begin{array}{c}\text { Nonhighway } \\
\text { total }\end{array}$ & $\begin{array}{c}\text { Transportation } \\
\text { total (trillion Btu) }\end{array}$ \\
\hline 1970 & $8.5 \%$ & $5.4 \%$ & $6.4 \%$ & $3.6 \%$ & $24.0 \%$ & 15,395 \\
\hline 1971 & $8.1 \%$ & $4.8 \%$ & $6.3 \%$ & $3.5 \%$ & $22.8 \%$ & 16,015 \\
\hline 1972 & $7.7 \%$ & $4.6 \%$ & $6.1 \%$ & $3.4 \%$ & $21.9 \%$ & 17,036 \\
\hline 1973 & $7.7 \%$ & $5.0 \%$ & $5.6 \%$ & $3.4 \%$ & $21.7 \%$ & 17,874 \\
\hline 1974 & $7.3 \%$ & $5.1 \%$ & $5.4 \%$ & $3.6 \%$ & $21.5 \%$ & 17,174 \\
\hline 1975 & $7.3 \%$ & $5.3 \%$ & $4.8 \%$ & $3.2 \%$ & $20.7 \%$ & 17,424 \\
\hline 1976 & $7.2 \%$ & $5.9 \%$ & $4.3 \%$ & $3.1 \%$ & $20.6 \%$ & 18,491 \\
\hline 1977 & $7.1 \%$ & $6.2 \%$ & $4.1 \%$ & $3.1 \%$ & $20.4 \%$ & 19,126 \\
\hline 1978 & $7.1 \%$ & $6.9 \%$ & $3.9 \%$ & $2.9 \%$ & $20.8 \%$ & 20,097 \\
\hline 1979 & $7.6 \%$ & $5.8 \%$ & $4.4 \%$ & $3.1 \%$ & $20.9 \%$ & 19,652 \\
\hline 1980 & $7.6 \%$ & $7.4 \%$ & $4.7 \%$ & $3.1 \%$ & $22.8 \%$ & 18,940 \\
\hline 1981 & $7.8 \%$ & $6.8 \%$ & $4.8 \%$ & $3.0 \%$ & $22.4 \%$ & 18,741 \\
\hline 1982 & $7.9 \%$ & $5.8 \%$ & $4.7 \%$ & $2.6 \%$ & $21.1 \%$ & 18,237 \\
\hline 1983 & $7.8 \%$ & $5.3 \%$ & $4.0 \%$ & $2.6 \%$ & $19.8 \%$ & 18,368 \\
\hline 1984 & $8.5 \%$ & $5.1 \%$ & $4.1 \%$ & $2.8 \%$ & $20.5 \%$ & 18,962 \\
\hline 1985 & $8.7 \%$ & $4.5 \%$ & $3.9 \%$ & $2.6 \%$ & $19.8 \%$ & 19,205 \\
\hline 1986 & $9.0 \%$ & $6.5 \%$ & $3.6 \%$ & $2.4 \%$ & $21.5 \%$ & 20,276 \\
\hline 1987 & $9.1 \%$ & $6.6 \%$ & $3.7 \%$ & $2.4 \%$ & $21.9 \%$ & 20,771 \\
\hline 1988 & $9.3 \%$ & $6.6 \%$ & $4.1 \%$ & $2.4 \%$ & $22.4 \%$ & 21,327 \\
\hline 1989 & $9.1 \%$ & $7.0 \%$ & $4.1 \%$ & $2.4 \%$ & $22.6 \%$ & 21,685 \\
\hline 1990 & $9.6 \%$ & $6.7 \%$ & $4.3 \%$ & $2.3 \%$ & $22.9 \%$ & 21,613 \\
\hline 1991 & $9.1 \%$ & $7.2 \%$ & $4.1 \%$ & $2.3 \%$ & $22.6 \%$ & 21,205 \\
\hline 1992 & $9.0 \%$ & $7.3 \%$ & $3.9 \%$ & $2.2 \%$ & $22.4 \%$ & 21,866 \\
\hline 1993 & $8.9 \%$ & $6.4 \%$ & $4.0 \%$ & $2.3 \%$ & $21.6 \%$ & 22,322 \\
\hline 1994 & $9.0 \%$ & $6.1 \%$ & $4.1 \%$ & $2.3 \%$ & $21.6 \%$ & 22,926 \\
\hline 1995 & $9.1 \%$ & $6.3 \%$ & $4.1 \%$ & $2.4 \%$ & $21.9 \%$ & 23,465 \\
\hline 1996 & $9.2 \%$ & $5.9 \%$ & $4.1 \%$ & $2.4 \%$ & $21.6 \%$ & 23,973 \\
\hline 1997 & $9.5 \%$ & $5.1 \%$ & $4.2 \%$ & $2.4 \%$ & $21.2 \%$ & 24,327 \\
\hline 1998 & $9.6 \%$ & $5.0 \%$ & $3.6 \%$ & $2.3 \%$ & $20.5 \%$ & 24,757 \\
\hline 1999 & $9.5 \%$ & $5.3 \%$ & $3.5 \%$ & $2.3 \%$ & $20.6 \%$ & 25,948 \\
\hline 2000 & $9.7 \%$ & $5.5 \%$ & $3.4 \%$ & $2.3 \%$ & $21.0 \%$ & 26,268 \\
\hline 2001 & $9.3 \%$ & $4.6 \%$ & $3.4 \%$ & $2.3 \%$ & $19.6 \%$ & 25,958 \\
\hline 2002 & $8.3 \%$ & $4.7 \%$ & $3.5 \%$ & $2.3 \%$ & $18.8 \%$ & 26,521 \\
\hline 2003 & $8.3 \%$ & $4.0 \%$ & $3.2 \%$ & $2.3 \%$ & $17.8 \%$ & 26,672 \\
\hline 2004 & $8.7 \%$ & $4.8 \%$ & $3.0 \%$ & $2.4 \%$ & $18.9 \%$ & 27,065 \\
\hline 2005 & $9.0 \%$ & $5.0 \%$ & $3.1 \%$ & $2.4 \%$ & $19.4 \%$ & 27,526 \\
\hline 2006 & $9.0 \%$ & $5.2 \%$ & $3.0 \%$ & $2.4 \%$ & $19.7 \%$ & 27,759 \\
\hline 2007 & $9.0 \%$ & $5.6 \%$ & $3.1 \%$ & $2.3 \%$ & $20.0 \%$ & 28,013 \\
\hline 2008 & $8.9 \%$ & $4.6 \%$ & $3.4 \%$ & $2.4 \%$ & $19.3 \%$ & 26,823 \\
\hline
\end{tabular}

Source:

See Appendix A for Nonhighway Energy Use. 
These data include ALL international and domestic certificated route air carrier statistics; therefore, the data are different than those in Chapter 2. Revenue aircraft-miles, passenger-miles, and seat-miles declined in 2008. Passenger load factor was over $79 \%$ in 2007 and 2008.

Table 9.2

Summary Statistics for U.S. Domestic and International Certificated Route Air Carriers (Combined Totals), 1970-2008 ${ }^{\mathrm{a}}$

\begin{tabular}{|c|c|c|c|c|c|c|c|}
\hline Year & $\begin{array}{l}\text { Revenue } \\
\text { aircraft- } \\
\text { miles } \\
\text { (millions) }\end{array}$ & $\begin{array}{c}\text { Revenue } \\
\text { passenger-miles } \\
\text { (millions) }\end{array}$ & $\begin{array}{l}\text { Available } \\
\text { seat-miles } \\
\text { (millions) } \\
\end{array}$ & $\begin{array}{c}\text { Available } \\
\text { seats per } \\
\text { aircraft }\end{array}$ & $\begin{array}{l}\text { Passenger load } \\
\text { factor } \\
\text { (percentage) }^{\mathrm{c}}\end{array}$ & $\begin{array}{c}\text { Revenue cargo } \\
\text { ton-miles } \\
\text { (millions) }\end{array}$ & $\begin{array}{c}\text { Energy use } \\
\text { (trillion } \\
\text { Btu })^{\mathrm{d}} \\
\end{array}$ \\
\hline 1970 & 2,542 & 148,137 & 264,904 & 104 & $55.9 \%$ & 3,755 & $1,363.4$ \\
\hline 1975 & 2,241 & 173,324 & 315,823 & 141 & $54.9 \%$ & $5,062$. & $1,283.4$ \\
\hline 1980 & 2,924 & 267,722 & 448,479 & 153 & $59.7 \%$ & 7,885 & $1,386.0$ \\
\hline 1981 & 2,703 & 260,063 & 438,778 & 162 & $59.3 \%$ & 7,917 & $1,429.3$ \\
\hline 1982 & 2,804 & 272,435 & 455,938 & 163 & $59.8 \%$ & 7,807 & $1,404.0$ \\
\hline 1983 & 2,923 & 295,144 & 480,977 & 165 & $61.4 \%$ & 8,497 & $1,440.6$ \\
\hline 1984 & 3,264 & 319,504 & 534,104 & 164 & $59.8 \%$ & 9,328 & $1,608.2$ \\
\hline 1985 & 3,462 & 351,073 & 565,677 & 163 & $62.1 \%$ & 9,048 & $1,701.4$ \\
\hline 1986 & 3,873 & 378,923 & 623,075 & 161 & $60.8 \%$ & 10,987 & $1,847.1$ \\
\hline 1987 & 4,182 & 417,808 & 670,825 & 160 & $62.3 \%$ & 13,137 & $1,954.9$ \\
\hline 1988 & 4,354 & 437,649 & 696,337 & 160 & $62.9 \%$ & 14,632 & $2,049.4$ \\
\hline 1989 & 4,442 & 447,480 & 703,888 & 158 & $63.6 \%$ & 16,347 & $2,087.4$ \\
\hline 1990 & 4,724 & 472,236 & 753,211 & 159 & $62.7 \%$ & 16,403 & $2,213.0$ \\
\hline 1991 & 4,661 & 463,296 & 738,030 & 158 & $62.8 \%$ & 16,149 & $2,085.2$ \\
\hline 1992 & 4,899 & 493,715 & 772,869 & 158 & $63.9 \%$ & 17,306 & $2,144.2$ \\
\hline 1993 & 5,118 & 505,996 & 793,959 & 155 & $63.7 \%$ & 19,083 & $2,169.7$ \\
\hline 1994 & 5,360 & 537,518 & 809,259 & 151 & $66.4 \%$ & 21,773 & $2,266.2$ \\
\hline 1995 & 5,627 & 558,794 & 832,081 & 148 & $67.2 \%$ & 23,375 & $2,338.6$ \\
\hline 1996 & 5,855 & 596,164 & 859,721 & 147 & $69.3 \%$ & 24,892 & $2,409.1$ \\
\hline 1997 & 6,025 & 620,029 & 880,715 & 146 & $70.4 \%$ & 27,610 & $2,514.2$ \\
\hline 1998 & 6,220 & 634,933 & 899,029 & 145 & $70.6 \%$ & 28,015 & $2,573.4$ \\
\hline 1999 & 6,558 & 668,626 & 942,311 & 144 & $71.0 \%$ & 25,147 & $2,653.1$ \\
\hline 2000 & 6,946 & 708,926 & 981,080 & 141 & $72.3 \%$ & 30,221 & $2,743.1$ \\
\hline 2001 & 6,814 & 664,849 & 950,519 & 139 & $69.9 \%$ & 27,882 & $2,599.4$ \\
\hline 2002 & 6,834 & 655,215 & 913,898 & 134 & $71.7 \%$ & 30,507 & $2,408.3$ \\
\hline 2003 & 7,367 & 674,160 & 922,440 & 125 & $73.1 \%$ & 32,446 & $2,402.3$ \\
\hline 2004 & 7,479 & 752,341 & $1,000,193$ & 134 & $75.2 \%$ & 37,958 & $2,504.8$ \\
\hline 2005 & 7,716 & 795,117 & $1,029,316$ & 133 & $77.2 \%$ & 39,286 & $2,606.8$ \\
\hline 2006 & 8,220 & 810,086 & $1,027,526$ & 125 & $78.8 \%$ & 38,251 & $2,661.1$ \\
\hline 2007 & 8,415 & 842,007 & $1,060,093$ & 126 & $79.4 \%$ & 38,433 & $2,684.6$ \\
\hline 2008 & 8,145 & 823,770 & $1,040,618$ & 128 & $79.2 \%$ & 35,529 & $2,542.5$ \\
\hline \multicolumn{8}{|c|}{ Average annual percentage change } \\
\hline 1970-2008 & $3.1 \%$ & $4.6 \%$ & $3.7 \%$ & $0.5 \%$ & & $6.1 \%$ & $1.7 \%$ \\
\hline $1998-2008$ & $2.7 \%$ & $2.6 \%$ & $1.5 \%$ & $-1.2 \%$ & & $2.4 \%$ & $-0.1 \%$ \\
\hline
\end{tabular}

Sources:

U.S. Department of Transportation, Bureau of Transportation Statistics, www.transtats.bts.gov. (Additional resources: www.bts.gov/programs/airline_information/air_carrier_traffic_statistics)

1970-76 Energy Use - Department of Transportation, Civil Aeronautics Board, Fuel Cost and Consumption, Washington, DC, 1981, and annual.

${ }^{a}$ Data are for all U.S. air carriers reporting on Form 41.

${ }^{\mathrm{b}}$ Available seats per aircraft is calculated as the ratio of available seat-miles to revenue aircraft-miles.

${ }^{c}$ Passenger load factor is calculated as the ratio of revenue passenger-miles to available seat-miles for scheduled and nonscheduled services.

${ }^{\mathrm{d}}$ Energy use includes fuel purchased abroad for international flights. 
General aviation includes: (1) aircraft operating under general operating and flight rules; (2) not-for-hire airplanes with a seating capacity of 20 or more or a maximum payload capacity of 6,000 lbs. or more; (3) rotocraft external load operations; (4) on-demand and commuter operations not covered under Federal Aviation Regulations Part 121; and (5) agricultural aircraft operations.

Table 9.3

Summary Statistics for General Aviation, 1970-2008

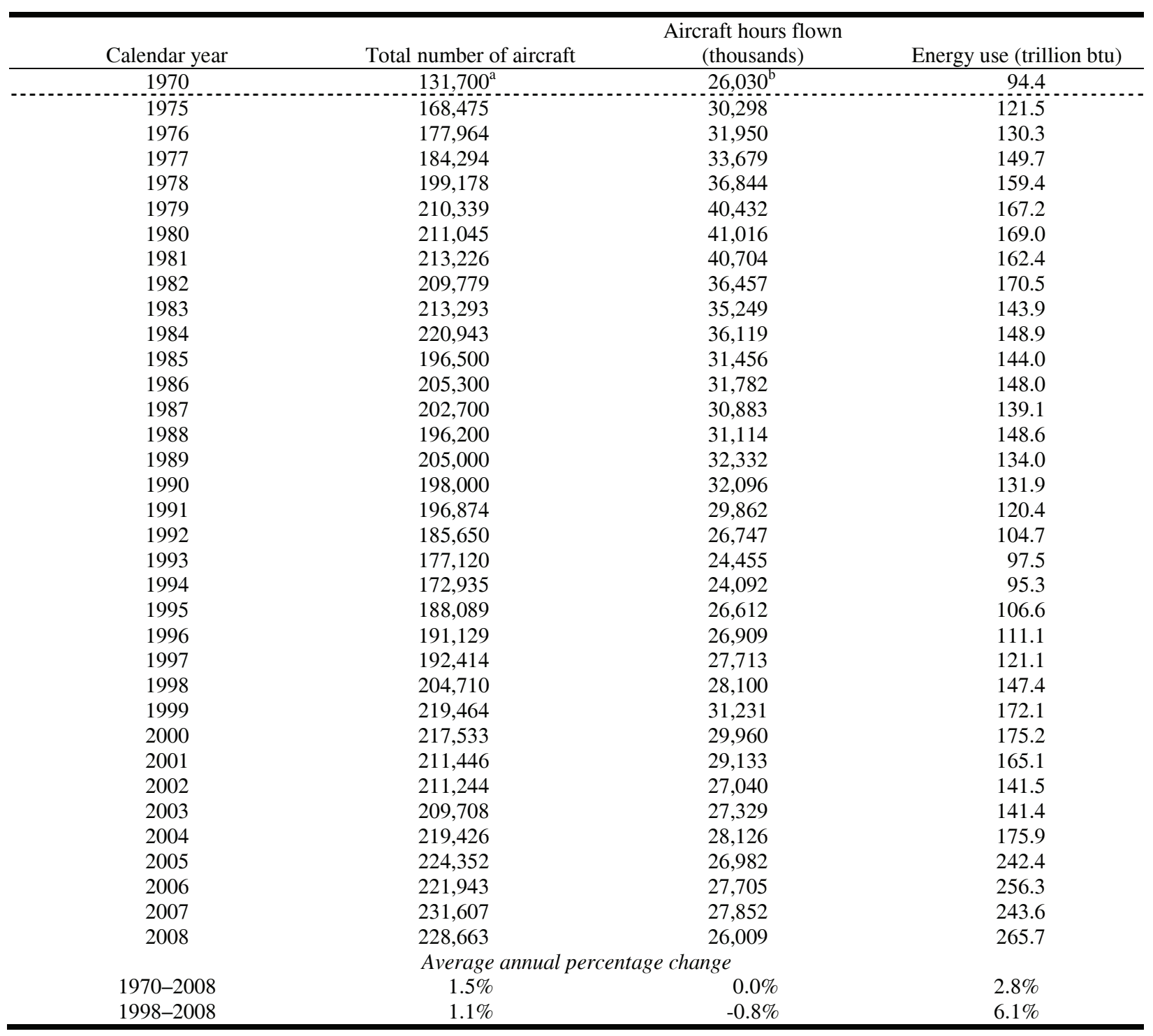

\section{Sources:}

U.S. Department of Transportation, Federal Aviation Administration, General Aviation Activity and Avionics Survey: Calendar Year 2008, Tables 1.2, 1.5, 5.1, and annual. (Additional resources: www.faa.gov/data_statistics/aviation_data_statistics/general_aviation/CY2008/)

${ }^{a}$ Active fixed-wing general aviation aircraft only.

${ }^{\mathrm{b}}$ Includes rotocraft. 
In the early seventies, domestic waterborne commerce accounted for over $60 \%$ of total tonnage, but by 1994 foreign tonnage grew to more than half of all waterborne tonnage. Total foreign and domestic tons shipped was almost 2.5 billion tons in 2008.

Table 9.4

Tonnage Statistics for Domestic and

International Waterborne Commerce, 1970-2008

(million tons shipped)

\begin{tabular}{|c|c|c|c|c|}
\hline Year & $\begin{array}{c}\text { Foreign and domestic } \\
\text { total }\end{array}$ & Foreign total $^{\mathrm{a}}$ & Domestic total $^{\mathrm{b}}$ & Percent domestic of total \\
\hline 1970 & 1,532 & 581 & 951 & $62.1 \%$ \\
\hline 1975 & $1,695^{\circ}$ & $749^{\circ}$ & $9 \overline{4} 6$ & $55.8 \%$ \\
\hline 1976 & 1,835 & 856 & 979 & $53.4 \%$ \\
\hline 1977 & 1,908 & 935 & 973 & $51.0 \%$ \\
\hline 1978 & 2,021 & 946 & 1,075 & $53.2 \%$ \\
\hline 1979 & 2,073 & 993 & 1,080 & $52.1 \%$ \\
\hline 1980 & 1,999 & 921 & 1,077 & $53.9 \%$ \\
\hline 1981 & 1,942 & 887 & 1,054 & $54.3 \%$ \\
\hline 1982 & 1,777 & 820 & 957 & $53.9 \%$ \\
\hline 1983 & 1,708 & 751 & 957 & $56.0 \%$ \\
\hline 1984 & 1,836 & 803 & 1,033 & $56.3 \%$ \\
\hline 1985 & 1,788 & 774 & 1,014 & $56.7 \%$ \\
\hline 1986 & 1,874 & 837 & 1,037 & $55.3 \%$ \\
\hline 1987 & 1,967 & 891 & 1,076 & $54.7 \%$ \\
\hline 1988 & 2,088 & 976 & 1,112 & $53.3 \%$ \\
\hline 1989 & 2,140 & 1,038 & 1,103 & $51.5 \%$ \\
\hline 1990 & 2,164 & 1,042 & 1,122 & $51.8 \%$ \\
\hline 1991 & 2,092 & 1,014 & 1,079 & $51.6 \%$ \\
\hline 1992 & 2,132 & 1,037 & 1,095 & $51.4 \%$ \\
\hline 1993 & 2,128 & 1,060 & 1,068 & $50.2 \%$ \\
\hline 1994 & 2,215 & 1,116 & 1,099 & $49.6 \%$ \\
\hline 1995 & 2,240 & 1,147 & 1,093 & $48.8 \%$ \\
\hline 1996 & 2,284 & 1,183 & 1,101 & $48.2 \%$ \\
\hline 1997 & 2,333 & 1,221 & 1,113 & $47.7 \%$ \\
\hline 1998 & 2,340 & 1,245 & 1,094 & $46.8 \%$ \\
\hline 1999 & 2,323 & 1,261 & 1,062 & $45.7 \%$ \\
\hline 2000 & 2,425 & 1,355 & 1,070 & $44.1 \%$ \\
\hline 2001 & 2,393 & 1,351 & 1,042 & $43.5 \%$ \\
\hline 2002 & 2,340 & 1,319 & 1,021 & $43.6 \%$ \\
\hline 2003 & 2,394 & 1,378 & 1,016 & $42.4 \%$ \\
\hline 2004 & 2,552 & 1,505 & 1,047 & $41.0 \%$ \\
\hline 2005 & 2,527 & 1,499 & 1,029 & $40.7 \%$ \\
\hline 2006 & 2,588 & 1,565 & 1,023 & $39.5 \%$ \\
\hline 2007 & 2,564 & 1,543 & 1,022 & $39.9 \%$ \\
\hline 2008 & 2,477 & 1,521 & 956 & $38.6 \%$ \\
\hline \multicolumn{5}{|c|}{ Average annual percentage change } \\
\hline 1970-2008 & $1.3 \%$ & $2.6 \%$ & $0.0 \%$ & \\
\hline 1998-2008 & $0.6 \%$ & $2.0 \%$ & $-1.3 \%$ & \\
\hline
\end{tabular}

Source:

U.S. Department of the Army, Corps of Engineers, Waterborne Commerce of the United States, Calendar Year 2008, New Orleans, Louisiana, 2009, Table 1-1. (Additional resources: www.ndc.iwr.usace.army.mil)

a All movements between the United States and foreign countries and between Puerto Rico and the Virgin Islands and foreign countries are classified as foreign trade.

b All movements between U.S. ports, continental and noncontiguous, and on the inland rivers, canals, and connecting channels of the United States, Puerto Rico, and the Virgin Islands, excluding the Panama Canal. Beginning in 1996, fish was excluded for internal and intra port domestic traffic. 
Table 9.5

Summary Statistics for Domestic Waterborne Commerce, 1970-2008

\begin{tabular}{|c|c|c|c|c|c|c|}
\hline Year & $\begin{array}{c}\text { Number of } \\
\text { vessels }^{\mathrm{a}}\end{array}$ & $\begin{array}{l}\text { Ton-miles } \\
\text { (billions) }\end{array}$ & $\begin{array}{l}\text { Tons shipped }^{\mathrm{b}} \\
\text { (millions) }\end{array}$ & $\begin{array}{c}\text { Average length of } \\
\text { haul (miles) }\end{array}$ & $\begin{array}{l}\text { Energy intensity } \\
\text { (Btu/ton-mile) }\end{array}$ & $\begin{array}{l}\text { Energy use } \\
\text { (trillion Btu) }\end{array}$ \\
\hline 1970 & 25,832 & 596 & 949 & 628.2 & 545 & 324.8 \\
\hline 1975 & 31,666 & $566^{-}$ & $944^{-}$ & 599.9 & 549 & 311.0 \\
\hline 1976 & 33,204 & 592 & 976 & 606.3 & 468 & 277.3 \\
\hline 1977 & 35,333 & 599 & 969 & 618.0 & 458 & 274.3 \\
\hline 1978 & 35,723 & 827 & 1,072 & 771.6 & 383 & 316.6 \\
\hline 1979 & 36,264 & 829 & 1,076 & 770.0 & 436 & 361.8 \\
\hline 1980 & 38,792 & 922 & 1,074 & 856.4 & 358 & 329.8 \\
\hline 1981 & 42,079 & 929 & 1,051 & 884.0 & 360 & 334.5 \\
\hline 1982 & 42,079 & 886 & 954 & 929.0 & 310 & 274.9 \\
\hline 1983 & 41,784 & 920 & 953 & 964.6 & 286 & 262.7 \\
\hline 1984 & 41,784 & 888 & 1,029 & 862.5 & 346 & 307.3 \\
\hline 1985 & 41,672 & 893 & 1,011 & 883.5 & 446 & 398.6 \\
\hline 1986 & 40,308 & 873 & 1,033 & 845.3 & 463 & 404.0 \\
\hline 1987 & 40,000 & 895 & 1,072 & 835.0 & 414 & 370.7 \\
\hline 1988 & 39,192 & 890 & 1,106 & 804.3 & 373 & 332.2 \\
\hline 1989 & 39,209 & 816 & 1,097 & 743.2 & 423 & 345.5 \\
\hline 1990 & 39,233 & 834 & 1,118 & 745.7 & 387 & 323.1 \\
\hline 1991 & 39,233 & 848 & 1,074 & 789.9 & 386 & 327.5 \\
\hline 1992 & 39,210 & 857 & 1,090 & 785.7 & 398 & 341.0 \\
\hline 1993 & 39,064 & 790 & 1,063 & 742.7 & 389 & 307.0 \\
\hline 1994 & 39,064 & 815 & 1,093 & 745.5 & 369 & 300.6 \\
\hline 1995 & 39,641 & 808 & 1,086 & 743.6 & 374 & 302.2 \\
\hline 1996 & 41,104 & 765 & 1,093 & 699.4 & 412 & 314.9 \\
\hline 1997 & 41,419 & 707 & 1,106 & 639.5 & 415 & 293.2 \\
\hline 1998 & 42,032 & 673 & 1,087 & 619.0 & 435 & 293.1 \\
\hline 1999 & 41,766 & 656 & 1,056 & 621.1 & 457 & 299.9 \\
\hline 2000 & 41,354 & 646 & 1,064 & 606.8 & 473 & 305.6 \\
\hline 2001 & 41,588 & 622 & 1,037 & 599.7 & 460 & 286.1 \\
\hline 2002 & 41,002 & 612 & 1,016 & 602.5 & 470 & 287.7 \\
\hline 2003 & 39,983 & 606 & 1,010 & 600.3 & 418 & 253.2 \\
\hline 2004 & 40,290 & 621 & 1,042 & 596.4 & 510 & 316.7 \\
\hline 2005 & 41,028 & 591 & 1,024 & 577.4 & 515 & 304.4 \\
\hline 2006 & 41,109 & 562 & 1,018 & 548.7 & 571 & 320.7 \\
\hline 2007 & 40,695 & 553 & 1,016 & 544.2 & 590 & 326.4 \\
\hline 2008 & 40,301 & 521 & 952 & 546.7 & 418 & 218.0 \\
\hline \multicolumn{7}{|c|}{ Average annual percentage change } \\
\hline 1970-2008 & $1.2 \%$ & $-0.2 \%$ & $0.2 \%$ & $-0.4 \%$ & $-0.7 \%$ & $-1.0 \%$ \\
\hline $1998-2008$ & $-0.4 \%$ & $-2.5 \%$ & $-1.3 \%$ & $-1.2 \%$ & $-0.4 \%$ & $-2.9 \%$ \\
\hline
\end{tabular}

Sources:

Number of vessels -1970-92, 1995-2006 - U.S. Department of the Army, Corps of Engineers, Waterborne Transportation Lines of the United States, 2008, New Orleans, LA, 2009, Table 2, p. 6, and annual. 1993-94 - U.S. Department of the Army, Corps of Engineers, The U.S. Waterway System-Facts, Navigation Data Center, New Orleans, Louisiana, January 1996.

Ton-miles, tons shipped, average length of haul - U.S. Department of the Army, Corps of Engineers, Waterborne Commerce of the United States, Calendar Year 2008, Part 5: National Summaries, New Orleans, LA, 2009, Table 1-4, pp. 1-6, 1-7, and annual.

Energy use - See Appendix A for Water Energy Use. (Additional resources: www.iwr.usace.army.mil/ndc)

${ }^{\text {a }}$ Grand total for self-propelled and non-self-propelled.

${ }^{b}$ These figures are not consistent with the figures on Table 9.3 because intra-territory tons are not included in this table. Intra-territory traffic is traffic between ports in Puerto Rico and the Virgin Islands. 
The data displayed in this table come from the Environmental Protection Agency's NONROAD2008a model.

Table 9.6

Recreational Boat Energy Use, 1970-2008

\begin{tabular}{|c|c|c|c|c|}
\hline & Number of boats & Diesel fuel & Gasoline & Total energy use \\
\hline Year & (thousands) & & (trillion Btu) & \\
\hline 1970 & 10,087 & 5.5 & 151.7 & 157.2 \\
\hline 1971 & 10,137 & 6.5 & 152.6 & 159.2 \\
\hline 1972 & 10,187 & 7.6 & 153.6 & 161.2 \\
\hline 1973 & 10,237 & 8.6 & 154.5 & 163.2 \\
\hline 1974 & 10,287 & 9.7 & 155.5 & 165.1 \\
\hline 1975 & 10,337 & 10.7 & 156.4 & 167.1 \\
\hline 1976 & 10,387 & 11.8 & 157.4 & 169.1 \\
\hline 1977 & 10,437 & 12.8 & 158.3 & 171.1 \\
\hline 1978 & 10,487 & 13.9 & 159.3 & 173.1 \\
\hline 1979 & 10,537 & 14.9 & 160.2 & 175.1 \\
\hline 1980 & 10,587 & 16.0 & 161.2 & 177.1 \\
\hline 1981 & 10,637 & 17.0 & 162.1 & 179.1 \\
\hline 1982 & 10,687 & 18.0 & 163.1 & 181.1 \\
\hline 1983 & 10,737 & 19.1 & 164.0 & 183.1 \\
\hline 1984 & 10,787 & 20.1 & 165.0 & 185.1 \\
\hline 1985 & 10,837 & 21.2 & 165.9 & 187.1 \\
\hline 1986 & 10,887 & 22.2 & 166.9 & 189.1 \\
\hline 1987 & 10,937 & 23.3 & 167.8 & 191.1 \\
\hline 1988 & 11,030 & 24.3 & 170.4 & 194.7 \\
\hline 1989 & 11,122 & 25.4 & 172.9 & 198.3 \\
\hline 1990 & 11,215 & 26.4 & 175.4 & 201.8 \\
\hline 1991 & 11,327 & 27.5 & 178.7 & 206.2 \\
\hline 1992 & 11,440 & 28.5 & 182.0 & 210.5 \\
\hline 1993 & 11,553 & 29.5 & 185.3 & 214.8 \\
\hline 1994 & 11,770 & 30.6 & 192.5 & 223.1 \\
\hline 1995 & 11,988 & 31.6 & 199.7 & 231.3 \\
\hline 1996 & 12,206 & 32.7 & 206.8 & 239.5 \\
\hline 1997 & 12,244 & 33.7 & 207.2 & 240.9 \\
\hline 1998 & 12,283 & 34.8 & 207.4 & 242.2 \\
\hline 1999 & 12,321 & 35.8 & 207.1 & 243.0 \\
\hline 2000 & 12,359 & 36.8 & 206.6 & 243.4 \\
\hline 2001 & 12,464 & 37.9 & 206.9 & 244.9 \\
\hline 2002 & 12,568 & 39.0 & 206.7 & 245.7 \\
\hline 2003 & 12,673 & 40.2 & 206.0 & 246.2 \\
\hline 2004 & 12,777 & 41.3 & 205.0 & 246.2 \\
\hline 2005 & 12,882 & 42.4 & 203.7 & 246.1 \\
\hline 2006 & 12,984 & 43.5 & 202.5 & 245.9 \\
\hline 2007 & 13,086 & 44.6 & 201.2 & 245.8 \\
\hline 2008 & 13,189 & 45.7 & 200.0 & 245.7 \\
\hline \multicolumn{5}{|c|}{ Average annual percentage change } \\
\hline 1970-2008 & $0.7 \%$ & $5.7 \%$ & $0.7 \%$ & $1.2 \%$ \\
\hline 1998-2008 & $0.7 \%$ & $2.8 \%$ & $-0.4 \%$ & $0.1 \%$ \\
\hline
\end{tabular}

Source:

U.S. Environmental Protection Agency, NONROAD2008a model, downloadable file from http://www.epa.gov/otaq/nonrdmdl.htm. 
The Interstate Commerce Commission designates Class I railroads on the basis of annual gross revenues. In 2008, seven railroads were given this designation. The number of railroads designated as Class I has changed considerably in the last 30 years; in 1976 there were 52 railroads given Class I designation.

Table 9.7

Class I Railroad Freight Systems in the United States Ranked by Revenue Ton-Miles, 2008

\begin{tabular}{lcc}
\hline \multicolumn{1}{c}{ Railroad } & $\begin{array}{c}\text { Revenue ton-miles } \\
\text { (billions) }\end{array}$ & Percent \\
\hline Burlington Northern and Santa Fe Railway Company & 664 & $37.4 \%$ \\
Union Pacific Railroad Company & 563 & $31.7 \%$ \\
CSX Transportation & 248 & $14.0 \%$ \\
Norfolk Southern Railway & 195 & $11.0 \%$ \\
Canadian National, Grand Trunk Corporation & 53 & $3.0 \%$ \\
Kansas City Southern Railway Company & 30 & $1.7 \%$ \\
Soo Line Railroad Company & 24 & $1.4 \%$ \\
& & \\
Total & $\mathbf{1 , 7 7 7}$ & $\mathbf{1 0 0 . 0 \%}$ \\
\hline
\end{tabular}

\section{Source:}

Association of American Railroads, Railroad Facts, 2009 Edition, Washington, DC, November 2009, p. 66. (Additional resources: www.aar.org) 
Revenue ton-miles for Class I freight railroads was over 1.7 trillion in 2008. Though there are many regional and local freight railroads, the Class I freight railroads accounted for $94 \%$ of the railroad industry's freight revenue in 2008 and 67\% of the industry's mileage operated. The energy intensity of Class I railroads hit an all-time low of 305 btu/ton-mile in 2008.

Table 9.8

Summary Statistics for Class I Freight Railroads, 1970-2008

\begin{tabular}{|c|c|c|c|c|c|c|c|c|c|}
\hline Year & $\begin{array}{l}\text { Number of } \\
\text { locomotives } \\
\text { in service }^{\mathrm{a}}\end{array}$ & $\begin{array}{l}\text { Number of } \\
\text { freight cars } \\
\text { (thousands) }^{\mathrm{b}}\end{array}$ & $\begin{array}{c}\text { Train- } \\
\text { miles } \\
\text { (millions) }\end{array}$ & $\begin{array}{l}\text { Car-miles } \\
\text { (millions) }\end{array}$ & $\begin{array}{l}\text { Tons } \\
\text { originated }^{\mathrm{c}} \\
\text { (millions) }^{\text {milions }}\end{array}$ & $\begin{array}{c}\text { Average } \\
\text { length of } \\
\text { haul } \\
\text { (miles) }\end{array}$ & $\begin{array}{l}\text { Revenue } \\
\text { ton-miles } \\
\text { (millions) }\end{array}$ & $\begin{array}{c}\text { Energy } \\
\text { intensity } \\
\text { (Btu/ton- } \\
\text { mile) }\end{array}$ & $\begin{array}{c}\text { Energy } \\
\text { use } \\
\text { (trillion } \\
\text { Btu) }\end{array}$ \\
\hline 1970 & $27,077^{d}$ & 1,424 & 427 & 29,890 & 1,485 & 515 & 764,809 & 691 & 528.1 \\
\hline 1975 & 27,846 & 1,359 & 403 & 27,656 & 1,395 & 541 & 754,252 & 687 & 518.3 \\
\hline 1980 & 28,094 & 1,168 & 428 & 29,277 & 1,492 & 616 & 918,958 & 597 & 548.7 \\
\hline 1981 & 27,421 & 1,111 & 408 & 27,968 & 1,453 & 626 & 910,169 & 572 & 521.0 \\
\hline 1983 & 25,448 & 1,007 & 346 & 24,358 & 1,293 & 641 & 828,275 & 525 & 435.1 \\
\hline 1984 & 24,117 & 948 & 369 & 26,409 & 1,429 & 645 & 921,542 & 510 & 469.9 \\
\hline 1985 & 22,548 & 867 & 347 & 24,920 & 1,320 & 665 & 876,984 & 497 & 436.1 \\
\hline 1986 & 20,790 & 799 & 347 & 24,414 & 1,306 & 664 & 867,722 & 486 & 421.5 \\
\hline 1987 & 19,647 & 749 & 361 & 25,627 & 1,372 & 688 & 943,747 & 456 & 430.3 \\
\hline 1988 & 19,364 & 725 & 379 & 26,339 & 1,430 & 697 & 996,182 & 443 & 441.4 \\
\hline 1989 & 19,015 & 682 & 383 & 26,196 & 1,403 & 723 & $1,013,841$ & 437 & 442.6 \\
\hline 1990 & 18,835 & 659 & 380 & 26,159 & 1,425 & 726 & $1,033,969$ & 420 & 434.7 \\
\hline 1992 & 18,004 & 605 & 390 & 26,128 & 1,399 & 763 & $1,066,781$ & 393 & 419.2 \\
\hline 1993 & 18,161 & 587 & 405 & 26,883 & 1,397 & 794 & $1,109,309$ & 389 & 431.6 \\
\hline 1994 & 18,505 & 591 & 441 & 28,485 & 1,470 & 817 & $1,200,701$ & 388 & 465.4 \\
\hline 1995 & 18,812 & 583 & 458 & 30,383 & 1,550 & 843 & $1,305,688$ & 372 & 485.9 \\
\hline 1996 & 19,269 & 571 & 469 & 31,715 & 1,611 & 842 & $1,355,975$ & 368 & 499.4 \\
\hline 1997 & 19,684 & 568 & 475 & 31,660 & 1,585 & 851 & $1,348,926$ & 370 & 499.7 \\
\hline 1998 & 20,261 & 576 & 475 & 32,657 & 1,649 & 835 & $1,376,802$ & 365 & 502.0 \\
\hline 1999 & 20,256 & 579 & 490 & 33,851 & 1,717 & 835 & $1,433,461$ & 363 & 520.0 \\
\hline 2000 & 20,028 & 560 & 504 & 34,590 & 1,738 & 843 & $1,465,960$ & 352 & 516.0 \\
\hline 2001 & 19,745 & 500 & 500 & 34,243 & 1,742 & 859 & $1,495,472$ & 346 & 517.3 \\
\hline 2002 & 20,506 & 478 & 500 & 34,680 & 1,767 & 853 & $1,507,011$ & 345 & 520.3 \\
\hline 2003 & 20,774 & 467 & 516 & 35,555 & 1,799 & 862 & $1,551,438$ & 344 & 533.9 \\
\hline 2004 & 22,015 & 474 & 535 & 37,071 & 1,844 & 902 & $1,662,598$ & 341 & 566.2 \\
\hline 2005 & 22,779 & 475 & 548 & 37,712 & 1,899 & 894 & $1,696,425$ & 337 & 571.4 \\
\hline 2006 & 23,732 & 475 & 563 & 38,995 & 1,957 & 906 & $1,771,897$ & 330 & 584.5 \\
\hline 2007 & 24,143 & 460 & 543 & 38,186 & 1,940 & 913 & $1,770,545$ & 320 & 566.9 \\
\hline 1970-2008 & $-0.3 \%$ & $-3.0 \%$ & $0.5 \%$ & $0.6 \%$ & $0.7 \%$ & $1.5 \%$ & $2.2 \%$ & $-2.1 \%$ & $0.1 \%$ \\
\hline 1998-2008 & $1.7 \%$ & $-2.4 \%$ & $1.0 \%$ & $1.3 \%$ & $1.6 \%$ & $1.0 \%$ & $2.6 \%$ & $-1.8 \%$ & $0.4 \%$ \\
\hline
\end{tabular}

\section{Source:}

Association of American Railroads, Railroad Facts, 2008 Edition, Washington, DC, November 2009, pp. 27, 28, 33, 34, 36, 49, 52, 61. (Additional resources: www.aar.org)

${ }^{a}$ Does not include self-powered units.

${ }^{\mathrm{b}}$ Does not include private or shipper-owned cars. Beginning in 2001, Canadian-owned U.S. railroads are excluded.

${ }^{\mathrm{c}}$ Tons originated is a more accurate representation of total tonnage than revenue tons. Revenue tons often produces double-counting of loads switched between rail companies.

${ }^{\mathrm{d}}$ Data represent total locomotives used in freight and passenger service. Separate estimates are not available. 
According to the 2002 Commodity Flow Survey, $5 \%$ of all freight ton-miles are rail intermodal shipments (truck/rail or rail/water). See Table 5.13 for details. The number of trailers and containers moved by railroads has increased almost seven-fold from 1965 to 2008. Containerization has increased in recent years, evidenced by the 293\% increase in the number of containers from 1988 to 2008.

Table 9.9

Intermodal Rail Traffic, $1965-2008^{a}$

\begin{tabular}{|c|c|c|c|}
\hline Year & Trailers \& containers & Trailers & Containers \\
\hline 1965 & $1,664,929$ & $\mathrm{~b}$ & $\mathrm{~b}$ \\
\hline 1970 & $2,363,200$ & $b$ & \\
\hline 1975 & $2,238,117$ & $\mathrm{~b}$ & $\mathrm{~b}$ \\
\hline 1980 & $3,059,402$ & $b^{-7}+a$ & $b^{-}$ \\
\hline 1981 & $3,150,522$ & b & b \\
\hline 1982 & $3,396,973$ & $\mathrm{~b}$ & b \\
\hline 1983 & $4,090,078$ & b & $\mathrm{b}$ \\
\hline 1984 & $4,565,743$ & b & b \\
\hline 1985 & $4,590,952$ & b & b \\
\hline 1986 & $4,997,229$ & b & $\mathrm{b}$ \\
\hline 1987 & $5,503,819$ & $\mathrm{~b}$ & $\mathrm{~b}$ \\
\hline 1988 & $5,779,547$ & $3,481,020$ & $2,298,527$ \\
\hline 1989 & $5,987,355$ & $3,496,262$ & $2,491,093$ \\
\hline 1990 & $6,206,782$ & $3,451,953$ & $2,754,829$ \\
\hline 1991 & $6,246,134$ & $3,201,560$ & $3,044,574$ \\
\hline 1992 & $6,627,841$ & $3,264,597$ & $3,363,244$ \\
\hline 1993 & $7,156,628$ & $3,464,126$ & $3,692,502$ \\
\hline 1994 & $8,128,228$ & $3,752,502$ & $4,375,726$ \\
\hline 1995 & $7,936,172$ & $3,492,463$ & $4,443,709$ \\
\hline 1996 & $8,143,258$ & $3,302,128$ & $4,841,130$ \\
\hline 1997 & $8,698,308$ & $3,453,907$ & $5,244,401$ \\
\hline 1998 & $8,772,663$ & $3,353,032$ & $5,419,631$ \\
\hline 1999 & $8,907,626$ & $3,207,407$ & $5,700,219$ \\
\hline 2000 & $9,176,890$ & $2,888,630$ & $6,288,260$ \\
\hline 2001 & $8,935,444$ & $2,603,423$ & $6,332,021$ \\
\hline 2002 & $9,312,360$ & $2,531,338$ & $6,781,022$ \\
\hline 2003 & $9,955,605$ & $2,625,837$ & $7,329,768$ \\
\hline 2004 & $10,993,662$ & $2,928,123$ & $8,065,539$ \\
\hline 2005 & $11,693,512$ & $2,979,906$ & $8,713,606$ \\
\hline 2006 & $12,282,221$ & $2,882,699$ & $9,399,522$ \\
\hline 2007 & $12,026,631$ & $2,600,635$ & $9,425,996$ \\
\hline 2008 & $11,517,240$ & $2,483,940$ & $9,033,300$ \\
\hline \multicolumn{4}{|c|}{ Average annual percentage change } \\
\hline 1965-2008 & $4.6 \%$ & $b^{\circ}$ & $\mathrm{b}$ \\
\hline 1998-2008 & $2.8 \%$ & $-3.0 \%$ & $5.2 \%$ \\
\hline
\end{tabular}

Source:

Association of American Railroads, Railroad Facts, 2008 Edition, Washington, DC, November 2009, p. 26. Additional resources: www.aar.org)

${ }^{a}$ Beginning in 1995, the Grand Trunk Western Railroad and the Soo Line Railroad Company are excluded. Beginning in 1999, the Illinois Central data are excluded. Beginning in 2002, the Wisconsin Central data are excluded.

${ }^{\mathrm{b}}$ Data are not available. 
The National Railroad Passenger Corporation, known as Amtrak, began operation in 1971. Amtrak revenue passenger-miles have grown at an average annual rate of $3.1 \%$ from 1971 to 2008.

Table 9.10

Summary Statistics for the National Railroad Passenger Corporation (Amtrak), 1971-2008

\begin{tabular}{|c|c|c|c|c|c|c|c|c|}
\hline Year & $\begin{array}{c}\text { Number of } \\
\text { locomotives } \\
\text { in service }\end{array}$ & $\begin{array}{c}\text { Number of } \\
\text { passenger } \\
\text { cars }\end{array}$ & $\begin{array}{l}\text { Train-miles } \\
\text { (thousands) }\end{array}$ & $\begin{array}{l}\text { Car-miles } \\
\text { (thousands) }\end{array}$ & $\begin{array}{c}\text { Revenue } \\
\text { passenger- } \\
\text { miles } \\
\text { (millions) }\end{array}$ & $\begin{array}{l}\text { Average } \\
\text { trip length } \\
\text { (miles) }\end{array}$ & $\begin{array}{l}\text { Energy intensity } \\
\text { (Btu per revenue } \\
\text { passenger-mile) }\end{array}$ & $\begin{array}{c}\text { Energy } \\
\text { use } \\
\text { (trillion } \\
\text { Btu) }\end{array}$ \\
\hline 1971 & $\mathrm{a}$ & 1,165 & 16,537 & 140,147 & 1,993 & 188 & a & $\mathrm{a}$ \\
\hline 1975 & $355^{-}$ & 1,913 & 30,166 & 253,898 & 3,753 & 224 & 3,548 & 13.3 \\
\hline $1980^{-}$ & 448 & 2,128 & 29,487 & 235,235 & 4,503 & 217 & 3,065 & 13.8 \\
\hline 1981 & 398 & 1,830 & 30,380 & 222,753 & 4,397 & 226 & 2,883 & 12.7 \\
\hline 1983 & 388 & 1,880 & 28,805 & 223,509 & 4,227 & 223 & 2,875 & 12.2 \\
\hline 1984 & 387 & 1,844 & 29,133 & 234,557 & 4,427 & 227 & 2,923 & 12.9 \\
\hline 1985 & 382 & 1,818 & 30,038 & 250,642 & 4,785 & 238 & 2,703 & 12.9 \\
\hline 1986 & 369 & 1,793 & 28,604 & 249,665 & 5,011 & 249 & 2,481 & 12.4 \\
\hline 1987 & 381 & 1,850 & 29,515 & 261,054 & 5,361 & 259 & 2,450 & 13.1 \\
\hline 1988 & 391 & 1,845 & 30,221 & 277,774 & 5,686 & 265 & 2,379 & 13.5 \\
\hline 1989 & 312 & 1,742 & 31,000 & 285,255 & 5,859 & 274 & 2,614 & 15.3 \\
\hline 1990 & 318 & 1,863 & 33,000 & 300,996 & 6,057 & 273 & 2,505 & 15.2 \\
\hline 1992 & 336 & 1,796 & 34,000 & 307,282 & 6,091 & 286 & 2,534 & 15.4 \\
\hline 1993 & 360 & 1,853 & 34,936 & 302,739 & 6,199 & 280 & 2,565 & 15.9 \\
\hline 1994 & 411 & 1,874 & 34,940 & 305,600 & 5,869 & 276 & 2,282 & $13.4^{\mathrm{b}}$ \\
\hline 1995 & 422 & 1,907 & 31,579 & 282,579 & 5,401 & 266 & 2,501 & 13.5 \\
\hline 1996 & 348 & 1,501 & 30,542 & 277,750 & 5,066 & 257 & 2,690 & 13.6 \\
\hline 1997 & 292 & 1,572 & 32,000 & 287,760 & 5,166 & 255 & 2,811 & 14.5 \\
\hline 1998 & 362 & 1,347 & 32,926 & 315,823 & 5,325 & 251 & 2,788 & 14.8 \\
\hline 1999 & 385 & 1,285 & 34,080 & 349,337 & 5,289 & 245 & 2,943 & 15.6 \\
\hline 2000 & 385 & 1,891 & 35,404 & 371,215 & 5,574 & 243 & 3,235 & 18.0 \\
\hline 2001 & 401 & 2,084 & 36,512 & 377,705 & 5,571 & 238 & 3,257 & 18.1 \\
\hline 2002 & 372 & 2,896 & 37,624 & 378,542 & 5,314 & 228 & 3,212 & 17.1 \\
\hline 2003 & 442 & 1,623 & 37,459 & 331,864 & 5,680 & 231 & 2,800 & 15.9 \\
\hline 2004 & 276 & 1,211 & 37,159 & 308,437 & 5,511 & 219 & 2,760 & 15.2 \\
\hline 2005 & 258 & 1,186 & 36,199 & 264,796 & 5,381 & 215 & 2,709 & 14.6 \\
\hline 2006 & 319 & 1,191 & 36,083 & 263,908 & 5,410 & 220 & 2,650 & 14.3 \\
\hline 2007 & 270 & 1,164 & 37,484 & 266,545 & 5,784 & 218 & 2,516 & 14.5 \\
\hline 1971-2008 & a & $0.0 \%$ & $2.3 \%$ & $1.8 \%$ & $3.1 \%$ & $0.4 \%$ & $\mathrm{a}$ & $\mathrm{a}$ \\
\hline 1998-2008 & $-2.6 \%$ & $-1.3 \%$ & $1.4 \%$ & $-1.5 \%$ & $1.5 \%$ & $-1.5 \%$ & $-1.5 \%$ & $0.0 \%$ \\
\hline
\end{tabular}

\section{Sources:}

1971-83- Association of American Railroads, Economics and Finance Department, Statistics of Class I Railroads, Washington, DC, and annual.

1984-88- Association of American Railroads, Railroad Facts, 1988 Edition, Washington, DC, December 1989, p. 61 , and annual.

1989-93- Personal communication with the Corporate Accounting Office of Amtrak, Washington, D.C.

1994-2008 - Number of locomotives in service, number of passenger cars, train-miles, car-miles, revenue passenger-miles, and average trip length - Association of American Railroads, Railroad Facts, 2009 Edition, Washington, DC, 2009, p. 77.

Energy use - Personal communication with the Amtrak, Washington, DC. (Additional resources: www.amtrak.com, www.aar.org)

${ }^{a}$ Data are not available.

${ }^{\mathrm{b}}$ Energy use for 1994 on is not directly comparable to earlier years. Some commuter rail energy use may have been inadvertently included in earlier years. 
Commuter rail, which is also known as regional rail or suburban rail, is long-haul rail passenger service operating between metropolitan and suburban areas, whether within or across state lines. Commuter rail lines usually have reduced fares for multiple rides and commutation tickets for regular, recurring riders.

Table 9.11

Summary Statistics for Commuter Rail Operations, 1984-2008

\begin{tabular}{cccccccc}
\hline & $\begin{array}{c}\text { Number of } \\
\text { passenger } \\
\text { vehicles }\end{array}$ & $\begin{array}{c}\text { Vehicle- } \\
\text { miles } \\
\text { (millions) }\end{array}$ & $\begin{array}{c}\text { Passenger } \\
\text { trips } \\
\text { (millions) }\end{array}$ & $\begin{array}{c}\text { Passenger- } \\
\text { miles } \\
\text { (millions) }\end{array}$ & $\begin{array}{c}\text { Average } \\
\text { trip length } \\
\text { (miles) }\end{array}$ & $\begin{array}{c}\text { Energy intensity } \\
\text { (Btu/passenger- } \\
\text { mile) }\end{array}$ & $\begin{array}{c}\text { Energy } \\
\text { use } \\
\text { (trillion } \\
\text { Btu) }\end{array}$ \\
\hline 1984 & 4,075 & 167.9 & 267 & 6,207 & 23.2 & 2,804 & 17.4 \\
1985 & 4,035 & 182.7 & 275 & 6,534 & 23.8 & 2,826 & 18.5 \\
1986 & 4,440 & 188.6 & 306 & 6,723 & 22.0 & 2,926 & 19.7 \\
1987 & 4,686 & 188.9 & 311 & 6,818 & 21.9 & 2,801 & 19.1 \\
1988 & 4,649 & 202.2 & 325 & 6,964 & 21.4 & 2,872 & 19.7 \\
1989 & 4,472 & 209.6 & 330 & 7,211 & 21.9 & 2,864 & 20.7 \\
1990 & 4,982 & 212.7 & 328 & 7,082 & 21.6 & 2,822 & 20.0 \\
1991 & 5,126 & 214.9 & 318 & 7,344 & 23.1 & 2,770 & 20.3 \\
1992 & 5,164 & 218.8 & 314 & 7,320 & 23.3 & 2,629 & 19.2 \\
1993 & 4,982 & 223.9 & 322 & 6,940 & 21.6 & 2,976 & 20.7 \\
1994 & 5,126 & 230.8 & 339 & 7,996 & 23.6 & 2,682 & 21.4 \\
1995 & 5,164 & 237.7 & 344 & 8,244 & 24.0 & 2,632 & 21.7 \\
1996 & 5,240 & 241.9 & 352 & 8,351 & 23.7 & 2,582 & 21.6 \\
1997 & 5,426 & 250.7 & 357 & 8,038 & 22.5 & 2,724 & 21.9 \\
1998 & 5,536 & 259.5 & 381 & 8,704 & 22.8 & 2,646 & 23.0 \\
1999 & 5,550 & 265.9 & 396 & 8,766 & 22.1 & 2,714 & 23.8 \\
2000 & 5,498 & 270.9 & 413 & 9,402 & 22.8 & 2,551 & 24.0 \\
2001 & 5,572 & 277.3 & 419 & 9,548 & 22.8 & 2,515 & 24.0 \\
2002 & 5,724 & 283.7 & 414 & 9,504 & 22.9 & 2,514 & 23.9 \\
2003 & 5,959 & 286.0 & 410 & 9,559 & 23.3 & 2,545 & 24.3 \\
2004 & 6,228 & 294.7 & 414 & 9,719 & 23.5 & 2,569 & 25.0 \\
2005 & 6,392 & 303.4 & 423 & 9,473 & 22.4 & 2,743 & 26.0 \\
2006 & 6,403 & 314.7 & 441 & 10,361 & 23.5 & 2,527 & 26.2 \\
2007 & 6,391 & 325.7 & 459 & 11,153 & 24.3 & 2,638 & 29.4 \\
2008 & 6,617 & 310.2 & 472 & 11,049 & 23.4 & 2,656 & 29.3 \\
$1984-2008$ & $2.0 \%$ & $2.6 \%$ & $2.4 \%$ & $2.4 \%$ & $0.0 \%$ & & \\
$1998-2008$ & $1.8 \%$ & $1.8 \%$ & $2.2 \%$ & $2.4 \%$ & $0.3 \%$ & & \\
\hline
\end{tabular}

Source:

American Public Transportation Association, 2010 Public Transportation Fact Book, Washington, DC, April 2010, Tables 5, 6, 8, and 9. (Additional resources: www.apta.com) 
This table on transit rail operations includes data on light rail and heavy rail systems. Light rail vehicles are usually single vehicles driven electrically with power drawn from overhead wires. Heavy rail is characterized by high speed and rapid acceleration of rail cars operating on a separate right-of-way.

Table 9.12

Summary Statistics for Rail Transit Operations, 1970-2008

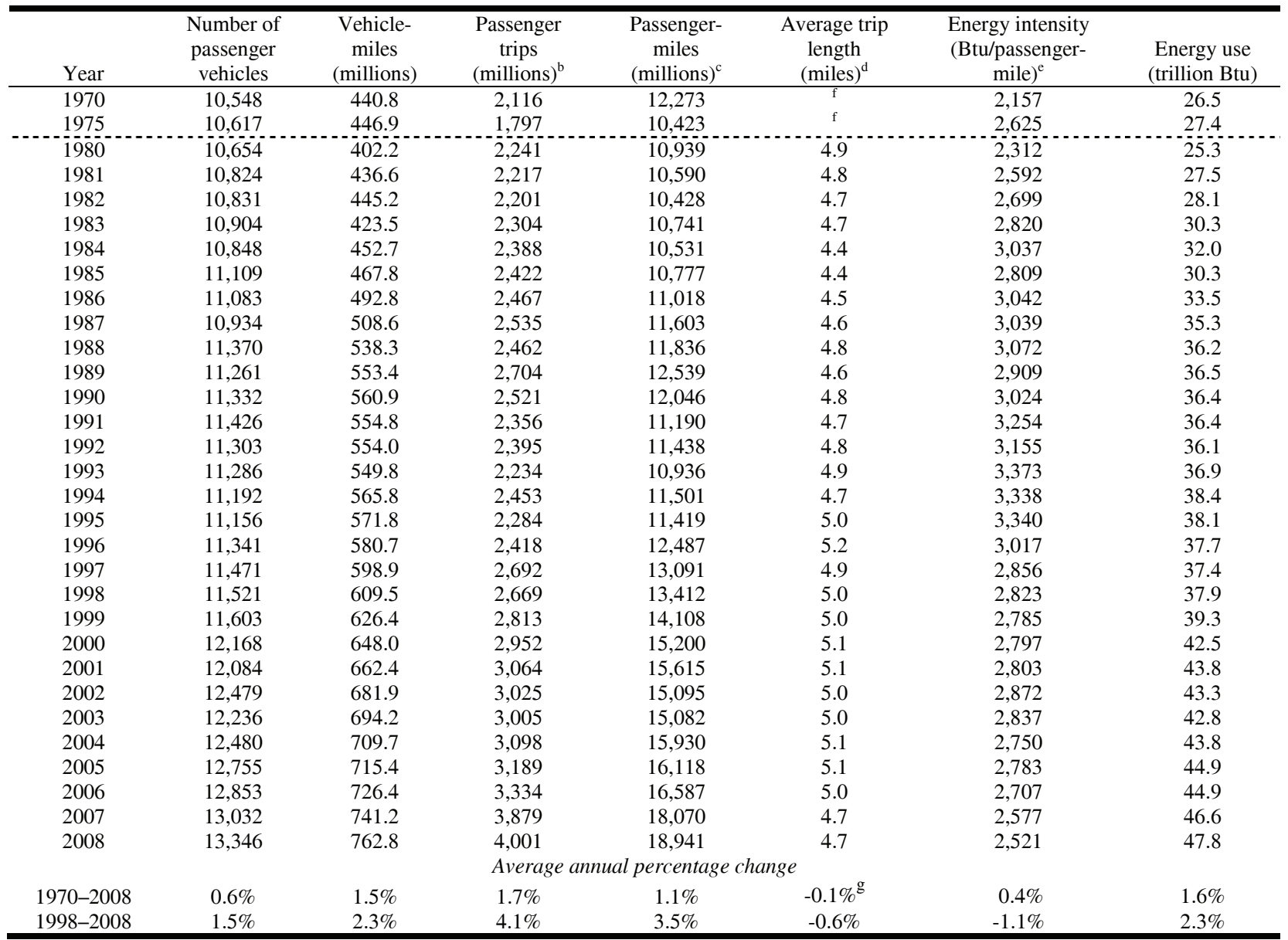

\section{Sources:}

American Public Transportation Association, 2010 Public Transportation Fact Book, Washington, DC, April 2010, Table 27. (Additional resources: www.apta.com)

Energy use - See Appendix A for Rail Transit Energy Use.

${ }^{a}$ Heavy rail and light rail. Series not continuous between 1983 and 1984 because of a change in data source by the American Public Transit Association (APTA). Beginning in 1984, data provided by APTA are taken from mandatory reports filed with the Urban Mass Transit Administration (UMTA). Data for prior years were provided on a voluntary basis by APTA members and expanded statistically.

${ }^{\mathrm{b}}$ 1970-79 data represents total passenger rides; after 1979, data represents unlinked passenger trips.

${ }^{\mathrm{c}}$ Estimated for years 1970-76 based on an average trip length of 5.8 miles.

${ }^{\mathrm{d}}$ Calculated as the ratio of passenger-miles to passenger trips.

${ }^{\mathrm{e}}$ Large system-to-system variations exist within this category.

${ }^{\mathrm{f}}$ Data are not available.

${ }^{\mathrm{g}}$ Average annual percentage change is calculated for years 1980-2007. 
$9-14$ 


\section{Chapter 10 Transportation and the Economy}

Summary Statistics from Tables/Figures in this Chapter

\begin{tabular}{|c|c|c|}
\hline Source & & \\
\hline \multirow[t]{7}{*}{ Figure 10.2} & Share of gasoline cost attributed to taxes, 2008 & \\
\hline & Canada & $28 \%$ \\
\hline & France & $73 \%$ \\
\hline & Germany & $65 \%$ \\
\hline & Japan & $39 \%$ \\
\hline & United Kingdom & $67 \%$ \\
\hline & United States & $18 \%$ \\
\hline \multirow[t]{3}{*}{ Table 10.12} & Average price of a new car, 2008 (current dollars) & 23,051 \\
\hline & Domestic & 20,490 \\
\hline & Import & 29,174 \\
\hline \multirow[t]{3}{*}{ Table 10.13} & Car operating costs, 2009 & \\
\hline & Variable costs (constant 2009 dollars per 10,000 miles) & 1,542 \\
\hline & Fixed costs (constant 2009 dollars per 10,000 miles) & 5,526 \\
\hline \multirow[t]{3}{*}{ Table 10.17} & Transportation sector share of total employment & \\
\hline & 1999 & $8.4 \%$ \\
\hline & 2009 & $7.1 \%$ \\
\hline
\end{tabular}


The Transportation Services Index (TSI) was created by the U.S. Department of Transportation Bureau of Transportation Statistics (BTS). It is an index that measures the movement of freight and passengers. The Freight TSI consists of:

- for-hire trucking (parcel services are not included);

- freight railroad services (including rail-based intermodal shipments such as containers on flat cars); inland waterway traffic;

- pipeline movements (including principally petroleum and petroleum products and natural gas);

- and air freight.

The index does not include international or coastal steamship movements, private trucking, courier services, or the United States Postal Services.

The index does not include intercity bus, sightseeing services, taxi service, private automobile usage, or bicycling and other nonmotorized means of transportation.

Figure 10.1. Transportation Services Index, January 1990-January 2010

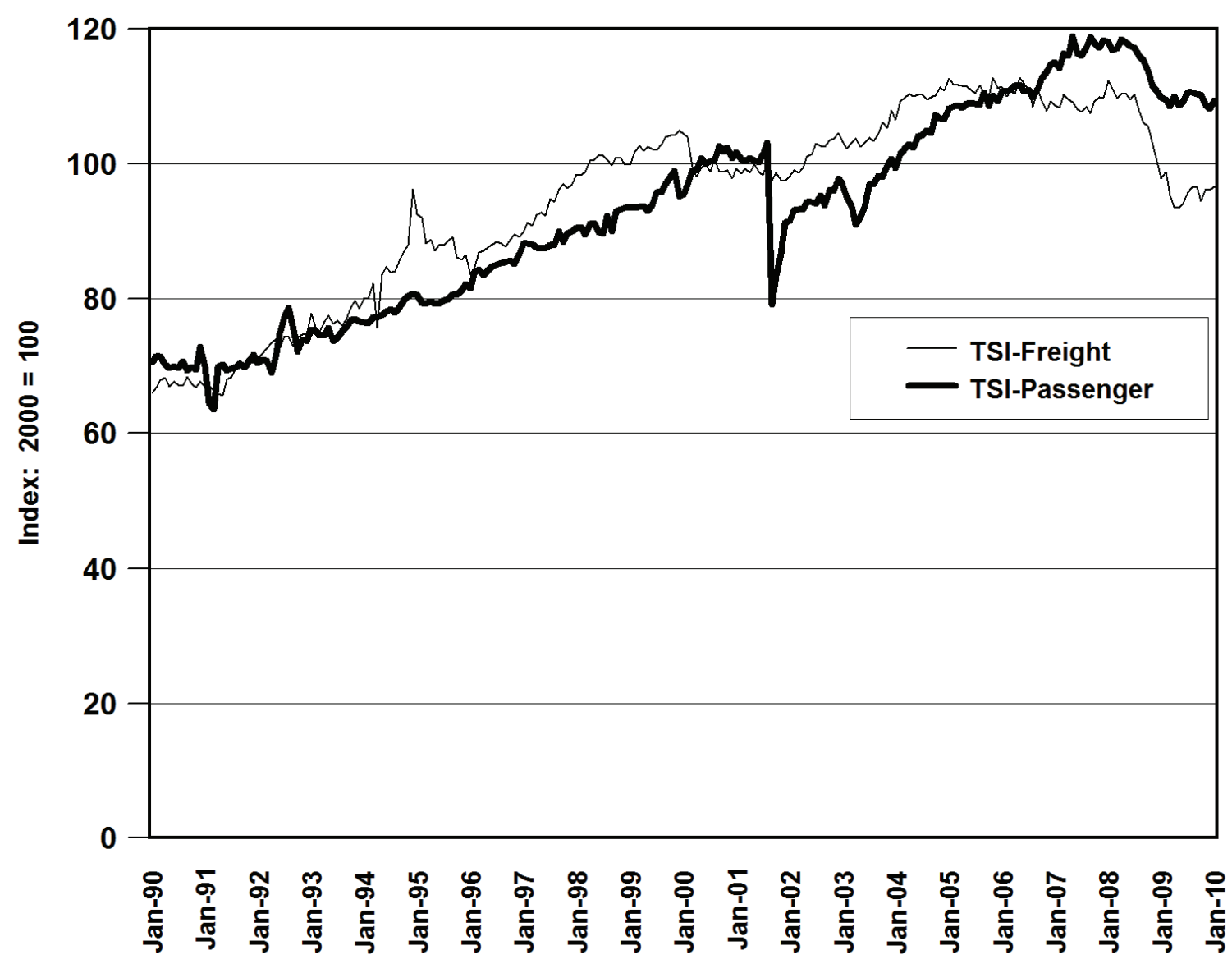

Source:

U.S. Department of Transportation, Bureau of Transportation Statistics, Transportation Services Index Web site, www.bts.gov/xml/tsi/src. (Additional resources: www.bts.gov.) 
The average price for a gallon of gasoline in China was 16 cents cheaper than in the United States in 2008. Those in France, the United Kingdom, and Germany paid, on average, more than seven dollars per gallon.

Table 10.1

Gasoline Prices ${ }^{\mathrm{a}}$ for Selected Countries, 1990-2008

\begin{tabular}{|c|c|c|c|c|c|c|c|c|}
\hline & \multicolumn{7}{|c|}{ Current dollars per gallon } & \multirow{2}{*}{$\begin{array}{c}\text { Average annual } \\
\text { percentage } \\
\text { change }\end{array}$} \\
\hline & 1990 & 1995 & 2000 & 2005 & 2006 & 2007 & 2008 & \\
\hline China & c & 1.03 & c & 1.70 & 2.11 & 2.29 & 3.11 & c \\
\hline Japan & 3.16 & 4.43 & 3.65 & 4.28 & 4.47 & 4.49 & 5.74 & $3.4 \%$ \\
\hline $\begin{array}{l}\text { France }^{b} \\
\text { United }\end{array}$ & 3.63 & 4.26 & 3.80 & 5.46 & 5.88 & 6.60 & 7.51 & $4.1 \%$ \\
\hline Kingdom $^{\mathrm{b}}$ & 2.82 & 3.21 & 4.58 & 5.97 & 6.36 & 7.13 & 7.42 & $5.5 \%$ \\
\hline Germany & 2.65 & 3.96 & 3.45 & 5.66 & 6.03 & 6.88 & 7.75 & $6.1 \%$ \\
\hline Canada & 1.87 & 1.53 & 1.86 & 2.89 & 3.26 & 3.59 & 4.08 & $4.4 \%$ \\
\hline \multirow[t]{3}{*}{ United States $^{\mathrm{d}}$} & 1.16 & 1.15 & 1.51 & 2.30 & 2.59 & 2.80 & 3.27 & $5.9 \%$ \\
\hline & \multicolumn{7}{|c|}{ Constant 2008 dollars $^{\mathrm{e}}$ per gallon } & $\begin{array}{l}\text { Average annual } \\
\text { percentage } \\
\text { change }\end{array}$ \\
\hline & 1990 & 1995 & 2000 & 2005 & 2006 & 2007 & 2008 & 1990-2008 \\
\hline China & c & 1.46 & c & 1.87 & 2.25 & 2.38 & 3.11 & $\mathrm{c}$ \\
\hline Japan & 5.21 & 6.26 & 4.56 & 4.72 & 4.77 & 4.66 & 5.74 & $0.5 \%$ \\
\hline $\begin{array}{l}\text { France } \\
\text { United }\end{array}$ & 5.98 & 6.02 & 4.75 & 6.02 & 6.28 & 6.85 & 7.51 & $1.3 \%$ \\
\hline Kingdom $^{\mathrm{b}}$ & 4.65 & 4.53 & 5.73 & 6.58 & 6.79 & 7.40 & 7.42 & $2.6 \%$ \\
\hline Germany & 4.37 & 5.59 & 4.31 & 6.24 & 6.44 & 7.14 & 7.25 & $2.9 \%$ \\
\hline Canada & 3.08 & 2.16 & 2.33 & 3.19 & 3.48 & 3.73 & 4.08 & $1.6 \%$ \\
\hline United States ${ }^{\mathrm{d}}$ & 1.91 & 1.62 & 1.89 & 2.54 & 2.77 & 2.91 & 3.27 & $3.0 \%$ \\
\hline
\end{tabular}

Source:

U.S. Department of Energy, Energy Information Administration, Annual Energy Review 2008, Washington, DC, June 2009. (Additional resources: www.eia.doe.gov)

Note: Comparisons between prices and price trends in different countries require care. They are of limited validity because of fluctuations in exchange rates; differences in product quality, marketing practices, and market structures; and the extent to which the standard categories of sales are representative of total national sales for a given period.

${ }^{a}$ Prices represent the retail prices (including taxes) for regular unleaded gasoline, except for France and the United Kingdom which are premium unleaded gasoline.

${ }^{\mathrm{b}}$ Premium gasoline.

${ }^{\mathrm{c}}$ Data are not available.

d These estimates are international comparisons only and do not necessarily correspond to gasoline price estimates in other sections of the book.

${ }^{\mathrm{e}}$ Adjusted by the U.S. Consumer Price Inflation Index. 
Of these selected countries, the United Kingdom had the highest diesel fuel price average in 2008, while China had the lowest.

Table 10.2

Diesel Fuel Prices ${ }^{\text {a }}$ for Selected Countries, 1998-2008

\begin{tabular}{|c|c|c|c|c|c|c|c|c|c|}
\hline & \multicolumn{8}{|c|}{ Current dollars per gallon } & \multirow{2}{*}{ 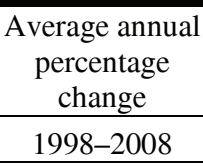 } \\
\hline & 1998 & 2000 & 2003 & 2004 & 2005 & 2006 & 2007 & 2008 & \\
\hline China & $\mathrm{b}$ & $\mathrm{b}$ & 1.32 & 1.47 & 1.69 & 2.10 & 2.42 & 3.15 & $\mathrm{~b}$ \\
\hline Japan & 2.25 & 2.85 & 2.76 & 3.09 & 3.45 & 3.68 & 3.82 & 5.14 & $8.6 \%$ \\
\hline France & 2.71 & 2.95 & 3.39 & 4.16 & 4.81 & 5.13 & 5.64 & 7.03 & $10.0 \%$ \\
\hline United Kingdom & 4.10 & 4.66 & 4.82 & 5.68 & 6.26 & 6.64 & 7.33 & 8.14 & $7.1 \%$ \\
\hline Germany & 2.45 & 2.79 & 3.79 & 4.41 & 5.01 & 5.30 & 6.06 & 7.37 & $11.6 \%$ \\
\hline \multirow[t]{3}{*}{ United States $^{\mathrm{c}}$} & 1.04 & 1.50 & 1.51 & 1.81 & 2.41 & 2.70 & 2.88 & 3.80 & $13.8 \%$ \\
\hline & \multicolumn{8}{|c|}{ Constant 2008 dollars $^{\mathrm{d}}$ per gallon } & $\begin{array}{l}\text { Average annual } \\
\text { percentage } \\
\text { change }\end{array}$ \\
\hline & 1998 & 2000 & 2003 & 2004 & 2005 & 2006 & 2007 & 2008 & 1998-2008 \\
\hline China & $\mathrm{b}$ & $\mathrm{b}$ & 1.55 & 1.68 & 1.86 & 2.25 & 2.51 & 3.15 & $\mathrm{~b}$ \\
\hline Japan & 2.98 & 3.57 & 3.23 & 3.52 & 3.80 & 3.93 & 3.97 & 5.14 & $5.6 \%$ \\
\hline France & 3.58 & 3.69 & 3.97 & 4.74 & 5.30 & 5.47 & 5.86 & 7.03 & $7.0 \%$ \\
\hline United Kingdom & 5.42 & 5.82 & 5.64 & 6.47 & 6.90 & 7.09 & 7.61 & 8.14 & $4.2 \%$ \\
\hline Germany & 3.24 & 3.49 & 4.43 & 5.02 & 5.52 & 5.66 & 6.29 & 7.37 & $8.6 \%$ \\
\hline United States $^{\mathrm{c}}$ & 1.38 & 1.87 & 1.76 & 2.07 & 2.65 & 2.89 & 3.00 & 3.80 & $10.7 \%$ \\
\hline
\end{tabular}

Source:

International Energy Agency, Energy Prices and Taxes, Fourth Quarter, 2009, Paris, France, 2010 (Additional resources: www.iea.org)

Note: Comparisons between prices and price trends in different countries require care. They are of limited validity because of fluctuations in exchange rates; differences in product quality, marketing practices, and market structures; and the extent to which the standard categories of sales are representative of total national sales for a given period.

a Prices represent the retail prices (including taxes) for automotive diesel fuel for non-commercial (household) use.

${ }^{\mathrm{b}}$ Data are not available.

${ }^{c}$ These estimates are for international comparisons only and do not necessarily correspond to gasoline price estimates in other sections of the book.

${ }^{\mathrm{d}}$ Adjusted by the U.S. Consumer Price Inflation Index. 
In 2008 more than sixty percent of the cost of gasoline in France, Germany, and the United Kingdom went for taxes.

Of the listed countries, the United States has the lowest percentage of taxes.

Figure 10.2. Gasoline Prices for Selected Countries, 1990 and 2008

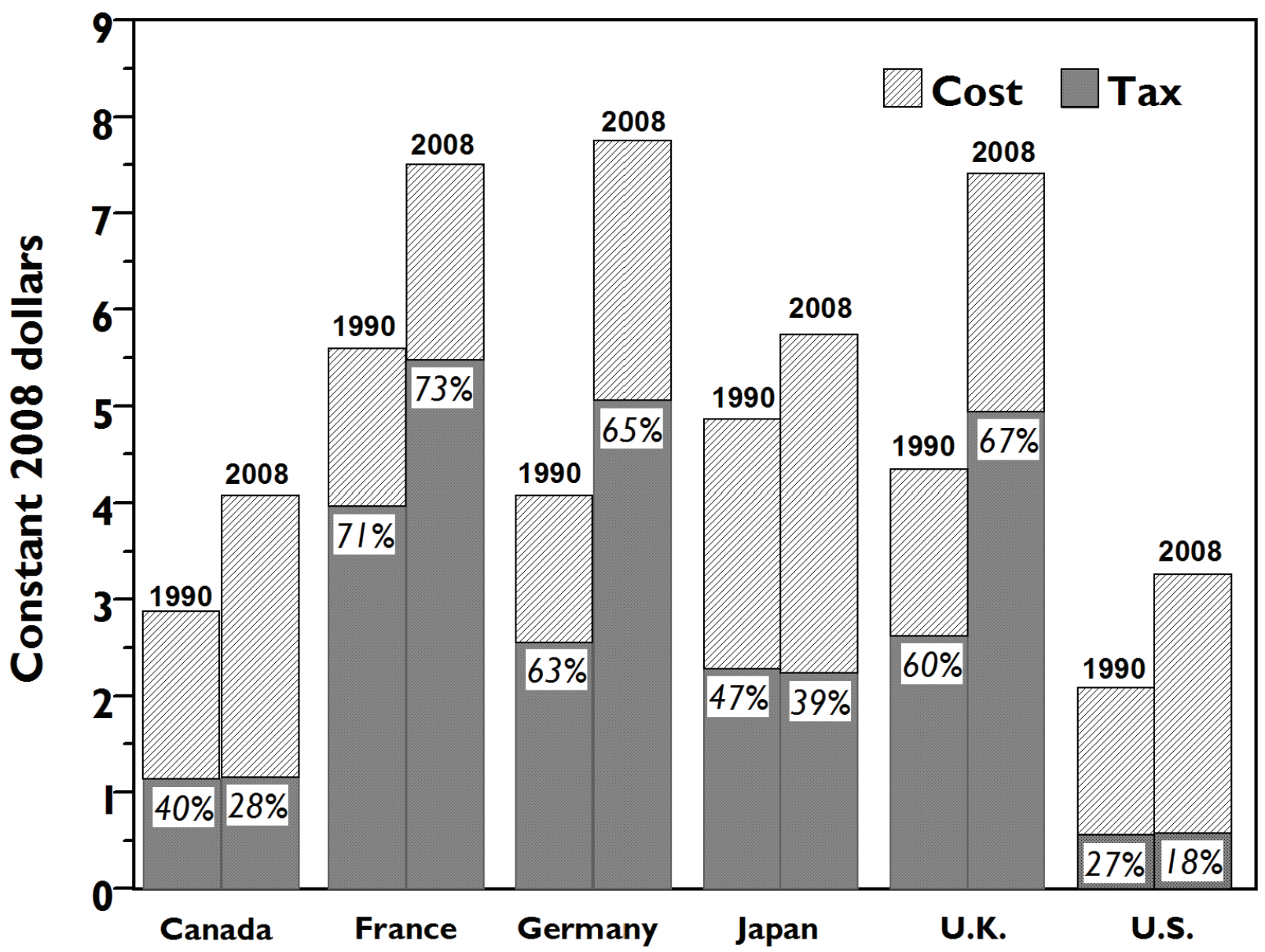

Source:

Table 10.1 and International Energy Agency, Energy Prices \& Taxes, Fourth Quarter, 2009, Paris, France, 2010. (Additional resources: www.iea.org.) 
Diesel fuel is taxed heavily in the European countries shown here. The U.S. diesel fuel tax share is the lowest of the listed countries.

Figure 10.3. Diesel Prices for Selected Countries, 1990 and 2008

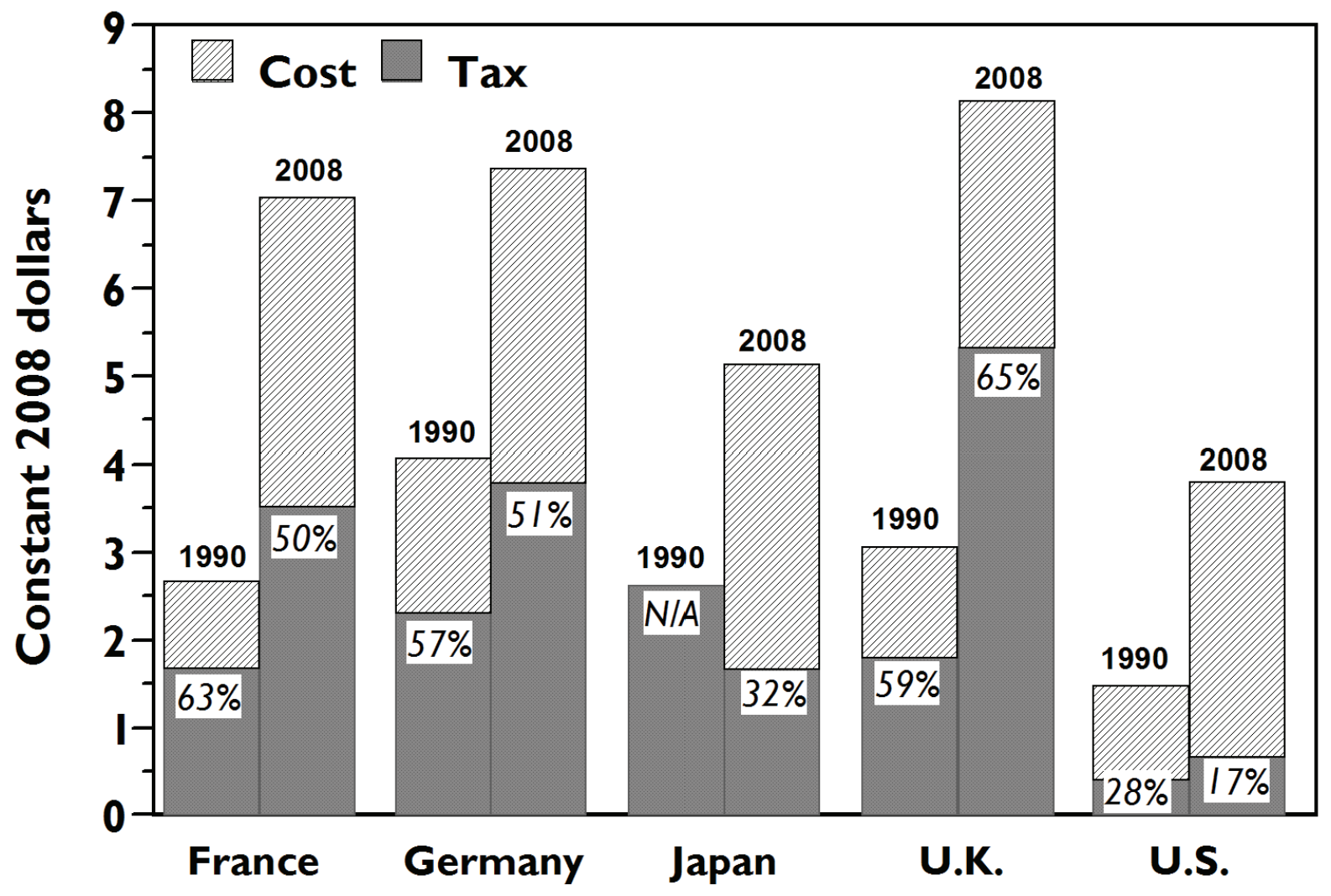

Source:

Table 10.2 and International Energy Agency, Energy Prices \& Taxes, Fourth Quarter, 2009, Paris, France, 2010. (Additional resources: www.iea.org.) 
Though the cost of crude oil certainly influences the price of gasoline, it is not the only factor which determines the price at the pump. Processing cost, transportation cost, and taxes also play a major part of the cost of a gallon of gasoline. The average price of a barrel of crude oil (in constant 2009 dollars) increased by 68\% from 2000 to 2009, while the average price of a gallon of gasoline increased 23\% in this same time period.

Table 10.3

Prices for a Barrel of Crude Oil and a Gallon of Gasoline, 1978-2009

\begin{tabular}{|c|c|c|c|c|c|}
\hline \multirow[b]{2}{*}{ Year } & \multicolumn{2}{|c|}{$\begin{array}{c}\text { Crude oil }^{\mathrm{a}} \\
\text { (dollars per barrel) }\end{array}$} & \multicolumn{2}{|c|}{$\begin{array}{c}\text { Gasoline } \\
\text { (cents per gallon) }\end{array}$} & \multirow{2}{*}{$\begin{array}{l}\text { Ratio of } \\
\text { gasoline to } \\
\text { crude oil }\end{array}$} \\
\hline & Current & Constant $2009^{c}$ & Current & Constant $2009^{c}$ & \\
\hline 1978 & 12.5 & 41.0 & 65.2 & 214.5 & 219.8 \\
\hline 1979 & 17.7 & 52.4 & 88.2 & 260.0 & 209.1 \\
\hline 1980 & 28.1 & 73.1 & 122.1 & 317.9 & 182.7 \\
\hline 1981 & 35.2 & 83.2 & 135.3 & 319.3 & 161.3 \\
\hline 1982 & 31.9 & 70.9 & 128.1 & 284.8 & 168.8 \\
\hline 1983 & 29.0 & 62.4 & 122.5 & 263.9 & 177.5 \\
\hline 1984 & 28.6 & 59.1 & 119.8 & 247.4 & 175.7 \\
\hline 1985 & 26.8 & 53.3 & 119.6 & 238.5 & 187.8 \\
\hline 1986 & 14.6 & 28.5 & 93.1 & 182.2 & 268.7 \\
\hline 1987 & 17.9 & 33.8 & 95.7 & 180.7 & 224.5 \\
\hline 1988 & 14.7 & 26.6 & 96.3 & 174.6 & 275.7 \\
\hline 1989 & 18.0 & 31.1 & 106.0 & 183.4 & 247.7 \\
\hline 1990 & 22.2 & 36.5 & 121.7 & 199.8 & 230.0 \\
\hline 1991 & 19.1 & 30.0 & 119.6 & 188.4 & 263.5 \\
\hline 1992 & 18.4 & 28.2 & 119.0 & 182.0 & 271.2 \\
\hline 1993 & 16.4 & 24.4 & 117.3 & 174.2 & 300.2 \\
\hline 1994 & 15.6 & 22.6 & 117.4 & 170.0 & 316.3 \\
\hline 1995 & 17.2 & 24.3 & 120.5 & 169.6 & 293.7 \\
\hline 1996 & 20.7 & 28.3 & 128.8 & 176.1 & 261.2 \\
\hline 1997 & 19.0 & 25.5 & 129.1 & 172.6 & 284.8 \\
\hline 1998 & 12.5 & 16.5 & 111.5 & 146.8 & 374.0 \\
\hline 1999 & 17.5 & 22.5 & 122.1 & 157.2 & 292.9 \\
\hline 2000 & 28.3 & 35.2 & 156.3 & 194.7 & 232.3 \\
\hline 2001 & 23.0 & 27.8 & 153.1 & 185.5 & 280.2 \\
\hline 2002 & 24.1 & 28.7 & 144.1 & 171.8 & 251.1 \\
\hline 2003 & 28.5 & 33.3 & 163.8 & 191.0 & 241.1 \\
\hline 2004 & 37.0 & 42.0 & 192.3 & 218.4 & 218.4 \\
\hline 2005 & 50.2 & 55.2 & 233.8 & 256.8 & 195.5 \\
\hline 2006 & 60.2 & 64.1 & 263.5 & 280.4 & 183.7 \\
\hline 2007 & 67.9 & 70.3 & 284.9 & 294.8 & 176.1 \\
\hline 2008 & 94.7 & 94.4 & 331.7 & 330.5 & 147.1 \\
\hline 2009 & 59.3 & 59.3 & 240.1 & 240.1 & 170.1 \\
\hline \multicolumn{6}{|c|}{ Average annual percentage change } \\
\hline 1978-2009 & $5.2 \%$ & $1.2 \%$ & $4.3 \%$ & $0.4 \%$ & \\
\hline 1999-2009 & $13.0 \%$ & $10.2 \%$ & $7.0 \%$ & $4.3 \%$ & \\
\hline
\end{tabular}

\section{Sources:}

Crude oil - U.S. Department of Energy, Energy Information Administration, Monthly Energy Review, March 2010, Washington, DC, Table 9.1.

Gasoline - U.S. Department of Energy, Energy Information Administration, Monthly Energy Review, March 2010, Washington, DC, Table 9.4. (Additional resources: www.eia.doe.gov)

${ }^{a}$ Refiner acquisition cost of composite (domestic and imported) crude oil.

${ }^{\mathrm{b}}$ Average for all types. These prices were collected from a sample of service stations in 85 urban areas selected to represent all urban consumers. Urban consumers make up about $80 \%$ of the total U.S. population.

${ }^{c}$ Adjusted by the Consumer Price Inflation Index. 
Diesel fuel price has generally been lower than gasoline; however, from 2005 through 2009 the price of diesel fuel was higher than that of gasoline.

Table 10.4

Retail Prices for Motor Fuel, 1978-2009 (cents per gallon, including tax)

\begin{tabular}{|c|c|c|c|c|}
\hline \multirow[b]{2}{*}{ Year } & \multicolumn{2}{|c|}{ Diesel fuel $^{\mathrm{a}}$} & \multicolumn{2}{|c|}{ Average for all gasoline types ${ }^{\mathrm{b}}$} \\
\hline & Current & $\begin{array}{c}\text { Constant } \\
2009^{c}\end{array}$ & Current & $\begin{array}{c}\text { Constant } \\
2009^{c}\end{array}$ \\
\hline 1978 & d & d & 65 & 215 \\
\hline 1979 & d & d & 88 & 261 \\
\hline 1980 & 101 & 263 & 122 & 318 \\
\hline 1981 & 118 & 278 & 135 & 319 \\
\hline 1982 & 116 & 258 & 128 & 285 \\
\hline 1983 & 120 & 258 & 123 & 264 \\
\hline 1984 & 122 & 252 & 120 & 247 \\
\hline 1985 & 122 & 243 & 120 & 238 \\
\hline 1986 & 94 & 184 & 93 & 182 \\
\hline 1987 & 96 & 181 & 96 & 181 \\
\hline 1988 & 95 & 172 & 96 & 175 \\
\hline 1989 & 102 & 176 & 106 & 183 \\
\hline 1990 & 107 & 176 & 122 & 200 \\
\hline 1991 & 91 & 143 & 120 & 188 \\
\hline 1992 & 106 & 162 & 119 & 182 \\
\hline 1993 & 98 & 145 & 117 & 174 \\
\hline 1994 & 111 & 161 & 117 & 170 \\
\hline 1995 & 111 & 156 & 121 & 170 \\
\hline 1996 & 124 & 169 & 129 & 176 \\
\hline 1997 & 120 & 160 & 129 & 173 \\
\hline 1998 & 104 & 137 & 112 & 147 \\
\hline 1999 & 112 & 144 & 122 & 157 \\
\hline 2000 & 149 & 186 & 156 & 195 \\
\hline 2001 & 140 & 170 & 153 & 185 \\
\hline 2002 & 132 & 157 & 144 & 172 \\
\hline 2003 & 151 & 176 & 164 & 191 \\
\hline 2004 & 181 & 206 & 192 & 218 \\
\hline 2005 & 240 & 264 & 234 & 257 \\
\hline 2006 & 271 & 288 & 264 & 280 \\
\hline 2007 & 289 & 299 & 285 & 295 \\
\hline 2008 & 380 & 379 & 332 & 331 \\
\hline 2009 & 247 & 247 & 240 & 240 \\
\hline \multicolumn{5}{|c|}{ Average annual percentage change } \\
\hline 1978-2009 & $3.1 \% \mathrm{e}^{\mathrm{e}}$ & $-0.2 \%^{\mathrm{e}}$ & $4.3 \%$ & $0.4 \%$ \\
\hline 1999-2009 & $8.2 \%$ & $5.5 \%$ & $7.0 \%$ & $4.3 \%$ \\
\hline
\end{tabular}

\section{Sources:}

Gasoline - U.S. Department of Energy, Energy Information Administration, Monthly Energy Review, February 2010, Washington, DC, Table 9.4.

Diesel - U.S. Department of Energy, Energy Information Administration, International Energy Annual 2004, Washington, DC, June 2004, Table 7.2. 2005-2009 data from EIA Petroleum Navigator Web site. (Additional resources: www.eia.doe.gov)

a 1980-1993: Collected from a survey of prices on January 1 of the current year. 1994-on: Annual average.

${ }^{b}$ These prices were collected from a sample of service stations in 85 urban areas selected to represent all urban consumers. Urban consumers make up about 80 percent of the total U.S. population.

${ }^{\mathrm{c}}$ Adjusted by the Consumer Price Inflation Index.

${ }^{\mathrm{d}}$ Data are not available.

${ }^{\mathrm{e}}$ Average annual percentage change is from the earliest year possible to 2007. 
The fuel prices shown here are refiner sales prices of transportation fuels to end users, excluding tax. Sales to end users are those made directly to the ultimate consumer, including bulk consumers. Bulk sales to utility, industrial, and commercial accounts previously included in the wholesale category are now counted as sales to end users.

Table 10.5

Refiner Sales Prices for Propane and No. 2 Diesel, 1978-2009 (cents per gallon, excluding tax)

\begin{tabular}{|c|c|c|c|c|}
\hline \multirow[b]{3}{*}{ Year } & \multicolumn{2}{|c|}{ Propane $^{\mathrm{a}}$} & \multicolumn{2}{|c|}{ No 2. diesel fuel } \\
\hline & & Constant & & Constant \\
\hline & Current & $2009^{\mathrm{b}}$ & Current & $2009^{\mathrm{b}}$ \\
\hline 1978 & 33.5 & 110.2 & 37.7 & 124.0 \\
\hline 1979 & 35.7 & 105.5 & 58.5 & 172.9 \\
\hline 1980 & 48.2 & 125.5 & 81.8 & 213.0 \\
\hline 1981 & 56.5 & 133.3 & 99.5 & 234.8 \\
\hline 1982 & 59.2 & 131.6 & 94.2 & 209.4 \\
\hline 1983 & 70.9 & 152.7 & 82.6 & 177.9 \\
\hline 1984 & 73.7 & 152.2 & 82.3 & 169.9 \\
\hline 1985 & 71.7 & 143.0 & 78.9 & 157.3 \\
\hline 1986 & 74.5 & 145.8 & 47.8 & 93.6 \\
\hline 1987 & 70.1 & 132.4 & 55.1 & 104.1 \\
\hline 1988 & 71.4 & 129.5 & 50.0 & 90.7 \\
\hline 1989 & 61.5 & 106.4 & 58.5 & 101.2 \\
\hline 1990 & 74.5 & 122.3 & 72.5 & 119.0 \\
\hline 1991 & 73.0 & 115.0 & 64.8 & 102.1 \\
\hline 1992 & 64.3 & 98.3 & 61.9 & 94.7 \\
\hline 1993 & 67.3 & 99.9 & 60.2 & 89.4 \\
\hline 1994 & 53.0 & 76.7 & 55.4 & 80.2 \\
\hline 1995 & 49.2 & 69.3 & 56.0 & 78.8 \\
\hline 1996 & 60.5 & 82.7 & 68.1 & 93.1 \\
\hline 1997 & 55.2 & 73.8 & 64.2 & 85.8 \\
\hline 1998 & 40.5 & 53.3 & 49.4 & 65.0 \\
\hline 1999 & 45.8 & 59.0 & 58.4 & 75.2 \\
\hline 2000 & 60.3 & 75.1 & 93.5 & 116.5 \\
\hline 2001 & 50.6 & 61.3 & 84.2 & 102.0 \\
\hline 2002 & 41.9 & 50.0 & 76.2 & 90.9 \\
\hline 2003 & 57.7 & 67.3 & 94.4 & 110.1 \\
\hline 2004 & 83.9 & 95.3 & 124.3 & 141.2 \\
\hline 2005 & 108.9 & 119.6 & 178.6 & 196.2 \\
\hline 2006 & 135.8 & 144.5 & 209.6 & 223.1 \\
\hline 2007 & 148.8 & 154.1 & 226.7 & 234.6 \\
\hline 2008 & 184.2 & 188.5 & 315.0 & 313.9 \\
\hline 2009 & 123.6 & 123.6 & 183.3 & 183.3 \\
\hline \multicolumn{5}{|c|}{ Average annual percentage change } \\
\hline 1978-2009 & $4.3 \%$ & $0.4 \%$ & $5.2 \%$ & $1.3 \%$ \\
\hline 1999-2009 & $10.4 \%$ & $7.7 \%$ & $12.1 \%$ & $9.3 \%$ \\
\hline
\end{tabular}

Source:

U.S. Department of Energy, Energy Information Administration, Monthly Energy Review, March 2010, Washington, DC, Table 9.7. (Additional resources: www.eia.doe.gov)

${ }^{\mathrm{a}}$ Consumer grade.

${ }^{\mathrm{b}}$ Adjusted by the Consumer Price Inflation Index. 
Prices of finished aviation gasoline and kerosene-type jet fuel began climbing in 1999 and peaked in 2008. In 2009 the prices declined to be near the 2005 levels.

Table 10.6

Refiner Sales Prices for Aviation Gasoline and Jet Fuel, 1978-2009 (cents per gallon, excluding tax)

\begin{tabular}{|c|c|c|c|c|}
\hline \multirow[b]{3}{*}{ Year } & \multicolumn{2}{|c|}{ Finished aviation gasoline } & \multicolumn{2}{|c|}{$\begin{array}{c}\text { Kerosene-type } \\
\text { jet fuel }\end{array}$} \\
\hline & & Constant & & Constant \\
\hline & Current & $2009^{\mathrm{a}}$ & Current & $2009^{a}$ \\
\hline 1978 & 51.6 & 169.8 & 38.7 & 127.3 \\
\hline 1979 & 68.9 & 203.6 & 54.7 & 161.6 \\
\hline 1980 & 108.4 & 282.2 & 86.6 & 225.5 \\
\hline 1981 & 130.3 & 307.5 & 102.4 & 241.7 \\
\hline 1982 & 131.2 & 291.7 & 96.3 & 214.1 \\
\hline 1983 & 125.5 & 270.3 & 87.8 & 189.1 \\
\hline 1984 & 123.4 & 254.8 & 84.2 & 173.9 \\
\hline 1985 & 120.1 & 239.5 & 79.6 & 158.7 \\
\hline 1986 & 101.1 & 197.9 & 52.9 & 103.5 \\
\hline 1987 & 90.7 & 171.3 & 54.3 & 102.5 \\
\hline 1988 & 89.1 & 161.6 & 51.3 & 93.0 \\
\hline 1989 & 99.5 & 172.1 & 59.2 & 102.4 \\
\hline 1990 & 112.0 & 183.8 & 76.6 & 125.7 \\
\hline 1991 & 104.7 & 164.9 & 65.2 & 102.7 \\
\hline 1992 & 102.7 & 157.0 & 61.0 & 93.3 \\
\hline 1993 & 99.0 & 147.0 & 58.0 & 86.1 \\
\hline 1994 & 95.7 & 138.5 & 53.4 & 77.3 \\
\hline 1995 & 100.5 & 141.5 & 54.0 & 76.0 \\
\hline 1996 & 111.6 & 152.6 & 65.1 & 89.0 \\
\hline 1997 & 112.8 & 150.8 & 61.3 & 81.9 \\
\hline 1998 & 97.5 & 128.3 & 45.2 & 59.5 \\
\hline 1999 & 105.9 & 136.4 & 54.3 & 69.9 \\
\hline 2000 & 130.6 & 162.7 & 89.9 & 112.0 \\
\hline 2001 & 132.3 & 160.3 & 77.5 & 93.9 \\
\hline 2002 & 128.8 & 153.6 & 72.1 & 86.0 \\
\hline 2003 & 149.3 & 174.1 & 87.2 & 101.7 \\
\hline 2004 & 181.9 & 206.6 & 120.7 & 137.1 \\
\hline 2005 & 223.1 & 245.1 & 173.5 & 190.6 \\
\hline 2006 & 268.2 & 285.4 & 199.8 & 212.6 \\
\hline 2007 & 284.9 & 294.8 & 216.5 & 224.0 \\
\hline 2008 & 327.3 & 326.1 & 305.2 & 304.1 \\
\hline 2009 & 243.8 & 243.8 & 170.4 & 170.4 \\
\hline \multicolumn{5}{|c|}{ Average annual percentage change } \\
\hline 1978-2009 & $5.1 \%$ & $1.2 \%$ & $4.9 \%$ & $0.9 \%$ \\
\hline 1999-2009 & $8.7 \%$ & $6.0 \%$ & $12.1 \%$ & $9.3 \%$ \\
\hline
\end{tabular}

Source:

U.S. Department of Energy, Energy Information Administration, Monthly Energy Review, March 2010, Washington, DC, Table 9.7. (Additional resources: www.eia.doe.gov)

${ }^{a}$ Adjusted by the Consumer Price Inflation Index. 
At the end of 2009, only four states offered tax exemptions to encourage the use of gasohol for transportation purposes. This list is quite short compared to the 30 states which offered gasohol tax exemptions twenty years ago.

Table 10.7

State Tax Exemptions for Gasohol, 2008

\begin{tabular}{lc}
\hline State & $\begin{array}{c}\text { Exemption } \\
\text { (cents/gallon of gasohol) }\end{array}$ \\
\hline Idaho & 2.5 \\
Iowa & 2.0 \\
Maine & 5.4 \\
South Dakota & 2.0 \\
\hline
\end{tabular}

\section{Source:}

U.S. Department of Transportation, Federal Highway Administration, Highway Statistics 2008, December 2009, Washington, DC, Table MF-121T. (Additional resources: www.fhwa.dot.gov)

Table 10.8

Federal Excise Taxes on Motor Fuels, 2008

\begin{tabular}{lc}
\hline Fuel & Cents per gallon \\
\hline Gasoline $^{\mathrm{a}}$ & 18.40 \\
Diesel & 24.40 \\
Gasohol $^{\mathrm{b}}$ & 18.40 \\
Other special fuels $^{\mathrm{b}}$ & 18.40 \\
CNG & 18.30 \\
LNG & 24.30 \\
LPG & 18.30 \\
\hline
\end{tabular}

Source:

U.S. Department of Transportation, Federal Highway Administration, Highway Statistics 2008, December 2009, Washington, DC, Table FE-21B. (Additional resources: www.fhwa.dot.gov)

${ }^{a}$ All gasohol blends are taxed at the same rate.

${ }^{\mathrm{b}}$ Includes benzol, benzene, naphtha, and other liquid used as a motor fuel. 
These states have laws and incentives for alternative fuels production and/or use.

Table 10.9

Federal and State Alternative Fuel Incentives, 2010

\begin{tabular}{|c|c|c|c|c|c|c|c|c|}
\hline State & Biodiesel & Ethanol & $\begin{array}{c}\text { Natural } \\
\text { Gas }\end{array}$ & $\begin{array}{l}\text { Liquefied } \\
\text { petroleum } \\
\text { gas (LPG) }\end{array}$ & $\begin{array}{c}\text { Electric } \\
\text { vehicles } \\
\text { (EVs) }\end{array}$ & $\begin{array}{l}\text { Neighborhood } \\
\text { Electric } \\
\text { vehicles } \\
\text { (NEVs) }\end{array}$ & $\begin{array}{l}\text { Hydrogen } \\
\text { fuel cells }\end{array}$ & $\begin{array}{l}\text { Aftermarket } \\
\text { conversions }\end{array}$ \\
\hline Federal & 33 & 28 & 27 & 27 & 22 & 2 & 27 & 6 \\
\hline Alabama & 7 & 5 & 4 & 4 & 3 & 0 & 3 & 0 \\
\hline Alaska & 2 & 3 & 2 & 2 & 2 & 1 & 2 & 1 \\
\hline Arizona & 5 & 3 & 9 & 9 & 8 & 1 & 6 & 0 \\
\hline Arkansas & 4 & 3 & 4 & 3 & 2 & 0 & 2 & 1 \\
\hline California & 15 & 12 & 30 & 18 & 32 & 3 & 25 & 1 \\
\hline Colorado & 9 & 9 & 12 & 10 & 8 & 1 & 9 & 3 \\
\hline Connecticut & 5 & 4 & 7 & 5 & 5 & 0 & 7 & 3 \\
\hline Delaware & 1 & 1 & 1 & 3 & 1 & 1 & 1 & 0 \\
\hline Dist. of Columbia & 1 & 2 & 4 & 3 & 3 & 0 & 3 & 0 \\
\hline Florida & 12 & 14 & 3 & 3 & 5 & 1 & 8 & 0 \\
\hline Georgia & 8 & 9 & 8 & 4 & 6 & 0 & 5 & 1 \\
\hline Hawaii & 8 & 10 & 5 & 5 & 6 & 1 & 6 & 0 \\
\hline Idaho & 5 & 3 & 2 & 2 & 0 & 1 & 1 & 0 \\
\hline Illinois & 19 & 18 & 10 & 9 & 9 & 2 & 8 & 3 \\
\hline Indiana & 12 & 18 & 9 & 6 & 5 & 1 & 5 & 2 \\
\hline Iowa & 13 & 17 & 6 & 5 & 7 & 1 & 5 & 1 \\
\hline Kansas & 8 & 11 & 4 & 4 & 0 & 1 & 0 & 1 \\
\hline Kentucky & 7 & 7 & 6 & 4 & 1 & 1 & 1 & 0 \\
\hline Louisiana & 5 & 9 & 5 & 4 & 3 & 1 & 1 & 1 \\
\hline Maine & 7 & 7 & 4 & 4 & 3 & 1 & 3 & 0 \\
\hline Maryland & 3 & 3 & 1 & 1 & 2 & 1 & 1 & 0 \\
\hline Massachusetts & 9 & 9 & 7 & 5 & 5 & 0 & 5 & 0 \\
\hline Michigan & 12 & 12 & 6 & 6 & 7 & 0 & 7 & 0 \\
\hline Minnesota & 10 & 11 & 4 & 4 & 6 & 2 & 5 & 0 \\
\hline Mississippi & 2 & 2 & 5 & 4 & 1 & 0 & 1 & 1 \\
\hline Missouri & 8 & 6 & 7 & 6 & 4 & 1 & 5 & 0 \\
\hline Montana & 9 & 7 & 4 & 4 & 2 & 1 & 2 & 1 \\
\hline Nebraska & 4 & 5 & 3 & 3 & 2 & 0 & 2 & 1 \\
\hline Nevada & 6 & 5 & 7 & 7 & 6 & 1 & 6 & 0 \\
\hline New Hampshire & 7 & 3 & 3 & 3 & 3 & 1 & 3 & 0 \\
\hline New Jersey & 3 & 3 & 6 & 5 & 5 & 1 & 3 & 0 \\
\hline New Mexico & 14 & 12 & 7 & 6 & 7 & 1 & 9 & 1 \\
\hline New York & 10 & 12 & 16 & 10 & 10 & 1 & 11 & 1 \\
\hline North Carolina & 13 & 11 & 6 & 6 & 6 & 0 & 5 & 1 \\
\hline North Dakota & 11 & 8 & 2 & 2 & 0 & 1 & 3 & 0 \\
\hline Ohio & 5 & 6 & 3 & 3 & 2 & 0 & 3 & 1 \\
\hline Oklahoma & 10 & 12 & 10 & 9 & 8 & 2 & 8 & 5 \\
\hline Oregon & 11 & 10 & 6 & 5 & 7 & 2 & 6 & 1 \\
\hline Pennsylvania & 8 & 7 & 5 & 4 & 4 & 0 & 4 & 0 \\
\hline Rhode Island & 2 & 1 & 3 & 2 & 2 & 0 & 2 & 0 \\
\hline South Carolina & 10 & 8 & 3 & 4 & 2 & 1 & 5 & 0 \\
\hline South Dakota & 8 & 9 & 1 & 2 & 0 & 0 & 0 & 0 \\
\hline Tennessee & 12 & 9 & 6 & 6 & 5 & 1 & 4 & 0 \\
\hline Texas & 9 & 9 & 13 & 11 & 7 & 1 & 7 & 2 \\
\hline Utah & 1 & 1 & 11 & 7 & 7 & 0 & 5 & 1 \\
\hline Vermont & 6 & 5 & 5 & 4 & 4 & 1 & 4 & 1 \\
\hline Virginia & 9 & 6 & 9 & 7 & 6 & 1 & 7 & 2 \\
\hline Washington & 16 & 14 & 8 & 8 & 15 & 1 & 5 & 3 \\
\hline West Virginia & 5 & 5 & 5 & 5 & 5 & 1 & 5 & 0 \\
\hline Wisconsin & 11 & 8 & 7 & 7 & 5 & 2 & 6 & 1 \\
\hline Wyoming & 0 & 1 & 1 & 0 & 0 & 0 & 0 & 0 \\
\hline Totals & 430 & 413 & 342 & 290 & 276 & 44 & 267 & 47 \\
\hline
\end{tabular}

Source:

U.S. Department of Energy, Energy Efficiency and Renewable Energy, Alternative Fuels Data Center. Data downloaded March 2010. (Additional resources: www.eere.energy.gov/afdc/laws/matrix/tech) 
Table 10.10

Federal and State Advanced Technology Incentives, 2010

\begin{tabular}{|c|c|c|c|c|}
\hline State & $\begin{array}{l}\text { Hybrid Electric Vehicles (HEV) or } \\
\text { Plug-in Hybrid Vehicles (PHEVs) }\end{array}$ & $\begin{array}{l}\text { Fuel Economy or } \\
\text { Efficiency }\end{array}$ & Idle Reduction & Other $^{\mathrm{a}}$ \\
\hline Federal & 8 & 13 & 6 & 7 \\
\hline Alabama & 1 & 1 & 1 & 0 \\
\hline Alaska & 0 & 1 & 0 & 0 \\
\hline Arizona & 1 & 1 & 3 & 1 \\
\hline Arkansas & 0 & 1 & 2 & 0 \\
\hline California & 23 & 3 & 5 & 3 \\
\hline Colorado & 4 & 2 & 4 & 0 \\
\hline Connecticut & 3 & 2 & 3 & 2 \\
\hline Delaware & 0 & 0 & 2 & 0 \\
\hline Dist. of Columbia & 1 & 2 & 1 & 1 \\
\hline Florida & 2 & 1 & 1 & 1 \\
\hline Georgia & 2 & 1 & 1 & 0 \\
\hline Hawaii & 1 & 1 & 1 & 0 \\
\hline Idaho & 1 & 1 & 0 & 0 \\
\hline Illinois & 5 & 3 & 3 & 0 \\
\hline Indiana & 4 & 1 & 0 & 0 \\
\hline Iowa & 0 & 1 & 0 & 0 \\
\hline Kansas & 0 & 1 & 1 & 0 \\
\hline Kentucky & 1 & 1 & 0 & 0 \\
\hline Louisiana & 2 & 1 & 0 & 0 \\
\hline Maine & 0 & 2 & 2 & 1 \\
\hline Maryland & 2 & 0 & 1 & 1 \\
\hline Massachusetts & 4 & 0 & 1 & 1 \\
\hline Michigan & 8 & 2 & 1 & 0 \\
\hline Minnesota & 5 & 2 & 4 & 0 \\
\hline Mississippi & 1 & 1 & 0 & 0 \\
\hline Missouri & 0 & 0 & 3 & 0 \\
\hline Montana & 0 & 1 & 0 & 0 \\
\hline Nebraska & 0 & 0 & 1 & 0 \\
\hline Nevada & 6 & 0 & 1 & 0 \\
\hline New Hampshire & 2 & 1 & 3 & 0 \\
\hline New Jersey & 4 & 1 & 1 & 1 \\
\hline New Mexico & 2 & 1 & 1 & 1 \\
\hline New York & 3 & 2 & 4 & 1 \\
\hline North Carolina & 3 & 1 & 3 & 0 \\
\hline North Dakota & 0 & 0 & 0 & 0 \\
\hline Ohio & 0 & 0 & 2 & 0 \\
\hline Oklahoma & 3 & 0 & 1 & 0 \\
\hline Oregon & 2 & 4 & 4 & 4 \\
\hline Pennsylvania & 2 & 1 & 6 & 1 \\
\hline Rhode Island & 1 & 1 & 1 & 1 \\
\hline South Carolina & 2 & 0 & 2 & 0 \\
\hline South Dakota & 0 & 0 & 0 & 0 \\
\hline Tennessee & 4 & 2 & 0 & 0 \\
\hline Texas & 4 & 0 & 5 & 1 \\
\hline Utah & 2 & 0 & 3 & 0 \\
\hline Vermont & 3 & 3 & 2 & 1 \\
\hline Virginia & 1 & 2 & 2 & 0 \\
\hline Washington & 4 & 1 & 3 & 3 \\
\hline West Virginia & 1 & 1 & 0 & 0 \\
\hline Wisconsin & 3 & 1 & 3 & 0 \\
\hline Wyoming & 0 & 0 & 0 & 0 \\
\hline Totals & 131 & 68 & 94 & 32 \\
\hline
\end{tabular}

Source:

U.S. Department of Energy, Energy Efficiency and Renewable Energy, Alternative Fuels Data Center. Data downloaded March 2010. (Additional resources: www.eere.energy.gov/afdc/laws/matrix/tech)

${ }^{a}$ Includes Clean Fuel Initiatives and Pollution Prevention. 
The average price of a new car in 2008 (\$23,051) was very close to the average price in $1916(\$ 21,348)$ when adjusted for inflation. Average new car prices were at their lowest in 1940 (\$11,941). Since 1914 the highest average price was in the year 1998 (\$26,898).

Table 10.11

Average Price of a New Car, 1906-2008

\begin{tabular}{cccccccc}
\hline & $\begin{array}{c}2008 \\
\text { Constant }\end{array}$ & \multicolumn{3}{c}{$\begin{array}{c}2008 \\
\text { Constant }\end{array}$} & & $\begin{array}{c}2008 \\
\text { Constant }\end{array}$ \\
Year & Dollars & Year & Dollars & Year & Dollars & Year & $\begin{array}{c}\text { Constant } \\
\text { Dollars }\end{array}$ \\
\hline 1906 & $\$ 36,588$ & 1934 & $\$ 16,645$ & 1959 & $\$ 21,259$ & 1984 & $\$ 23,571$ \\
1908 & $\$ 32,755$ & 1935 & $\$ 14,835$ & 1960 & $\$ 20,457$ & 1985 & $\$ 23,687$ \\
1910 & $\$ 27,877$ & 1936 & $\$ 13,026$ & 1961 & $\$ 19,479$ & 1986 & $\$ 24,854$ \\
1912 & $\$ 28,922$ & 1937 & $\$ 13,388$ & 1962 & $\$ 19,364$ & 1987 & $\$ 25,370$ \\
1913 & $\$ 31,118$ & 1938 & $\$ 13,750$ & 1963 & $\$ 19,151$ & 1988 & $\$ 25,356$ \\
1914 & $\$ 32,204$ & 1939 & $\$ 12,845$ & 1964 & $\$ 19,246$ & 1989 & $\$ 24,953$ \\
1915 & $\$ 26,776$ & 1940 & $\$ 11,941$ & 1965 & $\$ 18,882$ & 1990 & $\$ 24,779$ \\
1916 & $\$ 21,348$ & 1941 & $\$ 12,095$ & 1966 & $\$ 18,867$ & 1991 & $\$ 24,463$ \\
1917 & $\$ 19,720$ & 1942 & $\$ 12,250$ & 1967 & $\$ 18,957$ & 1992 & $\$ 25,069$ \\
1918 & $\$ 18,092$ & 1943 & $\$ 12,405$ & 1968 & $\$ 19,534$ & 1993 & $\$ 25,138$ \\
1919 & $\$ 17,911$ & 1944 & $\$ 12,560$ & 1969 & $\$ 19,535$ & 1994 & $\$ 26,009$ \\
1920 & $\$ 17,730$ & 1945 & $\$ 12,715$ & 1970 & $\$ 19,655$ & 1995 & $\$ 25,372$ \\
1921 & $\$ 18,816$ & 1946 & $\$ 12,870$ & 1971 & $\$ 19,893$ & 1996 & $\$ 25,766$ \\
1922 & $\$ 19,901$ & 1947 & $\$ 13,025$ & 1972 & $\$ 19,980$ & 1997 & $\$ 26,200$ \\
1923 & $\$ 18,092$ & 1948 & $\$ 13,641$ & 1973 & $\$ 19,649$ & 1998 & $\$ 26,898$ \\
1924 & $\$ 16,283$ & 1949 & $\$ 15,895$ & 1974 & $\$ 19,390$ & 1999 & $\$ 26,339$ \\
1925 & $\$ 16,102$ & 1950 & $\$ 16,290$ & 1975 & $\$ 19,809$ & 2000 & $\$ 25,756$ \\
1926 & $\$ 15,921$ & 1951 & $\$ 16,567$ & 1976 & $\$ 20,501$ & 2001 & $\$ 25,463$ \\
1927 & $\$ 15,740$ & 1952 & $\$ 17,946$ & 1977 & $\$ 20,656$ & 2002 & $\$ 25,431$ \\
1928 & $\$ 15,559$ & 1953 & $\$ 17,968$ & 1978 & $\$ 21,065$ & 2003 & $\$ 24,770$ \\
1929 & $\$ 15,378$ & 1954 & $\$ 17,642$ & 1979 & $\$ 20,306$ & 2004 & $\$ 24,661$ \\
1930 & $\$ 15,197$ & 1955 & $\$ 17,546$ & 1980 & $\$ 19,790$ & 2005 & $\$ 24,801$ \\
1931 & $\$ 17,006$ & 1956 & $\$ 18,052$ & 1981 & $\$ 21,104$ & 2006 & $\$ 24,713$ \\
1932 & $\$ 18,816$ & 1957 & $\$ 20,053$ & 1982 & $\$ 22,066$ & 2007 & $\$ 24,232$ \\
1933 & $\$ 17,730$ & 1958 & $\$ 21,214$ & 1983 & $\$ 22,927$ & 2008 & $\$ 23,051$ \\
\hline
\end{tabular}

Sources:

Compiled by Jacob Ward, Vehicle Technologies Program, U.S. Department of Energy, from the following sources. Raff, D.M.G. \& Trajtenberg, M. (1995), "Quality-Adjusted Prices for the American Automobile Industry: 1906-1940," National Bureau of Economic Research, Inc.; Gordon, R.J. (1990), The Measurement of Durable Goods Prices, National Bureau of Economic Research, Inc.; and U.S. Department of Commerce, Bureau of Economic Analysis (2010), National Income and Product Accounts.

Note: Estimations were used for years 1941-1946. 
In current dollars, import cars, on average, were less expensive than domestic cars until 1982. Since then, import prices have tripled, while domestic prices have doubled (current dollars).

Table 10.12

Average Price of a New Car (Domestic and Import), 1970-2008

\begin{tabular}{|c|c|c|c|c|c|c|}
\hline \multirow[b]{2}{*}{ Year } & \multicolumn{2}{|c|}{ Domestic $^{\mathrm{a}}$} & \multicolumn{2}{|c|}{ Import } & \multicolumn{2}{|c|}{ Total } \\
\hline & $\begin{array}{c}\text { Current } \\
\text { dollars }\end{array}$ & $\begin{array}{c}\text { Constant } 2008 \\
\text { dollars }^{\mathrm{b}}\end{array}$ & $\begin{array}{c}\text { Current } \\
\text { dollars }\end{array}$ & $\begin{array}{c}\text { Constant } 2008 \\
\text { dollars }\end{array}$ & $\begin{array}{c}\text { Current } \\
\text { dollars }\end{array}$ & $\begin{array}{c}\text { Constant } 2008 \\
\text { dollars }^{\mathrm{b}}\end{array}$ \\
\hline 1970 & 3,708 & 20,576 & 2,648 & 14,694 & 3,542 & 19,655 \\
\hline 1971 & 3,919 & 20,834 & 2,769 & 14,720 & 3,742 & 19,893 \\
\hline 1972 & 4,034 & 20,778 & 2,994 & 15,421 & 3,879 & 19,980 \\
\hline 1973 & 4,181 & 20,274 & 3,344 & 16,216 & 4,052 & 19,649 \\
\hline 1974 & 4,524 & 19,757 & 4,026 & 17,582 & 4,440 & 19,390 \\
\hline 1975 & 5,084 & 20,346 & 4,384 & 17,544 & 4,950 & 19,809 \\
\hline 1976 & 5,506 & 20,834 & 4,923 & 18,628 & 5,418 & 20,501 \\
\hline 1977 & 5,985 & 21,264 & 5,072 & 18,020 & 5,814 & 20,656 \\
\hline 1978 & 6,478 & 21,392 & 5,934 & 19,595 & 6,379 & 21,065 \\
\hline 1979 & 6,889 & 20,430 & 6,704 & 19,881 & 6,847 & 20,306 \\
\hline 1980 & 7,609 & 19,882 & 7,482 & 19,550 & 7,574 & 19,790 \\
\hline 1981 & 8,912 & 21,109 & 8,896 & 21,071 & 8,910 & 21,104 \\
\hline 1982 & 9,865 & 22,010 & 9,957 & 22,215 & 9,890 & 22,066 \\
\hline 1983 & 10,516 & 22,732 & 10,868 & 23,493 & 10,606 & 22,927 \\
\hline 1984 & 11,079 & 22,958 & 12,336 & 25,563 & 11,375 & 23,571 \\
\hline 1985 & 11,589 & 23,189 & 12,853 & 25,718 & 11,838 & 23,687 \\
\hline 1986 & 12,319 & 24,200 & 13,670 & 26,854 & 12,652 & 24,854 \\
\hline 1987 & 12,922 & 24,491 & 14,470 & 27,425 & 13,386 & 25,370 \\
\hline 1988 & 13,418 & 24,420 & 15,221 & 27,702 & 13,932 & 25,356 \\
\hline 1989 & 13,936 & 24,197 & 15,510 & 26,930 & 14,371 & 24,953 \\
\hline 1990 & 14,489 & 23,868 & 16,640 & 27,411 & 15,042 & 24,779 \\
\hline 1991 & 15,192 & 24,015 & 16,327 & 25,809 & 15,475 & 24,463 \\
\hline 1992 & 15,644 & 24,007 & 18,593 & 28,533 & 16,336 & 25,069 \\
\hline 1993 & 15,976 & 23,804 & 20,261 & 30,189 & 16,871 & 25,138 \\
\hline 1994 & 16,930 & 24,596 & 21,989 & 31,945 & 17,903 & 26,009 \\
\hline 1995 & 16,864 & 23,825 & 23,202 & 32,779 & 17,959 & 25,372 \\
\hline 1996 & 17,468 & 23,970 & 26,205 & 35,959 & 18,777 & 25,766 \\
\hline 1997 & 17,907 & 24,021 & 27,722 & 37,188 & 19,531 & 26,200 \\
\hline 1998 & 18,479 & 24,408 & 29,614 & 39,116 & 20,364 & 26,898 \\
\hline 1999 & 18,339 & 23,700 & 28,695 & 37,084 & 20,381 & 26,339 \\
\hline 2000 & 18,577 & 23,227 & 27,447 & 34,317 & 20,600 & 25,756 \\
\hline 2001 & 18,755 & 22,801 & 27,539 & 33,480 & 20,945 & 25,463 \\
\hline 2002 & 18,897 & 22,616 & 27,440 & 32,840 & 21,249 & 25,431 \\
\hline 2003 & 18,536 & 21,689 & 28,139 & 32,926 & 21,169 & 24,770 \\
\hline 2004 & 18,910 & 21,553 & 28,409 & 32,380 & 21,637 & 24,661 \\
\hline 2005 & 19,833 & 21,864 & 29,172 & 32,160 & 22,497 & 24,801 \\
\hline 2006 & 20,342 & 21,725 & 29,625 & 31,639 & 23,140 & 24,713 \\
\hline 2007 & 20,434 & 21,219 & 29,735 & 30,877 & 23,336 & 24,232 \\
\hline 2008 & 20,490 & 20,490 & 28,174 & 28,174 & 23,051 & 23,051 \\
\hline \multicolumn{7}{|c|}{ Average annual percentage change } \\
\hline 1970-2008 & $4.6 \%$ & $0.0 \%$ & $6.4 \%$ & $1.7 \%$ & $5.1 \%$ & $0.0 \%$ \\
\hline $1998-2008$ & $1.0 \%$ & $-1.7 \%$ & $-0.5 \%$ & $-3.2 \%$ & $1.2 \%$ & $-1.5 \%$ \\
\hline
\end{tabular}

\section{Source:}

U.S. Department of Commerce, Bureau of Economic Analysis, National Income and Product Accounts, underlying detail estimates for Motor Vehicle Output, Washington, DC, 2009. (Additional resources: www.statusa.gov)

\footnotetext{
${ }^{a}$ Includes transplants.

${ }^{\mathrm{b}}$ Adjusted by the Consumer Price Inflation Index.
} 
The total cost of operating a car is the sum of the fixed cost (depreciation, insurance, finance charge, and license fee) and the variable cost (gas and oil, tires, and maintenance), which is related to the amount of travel. The gas and oil share of total cost was $14.3 \%$ in 2009.

Table 10.13

Car Operating Cost per Mile, 1985-2009

\begin{tabular}{|c|c|c|c|c|c|}
\hline \multirow[b]{2}{*}{ Model year } & \multicolumn{3}{|c|}{ Constant 2009 dollars per 10,000 miles $^{\mathrm{a}}$} & \multirow{2}{*}{$\begin{array}{l}\text { Total cost per } \\
\text { mile }^{\mathrm{b}}(\text { constant } \\
\left.2009 \text { cents }^{\mathrm{a}}\right)\end{array}$} & \multirow{2}{*}{$\begin{array}{l}\text { Percentage gas } \\
\text { and oil of total } \\
\text { cost }\end{array}$} \\
\hline & Variable cost & Fixed cost & Total cost & & \\
\hline 1985 & 1,479 & 4,109 & 5,589 & 55.89 & $19.9 \%$ \\
\hline 1986 & 1,276 & 4,516 & 5,792 & 57.92 & $15.1 \%$ \\
\hline 1987 & 1,265 & 4,396 & 5,662 & 56.62 & $14.7 \%$ \\
\hline 1988 & 1,433 & 5,495 & 6,928 & 69.28 & $13.6 \%$ \\
\hline 1989 & 1,384 & 5,052 & 6,436 & 64.36 & $14.2 \%$ \\
\hline 1990 & 1,379 & 5,345 & 6,723 & 67.23 & $13.2 \%$ \\
\hline 1991 & 1,528 & 5,617 & 7,145 & 71.45 & $14.6 \%$ \\
\hline 1992 & 1,376 & 5,786 & 7,162 & 71.62 & $12.6 \%$ \\
\hline 1993 & 1,366 & 5,526 & 6,892 & 68.92 & $12.7 \%$ \\
\hline 1994 & 1,317 & 5,553 & 6,870 & 68.70 & $11.8 \%$ \\
\hline 1995 & 1,351 & 5,638 & 6,989 & 69.89 & $11.7 \%$ \\
\hline 1996 & 1,313 & 5,733 & 7,046 & 70.46 & $10.9 \%$ \\
\hline 1997 & 1,444 & 5,812 & 7,269 & 72.69 & $12.1 \%$ \\
\hline 1998 & 1,408 & 5,960 & 7,355 & 73.55 & $11.1 \%$ \\
\hline 1999 & 1,365 & 6,001 & 7,366 & 73.66 & $9.8 \%$ \\
\hline 2000 & 1,520 & 5,885 & 7,405 & 74.05 & $11.6 \%$ \\
\hline 2001 & 1,647 & 5,598 & 7,245 & 72.45 & $13.2 \%$ \\
\hline 2002 & 1,407 & 5,812 & 7,220 & 72.20 & $9.7 \%$ \\
\hline 2003 & 1,527 & 5,695 & 7,222 & 72.22 & $11.6 \%$ \\
\hline 2004 & 1,431 & 6,397 & 7,828 & 78.28 & $9.4 \%$ \\
\hline 2005 & 1,549 & 5,945 & 7,494 & 74.94 & $12.0 \%$ \\
\hline 2006 & 1,607 & 4,987 & 6,594 & 65.94 & $15.3 \%$ \\
\hline 2007 & 1,500 & 4,930 & 6,431 & 64.31 & $14.3 \%$ \\
\hline 2008 & 1,690 & 5,380 & 7,070 & 70.70 & $16.4 \%$ \\
\hline 2009 & 1,542 & 5,526 & 7,068 & 70.68 & $14.3 \%$ \\
\hline \multicolumn{6}{|c|}{ Average annual percentage change } \\
\hline 1985-2009 & $0.2 \%$ & $1.2 \%$ & $1.0 \%$ & $1.0 \%$ & \\
\hline
\end{tabular}

Source:

Ward's Communications, Motor Vehicle Facts and Figures 2009, Southfield, Michigan, 2009, p. 66, and annual. Original data from AAA "Your Driving Costs."

${ }^{a}$ Adjusted by the Consumer Price Inflation Index.

${ }^{\mathrm{b}}$ Based on 10,000 miles per year. 
While the previous table shows costs per mile, this table presents costs per year for fixed costs associated with car operation. For 2009 model year autos, the fixed cost is almost \$16 per day.

Table 10.14

Fixed Car Operating Costs per Year, 1975-2009 (constant 2009 dollars) $^{a}$

\begin{tabular}{|c|c|c|c|c|c|c|}
\hline Model year & Insurance $^{\mathrm{b}}$ & $\begin{array}{l}\text { License, } \\
\text { registration } \\
\text { \& taxes }\end{array}$ & Depreciation & $\begin{array}{c}\text { Finance } \\
\text { charge }\end{array}$ & Total & $\begin{array}{l}\text { Average } \\
\text { fixed cost } \\
\text { per day }\end{array}$ \\
\hline 1975 & 1,527 & 120 & 3,082 & $\mathrm{c}$ & 4,729 & 12.96 \\
\hline 1977 & 1,834 & 262 & 2,999 & $\mathrm{c}$ & 5,094 & 13.95 \\
\hline 1978 & 1,395 & 243 & 2,942 & $\mathrm{c}$ & 4,580 & 12.54 \\
\hline 1979 & 1,427 & 266 & 2,784 & c & 5,352 & 14.66 \\
\hline 1980 & 1,276 & 213 & 2,703 & $\mathrm{c}$ & 5,293 & 14.50 \\
\hline 1981 & 1,204 & 208 & 3,038 & c & 5,605 & 15.36 \\
\hline 1982 & 998 & 120 & 3,015 & c & 5,331 & 14.61 \\
\hline 1983 & 1,012 & 209 & 2,796 & c & 5,157 & 14.13 \\
\hline 1984 & 1,043 & 219 & 2,492 & $\mathrm{c}$ & 4,844 & 13.28 \\
\hline 1985 & 927 & 219 & 2,516 & 1,065 & 4,727 & 12.96 \\
\hline 1986 & 996 & 254 & 2,584 & 1,247 & 5,082 & 13.92 \\
\hline 1987 & 1,010 & 242 & 2,821 & 993 & 5,067 & 13.88 \\
\hline 1988 & 1,039 & 252 & 3,235 & 1,025 & 5,551 & 15.22 \\
\hline 1989 & 1,116 & 249 & 3,491 & 1,017 & 5,874 & 16.09 \\
\hline 1990 & 1,105 & 271 & 3,869 & 1,116 & 6,361 & 17.43 \\
\hline 1991 & 1,115 & 265 & 3,944 & 419 & 5,743 & 15.74 \\
\hline 1992 & 1,203 & 266 & 4,155 & 1,217 & 6,841 & 18.75 \\
\hline 1993 & 1,105 & 264 & 4,202 & 995 & 6,565 & 17.99 \\
\hline 1994 & 1,113 & 281 & 4,256 & 938 & 6,588 & 18.05 \\
\hline 1995 & 1,102 & 286 & 4,326 & 966 & 6,680 & 18.30 \\
\hline 1996 & 1,155 & 294 & 4,334 & 982 & 6,766 & 18.54 \\
\hline 1997 & 1,132 & 289 & 4,374 & 1,027 & 6,821 & 18.69 \\
\hline 1998 & 1,185 & 297 & 4,428 & 1,070 & 6,980 & 19.12 \\
\hline 1999 & 1,249 & 291 & 4,425 & 1,066 & 7,031 & 19.26 \\
\hline 2000 & 1,208 & 278 & 4,351 & 1,058 & 6,895 & 18.89 \\
\hline 2001 & 1,200 & 252 & 4,298 & 1,049 & 6,800 & 18.63 \\
\hline 2002 & 1,209 & 240 & 4,437 & 987 & 6,874 & 18.83 \\
\hline 2003 & 1,285 & 239 & 4,358 & 867 & 6,750 & 18.49 \\
\hline 2004 & 1,821 & 471 & 4,295 & 842 & 7,429 & 20.35 \\
\hline 2005 & 1,415 & 427 & 4,261 & 812 & 6,915 & 18.95 \\
\hline 2006 & 985 & 569 & 3,610 & 762 & 5,926 & 16.24 \\
\hline 2007 & 1,019 & 557 & 3,510 & 758 & 5,844 & 16.01 \\
\hline 2008 & 940 & 552 & 3,309 & 755 & 5,556 & 15.23 \\
\hline 2009 & 976 & 567 & 3,461 & 779 & 5,783 & 15.84 \\
\hline \multicolumn{7}{|c|}{ Average annual percentage change } \\
\hline 1975-2009 & $-1.3 \%$ & $4.7 \%$ & $0.3 \%$ & $\mathrm{c}$ & $0.6 \%$ & $0.6 \%$ \\
\hline 1999-2009 & $-2.4 \%$ & $6.9 \%$ & $-2.4 \%$ & $-3.1 \%$ & $-1.9 \%$ & $-1.9 \%$ \\
\hline
\end{tabular}

Source:

Ward's Communications, Motor Vehicle Facts and Figures 2009, Southfield, Michigan, 2009, p. 66 and annual. Original data from AAA "Your Driving Costs."

${ }^{a}$ Adjusted by the Consumer Price Inflation Index.

${ }^{\mathrm{b}}$ Fire \& Theft: $\$ 50$ deductible 1975 through 1977; \$100 deductible 1978 through 1992; \$250 deductible for 1993 - on. Collision: $\$ 100$ deductible through 1977; \$250 deductible 1978 through 1992; \$500 deductible for 1993 - on. Property Damage \& Liability: coverage $=\$ 100,000 / \$ 300,000$.

${ }^{\mathrm{c}}$ Data are not available. 
Table 10.15

Personal Consumption Expenditures, 1970-2009

(billion dollars)

\begin{tabular}{|c|c|c|c|c|c|}
\hline \multirow[b]{2}{*}{ Year } & \multicolumn{2}{|c|}{ Personal consumption expenditures } & \multicolumn{2}{|c|}{$\begin{array}{c}\text { Transportation personal } \\
\text { consumption expenditures }\end{array}$} & \multirow[b]{2}{*}{$\begin{array}{l}\text { Transportation PCE } \\
\text { as a percent of PCE }\end{array}$} \\
\hline & Current & $\begin{array}{c}\text { Constant } \\
2009^{\mathrm{a}}\end{array}$ & Current & $\begin{array}{c}\text { Constant } \\
2009^{\mathrm{a}} \\
\end{array}$ & \\
\hline 1970 & 648.3 & $2,928.5$ & 80.8 & 365.0 & $12.5 \%$ \\
\hline 1980 & $1,755.8$ & $4,038.0$ & 241.7 & 555.9 & $13.8 \%$ \\
\hline 1990 & $3,835.5$ & $5,831.1$ & 455.7 & 692.8 & $11.9 \%$ \\
\hline 2000 & $6,830.4$ & $8,457.7$ & 814.3 & $1,008.3$ & $11.9 \%$ \\
\hline 2001 & $7,148.8$ & $8,656.4$ & 829.6 & $1,004.5$ & $11.6 \%$ \\
\hline 2002 & $7,439.2$ & $8,864.7$ & 832.6 & 992.1 & $11.2 \%$ \\
\hline 2003 & $7,804.0$ & $9,103.5$ & 874.2 & $1,019.8$ & $11.2 \%$ \\
\hline 2004 & $8,285.1$ & $9,397.9$ & 927.8 & $1,052.4$ & $11.2 \%$ \\
\hline 2005 & $8,819.0$ & $9,680.1$ & $1,000.3$ & $1,098.0$ & $11.3 \%$ \\
\hline 2006 & $9,322.7$ & $9,909.9$ & $1,031.0$ & $1,095.9$ & $11.1 \%$ \\
\hline 2007 & $9,826.4$ & $10,154.7$ & $1,075.7$ & $1,111.6$ & $10.9 \%$ \\
\hline 2008 & $10,129.9$ & $10,249.2$ & $1,063.1$ & $1,075.6$ & $10.5 \%$ \\
\hline 2009 & $10,088.5$ & $10,088.5$ & 921.9 & 921.9 & $9.1 \%$ \\
\hline
\end{tabular}

\section{Source:}

U.S. Department of Commerce, Bureau of Economic Analysis, National Income and Product Accounts, Table 2.3.5, http://www.bea.doc.gov/bea/dn/nipaweb.

Note: Transportation PCE includes the following categories: transportation, motor vehicles and parts, and gasoline and oil.

Table 10.16

Consumer Price Indices, 1970-2009

$(1970=1.000)$

\begin{tabular}{cccccc}
\hline Year & $\begin{array}{c}\text { Consumer } \\
\text { Price Index }\end{array}$ & $\begin{array}{c}\text { Transportation } \\
\text { Consumer Price } \\
\text { Index }^{\mathrm{b}}\end{array}$ & $\begin{array}{c}\text { New car } \\
\text { Consumer } \\
\text { Price Index }\end{array}$ & $\begin{array}{c}\text { Used car } \\
\text { Consumer } \\
\text { Price Index }\end{array}$ & $\begin{array}{c}\text { Gross National } \\
\text { Product Index }\end{array}$ \\
\hline 1970 & 1.000 & 1.000 & 1.000 & 1.000 & 1.000 \\
1980 & 2.124 & 2.216 & 1.667 & 1.997 & 2.702 \\
1990 & 3.369 & 3.213 & 2.286 & 3.769 & 5.585 \\
2000 & 4.438 & 4.088 & 2.689 & 4.994 & 9.562 \\
2005 & 5.034 & 4.637 & 2.597 & 4.468 & 12.191 \\
2006 & 5.196 & 4.824 & 2.591 & 4.487 & 13.895 \\
2007 & 5.344 & 4.925 & 2.566 & 4.351 & 13.986 \\
2008 & 5.549 & 5.215 & 2.527 & 4.293 & 13.747 \\
2009 & 5.529 & 4.780 & 2.554 & 4.070 & \\
\hline
\end{tabular}

\section{Sources:}

Bureau of Labor Statistics, Consumer Price Index Table 1A for 2009, and annual.

(Additional resources: www.bls.gov)

GNP - U.S. Department of Commerce, Bureau of Economic Analysis, National Income and Product Accounts, Table 1.7.5. (Additional resources: www.bea.doc.gov)

\footnotetext{
${ }^{a}$ Adjusted by the GNP price deflator.

b Transportation Consumer Price Index includes new and used cars, gasoline, auto insurance rates, intracity mass transit, intracity bus fare, and airline fares.
} 
The data below were summarized from the Bureau of Labor Statistics (BLS) Current Employment Statistics Survey data using the North American Industry Classification System (NAICS). Transportation-related employment was $7.1 \%$ of total employment in 2009.

Table 10.17

Transportation-related Employment, 1999 and 2009 (thousands)

\begin{tabular}{lrrr}
\hline & & & Percent \\
& 1999 & 2009 & Change \\
\hline Truck transportation & $1,391.5$ & $1,265.9$ & $-9.0 \%$ \\
Transit and ground transportation & 371.0 & 419.3 & $13.0 \%$ \\
Air transportation & 586.3 & 459.7 & $-21.6 \%$ \\
Rail transportation & 228.8 & 219.4 & $-4.1 \%$ \\
Water transportation & 51.7 & 63.7 & $23.2 \%$ \\
Pipeline transportation & 46.9 & 41.7 & $-11.1 \%$ \\
Motor vehicle and parts - retail & $1,796.6$ & $1,640.0$ & $-8.7 \%$ \\
Motor vehicles and parts - wholesale & 359.5 & 316.2 & $-12.0 \%$ \\
Gasoline stations - retail & 943.5 & 827.0 & $-12.3 \%$ \\
Automotive repair and maintenance & 864.2 & 806.1 & $-6.7 \%$ \\
Automotive equipment rental and leasing & 198.6 & 168.8 & $-15.0 \%$ \\
Manufacturing & $2,175.3$ & $1,405.4$ & $-35.4 \%$ \\
$\quad$ Autos and light trucks & 236.7 & 119.5 & $-49.5 \%$ \\
$\quad$ Heavy-duty trucks & 54.6 & 23.0 & $-57.9 \%$ \\
Motor vehicle bodies and trailers & 184.2 & 105.2 & $-42.9 \%$ \\
Motor vehicle parts & 837.1 & 418.7 & $-50.0 \%$ \\
Aerospace products and parts & 547.1 & 492.8 & $-9.9 \%$ \\
$\quad$ Railroad rolling stock & 35.3 & 22.9 & $-35.1 \%$ \\
Ship \& boat building & 153.9 & 132.1 & $-14.2 \%$ \\
All other transportation equipment & 39.8 & 38.8 & $-2.5 \%$ \\
$\quad$ Tires & 86.6 & 52.4 & $-39.5 \%$ \\
Oil and gas pipeline construction & 72.9 & 107.1 & $46.9 \%$ \\
Highway street and bridge construction & 336.3 & 286.4 & $-14.8 \%$ \\
Scenic \& sightseeing & 26.1 & 27.8 & $6.5 \%$ \\
Support activities for transportation & 518.1 & 549.0 & $6.0 \%$ \\
Couriers and messengers & 585.9 & 547.1 & $-6.6 \%$ \\
Travel arrangement and reservation services & 297.0 & 196.0 & $-34.0 \%$ \\
\hline Total transportation-related employment & $\mathbf{1 0 , 8 5 0 . 2}$ & $\mathbf{9 , 3 4 6 . 6}$ & $-13.9 \%$ \\
\hline Total nonfarm employment & $128,993.0$ & $130,920.0$ & $1.5 \%$ \\
Transportation-related to total employment & $8.4 \%$ & $7.1 \%$ & \\
\hline & & & \\
\hline
\end{tabular}

Source:

Bureau of Labor Statistics Web site query system: www.bls.gov/ces/cesnaics.htm, (Additional resources: www.bls.gov)

\footnotetext{
${ }^{\mathrm{a}}$ Not seasonally adjusted.
} 
The total number of employees involved in the manufacture of motor vehicles decreased by almost $48 \%$ from 1990 to 2009 and by about $36 \%$ for those involved in the manufacturer of motor vehicle parts. In 2008, the share of production workers fell below $80 \%$ for manufacturers of both vehicles and parts.

Table 10.18

U.S. Employment for Motor Vehicles and Motor Vehicle Parts Manufacturing, 1990-2009ª

\begin{tabular}{|c|c|c|c|}
\hline Year & All Employees & Production Workers & $\begin{array}{c}\text { Share of Production } \\
\text { Workers to Total } \\
\text { Employees }\end{array}$ \\
\hline \multicolumn{4}{|c|}{ Motor Vehicles } \\
\hline 1990 & 271.4 & 243.4 & $89.68 \%$ \\
\hline 1991 & 258.4 & 234.8 & $90.87 \%$ \\
\hline 1992 & 259.9 & 234.0 & $90.03 \%$ \\
\hline 1993 & 263.7 & 234.8 & $89.04 \%$ \\
\hline 1994 & 281.5 & 250.9 & $89.13 \%$ \\
\hline 1995 & 294.7 & 273.7 & $92.87 \%$ \\
\hline 1996 & 285.3 & 271.2 & $95.06 \%$ \\
\hline 1997 & 286.8 & 273.6 & $95.40 \%$ \\
\hline 1998 & 283.6 & 254.8 & $89.84 \%$ \\
\hline 1999 & 291.3 & 254.3 & $87.30 \%$ \\
\hline 2000 & 291.4 & 251.0 & $86.14 \%$ \\
\hline 2001 & 278.7 & 236.4 & $84.82 \%$ \\
\hline 2002 & 265.4 & 220.8 & $83.20 \%$ \\
\hline 2003 & 264.6 & 217.1 & $82.05 \%$ \\
\hline 2004 & 255.9 & 208.0 & $81.28 \%$ \\
\hline 2005 & 247.6 & 198.6 & $80.21 \%$ \\
\hline 2006 & 236.5 & 191.8 & $81.10 \%$ \\
\hline 2007 & 220.0 & 177.3 & $80.59 \%$ \\
\hline 2008 & 191.6 & 151.1 & $78.86 \%$ \\
\hline 2009 & 142.5 & 111.0 & $77.89 \%$ \\
\hline \multicolumn{4}{|c|}{ Motor Vehicle Parts } \\
\hline 1990 & 653.0 & 527.4 & $80.77 \%$ \\
\hline 1991 & 638.9 & 514.7 & $80.56 \%$ \\
\hline 1992 & 661.2 & 537.0 & $81.22 \%$ \\
\hline 1993 & 677.8 & 554.7 & $81.84 \%$ \\
\hline 1994 & 735.6 & 606.9 & $82.50 \%$ \\
\hline 1995 & 786.9 & 647.7 & $82.31 \%$ \\
\hline 1996 & 799.9 & 657.4 & $82.19 \%$ \\
\hline 1997 & 808.9 & 662.4 & $81.89 \%$ \\
\hline 1998 & 818.2 & 660.3 & $80.70 \%$ \\
\hline 1999 & 837.1 & 674.2 & $80.54 \%$ \\
\hline 2000 & 839.5 & 676.7 & $80.61 \%$ \\
\hline 2001 & 774.7 & 624.9 & $80.66 \%$ \\
\hline 2002 & 733.6 & 590.9 & $80.55 \%$ \\
\hline 2003 & 707.8 & 567.6 & $80.19 \%$ \\
\hline 2004 & 692.1 & 561.6 & $81.14 \%$ \\
\hline 2005 & 678.1 & 553.9 & $81.68 \%$ \\
\hline 2006 & 654.7 & 533.7 & $81.52 \%$ \\
\hline 2007 & 607.9 & 488.9 & $80.42 \%$ \\
\hline 2008 & 543.7 & 430.6 & $79.20 \%$ \\
\hline 2009 & 418.7 & 321.8 & $76.86 \%$ \\
\hline
\end{tabular}

Source:

Tabulated from the U.S. Department of Labor, Bureau of Labor Statistics, www.bls.gov, March 2010.

${ }^{\mathrm{a}}$ Not seasonally adjusted. 


\section{Chapter 11 Greenhouse Gas Emissions}

Summary Statistics from Tables/Figures in this Chapter

\begin{tabular}{|c|c|c|c|}
\hline Source & & & \\
\hline \multirow[t]{8}{*}{ Table 11.1} & Carbon dioxide emissions (million metric tonnes) & 1990 & 2006 \\
\hline & United States & 4,989 & 5,907 \\
\hline & OECD Europe & 4,149 & 4,429 \\
\hline & China & 2,293 & 6,018 \\
\hline & Russia & 2,393 & 1,704 \\
\hline & Japan & 1,054 & 1,247 \\
\hline & Non-OECD Europe & 1,853 & 1,182 \\
\hline & India & 573 & 1,292 \\
\hline \multirow[t]{5}{*}{ Table 11.5} & \multicolumn{3}{|c|}{$\begin{array}{l}\text { Transportation share of U.S. carbon dioxide emissions from fossil fuel } \\
\text { consumption }\end{array}$} \\
\hline & 1990 & & $31.6 \%$ \\
\hline & 1995 & & $31.7 \%$ \\
\hline & 2000 & & $32.0 \%$ \\
\hline & 2008 & & $33.2 \%$ \\
\hline Table 11.6 & \multicolumn{2}{|c|}{ Motor gasoline share of transportation carbon dioxide emissions } & $58.8 \%$ \\
\hline \multirow[t]{3}{*}{ Table 11.10} & Average annual carbon footprint (short tons of $\mathrm{CO}$ & & \\
\hline & Cars & & 6.1 \\
\hline & Light trucks & & 8.2 \\
\hline
\end{tabular}


The U. S. accounted for $23.2 \%$ of the World's carbon dioxide emissions in 1990 and $21.1 \%$ in 2006. Nearly half (44\%) of the U.S. carbon emissions are from oil use.

Table 11.1

World Carbon Dioxide Emissions, 1990 and 2006

\begin{tabular}{|c|c|c|c|c|}
\hline & \multicolumn{2}{|c|}{1990} & \multicolumn{2}{|c|}{2006} \\
\hline & $\begin{array}{l}\text { Million } \\
\text { metric tons }\end{array}$ & $\begin{array}{l}\text { Percent of } \\
\text { emissions } \\
\text { from oil use }\end{array}$ & $\begin{array}{c}\text { Million } \\
\text { metric tons }\end{array}$ & $\begin{array}{l}\text { Percent of } \\
\text { emissions } \\
\text { from oil use } \\
\end{array}$ \\
\hline United States & 4,989 & $44 \%$ & 5,907 & $44 \%$ \\
\hline Canada & 471 & $48 \%$ & 611 & $48 \%$ \\
\hline Mexico & 302 & $77 \%$ & 431 & $62 \%$ \\
\hline OECD $^{\mathrm{a}}$ Europe & 4,149 & $45 \%$ & 4,429 & $48 \%$ \\
\hline OECD $^{\mathrm{a}}$ Asia & 243 & $59 \%$ & 515 & $46 \%$ \\
\hline Japan & 1,054 & $65 \%$ & 1,247 & $50 \%$ \\
\hline Australia/New Zealand & 298 & $38 \%$ & 455 & $33 \%$ \\
\hline Russia & 2,393 & $33 \%$ & 1,704 & $23 \%$ \\
\hline Non-OECD ${ }^{\mathrm{a}}$ Europe & 1,853 & $32 \%$ & 1,182 & $26 \%$ \\
\hline China & 2,293 & $15 \%$ & 6,018 & $16 \%$ \\
\hline India & 573 & $28 \%$ & 1,292 & $26 \%$ \\
\hline Non-OECD ${ }^{\mathrm{a}}$ Asia & 811 & $57 \%$ & 1,678 & $52 \%$ \\
\hline Middle East & 704 & $70 \%$ & 1,456 & $58 \%$ \\
\hline Africa & 659 & $46 \%$ & 982 & $43 \%$ \\
\hline Central \& South America & 695 & $76 \%$ & 1,123 & $70 \%$ \\
\hline Total World & 21,488 & $42 \%$ & 28,028 & $39 \%$ \\
\hline
\end{tabular}

Source:

U.S. Department of Energy, Energy Information Administration, International Energy Outlook 2009, Washington, DC, May 2009, Tables A10 and A11. (Additional resources: www.eia.doe.gov)

${ }^{a}$ OECD is the Organization for Economic Cooperation and Development. See Glossary for included countries. 
Global Warming Potentials (GWP) were developed to allow comparison of the ability of each greenhouse gas to trap heat in the atmosphere relative to carbon dioxide. Extensive research has been performed and it has been discovered that the effects of various gases on global warming are too complex to be precisely summarized by a single number. Further understanding of the subject also causes frequent changes to estimates. Despite that, the scientific community has developed approximations, the latest of which are shown below. Most analysts use the 100-year time horizon.

Table 11.2

Numerical Estimates of Global Warming Potentials Compared with Carbon Dioxide (kilogram of gas per kilogram of carbon dioxide)

\begin{tabular}{|c|c|c|c|c|}
\hline \multirow[b]{2}{*}{ Gas } & \multirow{2}{*}{$\begin{array}{c}\text { Lifetime } \\
\text { (years) }\end{array}$} & \multicolumn{3}{|c|}{$\begin{array}{c}\text { Global warming potential } \\
\text { direct effect for time horizons of }\end{array}$} \\
\hline & & 20 years & 100 years & 500 years \\
\hline Carbon Dioxide $\left(\mathrm{CO}_{2}\right)$ & $5-200^{\mathrm{a}}$ & 1 & 1 & 1 \\
\hline Methane $\left(\mathrm{CH}_{4}\right)$ & 12 & 72 & 25 & 8 \\
\hline Nitrous Oxide $\left(\mathrm{N}_{2} \mathrm{O}\right)$ & 114 & 289 & 298 & 153 \\
\hline \multicolumn{5}{|c|}{$\mathrm{HFCs}^{\mathrm{b}}, \mathrm{PFCs}^{\mathrm{c}}$, and Sulfur Hexafluoride } \\
\hline HFC-23 & 270 & 12,000 & 14,800 & 12,200 \\
\hline HFC-125 & 29 & 6,350 & 3,500 & 1,100 \\
\hline HFC-134a & 14 & 3,830 & 1,430 & 435 \\
\hline HFC-152a & 1 & 437 & 124 & 38 \\
\hline HFC-227ea & 34 & 5,310 & 3,220 & 1,040 \\
\hline Perfluoromethane $\left(\mathrm{CF}_{4}\right)$ & 50,000 & 5,210 & 7,390 & 11,200 \\
\hline Perfluoroethane $\left(\mathrm{C}_{2} \mathrm{~F}_{6}\right)$ & 10,000 & 8,630 & 12,200 & 18,200 \\
\hline Sulfur hexafluoride $\left(\mathrm{SF}_{6}\right)$ & 3,200 & 16,300 & 22,800 & 32,600 \\
\hline
\end{tabular}

\section{Source:}

Solomon, S. et al., "Technical Summary," in Climate Change 2007: The Physical Science Basis, Contribution of Working Group I to the Fourth Assessment Report of the Intergovernmental Panel on Climate Change, Cambridge University Press, Cambridge, United Kingdom and New York, NY, USA, 2007.

Note: The typical uncertainty for global warming potentials is estimated by the Intergovernmental Panel on Climate Change \pm 35 percent.

${ }^{\text {a }}$ No single lifetime can be defined for carbon dioxide due to different rates of uptake by different removal processes.

${ }^{\mathrm{b}}$ Hydrofluorocarbons

${ }^{\mathrm{c}}$ Perfluorocarbons 
Carbon dioxide emissions in 2008 were 16\% higher than in 1990. Carbon dioxide accounts for the majority of greenhouse gases.

Table 11.3

U.S. Emissions of Greenhouse Gases, based on Global Warming Potential, 1990-2008 (million metric tonnes carbon dioxide equivalent ${ }^{\mathrm{a}}$ )

\begin{tabular}{cccccc}
\hline & Carbon Dioxide & Methane & Nitrous Oxide & High GWP Gases $^{\mathrm{b}}$ & Total \\
\hline 1990 & $5,022.3$ & 783.5 & 279.3 & 102.3 & $6,187.4$ \\
1991 & $4,976.4$ & 780.5 & 287.8 & 93.1 & $6,137.7$ \\
1992 & $5,082.5$ & 784.9 & 292.9 & 97.7 & $6,258.0$ \\
1993 & $5,203.4$ & 759.7 & 292.9 & 97.2 & $6,353.2$ \\
1994 & $5,289.7$ & 762.7 & 313.5 & 99.9 & $6,465.8$ \\
1995 & $5,341.5$ & 756.2 & 305.6 & 119.0 & $6,522.3$ \\
1996 & $5,526.4$ & 731.3 & 307.5 & 129.7 & $6,694.9$ \\
1997 & $5,594.8$ & 729.2 & 298.0 & 137.2 & $6,759.2$ \\
1998 & $5,627.0$ & 695.7 & 296.7 & 152.2 & $6,771.6$ \\
1999 & $5,695.2$ & 690.3 & 294.4 & 148.9 & $6,828.9$ \\
2000 & $5,886.4$ & 683.0 & 289.8 & 150.5 & $7,009.8$ \\
2001 & $5,796.5$ & 668.5 & 285.5 & 137.7 & $6,888.2$ \\
2002 & $5,849.1$ & 673.3 & 283.7 & 148.1 & $6,954.2$ \\
2003 & $5,908.8$ & 681.6 & 283.0 & 141.8 & $7,015.2$ \\
2004 & $6,009.9$ & 686.6 & 302.2 & 153.5 & $7,152.0$ \\
2005 & $6,029.0$ & 691.8 & 304.0 & 157.8 & $7,182.6$ \\
2006 & $5,928.7$ & 706.3 & 305.2 & 160.5 & $7,100.9$ \\
2007 & $6,017.0$ & 722.7 & 299.8 & 170.3 & $7,209.8$ \\
2008 & $5,839.3$ & 737.4 & 300.3 & 175.6 & $7,052.6$ \\
\hline
\end{tabular}

Source:

U.S. Department of Energy, Energy Information Administration, Emissions of Greenhouse Gases in the United States, 2008, Washington, DC, December 2009, Table 1. (Additional resources: www.eia.doe.gov)

Note: This greenhouse gas emissions inventory includes two "adjustments to energy consumption" which make the data different from Table 11.5. The adjustments are as follows:

1) Emissions from U.S. Territories are included.

2) International bunker fuels and military bunker fuels are excluded from the U.S. total.

${ }^{a}$ Carbon dioxide equivalents are computed by multiplying the weight of the gas being measured by its estimated Global Warming Potential (See Table 11.2).

${ }^{\mathrm{b}} \mathrm{GWP}=$ Global warming potential. Includes HFC-hydrofluorocarbons; PFC-perfluorocarbons; and $\mathrm{SF}_{6^{-}}$ sulfur hexaflouride. 
Though the transportation sector accounts for the largest share of carbon dioxide emissions, the industrial sector accounts for the largest share of total greenhouse gas emissions.

Table 11.4

Total U.S. Greenhouse Emissions by End-Use Sector, 2008 (million metric tonnes carbon dioxide equivalent ${ }^{a}$ )

\begin{tabular}{lrrrrr}
\hline \multicolumn{1}{c}{ Greenhouse gas and source } & Residential & Commercial & Industrial & Transportation & Total \\
\hline Carbon dioxide & $1,230.3$ & $1,084.1$ & $1,760.1$ & $1,818.8$ & $5,839.2$ \\
Methane & 4.8 & 203.2 & 524.8 & 4.6 & 737.4 \\
Nitrous oxide & 4.6 & 6.9 & 239.9 & 48.8 & 300.2 \\
Hydrofluorocarbons & 0.0 & 47.2 & 21.9 & 73.6 & 142.7 \\
Perfluorocarbons & 0.0 & 0.0 & 10.1 & 0.0 & 10.1 \\
Other hydrofluorocarbons, & & & & & \\
perfluorocarbons/perfluoropolyether & 0.0 & 7.1 & 0.0 & 0.0 & 7.1 \\
Sulfur hexafluoride & 4.5 & 4.3 & 7.0 & 0.0 & 15.8 \\
\hline Total greenhouse gas emissions & $1,244.1$ & $1,352.8$ & $2,509.8$ & $1,945.8$ & $7,052.6$ \\
\hline
\end{tabular}

Source:

U.S. Department of Energy, Energy Information Administration, Emissions of Greenhouse Gases in the United States, 2008, Washington, DC, December 2009, and annual. (Additional resources: www.eia.doe.gov)

Note: This greenhouse gas emissions inventory includes two "adjustments to energy consumption" which make the data different from Table 11.5. The adjustments are as follows:

(1) Emissions from U.S. Territories are included.

(2) International bunker fuels and military bunker fuels are excluded from the U.S. total.

a Carbon dioxide equivalents are computed by multiplying the weight of the gas being measured by its estimated Global Warming Potential (See Table 11.2). 
Gases which contain carbon can be measured in terms of the full molecular weight of the gas or just in terms of their carbon content. This table presents carbon dioxide gas. The ratio of the weight of carbon to carbon dioxide is 0.2727. The transportation sector accounts for approximately one-third of carbon emissions.

Table 11.5

\section{U.S. Carbon Emissions from Fossil Energy Consumption by End-Use Sector, 1990-2008 (million metric tonnes of carbon dioxide)}

\begin{tabular}{|c|c|c|c|c|c|c|}
\hline & \multicolumn{4}{|c|}{ End Use Sector } & \multirow{2}{*}{$\begin{array}{l}\text { Transportation } \\
\text { Percentage }\end{array}$} & \multirow{2}{*}{$\begin{array}{l}\mathrm{CO}_{2} \text { From } \\
\text { All Sectors }\end{array}$} \\
\hline & Residential & Commercial & Industrial & Transportation & & \\
\hline 1990 & 958.6 & 785.1 & $1,689.5$ & $1,586.9$ & $31.6 \%$ & $5,020.1$ \\
\hline 1991 & 974.8 & 786.3 & $1,647.4$ & $1,566.4$ & $31.5 \%$ & $4,974.9$ \\
\hline 1992 & 976.0 & 787.6 & $1,717.6$ & $1,587.9$ & $31.3 \%$ & $5,069.1$ \\
\hline 1993 & $1,036.0$ & 813.2 & $1,711.9$ & $1,610.7$ & $31.1 \%$ & $5,171.8$ \\
\hline 1994 & $1,029.2$ & 827.6 & $1,742.1$ & $1,651.9$ & $31.5 \%$ & $5,250.7$ \\
\hline 1995 & $1,035.5$ & 845.1 & $1,739.5$ & $1,682.2$ & $31.7 \%$ & $5,302.3$ \\
\hline 1996 & $1,094.9$ & 876.0 & $1,791.5$ & $1,725.4$ & $31.4 \%$ & $5,487.8$ \\
\hline 1997 & $1,086.2$ & 919.8 & $1,812.2$ & $1,743.8$ & $31.4 \%$ & $5,561.9$ \\
\hline 1998 & $1,093.4$ & 939.7 & $1,792.3$ & $1,779.6$ & $31.8 \%$ & $5,605.0$ \\
\hline 1999 & $1,116.7$ & 952.2 & $1,768.0$ & $1,828.4$ & $32.3 \%$ & $5,665.3$ \\
\hline 2000 & $1,179.8$ & $1,013.1$ & $1,784.7$ & $1,872.7$ & $32.0 \%$ & $5,850.4$ \\
\hline 2001 & $1,167.3$ & $1,019.5$ & $1,707.2$ & $1,851.4$ & $32.2 \%$ & $5,745.4$ \\
\hline 2002 & $1,197.6$ & $1,018.0$ & $1,683.3$ & $1,890.7$ & $32.7 \%$ & $5,789.6$ \\
\hline 2003 & $1,224.9$ & $1,026.1$ & $1,690.3$ & $1,897.4$ & $32.5 \%$ & $5,838.6$ \\
\hline 2004 & $1,221.9$ & $1,043.3$ & $1,728.5$ & $1,958.9$ & $32.9 \%$ & $5,952.5$ \\
\hline 2005 & $1,254.5$ & $1,059.6$ & $1,671.4$ & $1,988.7$ & $33.3 \%$ & $5,974.3$ \\
\hline 2006 & $1,186.7$ & $1,034.9$ & $1,657.8$ & $2,014.3$ & $34.2 \%$ & $5,893.7$ \\
\hline 2007 & $1,235.1$ & $1,070.3$ & $1,655.2$ & $2,025.7$ & $33.8 \%$ & $5,986.4$ \\
\hline 2008 & $1,220.1$ & $1,075.1$ & $1,589.1$ & $1,930.1$ & $33.2 \%$ & $5,814.4$ \\
\hline \multicolumn{7}{|c|}{ Average annual percentage change } \\
\hline 1990-2008 & $1.3 \%$ & $1.8 \%$ & $-0.3 \%$ & $1.1 \%$ & & $0.8 \%$ \\
\hline 1998-2008 & $1.1 \%$ & $1.4 \%$ & $-1.2 \%$ & $0.8 \%$ & & $0.4 \%$ \\
\hline
\end{tabular}

Source:

U.S. Department of Energy, Energy Information Administration, Emissions of Greenhouse Gases in the United States, 2008, Washington, DC, December 2009, Table 6. (Additional resources: www.eia.doe.gov)

Note: Emissions from U.S. Territories are not included. International bunker fuels and military bunker fuels are included in these data.

${ }^{a}$ Includes energy from petroleum, coal, and natural gas. Electric utility emissions are distributed across consumption sectors. 
Most U.S. transportation sector carbon dioxide emissions come from petroleum fuels (98\%). Motor gasoline has been responsible for about $60 \%$ of U.S. carbon dioxide emissions over the last twenty years.

Table 11.6

U.S. Carbon Emissions from Energy Use in the Transportation Sector, 1990-2008 (million metric tonnes of carbon dioxide)

\begin{tabular}{|c|c|c|c|c|c|c|}
\hline \multirow[b]{2}{*}{ Fuel } & \multicolumn{2}{|c|}{1990} & \multicolumn{2}{|c|}{2000} & \multicolumn{2}{|c|}{2008} \\
\hline & Emissions & Percentage & Emissions & Percentage & Emissions & Percentage \\
\hline & \multicolumn{6}{|c|}{ Petroleum } \\
\hline Motor gasoline & 966.2 & $60.9 \%$ & $1,122.0$ & $59.9 \%$ & $1,134.9$ & $58.8 \%$ \\
\hline $\mathrm{LPG}^{\mathrm{a}}$ & 1.4 & $0.1 \%$ & 0.7 & $0.0 \%$ & 1.2 & $0.1 \%$ \\
\hline Jet fuel & 222.6 & $14.0 \%$ & 253.8 & $13.6 \%$ & 226.3 & $11.7 \%$ \\
\hline Distillate fuel & 267.8 & $16.9 \%$ & 377.8 & $20.2 \%$ & 445.7 & $23.1 \%$ \\
\hline Residual fuel & 80.1 & $5.0 \%$ & 69.9 & $3.7 \%$ & 74.1 & $3.8 \%$ \\
\hline Lubricants & 6.5 & $0.4 \%$ & 6.7 & $0.4 \%$ & 5.2 & $0.3 \%$ \\
\hline Aviation gas & 3.1 & $0.2 \%$ & 2.5 & $0.1 \%$ & 2.0 & $0.1 \%$ \\
\hline \multirow[t]{2}{*}{ Subtotal } & $1,547.7$ & $97.5 \%$ & $1,833.4$ & $97.9 \%$ & $1,889.4$ & $97.9 \%$ \\
\hline & \multicolumn{6}{|c|}{ Other energy } \\
\hline Natural gas & 36.1 & $2.3 \%$ & 35.7 & $1.9 \%$ & 35.9 & $1.9 \%$ \\
\hline Electricity $^{\mathrm{b}}$ & 3.2 & $0.2 \%$ & 3.6 & $0.2 \%$ & 4.9 & $0.3 \%$ \\
\hline Total $^{\mathrm{c}}$ & $1,586.9$ & $100.0 \%$ & $1,872.7$ & $100.0 \%$ & $1,930.1$ & $100.0 \%$ \\
\hline
\end{tabular}

Source:

U.S. Department of Energy, Energy Information Administration, Emissions of Greenhouse Gases in the United States, 2008, Washington, DC, December 2009, Table 10 and annual. (Additional resources: www.eia.doe.gov)

\footnotetext{
${ }^{\text {a }}$ Liquified petroleum gas.

${ }^{\mathrm{b}}$ Share of total electric utility carbon dioxide emissions weighted by sales to the transportation sector.

${ }^{\mathrm{c}}$ Totals may not equal sum of components due to independent rounding.
} 
Highway vehicles are responsible for the majority of greenhouse gas emissions in the transportation sector.

Table 11.7

Transportation Greenhouse Gas Emissions by Mode, 1990 and 2008 (Million metric tonnes of carbon dioxide equivalent)

\begin{tabular}{|c|c|c|c|}
\hline & Carbon Dioxide & Methane & Nitrous Oxide \\
\hline \multicolumn{4}{|c|}{1990} \\
\hline Highway Total & $1,190.2$ & 4.2 & 40.4 \\
\hline Cars, light trucks, motorcycles & 951.9 & 4.0 & 39.6 \\
\hline Medium \& heavy trucks and buses & 238.3 & 0.2 & 0.8 \\
\hline Water & 44.5 & 0.0 & 0.6 \\
\hline Air & 179.3 & 0.2 & 1.7 \\
\hline Rail & 38.5 & 0.1 & 0.3 \\
\hline Pipeline & 36.1 & 0.0 & 0.0 \\
\hline Other & 0.0 & 0.2 & 0.9 \\
\hline Total $^{\mathrm{a}}$ & $1,488.8$ & 4.7 & 43.9 \\
\hline \multicolumn{4}{|c|}{2008} \\
\hline Highway Total & $1,513.5$ & 1.6 & 22.3 \\
\hline Cars, light trucks, motorcycles & $1,113.3$ & 1.5 & 21.4 \\
\hline Medium \& heavy trucks and buses & 400.2 & 0.1 & 0.9 \\
\hline Water & 38.0 & 0.0 & 0.5 \\
\hline Air & 155.6 & 0.1 & 1.5 \\
\hline Rail & 47.8 & 0.1 & 0.3 \\
\hline Pipeline & 34.9 & 0.0 & 0.0 \\
\hline Other & 0.0 & 0.3 & 1.5 \\
\hline Total $^{\mathrm{a}}$ & $1,789.9$ & 2.0 & 26.1 \\
\hline \multicolumn{4}{|c|}{ Percent change $1990-2008$} \\
\hline Highway Total & $27.2 \%$ & $-61.9 \%$ & $-44.8 \%$ \\
\hline Cars, light trucks, motorcycles & $17.0 \%$ & $-62.5 \%$ & $-46.0 \%$ \\
\hline Medium \& heavy trucks and buses & $67.9 \%$ & $-50.0 \%$ & $12.5 \%$ \\
\hline Water & $-14.6 \%$ & $0.0 \%$ & $-16.7 \%$ \\
\hline Air & $-13.2 \%$ & $-50.0 \%$ & $-11.8 \%$ \\
\hline Rail & $24.2 \%$ & $0.0 \%$ & $0.0 \%$ \\
\hline Pipeline & $-3.3 \%$ & $0.0 \%$ & $0.0 \%$ \\
\hline Other & $0.0 \%$ & $0.0 \%$ & $0.0 \%$ \\
\hline Total $^{\mathrm{a}}$ & $20.2 \%$ & $-57.4 \%$ & $-40.5 \%$ \\
\hline
\end{tabular}

\section{Source:}

U.S. Environmental Protection Agency, Draft Inventory of U.S. Greenhouse Gas Emissions and Sinks: 1990-2008, Tables 3-12, 3-13, 3-14, March 2010. (Additional resources: www.epa.gov/climatechange/emissions)

Note: Emissions from U.S. Territories, International bunker fuels, and military bunker fuels are not included.

\footnotetext{
${ }^{\mathrm{a}}$ The sums of subcategories may not equal due to rounding.
} 


\section{The Greenhouse Gases, Regulated Emissions, and Energy Use in Transportation (GREET) Model}

http://www.transportation.anl.gov/modeling_simulation/GREET/

Sponsored by the U.S. Department of Energy's Office of Energy Efficiency and Renewable Energy (EERE), Argonne has developed a full life-cycle model called GREET (Greenhouse gases, Regulated Emissions, and Energy use in Transportation). It allows researchers and analysts to evaluate energy and emission impacts of various vehicle and fuel combinations on a full fuel-cycle/vehicle-cycle basis. The first version of GREET was released in 1996. Since then, Argonne has continued to update and expand the model. The most recent GREET versions are GREET $1.8 \mathrm{c} .0$ version for fuel-cycle analysis and GREET 2.7 version for vehicle-cycle analysis.

Figure 11.1. GREET Model

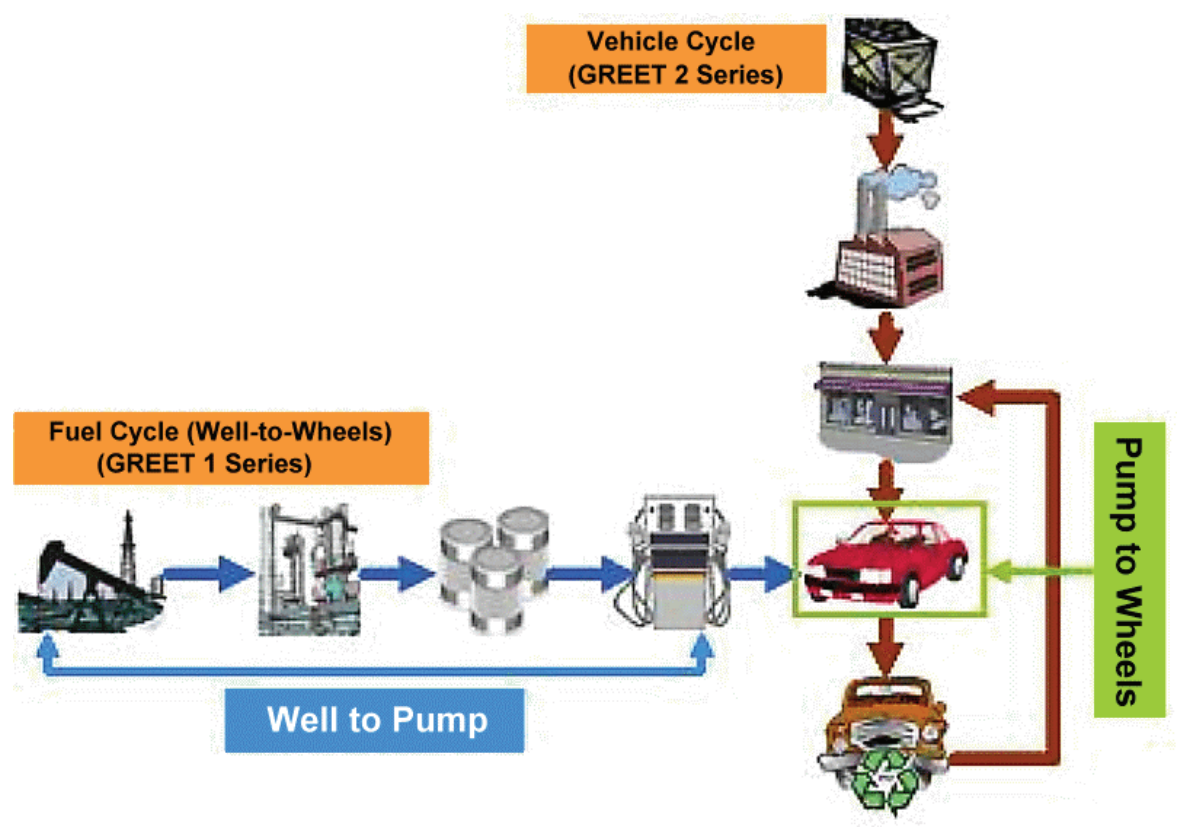

For a given vehicle and fuel system, GREET separately calculates the following:

- Consumption of total energy (energy in non-renewable and renewable sources), fossil fuels (petroleum, natural gas, and coal together), petroleum, coal and natural gas.

- Emissions of $\mathrm{CO}_{2}$-equivalent greenhouse gases - primarily carbon dioxide $\left(\mathrm{CO}_{2}\right)$, methane $\left(\mathrm{CH}_{4}\right)$, and nitrous oxide $\left(\mathrm{N}_{2} \mathrm{O}\right)$. 
- Emissions of six criteria pollutants: volatile organic compounds (VOCs), carbon monoxide $(\mathrm{CO})$, nitrogen oxide $(\mathrm{NOx})$, particulate matter with size smaller than 10 micron $\left(\mathrm{PM}_{10}\right)$, particulate matter with size smaller than 2.5 micron $\left(\mathrm{PM}_{2.5}\right)$,and sulfur oxides (SOx).

GREET includes more than 100 fuel production pathways and more than 70 vehicle/fuel systems. These vehicle/fuel systems cover all major vehicle technologies in the market and R\&D arena:

- Conventional spark-ignition engines

- Direct-injection, spark-ignition engines

- Direct injection, compression-ignition engines

- Grid-independent hybrid electric vehicles

- Grid-connected (or plug-in) hybrid electric vehicles

- Battery-powered electric vehicles

- Fuel-cell vehicles

Figure 11.2. GREET Model Feedstocks and Fuels

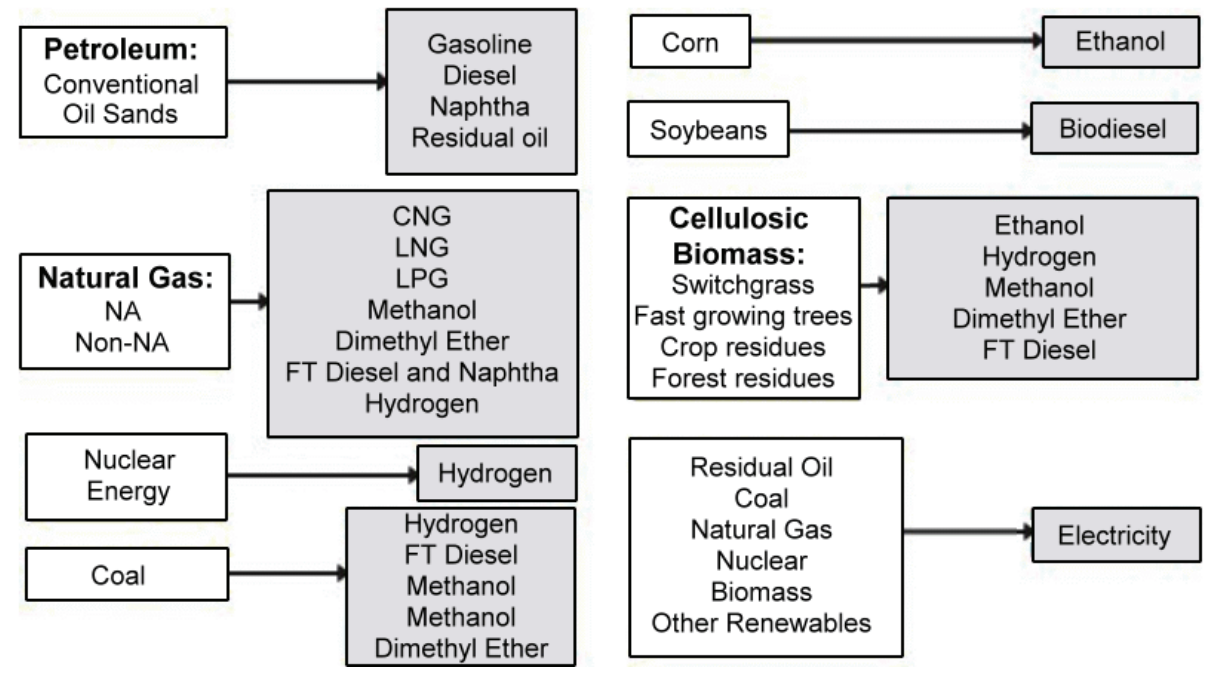

To address technology improvements over time, GREET simulates vehicle/fuel systems over the period from 1990 to 2020, in five-year intervals.

For additional information about the GREET model, see the GREET Web site, or contact:

Michael Q. Wang

Argonne National Laboratory

9700 South Cass Avenue, ES/362

Argonne, IL 60439-4815

phone: 630-252-2819

fax: 630-252-3443

email: mqwang@anl.gov 


\section{Carbon Footprint}

The carbon footprint measures a vehicle's impact on climate change in tons of carbon dioxide $\left(\mathrm{CO}_{2}\right)$ emitted annually. The following three tables show the carbon footprint for various vehicle classes. The sales-weighted average fuel economy rating for each vehicle class, based on $45 \%$ highway and 55\% city driving, is used to determine the average annual carbon footprint for vehicles in the class. An estimate of 15,000 annual miles is used for each vehicle class and for each year in the series. The equation to calculate carbon footprint uses results of the GREET model version 1.8.

CarbonFootprint $=\left(\mathrm{CO}_{2} \times L \mathrm{HV} \times \frac{\text { AnnualMiles }}{\text { CombinedMPG }}\right)+\left(\mathrm{CH}_{4}+\mathrm{N}_{2} \mathrm{O}\right) \times$ AnnualMiles

where:

$\mathrm{CO}_{2}=\left(\right.$ Tailpipe $\mathrm{CO}_{2}+$ Upstream Greenhouse Gases $)$ in grams per million Btu

LHV = Lower (or net) Heating Value in million Btu per gallon

$\mathrm{CH}_{4}=$ Tailpipe ${\underline{\mathrm{CO}_{2}}}_{2}$ equivalent methane in grams per mile

$\mathrm{N}_{2} \mathrm{O}=$ Tailpipe $\underline{\mathrm{CO}}_{2}$ equivalent nitrous oxide in grams per mile 
The carbon footprint for all classifications of cars declined between 1975 and 2009. Midsize cars have experienced the greatest reduction in carbon footprint with a decrease of almost $57 \%$.

Table 11.8

Sales-Weighted Annual Carbon Footprint of New Domestic and Import Cars by Size Class, Model Years 1975-2009

(short tons of $\mathrm{CO}_{2}$ )

\begin{tabular}{|c|c|c|c|c|c|c|}
\hline \multirow[b]{2}{*}{ Sales period } & \multicolumn{3}{|c|}{ Cars } & \multicolumn{3}{|c|}{ Wagons } \\
\hline & Small & Midsize & Large & Small & Midsize & Large \\
\hline 1975 & 10.2 & 13.7 & 14.2 & 8.3 & 14.1 & 15.6 \\
\hline 1976 & 9.4 & 11.9 & 13.1 & 7.8 & 11.6 & 13.8 \\
\hline 1977 & 9.0 & 11.4 & 11.7 & 7.3 & 11.4 & 12.0 \\
\hline 1978 & 8.1 & 10.0 & 11.1 & 7.7 & 10.0 & 11.7 \\
\hline 1979 & 8.0 & 9.8 & 10.7 & 7.3 & 9.8 & 11.6 \\
\hline 1980 & 7.2 & 8.6 & 9.8 & 6.5 & 8.8 & 9.8 \\
\hline 1981 & 6.6 & 8.2 & 9.2 & 6.2 & 8.1 & 9.4 \\
\hline 1982 & 6.4 & 7.8 & 9.0 & 6.1 & 7.9 & 9.7 \\
\hline 1983 & 6.4 & 7.8 & 9.2 & 5.8 & 7.7 & 9.5 \\
\hline 1984 & 6.4 & 7.8 & 9.1 & 5.9 & 7.5 & 9.4 \\
\hline 1985 & 6.3 & 7.5 & 8.4 & 5.8 & 7.4 & 8.9 \\
\hline 1986 & 6.2 & 7.2 & 7.8 & 6.0 & 7.2 & 8.5 \\
\hline 1987 & 6.2 & 7.2 & 7.8 & 6.1 & 7.3 & 8.5 \\
\hline 1988 & 6.1 & 7.0 & 7.7 & 6.0 & 7.1 & 8.2 \\
\hline 1989 & 6.2 & 7.0 & 7.8 & 5.9 & 7.3 & 8.3 \\
\hline 1990 & 6.3 & 7.1 & 7.9 & 6.3 & 7.4 & 8.2 \\
\hline 1991 & 6.2 & 7.2 & 7.9 & 6.1 & 7.2 & 8.2 \\
\hline 1992 & 6.2 & 7.2 & 7.9 & 6.2 & 7.1 & 8.2 \\
\hline 1993 & 6.1 & 7.2 & 7.7 & 5.8 & 7.1 & 8.3 \\
\hline 1994 & 6.2 & 7.2 & 7.8 & 5.7 & 7.2 & 8.2 \\
\hline 1995 & 6.1 & 7.2 & 7.7 & 5.6 & 7.0 & 8.2 \\
\hline 1996 & 6.1 & 7.1 & 7.7 & 5.9 & 7.1 & 8.1 \\
\hline 1997 & 6.1 & 7.1 & 7.6 & 5.8 & 7.1 & b \\
\hline 1998 & 6.1 & 6.9 & 7.6 & 5.8 & 7.1 & $\mathrm{~b}$ \\
\hline 1999 & 6.2 & 6.9 & 7.5 & 5.9 & 7.1 & b \\
\hline 2000 & 6.2 & 6.9 & 7.3 & 6.4 & 6.9 & b \\
\hline 2001 & 6.1 & 6.9 & 7.4 & 6.9 & 7.0 & b \\
\hline 2002 & 6.1 & 6.8 & 7.2 & 7.2 & 6.8 & b \\
\hline 2003 & 6.1 & 6.6 & 7.2 & 6.2 & 6.9 & b \\
\hline 2004 & 6.1 & 6.5 & 7.2 & 6.0 & 7.1 & 8.5 \\
\hline 2005 & 6.0 & 6.3 & 7.1 & 5.8 & 7.2 & 8.4 \\
\hline 2006 & 6.0 & 6.3 & 7.2 & 6.0 & 7.1 & 8.5 \\
\hline 2007 & 5.9 & 6.0 & 7.2 & 5.9 & 6.9 & 8.5 \\
\hline 2008 & 5.9 & 6.0 & 6.9 & 5.8 & 6.9 & 8.6 \\
\hline \multirow[t]{2}{*}{2009} & 5.8 & 5.9 & 6.8 & 5.8 & 6.8 & 8.6 \\
\hline & \multicolumn{6}{|c|}{ Average annual percentage change } \\
\hline 1975-2009 & $-1.6 \%$ & $-2.4 \%$ & $-2.1 \%$ & $-1.0 \%$ & $-2.1 \%$ & $-1.7 \%$ \\
\hline 1999-2009 & $-0.7 \%$ & $-1.6 \%$ & $-1.0 \%$ & $-0.2 \%$ & $-0.4 \%$ & $c$ \\
\hline
\end{tabular}

\section{Source:}

Calculated using fuel economy from the U.S. Environmental Protection Agency, Light-Duty Automotive Technology, Carbon Dioxide Emissions, and Fuel Economy Trends: 1975 Through 2009, November 2009. See page 11-10 for details.

${ }^{a}$ Annual carbon footprint is based on 15,000 miles of annual driving.

${ }^{\mathrm{b}}$ No vehicles in this category were sold in this model year.

${ }^{\mathrm{c}}$ Data are not available. 
The annual carbon footprint of light trucks decreased for all classes of light trucks between 1975 and 2009. In the last ten years, midsize SUVs experienced the greatest decline with about $17 \%$ while small SUVs experienced a slight gain in carbon emissions.

Table 11.9

Sales-Weighted Annual Carbon Footprint of New Domestic and Import Light Trucks by Size Class, Model Years 1975-2009 (short tons of $\mathrm{CO}_{2}$ )

\begin{tabular}{|c|c|c|c|c|c|c|c|c|c|}
\hline \multirow[b]{2}{*}{ Sales period } & \multicolumn{3}{|c|}{ Pickups } & \multicolumn{3}{|c|}{ Vans } & \multicolumn{3}{|c|}{ SUVs } \\
\hline & Small & Midsize & Large & Small & Midsize & Large & Small & Midsize & Large \\
\hline 1975 & 8.3 & 8.8 & 14.2 & 9.1 & 14.0 & 14.8 & 11.6 & 15.4 & 15.3 \\
\hline 1980 & 7.7 & 7.2 & 10.8 & 9.8 & 11.0 & 11.6 & 9.9 & 13.0 & 13.0 \\
\hline 1981 & 6.6 & 7.1 & 10.0 & 10.1 & 10.4 & 11.1 & 9.2 & 11.9 & 12.2 \\
\hline 1982 & 6.9 & 7.0 & 10.0 & 8.6 & 10.4 & 11.6 & 9.1 & 11.3 & 9.9 \\
\hline 1983 & 7.0 & 7.1 & 10.3 & 9.5 & 10.0 & 11.5 & 8.7 & 9.9 & 10.6 \\
\hline 1984 & 7.2 & 7.3 & 10.5 & 7.3 & 9.7 & 11.4 & 8.6 & 9.9 & 11.0 \\
\hline 1985 & 7.0 & 7.3 & 10.5 & 7.3 & 9.4 & 11.6 & 8.5 & 9.5 & 11.0 \\
\hline 1986 & 7.2 & 7.2 & 10.2 & 7.3 & 9.0 & 10.6 & 7.9 & 9.5 & 11.1 \\
\hline 1987 & 7.2 & 7.4 & 10.5 & 7.7 & 8.8 & 11.0 & 7.7 & 9.4 & 11.0 \\
\hline 1988 & 7.5 & 7.4 & 10.4 & 7.6 & 8.6 & 11.0 & 7.7 & 9.6 & 11.2 \\
\hline 1989 & 7.8 & 7.5 & 10.3 & 7.5 & 8.6 & 11.1 & 8.2 & 9.6 & 11.2 \\
\hline 1990 & 7.5 & 7.6 & 10.4 & 7.8 & 8.6 & 11.3 & 8.0 & 9.8 & 11.2 \\
\hline 1991 & 7.5 & 7.6 & 10.2 & 7.8 & 8.5 & 11.2 & 7.9 & 9.2 & 11.5 \\
\hline 1992 & 7.6 & 7.9 & 10.2 & 6.9 & 8.6 & 11.0 & 8.0 & 9.4 & 11.9 \\
\hline 1993 & 7.1 & 7.9 & 10.0 & 6.6 & 8.4 & 11.0 & 8.1 & 9.3 & 11.4 \\
\hline 1994 & 7.5 & 7.8 & 10.1 & 6.9 & 8.5 & 11.0 & 7.8 & 9.4 & 11.4 \\
\hline 1995 & 7.7 & 7.6 & 10.4 & 7.1 & 8.4 & 10.9 & 7.7 & 9.5 & 11.2 \\
\hline 1996 & 7.6 & 7.5 & 10.2 & 7.1 & 8.2 & 10.9 & 6.6 & 9.3 & 10.8 \\
\hline 1997 & 7.5 & 7.7 & 9.9 & $\mathrm{~b}$ & 8.3 & 10.0 & 8.2 & 9.1 & 10.7 \\
\hline 1998 & 7.6 & 7.8 & 10.0 & b & 8.0 & 10.2 & 7.9 & 9.0 & 10.7 \\
\hline 1999 & 8.1 & 8.3 & 10.1 & b & 8.1 & 10.4 & 7.8 & 8.9 & 10.8 \\
\hline 2000 & 7.1 & 8.2 & 9.7 & b & 8.0 & 10.4 & 8.3 & 8.9 & 10.6 \\
\hline 2001 & 7.1 & 8.6 & 9.9 & b & 7.8 & 10.5 & 7.5 & 8.6 & 10.1 \\
\hline 2002 & 8.1 & 8.8 & 10.0 & b & 7.9 & 10.4 & 7.6 & 8.6 & 9.8 \\
\hline 2003 & 8.1 & 8.2 & 9.9 & b & 7.8 & 10.0 & 7.4 & 8.3 & 9.9 \\
\hline 2004 & 8.3 & 8.6 & 9.8 & b & 7.8 & 9.6 & 7.6 & 8.3 & 9.9 \\
\hline 2005 & 7.2 & 7.9 & 9.6 & b & 7.7 & 9.6 & 7.7 & 8.1 & 9.4 \\
\hline 2006 & 7.0 & 7.8 & 9.5 & b & 7.6 & 9.6 & 8.7 & 7.9 & 9.2 \\
\hline 2007 & b & 8.0 & 9.5 & b & 7.7 & 9.4 & 8.3 & 7.6 & 8.9 \\
\hline 2008 & b & 7.8 & 9.3 & b & 7.6 & 9.3 & 8.2 & 7.3 & 8.8 \\
\hline \multirow[t]{2}{*}{2009} & b & 7.6 & 9.3 & b & 7.5 & 9.5 & 8.0 & 7.4 & 8.4 \\
\hline & \multicolumn{9}{|c|}{ Average annual percentage change } \\
\hline 1975-2009 & $\mathrm{c}$ & $-0.4 \%$ & $-1.2 \%$ & $\mathrm{c}$ & $-1.8 \%$ & $-1.3 \%$ & $-1.1 \%$ & $-2.1 \%$ & $-1.7 \%$ \\
\hline 1999-2009 & c & $-0.9 \%$ & $-0.8 \%$ & $\mathrm{c}$ & $-0.8 \%$ & $-0.9 \%$ & $-0.3 \%$ & $-1.8 \%$ & $-2.5 \%$ \\
\hline
\end{tabular}

Source:

Calculated using fuel economy from the U.S. Environmental Protection Agency, Light-Duty Automotive Technology, Carbon Dioxide Emissions, and Fuel Economy Trends: 1975 Through 2009, November 2009. See page 11-10 for details.

Note: Includes light trucks of 8,500 lbs. or less.

\footnotetext{
a Annual carbon footprint is based on 15,000 miles of annual driving.

${ }^{\mathrm{b}}$ No vehicles in this category were sold in this model year.

${ }^{\mathrm{c}}$ Data are not available.
} 
Between 1975 and 2009, the carbon footprint for light vehicles sold in the United States dropped dramatically. Cars experienced the greatest decrease at $48.3 \%$ while the carbon footprint for light trucks decreased by $39.7 \%$.

Table 11.10

Average Annual Carbon Footprint by Vehicle Classification, 1975 and 2009 (short tons of $\mathrm{CO}_{2}$ )

\begin{tabular}{|c|c|c|c|c|c|}
\hline \multirow[b]{2}{*}{ Fuel } & \multicolumn{2}{|c|}{ Market Share } & \multicolumn{2}{|c|}{ Carbon Footprint } & \multirow{2}{*}{$\begin{array}{c}\text { Percent Change } \\
1975-2009\end{array}$} \\
\hline & 1975 & 2009 & 1975 & 2009 & \\
\hline \multicolumn{6}{|c|}{ Cars } \\
\hline Small & $40.0 \%$ & $19.0 \%$ & 10.2 & 5.8 & $-43.1 \%$ \\
\hline Midsize & $16.0 \%$ & $16.3 \%$ & 13.6 & 5.9 & $-56.9 \%$ \\
\hline Large & $15.2 \%$ & $11.7 \%$ & 14.2 & 6.8 & $-52.1 \%$ \\
\hline Small Wagon & $4.7 \%$ & $3.4 \%$ & 8.3 & 5.8 & $-30.1 \%$ \\
\hline Midsize Wagon & $2.8 \%$ & $0.8 \%$ & 14.1 & 6.8 & $-51.8 \%$ \\
\hline Large Wagon & $1.9 \%$ & $0.0 \%$ & 15.6 & 8.6 & $-44.9 \%$ \\
\hline Total Cars & $80.6 \%$ & $51.3 \%$ & 11.8 & 6.1 & $-48.3 \%$ \\
\hline \multicolumn{6}{|c|}{ Light Trucks } \\
\hline Small Van & $0.0 \%$ & $0.0 \%$ & 9.0 & 0.0 & $\mathrm{~b}$ \\
\hline Midsize Van & $3.0 \%$ & $4.4 \%$ & 14.0 & 7.5 & $-46.4 \%$ \\
\hline Large Van & $1.5 \%$ & $0.2 \%$ & 14.7 & 9.5 & $-35.8 \%$ \\
\hline Small SUV & $0.5 \%$ & $1.2 \%$ & 11.5 & 8.0 & $-31.0 \%$ \\
\hline Midsize SUV & $1.2 \%$ & $15.4 \%$ & 15.3 & 7.4 & $-51.9 \%$ \\
\hline Large SUV & $0.1 \%$ & $15.5 \%$ & 15.2 & 8.4 & $-45.1 \%$ \\
\hline Small Pickup & $1.6 \%$ & $0.0 \%$ & 8.3 & 0.0 & $\mathrm{~b}$ \\
\hline Midsize Pickup & $0.5 \%$ & $2.0 \%$ & 8.8 & 7.6 & $-13.6 \%$ \\
\hline Large Pickup & $11.0 \%$ & $10.1 \%$ & 14.2 & 9.3 & $-34.5 \%$ \\
\hline Total Light Trucks & $19.4 \%$ & $48.7 \%$ & 13.5 & 8.2 & $-39.7 \%$ \\
\hline
\end{tabular}

Source:

Calculated using fuel economy from the U.S. Environmental Protection Agency, Light-Duty Automotive Technology, Carbon Dioxide Emissions, and Fuel Economy Trends: 1975 Through 2009, November 2009. See page 11-10 for details.

\footnotetext{
${ }^{\text {a }}$ Annual carbon footprint is based on 15,000 miles of annual driving.

${ }^{\mathrm{b}}$ Data are not available.
} 
The amount of carbon dioxide released into the atmosphere by a vehicle is primarily determined by the carbon content of the fuel. However, there is a small portion of the fuel that is not oxidized into carbon dioxide when the fuel is burned. The Environmental Protection Agency (EPA) has published information on carbon dioxide emissions from gasoline and diesel which takes the oxidation factor into account and is based on the carbon content used in EPA's fuel economy analyses. The other fuels listed come from the Energy Information Administration.

Table 11.11

Carbon Dioxide Emissions from a Gallon of Fuel

\begin{tabular}{lccc}
\hline & $\begin{array}{c}\text { Grams } \\
\text { per gallon }\end{array}$ & $\begin{array}{c}\text { Kilograms } \\
\text { per gallon }\end{array}$ & $\begin{array}{c}\text { Pounds } \\
\text { per gallon }\end{array}$ \\
\hline Gasoline & 8,788 & 8.8 & 19.4 \\
Diesel & 10,084 & 10.1 & 22.2 \\
LPG & 5,805 & 5.8 & 12.8 \\
Propane & 5,760 & 5.8 & 12.7 \\
Aviation gasoline & 8,345 & 8.3 & 18.4 \\
Jet fuel & 9,569 & 9.6 & 21.1 \\
Keosene & 9,751 & 9.8 & 21.5 \\
Residual fuel & 11,791 & 11.8 & 26.0 \\
\hline
\end{tabular}

\section{Sources:}

Gasoline and Diesel: U.S. Environmental Protection Agency, "Emission Facts: Average Carbon Dioxide Emissions Resulting from Gasoline and Diesel Fuel," February 2009. (Additional resources: www.epa.gov/OMS)

All others: Energy Information Administration, Voluntary Reporting of Greenhouse Gases Program, Fuel and Energy Source Codes and Emission Coefficients. 
$11-16$

TRANSPORTATION ENERGY DATA BOOK: EDITION 29—2010 


\section{Chapter 12 \\ Criteria Air Pollutants}

Summary Statistics from Tables in this Chapter

\begin{tabular}{|c|c|c|}
\hline Source & & \\
\hline \multirow[t]{7}{*}{ Table 12.1} & Transportation's share of U.S. emissions, 2008 & \\
\hline & $\mathrm{CO}$ & $73.2 \%$ \\
\hline & $N O_{X}$ & $57.9 \%$ \\
\hline & VOC & $37.7 \%$ \\
\hline & $P M-2.5$ & $.2 \%$ \\
\hline & $\mathrm{SO}_{2}$ & $4.5 \%$ \\
\hline & $P M-10$ & $3.2 \%$ \\
\hline
\end{tabular}


Transportation accounts for the majority of carbon monoxide and nitrogen oxide emissions. Highway vehicles are responsible for the largest share of transportation emissions.

Table 12.1

Total National Emissions of the Criteria Air Pollutants by Sector, 2008 (millions of short tons/percentage)

\begin{tabular}{lrrrrrr}
\hline Sector & \multicolumn{1}{c}{ CO } & \multicolumn{1}{c}{ NOx } & \multicolumn{1}{c}{ VOC } & PM-10 & PM-2.5 & \multicolumn{1}{c}{ SO $_{2}$} \\
\hline Highway vehicles & $\mathbf{3 8 . 8 7}$ & $\mathbf{5 . 2 1}$ & $\mathbf{3 . 4 2}$ & $\mathbf{0 . 1 7}$ & $\mathbf{0 . 1 1}$ & $\mathbf{0 . 0 6}$ \\
& $50.0 \%$ & $31.9 \%$ & $21.5 \%$ & $1.2 \%$ & $0.1 \%$ & $0.6 \%$ \\
Other off-highway & $\mathbf{1 8 . 0 4}$ & $\mathbf{4 . 2 6}$ & $\mathbf{2 . 5 9}$ & $\mathbf{0 . 3 0}$ & $\mathbf{0 . 2 8}$ & $\mathbf{0 . 4 6}$ \\
& $23.2 \%$ & $26.0 \%$ & $16.2 \%$ & $2.1 \%$ & $5.1 \%$ & $4.0 \%$ \\
Transportation total & $\mathbf{5 6 . 9 0}$ & $\mathbf{9 . 4 6}$ & $\mathbf{6 . 0 0}$ & $\mathbf{0 . 4 8}$ & $\mathbf{0 . 3 9}$ & $\mathbf{0 . 5 2}$ \\
& $73.2 \%$ & $57.9 \%$ & $37.7 \%$ & $3.2 \%$ & $7.2 \%$ & $4.5 \%$ \\
Stationary source fuel combustion & $\mathbf{5 . 2 8}$ & $\mathbf{5 . 5 7}$ & $\mathbf{1 . 4 5}$ & $\mathbf{1 . 3 3}$ & $\mathbf{1 . 0 3}$ & $\mathbf{9 . 8 0}$ \\
& $6.8 \%$ & $34.1 \%$ & $9.1 \%$ & $9.0 \%$ & $19.0 \%$ & $85.7 \%$ \\
Industrial processes & $\mathbf{2 . 1 8}$ & $\mathbf{0 . 9 3}$ & $\mathbf{6 . 7 7}$ & $\mathbf{1 . 1 7}$ & $\mathbf{0 . 4 8}$ & $\mathbf{1 . 0 0}$ \\
& $2.8 \%$ & $5.7 \%$ & $42.5 \%$ & $7.9 \%$ & $8.8 \%$ & $8.7 \%$ \\
Waste disposal and recycling total & $\mathbf{1 . 5 8}$ & $\mathbf{0 . 1 2}$ & $\mathbf{0 . 3 7}$ & $\mathbf{0 . 2 9}$ & $\mathbf{0 . 2 7}$ & $\mathbf{0 . 0 3}$ \\
& $2.0 \%$ & $0.7 \%$ & $2.3 \%$ & $1.9 \%$ & $4.9 \%$ & $0.2 \%$ \\
Miscellaneous & $\mathbf{1 1 . 7 3}$ & $\mathbf{0 . 2 6}$ & $\mathbf{1 . 3 3}$ & $\mathbf{1 1 . 5 4}$ & $\mathbf{3 . 2 8}$ & $\mathbf{0 . 0 9}$ \\
Total of all sources & $15.1 \%$ & $1.6 \%$ & $8.4 \%$ & $77.9 \%$ & $60.2 \%$ & $0.7 \%$ \\
& $\mathbf{7 7 . 6 9}$ & $\mathbf{1 6 . 3 4}$ & $\mathbf{1 5 . 9 3}$ & $\mathbf{1 4 . 8 1}$ & $\mathbf{5 . 4 5}$ & $\mathbf{1 1 . 4 3}$ \\
& $100.0 \%$ & $100.0 \%$ & $100.0 \%$ & $100.0 \%$ & $100.0 \%$ & $100.0 \%$ \\
\hline
\end{tabular}

Source:

U. S. Environmental Protection Agency, National Emission Inventory Air Pollutant Emission Trends Web site www.epa.gov/ttn/chief/trends. (Additional resources: www.epa.gov/ttn/chief)

Note: $\mathrm{CO}=$ Carbon monoxide. $\mathrm{NO}_{\mathrm{x}}=$ Nitrogen oxides. $\mathrm{PM}-10=$ Particulate matter less than 10 microns. PM-2.5 = Particulate matter less than 2.5 microns. $\mathrm{SO}_{2}=$ Sulfur dioxide. $\mathrm{VOC}=$ Volatile organic compounds. $\mathrm{NH}_{3}=$ Ammonia. 
The transportation sector accounted for more than 73\% of the nation's carbon monoxide (CO) emissions in 2008. Highway vehicles are by far the source of the greatest amount of CO. For details on the highway emissions of CO,

see Table 12.3.

Table 12.2

Total National Emissions of Carbon Monoxide, 1970-2008

(million short tons)

\begin{tabular}{|c|c|c|c|c|c|c|c|}
\hline Source category & 1970 & 1980 & 1990 & 2000 & 2005 & 2008 & $\begin{array}{c}\text { Percent } \\
\text { of total, } \\
2008\end{array}$ \\
\hline Highway vehicles & 163.23 & 143.83 & 110.26 & 68.06 & 48.54 & 38.87 & $50.0 \%$ \\
\hline Other off-highway & 11.37 & 16.69 & 21.45 & 24.18 & 20.67 & 18.04 & $23.2 \%$ \\
\hline Transportation total & 174.60 & 160.51 & 131.70 & 92.24 & 69.22 & 56.90 & $73.2 \%$ \\
\hline Stationary fuel combustion total & 4.63 & 7.30 & 5.51 & 4.78 & 5.13 & 5.28 & $6.8 \%$ \\
\hline Industrial processes total & 9.84 & 6.95 & 4.77 & 2.63 & 2.03 & 2.18 & $2.8 \%$ \\
\hline Waste disposal and recycling total & 7.06 & 2.30 & 1.08 & 1.85 & 1.55 & 1.58 & $2.0 \%$ \\
\hline Miscellaneous total & 7.91 & 8.34 & 11.12 & 12.96 & 15.11 & 11.73 & $15.1 \%$ \\
\hline Total of all sources & 204.04 & 185.41 & 154.19 & 114.47 & 93.03 & 77.69 & $100.0 \%$ \\
\hline
\end{tabular}

\section{Source:}

U. S. Environmental Protection Agency, National Emission Inventory Air Pollutant Emission Trends Web site www.epa.gov/ttn/chief/trends (Additional resources: www.epa.gov/ttn/chief)

\footnotetext{
${ }^{a}$ The sums of subcategories may not equal total due to rounding.
} 
Though gasoline-powered light vehicles continue to be responsible for the majority of carbon monoxide emissions from highway vehicles, the total pollution from light vehicles in 2005 is about a third of what it was in 1970. This is despite the fact that there were many more light vehicles on the road in 2005.

Table 12.3

Emissions of Carbon Monoxide from Highway Vehicles, 1970-2005 ${ }^{\mathrm{a}}$ (million short tons)

\begin{tabular}{|c|c|c|c|c|c|c|c|}
\hline Source category & 1970 & 1980 & 1990 & 1995 & 2000 & 2005 & $\begin{array}{c}\text { Percent of } \\
\text { total, } \\
2005 \\
\end{array}$ \\
\hline \multicolumn{8}{|c|}{ Gasoline powered } \\
\hline $\begin{array}{l}\text { Light vehicles \& } \\
\text { motorcycles }\end{array}$ & 119.14 & 98.21 & 67.24 & 46.54 & 36.4 & 24.19 & $50.2 \%$ \\
\hline Light trucks ${ }^{\mathrm{b}}$ & 22.27 & 28.83 & 32.23 & 29.81 & 27.04 & 21.19 & $43.9 \%$ \\
\hline Heavy vehicles & 21.27 & 15.35 & 8.92 & 5.96 & 3.42 & 1.97 & $4.1 \%$ \\
\hline Total & 162.68 & 142.39 & 108.39 & 82.31 & 66.86 & 47.35 & $98.2 \%$ \\
\hline \multicolumn{8}{|c|}{ Diesel powered } \\
\hline Light vehicles & 0.01 & 0.03 & 0.04 & 0.02 & 0.01 & 0.01 & $0.0 \%$ \\
\hline Light trucks ${ }^{\mathrm{b}}$ & 0.06 & 0.05 & 0.03 & 0.02 & 0.01 & 0.01 & $0.0 \%$ \\
\hline Heavy vehicles & 0.49 & 1.36 & 1.81 & 1.53 & 1.19 & 0.85 & $1.8 \%$ \\
\hline Total & 0.56 & 1.43 & 1.87 & 1.57 & 1.2 & 0.87 & $1.8 \%$ \\
\hline \multicolumn{8}{|c|}{ Total } \\
\hline Highway vehicle total & 163.23 & 143.83 & 110.26 & 83.88 & 68.06 & 48.22 & $100.0 \%$ \\
\hline Percent diesel & $0.3 \%$ & $1.0 \%$ & $1.7 \%$ & $1.9 \%$ & $1.8 \%$ & $1.8 \%$ & \\
\hline
\end{tabular}

Source:

U. S. Environmental Protection Agency, National Emission Inventory Air Pollutant Emission Trends Web site www.epa.gov/ttn/chief/trends. (Additional resources: www.epa.gov/oar/oaqps)

Note: Data beyond 2005 are not available.

\footnotetext{
${ }^{\mathrm{a}}$ The sums of subcategories may not equal total due to rounding.

${ }^{\mathrm{b}}$ Less than 8,500 pounds.
} 
The transportation sector accounted for over half of the nation's nitrogen oxide (NOx) emissions in 2008, with the majority coming from highway vehicles. For details on the highway emissions of NOx, see Table 12.5.

Table 12.4

Total National Emissions of Nitrogen Oxides, 1970-2008 ${ }^{a}$

(million short tons)

\begin{tabular}{lrrrrrrr}
\hline & & & & & & \multicolumn{3}{c}{$\begin{array}{c}\text { Percent } \\
\text { of total, }\end{array}$} \\
Source category & 1970 & 1980 & 1990 & 2000 & 2005 & 2008 & 2008 \\
\hline Highway vehicles & 12.62 & 11.49 & 9.59 & 8.39 & 6.49 & 5.21 & $31.9 \%$ \\
$\quad$ Other off-highway & 2.65 & 3.35 & 3.78 & 4.17 & 4.89 & 4.26 & $26.0 \%$ \\
Transportation total & 15.28 & 14.85 & 13.37 & 12.56 & 11.38 & 9.46 & $57.9 \%$ \\
Stationary fuel combustion total & 10.06 & 11.32 & 10.89 & 8.82 & 6.34 & 5.57 & $34.1 \%$ \\
Industrial processes total & 0.78 & 0.56 & 0.80 & 0.81 & 0.98 & 0.93 & $5.7 \%$ \\
Waste disposal and recycling total & 0.44 & 0.11 & 0.09 & 0.13 & 0.15 & 0.12 & $0.7 \%$ \\
Miscellaneous total & 0.33 & 0.25 & 0.37 & 0.28 & 0.27 & 0.26 & $1.6 \%$ \\
Total of all sources & $\mathbf{2 6 . 8 8}$ & $\mathbf{2 7 . 0 8}$ & $\mathbf{2 5 . 5 3}$ & $\mathbf{2 2 . 6 0}$ & $\mathbf{1 9 . 1 2}$ & $\mathbf{1 6 . 3 4}$ & $\mathbf{1 0 0 . 0 \%}$ \\
\hline
\end{tabular}

Source:

U. S. Environmental Protection Agency, National Emission Inventory Air Pollutant Emission Trends Web site www.epa.gov/ttn/chief/trends (Additional resources: www.epa.gov/ttn/chief)

${ }^{a}$ The sums of subcategories may not equal total due to rounding. 
Heavy diesel-powered vehicles were responsible for nearly one-half (44.1\%) of highway vehicle nitrogen oxide emissions in 2005, while light gasoline vehicles were responsible for the rest.

Table 12.5

Emissions of Nitrogen Oxides from Highway Vehicles, 1970-2005 ${ }^{\mathrm{a}}$

(million short tons)

\begin{tabular}{|c|c|c|c|c|c|c|c|}
\hline Source category & 1970 & 1980 & 1990 & 1995 & 2000 & 2005 & $\begin{array}{c}\text { Percent } \\
\text { of total, } \\
2005\end{array}$ \\
\hline \multicolumn{8}{|c|}{ Gasoline powered } \\
\hline Light vehicles \& motorcycles & 8.54 & 6.63 & 4.26 & 3.05 & 2.31 & 1.63 & $25.5 \%$ \\
\hline Light trucks $^{\mathrm{b}}$ & 1.54 & 1.58 & 1.50 & 1.46 & 1.44 & 1.56 & $24.4 \%$ \\
\hline Heavy vehicles & 0.72 & 0.62 & 0.57 & 0.52 & 0.45 & 0.38 & $5.9 \%$ \\
\hline Total & 10.81 & 8.83 & 6.33 & 5.03 & 4.20 & 3.57 & $55.9 \%$ \\
\hline \multicolumn{8}{|c|}{ Diesel powered } \\
\hline Light vehicles & 0.00 & 0.03 & 0.04 & 0.02 & 0.01 & 0.00 & $0.0 \%$ \\
\hline Light trucks ${ }^{\mathrm{b}}$ & 0.07 & 0.05 & 0.02 & 0.01 & 0.01 & 0.01 & $0.2 \%$ \\
\hline Heavy vehicles & 1.76 & 2.59 & 3.19 & 3.82 & 4.18 & 2.81 & $44.0 \%$ \\
\hline Total & 1.83 & 2.66 & 3.26 & 3.85 & 4.19 & 2.82 & $44.1 \%$ \\
\hline \multicolumn{8}{|c|}{ Total } \\
\hline Highway vehicle total & 12.64 & 11.49 & 9.59 & 8.88 & 8.39 & 6.39 & $100.0 \%$ \\
\hline Percent diesel & $14.5 \%$ & $23.1 \%$ & $34.0 \%$ & $43.4 \%$ & $49.9 \%$ & $44.1 \%$ & \\
\hline
\end{tabular}

Source:

U. S. Environmental Protection Agency, National Emission Inventory Air Pollutant Emission Trends Web site www.epa.gov/ttn/chief/trends. (Additional resources: www.epa.gov/oar/oaqps)

Note: Data beyond 2005 are not available.

\footnotetext{
${ }^{a}$ The sums of subcategories may not equal total due to rounding.

${ }^{\mathrm{b}}$ Less than 8,500 pounds.
} 
The transportation sector accounted for almost $38 \%$ of the nation's volatile organic compound (VOC) emissions in 2008, with the majority coming from highway vehicles. For details on the highway emissions of VOC, see Table 12.7.

Table 12.6

Total National Emissions of Volatile Organic Compounds, 1970-2008 (million short tons)

\begin{tabular}{|c|c|c|c|c|c|c|c|}
\hline Source category & 1970 & 1980 & 1990 & 2000 & 2005 & 2008 & $\begin{array}{l}\text { Percent of } \\
\text { total, } 2008\end{array}$ \\
\hline Highway vehicles & 16.91 & 13.87 & 9.39 & 5.33 & 4.11 & 3.42 & $21.5 \%$ \\
\hline Off-highway & 1.62 & 2.19 & 2.66 & 2.64 & 2.87 & 2.59 & $16.2 \%$ \\
\hline Transportation total & 18.53 & 16.06 & 12.05 & 7.97 & 6.98 & 6.00 & $37.7 \%$ \\
\hline Stationary fuel combustion total & 0.72 & 1.05 & 1.01 & 1.18 & 1.77 & 1.45 & $9.1 \%$ \\
\hline Industrial processes total & 12.33 & 12.10 & 9.01 & 7.21 & 6.99 & 6.79 & $42.5 \%$ \\
\hline Waste disposal and recycling total & 1.98 & 0.76 & 0.99 & 0.42 & 0.39 & 0.37 & $2.3 \%$ \\
\hline Miscellaneous total & 1.10 & 1.13 & 1.06 & 0.73 & 3.29 & 1.33 & $8.4 \%$ \\
\hline Total of all sources & 34.66 & 31.11 & 24.11 & 17.51 & 18.42 & 15.93 & $100.0 \%$ \\
\hline
\end{tabular}

Source:

U. S. Environmental Protection Agency, National Emission Inventory Air Pollutant Emission Trends Web site www.epa.gov/ttn/chief/trends (Additional resources: www.epa.gov/ttn/chief)

${ }^{a}$ The sum of subcategories may not equal total due to rounding. The EPA's definition of volatile organic compounds excludes methane, ethane, and certain other nonphotochemically reactive organic compounds. 
Gasoline-powered vehicles are responsible for over $95 \%$ of highway vehicle emissions of volatile organic compounds. VOC emissions from highway vehicles in 2005 were about one-quarter of the 1990 level.

Table 12.7

Emissions of Volatile Organic Compounds from Highway Vehicles, 1970-2005 (thousand short tons)

\begin{tabular}{|c|c|c|c|c|c|c|c|}
\hline Source category & 1970 & 1980 & 1990 & 1995 & 2000 & 2005 & $\begin{array}{c}\text { Percent of } \\
\text { total, } \\
2005\end{array}$ \\
\hline \multicolumn{8}{|c|}{ Gasoline powered } \\
\hline Light vehicles \& motorcycles & 11,996 & 9,304 & 5,690 & 3,768 & 2,903 & 2,111 & $51.8 \%$ \\
\hline Light trucks $^{\mathrm{b}}$ & 2,776 & 2,864 & 2,617 & 2,225 & 1,929 & 1,629 & $39.9 \%$ \\
\hline Heavy vehicles & 1,679 & 1,198 & 633 & 421 & 256 & 171 & $4.2 \%$ \\
\hline Total & 16,451 & 13,366 & 8,940 & 6,414 & 5,088 & 3,911 & $95.9 \%$ \\
\hline \multicolumn{8}{|c|}{ Diesel powered } \\
\hline Light vehicles & 8 & 16 & 18 & 9 & 3 & 2 & $0.0 \%$ \\
\hline Light trucks ${ }^{\mathrm{b}}$ & 41 & 28 & 15 & 10 & 4 & 6 & $0.1 \%$ \\
\hline Heavy vehicles & 411 & 459 & 415 & 315 & 230 & 159 & $3.9 \%$ \\
\hline Total & 460 & 503 & 448 & 335 & 238 & 167 & $4.1 \%$ \\
\hline \multicolumn{8}{|c|}{ Total } \\
\hline Highway vehicle total & 16,911 & 13,869 & 9,388 & 6,749 & 5,326 & 4,078 & $100.0 \%$ \\
\hline Percent diesel & $2.7 \%$ & $3.6 \%$ & $4.8 \%$ & $5.0 \%$ & $4.5 \%$ & $4.1 \%$ & \\
\hline
\end{tabular}

Source:

U. S. Environmental Protection Agency, National Emission Inventory Air Pollutant Emission Trends Web site www.epa.gov/ttn/chief/trends (Additional resources: www.epa.gov/oar/oaqps)

Note: Data beyond 2005 are not available.

\footnotetext{
${ }^{a}$ The sums of subcategories may not equal total due to rounding.

${ }^{\mathrm{b}}$ Less than 8,500 pounds.
} 
The transportation sector accounted for just over 3\% of the nation's particulate matter (PM-10) emissions in 2008.

For details on the highway emissions of PM-10, see Table 12.9.

Table 12.8

Total National Emissions of Particulate Matter (PM-10), 1970-2008 (million short tons)

\begin{tabular}{lrrrrrrr}
\hline Source category & 1970 & 1980 & 1990 & 2000 & 2005 & $\begin{array}{r}\text { Percent of } \\
\text { total, 2008 }\end{array}$ \\
\hline \multicolumn{1}{c}{ Highway vehicles } & 0.48 & 0.43 & 0.39 & 0.23 & 0.19 & 0.17 & $1.2 \%$ \\
$\quad$ Off-highway & 0.16 & 0.26 & 0.33 & 0.32 & 0.35 & 0.30 & $2.1 \%$ \\
Transportation total & 0.64 & 0.69 & 0.72 & 0.55 & 0.54 & 0.48 & $3.2 \%$ \\
Stationary fuel combustion total & 2.87 & 2.45 & 1.20 & 1.47 & 1.44 & 1.33 & $9.0 \%$ \\
Industrial processes total & 7.67 & 2.75 & 1.04 & 0.71 & 1.22 & 1.17 & $7.9 \%$ \\
Waste disposal and recycling total & 1.00 & 0.27 & 0.27 & 0.36 & 0.29 & 0.29 & $1.9 \%$ \\
Miscellaneous total & 0.84 & 0.85 & 24.54 & 20.65 & 17.66 & 11.54 & $\mathbf{7 7 . 9 \%}$ \\
Total of all sources & $\mathbf{1 3 . 0 2}$ & $\mathbf{7 . 0 1}$ & $\mathbf{2 7 . 7 5}$ & $\mathbf{2 3 . 7 5}$ & $\mathbf{2 1 . 1 5}$ & $\mathbf{1 4 . 8 1}$ & $\mathbf{1 0 0 . 0 \%}$ \\
\hline
\end{tabular}

\section{Source:}

U. S. Environmental Protection Agency, National Emission Inventory Air Pollutant Emission Trends Web site www.epa.gov/ttn/chief/trends (Additional resources: www.epa.gov/ttn/chief)

Note: Because PM-10 is fine particle matter less than 10 microns, it also includes PM-2.5. Specific data for PM-2.5 are shown on Tables 12.10 and 12.11.

\footnotetext{
${ }^{a}$ Fine particle matter less than 10 microns. The sums of subcategories may not equal total due to rounding.
}

${ }^{\mathrm{b}}$ Data are not available. 
Since the mid-1980's, diesel-powered vehicles have been responsible for more than half of highway vehicle emissions of particulate matter (PM-10). Heavy vehicles are clearly the main source.

Table 12.9

Emissions of Particulate Matter (PM-10) from Highway Vehicles, 1970-2005 ${ }^{\text {a }}$ (thousand short tons)

\begin{tabular}{|c|c|c|c|c|c|c|c|}
\hline Source category & 1970 & 1980 & 1990 & 1995 & 2000 & 2005 & $\begin{array}{c}\text { Percent of total, } \\
2005\end{array}$ \\
\hline \multicolumn{8}{|c|}{ Gasoline powered } \\
\hline Light vehicles \& motorcycles & 249 & 141 & 56 & 53 & 51 & 46 & $25.1 \%$ \\
\hline Light trucks ${ }^{b}$ & 74 & 49 & 31 & 32 & 31 & 35 & $19.1 \%$ \\
\hline Heavy vehicles & 44 & 30 & 17 & 13 & 10 & 8 & $4.4 \%$ \\
\hline Total & 367 & 220 & 104 & 98 & 92 & 89 & $48.6 \%$ \\
\hline \multicolumn{8}{|c|}{ Diesel powered } \\
\hline Light vehicles & 2 & 9 & 11 & 4 & 1 & 1 & $0.5 \%$ \\
\hline Light trucks ${ }^{\mathrm{b}}$ & 19 & 12 & 5 & 3 & 1 & 1 & $0.5 \%$ \\
\hline Heavy vehicles & 92 & 191 & 268 & 199 & 135 & 92 & $50.3 \%$ \\
\hline Total & 113 & 212 & 284 & 206 & 137 & 94 & $51.4 \%$ \\
\hline \multicolumn{8}{|c|}{ Total } \\
\hline Highway vehicle total & 480 & 432 & 387 & 304 & 230 & 183 & $100.0 \%$ \\
\hline Percent diesel & $23.5 \%$ & $49.1 \%$ & $73.4 \%$ & $67.8 \%$ & $59.6 \%$ & $51.4 \%$ & \\
\hline
\end{tabular}

Source:

U. S. Environmental Protection Agency, National Emission Inventory Air Pollutant Emission Trends Web site www.epa.gov/ttn/chief/trends (Additional resources: www.epa.gov/oar/oaqps)

Note: Because PM-10 is fine particle matter less than 10 microns, it also includes PM-2.5. Specific data for PM-2.5 are shown on Tables 12.10 and 12.11. Data beyond 2005 are not available.

\footnotetext{
${ }^{a}$ The sums of subcategories may not equal total due to rounding.

${ }^{\mathrm{b}}$ Less than 8,500 pounds.
} 
The transportation sector accounted for only $4 \%$ of the nation's particulate matter (PM-2.5) emissions in 2008. For details on the highway emissions of PM-2.5, see Table 12.11 .

Table 12.10

Total National Emissions of Particulate Matter (PM-2.5), 1990-2008 (million short tons)

\begin{tabular}{lccccccr}
\hline & & & & & & \multicolumn{3}{c}{$\begin{array}{c}\text { Percent } \\
\text { of total, }\end{array}$} \\
Source category & 1990 & 1995 & 2000 & 2005 & 2006 & 2008 & \multicolumn{1}{c}{2008} \\
\hline Highway vehicles & 0.32 & 0.25 & 0.17 & 0.14 & 0.13 & 0.11 & $2.2 \%$ \\
$\quad$ Off-highway & 0.30 & 0.31 & 0.30 & 0.32 & 0.31 & 0.28 & $5.8 \%$ \\
Transportation total & 0.62 & 0.56 & 0.47 & 0.46 & 0.44 & 0.39 & $8.0 \%$ \\
Stationary fuel combustion total & 0.91 & 0.90 & 1.29 & 1.13 & 1.09 & 1.01 & $20.6 \%$ \\
Industrial processes total & 0.56 & 0.50 & 0.50 & 0.53 & 0.52 & 0.48 & $9.9 \%$ \\
Waste disposal and recycling total & 0.23 & 0.25 & 0.33 & 0.27 & 0.27 & 0.27 & $5.5 \%$ \\
Miscellaneous total & 5.23 & 4.73 & 4.69 & 3.07 & 2.96 & 2.74 & $56.1 \%$ \\
Total of all sources & $\mathbf{7 . 5 6}$ & $\mathbf{6 . 9 3}$ & $\mathbf{7 . 2 9}$ & $\mathbf{5 . 4 6}$ & $\mathbf{5 . 2 7}$ & $\mathbf{4 . 8 9}$ & $\mathbf{1 0 0 . 0 \%}$ \\
\hline
\end{tabular}

Source:

U. S. Environmental Protection Agency, National Emission Inventory Air Pollutant Emission Trends Web site www.epa.gov/ttn/chief/trends (Additional resources: www.epa.gov/ttn/chief) 
Diesel vehicles are responsible for the majority of highway vehicle PM-2.5 emissions. Nearly two-thirds of the highway vehicles' PM-2.5 emissions are from heavy diesel trucks.

Table 12.11

Emissions of Particulate Matter (PM-2.5) from Highway Vehicles, 1990-2005 ${ }^{\mathrm{a}}$ (thousand short tons)

\begin{tabular}{|c|c|c|c|c|c|}
\hline Source category & 1990 & 1995 & 2000 & 2005 & $\begin{array}{l}\text { Percent of } \\
\text { total, } 2005\end{array}$ \\
\hline \multicolumn{6}{|c|}{ Gasoline powered } \\
\hline Light vehicles \& motorcycles & 35 & 30 & 27 & 23 & $18.0 \%$ \\
\hline Light trucks ${ }^{b}$ & 21 & 20 & 18 & 18 & $14.1 \%$ \\
\hline Heavy vehicles & 11 & 9 & 7 & 6 & $4.7 \%$ \\
\hline Total & 67 & 59 & 52 & 47 & $36.7 \%$ \\
\hline \multicolumn{6}{|c|}{ Diesel powered } \\
\hline Light vehicles & 9 & 4 & 1 & 1 & $0.8 \%$ \\
\hline Light trucks ${ }^{\mathrm{b}}$ & 4 & 2 & 1 & 1 & $0.8 \%$ \\
\hline Heavy vehicles & 243 & 179 & 119 & 79 & $61.7 \%$ \\
\hline Total & 256 & 185 & 121 & 81 & $63.3 \%$ \\
\hline \multicolumn{6}{|c|}{ Total } \\
\hline Highway vehicle total & 323 & 244 & 173 & 128 & $100.0 \%$ \\
\hline Percent diesel & $79.3 \%$ & $75.8 \%$ & $69.9 \%$ & $63.3 \%$ & \\
\hline
\end{tabular}

Source:

U. S. Environmental Protection Agency, National Emission Inventory Air Pollutant Emission Trends Web site www.epa.gov/ttn/chief/trends (Additional resources: www.epa.gov/oar/oaqps)

Note: Data beyond 2005 are not available.

\footnotetext{
a The sums of subcategories may not equal total due to rounding.

${ }^{\mathrm{b}}$ Less than 8,500 pounds.
} 
Table 12.12

U.S. Tier 2 Emission Standards for Cars and Light Trucks (grams/mile)

\begin{tabular}{cccccc}
\hline Bin & NMOG & CO & Nox & PM & HCHO \\
\hline \multicolumn{7}{c}{50,000 miles } & & & \\
\hline $10^{\mathrm{a}}$ & 0.125 & 3.4 & 0.4 & $\mathrm{~b}$ & 0.015 \\
$9^{\mathrm{a}}$ & 0.075 & 3.4 & 0.2 & $\mathrm{~b}$ & 0.015 \\
8 & 0.100 & 3.4 & 0.14 & $\mathrm{~b}$ & 0.015 \\
7 & 0.075 & 3.4 & 0.11 & $\mathrm{~b}$ & 0.015 \\
6 & 0.075 & 3.4 & 0.08 & $\mathrm{~b}$ & 0.015 \\
5 & 0.075 & 3.4 & 0.05 & $\mathrm{~b}$ & 0.015 \\
\hline \multicolumn{7}{c}{} & 120,000 miles & & & \\
\hline MDPV $^{\mathrm{a}}$ & 0.280 & 7.3 & 0.9 & 0.12 & 0.032 \\
$10^{\mathrm{a}}$ & 0.156 & 4.2 & 0.6 & 0.08 & 0.018 \\
8 & 0.090 & 4.2 & 0.3 & 0.06 & 0.018 \\
7 & 0.125 & 4.2 & 0.2 & 0.02 & 0.018 \\
6 & 0.090 & 4.2 & 0.15 & 0.02 & 0.018 \\
5 & 0.090 & 4.2 & 0.10 & 0.01 & 0.018 \\
4 & 0.090 & 4.2 & 0.07 & 0.01 & 0.018 \\
3 & 0.070 & 2.1 & 0.04 & 0.01 & 0.011 \\
2 & 0.055 & 2.1 & 0.03 & 0.01 & 0.011 \\
1 & 0.010 & 2.1 & 0.02 & 0.01 & 0.004 \\
\hline
\end{tabular}

Source:

Federal Register, Vol. 65, No. 28, Thursday, February 10, 2000, pp. 6822-6870.

\begin{tabular}{|ll|}
\hline \multicolumn{2}{|c|}{ Acronyms Used on Tables 12.12 and 12.13 } \\
CO & Carbon Monoxide \\
GVW & Gross vehicle weight \\
HC & Hydrocarbons \\
HCHO & Formaldehyde \\
LDT & Light-duty truck \\
LEV & Low-emission vehicle \\
LVW & Loaded vehicle weight \\
MDPV & Medium-duty passenger vehicle \\
& (8,500-10,000 lbs. GVWR) \\
NMOG & Non-methane organic gases \\
NOx & Nitrogen oxides \\
PM & Particulate matter \\
SULEV & Super-ultra-low-emission vehicle \\
ULEV & Ultra-low-emission vehicle \\
ZEV & Zero-emission vehicle \\
\hline
\end{tabular}

\footnotetext{
${ }^{\mathrm{a}}$ Bin expired after 2008.

${ }^{\mathrm{b}}$ No Standard.
} 
Table 12.13

Light Vehicle Exhaust Emission Standards in Effect in 2009

when U.S. Tier 2 Standards are Final

(grams/mile)

Vehicle fuels: Gasoline AND diesel

Vehicle size: Up to 8,500 lbs. GVW unless noted otherwise

unless noted otherwise

\begin{tabular}{|c|c|c|c|c|c|c|}
\hline \multirow[b]{2}{*}{ Useful life: } & \multirow[b]{2}{*}{ Bins, category, size } & \multicolumn{5}{|c|}{120,000 miles } \\
\hline & & NMOG & $\mathrm{CO}$ & NOx & $\mathrm{PM}$ & $\mathrm{HCHO}$ \\
\hline U.S. & Bins & & & & & \\
\hline emission & 8 & 0.125 & 4.2 & 0.20 & 0.02 & 0.018 \\
\hline \multirow[t]{9}{*}{ standards } & 7 & 0.090 & 4.2 & 0.15 & 0.02 & 0.018 \\
\hline & 6 & 0.090 & 4.2 & 0.10 & 0.01 & 0.018 \\
\hline & 5 & 0.090 & 4.2 & 0.07 & 0.01 & 0.018 \\
\hline & 4 & 0.070 & 2.1 & 0.04 & 0.01 & 0.011 \\
\hline & 3 & 0.055 & 2.1 & 0.03 & 0.01 & 0.011 \\
\hline & 2 & 0.010 & 2.1 & 0.02 & 0.01 & 0.004 \\
\hline & 1 & 0.000 & 0.0 & 0.00 & 0.00 & 0.000 \\
\hline & Average $^{\mathrm{a}}$ & - & - & 0.07 & - & - \\
\hline & Category & \multicolumn{5}{|c|}{ (Diesel only) } \\
\hline California & $\mathrm{LEV}^{\mathrm{b}}$ & 0.090 & 4.2 & 0.07 & 0.01 & 0.018 \\
\hline LEV II & ULEV & 0.055 & 2.1 & 0.07 & 0.01 & 0.011 \\
\hline emission & SULEV & 0.010 & 1.0 & 0.02 & 0.01 & 0.004 \\
\hline standards & $\mathrm{ZEV}^{\mathrm{c}}$ & 0.000 & 0.0 & 0.00 & 0.00 & 0.000 \\
\hline
\end{tabular}

\section{Source:}

U.S.: Federal Register, Vol. 65, No. 28, Thursday, February 10, 2000, pp. 6822-6870.

California Exhaust Emission Standards and Test Procedures for 2001 and Subsequent Model Passenger Cars, Light-Duty Trucks and Medium-Duty Vehicles, as of December 1, 1999 (adopted August 5, 1999), incorporated by reference in section 1961(d), title 13, CCR.

Note: See acronym list on previous page.

\footnotetext{
${ }^{\mathrm{a}}$ Includes medium-duty passenger vehicles which are also required to meet bin standards.

${ }^{\mathrm{b}}$ A LEV Option 1 with higher NOx levels also exists for up to 4\% of LDTs above 3,750 lbs.

${ }^{c}$ Only apply to cars and LDTs 0-3750 lbs LVW.
} 
Table 12.14

California Cars and Light Trucks Emission Certification Standards (grams/mile)

\begin{tabular}{llccccccc}
\hline & & \multicolumn{7}{c}{ Vehicle Useful Life } \\
\cline { 3 - 9 } Vehicle & Emission & \multicolumn{7}{c}{10 Years / 100,000 Miles } \\
\cline { 3 - 9 } Type & Category & THC $^{\mathrm{a}}$ & NMHC $^{\mathrm{b}}$ & NMOG $^{\mathrm{c}}$ & $\mathrm{CO}$ & $\mathrm{NO}_{\mathrm{x}}$ & $\mathrm{PM}$ & HCHO \\
\hline Car & Tier 1 & - & 0.31 & - & 4.2 & 0.6 & - & - \\
& TLEV & - & - & 0.156 & 4.2 & 0.6 & $0.08^{\mathrm{d}}$ & 0.018 \\
& LEV & - & - & 0.090 & 4.2 & 0.3 & $0.08^{\mathrm{d}}$ & 0.018 \\
& ULEV & - & - & 0.055 & 2.1 & 0.3 & $0.04^{\mathrm{d}}$ & 0.011 \\
& ZEV & 0.00 & 0.00 & 0.000 & 0.0 & 0.0 & 0.00 & 0.000 \\
\hline LDT1 & Tier 1 & - & 0.31 & - & 4.2 & 0.6 & - & - \\
& TLEV & - & - & 0.156 & 4.2 & 0.6 & $0.08^{\mathrm{d}}$ & 0.018 \\
& LEV & - & - & 0.090 & 4.2 & 0.3 & $0.08^{\mathrm{d}}$ & 0.018 \\
& ULEV & - & - & 0.055 & 2.1 & 0.3 & $0.04^{\mathrm{d}}$ & 0.011 \\
& ZEV & 0.00 & 0.00 & 0.000 & 0.0 & 0.0 & 0.00 & 0.000 \\
\hline LDT2 & Tier 1 & - & 0.40 & - & 5.5 & 0.9 & - & - \\
& TLEV & - & - & 0.200 & 5.5 & 0.9 & $0.10^{\mathrm{d}}$ & 0.023 \\
& LEV & - & - & 0.130 & 5.5 & 0.5 & $0.10^{\mathrm{d}}$ & 0.023 \\
& ULEV & - & - & 0.070 & 2.8 & 0.5 & $0.05^{\mathrm{d}}$ & 0.013 \\
\hline
\end{tabular}

Source:

U.S. Environmental Protection Agency, Office of Transportation and Air Quality, EPA 420-B-00-001. (Additional resources: www.epa.gov/otag)

Note: After 2003, Tier 1 and TLEV standards were eliminated. LDT1 = light truck (6,000 lbs. or less GVWR) up through 3,750 lbs. loaded vehicle weight; LDT2 = light truck $(6,000 \mathrm{lbs}$. or less GVWR) greater than 3,750 lbs. loaded vehicle weight.

a THCE for methanol vehicles. Does not apply to CNG vehicles.

${ }^{b}$ THCE for Tier 0 methanol vehicles. NMHCE for other alcohol vehicles.

${ }^{\mathrm{c}} \mathrm{NMHC}$ for diesel-fueled vehicles.

${ }^{\mathrm{d}}$ Diesel-fueled vehicles only. 
$12-16$

TRANSPORTATION ENERGY DATA BOOK: EDITION 29-2010 
APPENDIX A

SOURCES \& METHODOLOGIES 
A-2

TRANSPORTATION ENERGY DATA BOOK: EDITION 29-2010 


\section{SOURCES \& METHODOLOGIES}

This appendix contains documentation of the estimation procedures used by ORNL. The reader can examine the methodology behind the estimates and form an opinion as to their utility. The appendix is arranged by subject heading. Only tables which contain ORNL estimations are documented in Appendix A; all other tables have sources listed at the bottom of the table. Since abbreviations are used throughout the appendix, a list of abbreviations is also included.

\section{Contents of Appendix A}

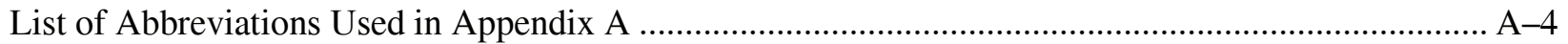

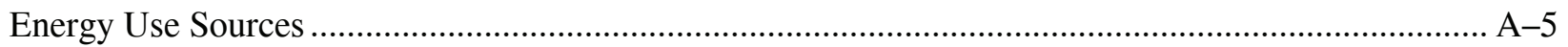

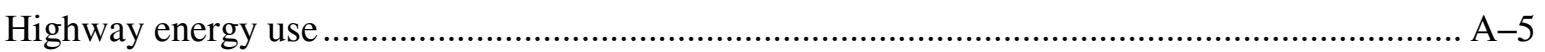

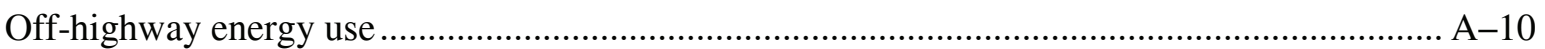

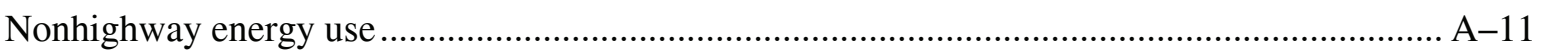

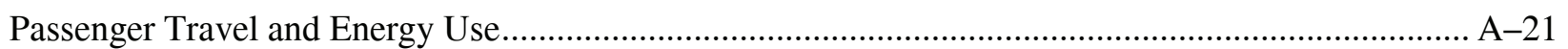

Highway Passenger Mode Energy Intensities................................................................................ A-25

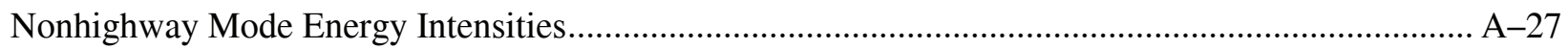

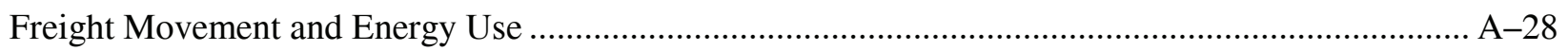

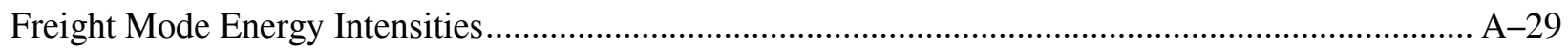




\section{List of Abbreviations Used in Appendix A}

\begin{tabular}{ll} 
AAMA & American Automobile Manufacturers Association \\
AAR & Association of American Railroads \\
APTA & American Public Transportation Association \\
Amtrak & National Railroad Passenger Corporation \\
Btu & British thermal unit \\
DOC & Department of Commerce \\
DOE & Department of Energy \\
DOT & Department of Transportation \\
EIA & Energy Information Administration \\
EPA & Environmental Protection Agency \\
FAA & Federal Aviation Administration \\
FHWA & Federal Highway Administration \\
GSA & General Services Administration \\
gvw & gross vehicle weight \\
lpg & liquefied petroleum gas \\
mpg & miles per gallon \\
NHTS & National Household Travel Survey \\
NHTSA & National Highway Traffic Safety Administration \\
NPTS & Nationwide Personal Transportation Survey \\
NVPP & National Vehicle Population Profile \\
ORNL & Oak Ridge National Laboratory \\
pmt & passenger-miles traveled \\
RECS & Residential Energy Consumption Survey \\
RTECS & Residential Transportation Energy Consumption Survey \\
TIUS & Truck Inventory and Use Survey \\
TSC & Transportation Systems Center \\
\hline vidnentory and Use Survey \\
NHiles traveled
\end{tabular}




\section{Energy Use Sources}

\section{Highway energy use}

\section{Automobiles}

Fuel use in gallons from: DOT, FHWA, Highway Statistics 2008, Table VM-1 and annual editions back to 1996; DOT, FHWA, Highway Statistics Summary to 1995. Fuel use was distributed among fuel types using the percentages shown in Table A.1. The Federal Highway Administration discontinued publication of gasohol data in 2005. Therefore, a new methodology was developed using data from EIA, Alternatives to Traditional Transportation Fuels, 2006, Table C1.

Table A.1

Automobile Fuel Use and Fuel Type Shares for Calculation of Energy Use

\begin{tabular}{|c|c|c|c|c|c|c|}
\hline \multirow[b]{2}{*}{ Year } & \multirow{2}{*}{$\begin{array}{c}\text { Fuel use } \\
\text { (million gallons) }\end{array}$} & \multirow{2}{*}{$\begin{array}{c}\text { Source for } \\
\text { Gasohol shares }\end{array}$} & \multirow{2}{*}{$\begin{array}{c}\text { Source for } \\
\text { gasoline/diesel shares }\end{array}$} & \multicolumn{3}{|c|}{ Shares by fuel type } \\
\hline & & & & Gasoline & Gasohol & Diesel \\
\hline 1970 & 67,820 & & 1984 NVPP & $99.8 \%$ & $0.0 \%$ & $0.2 \%$ \\
\hline 1971 & 71,346 & & interpolated & $99.2 \%$ & $0.0 \%$ & $0.8 \%$ \\
\hline 1972 & 75,937 & & interpolated & $98.7 \%$ & $0.0 \%$ & $1.3 \%$ \\
\hline 1973 & 78,233 & & interpolated & $98.1 \%$ & $0.0 \%$ & $1.9 \%$ \\
\hline 1974 & 74,229 & & interpolated & $97.5 \%$ & $0.0 \%$ & $2.5 \%$ \\
\hline 1975 & 74,140 & & interpolated & $97.0 \%$ & $0.0 \%$ & $3.0 \%$ \\
\hline 1976 & 78,297 & & interpolated & $96.4 \%$ & $0.0 \%$ & $3.6 \%$ \\
\hline 1977 & 79,060 & & interpolated & $95.8 \%$ & $0.0 \%$ & $4.2 \%$ \\
\hline 1978 & 80,652 & & interpolated & $95.3 \%$ & $0.0 \%$ & $4.7 \%$ \\
\hline 1979 & 76,588 & & 1979 RTECS & $94.7 \%$ & $0.0 \%$ & $5.3 \%$ \\
\hline 1980 & 69,981 & FHWA, MF-33e & interpolated & $93.9 \%$ & $0.5 \%$ & $5.6 \%$ \\
\hline 1981 & 69,112 & FHWA, MF-33e & 1981 RTECS & $93.4 \%$ & $0.7 \%$ & $5.9 \%$ \\
\hline 1982 & 69,116 & FHWA, MF-33e & interpolated & $93.5 \%$ & $2.3 \%$ & $4.2 \%$ \\
\hline 1983 & 70,322 & FHWA, MF-33e & 1983 RTECS & $93.2 \%$ & $4.3 \%$ & $2.5 \%$ \\
\hline 1984 & 70,663 & FHWA, MF-33e & interpolated & $92.7 \%$ & $5.3 \%$ & $2.0 \%$ \\
\hline 1985 & 71,518 & FHWA, MF-33e & 1985 RTECS & $90.8 \%$ & $7.7 \%$ & $1.5 \%$ \\
\hline 1986 & 73,174 & FHWA, MF-33e & interpolated & $91.0 \%$ & $7.6 \%$ & $1.4 \%$ \\
\hline 1987 & 73,308 & FHWA, MF-33e & interpolated & $92.4 \%$ & $6.3 \%$ & $1.3 \%$ \\
\hline 1988 & 73,345 & FHWA, MF-33e & 1988 RTECS & $91.4 \%$ & $7.4 \%$ & $1.2 \%$ \\
\hline 1989 & 73,913 & FHWA, MF-33e & interpolated & $92.6 \%$ & $6.2 \%$ & $1.2 \%$ \\
\hline 1990 & 69,568 & FHWA, MF-33e & interpolated & $92.0 \%$ & $6.8 \%$ & $1.2 \%$ \\
\hline 1991 & 64,318 & FHWA, MF-33e & 1991 RTECS & $90.8 \%$ & $8.0 \%$ & $1.2 \%$ \\
\hline 1992 & 65,436 & FHWA, MF-33e & interpolated & $90.8 \%$ & $7.9 \%$ & $1.2 \%$ \\
\hline 1993 & 67,047 & FHWA, MF-33e & interpolated & $89.7 \%$ & $9.1 \%$ & $1.3 \%$ \\
\hline 1994 & 67,874 & FHWA, MF-33e & 1994 RTECS & $89.1 \%$ & $9.6 \%$ & $1.3 \%$ \\
\hline 1995 & 68,072 & FHWA, MF-33e & interpolated & $87.6 \%$ & $11.2 \%$ & $1.2 \%$ \\
\hline 1996 & 69,221 & FHWA, MF-33e & interpolated & $88.8 \%$ & $10.1 \%$ & $1.0 \%$ \\
\hline 1997 & 69,892 & FHWA, MF-33e & interpolated & $86.9 \%$ & $12.2 \%$ & $0.9 \%$ \\
\hline 1998 & 71,695 & FHWA, MF-33e & interpolated & $88.0 \%$ & $11.2 \%$ & $0.8 \%$ \\
\hline 1999 & 73,283 & FHWA, MF-33e & interpolated & $88.3 \%$ & $11.0 \%$ & $0.6 \%$ \\
\hline 2000 & 73,065 & FHWA, MF-33e & 2000 NVPP & $86.9 \%$ & $12.6 \%$ & $0.5 \%$ \\
\hline 2001 & 73,559 & FHWA, MF-33e & 2001 NVPP & $86.5 \%$ & $13.0 \%$ & $0.5 \%$ \\
\hline 2002 & 75,471 & FHWA, MF-33e & 2001 NVPP & $83.9 \%$ & $15.6 \%$ & $0.5 \%$ \\
\hline 2003 & 74,590 & FHWA, MF-33e & 2001 NVPP & $75.3 \%$ & $24.2 \%$ & $0.5 \%$ \\
\hline 2004 & 75,402 & FHWA, MF-33e & 2001 NVPP & $67.2 \%$ & $32.3 \%$ & $0.5 \%$ \\
\hline 2005 & 77,418 & FHWA, MF-33e & 2001 NVPP & $66.9 \%$ & $32.6 \%$ & $0.5 \%$ \\
\hline 2006 & 75,009 & EIA, C1 & 2001 NVPP & $78.2 \%$ & $21.3 \%$ & $0.5 \%$ \\
\hline 2007 & 74,377 & EIA, C1 & 2001 NVPP & $72.9 \%$ & $26.6 \%$ & $0.5 \%$ \\
\hline 2008 & 71,497 & EIA, C1 & 2001 NVPP & $72.0 \%$ & $27.5 \%$ & $0.5 \%$ \\
\hline & \multicolumn{3}{|c|}{ Heat content used for conversion to btu: } & $\begin{array}{c}125,000 \\
\text { btu/gallon }\end{array}$ & $\begin{array}{c}120,900 \\
\text { btu/gallon }\end{array}$ & $\begin{array}{c}138,700 \\
\text { btu/gallon }\end{array}$ \\
\hline
\end{tabular}


Motorcycles

DOT, FHWA, Highway Statistics 2008, Table VM-1, and annual editions.

Table A.2

Motorcycle Fuel Use

\begin{tabular}{cccc}
\hline Year & $\begin{array}{c}\text { Fuel use } \\
\text { (thousand gallons) }\end{array}$ & Year & $\begin{array}{c}\text { Fuel use } \\
\text { (thousand gallons) }\end{array}$ \\
\hline 1970 & 59,580 & 1990 & 191,140 \\
1971 & 72,140 & 1991 & 183,560 \\
1972 & 86,620 & 1992 & 191,140 \\
1973 & 103,880 & 1993 & 198,120 \\
1974 & 108,900 & 1994 & 204,800 \\
1975 & 112,580 & 1995 & 198,262 \\
1976 & 120,060 & 1996 & 195,940 \\
1977 & 126,980 & 1997 & 201,620 \\
1978 & 143,160 & 1998 & 205,660 \\
1979 & 172,740 & 1999 & 211,680 \\
1980 & 204,280 & 2000 & 209,380 \\
1981 & 213,800 & 2001 & 192,780 \\
1982 & 198,200 & 2002 & 191,040 \\
1983 & 175,200 & 2003 & 190,780 \\
1984 & 175,680 & 2004 & 202,447 \\
1985 & 181,720 & 2005 & 189,495 \\
1986 & 187,940 & 2006 & 221,030 \\
1987 & 190,120 & 2007 & 242,241 \\
1988 & 200,480 & 2008 & 256,358 \\
1989 & 207,420 & & \\
\hline \multicolumn{4}{r}{ Heat content used for conversion to btu: } \\
\hline
\end{tabular}

\section{Buses}

Transit:

APTA, 2010 Public Transportation Fact Book, Washington, DC, 2010. Includes motorbus and trolley bus data.

Table A.3

Transit Bus Fuel Use

\begin{tabular}{|c|c|c|c|c|c|c|c|c|}
\hline Year & $\begin{array}{l}\text { LNG } \\
\text { (million } \\
\text { gallons) }\end{array}$ & $\begin{array}{c}\text { LPG } \\
\text { (million } \\
\text { gallons) }\end{array}$ & $\begin{array}{l}\text { CNG } \\
\text { (million } \\
\text { gallons) }\end{array}$ & $\begin{array}{l}\text { Gasoline } \\
\text { (million } \\
\text { gallons) }\end{array}$ & $\begin{array}{c}\text { Diesel } \\
\text { fuel } \\
\text { (million } \\
\text { gallons) }\end{array}$ & $\begin{array}{c}\text { Electricity } \\
\text { (thousand } \\
\text { kilowatt } \\
\text { hours) }\end{array}$ & $\begin{array}{l}\text { Biodiesel } \\
\text { (million } \\
\text { gallons) } \\
\end{array}$ & $\begin{array}{l}\text { Methanol } \\
\text { (million } \\
\text { gallons) }\end{array}$ \\
\hline 1994 & 1.1 & 0.2 & 3.1 & 2.1 & 565.1 & 102.9 & & 12.5 \\
\hline 1995 & 1.7 & 0.3 & 10.0 & 2.3 & 563.8 & 100.0 & & 12.0 \\
\hline 1996 & 2.3 & 0.6 & 11.5 & 1.8 & 577.7 & 69.0 & & 11.6 \\
\hline 1997 & 3.3 & 1.0 & 20.0 & 2.7 & 597.6 & 78.0 & & 8.7 \\
\hline 1998 & 3.1 & 0.9 & 32.6 & 2.0 & 606.6 & 74.0 & & 5.0 \\
\hline 1999 & 5.3 & 0.8 & 39.9 & 1.4 & 618.0 & 75.0 & & 2.7 \\
\hline 2000 & 10.5 & 0.7 & 50.4 & 1.3 & 635.2 & 77.0 & & 0.8 \\
\hline 2001 & 11.7 & 1.2 & 60.9 & 1.5 & 587.2 & 74.0 & & 0.8 \\
\hline 2002 & 16.8 & 1.8 & 77.8 & 1.3 & 559.0 & 73.0 & & 1.8 \\
\hline 2003 & 14.2 & 1.8 & 94.9 & 1.1 & 536.0 & 69.0 & & 1.9 \\
\hline 2004 & 16.5 & 1.7 & 106.7 & 1.8 & 550.5 & 68.0 & & 4.7 \\
\hline 2005 & 18.3 & 2.0 & 117.2 & 1.0 & 533.8 & 67.0 & & 8.1 \\
\hline 2006 & 19.6 & 1.6 & 138.8 & 2.3 & 536.7 & 62.0 & 20.5 & 0.9 \\
\hline 2007 & 18.3 & a & 129.1 & 2.5 & 494.1 & 61.0 & 25.8 & 1.3 \\
\hline 2008 & 17.9 & a & 135.5 & 3.8 & 493.3 & 62.2 & 41.8 & 0.9 \\
\hline $\begin{array}{l}\text { Heat content used } \\
\text { for conversion } \\
\text { to btu: }\end{array}$ & $\begin{array}{r}84,800 \\
\text { btu/gallon }\end{array}$ & $\begin{array}{r}91,300 \\
\text { btu/gallon }\end{array}$ & $\begin{array}{r}138,700 \\
\text { btu/gallon }\end{array}$ & $\begin{array}{r}125,000 \\
\text { btu/gallon }\end{array}$ & $\begin{array}{r}138,700 \\
\text { btu/gallon }\end{array}$ & $\begin{array}{r}64,600 \\
\text { btu/gallon }\end{array}$ & & $\begin{array}{r}10,339 \\
\text { but/kWhr }\end{array}$ \\
\hline
\end{tabular}

Note: $\mathrm{CNG}$ is reported in diesel-gallon equivalents.

${ }^{\mathrm{a}}$ Data are not available. 
Intercity and School:

Eno Transportation Foundation, Transportation in America, 2001, Nineteenth Edition, 2003, Washington, DC, pp. 20-23. School bus fuel was assumed to be $90 \%$ diesel fuel and $10 \%$ gasoline based on estimates from the National Association of State Directors of Pupil Transportation Services. Intercity bus fuel was assumed to be $100 \%$ diesel.

\section{Table A.4}

Intercity and School Bus Fuel Use

\begin{tabular}{|c|c|c|}
\hline Year & $\begin{array}{c}\text { Intercity } \\
\text { (million gallons) }\end{array}$ & $\begin{array}{c}\text { School } \\
\text { (million gallons) }\end{array}$ \\
\hline 1970 & 305.34 & 299.88 \\
\hline 1971 & 296.73 & 309.75 \\
\hline 1972 & 288.12 & 319.62 \\
\hline 1973 & 252.42 & 327.04 \\
\hline 1974 & 216.72 & 334.46 \\
\hline 1975 & 181.02 & 341.88 \\
\hline 1976 & 182.28 & 389.76 \\
\hline 1977 & 181.86 & 401.52 \\
\hline 1978 & 180.18 & 406.98 \\
\hline 1979 & 205.38 & 404.88 \\
\hline 1980 & 213.78 & 379.68 \\
\hline 1981 & 205.38 & 386.82 \\
\hline 1982 & 227.22 & 398.58 \\
\hline 1983 & 237.30 & 400.68 \\
\hline 1984 & 169.26 & 375.06 \\
\hline 1985 & 165.48 & 425.04 \\
\hline 1986 & 148.68 & 462.42 \\
\hline 1987 & 155.82 & 487.20 \\
\hline 1988 & 160.44 & 511.14 \\
\hline 1989 & 166.74 & 498.12 \\
\hline 1990 & 159.60 & 472.08 \\
\hline 1991 & 160.44 & 533.40 \\
\hline 1992 & 157.08 & 546.00 \\
\hline 1993 & 171.36 & 533.40 \\
\hline 1994 & 195.30 & 546.00 \\
\hline 1995 & 195.30 & 545.16 \\
\hline 1996 & 199.92 & 545.16 \\
\hline 1997 & 212.52 & 544.74 \\
\hline 1998 & 220.08 & 550.20 \\
\hline 1999 & 241.08 & 555.66 \\
\hline 2000 & 233.10 & 577.08 \\
\hline 2001 & $217.35^{*}$ & $538.08 *$ \\
\hline 2002 & $210.22 *$ & $520.44 *$ \\
\hline 2003 & $208.32 *$ & $515.72 *$ \\
\hline 2004 & $208.87 *$ & $517.09 *$ \\
\hline 2005 & $214.37 *$ & $530.70 *$ \\
\hline 2006 & $208.32 *$ & $515.72 *$ \\
\hline 2007 & $214.37 *$ & $530.70 *$ \\
\hline 2008 & 218.48 & 540.89 \\
\hline Fuel type shares & $100 \%$ diesel & $\begin{array}{c}90 \% \text { diesel } \\
10 \% \text { gasoline }\end{array}$ \\
\hline $\begin{array}{l}\text { Heat content used for } \\
\text { conversion to btu: }\end{array}$ & $\begin{array}{c}\text { 138,700 } \\
\text { btu/gallon }\end{array}$ & $\begin{array}{l}138,700 \mathrm{btu} / \text { gallon } \\
125,000 \mathrm{btu} / \text { gallon }\end{array}$ \\
\hline
\end{tabular}

*Estimated using the rate of change of bus vehicle-miles traveled from FHWA Highway Statistics, Table VM-1. 


\section{Trucks}

Light Trucks:

DOT, FHWA, Highway Statistics 2008, Table VM-1 and annual editions back to 1996; DOT, FHWA, Highway Statistics Summary to 1995. The Federal Highway Administration discontinued publication of gasohol data in 2005. Therefore, a new methodology was developed using data from EIA, Alternatives to Traditional Transportation Fuels, 2006, Table C1.

\section{Table A.5}

Light Truck Fuel Use and Fuel Type Shares for Calculation of Energy Use

\begin{tabular}{|c|c|c|c|c|c|c|c|}
\hline \multirow[b]{2}{*}{ Year } & \multirow{2}{*}{$\begin{array}{l}\text { Fuel use } \\
\text { (million } \\
\text { gallons) }\end{array}$} & \multirow{2}{*}{$\begin{array}{c}\text { Source for } \\
\text { gasohol shares }\end{array}$} & \multirow{2}{*}{$\begin{array}{c}\text { Source for } \\
\text { gasoline/diesel } \\
\text { /lpg shares }\end{array}$} & \multicolumn{4}{|c|}{ Shares by fuel type } \\
\hline & & & & Gasoline & Gasohol & Diesel & Lpg \\
\hline 1970 & 12,313 & & 1977 TIUS & $97.6 \%$ & $0.0 \%$ & $1.6 \%$ & $0.8 \%$ \\
\hline 1971 & 13,484 & & 1977 TIUS & $97.6 \%$ & $0.0 \%$ & $1.6 \%$ & $0.8 \%$ \\
\hline 1972 & 15,150 & & 1977 TIUS & $97.6 \%$ & $0.0 \%$ & $1.6 \%$ & $0.8 \%$ \\
\hline 1973 & 16,828 & & 1977 TIUS & $97.6 \%$ & $0.0 \%$ & $1.6 \%$ & $0.8 \%$ \\
\hline 1974 & 16,657 & & 1977 TIUS & $97.6 \%$ & $0.0 \%$ & $1.6 \%$ & $0.8 \%$ \\
\hline 1975 & 19,081 & & 1977 TIUS & $97.6 \%$ & $0.0 \%$ & $1.6 \%$ & $0.8 \%$ \\
\hline 1976 & 20,828 & & 1977 TIUS & $97.6 \%$ & $0.0 \%$ & $1.6 \%$ & $0.8 \%$ \\
\hline 1977 & 22,383 & & 1977 TIUS & $97.6 \%$ & $0.0 \%$ & $1.6 \%$ & $0.8 \%$ \\
\hline 1978 & 24,162 & & Interpolated & $97.1 \%$ & $0.0 \%$ & $2.0 \%$ & $0.9 \%$ \\
\hline 1979 & 24,445 & & Interpolated & $96.7 \%$ & $0.0 \%$ & $2.4 \%$ & $1.0 \%$ \\
\hline 1980 & 23,796 & FHWA, MF-33e & Interpolated & $95.7 \%$ & $0.5 \%$ & $2.7 \%$ & $1.0 \%$ \\
\hline 1981 & 23,697 & FHWA, MF-33e & Interpolated & $95.1 \%$ & $0.7 \%$ & $3.1 \%$ & $1.1 \%$ \\
\hline 1982 & 22,702 & FHWA, MF-33e & 1982 TIUS & $93.0 \%$ & $2.3 \%$ & $3.5 \%$ & $1.2 \%$ \\
\hline 1983 & 23,945 & FHWA, MF-33e & Interpolated & $91.0 \%$ & $4.3 \%$ & $3.5 \%$ & $1.2 \%$ \\
\hline 1984 & 25,604 & FHWA, MF-33e & Interpolated & $90.0 \%$ & $5.3 \%$ & $3.5 \%$ & $1.2 \%$ \\
\hline 1985 & 27,363 & FHWA, MF-33e & Interpolated & $87.6 \%$ & $7.7 \%$ & $3.5 \%$ & $1.2 \%$ \\
\hline 1986 & 29,074 & FHWA, MF-33e & Interpolated & $87.7 \%$ & $7.6 \%$ & $3.5 \%$ & $1.2 \%$ \\
\hline 1987 & 30,598 & FHWA, MF-33e & 1987 TIUS & $89.0 \%$ & $6.3 \%$ & $3.5 \%$ & $1.2 \%$ \\
\hline 1988 & 32,653 & FHWA, MF-33e & Interpolated & $88.2 \%$ & $7.4 \%$ & $3.5 \%$ & $1.0 \%$ \\
\hline 1989 & 33,271 & FHWA, MF-33e & Interpolated & $89.5 \%$ & $6.2 \%$ & $3.4 \%$ & $0.8 \%$ \\
\hline 1990 & 35,611 & FHWA, MF-33e & Interpolated & $89.2 \%$ & $6.8 \%$ & $3.4 \%$ & $0.7 \%$ \\
\hline 1991 & 38,217 & FHWA, MF-33e & Interpolated & $88.1 \%$ & $8.0 \%$ & $3.3 \%$ & $0.5 \%$ \\
\hline 1992 & 40,929 & FHWA, MF-33e & 1992 TIUS & $88.5 \%$ & $7.9 \%$ & $3.3 \%$ & $0.3 \%$ \\
\hline 1993 & 42,851 & FHWA, MF-33e & Interpolated & $87.3 \%$ & $9.1 \%$ & $3.3 \%$ & $0.3 \%$ \\
\hline 1994 & 44,112 & FHWA, MF-33e & Interpolated & $86.8 \%$ & $9.6 \%$ & $3.3 \%$ & $0.3 \%$ \\
\hline 1995 & 45,605 & FHWA, MF-33e & Interpolated & $85.1 \%$ & $11.2 \%$ & $3.4 \%$ & $0.3 \%$ \\
\hline 1996 & 47,354 & FHWA, MF-33e & Interpolated & $86.2 \%$ & $10.1 \%$ & $3.4 \%$ & $0.3 \%$ \\
\hline 1997 & 49,388 & FHWA, MF-33e & 1997 VIUS & $84.2 \%$ & $12.2 \%$ & $3.4 \%$ & $0.2 \%$ \\
\hline 1998 & 50,462 & FHWA, MF-33e & Interpolated & $85.0 \%$ & $11.2 \%$ & $3.5 \%$ & $0.3 \%$ \\
\hline 1999 & 52,859 & FHWA, MF-33e & Interpolated & $84.9 \%$ & $11.0 \%$ & $3.6 \%$ & $0.4 \%$ \\
\hline 2000 & 52,939 & FHWA, MF-33e & Interpolated & $83.1 \%$ & $12.6 \%$ & $3.8 \%$ & $0.6 \%$ \\
\hline 2001 & 53,522 & FHWA, MF-33e & Interpolated & $82.4 \%$ & $13.0 \%$ & $3.9 \%$ & $0.7 \%$ \\
\hline 2002 & 55,220 & FHWA, MF-33e & 2002 VIUS & $79.6 \%$ & $15.6 \%$ & $4.0 \%$ & $0.8 \%$ \\
\hline 2003 & 60,758 & FHWA, MF-33e & 2002 VIUS & $71.0 \%$ & $24.2 \%$ & $4.0 \%$ & $0.8 \%$ \\
\hline 2004 & 63,417 & FHWA, MF-33e & 2002 VIUS & $62.9 \%$ & $32.3 \%$ & $4.0 \%$ & $0.8 \%$ \\
\hline 2005 & 58,869 & FHWA, MF-33e & 2002 VIUS & $62.6 \%$ & $32.6 \%$ & $4.0 \%$ & $0.8 \%$ \\
\hline 2006 & 60,685 & EIA, C1 & 2002 VIUS & $73.9 \%$ & $21.3 \%$ & $4.0 \%$ & $0.8 \%$ \\
\hline 2007 & 61,836 & EIA, C1 & 2002 VIUS & $68.6 \%$ & $26.6 \%$ & $4.0 \%$ & $0.8 \%$ \\
\hline 2008 & 61,199 & EIA, C1 & 2002 VIUS & $57.5 \%$ & $37.7 \%$ & $4.0 \%$ & $0.8 \%$ \\
\hline \multicolumn{4}{|c|}{ Heat content used for conversion to btu: } & $\begin{array}{c}125,000 \\
\text { btu/gallon }\end{array}$ & $\begin{array}{c}120,900 \\
\text { btu/gallon }\end{array}$ & $\begin{array}{c}138,700 \\
\text { btu/gallon }\end{array}$ & $\begin{array}{c}90,800 \\
\text { btu/gallon }\end{array}$ \\
\hline
\end{tabular}


Medium/Heavy Trucks:

DOT, FHWA, Highway Statistics 2008, Table VM-1 and annual editions back to 1996; DOT, FHWA, Highway Statistics Summary to 1995. Total gallons for other trucks was the difference between total trucks and 2-axle, 4-tire trucks.

Table A.6

Medium/Heavy Truck Fuel Use and Fuel Type Shares for Calculation of Energy Use

\begin{tabular}{|c|c|c|c|c|c|}
\hline \multirow[b]{2}{*}{ Year } & \multirow{2}{*}{$\begin{array}{c}\text { Fuel use } \\
\text { (million gallons) } \\
\end{array}$} & \multirow{2}{*}{$\begin{array}{c}\text { Source for } \\
\text { gasoline/diesel/lpg } \\
\text { shares }\end{array}$} & \multicolumn{3}{|c|}{ Shares by fuel type } \\
\hline & & & Gasoline & Diesel & Lpg \\
\hline 1970 & 11,316 & 1977 TIUS & $10.4 \%$ & $89.5 \%$ & $0.1 \%$ \\
\hline 1971 & 11,812 & 1977 TIUS & $10.4 \%$ & $89.5 \%$ & $0.1 \%$ \\
\hline 1972 & 12,964 & 1977 TIUS & $10.4 \%$ & $89.5 \%$ & $0.1 \%$ \\
\hline 1973 & 14,320 & 1977 TIUS & $10.4 \%$ & $89.5 \%$ & $0.1 \%$ \\
\hline 1974 & 14,341 & 1977 TIUS & $10.4 \%$ & $89.5 \%$ & $0.1 \%$ \\
\hline 1975 & 14,598 & 1977 TIUS & $10.4 \%$ & $89.5 \%$ & $0.1 \%$ \\
\hline 1976 & 15,408 & 1977 TIUS & $10.4 \%$ & $89.5 \%$ & $0.1 \%$ \\
\hline 1977 & 17,082 & 1977 TIUS & $10.4 \%$ & $89.5 \%$ & $0.1 \%$ \\
\hline 1978 & 19,121 & Interpolated & $16.2 \%$ & $83.5 \%$ & $0.3 \%$ \\
\hline 1979 & 19,913 & Interpolated & $22.1 \%$ & $77.5 \%$ & $0.5 \%$ \\
\hline 1980 & 19,960 & Interpolated & $27.9 \%$ & $71.4 \%$ & $0.6 \%$ \\
\hline 1981 & 20,376 & Interpolated & $33.8 \%$ & $65.4 \%$ & $0.8 \%$ \\
\hline 1982 & 20,386 & 1982 TIUS & $39.6 \%$ & $59.4 \%$ & $1.0 \%$ \\
\hline 1983 & 20,761 & Interpolated & $35.6 \%$ & $63.6 \%$ & $0.8 \%$ \\
\hline 1984 & 21,428 & Interpolated & $31.5 \%$ & $67.8 \%$ & $0.7 \%$ \\
\hline 1985 & 21,405 & Interpolated & $27.5 \%$ & $72.0 \%$ & $0.5 \%$ \\
\hline 1986 & 21,861 & Interpolated & $23.4 \%$ & $76.2 \%$ & $0.4 \%$ \\
\hline 1987 & 22,513 & 1987 TIUS & $19.4 \%$ & $80.4 \%$ & $0.2 \%$ \\
\hline 1988 & 22,925 & Interpolated & $18.8 \%$ & $81.0 \%$ & $0.3 \%$ \\
\hline 1989 & 23,512 & Interpolated & $18.1 \%$ & $81.6 \%$ & $0.3 \%$ \\
\hline 1990 & 24,490 & Interpolated & $17.5 \%$ & $82.1 \%$ & $0.4 \%$ \\
\hline 1991 & 24,981 & Interpolated & $16.8 \%$ & $82.7 \%$ & $0.4 \%$ \\
\hline 1992 & 25,453 & 1992 TIUS & $16.2 \%$ & $83.3 \%$ & $0.5 \%$ \\
\hline 1993 & 26,236 & Interpolated & $15.4 \%$ & $84.1 \%$ & $0.5 \%$ \\
\hline 1994 & 27,685 & Interpolated & $14.7 \%$ & $84.8 \%$ & $0.5 \%$ \\
\hline 1995 & 28,828 & Interpolated & $13.9 \%$ & $85.6 \%$ & $0.5 \%$ \\
\hline 1996 & 29,601 & Interpolated & $13.2 \%$ & $86.3 \%$ & $0.5 \%$ \\
\hline 1997 & 29,878 & 1997 VIUS & $12.4 \%$ & $87.1 \%$ & $0.5 \%$ \\
\hline 1998 & 30,841 & Interpolated & $12.1 \%$ & $87.4 \%$ & $0.5 \%$ \\
\hline 1999 & 33,909 & Interpolated & $11.8 \%$ & $87.6 \%$ & $0.5 \%$ \\
\hline 2000 & 35,229 & Interpolated & $11.6 \%$ & $87.9 \%$ & $0.5 \%$ \\
\hline 2001 & 35,179 & Interpolated & $11.3 \%$ & $88.1 \%$ & $0.5 \%$ \\
\hline 2002 & 36,800 & 2002 VIUS & $11.0 \%$ & $88.4 \%$ & $0.5 \%$ \\
\hline 2003 & 35,775 & 2002 VIUS & $11.0 \%$ & $88.4 \%$ & $0.5 \%$ \\
\hline 2004 & 33,150 & 2002 VIUS & $11.0 \%$ & $88.4 \%$ & $0.5 \%$ \\
\hline 2005 & 37,190 & 2002 VIUS & $11.0 \%$ & $88.4 \%$ & $0.5 \%$ \\
\hline 2006 & 37,959 & 2002 VIUS & $11.0 \%$ & $88.4 \%$ & $0.5 \%$ \\
\hline 2007 & 38,589 & 2002 VIUS & $11.0 \%$ & $88.4 \%$ & $0.5 \%$ \\
\hline 2008 & 36,703 & 2002 VIUS & $11.0 \%$ & $88.4 \%$ & $0.5 \%$ \\
\hline \multicolumn{3}{|c|}{ Heat content used for conversion to btu: } & $\begin{array}{c}125,000 \\
\text { btu/gallon }\end{array}$ & $\begin{array}{c}138,700 \\
\text { btu/gallon }\end{array}$ & $\begin{array}{c}90,800 \\
\text { btu/gallon }\end{array}$ \\
\hline
\end{tabular}




\section{Off-highway energy use}

U.S. Environmental Protection Agency, NONROAD2008a model, results generated April 2010. Gallons of fuel by fuel type were produced for agricultural equipment, airport equipment, construction and mining equipment, industrial equipment, lawn and garden equipment, logging equipment, railroad maintenance equipment, and recreational equipment. Some non-transportation-related equipment, such as generators, chain saws, compressors, and pumps, were excluded from the data. 


\section{Nonhighway energy use}

Air

General Aviation:

DOT, FAA, General Aviation Activity and Avionics Survey: Annual Summary Report Calendar Year 2008, Table 5.1, and annual.

Table A.7

General Aviation Fuel Use

\begin{tabular}{|c|c|c|}
\hline Year & $\begin{array}{c}\text { Jet fuel } \\
\text { (million gallons) }\end{array}$ & $\begin{array}{l}\text { Aviation gasoline } \\
\text { (million gallons) }\end{array}$ \\
\hline 1970 & 208.0 & 551.0 \\
\hline 1971 & 226.0 & 508.0 \\
\hline 1972 & 245.0 & 584.0 \\
\hline 1973 & 304.0 & 411.0 \\
\hline 1974 & 357.0 & 443.0 \\
\hline 1975 & 453.0 & 412.0 \\
\hline 1976 & 495.0 & 432.0 \\
\hline 1977 & 536.0 & 456.0 \\
\hline 1978 & 763.0 & 518.0 \\
\hline 1979 & 736.0 & 570.0 \\
\hline 1980 & 766.0 & 520.0 \\
\hline 1981 & 759.0 & 489.0 \\
\hline 1982 & 887.0 & 448.0 \\
\hline 1983 & 613.0 & 428.0 \\
\hline 1984 & 738.9 & 462.4 \\
\hline 1985 & 691.0 & 421.0 \\
\hline 1986 & 732.1 & 408.6 \\
\hline 1987 & 672.7 & 401.8 \\
\hline 1988 & 746.0 & 398.0 \\
\hline 1989 & 688.0 & 342.8 \\
\hline 1990 & 662.0 & 353.0 \\
\hline 1991 & 579.0 & 348.0 \\
\hline 1992 & 496.0 & 306.0 \\
\hline 1993 & 454.1 & 268.4 \\
\hline 1994 & 470.8 & 264.1 \\
\hline 1995 & 544.0 & 276.0 \\
\hline 1996 & 567.5 & 286.5 \\
\hline 1997 & 639.4 & 289.7 \\
\hline 1998 & 814.6 & 311.4 \\
\hline 1999 & 967.2 & 345.4 \\
\hline 2000 & 998.1 & 336.3 \\
\hline 2001 & 938.7 & 319.3 \\
\hline 2002 & 815.5 & 261.4 \\
\hline 2003 & 820.0 & 255.5 \\
\hline 2004 & $1,075.2$ & 256.1 \\
\hline 2005 & $1,507.4$ & 323.6 \\
\hline 2006 & $1,636.3$ & 294.7 \\
\hline 2007 & $1,516.3$ & 314.8 \\
\hline 2008 & $1,688.6$ & 306.3 \\
\hline $\begin{array}{l}\text { Heat content used for } \\
\text { conversion to btu: }\end{array}$ & $135,000 \mathrm{btu} /$ gallon & $\begin{array}{c}\text { 120,200 } \\
\text { btu/gallon }\end{array}$ \\
\hline
\end{tabular}




\section{Domestic and International Air Carrier:}

DOT, Bureau of Transportation Statistics, "Fuel Cost and Consumption Tables," www.bts.gov/xml/fuel/report/src/index.xml. The table below shows all international fuel use. Because the data for international include fuel purchased abroad, for the tables in Chapter 2, the international total was divided in half to estimate domestic fuel use for international flights.

Table A.8

Air Carrier Fuel Use

\begin{tabular}{|c|c|c|c|}
\hline Year & $\begin{array}{c}\text { Domestic } \\
\text { (thousand gallons) }\end{array}$ & $\begin{array}{c}\text { International } \\
\text { (thousand gallons) }\end{array}$ & $\begin{array}{c}\text { Total } \\
\text { (thousand gallons) }\end{array}$ \\
\hline 1970 & \multirow{7}{*}{\multicolumn{2}{|c|}{$\begin{array}{l}\text { Separate estimates for domestic } \\
\text { and international are not available } \\
\text { from 1970-1976. }\end{array}$}} & $10,085,000$ \\
\hline 1971 & & & $10,140,000$ \\
\hline 1972 & & & $10,302,000$ \\
\hline 1973 & & & $10,671,000$ \\
\hline 1974 & & & $10,417,260$ \\
\hline 1975 & & & $10,412,640$ \\
\hline 1976 & & & $10,400,040$ \\
\hline 1977 & $8,202,051$ & $1,708,376$ & $9,910,427$ \\
\hline 1978 & $8,446,117$ & $1,741,918$ & $10,188,035$ \\
\hline 1979 & $8,865,885$ & $1,828,435$ & $10,694,320$ \\
\hline 1980 & $8,519,233$ & $1,747,306$ & $10,266,539$ \\
\hline 1981 & $8,555,249$ & $2,032,520$ & $10,587,769$ \\
\hline 1982 & $8,432,465$ & $1,967,733$ & $10,400,198$ \\
\hline 1983 & $8,672,574$ & $1,998,289$ & $10,670,863$ \\
\hline 1984 & $9,625,958$ & $2,286,407$ & $11,912,365$ \\
\hline 1985 & $10,115,007$ & $2,487,929$ & $12,602,936$ \\
\hline 1986 & $11,137,331$ & $2,544,996$ & $13,682,327$ \\
\hline 1987 & $11,586,838$ & $2,893,617$ & $14,480,455$ \\
\hline 1988 & $11,917,904$ & $3,262,824$ & $15,180,728$ \\
\hline 1989 & $11,905,144$ & $3,557,294$ & $15,462,438$ \\
\hline 1990 & $12,429,305$ & $3,963,081$ & $16,392,386$ \\
\hline 1991 & $11,506,477$ & $3,939,666$ & $15,446,144$ \\
\hline 1992 & $11,762,852$ & $4,120,132$ & $15,882,983$ \\
\hline 1993 & $11,958,663$ & $4,113,321$ & $16,071,984$ \\
\hline 1994 & $12,475,549$ & $4,310,879$ & $16,786,428$ \\
\hline 1995 & $12,811,717$ & $4,511,418$ & $17,323,135$ \\
\hline 1996 & $13,187,305$ & $4,658,093$ & $17,845,398$ \\
\hline 1997 & $13,659,581$ & $4,964,181$ & $18,623,762$ \\
\hline 1998 & $13,876,971$ & $5,185,562$ & $19,062,533$ \\
\hline 1999 & $14,402,127$ & $5,250,492$ & $19,652,619$ \\
\hline 2000 & $14,844,592$ & $5,474,685$ & $20,319,277$ \\
\hline 2001 & $14,017,461$ & $5,237,487$ & $19,254,948$ \\
\hline 2002 & $12,848,329$ & $4,990,798$ & $17,839,127$ \\
\hline 2003 & $12,958,581$ & $4,836,356$ & $17,794,936$ \\
\hline 2004 & $13,622,603$ & $4,931,546$ & $18,554,149$ \\
\hline 2005 & $13,778,869$ & $5,520,889$ & $19,309,758$ \\
\hline 2006 & $13,694,437$ & $6,017,638$ & $19,712,075$ \\
\hline 2007 & $13,681,664$ & $6,204,502$ & $19,886,165$ \\
\hline 2008 & $12,666,911$ & $6,186,747$ & $18,853,658$ \\
\hline $\begin{array}{l}\text { Heat content used for } \\
\text { conversion to btu: }\end{array}$ & $\begin{array}{c}135,000 \\
\text { btu/gallon }\end{array}$ & $\begin{array}{c}135,000 \\
\text { btu/gallon }\end{array}$ & $\begin{array}{c}135,000 \\
\text { btu/gallon }\end{array}$ \\
\hline
\end{tabular}




\section{Water}

Freight:

Total - DOE, EIA, Fuel Oil and Kerosene Sales 2008, Table 13. Adjusted sales of distillate and residual fuel oil for vessel bunkering. (This may include some amounts of bunker fuels used for recreational purposes.)

Table A.9

Diesel and Residual Fuel Oil for Vessel Bunkering

\begin{tabular}{|c|c|c|}
\hline Year & $\begin{array}{l}\text { Distillate fuel oil } \\
\text { (thousand gallons) }\end{array}$ & $\begin{array}{l}\text { Residual fuel oil } \\
\text { (thousand gallons) }\end{array}$ \\
\hline 1970 & 819,000 & $3,774,120$ \\
\hline 1971 & 880,000 & $3,307,000$ \\
\hline 1972 & $1,013,000$ & $3,273,000$ \\
\hline 1973 & $1,125,000$ & $3,859,000$ \\
\hline 1974 & $1,018,920$ & $3,827,040$ \\
\hline 1975 & $1,097,880$ & $4,060,140$ \\
\hline 1976 & $1,220,100$ & $4,977,000$ \\
\hline 1977 & $1,407,420$ & $5,416,740$ \\
\hline 1978 & $1,578,822$ & $6,614,790$ \\
\hline 1979 & $1,630,858$ & $8,002,672$ \\
\hline 1980 & 717,376 & $7,454,242$ \\
\hline 1981 & $1,723,143$ & $7,922,512$ \\
\hline 1982 & $1,423,216$ & $6,408,818$ \\
\hline 1983 & $1,418,890$ & $5,724,115$ \\
\hline 1984 & $1,692,045$ & $5,688,931$ \\
\hline 1985 & $1,894,265$ & $5,269,733$ \\
\hline 1986 & $2,034,215$ & $5,690,250$ \\
\hline 1987 & $2,223,258$ & $5,869,154$ \\
\hline 1988 & $2,310,367$ & $6,025,511$ \\
\hline 1989 & $2,356,444$ & $6,621,100$ \\
\hline 1990 & $2,197,004$ & $6,248,095$ \\
\hline 1991 & $2,167,640$ & $6,786,055$ \\
\hline 1992 & $2,240,170$ & $7,199,078$ \\
\hline 1993 & $2,043,745$ & $6,269,882$ \\
\hline 1994 & $2,026,899$ & $5,944,383$ \\
\hline 1995 & $1,978,105$ & $6,431,238$ \\
\hline 1996 & $2,177,608$ & $5,804,977$ \\
\hline 1997 & $2,107,561$ & $4,789,861$ \\
\hline 1998 & $2,125,568$ & $4,640,153$ \\
\hline 1999 & $2,064,590$ & $5,598,630$ \\
\hline 2000 & $2,041,433$ & $6,192,294$ \\
\hline 2001 & $2,099,011$ & $4,345,284$ \\
\hline 2002 & $2,056,465$ & $4,783,956$ \\
\hline 2003 & $1,863,150$ & $3,801,425$ \\
\hline 2004 & $2,313,448$ & $4,886,978$ \\
\hline 2005 & $2,115,381$ & $5,533,552$ \\
\hline 2006 & $2,206,690$ & $6,000,434$ \\
\hline 2007 & $2,158,930$ & $6,773,950$ \\
\hline 2008 & $1,339,546$ & $5,315,722$ \\
\hline $\begin{array}{l}\text { Heat content used for } \\
\text { conversion to btu: }\end{array}$ & $\begin{array}{c}138,700 \\
\text { btu/gallon }\end{array}$ & $\begin{array}{c}149,700 \\
\text { btu/gallon }\end{array}$ \\
\hline $\begin{array}{l}\text { Domestic share of total } \\
\text { fuel use }\end{array}$ & $77.5 \%$ & $9.3 \%$ \\
\hline
\end{tabular}




\section{Recreational Boating:}

Fuel use by recreational boating comes from the EPA's NONROAD2005 model.

Table A.10

Recreational Boating Fuel Use

\begin{tabular}{|c|c|c|}
\hline Year & $\begin{array}{l}\text { Diesel use } \\
\text { (gallons) }\end{array}$ & $\begin{array}{c}\text { Gasoline use } \\
\text { (gallons) }\end{array}$ \\
\hline 1970 & $39,589,953$ & $1,213,397,311$ \\
\hline 1971 & $47,130,906$ & $1,220,995,448$ \\
\hline 1972 & $54,671,856$ & $1,228,593,572$ \\
\hline 1973 & $62,212,803$ & $1,236,191,635$ \\
\hline 1974 & $69,753,735$ & $1,243,789,752$ \\
\hline 1975 & $77,294,680$ & $1,251,387,972$ \\
\hline 1976 & $84,835,632$ & $1,258,986,070$ \\
\hline 1977 & $92,376,573$ & $1,266,584,111$ \\
\hline 1978 & $99,917,523$ & $1,274,182,341$ \\
\hline 1979 & $107,458,470$ & $1,281,780,460$ \\
\hline 1980 & $114,999,421$ & $1,289,378,532$ \\
\hline 1981 & $122,540,357$ & $1,296,976,672$ \\
\hline 1982 & $130,081,302$ & $1,304,574,832$ \\
\hline 1983 & $137,622,248$ & $1,312,172,890$ \\
\hline 1984 & $145,163,202$ & $1,319,771,007$ \\
\hline 1985 & $152,704,140$ & $1,327,369,146$ \\
\hline 1986 & $160,245,074$ & $1,334,967,322$ \\
\hline 1987 & $167,786,030$ & $1,342,565,455$ \\
\hline 1988 & $175,326,970$ & $1,362,856,034$ \\
\hline 1989 & $182,867,916$ & $1,383,146,636$ \\
\hline 1990 & $190,408,869$ & $1,403,437,194$ \\
\hline 1991 & $197,949,808$ & $1,429,688,292$ \\
\hline 1992 & $205,490,749$ & $1,455,939,504$ \\
\hline 1993 & $213,031,707$ & $1,482,190,597$ \\
\hline 1994 & $220,572,649$ & $1,539,794,180$ \\
\hline 1995 & $228,113,596$ & $1,597,269,921$ \\
\hline 1996 & $235,654,521$ & $1,654,446,069$ \\
\hline 1997 & $243,195,481$ & $1,657,737,628$ \\
\hline 1998 & $250,736,414$ & $1,659,056,085$ \\
\hline 1999 & $258,159,525$ & $1,657,198,161$ \\
\hline 2000 & $265,582,657$ & $1,652,906,973$ \\
\hline 2001 & $273,547,835$ & $1,655,303,922$ \\
\hline 2002 & $281,512,965$ & $1,653,583,696$ \\
\hline 2003 & $289,478,093$ & $1,648,070,959$ \\
\hline 2004 & $297,443,197$ & $1,639,713,127$ \\
\hline 2005 & $305,408,463$ & $1,629,873,278$ \\
\hline 2006 & $313,420,594$ & $1,619,603,593$ \\
\hline 2007 & $321,432,801$ & $1,609,567,873$ \\
\hline 2008 & $329,445,068$ & $1,599,830,522$ \\
\hline $\begin{array}{l}\text { Heat content used for } \\
\text { conversion to btu: }\end{array}$ & $\begin{array}{c}138,700 \\
\text { btu/gallon }\end{array}$ & $\begin{array}{c}125,000 \\
\text { btu/gallon }\end{array}$ \\
\hline
\end{tabular}




\section{Pipeline}

The sum of natural gas, crude petroleum and petroleum product, and coal slurry and water.

\section{Natural Gas:}

The amount of natural gas used to transport natural gas was defined as "pipeline fuel" as reported in DOE, EIA, Natural Gas Annual 2008, Table 1. Cubic feet were converted to Btu using 1,031 Btu/ft3. Electricity use was estimated using the following procedure as reported on p. 5-110 of J. N. Hooker et al., End Use Energy Consumption DataBase: Transportation Sector. The energy consumption of a natural gas pipeline was taken to be the energy content of the fuel used to drive the pumps. Some $94 \%$ of the installed pumping horsepower was supplied by natural gas. The remaining $6 \%$ of the horsepower was generated more efficiently, mostly by electric motors. The energy consumed by natural gas pipeline pumps that were electrically powered was not known. In order to estimate the electricity consumed, the Btu of natural gas pipeline fuel consumed was multiplied by a factor of 0.015 . From this computed value, electricity efficiency and generation loss must be taken into account. The electricity energy use in Btu must be converted to $\mathrm{kWhr}$, using the conversion factor $29.305 \times 10-5 \mathrm{kWhr} /$ Btu. Electricity generation and distribution efficiency was $29 \%$. When generation and distribution efficiency are taken into account, $1 \mathrm{kWhr}$ equals 10,339 Btu.

\section{Crude petroleum and petroleum product:}

J. N. Hooker, Oil Pipeline Energy Consumption and Efficiency, ORNL-5697, ORNL, Oak Ridge, TN, 1981. (Data held constant; Latest available data.)

\section{Coal slurry and water:}

W. F. Banks, Systems, Science and Software, Energy Consumption in the Pipeline Industry, LaJolla, CA, October 1977. (Data held constant; Latest available data.) 
A-16

Table A.11

Pipeline Fuel Use

\begin{tabular}{|c|c|c|c|}
\hline Year & $\begin{array}{l}\text { Natural gas } \\
\text { (million cubic } \\
\text { feet) }\end{array}$ & $\begin{array}{l}\text { Estimated natural } \\
\text { gas pipeline } \\
\text { electricity use } \\
\text { (million kWhr) }\end{array}$ & $\begin{array}{c}\text { Electricity } \\
\text { constant (btu) }\end{array}$ \\
\hline 1970 & 722,166 & $3,272.9$ & 212.1 \\
\hline 1971 & 742,592 & $3,365.4$ & 212.1 \\
\hline 1972 & 766,156 & $3,472.2$ & 212.1 \\
\hline 1973 & 728,177 & $3,300.1$ & 212.1 \\
\hline 1974 & 668,792 & $3,031.0$ & 212.1 \\
\hline 1975 & 582,963 & $2,642.0$ & 212.1 \\
\hline 1976 & 548,323 & $2,485.0$ & 212.1 \\
\hline 1977 & 532,669 & $2,414.1$ & 212.1 \\
\hline 1978 & 530,451 & $2,404.0$ & 212.1 \\
\hline 1979 & 600,964 & $2,723.6$ & 212.1 \\
\hline 1980 & 634,622 & $2,876.1$ & 212.1 \\
\hline 1981 & 642,325 & $2,911.0$ & 212.1 \\
\hline 1982 & 596,411 & $2,703.0$ & 212.1 \\
\hline 1983 & 490,042 & $2,220.9$ & 212.1 \\
\hline 1984 & 528,754 & $2,396.3$ & 212.1 \\
\hline 1985 & 503,766 & $2,283.1$ & 212.1 \\
\hline 1986 & 485,041 & $2,198.2$ & 212.1 \\
\hline 1987 & 519,170 & $2,352.9$ & 212.1 \\
\hline 1988 & 613,912 & $2,782.3$ & 212.1 \\
\hline 1989 & 629,308 & $2,852.0$ & 212.1 \\
\hline 1990 & 659,816 & $2,990.3$ & 212.1 \\
\hline 1991 & 601,305 & $2,725.1$ & 212.1 \\
\hline 1992 & 587,710 & $2,663.5$ & 212.1 \\
\hline 1993 & 624,308 & $2,829.4$ & 212.1 \\
\hline 1994 & 685,362 & $3,106.1$ & 212.1 \\
\hline 1995 & 700,335 & $3,173.9$ & 212.1 \\
\hline 1996 & 711,446 & $3,224.3$ & 212.1 \\
\hline 1997 & 751,470 & $3,405.7$ & 212.1 \\
\hline 1998 & 635,477 & $2,880.0$ & 212.1 \\
\hline 1999 & 645,319 & $2,924.6$ & 212.1 \\
\hline 2000 & 642,210 & $2,910.5$ & 212.1 \\
\hline 2001 & 624,964 & $2,832.3$ & 212.1 \\
\hline 2002 & 666,920 & $3,022.5$ & 212.1 \\
\hline 2003 & 591,492 & $2,680.7$ & 212.1 \\
\hline 2004 & 566,187 & $2,566.0$ & 212.1 \\
\hline 2005 & 584,026 & $2,646.8$ & 212.1 \\
\hline 2006 & 584,213 & $2,647.7$ & 212.1 \\
\hline 2007 & 621,364 & $2,816.0$ & 212.1 \\
\hline 2008 & 647,958 & $2,936.6$ & 212.1 \\
\hline $\begin{array}{l}\text { Heat content used for } \\
\text { conversion to btu: }\end{array}$ & $\begin{array}{c}1,031 \\
\text { btu/cubic foot }\end{array}$ & $\begin{array}{c}10,339 \\
\text { Btu/kWhr }\end{array}$ & \\
\hline
\end{tabular}

Note: Formula for estimating electricity use for natural gas pipelines is:

Natural gas use (in million cubic ft) $\times 1,031 \mathrm{btu} /$ cubic $\mathrm{ft} \times 0.015 \times 29.305 \times 10-5 \mathrm{kWhr} / \mathrm{btu}$ 


\section{Rail}

\section{Freight:}

AAR, Railroad Facts, 2009 Edition, Washington, DC, 2009.

Table A.12

Class I Freight Railroad Fuel Use

\begin{tabular}{cc}
\hline Year & $\begin{array}{c}\text { Diesel fuel } \\
\text { (thousand gallons) }\end{array}$ \\
\hline 1970 & $3,807,663$ \\
1971 & $3,822,907$ \\
1972 & $3,996,985$ \\
1973 & $4,160,730$ \\
1974 & $4,175,375$ \\
1975 & $3,736,484$ \\
1976 & $3,895,542$ \\
1977 & $3,985,069$ \\
1978 & $3,968,007$ \\
1979 & $4,072,187$ \\
1980 & $3,955,996$ \\
1981 & $3,756,439$ \\
1982 & $3,178,116$ \\
1983 & $3,137,295$ \\
1984 & $3,388,173$ \\
1985 & $3,144,190$ \\
1986 & $3,039,069$ \\
1987 & $3,102,227$ \\
1988 & $3,182,267$ \\
1989 & $3,190,815$ \\
1990 & $3,134,446$ \\
1991 & $2,925,970$ \\
1992 & $3,022,108$ \\
1993 & $3,111,981$ \\
1994 & $3,355,802$ \\
1995 & $3,503,096$ \\
1996 & $3,600,649$ \\
1997 & $3,602,793$ \\
1998 & $3,619,341$ \\
1999 & $3,749,428$ \\
2000 & $3,720,107$ \\
2001 & $3,729,985$ \\
2002 & $3,751,413$ \\
2003 & $3,849,229$ \\
2004 & $4,082,236$ \\
2005 & $4,119,879$ \\
2006 & $4,214,459$ \\
2007 & $4,087,405$ \\
2008 & $3,911,178$ \\
\hline content used for & 138,700 \\
Bion to btu: & \\
\hline & \\
\hline &
\end{tabular}




\section{Passenger:}

Commuter - APTA, Public Transportation Fact Book, 2010, Washington, DC, 2010.

Table A.13

Commuter Rail Fuel Use

\begin{tabular}{ccc}
\hline Year & $\begin{array}{c}\text { Diesel } \\
\text { (thousand gallons) }\end{array}$ & $\begin{array}{c}\text { Electricity } \\
\text { (million kWhr) }\end{array}$ \\
\hline 1984 & 58,320 & 901 \\
1985 & 55,372 & 1,043 \\
1986 & 54,608 & 1,170 \\
1987 & 51,594 & 1,155 \\
1988 & 53,054 & 1,195 \\
1989 & 52,516 & 1,293 \\
1990 & 52,681 & 1,226 \\
1991 & 54,315 & 1,239 \\
1992 & 54,951 & 1,124 \\
1993 & 59,766 & 1,196 \\
1994 & 61,900 & 1,244 \\
1995 & 63,064 & 1,253 \\
1996 & 61,888 & 1,255 \\
1997 & 63,195 & 1,270 \\
1998 & 69,200 & 1,299 \\
1999 & 73,005 & 1,322 \\
2000 & 70,818 & 1,370 \\
2001 & 72,204 & 1,354 \\
2002 & 72,847 & 1,334 \\
2003 & 72,264 & 1,383 \\
2004 & 71,999 & 1,449 \\
2005 & 76,714 & 1,484 \\
2006 & 78,600 & 1,478 \\
2007 & 80,700 & 1,763 \\
2008 & 83,500 & 1,718 \\
\hline conversion to btu: & 138,700 & 10,339 \\
& Btu/gallon & Btu/kWhr \\
\hline
\end{tabular}


Transit - APTA, Public Transportation Fact Book, 2010, Washington, DC, 2010. Includes light rail and heavy rail.

Table A.14

Transit Rail Fuel Use

\begin{tabular}{|c|c|c|c|}
\hline \multirow[b]{2}{*}{ Year } & \multicolumn{3}{|c|}{ Electricity (million kWhr) } \\
\hline & Light rail & Heavy rail & Total \\
\hline 1970 & & & 2,561 \\
\hline 1971 & & & 2,556 \\
\hline 1972 & & & 2,428 \\
\hline 1973 & & & 2,331 \\
\hline 1974 & & & 2,630 \\
\hline 1975 & & & 2,646 \\
\hline 1976 & \multirow{3}{*}{\multicolumn{2}{|c|}{$\begin{array}{l}\text { Light rail and heavy rail data are } \\
\text { not available separately from }\end{array}$}} & 2,576 \\
\hline 1977 & & & 2,303 \\
\hline 1978 & & & 2,223 \\
\hline 1979 & & & 2,473 \\
\hline 1980 & & & 2,446 \\
\hline 1981 & & & 2,655 \\
\hline 1982 & & & 2,722 \\
\hline 1983 & & & 2,930 \\
\hline 1984 & & & 3,092 \\
\hline 1985 & & & 2,928 \\
\hline 1986 & 173 & 3,066 & 3,239 \\
\hline 1987 & 191 & 3,219 & 3,410 \\
\hline 1988 & 243 & 3,256 & 3,499 \\
\hline 1989 & 242 & 3,286 & 3,528 \\
\hline 1990 & 239 & 3,284 & 3,523 \\
\hline 1991 & 274 & 3,248 & 3,522 \\
\hline 1992 & 297 & 3,193 & 3,490 \\
\hline 1993 & 281 & 3,287 & 3,568 \\
\hline 1994 & 282 & 3,431 & 3,713 \\
\hline 1995 & 288 & 3,401 & 3,689 \\
\hline 1996 & 321 & 3,322 & 3,643 \\
\hline 1997 & 363 & 3,253 & 3,616 \\
\hline 1998 & 382 & 3,280 & 3,662 \\
\hline 1999 & 416 & 3,385 & 3,801 \\
\hline 2000 & 563 & 3,549 & 4,112 \\
\hline 2001 & 587 & 3,646 & 4,233 \\
\hline 2002 & 510 & 3,683 & 4,193 \\
\hline 2003 & 507 & 3,632 & 4,138 \\
\hline 2004 & 553 & 3,684 & 4,237 \\
\hline 2005 & 571 & 3,769 & 4,339 \\
\hline 2006 & 634 & 3,709 & 4,343 \\
\hline 2007 & 687 & 3,817 & 4,505 \\
\hline 2008 & 721 & 3,898 & 4,619 \\
\hline Heat content used for & 10,339 & 10,339 & 10,339 \\
\hline conversion to btu: & Btu/kWhr & Btu/kWhr & Btu/kWhr \\
\hline
\end{tabular}


Intercity - Personal communication with Amtrak, Washington, DC, 2009.

Table A.15

Intercity Rail Fuel Use

\begin{tabular}{ccc}
\hline Year & $\begin{array}{c}\text { Diesel fuel } \\
\text { (thousand } \\
\text { gallons) }\end{array}$ & $\begin{array}{c}\text { Electricity } \\
\text { (thousand kWhr) }\end{array}$ \\
\hline 1994 & 73,516 & 308,948 \\
1995 & 72,371 & 335,818 \\
1996 & 71,226 & 362,689 \\
1997 & 75,656 & 389,559 \\
1998 & 75,999 & 416,429 \\
1999 & 79,173 & 443,300 \\
2000 & 94,968 & 470,170 \\
2001 & 96,846 & 455,703 \\
2002 & 84,432 & 518,306 \\
2003 & 74,621 & 536,950 \\
2004 & 68,605 & 550,695 \\
2005 & 65,477 & 531,377 \\
2006 & 62,463 & 548,856 \\
2007 & 61,824 & 577,864 \\
2008 & 63,428 & 582,022 \\
\hline Heat content used for & 138,700 & 10,339 \\
conversion to Btu & Btu/gallon & Btu/kWhr \\
\hline
\end{tabular}

\section{Calculation of}

Million Barrels per Day

Crude Oil Equivalent

One gallon of gasoline, diesel fuel, or lpg is estimated to be the equivalent of one gallon of crude oil. Petroleum used for electricity was calculated using the following formula:

$(\{[(\mathrm{BTU} * \mathrm{~S}) / \mathrm{G}] / \mathrm{P}\} / 365) / 1000$

$\mathrm{BTU}=$ Btus of electricity from Table 2.4

$\mathrm{S}=$ Share of petroleum used in making primary electricity (Calculated from Table 2.6 from the EIA, Monthly Energy Review)

$\mathrm{G}=$ Electricity generation and distribution (assumed 29\%)

$\mathrm{P}=\quad$ Btus per barrel of petroleum product (Table A3 from the EIA, Monthly Energy Review). 


\section{Passenger Travel and Energy Use}

\section{Automobiles}

Number of vehicles, vehicle-miles - DOT, FHWA, Highway Statistics, 2008, Table VM-1. Data series shown in Table 4.1.

Passenger-miles - Vehicle-miles multiplied by an average load factor.

Load factor - 2009 NHTS shows automobile load factor as 1.59 persons per vehicle (preliminary).

Energy intensities -

Btu per vehicle-mile - Automobile energy use divided by vehicle-miles.

Btu per passenger-mile - Automobile energy use divided by passenger-miles.

Energy use - See Energy Use Sources, p. A-4. Data series shown in Table 2.7.

\section{Light trucks}

Number of vehicles, vehicle-miles - DOT, FHWA, Highway Statistics 2008, Table VM-1. Data by truck type were multiplied by the shares of trucks/truck travel which are for personal use (Table A.16).

Passenger-miles - Vehicle-miles multiplied by an average load factor.

Load factor - 2009 NHTS shows personal light truck load factor as 1.84 persons per vehicle (preliminary).

Energy intensities -

Btu per vehicle-mile - Personal light truck energy use divided by personal light truck vehicle-miles.

Btu per passenger-mile - Personal light truck energy use divided by personal light truck passengermiles.

Energy use - See Energy Use Sources, p. A-7, A-8 (light trucks, medium/heavy trucks). Data by truck type were multiplied by the shares of truck fuel use which are for personal use (Table A.16) which were derived by ORNL from the 2002 VIUS Micro Data File on CD.

Table A.16

Share of Trucks, Truck Travel, and Fuel Use for Personal Travel

\begin{tabular}{cl}
\hline Personal trucks & \\
$85.6 \%$ & 2-axle, 4-tire trucks \\
$26.9 \%$ & Other single-unit and combination trucks \\
Personal truck travel & \\
$80.9 \%$ & 2-axle, 4-tire trucks \\
$13.1 \%$ & Other single-unit and combination trucks \\
Personal truck fuel use & \\
$78.0 \%$ & 2-axle, 4-tire trucks \\
$6.0 \%$ & Other single-unit and combination trucks \\
\hline
\end{tabular}

Note: Since these shares come from the 2002 VIUS, they may underestimate the amount of personal trucks, truck travel, and energy use for 2008. 


\section{Motorcycles}

Number of vehicles, vehicle-miles - DOT, FHWA, Highway Statistics 2008, Table VM-1.

Passenger-miles - Vehicle-miles multiplied by an average load factor.

Load factor - 2009 NHTS shows motorcycle load factor as 1.18 persons per vehicle (preliminary).

Energy intensities -

Btu per vehicle-mile - Motorcycle energy use divided by vehicle-miles.

Btu per passenger-mile - Motorcycle energy use divided by passenger-miles.

Energy use - See Energy Use Sources, p. A-6. Data series shown in Table 2.7.

\section{Demand Response}

Number of vehicles, vehicle-miles, passenger-miles - APTA, 2010 Public Transportation Fact Book, Washington, DC, 2010.

Load factor - Passenger-miles divided by vehicle-miles.

Energy intensities -

Btu per vehicle-mile - Energy use divided by vehicle-miles.

Btu per passenger-mile - Energy use divided by passenger-miles.

Energy use - APTA, 2010 Public Transportation Fact Book, Washington, DC, 2010.

\section{Vanpool}

Number of vehicles, vehicle-miles, passenger-miles - APTA, 2010 Public Transportation Fact Book, Washington, DC, 2010.

Load factor - Passenger-miles divided by vehicle-miles.

Energy intensities -

Btu per vehicle-mile - Energy use divided by vehicle-miles.

Btu per passenger-mile - Energy use divided by passenger-miles.

Energy use - APTA, 2010 Public Transportation Fact Book, Washington, DC, 2010.

\section{Buses}

\section{Transit}

Number of vehicles, vehicle-miles, passenger-miles - APTA, 2010 Public Transportation Fact Book, Washington, DC, 2010. Data series shown on Table 5.16.

Load factor - Passenger-miles divided by vehicle-miles.

Energy intensities -

Btu per vehicle-mile - Transit bus energy use divided by transit bus vehicle-miles.

Btu per passenger-mile - Transit bus energy use divided by transit bus passenger-miles.

Energy use - See Energy Use Sources, p. A-6. Data series shown in Table 5.16. 


\section{Intercity}

Energy use - See Energy Use Sources, p. A-7. Because the 2001 and 2002 data are not available, the rate of change in bus VMT from FHWA, Highway Statistics 2008, was used to estimate the change in energy use.

\section{School}

Number of vehicles - DOT, FHWA, Highway Statistics 2008, Table MV-10.

Energy use - See Energy Use Sources, p. A-7. Because the data past 2000 are not available, the rate of change in bus VMT from FHWA, Highway Statistics 2008, was used to estimate the change in energy use.

$\underline{\text { Air }}$

\section{Certificated air carriers}

Aircraft-miles, passenger-miles - DOT, BTS, Air Carrier Traffic Statistics, www.bts.gov/programs/airline_information/air_carrier_traffic_statistics, Washington, DC.

Load factor - Passenger-miles divided by aircraft-miles.

Energy intensities Btu per passenger-mile - Certificated air carrier energy use divided by passenger-miles.

Energy use - See Energy Use Sources, p. A-11. All of domestic fuel use and half of international fuel use was considered to be domestic use.

Note: These data differ from the data in Table 9.2 because that table contains data on ALL domestic AND international air carrier energy use and passenger-miles.

\section{General aviation}

Number of vehicles - DOT, FAA, General Aviation Activity and Avionics Survey: Calendar Year 2008, Data series shown in Table 9.3.

Energy intensities -

Btu per passenger-mile - General aviation energy use divided by passenger-miles.

Energy use - See Energy Use Sources, p. A-11. Data series shown in Table 9.3.

\section{$\underline{\text { Recreational boating }}$}

Number of vehicles and energy use - U.S. EPA, NONROAD2008a model. 


\section{Rail}

\section{Intercity}

Number of vehicles, vehicle-miles, passenger-miles - AAR, Railroad Facts, 2009 Edition, Washington, DC, 2009.

Load factor - Passenger-miles divided by vehicle-miles.

Energy Intensities -

Btu per vehicle-mile - Intercity rail energy use divided by vehicle-miles.

Btu per passenger-mile - Intercity rail energy use divided by passenger-miles.

Energy use - See Energy Use Sources, p. A-20. Data series shown in Table 9.10.

\section{Transit}

Number of vehicles, vehicle-miles, passenger-miles - APTA, 2010 Public Transportation Fact Book, Washington, DC, 2010. Sum of light and heavy rail transit. Data series shown on Table 9.12.

Load factor - Passenger-miles divided by vehicle-miles.

\section{Energy intensities -}

Btu per vehicle-mile - Light and heavy transit rail energy use divided by vehicle-miles.

Btu per passenger-mile - Light and heavy transit rail energy use divided by passenger-miles.

Energy use - See Energy Use Sources, p. A-19. Data series shown in Table 9.12.

\section{Commuter}

Number of vehicles, vehicle-miles, passenger-miles - APTA, 2010 Public Transportation Fact Book, Washington, DC, 2010. Data series shown on Table 9.11.

Load factor - Passenger-miles divided by vehicle-miles.

Energy intensities -

Btu per vehicle-mile - Commuter rail energy use divided by vehicle-miles.

Btu per passenger-mile - Commuter rail energy use divided by passenger-miles.

Energy use - See Energy Use Sources, p. A-18. Data series shown in Table 9.11. 


\section{Highway Passenger Mode Energy Intensities}

\section{Automobiles}

Btu per vehicle-mile - Automobile energy use divided by automobile vehicle miles of travel.

Energy use - See Energy Use Sources, p. A-5. Data series shown in Table 2.7.

Vehicle-miles - DOT, FHWA, Highway Statistics 2008, Table VM-1 and annual editions back to 1996; DOT, FHWA, Highway Statistics Summary to 1995. Data series shown in Table 4.1.

Btu per passenger-mile - Automobile energy use divided by automobile passenger-miles.

Energy use - See Energy Use Sources, p. A-5. Data series shown in Table 2.7.

Passenger miles - Vehicle miles multiplied by an average load factor.

Vehicle-miles - DOT, FHWA, Highway Statistics 2008, Table VM-1 and annual editions back to 1996; DOT, FHWA, Highway Statistics Summary to 1995. Data series shown in Table 4.1.

Load factor - NPTS 1969, 1977, 1983/84, 1990, and 1995; NHTS 2001 and 2009.

Table A.17

Automobile Load Factor used to calculate Passenger-Miles

\begin{tabular}{|c|c|c|}
\hline Year & Source & Load Factor \\
\hline 1970 & 1969 NPTS & 1.90 \\
\hline 1971 & Interpolated & 1.90 \\
\hline 1972 & Interpolated & 1.90 \\
\hline 1973 & Interpolated & 1.90 \\
\hline 1974 & Interpolated & 1.90 \\
\hline 1975 & Interpolated & 1.90 \\
\hline 1976 & Interpolated & 1.90 \\
\hline 1977 & 1977 NPTS & 1.90 \\
\hline 1978 & Interpolated & 1.88 \\
\hline 1979 & Interpolated & 1.87 \\
\hline 1980 & Interpolated & 1.85 \\
\hline 1981 & Interpolated & 1.83 \\
\hline 1982 & Interpolated & 1.82 \\
\hline 1983 & $1983 / 84$ NPTS & 1.80 \\
\hline 1984 & Interpolated & 1.77 \\
\hline 1985 & Interpolated & 1.74 \\
\hline 1986 & Interpolated & 1.71 \\
\hline 1987 & Interpolated & 1.69 \\
\hline 1988 & Interpolated & 1.66 \\
\hline 1989 & Interpolated & 1.63 \\
\hline 1990 & 1990 NPTS & 1.60 \\
\hline 1991 & Interpolated & 1.60 \\
\hline 1992 & Interpolated & 1.60 \\
\hline 1993 & Interpolated & 1.60 \\
\hline 1994 & Interpolated & 1.60 \\
\hline 1995 & 1995 NPTS & 1.60 \\
\hline 1996 & Interpolated & 1.60 \\
\hline 1997 & Interpolated & 1.59 \\
\hline 1998 & Interpolated & 1.59 \\
\hline 1999 & Interpolated & 1.58 \\
\hline 2000 & Interpolated & 1.58 \\
\hline 2001 & 2001 NHTS & 1.57 \\
\hline 2002 & 2001 NHTS & 1.57 \\
\hline 2003 & 2001 NHTS & 1.57 \\
\hline 2004 & 2001 NHTS & 1.57 \\
\hline 2005 & 2001 NHTS & 1.57 \\
\hline 2006 & 2001 NHTS & 1.57 \\
\hline 2007 & 2001 NHTS & 1.57 \\
\hline 2008 & 2009 NHTS & 1.59 \\
\hline
\end{tabular}




\section{Light trucks}

Btu per vehicle-mile - Light truck energy use divided by light truck vehicle miles of travel.

Energy use - See Energy Use Sources, p. A-8. Data series shown in Table 2.7.

Vehicle-miles - DOT, FHWA, Highway Statistics 2008, Table VM-1 and annual editions back to 1996; DOT, FHWA, Highway Statistics Summary to 1995. Data series shown in Table 4.2.

\section{Buses}

\section{Transit}

Btu per vehicle-mile - Transit bus energy use divided by transit bus vehicle-miles.

Energy use - See Energy Use Sources, p. A-6. Data series shown in Table 5.16.

Vehicle-miles - APTA, 2010 Public Transportation Fact Book, Washington, DC, 2010. Data series shown on Table 5.16.

Btu per passenger-mile - Transit bus energy use divided by transit bus passenger-miles.

Energy use - See Energy Use Sources, p. A-6. Data series shown in Table 5.16.

Passenger-miles - APTA, 2010 Public Transportation Fact Book, Washington, DC, 2010. Data series shown on Table 5.16.

\section{Intercity}

Btu per passenger-mile - Intercity bus energy use divided by intercity bus passenger-miles.

Energy use - See Energy Use Sources, p. A-7. Because the data past 2000 are not available, the rate of change in bus VMT from FHWA, Highway Statistics 2008, was used to estimate the change in energy use.

Passenger-miles - (Data past 2000 are not available.) Eno Foundation for Transportation, Transportation in America 2001, Nineteenth edition, Washington, DC. 


\section{Nonhighway Mode Energy Intensities}

\section{$\underline{\text { Air }}$}

\section{Certificated air carriers}

Btu per passenger-mile - Certificated air carrier energy use divided by passenger-miles.

Energy use - See Energy Use Sources, p. A-12. All of domestic fuel use and half of international fuel use was considered to be domestic use.

Passenger-miles - DOT, BTS, Air Carrier Traffic Statistics, Washington, DC, ww.bts.gov/programs/airline_information/air_carrier_traffic_statistics. Pre-1994 data are from various editions of the FAA Statistical Handbook of Aviation (no longer published). Scheduled service passenger-miles of domestic air carriers and half of international air carriers were used to coincide with fuel use.

Note: These data differ from the data in Table 9.2 because that table contains data on ALL domestic AND international air carrier energy use and passenger-miles.

\section{General aviation}

Btu per passenger-mile - General aviation energy use divided by passenger-miles.

Energy use - See Energy Use Sources, p. A-11. Data series shown in Table 9.3.

Passenger-miles - (Data past 2000 not available.) Eno Foundation for Transportation, Transportation in America 2001, Nineteenth edition, Washington, DC.

$\underline{\text { Rail }}$

Intercity

Btu per passenger-mile - Intercity rail energy use divided by passenger-miles.

Energy use - See Energy Use Sources, p. A-20. Data series shown in Table 9.10.

Passenger-miles - AAR, Railroad Facts, 2009 Edition, and previous annual editions.

\section{Transit}

Btu per passenger-mile - Transit rail energy use divided by passenger-miles.

Energy use - See Energy Use Sources, p. A-19. Data series shown in Table 9.12.

Passenger-miles - APTA, 2010 Public Transportation Fact Book, Washington, DC, 2010. Data series shown on Table 9.12.

\section{Commuter}

Btu per passenger-mile - Commuter rail energy use divided by passenger-miles.

Energy use - See Energy Use Sources, p. A-18. Data series shown in Table 9.11.

Passenger-miles - APTA, 2010 Public Transportation Fact Book, Washington, DC, 2010. Data series shown on Table 9.11. 


\section{Freight Movement and Energy Use}

$\underline{\text { Rail }}$

Number of locomotives, ton-miles, tons shipped, average length of haul - AAR, Railroad Facts, 2009 Edition, Washington, DC, 2009. Data series shown in Table 9.8.

Energy intensity - Class I rail energy use divided by freight car-miles.

Energy use - See Energy Use Sources, p. A-16. Data series shown in Table 9.8.

\section{Water}

Number of vehicles - U.S. Department of the Army, Army Corps of Engineers, Waterborne Transportation Lines of the United States, Calendar Year 2008, Volume 1-National Summaries, New Orleans, LA, 2009.

Ton-miles, tons shipped, average length of haul - U.S. Department of the Army, Army Corps of Engineers, Waterborne Commerce of the United States, Calendar Year 2008, Part 5: National Summaries, New Orleans, LA, 2009. Data series shown in Table 9.5.

Btu per ton-mile - Domestic waterborne commerce energy use divided by ton-miles.

Energy use - See Energy Use Sources, p. A-12. Data series shown in Table 9.5. 


\section{Freight Mode Energy Intensities}

\section{Truck}

Btu per vehicle-mile - Heavy single-unit and combination truck energy use divided by vehicle miles Energy use - See Energy Use Sources (medium/heavy trucks), p. A-9.

Vehicle-miles - DOT, FHWA, Highway Statistics 2008, Table VM-1 and annual editions back to 1996; DOT, FHWA, Highway Statistics Summary to 1995. Data series is the total of vehicle travel data on Tables 5.1 and 5.2.

\section{Rail}

Btu per freight car-mile - Class I rail energy use divided by freight car-miles.

Energy use - See Energy Use Sources, p. A-17. Data series shown in Table 9.8.

Freight car miles - AAR, Railroad Facts, 2009 Edition, Washington, DC, 2009. Data series shown in Table 9.8.

Btu per ton-mile - Class I rail energy use divided by ton-miles.

Energy use - See Energy Use Sources, p. A-16. Data series shown in Table 9.8.

Ton-miles - AAR, Railroad Facts, 2009 Edition, Washington, DC, 2009. Data series shown in Table 9.8.

\section{Water}

Btu per ton-mile - Domestic waterborne commerce energy use divided by ton-miles.

Energy use - See Energy Use Sources, p. A-13. Data series shown in Table 9.5.

Ton-miles - U.S. Department of the Army, Army Corps of Engineers, Waterborne Commerce of the United States, Calendar Year 2008, Part 5: National Summaries, New Orleans, LA, 2009. Data series shown in Table 9.5. 
A-30

TRANSPORTATION ENERGY DATA BOOK: EDITION 29-2010 
APPENDIX B

CONVERSIONS 
B-2

TRANSPORTATION ENERGY DATA BOOK: EDITION 29-2010 


\section{CONVERSIONS}

\section{A Note about Heating Values}

The heat content of a fuel is the quantity of energy released by burning a unit amount of that fuel. However, this value is not absolute and can vary according to several factors. For example, empirical formulae for determining the heating value of liquid fuels depend on the fuels' American Petroleum Institute (API) gravity. The API gravity varies depending on the percent by weight of the chemical constituents and impurities in the fuel, both of which are affected by the combination of raw materials used to produce the fuel and by the type of manufacturing process. Temperature and climatic conditions are also factors.

Because of these variations, the heating values in Table B.4 may differ from values in other publications. The figures in this report are representative or average values, not absolute ones. The gross (higher) heating values used here agree with those used by the Energy Information Administration (EIA).

Heating values fall into two categories, usually referred to as "higher" (or gross) and "lower" (or net). If the products of fuel combustion are cooled back to the initial fuel-air or fuel-oxidizer mixture temperature and the water formed during combustion is condensed, the energy released by the process is the higher (gross) heating value. If the products of combustion are cooled to the initial fuel-air temperature, but the water is considered to remain as a vapor, the energy released by the process is the lower (net) heating value. Usually the difference between the gross and net heating values for fuels used in transportation is around 5 to 8 percent; however, it is important to be consistent in their use. 
Table B.1

Hydrogen Heat Content

\begin{tabular}{cc}
\hline \multicolumn{2}{c}{1 kilogram hydrogen $=$} \\
\hline Higher heating value & Lower heating value \\
$134,200 \mathrm{Btu}$ & $113,400 \mathrm{Btu}$ \\
$39.3 \mathrm{kWhr}$ & $33.2 \mathrm{kWhr}$ \\
$141,600 \mathrm{~kJ}$ & $119,600 \mathrm{~kJ}$ \\
$33,800 \mathrm{kCal}$ & $28,560 \mathrm{kCal}$ \\
\hline
\end{tabular}

Table B.2

Hydrogen Conversions

\begin{tabular}{|c|c|c|c|c|c|c|}
\hline & \multicolumn{2}{|c|}{ Weight } & \multicolumn{2}{|c|}{ Gas } & \multicolumn{2}{|c|}{ Liquid } \\
\hline & $\begin{array}{l}\text { Pounds } \\
\text { (lb) }\end{array}$ & $\begin{array}{c}\text { Kilograms } \\
(\mathrm{kg})\end{array}$ & $\begin{array}{c}\text { Standard } \\
\text { cubic feet } \\
(\mathrm{SCF})\end{array}$ & $\begin{array}{c}\text { Normal } \\
\text { cubic meter } \\
\left(\mathrm{Nm}^{3}\right)\end{array}$ & $\begin{array}{l}\text { Gallons } \\
\text { (gal) }\end{array}$ & $\begin{array}{c}\text { Liters } \\
\text { (L) }\end{array}$ \\
\hline $1 \mathrm{lb}$ & 1.0 & 0.4536 & 192.00 & 5.047 & 1.6928 & 6.408 \\
\hline $1 \mathrm{~kg}$ & 2.205 & 1.0 & 423.3 & 11.126 & 3.733 & 14.128 \\
\hline $1 \mathrm{SCF}$ gas & 0.005209 & 0.002363 & 1.0 & 0.02628 & 0.00882 & 0.0339 \\
\hline $1 \mathrm{Nm}^{3}$ gas & 0.19815 & 0.08988 & 38.04 & 1.0 & 0.3355 & 1.2699 \\
\hline 1 gal liquid & 0.5906 & 0.2679 & 113.41 & 2.981 & 1.0 & 3.785 \\
\hline $1 \mathrm{~L}$ liquid & 0.15604 & 0.07078 & 29.99 & 0.77881 & 0.2642 & 1.0 \\
\hline
\end{tabular}

Table B.3

Pressure Conversions

\begin{tabular}{lllllll}
\hline & \multicolumn{2}{c}{ Weight } & \multicolumn{2}{c}{ Gas } & \multicolumn{2}{c}{ Liquid } \\
\hline & $\begin{array}{c}\text { Pounds } \\
(\mathrm{lb})\end{array}$ & $\begin{array}{c}\text { Kilograms } \\
(\mathrm{kg})\end{array}$ & $\begin{array}{c}\text { Standard } \\
\text { cubic feet } \\
(\mathrm{SCF})\end{array}$ & $\begin{array}{c}\text { Normal } \\
\text { cubic meter } \\
\left(\mathrm{Nm}^{3}\right)\end{array}$ & $\begin{array}{c}\text { Gallons } \\
(\mathrm{gal})\end{array}$ & $\begin{array}{c}\text { Liters } \\
(\mathrm{L})\end{array}$ \\
\hline $1 \mathrm{lb}$ & 1.0 & 0.4536 & 192.00 & 5.047 & 1.6928 & 6.408 \\
$1 \mathrm{~kg}$ & 2.205 & 1.0 & 423.3 & 11.126 & 3.733 & 14.128 \\
$1 \mathrm{SCF}$ gas & 0.005209 & 0.002363 & 1.0 & 0.02628 & 0.00882 & 0.0339 \\
$1 \mathrm{Nm}$ gas & 0.19815 & 0.08988 & 38.04 & 1.0 & 0.3355 & 1.2699 \\
1 gal liquid & 0.5906 & 0.2679 & 113.41 & 2.981 & 1.0 & 3.785 \\
$1 \mathrm{~L}$ liquid & 0.15604 & 0.07078 & 29.99 & 0.77881 & 0.2642 & 1.0 \\
\hline
\end{tabular}


Table B.4

Heat Content for Various Fuels

\section{Conventional gasoline}

Hydrogen

Diesel motor fuel

Biodiesel

Methanol

Ethanol

Gasohol

E85

Aviation gasoline

Liquefied petroleum gas (LPG)

Butane

Jet fuel (naphtha)

Jet fuel (kerosene)

Lubricants

Waxes

Asphalt and road oil

Liquefied natural gas (LNG)

Compressed natural gas (CNG)

Crude petroleum

\section{Fuel Oils}

Residual

Distillate

Coal

Anthracite - Consumption

Bituminous and lignite - Consumption

Production average

Consumption average
$125,000 \mathrm{Btu} / \mathrm{gal}($ gross$)=115,400 \mathrm{Btu} / \mathrm{gal}($ net $)$

$134,200 \mathrm{Btu} / \mathrm{kg}$ (gross) $=113,400 \mathrm{Btu} / \mathrm{kg}$ (net)

$138,700 \mathrm{Btu} / \mathrm{gal}($ gross$)=128,700 \mathrm{Btu} / \mathrm{gal}(\mathrm{net})$

$126,200 \mathrm{Btu} /$ gal $($ gross $)=117,100 \mathrm{Btu} /$ gal $($ net $)$

$64,600 \mathrm{Btu} / \mathrm{gal}($ gross $)=56,600 \mathrm{Btu} / \mathrm{gal}$ (net)

$84,600 \mathrm{Btu} / \mathrm{gal}($ gross $)=75,700 \mathrm{Btu} / \mathrm{gal}$ (net)

$120,900 \mathrm{Btu} / \mathrm{gal}($ gross$)=112,400 \mathrm{Btu} / \mathrm{gal}$ (net)

$90,700 \mathrm{Btu} /$ gal $($ gross $)=81,600 \mathrm{Btu} /$ gal $($ net $)$

$120,200 \mathrm{Btu} / \mathrm{gal}($ gross$)=112,000 \mathrm{Btu} / \mathrm{gal}(\mathrm{net})$

91,300 Btu/gal $($ gross $)=83,500 \mathrm{Btu} /$ gal $($ net $)$

103,000 Btu/gal (gross) = 93,000 Btu/gal (net)

$127,500 \mathrm{Btu} /$ gal $($ gross $)=118,700 \mathrm{Btu} /$ gal $($ net $)$

$135,000 \mathrm{Btu} / \mathrm{gal}($ gross $)=128,100 \mathrm{Btu} / \mathrm{gal}(\mathrm{net})$

$144,400 \mathrm{Btu} / \mathrm{gal}($ gross$)=130,900 \mathrm{Btu} / \mathrm{gal}(\mathrm{net})$

$131,800 \mathrm{Btu} /$ gal $($ gross) $=120,200 \mathrm{Btu} /$ gal $($ net $)$

$158,000 \mathrm{Btu} / \mathrm{gal}($ gross $)=157,700 \mathrm{Btu} / \mathrm{gal}(\mathrm{net})$

$84,800 \mathrm{Btu} / \mathrm{gal}$ (gross) $=74,700 \mathrm{Btu} / \mathrm{gal}$ (net)

$22,500 \mathrm{Btu} / \mathrm{gal}($ gross $)=20,300 \mathrm{Btu} / \mathrm{gal}($ net $)$

$138,100 \mathrm{Btu} /$ gal $($ gross $)=131,800 \mathrm{Btu} /$ gal $($ net $)$

$149,700 \mathrm{Btu} /$ gal $($ gross $)=138,400 \mathrm{Btu} /$ gal $($ net $)$

$138,700 \mathrm{Btu} / \mathrm{gal}($ gross $)=131,800 \mathrm{Btu} / \mathrm{gal}($ net $)$

$21.711 \times 10^{6} \mathrm{Btu} / \mathrm{short}$ ton

$21.012 \times 10^{6} \mathrm{Btu} / \mathrm{short}$ ton

$21.352 \times 10^{6} \mathrm{Btu} /$ short ton

$21.015 \times 10^{6} \mathrm{Btu} / \mathrm{short}$ ton 
Table B.5

Fuel Equivalents

\begin{tabular}{|c|c|}
\hline 1 million bbl crude oil/day & $\begin{array}{l}=0.365 \text { billion bbl crude oil/year } \\
=2.117 \text { quadrillion Btu/year } \\
=105.914 \text { million short tons coal/year } \\
=96.085 \text { million metric tons coal/year } \\
=2.059{\text { trillion } \mathrm{ft}^{3} \text { natural gas/year }}=2,233 \text { petajoules/year }\end{array}$ \\
\hline 1 billion bbl crude oil/year & $\begin{array}{l}=2.740 \text { million bbl crude oil/day } \\
=5.800 \text { quadrillion Btu/year } \\
=290.174 \text { million short tons coal/year } \\
=263.246 \text { million metric tons coal/year } \\
=5.642 \text { trillion } \mathrm{ft}^{3} \text { natural gas/year } \\
=6,119 \text { petajoules/year }\end{array}$ \\
\hline 1 quadrillion Btu/year & $\begin{array}{l}=0.5219 \text { gasoline gallon equivalents } \\
=0.472 \text { million bbl crude oil/day } \\
=172.414 \text { million bbl crude oil/year } \\
=50.030 \text { million short tons coal/year } \\
=45.387 \text { million metric tons coal/year } \\
=972.763 \text { billion } \mathrm{ft}^{3} \text { natural gas/year } \\
=1,055 \text { petajoules/year }\end{array}$ \\
\hline 1 billion short tons coal/year & $\begin{array}{l}=0.907 \text { billion metric tons coal/year } \\
=9.442 \text { million bbl crude oil/day } \\
=3.446 \text { billion bbl crude oil/year } \\
=19.988 \text { quadrillion Btu/year } \\
=19.444 \text { trillion } \mathrm{ft}^{3} \text { natural gas/year } \\
=21,087 \text { petajoules/year }\end{array}$ \\
\hline 1 billion metric tons coal/year & $\begin{array}{l}=1.102 \text { billion short tons coal/year } \\
=8.565 \text { million bbl crude oil/day } \\
=3.126 \text { billion bbl crude oil/year } \\
=18.133 \text { quadrillion btu/year } \\
=17.639 \text { trillion } \mathrm{ft}^{3} \text { natural gas/year } \\
=19,130 \text { petajoules/year }\end{array}$ \\
\hline 1 trillion $\mathrm{ft}^{3}$ natural gas/year & $\begin{array}{l}=0.486 \text { million bbl crude oil/day } \\
=0.177 \text { billion bbl crude oil/year } \\
=1.028 \text { quadrillion Btu/year } \\
=51.431 \text { million short tons coal/year } \\
=46.658 \text { million metric tons coal/year } \\
=1,085 \text { petajoules/year }\end{array}$ \\
\hline 1 petajoule/year & $\begin{array}{l}=447.741 \mathrm{bbl} \text { crude oil/day } \\
=163.425 \text { thousand bbl crude oil/year } \\
=0.948 \text { trillion Btu/year } \\
=47.421 \text { thousand short tons coal/year } \\
=43.021 \text { thousand metric tons coal/year } \\
=0.922 \text { billion } \mathrm{ft}^{3} \text { natural gas/year }\end{array}$ \\
\hline
\end{tabular}


Table B.6

Energy Unit Conversions

\begin{tabular}{|c|c|c|c|}
\hline $1 \mathrm{Btu}$ & $\begin{array}{l}=778.2 \mathrm{ft}-\mathrm{lb} \\
=107.6 \mathrm{~kg}-\mathrm{m} \\
=1055 \mathrm{~J} \\
=39.30 \times 10^{-5} \mathrm{hp}-\mathrm{h} \\
=39.85 \times 10^{-5} \text { metric hp-h } \\
=29.31 \times 10^{-5} \mathrm{kWhr}\end{array}$ & $1 \mathrm{kWhr}$ & $\begin{array}{l}=3412 \mathrm{Btu}^{\mathrm{a}} \\
=2.655 \times 10^{6} \mathrm{ft}-\mathrm{lb} \\
=3.671 \times 10^{5} \mathrm{~kg}-\mathrm{m} \\
=3.600 \times 10^{6} \mathrm{~J} \\
=1.341 \mathrm{hp}-\mathrm{h} \\
=1.360 \text { metric hp-h }\end{array}$ \\
\hline $1 \mathrm{~kg}-\mathrm{m}$ & $\begin{array}{l}=92.95 \times 10^{-4} \mathrm{Btu} \\
=7.233 \mathrm{ft}-\mathrm{lb} \\
=9.806 \mathrm{~J} \\
=36.53 \times 10^{-7} \mathrm{hp}-\mathrm{h} \\
=37.04 \times 10^{-7} \text { metric hp-h } \\
=27.24 \times 10^{-7} \mathrm{kWhr}\end{array}$ & 1 Joule & $\begin{array}{l}=94.78 \times 10^{-5} \mathrm{Btu} \\
=0.7376 \mathrm{ft}-\mathrm{lb} \\
=0.1020 \mathrm{~kg}-\mathrm{m} \\
=37.25 \times 10^{-8} \mathrm{hp}-\mathrm{h} \\
=37.77 \times 10^{-8} \text { metric hp-h } \\
=27.78 \times 10^{-8} \mathrm{kWhr}\end{array}$ \\
\hline $1 \mathrm{hp}-\mathrm{h}$ & $\begin{array}{l}=2544 \mathrm{Btu} \\
=1.98 \times 10^{6} \mathrm{ft}-\mathrm{lb} \\
=2.738 \times 10^{6} \mathrm{kgm} \\
=2.685 \times 10^{6} \mathrm{~J} \\
=1.014 \text { metric hp-h } \\
=0.7475 \mathrm{kWhr}\end{array}$ & 1 metric hp-h & $\begin{array}{l}=2510 \mathrm{Btu} \\
=1.953 \times 10^{6} \mathrm{ft}-\mathrm{lb} \\
=27.00 \times 10^{4} \mathrm{~kg}-\mathrm{m} \\
=2.648 \times 10^{6} \mathrm{~J} \\
=0.9863 \mathrm{hp}-\mathrm{h} \\
=0.7355 \mathrm{kWhr}\end{array}$ \\
\hline
\end{tabular}

${ }^{a}$ This figure does not take into account the fact that electricity generation and distribution efficiency is approximately $33 \%$. If generation and distribution efficiency are taken into account, $1 \mathrm{kWhr}=10,339 \mathrm{Btu}$.

Table B.7

International Energy Conversions

\begin{tabular}{rccccc}
\hline To: & Petajoules & $\begin{array}{c}\text { Giga- } \\
\text { calories }\end{array}$ & $\begin{array}{c}\text { Million } \\
\text { tonnes of oil } \\
\text { equivalent }\end{array}$ & $\begin{array}{c}\text { Million } \\
\text { Btu }\end{array}$ & $\begin{array}{c}\text { Gigawatt- } \\
\text { hours }\end{array}$ \\
\hline $\begin{array}{r}\text { From: } \\
\text { Petajoules }\end{array}$ & 1 & $238.8 \times 10^{3}$ & $2.388 \times 10^{-2}$ & $947.8 \times 10^{3}$ & 277.8 \\
$\begin{array}{r}\text { Gigacalories } \\
\text { Million tonnes }\end{array}$ & $4.1868 \times 10^{-6}$ & 1 & $10^{-7}$ & 3.968 & $1.163 \times 10^{-3}$ \\
$\begin{array}{r}\text { of oil equivalent } \\
\text { Million Btu }\end{array}$ & $1.0551 \times 10^{-6}$ & 0.252 & $2.52 \times 10^{-8}$ & 1 & $2.931 \times 10^{-4}$ \\
Gigawatthours & $3.6 \times 10^{-3}$ & 860 & $8.6 \times 10^{-5}$ & 3412 & 1 \\
\hline
\end{tabular}




\begin{tabular}{|c|c|c|c|}
\hline $1 \mathrm{in.}$ & $\begin{array}{l}=83.33 \times 10^{-3} \mathrm{ft} \\
=27.78 \times 10^{-3} \mathrm{yd} \\
=15.78 \times 10^{-6} \mathrm{mile} \\
=25.40 \times 10^{-3} \mathrm{~m} \\
=0.2540 \times 10^{-6} \mathrm{~km}\end{array}$ & $1 \mathrm{ft}$ & $\begin{array}{l}=12.0 \mathrm{in} . \\
=0.33 \mathrm{yd} \\
=189.4 \times 10^{-3} \mathrm{mile} \\
=0.3048 \mathrm{~m} \\
=0.3048 \times 10^{-3} \mathrm{~km}\end{array}$ \\
\hline 1 mile & $\begin{array}{l}=63360 \mathrm{in} . \\
=5280 \mathrm{ft} \\
=1760 \mathrm{yd} \\
=1609 \mathrm{~m} \\
=1.609 \mathrm{~km}\end{array}$ & $1 \mathrm{~km}$ & $\begin{array}{l}=39370 \mathrm{in} . \\
=3281 \mathrm{ft} \\
=1093.6 \mathrm{yd} \\
=0.6214 \mathrm{mile} \\
=1000 \mathrm{~m}\end{array}$ \\
\hline & $\begin{array}{l}1 \mathrm{ft} / \mathrm{sec}=0 \\
1 \mathrm{~m} / \mathrm{sec}= \\
1 \mathrm{~km} / \mathrm{h}=0 \\
1 \mathrm{mph}=1 .\end{array}$ & $\begin{array}{l}2 \mathrm{~km} / \mathrm{h} \\
\mathrm{m} / \mathrm{h} \\
\mathrm{mph} \\
\mathrm{h} / \mathrm{h}\end{array}$ & \\
\hline
\end{tabular}

Table B.9

Alternative Measures of Greenhouse Gases

1 pound methane, measured in carbon units $\left(\mathrm{CH}_{4}\right)$

1 pound carbon dioxide, measured in carbon units $\left(\mathrm{CO}_{2}-\mathrm{C}\right)$

1 pound carbon monoxide, measured in carbon units $(\mathrm{CO}-\mathrm{C})$

1 pound nitrous oxide, measured in nitrogen units $\left(\mathrm{N}_{2} \mathrm{O}-\mathrm{N}\right)$
1.333 pounds methane, measured at full

$=$ molecular weight $\left(\mathrm{CH}_{4}\right)$

3.6667 pounds carbon dioxide, measured at full $=$ molecular weight $\left(\mathrm{CO}_{2}\right)$

2.333 pounds carbon monoxide, measured at $=\quad$ full molecular weight $(\mathrm{CO})$

1.571 pounds nitrous oxide, measured at full $=\quad$ molecular weight $\left(\mathrm{N}_{2} \mathrm{O}\right)$ 
Table B.10

Volume and Flow Rate Conversions ${ }^{\mathrm{a}}$

\begin{tabular}{|c|c|c|c|}
\hline \multirow[t]{6}{*}{1 U.S. gal } & $=231$ in. $^{3}$ & 1 liter & $=61.02 \mathrm{in}^{3}$ \\
\hline & $=0.1337 \mathrm{ft}^{3}$ & & $=3.531 \times 10^{-2} \mathrm{ft}^{3}$ \\
\hline & $=3.785$ liters & & $=0.2624$ U.S. gal \\
\hline & $=0.8321$ imperial gal & & $=0.2200$ imperial gal \\
\hline & $=0.0238 \mathrm{bbl}$ & & $=6.29 \times 10^{-3} \mathrm{bbl}$ \\
\hline & $=0.003785 \mathrm{~m}^{3}$ & & $=0.001 \mathrm{~m}^{3}$ \\
\hline \multicolumn{4}{|c|}{ A U.S. gallon of gasoline weighs 6.2 pounds } \\
\hline \multirow[t]{6}{*}{1 imperial gal } & $=277.4$ in. $^{3}$ & $1 \mathrm{bbl}$ & $=9702$ in. $^{3}$ \\
\hline & $=0.1606 \mathrm{ft}^{3}$ & & $=5.615 \mathrm{ft}^{3}$ \\
\hline & $=4.545$ liters & & $=158.97$ liters \\
\hline & $=1.201$ U.S. gal & & $=42$ U.S. gal \\
\hline & $=0.0286 \mathrm{bbl}$ & & $=34.97$ imperial gal \\
\hline & $=0.004546 \mathrm{~m}^{3}$ & & $=0.15897 \mathrm{~m}^{3}$ \\
\hline \multirow[t]{4}{*}{1 U.S. gal/hr } & $=3.209 \mathrm{ft}^{3} /$ day & & $=1171 \mathrm{ft}^{3} /$ year \\
\hline & $=90.84$ liter/day & & $=33157$ liter/year \\
\hline & $=19.97 \mathrm{imperial} \mathrm{gal} / \mathrm{day}$ & & $=7289 \mathrm{imperial} \mathrm{gal} / \mathrm{year}$ \\
\hline & $=0.5712 \mathrm{bbl} / \mathrm{day}$ & & $=207.92 \mathrm{bbl} /$ year \\
\hline \multicolumn{4}{|c|}{ For Imperial gallons, multiply above values by 1.201} \\
\hline \multirow[t]{4}{*}{1 liter/hr } & $=0.8474 \mathrm{ft}^{3} /$ day & & $=309.3 \mathrm{ft}^{3} /$ year \\
\hline & $=6.298$ U.S. gal/day & & $=2299$ U.S. gal $/$ year \\
\hline & $=5.28 \mathrm{imperial} \mathrm{gal} / \mathrm{day}$ & & $=1927 \mathrm{imperial}$ gal $/$ year \\
\hline & $=0.1510 \mathrm{bbl} / \mathrm{day}$ & & $=55.10 \mathrm{bbl} /$ year \\
\hline \multirow[t]{4}{*}{$1 \mathrm{bbl} / \mathrm{hr}$} & $=137.8 \mathrm{ft}^{3} /$ year & & $=49187 \mathrm{ft}^{3}$ year \\
\hline & $=1008$ U.S. gal $/$ day & & $=3.679 \times 10^{5}$ U.S. gal $/$ year \\
\hline & $=839.3$ imperial gal $/$ day & & $=3.063 \times 10^{5}$ imperial gal $/$ year \\
\hline & $=3815$ liter $/$ day & & $=1.393 \times 10^{6}$ liter $/$ day \\
\hline
\end{tabular}

${ }^{\mathrm{a}}$ The conversions for flow rates are identical to those for volume measures, if the time units are identical. 
Table B.11

Power Conversions

\begin{tabular}{|c|c|c|c|c|c|c|}
\hline \multirow[b]{2}{*}{ FROM } & \multicolumn{6}{|c|}{ TO } \\
\hline & Horsepower & Kilowatts & $\begin{array}{c}\text { Metric } \\
\text { horsepower }\end{array}$ & Ft-lb per sec & $\begin{array}{c}\text { Kilocalories } \\
\text { per sec }\end{array}$ & Btu per sec \\
\hline Horsepower & 1 & 0.7457 & 1.014 & 550 & 0.1781 & 0.7068 \\
\hline Kilowatts & 1.341 & 1 & 1.360 & 737.6 & 0.239 & 0.9478 \\
\hline $\begin{array}{l}\text { Metric } \\
\text { horsepower }\end{array}$ & 0.9863 & 0.7355 & 1 & 542.5 & 0.1757 & 0.6971 \\
\hline Ft-lb per sec & $1.36 \times 10^{-3}$ & $1.356 \times 10^{-3}$ & $1.84 \times 10^{-3}$ & 1 & $0.3238 \times 10^{-3}$ & $1.285 \times 10^{-3}$ \\
\hline $\begin{array}{l}\text { Kilocalories } \\
\text { per sec }\end{array}$ & 5.615 & 4.184 & 5.692 & 3088 & 1 & 3.968 \\
\hline Btu per sec & 1.415 & 1.055 & 1.434 & 778.2 & 0.2520 & 1 \\
\hline
\end{tabular}

Table B.12

Mass Conversions

\begin{tabular}{llllll}
\hline & \multicolumn{5}{c}{ TO } \\
\cline { 2 - 6 } FROM & Pound & Kilogram & Short ton & Long ton & Metric ton \\
\hline Pound & 1 & 0.4536 & $5.0 \times 10^{-4}$ & $4.4643 \times 10^{-4}$ & $4.5362 \times 10^{-4}$ \\
Kilogram & 2.205 & 1 & $1.1023 \times 10^{-3}$ & $9.8425 \times 10^{-4}$ & $1.0 \times 10^{-3}$ \\
Short ton & 2,000 & 907.2 & 1 & 0.8929 & 0.9072 \\
Long ton & 2,240 & 1,106 & 1.12 & 1 & 1.016 \\
Metric ton & 2,205 & 1,000 & 1.102 & 0.9842 & 1 \\
\hline
\end{tabular}


Table B.13

Fuel Efficiency Conversions

\begin{tabular}{|c|c|c|c|c|c|}
\hline MPG & Miles/liter & Kilometers/L & $\begin{array}{c}\text { L/100 } \\
\text { kilometers }\end{array}$ & $\begin{array}{l}\text { Grams of } \\
\mathrm{CO}_{2} \\
\text { per mile }\end{array}$ & $\begin{array}{c}\text { Pounds of } \mathrm{CO}_{2} \\
\text { per mile }\end{array}$ \\
\hline 10 & 2.64 & 4.25 & 23.52 & 877.80 & 1.94 \\
\hline 15 & 3.96 & 6.38 & 15.68 & 585.20 & 1.29 \\
\hline 20 & 5.28 & 8.50 & 11.76 & 438.90 & 0.97 \\
\hline 25 & 6.60 & 10.63 & 9.41 & 351.12 & 0.78 \\
\hline 30 & 7.92 & 12.75 & 7.84 & 292.60 & 0.65 \\
\hline 35 & 9.25 & 14.88 & 6.72 & 250.80 & 0.55 \\
\hline 40 & 10.57 & 17.00 & 5.88 & 219.45 & 0.49 \\
\hline 45 & 11.89 & 19.13 & 5.23 & 195.07 & 0.43 \\
\hline 50 & 13.21 & 21.25 & 4.70 & 175.56 & 0.39 \\
\hline 55 & 14.53 & 23.38 & 4.28 & 159.60 & 0.35 \\
\hline 60 & 15.85 & 25.51 & 3.92 & 146.30 & 0.32 \\
\hline 65 & 17.17 & 27.63 & 3.62 & 135.05 & 0.30 \\
\hline 70 & 18.49 & 29.76 & 3.36 & 125.40 & 0.28 \\
\hline 75 & 19.81 & 31.88 & 3.14 & 117.04 & 0.26 \\
\hline 80 & 21.13 & 34.01 & 2.94 & 109.73 & 0.24 \\
\hline 85 & 22.45 & 36.13 & 2.77 & 103.27 & 0.23 \\
\hline 90 & 23.77 & 38.26 & 2.61 & 97.53 & 0.22 \\
\hline 95 & 25.09 & 40.38 & 2.48 & 92.40 & 0.20 \\
\hline 100 & 26.42 & 42.51 & 2.35 & 87.78 & 0.19 \\
\hline 105 & 27.74 & 44.64 & 2.24 & 83.60 & 0.18 \\
\hline 110 & 29.06 & 46.76 & 2.14 & 79.80 & 0.18 \\
\hline 115 & 30.38 & 48.89 & 2.05 & 76.33 & 0.17 \\
\hline 120 & 31.70 & 51.01 & 1.96 & 73.15 & 0.16 \\
\hline 125 & 33.02 & 53.14 & 1.88 & 70.22 & 0.16 \\
\hline 130 & 34.34 & 55.26 & 1.81 & 67.52 & 0.15 \\
\hline 135 & 35.66 & 57.39 & 1.74 & 65.02 & 0.14 \\
\hline 140 & 36.98 & 59.51 & 1.68 & 62.70 & 0.14 \\
\hline 145 & 38.30 & 61.64 & 1.62 & 60.54 & 0.13 \\
\hline 150 & 39.62 & 63.76 & 1.57 & 58.52 & 0.13 \\
\hline Formula & MPG/3.785 & MPG/[3.785/1.609] & 235.24/MPG & 8,778/MPG & 19.4/MPG \\
\hline
\end{tabular}

${ }^{\mathrm{a}}$ For gasoline-fueled vehicles. 
Table B.14

SI Prefixes and Their Values

\begin{tabular}{llll}
\hline & Value & Prefix & Symbol \\
\hline One million million millionth & $10^{-18}$ & atto & $\mathrm{a}$ \\
One thousand million millionth & $10^{-15}$ & femto & $\mathrm{f}$ \\
One million millionth & $10^{-12}$ & pico & $\mathrm{p}$ \\
One thousand millionth & $10^{-9}$ & nano & $\mathrm{n}$ \\
One millionth & $10^{-6}$ & micro & $\mathrm{m}$ \\
One thousandth & $10^{-3}$ & milli & $\mathrm{c}$ \\
One hundredth & $10^{-2}$ & centi & \\
One tenth & $10^{-1}$ & deci & \\
One & $10^{0}$ & & \\
Ten & $10^{1}$ & deca & \\
One hundred & $10^{2}$ & hecto & $\mathrm{k}$ \\
One thousand & $10^{3}$ & kilo & $\mathrm{M}$ \\
One million & $10^{6}$ & mega & $\mathrm{G}$ \\
One billion $^{\mathrm{a}}$ & $10^{9}$ & giga & $\mathrm{T}$ \\
One trillion $^{\mathrm{a}}$ & $10^{12}$ & tera & $\mathrm{P}$ \\
One quadrillion $^{\mathrm{a}}$ & $10^{15}$ & peta & $\mathrm{E}$ \\
One quintillion $^{\mathrm{a}}$ & $10^{18}$ & exa & \\
\hline
\end{tabular}

${ }^{a}$ Care should be exercised in the use of this nomenclature, especially in foreign correspondence, as it is either unknown or carries a different value in other countries. A "billion," for example, signifies a value of $10^{12}$ in most other countries.

Table B.15

Metric Units and Abbreviations

\begin{tabular}{lll}
\hline \multicolumn{1}{c}{ Quantity } & \multicolumn{1}{c}{ Unit name } & \multicolumn{1}{c}{ Symbol } \\
\hline & & $\mathrm{J}$ \\
Energy & joule & $\mathrm{J} / \mathrm{kg}$ \\
Specific energy & joule/kilogram & $\mathrm{J} /(\mathrm{kg} \bullet \mathrm{km})$ \\
Specific energy consumption & joule/kilogram•kilometer & $\mathrm{J} / \mathrm{km}$ \\
Energy consumption & joule/kilometer & $\mathrm{km} / \mathrm{kJ}$ \\
Energy economy & kilometer/kilojoule & $\mathrm{kW}$ \\
Power & kilowatt & $\mathrm{W} / \mathrm{kg}$ \\
Specific power & watt/kilogram & $\mathrm{W} / \mathrm{m}^{3}$ \\
Power density & watt/meter & $\mathrm{km} / \mathrm{h}$ \\
Speed & kilometer/hour & $\mathrm{m} / \mathrm{s}^{2}$ \\
Acceleration & meter/second ${ }^{2}$ & $\mathrm{~km}$ \\
Range (distance) & kilometer & $\mathrm{kg}$ \\
Weight & kilogram & $\mathrm{N} \bullet \mathrm{m}$ \\
Torque & newton•meter & $\mathrm{m}$ \\
Volume & meter ${ }^{3}$ & $\mathrm{~kg}$ \\
Mass; payload & kilogram & $\mathrm{m}$ \\
Length; width & meter & $\mathrm{kg} / \mathrm{J}$ \\
Brake specific fuel consumption & kilogram/joule & $\mathrm{L} / 100 \mathrm{~km}$ \\
Fuel economy (heat engine) & liters/100 km & \\
\hline
\end{tabular}


Table B.16

Carbon Coefficients, 2002

(Million metric tons carbon per quadrillion Btu)

\begin{tabular}{ll}
\hline \multicolumn{1}{c}{ Fuel Type } & \\
\hline Coal & \\
Coal (residential) & 26.04 \\
Coal (commercial) & 26.04 \\
Coal (industrial coking) & 25.63 \\
Coal (industrial other) & 25.74 \\
Coal (electric utility) & 25.98 \\
Natural gas & \\
Natural gas (pipeline) & 14.47 \\
Natural gas (flared) & 14.92 \\
Petroleum & \\
Asphalt and road oil & 20.62 \\
Aviation gasoline & 18.87 \\
Crude oil & 20.30 \\
Distillate fuel & 19.95 \\
Jet fuel & 19.33 \\
Kerosene & 19.72 \\
LPG & 16.99 \\
Lubricants & 20.24 \\
Motor gasoline & 19.34 \\
Petrochemical feed. & 19.37 \\
Petroleum coke & 27.85 \\
Residual fuel & 21.49 \\
Waxes & 19.81 \\
\hline
\end{tabular}

Note: All coefficients based on Higher Heating (Gross Calorific) Value and assume 100 percent combustion. 


\section{Conversion of Constant Dollar Values}

Many types of information in this data book are expressed in dollars. Generally, constant dollars are used-that is, dollars of a fixed value for a specific year, such as 1990 dollars. Converting current dollars to constant dollars, or converting constant dollars for one year to constant dollars for another year, requires conversion factors (Table B.17 and B.18). Table B.17 shows conversion factors for the Consumer Price Index inflation factors. Table B.18 shows conversion factors using the Gross National Product inflation factors. 
Table B.17

Consumer Price Inflation (CPI) Index

\begin{tabular}{|c|c|c|c|c|c|c|c|c|c|c|}
\hline From: & 1970 & 1971 & 1972 & 1973 & 1974 & 1975 & 1976 & 1977 & 1978 & 1979 \\
\hline 1970 & 1.000 & 1.044 & 1.077 & 1.144 & 1.271 & 1.387 & 1.466 & 1.562 & 1.680 & 1.871 \\
\hline 1971 & 0.958 & 1.000 & 1.032 & 1.096 & 1.217 & 1.328 & 1.405 & 1.496 & 1.610 & 1.793 \\
\hline 1972 & 0.928 & 0.969 & 1.000 & 1.062 & 1.179 & 1.287 & 1.361 & 1.450 & 1.560 & 1.737 \\
\hline 1973 & 0.874 & 0.912 & 0.941 & 1.000 & 1.110 & 1.212 & 1.282 & 1.365 & 1.468 & 1.635 \\
\hline 1974 & 0.787 & 0.822 & 0.848 & 0.901 & 1.000 & 1.091 & 1.154 & 1.229 & 1.323 & 1.473 \\
\hline 1975 & 0.721 & 0.753 & 0.777 & 0.825 & 0.916 & 1.000 & 1.058 & 1.126 & 1.212 & 1.349 \\
\hline 1976 & 0.682 & 0.712 & 0.735 & 0.780 & 0.866 & 0.946 & 1.000 & 1.065 & 1.146 & 1.276 \\
\hline 1977 & 0.640 & 0.668 & 0.690 & 0.733 & 0.814 & 0.888 & 0.939 & 1.000 & 1.076 & 1.198 \\
\hline 1978 & 0.595 & 0.621 & 0.641 & 0.681 & 0.756 & 0.825 & 0.873 & 0.929 & 1.000 & 1.113 \\
\hline 1979 & 0.534 & 0.558 & 0.576 & 0.612 & 0.679 & 0.741 & 0.784 & 0.835 & 0.898 & 1.000 \\
\hline 1980 & 0.471 & 0.492 & 0.507 & 0.539 & 0.598 & 0.653 & 0.691 & 0.735 & 0.791 & 0.881 \\
\hline 1981 & 0.427 & 0.446 & 0.460 & 0.488 & 0.542 & 0.592 & 0.626 & 0.667 & 0.717 & 0.799 \\
\hline 1982 & 0.402 & 0.420 & 0.433 & 0.460 & 0.511 & 0.558 & 0.590 & 0.628 & 0.676 & 0.752 \\
\hline 1983 & 0.390 & 0.407 & 0.420 & 0.446 & 0.495 & 0.540 & 0.571 & 0.608 & 0.655 & 0.729 \\
\hline 1984 & 0.373 & 0.390 & 0.402 & 0.427 & 0.474 & 0.518 & 0.548 & 0.583 & 0.628 & 0.699 \\
\hline 1985 & 0.361 & 0.376 & 0.388 & 0.413 & 0.458 & 0.500 & 0.529 & 0.563 & 0.606 & 0.675 \\
\hline 1986 & 0.354 & 0.370 & 0.381 & 0.405 & 0.450 & 0.491 & 0.519 & 0.553 & 0.595 & 0.662 \\
\hline 1987 & 0.342 & 0.357 & 0.368 & 0.391 & 0.434 & 0.474 & 0.501 & 0.533 & 0.574 & 0.639 \\
\hline 1988 & 0.328 & 0.342 & 0.353 & 0.375 & 0.417 & 0.455 & 0.481 & 0.512 & 0.551 & 0.614 \\
\hline 1989 & 0.313 & 0.327 & 0.337 & 0.358 & 0.398 & 0.434 & 0.459 & 0.489 & 0.526 & 0.585 \\
\hline 1990 & 0.297 & 0.310 & 0.320 & 0.340 & 0.377 & 0.412 & 0.435 & 0.464 & 0.499 & 0.555 \\
\hline 1991 & 0.285 & 0.297 & 0.307 & 0.326 & 0.362 & 0.395 & 0.418 & 0.445 & 0.479 & 0.533 \\
\hline 1992 & 0.277 & 0.289 & 0.298 & 0.316 & 0.351 & 0.383 & 0.406 & 0.432 & 0.465 & 0.517 \\
\hline 1993 & 0.269 & 0.280 & 0.289 & 0.307 & 0.341 & 0.372 & 0.394 & 0.419 & 0.451 & 0.502 \\
\hline 1994 & 0.262 & 0.273 & 0.282 & 0.300 & 0.333 & 0.363 & 0.384 & 0.409 & 0.440 & 0.490 \\
\hline 1995 & 0.255 & 0.266 & 0.274 & 0.291 & 0.323 & 0.353 & 0.373 & 0.398 & 0.428 & 0.476 \\
\hline 1996 & 0.247 & 0.258 & 0.266 & 0.283 & 0.314 & 0.343 & 0.363 & 0.386 & 0.416 & 0.463 \\
\hline 1997 & 0.242 & 0.252 & 0.260 & 0.277 & 0.307 & 0.335 & 0.355 & 0.378 & 0.406 & 0.452 \\
\hline 1998 & 0.238 & 0.248 & 0.256 & 0.272 & 0.302 & 0.330 & 0.349 & 0.372 & 0.400 & 0.445 \\
\hline 1999 & 0.233 & 0.243 & 0.251 & 0.267 & 0.296 & 0.323 & 0.342 & 0.364 & 0.391 & 0.436 \\
\hline 2000 & 0.225 & 0.235 & 0.243 & 0.258 & 0.286 & 0.312 & 0.330 & 0.352 & 0.379 & 0.422 \\
\hline 2001 & 0.219 & 0.229 & 0.236 & 0.251 & 0.278 & 0.304 & 0.321 & 0.342 & 0.368 & 0.410 \\
\hline 2002 & 0.216 & 0.225 & 0.232 & 0.247 & 0.274 & 0.299 & 0.316 & 0.337 & 0.362 & 0.404 \\
\hline 2003 & 0.211 & 0.220 & 0.227 & 0.241 & 0.268 & 0.292 & 0.309 & 0.329 & 0.354 & 0.395 \\
\hline 2004 & 0.205 & 0.214 & 0.221 & 0.235 & 0.261 & 0.285 & 0.301 & 0.321 & 0.345 & 0.384 \\
\hline 2005 & 0.199 & 0.207 & 0.214 & 0.227 & 0.252 & 0.275 & 0.291 & 0.310 & 0.334 & 0.372 \\
\hline 2006 & 0.192 & 0.201 & 0.207 & 0.220 & 0.245 & 0.267 & 0.282 & 0.301 & 0.323 & 0.360 \\
\hline 2007 & 0.187 & 0.195 & 0.202 & 0.214 & 0.238 & 0.259 & 0.274 & 0.292 & 0.314 & 0.350 \\
\hline 2008 & 0.180 & 0.188 & 0.194 & 0.206 & 0.229 & 0.250 & 0.264 & 0.281 & 0.303 & 0.337 \\
\hline 2009 & 0.181 & 0.189 & 0.195 & 0.207 & 0.230 & 0.251 & 0.265 & 0.282 & 0.304 & 0.338 \\
\hline
\end{tabular}


Table B.17

Consumer Price Inflation (CPI) Index (Continued)

\begin{tabular}{|c|c|c|c|c|c|c|c|c|c|c|}
\hline From: & 1980 & 1981 & 1982 & 1983 & 1984 & 1985 & 1986 & 1987 & 1988 & 1989 \\
\hline 1970 & 2.124 & 2.343 & 2.487 & 2.567 & 2.678 & 2.773 & 2.825 & 2.928 & 3.049 & 3.196 \\
\hline 1971 & 2.035 & 2.244 & 2.383 & 2.459 & 2.565 & 2.657 & 2.706 & 2.805 & 2.921 & 3.062 \\
\hline 1972 & 1.971 & 2.175 & 2.309 & 2.383 & 2.486 & 2.574 & 2.622 & 2.718 & 2.830 & 2.967 \\
\hline 1973 & 1.856 & 2.047 & 2.173 & 2.243 & 2.340 & 2.423 & 2.468 & 2.559 & 2.664 & 2.793 \\
\hline 1974 & 1.671 & 1.844 & 1.957 & 2.020 & 2.108 & 2.183 & 2.223 & 2.304 & 2.400 & 2.515 \\
\hline 1975 & 1.532 & 1.690 & 1.794 & 1.851 & 1.931 & 2.000 & 2.037 & 2.112 & 2.199 & 2.305 \\
\hline 1976 & 1.448 & 1.598 & 1.696 & 1.750 & 1.826 & 1.891 & 1.926 & 1.996 & 2.079 & 2.179 \\
\hline 1977 & 1.360 & 1.500 & 1.592 & 1.644 & 1.715 & 1.776 & 1.809 & 1.875 & 1.952 & 2.046 \\
\hline 1978 & 1.264 & 1.394 & 1.480 & 1.528 & 1.594 & 1.650 & 1.681 & 1.742 & 1.814 & 1.902 \\
\hline 1979 & 1.135 & 1.252 & 1.329 & 1.372 & 1.431 & 1.482 & 1.510 & 1.565 & 1.629 & 1.708 \\
\hline 1980 & 1.000 & 1.103 & 1.171 & 1.209 & 1.261 & 1.306 & 1.330 & 1.379 & 1.436 & 1.505 \\
\hline 1981 & 0.906 & 1.000 & 1.062 & 1.096 & 1.143 & 1.184 & 1.206 & 1.250 & 1.301 & 1.364 \\
\hline 1982 & 0.854 & 0.942 & 1.000 & 1.032 & 1.077 & 1.115 & 1.136 & 1.177 & 1.226 & 1.285 \\
\hline 1983 & 0.827 & 0.913 & 0.969 & 1.000 & 1.043 & 1.080 & 1.100 & 1.141 & 1.188 & 1.245 \\
\hline 1984 & 0.793 & 0.875 & 0.929 & 0.959 & 1.000 & 1.036 & 1.055 & 1.093 & 1.139 & 1.193 \\
\hline 1985 & 0.766 & 0.845 & 0.897 & 0.926 & 0.966 & 1.000 & 1.019 & 1.056 & 1.099 & 1.152 \\
\hline 1986 & 0.752 & 0.829 & 0.880 & 0.909 & 0.948 & 0.982 & 1.000 & 1.036 & 1.079 & 1.131 \\
\hline 1987 & 0.725 & 0.800 & 0.849 & 0.877 & 0.915 & 0.947 & 0.965 & 1.000 & 1.041 & 1.092 \\
\hline 1988 & 0.697 & 0.768 & 0.816 & 0.842 & 0.878 & 0.910 & 0.926 & 0.960 & 1.000 & 1.048 \\
\hline 1989 & 0.665 & 0.733 & 0.778 & 0.803 & 0.838 & 0.868 & 0.884 & 0.916 & 0.954 & 1.000 \\
\hline 1990 & 0.630 & 0.695 & 0.738 & 0.762 & 0.795 & 0.823 & 0.839 & 0.869 & 0.905 & 0.949 \\
\hline 1991 & 0.605 & 0.667 & 0.709 & 0.731 & 0.763 & 0.790 & 0.805 & 0.834 & 0.869 & 0.910 \\
\hline 1992 & 0.587 & 0.648 & 0.688 & 0.710 & 0.741 & 0.767 & 0.781 & 0.810 & 0.843 & 0.884 \\
\hline 1993 & 0.570 & 0.629 & 0.668 & 0.689 & 0.719 & 0.745 & 0.758 & 0.786 & 0.819 & 0.858 \\
\hline 1994 & 0.556 & 0.613 & 0.651 & 0.672 & 0.701 & 0.726 & 0.740 & 0.767 & 0.798 & 0.837 \\
\hline 1995 & 0.541 & 0.596 & 0.633 & 0.654 & 0.682 & 0.706 & 0.719 & 0.745 & 0.776 & 0.814 \\
\hline 1996 & 0.525 & 0.579 & 0.615 & 0.635 & 0.662 & 0.686 & 0.699 & 0.724 & 0.754 & 0.790 \\
\hline 1997 & 0.513 & 0.566 & 0.601 & 0.621 & 0.647 & 0.670 & 0.683 & 0.708 & 0.737 & 0.773 \\
\hline 1998 & 0.506 & 0.558 & 0.592 & 0.611 & 0.637 & 0.660 & 0.672 & 0.697 & 0.726 & 0.761 \\
\hline 1999 & 0.495 & 0.546 & 0.579 & 0.598 & 0.624 & 0.646 & 0.658 & 0.682 & 0.710 & 0.744 \\
\hline 2000 & 0.479 & 0.528 & 0.560 & 0.578 & 0.603 & 0.625 & 0.636 & 0.660 & 0.687 & 0.720 \\
\hline 2001 & 0.465 & 0.513 & 0.545 & 0.562 & 0.587 & 0.608 & 0.619 & 0.641 & 0.668 & 0.700 \\
\hline 2002 & 0.458 & 0.505 & 0.536 & 0.554 & 0.578 & 0.598 & 0.609 & 0.631 & 0.658 & 0.689 \\
\hline 2003 & 0.448 & 0.494 & 0.524 & 0.541 & 0.565 & 0.585 & 0.596 & 0.617 & 0.643 & 0.674 \\
\hline 2004 & 0.436 & 0.481 & 0.511 & 0.527 & 0.550 & 0.570 & 0.580 & 0.601 & 0.626 & 0.656 \\
\hline 2005 & 0.422 & 0.465 & 0.494 & 0.510 & 0.532 & 0.551 & 0.561 & 0.582 & 0.606 & 0.635 \\
\hline 2006 & 0.409 & 0.451 & 0.479 & 0.494 & 0.515 & 0.534 & 0.544 & 0.563 & 0.587 & 0.615 \\
\hline 2007 & 0.397 & 0.438 & 0.465 & 0.480 & 0.501 & 0.519 & 0.529 & 0.548 & 0.571 & 0.598 \\
\hline 2008 & 0.383 & 0.422 & 0.448 & 0.463 & 0.483 & 0.500 & 0.509 & 0.528 & 0.549 & 0.576 \\
\hline 2009 & 0.3840 & 0.424 & 0.450 & 0.464 & 0.484 & 0.502 & 0.511 & 0.530 & 0.551 & 0.578 \\
\hline
\end{tabular}


Table B.17

Consumer Price Inflation (CPI) Index (Continued)

\begin{tabular}{|c|c|c|c|c|c|c|c|c|c|c|}
\hline From: & 1990 & 1991 & 1992 & 1993 & 1994 & 1995 & 1996 & 1997 & 1998 & 1999 \\
\hline 1970 & 3.369 & 3.510 & 3.616 & 3.724 & 3.820 & 3.928 & 4.044 & 4.137 & 4.201 & 4.294 \\
\hline 1971 & 3.227 & 3.363 & 3.464 & 3.568 & 3.659 & 3.763 & 3.874 & 3.963 & 4.025 & 4.114 \\
\hline 1972 & 3.127 & 3.258 & 3.356 & 3.457 & 3.545 & 3.646 & 3.754 & 3.840 & 3.900 & 3.986 \\
\hline 1973 & 2.944 & 3.068 & 3.160 & 3.255 & 3.338 & 3.432 & 3.534 & 3.615 & 3.671 & 3.752 \\
\hline 1974 & 2.651 & 2.763 & 2.846 & 2.931 & 3.006 & 3.091 & 3.183 & 3.256 & 3.306 & 3.379 \\
\hline 1975 & 2.429 & 2.532 & 2.608 & 2.686 & 2.755 & 2.833 & 2.916 & 2.983 & 3.030 & 3.097 \\
\hline 1976 & 2.297 & 2.394 & 2.466 & 2.540 & 2.605 & 2.678 & 2.757 & 2.821 & 2.865 & 2.928 \\
\hline 1977 & 2.157 & 2.248 & 2.315 & 2.384 & 2.446 & 2.515 & 2.589 & 2.649 & 2.690 & 2.749 \\
\hline 1978 & 2.005 & 2.089 & 2.152 & 2.216 & 2.273 & 2.337 & 2.406 & 2.462 & 2.500 & 2.555 \\
\hline 1979 & 1.800 & 1.876 & 1.933 & 1.990 & 2.041 & 2.099 & 2.161 & 2.211 & 2.245 & 2.295 \\
\hline 1980 & 1.586 & 1.653 & 1.703 & 1.754 & 1.799 & 1.850 & 1.904 & 1.948 & 1.978 & 2.022 \\
\hline 1981 & 1.438 & 1.498 & 1.543 & 1.590 & 1.630 & 1.677 & 1.726 & 1.766 & 1.793 & 1.833 \\
\hline 1982 & 1.354 & 1.411 & 1.454 & 1.497 & 1.536 & 1.579 & 1.626 & 1.663 & 1.689 & 1.726 \\
\hline 1983 & 1.312 & 1.367 & 1.409 & 1.451 & 1.488 & 1.530 & 1.575 & 1.611 & 1.637 & 1.673 \\
\hline 1984 & 1.258 & 1.311 & 1.350 & 1.391 & 1.426 & 1.467 & 1.510 & 1.545 & 1.569 & 1.603 \\
\hline 1985 & 1.215 & 1.266 & 1.304 & 1.343 & 1.377 & 1.416 & 1.458 & 1.492 & 1.515 & 1.548 \\
\hline 1986 & 1.193 & 1.243 & 1.280 & 1.318 & 1.352 & 1.391 & 1.432 & 1.464 & 1.487 & 1.520 \\
\hline 1987 & 1.151 & 1.199 & 1.235 & 1.272 & 1.305 & 1.342 & 1.381 & 1.413 & 1.435 & 1.467 \\
\hline 1988 & 1.105 & 1.151 & 1.186 & 1.221 & 1.253 & 1.288 & 1.326 & 1.357 & 1.378 & 1.408 \\
\hline 1989 & 1.054 & 1.098 & 1.131 & 1.165 & 1.195 & 1.229 & 1.265 & 1.294 & 1.315 & 1.344 \\
\hline 1990 & 1.000 & 1.042 & 1.073 & 1.106 & 1.134 & 1.166 & 1.200 & 1.228 & 1.247 & 1.275 \\
\hline 1991 & 0.960 & 1.000 & 1.030 & 1.061 & 1.088 & 1.119 & 1.152 & 1.178 & 1.197 & 1.223 \\
\hline 1992 & 0.932 & 0.971 & 1.000 & 1.030 & 1.056 & 1.086 & 1.118 & 1.144 & 1.162 & 1.187 \\
\hline 1993 & 0.904 & 0.943 & 0.971 & 1.000 & 1.026 & 1.055 & 1.086 & 1.111 & 1.128 & 1.153 \\
\hline 1994 & 0.882 & 0.919 & 0.947 & 0.975 & 1.000 & 1.028 & 1.059 & 1.083 & 1.100 & 1.124 \\
\hline 1995 & 0.858 & 0.894 & 0.921 & 0.948 & 0.972 & 1.000 & 1.030 & 1.053 & 1.070 & 1.093 \\
\hline 1996 & 0.833 & 0.868 & 0.894 & 0.921 & 0.945 & 0.971 & 1.000 & 1.023 & 1.039 & 1.062 \\
\hline 1997 & 0.814 & 0.849 & 0.874 & 0.900 & 0.923 & 0.950 & 0.978 & 1.000 & 1.016 & 1.038 \\
\hline 1998 & 0.802 & 0.836 & 0.861 & 0.887 & 0.909 & 0.935 & 0.963 & 0.985 & 1.000 & 1.022 \\
\hline 1999 & 0.785 & 0.818 & 0.842 & 0.867 & 0.890 & 0.915 & 0.942 & 0.963 & 0.978 & 1.000 \\
\hline 2000 & 0.759 & 0.791 & 0.815 & 0.839 & 0.861 & 0.885 & 0.911 & 0.932 & 0.947 & 0.967 \\
\hline 2001 & 0.738 & 0.769 & 0.792 & 0.816 & 0.837 & 0.861 & 0.886 & 0.906 & 0.920 & 0.941 \\
\hline 2002 & 0.727 & 0.757 & 0.780 & 0.803 & 0.824 & 0.847 & 0.872 & 0.892 & 0.906 & 0.926 \\
\hline 2003 & 0.710 & 0.740 & 0.763 & 0.785 & 0.805 & 0.828 & 0.853 & 0.872 & 0.886 & 0.905 \\
\hline 2004 & 0.692 & 0.721 & 0.743 & 0.765 & 0.785 & 0.807 & 0.831 & 0.850 & 0.863 & 0.882 \\
\hline 2005 & 0.669 & 0.697 & 0.718 & 0.740 & 0.759 & 0.780 & 0.803 & 0.822 & 0.835 & 0.853 \\
\hline 2006 & 0.648 & 0.676 & 0.696 & 0.717 & 0.735 & 0.756 & 0.778 & 0.796 & 0.809 & 0.826 \\
\hline 2007 & 0.630 & 0.657 & 0.677 & 0.697 & 0.715 & 0.735 & 0.757 & 0.774 & 0.786 & 0.804 \\
\hline 2008 & 0.607 & 0.633 & 0.652 & 0.671 & 0.688 & 0.708 & 0.729 & 0.745 & 0.757 & 0.774 \\
\hline 2009 & 0.602 & 0.635 & 0.654 & 0.674 & 0.691 & 0.710 & 0.731 & 0.748 & 0.760 & 0.777 \\
\hline
\end{tabular}


Table B.17

Consumer Price Inflation (CPI) Index (Continued)

\begin{tabular}{|c|c|c|c|c|c|c|c|c|c|c|}
\hline From: & 2000 & 2001 & 2002 & 2003 & 2004 & 2005 & 2006 & 2007 & 2008 & 2009 \\
\hline 1970 & 4.438 & 4.564 & 4.637 & 4.742 & 4.869 & 5.034 & 5.196 & 5.344 & 5.549 & 5.529 \\
\hline 1971 & 4.252 & 4.373 & 4.442 & 4.543 & 4.664 & 4.822 & 4.978 & 5.120 & 5.316 & 5.297 \\
\hline 1972 & 4.120 & 4.237 & 4.304 & 4.402 & 4.519 & 4.672 & 4.823 & 4.960 & 5.151 & 5.132 \\
\hline 1973 & 3.878 & 3.989 & 4.052 & 4.144 & 4.255 & 4.399 & 4.541 & 4.670 & 4.849 & 4.832 \\
\hline 1974 & 3.493 & 3.592 & 3.649 & 3.732 & 3.832 & 3.961 & 4.089 & 4.206 & 4.367 & 4.352 \\
\hline 1975 & 3.201 & 3.292 & 3.344 & 3.420 & 3.511 & 3.630 & 3.747 & 3.854 & 4.002 & 3.988 \\
\hline 1976 & 3.026 & 3.112 & 3.162 & 3.234 & 3.320 & 3.432 & 3.543 & 3.644 & 3.784 & 3.770 \\
\hline 1977 & 2.842 & 2.922 & 2.969 & 3.036 & 3.117 & 3.223 & 3.327 & 3.421 & 3.553 & 3.540 \\
\hline 1978 & 2.641 & 2.716 & 2.759 & 2.822 & 2.897 & 2.995 & 3.092 & 3.180 & 3.302 & 3.290 \\
\hline 1979 & 2.372 & 2.439 & 2.478 & 2.534 & 2.602 & 2.690 & 2.777 & 2.856 & 2.966 & 2.955 \\
\hline 1980 & 2.090 & 2.149 & 2.183 & 2.233 & 2.292 & 2.370 & 2.447 & 2.516 & 2.613 & 2.604 \\
\hline 1981 & 1.894 & 1.948 & 1.979 & 2.024 & 2.078 & 2.149 & 2.218 & 2.281 & 2.369 & 2.360 \\
\hline 1982 & 1.784 & 1.835 & 1.864 & 1.907 & 1.958 & 2.024 & 2.089 & 2.149 & 2.231 & 2.223 \\
\hline 1983 & 1.729 & 1.778 & 1.806 & 1.847 & 1.897 & 1.961 & 2.024 & 2.082 & 2.162 & 2.154 \\
\hline 1984 & 1.657 & 1.705 & 1.731 & 1.771 & 1.818 & 1.880 & 1.940 & 1.996 & 2.072 & 2.065 \\
\hline 1985 & 1.600 & 1.646 & 1.672 & 1.710 & 1.756 & 1.815 & 1.874 & 1.927 & 2.001 & 1.994 \\
\hline 1986 & 1.571 & 1.616 & 1.641 & 1.679 & 1.724 & 1.782 & 1.839 & 1.892 & 1.964 & 1.957 \\
\hline 1987 & 1.516 & 1.559 & 1.584 & 1.620 & 1.663 & 1.719 & 1.775 & 1.825 & 1.895 & 1.889 \\
\hline 1988 & 1.456 & 1.497 & 1.521 & 1.555 & 1.597 & 1.651 & 1.704 & 1.753 & 1.820 & 1.813 \\
\hline 1989 & 1.389 & 1.428 & 1.451 & 1.484 & 1.523 & 1.575 & 1.626 & 1.672 & 1.736 & 1.730 \\
\hline 1990 & 1.318 & 1.355 & 1.376 & 1.408 & 1.445 & 1.494 & 1.542 & 1.586 & 1.647 & 1.641 \\
\hline 1991 & 1.264 & 1.300 & 1.321 & 1.351 & 1.387 & 1.434 & 1.480 & 1.522 & 1.581 & 1.575 \\
\hline 1992 & 1.227 & 1.262 & 1.282 & 1.311 & 1.346 & 1.392 & 1.437 & 1.478 & 1.535 & 1.529 \\
\hline 1993 & 1.192 & 1.226 & 1.245 & 1.273 & 1.307 & 1.352 & 1.395 & 1.435 & 1.490 & 1.485 \\
\hline 1994 & 1.162 & 1.195 & 1.214 & 1.242 & 1.275 & 1.318 & 1.360 & 1.399 & 1.453 & 1.448 \\
\hline 1995 & 1.130 & 1.162 & 1.180 & 1.207 & 1.240 & 1.281 & 1.323 & 1.360 & 1.413 & 1.408 \\
\hline 1996 & 1.098 & 1.129 & 1.147 & 1.173 & 1.204 & 1.245 & 1.285 & 1.321 & 1.372 & 1.367 \\
\hline 1997 & 1.073 & 1.103 & 1.121 & 1.146 & 1.177 & 1.217 & 1.256 & 1.292 & 1.341 & 1.337 \\
\hline 1998 & 1.056 & 1.087 & 1.104 & 1.129 & 1.159 & 1.198 & 1.237 & 1.272 & 1.321 & 1.316 \\
\hline 1999 & 1.034 & 1.063 & 1.080 & 1.104 & 1.134 & 1.172 & 1.210 & 1.245 & 1.292 & 1.288 \\
\hline 2000 & 1.000 & 1.028 & 1.045 & 1.069 & 1.097 & 1.134 & 1.171 & 1.204 & 1.250 & 1.246 \\
\hline 2001 & 0.972 & 1.000 & 1.016 & 1.039 & 1.067 & 1.103 & 1.138 & 1.171 & 1.216 & 1.211 \\
\hline 2002 & 0.957 & 0.984 & 1.000 & 1.023 & 1.050 & 1.086 & 1.121 & 1.153 & 1.197 & 1.193 \\
\hline 2003 & 0.936 & 0.963 & 0.978 & 1.000 & 1.027 & 1.061 & 1.096 & 1.127 & 1.170 & 1.166 \\
\hline 2004 & 0.912 & 0.938 & 0.952 & 0.974 & 1.000 & 1.034 & 1.067 & 1.098 & 1.140 & 1.136 \\
\hline 2005 & 0.882 & 0.907 & 0.921 & 0.942 & 0.967 & 1.000 & 1.032 & 1.062 & 1.102 & 1.098 \\
\hline 2006 & 0.854 & 0.878 & 0.892 & 0.913 & 0.937 & 0.969 & 1.000 & 1.028 & 1.068 & 1.064 \\
\hline 2007 & 0.831 & 0.854 & 0.868 & 0.887 & 0.911 & 0.942 & 0.972 & 1.000 & 1.038 & 1.035 \\
\hline 2008 & 0.800 & 0.823 & 0.836 & 0.855 & 0.877 & 0.907 & 0.936 & 0.963 & 1.000 & 0.996 \\
\hline 2009 & 0.803 & 0.825 & 0.839 & 0.858 & 0.881 & 0.910 & 0.940 & 0.966 & 1.004 & 1.000 \\
\hline
\end{tabular}

Source:

U.S. Bureau of Labor Statistics. 
Table B.18

Gross National Product Implicit Price Deflator

\begin{tabular}{|c|c|c|c|c|c|c|c|c|c|c|}
\hline From: & 1970 & 1971 & 1972 & 1973 & 1974 & 1975 & 1976 & 1977 & 1978 & 1979 \\
\hline 1970 & 1.000 & 1.050 & 1.096 & 1.157 & 1.261 & 1.380 & 1.460 & 1.553 & 1.662 & 1.800 \\
\hline 1971 & 0.952 & 1.000 & 1.043 & 1.102 & 1.201 & 1.315 & 1.391 & 1.479 & 1.583 & 1.714 \\
\hline 1972 & 0.913 & 0.958 & 1.000 & 1.056 & 1.151 & 1.260 & 1.333 & 1.418 & 1.517 & 1.643 \\
\hline 1973 & 0.864 & 0.908 & 0.947 & 1.000 & 1.090 & 1.193 & 1.262 & 1.342 & 1.437 & 1.556 \\
\hline 1974 & 0.793 & 0.833 & 0.869 & 0.917 & 1.000 & 1.094 & 1.158 & 1.231 & 1.318 & 1.427 \\
\hline 1975 & 0.724 & 0.761 & 0.794 & 0.838 & 0.914 & 1.000 & 1.058 & 1.125 & 1.204 & 1.304 \\
\hline 1976 & 0.685 & 0.719 & 0.750 & 0.792 & 0.864 & 0.945 & 1.000 & 1.064 & 1.138 & 1.233 \\
\hline 1977 & 0.644 & 0.676 & 0.705 & 0.745 & 0.812 & 0.889 & 0.940 & 1.000 & 1.070 & 1.159 \\
\hline 1978 & 0.602 & 0.632 & 0.659 & 0.696 & 0.759 & 0.830 & 0.878 & 0.934 & 1.000 & 1.083 \\
\hline 1979 & 0.555 & 0.583 & 0.609 & 0.643 & 0.701 & 0.767 & 0.811 & 0.863 & 0.923 & 1.000 \\
\hline 1980 & 0.509 & 0.535 & 0.558 & 0.589 & 0.642 & 0.703 & 0.744 & 0.791 & 0.847 & 0.917 \\
\hline 1981 & 0.466 & 0.489 & 0.510 & 0.539 & 0.587 & 0.643 & 0.680 & 0.723 & 0.774 & 0.838 \\
\hline 1982 & 0.439 & 0.461 & 0.481 & 0.508 & 0.553 & 0.606 & 0.641 & 0.682 & 0.729 & 0.790 \\
\hline 1983 & 0.422 & 0.443 & 0.462 & 0.488 & 0.532 & 0.583 & 0.616 & 0.656 & 0.702 & 0.760 \\
\hline 1984 & 0.407 & 0.427 & 0.446 & 0.471 & 0.513 & 0.562 & 0.594 & 0.632 & 0.676 & 0.732 \\
\hline 1985 & 0.395 & 0.415 & 0.433 & 0.457 & 0.498 & 0.545 & 0.576 & 0.613 & 0.656 & 0.711 \\
\hline 1986 & 0.386 & 0.406 & 0.423 & 0.447 & 0.487 & 0.533 & 0.564 & 0.600 & 0.642 & 0.695 \\
\hline 1987 & 0.376 & 0.395 & 0.412 & 0.435 & 0.747 & 0.519 & 0.549 & 0.584 & 0.625 & 0.677 \\
\hline 1988 & 0.364 & 0.382 & 0.398 & 0.421 & 0.459 & 0.502 & 0.531 & 0.565 & 0.604 & 0.654 \\
\hline 1989 & 0.350 & 0.368 & 0.384 & 0.405 & 0.442 & 0.483 & 0.511 & 0.544 & 0.582 & 0.631 \\
\hline 1990 & 0.337 & 0.354 & 0.369 & 0.390 & 0.425 & 0.465 & 0.492 & 0.524 & 0.561 & 0.607 \\
\hline 1991 & 0.326 & 0.342 & 0.357 & 0.377 & 0.411 & 0.450 & 0.476 & 0.506 & 0.542 & 0.587 \\
\hline 1992 & 0.319 & 0.334 & 0.349 & 0.369 & 0.402 & 0.440 & 0.465 & 0.495 & 0.530 & 0.573 \\
\hline 1993 & 0.311 & 0.327 & 0.341 & 0.360 & 0.393 & 0.430 & 0.455 & 0.483 & 0.517 & 0.560 \\
\hline 1994 & 0.305 & 0.320 & 0.334 & 0.353 & 0.384 & 0.421 & 0.445 & 0.473 & 0.507 & 0.549 \\
\hline 1995 & 0.299 & 0.314 & 0.327 & 0.346 & 0.377 & 0.412 & 0.436 & 0.464 & 0.497 & 0.538 \\
\hline 1996 & 0.293 & 0.308 & 0.321 & 0.339 & 0.370 & 0.405 & 0.428 & 0.455 & 0.487 & 0.528 \\
\hline 1997 & 0.288 & 0.303 & 0.316 & 0.334 & 0.364 & 0.398 & 0.421 & 0.448 & 0.479 & 0.519 \\
\hline 1998 & 0.285 & 0.299 & 0.312 & 0.330 & 0.360 & 0.394 & 0.416 & 0.443 & 0.474 & 0.513 \\
\hline 1999 & 0.281 & 0.295 & 0.308 & 0.325 & 0.355 & 0.388 & 0.410 & 0.437 & 0.467 & 0.506 \\
\hline 2000 & 0.275 & 0.289 & 0.301 & 0.318 & 0.347 & 0.380 & 0.402 & 0.427 & 0.457 & 0.495 \\
\hline 2001 & 0.269 & 0.282 & 0.294 & 0.311 & 0.339 & 0.371 & 0.392 & 0.417 & 0.447 & 0.484 \\
\hline 2002 & 0.264 & 0.277 & 0.289 & 0.306 & 0.333 & 0.365 & 0.386 & 0.410 & 0.439 & 0.475 \\
\hline 2003 & 0.259 & 0.272 & 0.283 & 0.299 & 0.326 & 0.357 & 0.378 & 0.402 & 0.430 & 0.465 \\
\hline 2004 & 0.251 & 0.264 & 0.276 & 0.291 & 0.317 & 0.347 & 0.367 & 0.391 & 0.418 & 0.453 \\
\hline 2005 & 0.244 & 0.256 & 0.267 & 0.282 & 0.308 & 0.337 & 0.356 & 0.379 & 0.406 & 0.439 \\
\hline 2006 & 0.236 & 0.248 & 0.259 & 0.273 & 0.298 & 0.326 & 0.345 & 0.367 & 0.392 & 0.425 \\
\hline 2007 & 0.230 & 0.241 & 0.252 & 0.266 & 0.290 & 0.317 & 0.335 & 0.357 & 0.382 & 0.413 \\
\hline 2008 & 0.225 & 0.236 & 0.246 & 0.260 & 0.283 & 0.310 & 0.328 & 0.349 & 0.374 & 0.405 \\
\hline 2009 & 0.221 & 0.232 & 0.242 & 0.256 & 0.279 & 0.306 & 0.323 & 0.344 & 0.368 & 0.399 \\
\hline
\end{tabular}


Table B.18

Gross National Product Implicit Price Deflator (Continued)

\begin{tabular}{|c|c|c|c|c|c|c|c|c|c|c|}
\hline From: & 1980 & 1981 & 1982 & 1983 & 1984 & 1985 & 1986 & 1987 & 1988 & 1989 \\
\hline 1970 & 1.963 & 2.148 & 2.279 & 2.369 & 2.458 & 2.533 & 2.589 & 2.660 & 2.751 & 2.855 \\
\hline 1971 & 1.870 & 2.046 & 2.170 & 2.256 & 2.341 & 2.413 & 2.466 & 2.533 & 2.620 & 2.719 \\
\hline 1972 & 1.792 & 1.960 & 2.080 & 2.162 & 2.244 & 2.312 & 2.363 & 2.428 & 2.510 & 2.606 \\
\hline 1973 & 1.697 & 1.857 & 1.970 & 2.048 & 2.125 & 2.190 & 2.238 & 2.299 & 2.378 & 2.468 \\
\hline 1974 & 1.557 & 1.703 & 1.807 & 1.879 & 1.949 & 2.009 & 2.053 & 2.109 & 2.181 & 2.264 \\
\hline 1975 & 1.422 & 1.556 & 1.651 & 1.716 & 1.781 & 1.835 & 1.876 & 1.927 & 1.993 & 2.068 \\
\hline 1976 & 1.344 & 1.471 & 1.561 & 1.623 & 1.683 & 1.735 & 1.773 & 1.822 & 1.884 & 1.955 \\
\hline 1977 & 1.264 & 1.383 & 1.467 & 1.525 & 1.583 & 1.631 & 1.667 & 1.713 & 1.771 & 1.838 \\
\hline 1978 & 1.181 & 1.292 & 1.371 & 1.425 & 1.479 & 1.524 & 1.557 & 1.600 & 1.655 & 1.717 \\
\hline 1979 & 1.091 & 1.193 & 1.266 & 1.316 & 1.366 & 1.407 & 1.438 & 1.478 & 1.528 & 1.586 \\
\hline 1980 & 1.000 & 1.094 & 1.161 & 1.207 & 1.252 & 1.290 & 1.319 & 1.355 & 1.401 & 1.454 \\
\hline 1981 & 0.914 & 1.000 & 1.061 & 1.103 & 1.144 & 1.179 & 1.205 & 1.238 & 1.281 & 1.329 \\
\hline 1982 & 0.861 & 0.943 & 1.000 & 1.040 & 1.079 & 1.112 & 1.136 & 1.167 & 1.207 & 1.253 \\
\hline 1983 & 0.829 & 0.907 & 0.962 & 1.000 & 1.038 & 1.069 & 1.093 & 1.123 & 1.161 & 1.205 \\
\hline 1984 & 0.799 & 0.874 & 0.927 & 0.964 & 1.000 & 1.031 & 1.053 & 1.082 & 1.119 & 1.161 \\
\hline 1985 & 0.775 & 0.848 & 0.900 & 0.935 & 0.970 & 1.000 & 1.022 & 1.050 & 1.086 & 1.127 \\
\hline 1986 & 0.758 & 0.830 & 0.880 & 0.915 & 0.950 & 0.978 & 1.000 & 1.027 & 1.063 & 1.103 \\
\hline 1987 & 0.738 & 0.808 & 0.857 & 0.891 & 0.924 & 0.952 & 0.973 & 1.000 & 1.034 & 1.073 \\
\hline 1988 & 0.714 & 0.781 & 0.828 & 0.861 & 0.894 & 0.921 & 0.941 & 0.967 & 1.000 & 1.038 \\
\hline 1989 & 0.688 & 0.752 & 0.798 & 0.830 & 0.861 & 0.887 & 0.907 & 0.932 & 0.963 & 1.000 \\
\hline 1990 & 0.662 & 0.724 & 0.768 & 0.799 & 0.829 & 0.854 & 0.873 & 0.897 & 0.928 & 0.963 \\
\hline 1991 & 0.640 & 0.700 & 0.743 & 0.772 & 0.801 & 0.825 & 0.844 & 0.867 & 0.896 & 0.930 \\
\hline 1992 & 0.625 & 0.684 & 0.726 & 0.755 & 0.783 & 0.807 & 0.825 & 0.847 & 0.876 & 0.909 \\
\hline 1993 & 0.611 & 0.669 & 0.709 & 0.738 & 0.765 & 0.789 & 0.806 & 0.828 & 0.856 & 0.889 \\
\hline 1994 & 0.598 & 0.655 & 0.695 & 0.722 & 0.749 & 0.772 & 0.789 & 0.811 & 0.838 & 0.870 \\
\hline 1995 & 0.586 & 0.642 & 0.681 & 0.708 & 0.734 & 0.757 & 0.773 & 0.794 & 0.822 & 0.853 \\
\hline 1996 & 0.575 & 0.630 & 0.668 & 0.694 & 0.721 & 0.743 & 0.759 & 0.780 & 0.806 & 0.837 \\
\hline 1997 & 0.566 & 0.619 & 0.657 & 0.683 & 0.709 & 0.730 & 0.746 & 0.767 & 0.793 & 0.823 \\
\hline 1998 & 0.560 & 0.613 & 0.650 & 0.676 & 0.701 & 0.722 & 0.738 & 0.759 & 0.784 & 0.814 \\
\hline 1999 & 0.552 & 0.604 & 0.641 & 0.666 & 0.691 & 0.712 & 0.728 & 0.748 & 0.773 & 0.803 \\
\hline 2000 & 0.540 & 0.591 & 0.627 & 0.652 & 0.676 & 0.697 & 0.712 & 0.732 & 0.757 & 0.785 \\
\hline 2001 & 0.528 & 0.577 & 0.612 & 0.637 & 0.660 & 0.681 & 0.696 & 0.715 & 0.739 & 0.767 \\
\hline 2002 & 0.518 & 0.567 & 0.602 & 0.626 & 0.649 & 0.669 & 0.684 & 0.702 & 0.726 & 0.754 \\
\hline 2003 & 0.508 & 0.555 & 0.589 & 0.613 & 0.636 & 0.655 & 0.669 & 0.688 & 0.711 & 0.738 \\
\hline 2004 & 0.494 & 0.540 & 0.573 & 0.596 & 0.618 & 0.637 & 0.651 & 0.669 & 0.692 & 0.718 \\
\hline 2005 & 0.479 & 0.524 & 0.556 & 0.578 & 0.600 & 0.618 & 0.632 & 0.649 & 0.671 & 0.697 \\
\hline 2006 & 0.463 & 0.507 & 0.538 & 0.559 & 0.580 & 0.598 & 0.611 & 0.628 & 0.649 & 0.674 \\
\hline 2007 & 0.451 & 0.493 & 0.523 & 0.544 & 0.564 & 0.582 & 0.594 & 0.611 & 0.632 & 0.656 \\
\hline 2008 & 0.441 & 0.483 & 0.512 & 0.533 & 0.533 & 0.569 & 0.582 & 0.598 & 0.618 & 0.642 \\
\hline 2009 & 0.435 & 0.476 & 0.505 & 0.525 & 0.544 & 0.561 & 0.573 & 0.590 & 0.610 & 0.633 \\
\hline
\end{tabular}


Table B.18

Gross National Product Implicit Price Deflator (Continued)

\begin{tabular}{|c|c|c|c|c|c|c|c|c|c|c|}
\hline From: & 1990 & 1991 & 1992 & 1993 & 1994 & 1995 & 1996 & 1997 & 1998 & 1999 \\
\hline 1970 & 2.966 & 3.069 & 3.140 & 3.212 & 3.281 & 3.348 & 3.412 & 3.468 & 3.507 & 3.557 \\
\hline 1971 & 2.824 & 2.923 & 2.990 & 3.059 & 3.124 & 3.189 & 3.249 & 3.303 & 3.340 & 3.388 \\
\hline 1972 & 2.707 & 2.801 & 2.865 & 2.932 & 2.994 & 3.056 & 3.114 & 3.165 & 3.200 & 3.247 \\
\hline 1973 & 2.563 & 2.653 & 2.714 & 2.777 & 2.836 & 2.894 & 2.949 & 2.998 & 3.031 & 3.075 \\
\hline 1974 & 2.351 & 2.433 & 2.489 & 2.547 & 2.601 & 2.655 & 2.705 & 2.750 & 2.780 & 2.821 \\
\hline 1975 & 2.148 & 2.224 & 2.274 & 2.327 & 2.377 & 2.426 & 2.472 & 2.513 & 2.540 & 2.577 \\
\hline 1976 & 2.031 & 2.102 & 2.150 & 2.200 & 2.247 & 2.293 & 2.336 & 2.375 & 2.401 & 2.436 \\
\hline 1977 & 1.909 & 1.976 & 2.021 & 2.068 & 2.112 & 2.156 & 2.197 & 2.233 & 2.258 & 2.290 \\
\hline 1978 & 1.784 & 1.846 & 1.889 & 1.932 & 1.974 & 2.014 & 2.052 & 2.086 & 2.109 & 2.140 \\
\hline 1979 & 1.647 & 1.705 & 1.744 & 1.785 & 1.822 & 1.860 & 1.895 & 1.927 & 1.948 & 1.976 \\
\hline 1980 & 1.510 & 1.563 & 1.599 & 1.636 & 1.671 & 1.705 & 1.738 & 1.767 & 1.786 & 1.812 \\
\hline 1981 & 1.381 & 1.429 & 1.462 & 1.496 & 1.527 & 1.559 & 1.588 & 1.615 & 1.633 & 1.656 \\
\hline 1982 & 1.301 & 1.347 & 1.378 & 1.410 & 1.440 & 1.469 & 1.497 & 1.522 & 1.539 & 1.561 \\
\hline 1983 & 1.252 & 1.295 & 1.325 & 1.356 & 1.385 & 1.413 & 1.440 & 1.464 & 1.480 & 1.501 \\
\hline 1984 & 1.206 & 1.249 & 1.277 & 1.307 & 1.335 & 1.362 & 1.388 & 1.411 & 1.426 & 1.447 \\
\hline 1985 & 1.171 & 1.212 & 1.239 & 1.268 & 1.295 & 1.322 & 1.347 & 1.369 & 1.384 & 1.404 \\
\hline 1986 & 1.145 & 1.186 & 1.213 & 1.241 & 1.267 & 1.293 & 1.318 & 1.340 & 1.354 & 1.374 \\
\hline 1987 & 1.115 & 1.154 & 1.180 & 1.208 & 1.233 & 1.259 & 1.283 & 1.304 & 1.318 & 1.337 \\
\hline 1988 & 1.078 & 1.116 & 1.141 & 1.168 & 1.193 & 1.217 & 1.240 & 1.261 & 1.275 & 1.293 \\
\hline 1989 & 1.039 & 1.075 & 1.100 & 1.125 & 1.149 & 1.173 & 1.195 & 1.215 & 1.228 & 1.246 \\
\hline 1990 & 1.000 & 1.035 & 1.059 & 1.083 & 1.106 & 1.129 & 1.150 & 1.170 & 1.182 & 1.200 \\
\hline 1991 & 0.966 & 1.000 & 1.023 & 1.047 & 1.069 & 1.091 & 1.112 & 1.130 & 1.143 & 1.159 \\
\hline 1992 & 0.945 & 0.978 & 1.000 & 1.023 & 1.045 & 1.066 & 1.087 & 1.105 & 1.117 & 1.133 \\
\hline 1993 & 0.923 & 0.955 & 0.977 & 1.000 & 1.021 & 1.042 & 1.062 & 1.080 & 1.092 & 1.107 \\
\hline 1994 & 0.904 & 0.935 & 0.957 & 0.979 & 1.000 & 1.021 & 1.040 & 1.057 & 1.069 & 1.084 \\
\hline 1995 & 0.886 & 0.917 & 0.938 & 0.959 & 0.980 & 1.000 & 1.019 & 1.036 & 1.047 & 1.062 \\
\hline 1996 & 0.869 & 0.900 & 0.920 & 0.942 & 0.962 & 0.981 & 1.000 & 1.017 & 1.028 & 1.043 \\
\hline 1997 & 0.855 & 0.885 & 0.905 & 0.926 & 0.946 & 0.965 & 0.984 & 1.000 & 1.011 & 1.026 \\
\hline 1998 & 0.846 & 0.875 & 0.895 & 0.916 & 0.936 & 0.955 & 0.973 & 0.989 & 1.000 & 1.014 \\
\hline 1999 & 0.834 & 0.863 & 0.883 & 0.903 & 0.922 & 0.941 & 0.959 & 0.975 & 0.986 & 1.000 \\
\hline 2000 & 0.816 & 0.844 & 0.864 & 0.884 & 0.903 & 0.921 & 0.939 & 0.954 & 0.965 & 0.979 \\
\hline 2001 & 0.797 & 0.825 & 0.844 & 0.863 & 0.882 & 0.900 & 0.917 & 0.932 & 0.942 & 0.956 \\
\hline 2002 & 0.783 & 0.811 & 0.829 & 0.848 & 0.866 & 0.884 & 0.901 & 0.916 & 0.926 & 0.939 \\
\hline 2003 & 0.767 & 0.794 & 0.812 & 0.831 & 0.848 & 0.866 & 0.882 & 0.897 & 0.907 & 0.920 \\
\hline 2004 & 0.746 & 0.772 & 0.789 & 0.808 & 0.825 & 0.842 & 0.858 & 0.872 & 0.882 & 0.894 \\
\hline 2005 & 0.724 & 0.749 & 0.766 & 0.784 & 0.801 & 0.817 & 0.833 & 0.846 & 0.856 & 0.868 \\
\hline 2006 & 0.700 & 0.724 & 0.741 & 0.758 & 0.774 & 0.790 & 0.805 & 0.819 & 0.828 & 0.840 \\
\hline 2007 & 0.681 & 0.705 & 0.721 & 0.738 & 0.753 & 0.769 & 0.783 & 0.796 & 0.805 & 0.817 \\
\hline 2008 & 0.667 & 0.690 & 0.706 & 0.722 & 0.737 & 0.753 & 0.767 & 0.780 & 0.788 & 0.800 \\
\hline 2009 & 0.658 & 0.681 & 0.697 & 0.713 & 0.728 & 0.743 & 0.757 & 0.770 & 0.779 & 0.790 \\
\hline
\end{tabular}


Table B.18

Gross National Product Implicit Price Deflator (Continued)

\begin{tabular}{|c|c|c|c|c|c|c|c|c|c|c|}
\hline From: & 2000 & 2001 & 2002 & 2003 & 2004 & 2005 & 2006 & 2007 & 2008 & 2009 \\
\hline 1970 & 3.635 & 3.722 & 3.787 & 3.867 & 3.977 & 4.097 & 4.237 & 4.355 & 4.449 & 4.517 \\
\hline 1971 & 3.462 & 3.544 & 3.606 & 3.683 & 3.787 & 3.902 & 4.035 & 4.147 & 4.237 & 4.302 \\
\hline 1972 & 3.317 & 3.397 & 3.456 & 3.529 & 3.630 & 3.739 & 3.867 & 3.975 & 4.061 & 4.124 \\
\hline 1973 & 3.142 & 3.217 & 3.273 & 3.343 & 3.438 & 3.542 & 3.662 & 3.764 & 3.846 & 3.907 \\
\hline 1974 & 2.882 & 2.951 & 3.002 & 3.066 & 3.153 & 3.249 & 3.359 & 3.453 & 3.528 & 3.582 \\
\hline 1975 & 2.633 & 2.696 & 2.743 & 2.802 & 2.881 & 2.968 & 3.069 & 3.155 & 3.223 & 3.273 \\
\hline 1976 & 2.489 & 2.549 & 2.593 & 2.648 & 2.723 & 2.806 & 2.901 & 2.982 & 3.047 & 3.094 \\
\hline 1977 & 2.340 & 2.396 & 2.438 & 2.490 & 2.561 & 2.638 & 2.728 & 2.804 & 2.865 & 2.909 \\
\hline 1978 & 2.186 & 2.239 & 2.278 & 2.326 & 2.392 & 2.465 & 2.548 & 2.620 & 2.676 & 2.718 \\
\hline 1979 & 2.019 & 2.067 & 2.103 & 2.148 & 2.209 & 2.276 & 2.353 & 2.419 & 2.472 & 2.509 \\
\hline 1980 & 1.851 & 1.896 & 1.929 & 1.970 & 2.026 & 2.087 & 2.158 & 2.218 & 2.266 & 2.300 \\
\hline 1981 & 1.692 & 1.733 & 1.763 & 1.800 & 1.852 & 1.908 & 1.972 & 2.027 & 2.071 & 2.103 \\
\hline 1982 & 1.595 & 1.633 & 1.662 & 1.697 & 1.745 & 1.798 & 1.859 & 1.911 & 1.952 & 1.982 \\
\hline 1983 & 1.534 & 1.571 & 1.598 & 1.632 & 1.679 & 1.729 & 1.788 & 1.838 & 1.878 & 1.906 \\
\hline 1984 & 1.479 & 1.514 & 1.540 & 1.573 & 1.618 & 1.667 & 1.723 & 1.772 & 1.810 & 1.837 \\
\hline 1985 & 1.435 & 1.469 & 1.495 & 1.527 & 1.570 & 1.617 & 1.672 & 1.719 & 1.756 & 1.783 \\
\hline 1986 & 1.404 & 1.438 & 1.463 & 1.494 & 1.536 & 1.583 & 1.636 & 1.682 & 1.719 & 1.745 \\
\hline 1987 & 1.366 & 1.399 & 1.424 & 1.454 & 1.495 & 1.540 & 1.593 & 1.637 & 1.673 & 1.695 \\
\hline 1988 & 1.321 & 1.353 & 1.377 & 1.406 & 1.446 & 1.490 & 1.540 & 1.583 & 1.617 & 1.639 \\
\hline 1989 & 1.273 & 1.304 & 1.326 & 1.355 & 1.393 & 1.435 & 1.484 & 1.525 & 1.558 & 1.579 \\
\hline 1990 & 1.226 & 1.255 & 1.277 & 1.304 & 1.341 & 1.382 & 1.429 & 1.468 & 1.500 & 1.520 \\
\hline 1991 & 1.184 & 1.213 & 1.234 & 1.260 & 1.296 & 1.335 & 1.380 & 1.419 & 1.450 & 1.468 \\
\hline 1992 & 1.158 & 1.185 & 1.206 & 1.232 & 1.267 & 1.305 & 1.349 & 1.387 & 1.417 & 1.434 \\
\hline 1993 & 1.131 & 1.159 & 1.179 & 1.204 & 1.238 & 1.275 & 1.319 & 1.356 & 1.385 & 1.403 \\
\hline 1994 & 1.108 & 1.134 & 1.154 & 1.179 & 1.212 & 1.249 & 1.291 & 1.327 & 1.356 & 1.374 \\
\hline 1995 & 1.086 & 1.112 & 1.131 & 1.155 & 1.188 & 1.224 & 1.265 & 1.301 & 1.329 & 1.346 \\
\hline 1996 & 1.065 & 1.091 & 1.110 & 1.134 & 1.166 & 1.201 & 1.242 & 1.276 & 1.304 & 1.321 \\
\hline 1997 & 1.048 & 1.073 & 1.092 & 1.115 & 1.147 & 1.181 & 1.222 & 1.256 & 1.283 & 1.298 \\
\hline 1998 & 1.037 & 1.061 & 1.080 & 1.103 & 1.134 & 1.168 & 1.208 & 1.242 & 1.269 & 1.284 \\
\hline 1999 & 1.022 & 1.046 & 1.064 & 1.087 & 1.118 & 1.152 & 1.191 & 1.224 & 1.251 & 1.265 \\
\hline 2000 & 1.000 & 1.024 & 1.042 & 1.064 & 1.094 & 1.127 & 1.166 & 1.198 & 1.224 & 1.238 \\
\hline 2001 & 0.977 & 1.000 & 1.017 & 1.039 & 1.069 & 1.101 & 1.138 & 1.170 & 1.195 & 1.211 \\
\hline 2002 & 0.960 & 0.983 & 1.000 & 1.021 & 1.050 & 1.082 & 1.119 & 1.150 & 1.175 & 1.192 \\
\hline 2003 & 0.940 & 0.962 & 0.979 & 1.000 & 1.028 & 1.059 & 1.096 & 1.126 & 1.150 & 1.167 \\
\hline 2004 & 0.914 & 0.936 & 0.952 & 0.972 & 1.000 & 1.030 & 1.065 & 1.095 & 1.118 & 1.134 \\
\hline 2005 & 0.887 & 0.908 & 0.924 & 0.944 & 0.970 & 1.000 & 1.031 & 1.060 & 1.083 & 1.098 \\
\hline 2006 & 0.858 & 0.878 & 0.894 & 0.913 & 0.939 & 0.969 & 1.000 & 1.027 & 1.049 & 1.063 \\
\hline 2007 & 0.835 & 0.855 & 0.870 & 0.888 & 0.914 & 0.943 & 0.974 & 1.000 & 1.022 & 1.033 \\
\hline 2008 & 0.817 & 0.837 & 0.851 & 0.869 & 0.894 & 0.923 & 0.953 & 0.979 & 1.000 & 1.012 \\
\hline 2009 & 0.808 & 0.826 & 0.839 & 0.857 & 0.882 & 0.911 & 0.941 & 0.968 & 0.988 & 1.000 \\
\hline
\end{tabular}

Source:

U.S. Department of Commerce, Bureau of Economic Analysis, Survey of Current Business, Washington, DC, monthly. 
APPENDIX C

MAPS 
C-2

TRANSPORTATION ENERGY DATA BOOK: EDITION 29-2010 
Table C.1

Census Regions and Divisions

\begin{tabular}{|c|c|c|c|}
\hline \multicolumn{4}{|c|}{ Northeast Region } \\
\hline \multicolumn{2}{|c|}{ Mid-Atlantic division } & \multicolumn{2}{|c|}{ New England division } \\
\hline $\begin{array}{l}\text { New Jersey } \\
\text { New York }\end{array}$ & Pennsylvania & $\begin{array}{l}\text { Connecticut } \\
\text { Maine } \\
\text { Massachusetts }\end{array}$ & $\begin{array}{l}\text { New Hampshire } \\
\text { Rhode Island } \\
\text { Vermont }\end{array}$ \\
\hline \multicolumn{4}{|c|}{ South Region } \\
\hline $\begin{array}{c}\text { West South Central } \\
\text { division }\end{array}$ & $\begin{array}{c}\text { East South Central } \\
\text { division }\end{array}$ & \multicolumn{2}{|c|}{$\begin{array}{c}\text { South Atlantic } \\
\text { division }\end{array}$} \\
\hline $\begin{array}{l}\text { Arkansas } \\
\text { Louisiana } \\
\text { Oklahoma } \\
\text { Texas }\end{array}$ & $\begin{array}{l}\text { Alabama } \\
\text { Kentucky } \\
\text { Mississippi } \\
\text { Tennessee }\end{array}$ & $\begin{array}{l}\text { Delaware } \\
\text { Florida } \\
\text { Georgia } \\
\text { Maryland } \\
\text { North Carolina }\end{array}$ & $\begin{array}{l}\text { South Carolina } \\
\text { Virginia } \\
\text { Washington, DC } \\
\text { West Virginia }\end{array}$ \\
\hline \multicolumn{4}{|c|}{ West Region } \\
\hline \multicolumn{2}{|c|}{ Pacific division } & \multicolumn{2}{|c|}{ Mountain division } \\
\hline $\begin{array}{l}\text { Alaska } \\
\text { California } \\
\text { Hawaii }\end{array}$ & $\begin{array}{l}\text { Oregon } \\
\text { Washington }\end{array}$ & $\begin{array}{l}\text { Arizona } \\
\text { Colorado } \\
\text { Idaho } \\
\text { Montana }\end{array}$ & $\begin{array}{l}\text { Nevada } \\
\text { New Mexico } \\
\text { Utah } \\
\text { Wyoming }\end{array}$ \\
\hline \multicolumn{4}{|c|}{ Midwest Region } \\
\hline \multicolumn{2}{|c|}{ West North Central division } & \multicolumn{2}{|c|}{ East North Central division } \\
\hline $\begin{array}{l}\text { Iowa } \\
\text { Kansas } \\
\text { Minnesota } \\
\text { Missouri }\end{array}$ & $\begin{array}{l}\text { Nebraska } \\
\text { North Dakota } \\
\text { South Dakota }\end{array}$ & $\begin{array}{l}\text { Illinois } \\
\text { Indiana } \\
\text { Michigan }\end{array}$ & $\begin{array}{l}\text { Ohio } \\
\text { Wisconsin }\end{array}$ \\
\hline
\end{tabular}

\section{Source:}

U.S. Census Bureau. 
Figure C1. Census Regions and Divisions

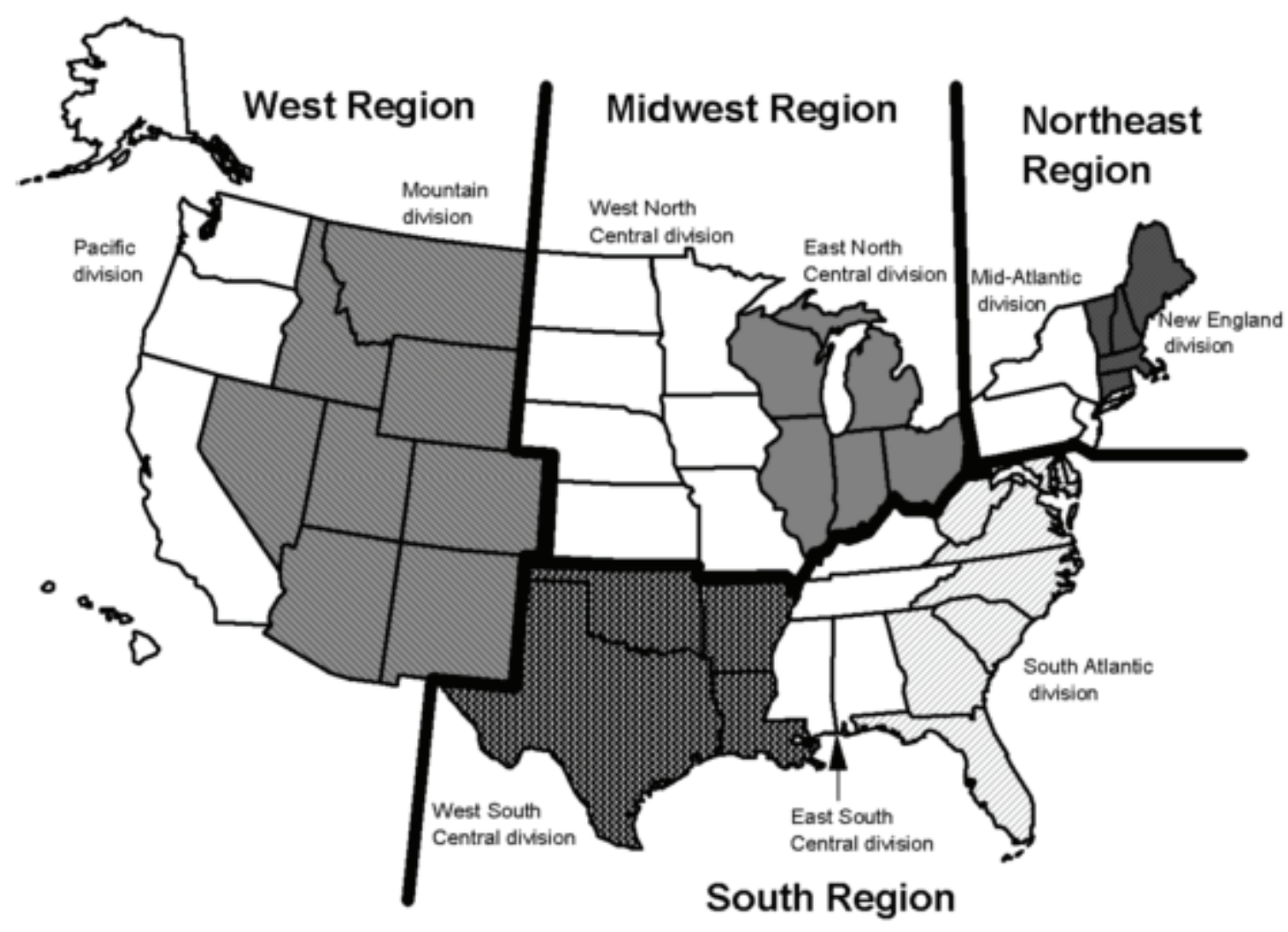

Source:

See Table C.1. 
Table C.2

Petroleum Administration for Defense Districts (PADD)

\begin{tabular}{|l|l|l|}
\hline District & Subdistrict & States \\
\hline $\begin{array}{l}\text { PAD District 1 } \\
\text { East Coast }\end{array}$ & $\begin{array}{l}\text { Subdistrict 1X } \\
\text { New England }\end{array}$ & $\begin{array}{l}\text { Connecticut, Maine, Massachusetts, New } \\
\text { Hampshire, Rhode Island, Vermont }\end{array}$ \\
\hline $\begin{array}{l}\text { Subdistrict 1Y } \\
\text { Central Atlantic }\end{array}$ & $\begin{array}{l}\text { Delaware, District of Columbia, Maryland, New } \\
\text { Jersey, New York, Pennsylvania }\end{array}$ \\
\hline $\begin{array}{l}\text { PAD District 2 } \\
\text { Midwest }\end{array}$ & $\begin{array}{l}\text { Subdistrict 1Z } \\
\text { Lower Atlantic }\end{array}$ & $\begin{array}{l}\text { Florida, Georgia, North Carolina, South Carolina, } \\
\text { Virginia, West Virginia }\end{array}$ \\
\hline $\begin{array}{l}\text { PAD District 3 } \\
\text { Gulf Coast }\end{array}$ & $\begin{array}{l}\text { Illinois, Indiana, Iowa, Kansas, Kentucky, } \\
\text { Michigan, Minnesota, Missouri, Nebraska, North } \\
\text { Dakota, South Dakota, Ohio, Oklahoma, } \\
\text { Tennessee, Wisconsin }\end{array}$ \\
\hline $\begin{array}{l}\text { PAD District 4 } \\
\text { Rocky Mountains }\end{array}$ & $\begin{array}{l}\text { Alabama, Arkansas, Louisiana, Mississippi, New } \\
\text { Mexico, Texas }\end{array}$ \\
\hline $\begin{array}{l}\text { PAD District 5 } \\
\text { West Coast }\end{array}$ & \begin{tabular}{l} 
Colorado Idaho, Montana, Utah, Wyoming \\
\hline
\end{tabular} & $\begin{array}{l}\text { Alaska, Arizona, California, Hawaii, Nevada, } \\
\text { Oregon, Washington }\end{array}$ \\
\hline
\end{tabular}

\section{Source:}

Energy Information Administration web site: http://tonto.eia.doe.gov/oog/info/twip/padddef.html 

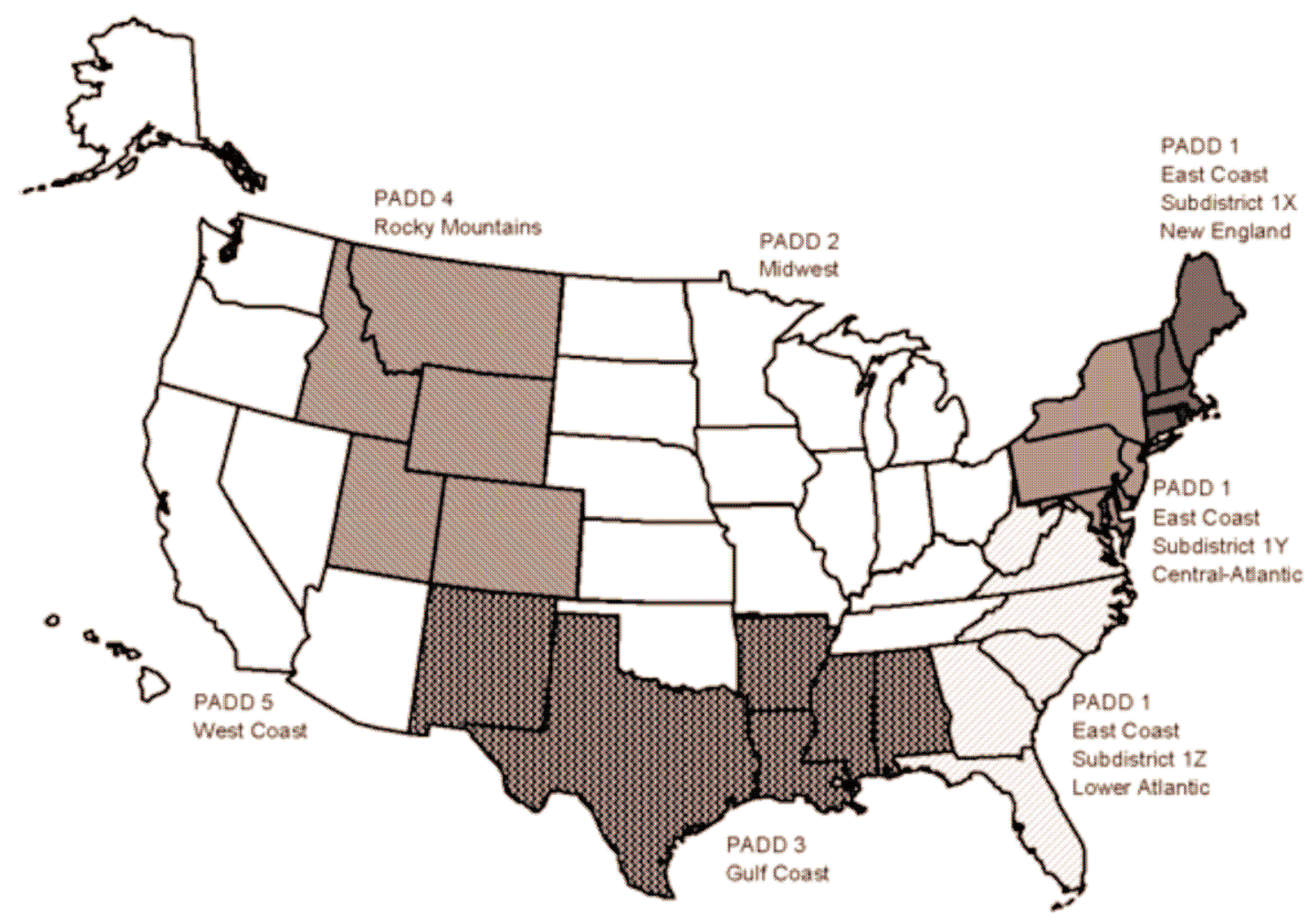

Source:

See Table C.2. 


\section{Figure C.3. Map of Places where Reformulated Gasoline is Sold}

\section{Source:}

U.S. Environmental Protection Agency, www.epa.gov/otaq/rfg/whereyoulive.htm.

Note: Reformulated gasoline is a motor gasoline specially formulated to achieve significant reductions in vehicle emissions of ozone-forming and toxic air pollutants. The Clean Air Act of 1990 mandates reformulated gasoline use in areas with ozone-air pollution problems. 
C-8

TRANSPORTATION ENERGY DATA BOOK: EDITION 29-2010 


\section{GLOSSARY}

Acceleration power - Measured in kilowatts. Pulse power obtainable from a battery used to accelerate a vehicle. This is based on a constant current pulse for 30 seconds at no less than $2 / 3$ of the maximum open-circuit-voltage, at $80 \%$ depth-of-discharge relative to the battery's rated capacity and at $20 \circ \mathrm{C}$ ambient temperature.

Air Carrier - The commercial system of air transportation consisting of certificated air carriers, air taxis (including commuters), supplemental air carriers, commercial operators of large aircraft, and air travel clubs.

Certificated route air carrier: An air carrier holding a Certificate of Public Convenience and Necessity issued by the Department of Transportation to conduct scheduled interstate services. Nonscheduled or charter operations may also be conducted by these carriers. These carriers operate large aircraft (30 seats or more, or a maximum payload capacity of 7,500 pounds or more) in accordance with Federal Aviation Regulation part 121.

Domestic air operator: Commercial air transportation within and between the 50 States and the District of Columbia. Includes operations of certificated route air carriers, Pan American, local service, helicopter, intra-Alaska, intra-Hawaii, all-cargo carriers and other carriers. Also included are transborder operations conducted on the domestic route segments of U.S. air carriers. Domestic operators are classified based on their operating revenue as follows:

Majors - over $\$ 1$ billion

Nationals - \$100-1,000 million

Large Regionals - \$10-99.9 million

Medium Regionals - \$0-9.99 million

International air operator: Commercial air transportation outside the territory of the United States, including operations between the U.S. and foreign countries and between the U.S. and its territories and possessions.

Supplemental air carrier: A class of air carriers which hold certificates authorizing them to perform passenger and cargo charter services supplementing the scheduled service of the certificated route air carriers. Supplemental air carriers are often referred to as nonscheduled air carriers or "nonskeds."

Alcohol - The family name of a group of organic chemical compounds composed of carbon, hydrogen, and oxygen. The molecules in the series vary in chain length and are composed of a hydrocarbon plus a hydroxyl group. Alcohol includes methanol and ethanol.

Amtrak - See Rail.

Anthropogenic - Human made. Usually used in the context of emissions that are produced as the result of human activities.

Automobile size classifications - Size classifications of automobiles are established by the Environmental Protection Agency (EPA) as follows:

Minicompact - less than 85 cubic feet of passenger and luggage volume.

Subcompact - between 85 to 100 cubic feet of passenger and luggage volume.

Compact - between 100 to 110 cubic feet of passenger and luggage volume.

Midsize - between 110 to 120 cubic feet of passenger and luggage volume.

Large - more than 120 cubic feet of passenger and luggage volume. 
Two seater - automobiles designed primarily to seat only two adults.

Station wagons are included with the size class for the sedan of the same name.

Aviation - See General aviation.

Aviation gasoline - All special grades of gasoline for use in aviation reciprocating engines, as given in the American Society for Testing and Materials (ASTM) Specification D 910. Includes all refinery products within the gasoline range that are to be marketed straight or in blends as aviation gasoline without further processing (any refinery operation except mechanical blending). Also included are finished components in the gasoline range which will be used for blending or compounding into aviation gasoline.

Barges - Shallow, nonself-propelled vessels used to carry bulk commodities on the rivers and the Great Lakes.

Battery efficiency - Measured in percentage. Net DC energy delivered on discharge, as a percentage of the total DC energy required to restore the initial state-of-charge. The efficiency value must include energy losses resulting from self-discharge, cell equalization, thermal loss compensation, and all battery-specific auxiliary equipment.

Btu - British thermal unit. The amount of energy required to raise the temperature of 1 pound of water 1 degree Fahrenheit at or near 39.2 degrees Fahrenheit. An average Btu content of fuel is the heat value per quantity of fuel as determined from tests of fuel samples.

Bunker - A storage tank.

Bunkering fuels - Fuels stored in ship bunkers.

Bus -

Intercity bus: A standard size bus equipped with front doors only, high backed seats, luggage compartments separate from the passenger compartment and usually with restroom facilities, for high-speed long distance service.

Motor bus: Rubber-tired, self-propelled, manually-steered bus with fuel supply on board the vehicle. Motor bus types include intercity, school, and transit.

School and other nonrevenue bus: Bus services for which passengers are not directly charged for transportation, either on a per passenger or per vehicle basis.

Transit bus: A bus designed for frequent stop service with front and center doors, normally with a rear-mounted diesel engine, low-back seating, and without luggage storage compartments or restroom facilities.

Trolley coach: Rubber-tired electric transit vehicle, manually-steered, propelled by a motor drawing current, normally through overhead wires, from a central power source not on board the vehicle.

Calendar year - The period of time between January 1 and December 31 of any given year.

Captive imports - Products produced overseas specifically for domestic manufacturers.

Carbon dioxide $\left(\mathrm{CO}_{2}\right)$ - A colorless, odorless, non-poisonous gas that is a normal part of the ambient air. Carbon dioxide is a product of fossil fuel combustion. 
Carbon monoxide (CO) - A colorless, odorless, highly toxic gas that is a by-product of incomplete fossil fuel combustion. Carbon monoxide, one of the major air pollutants, can be harmful in small amounts if breathed over a certain period of time.

Car-mile (railroad) - A single railroad car moved a distance of one mile.

Cargo ton-mile - See Ton-mile.

Certificated route air carriers - See Air carriers.

Class I freight railroad - See Rail.

Coal slurry - Finely crushed coal mixed with sufficient water to form a fluid.

Combination trucks - Consist of a power unit (a truck tractor) and one or more trailing units (a semitrailer or trailer). The most frequently used combination is popularly referred to as a "tractorsemitrailer" or "tractor trailer".

Commercial sector - An energy-consuming sector that consists of service-providing facilities of: businesses; Federal, State, and local governments; and other private and public organizations, such as religious, social or fraternal groups. Includes institutional living quarters.

Commuter railroad - See Rail.

Compact car - See Automobile size classifications.

Constant dollars - A time series of monetary figures is expressed in constant dollars when the effect of change over time in the purchasing power of the dollar has been removed. Usually the data are expressed in terms of dollars of a selected year or the average of a set of years.

Consumer Price Index (CPI) - An index issued by the U.S. Department of Labor, Bureau of Labor Statistics. The CPI is designed to measure changes in the prices of goods and services bought by wage earners and clerical workers in urban areas. It represents the cost of a typical consumption bundle at current prices as a ratio to its cost at a base year.

Continuous discharge capacity - Measured as percent of rated energy capacity. Energy delivered in a constant power discharge required by an electric vehicle for hill climbing and/or high-speed cruise, specified as the percent of its rated energy capacity delivered in a one hour constant-power discharge.

Corporate Average Fuel Economy (CAFE) Standards - CAFE standards were originally established by Congress for new automobiles, and later for light trucks, in Title V of the Motor Vehicle Information and Cost Savings Act (15 U.S.C.1901, et seq.) with subsequent amendments. Under CAFE, automobile manufacturers are required by law to produce vehicle fleets with a composite sales-weighted fuel economy which cannot be lower than the CAFE standards in a given year, or for every vehicle which does not meet the standard, a fine of $\$ 5.00$ is paid for every one-tenth of a mpg below the standard.

Crude oil - A mixture of hydrocarbons that exists in the liquid phase in natural underground reservoirs and remains liquid at atmospheric pressure after passing through surface separating facilities. Crude oil production is measured at the wellhead and includes lease condensate. 
Crude oil imports - The volume of crude oil imported into the 50 States and the District of Columbia, including imports from U.S. territories, but excluding imports of crude oil into the Hawaiian Foreign Trade Zone.

Curb weight - The weight of a vehicle including all standard equipment, spare tire and wheel, all fluids and lubricants to capacity, full tank of fuel, and the weight of major optional accessories normally found on the vehicle.

Current dollars - Represents dollars current at the time designated or at the time of the transaction. In most contexts, the same meaning would be conveyed by the use of the term "dollars." See also constant dollars.

Demand Response - A transit mode that includes passenger cars, vans, and small buses operating in response to calls from passengers to the transit operator who dispatches the vehicles. The vehicles do not operate over a fixed route on a fixed schedule. Can also be known as paratransit or dial-a-ride.

\section{Diesel fuel - See Distillate fuel oil.}

Disposable personal income - See Income.

Distillate fuel oil - The lighter fuel oils distilled off during the refining process. Included are products known as ASTM grades numbers 1 and 2 heating oils, diesel fuels, and number 4 fuel oil. The major uses of distillate fuel oils include heating, fuel for on-and off-highway diesel engines, and railroad diesel fuel.

Domestic air operator - See Air carrier.

E85 - 85\% ethanol and 15\% gasoline.

E95 - 95\% ethanol and 5\% gasoline.

Domestic water transportation - See Internal water transportation.

Electric utilities sector - Consists of privately and publicly owned establishments which generate electricity primarily for resale.

Emission standards - Standards for the levels of pollutants emitted from automobiles and trucks. Congress established the first standards in the Clean Air Act of 1963. Currently, standards are set for four vehicle classes-automobiles, light trucks, heavy-duty gasoline trucks, and heavy-duty diesel trucks.

Energy capacity - Measured in kilowatt hours. The energy delivered by the battery, when tested at C/3 discharge rate, up to termination of discharge specified by the battery manufacturer. The required acceleration power must be delivered by the battery at any point up to $80 \%$ of the battery's energy capacity rating.

Energy efficiency - In reference to transportation, the inverse of energy intensiveness: the ratio of outputs from a process to the energy inputs; for example, miles traveled per gallon of fuel (mpg).

Energy intensity - In reference to transportation, the ratio of energy inputs to a process to the useful outputs from that process; for example, gallons of fuel per passenger-mile or Btu per ton-mile. 
Ethanol $\left(\mathbf{C}_{2} \mathbf{H}_{5} \mathbf{O H}\right)$ - Otherwise known as ethyl alcohol, alcohol, or grain-spirit. A clear, colorless, flammable oxygenated hydrocarbon with a boiling point of 78.5 degrees Celsius in the anhydrous state. In transportation, ethanol is used as a vehicle fuel by itself (E100 - 100\% ethanol by volume), blended with gasoline (E85 - 85\% ethanol by volume), or as a gasoline octane enhancer and oxygenate ( $10 \%$ by volume).

Fixed operating cost - See Operating cost.

\section{Fleet vehicles -}

Private fleet vehicles: Ideally, a vehicle could be classified as a member of a fleet if it is:

a) operated in mass by a corporation or institution,

b) operated under unified control, or

c) used for non-personal activities.

However, the definition of a fleet is not consistent throughout the fleet industry. Some companies make a distinction between cars that were bought in bulk rather than singularly, or whether they are operated in bulk, as well as the minimum number of vehicles that constitute a fleet (i.e. 4 or 10).

Government fleet vehicles: Includes vehicles owned by all Federal, state, county, city, and metro units of government, including toll road operations.

Foreign freight - Movements between the United States and foreign countries and between Puerto Rico, the Virgin Islands, and foreign countries. Trade between U.S. territories and possessions (e.g. Guam, Wake, American Samoa) and foreign countries is excluded. Traffic to or from the Panama Canal Zone is included.

Gas Guzzler Tax - Originates from the 1978 Energy Tax Act (Public Law 95-618). A new car purchaser is required to pay the tax if the car purchased has a combined city/highway fuel economy rating that is below the standard for that year. For model years 1986 and later, the standard is $22.5 \mathrm{mpg}$.

Gasohol - A mixture of $10 \%$ anhydrous ethanol and $90 \%$ gasoline by volume; $7.5 \%$ anhydrous ethanol and $92.5 \%$ gasoline by volume; or $5.5 \%$ anhydrous ethanol and $94.5 \%$ gasoline by volume. There are other fuels that contain methanol and gasoline, but these fuels are not referred to as gasohol.

Gasoline - See Motor gasoline.

General aviation - That portion of civil aviation which encompasses all facets of aviation except air carriers. It includes any air taxis, commuter air carriers, and air travel clubs which do not hold Certificates of Public Convenience and Necessity.

Gross National Product - A measure of monetary value of the goods and services becoming available to the nation from economic activity. Total value at market prices of all goods and services produced by the nation's economy. Calculated quarterly by the Department of Commerce, the Gross National Product is the broadest available measure of the level of economic activity.

Gross vehicle weight (gvw) - The weight of the empty truck plus the maximum anticipated load weight.

Gross vehicle weight rating (gvwr) - The gross vehicle weight which is assigned to each new truck by the manufacturer. This rating may be different for trucks of the same model because of certain features, such as heavy-duty suspension. Passenger cars do not have gross vehicle weight ratings.

Heavy-heavy truck - See Truck size classifications. 
Household - Consists of all persons who occupy a housing unit, including the related family members and all unrelated persons, if any, who share the housing unit.

Housing unit - A house, apartment, a group of rooms, or a single room occupied or intended for occupancy as separate living quarters. Separate living quarters are those in which the occupants do not live and eat with any other persons in the structure and which have either (1) direct access from the outside of the building or through a common hallway intended to be used by the occupants of another unit or by the general public, or (2) complete kitchen facilities for the exclusive use of the occupants. The occupants may be a single family, one person living alone, two or more families living together, or any other group of related or unrelated persons who share living arrangements.

Hydrocarbon (HC) - A compound that contains only hydrogen and carbon. The simplest and lightest forms of hydrocarbon are gaseous. With greater molecular weights they are liquid, while the heaviest are solids.

\section{Income -}

Disposable personal income: Personal income less personal tax and non-tax payments.

National income: The aggregate earnings of labor and property which arise in the current production of goods and services by the nation's economy.

Personal income: The current income received by persons from all sources, net of contributions for social insurance.

Industrial sector - Construction, manufacturing, agricultural and mining establishments.

Inertia weight - The curb weight of a vehicle plus 300 pounds.

Intercity bus - See Bus.

Internal water transportation - Includes all local (intraport) traffic and traffic between ports or landings wherein the entire movement takes place on inland waterways. Also termed internal are movements involving carriage on both inland waterways and the water of the Great Lakes, and inland movements that cross short stretches of open water that link inland systems.

International air operator - See Air carrier.

International freight - See Foreign freight.

Jet fuel - Includes both naphtha-type and kerosene-type fuels meeting standards for use in aircraft turbine engines. Although most jet fuel is used in aircraft, some is used for other purposes such as generating electricity in gas turbines.

Kerosene-type jet fuel: A quality kerosene product with an average gravity of 40.7 degrees API and $10 \%$ to $90 \%$ distillation temperatures of 217 to 261 degrees centigrade. Used primarily as fuel for commercial turbojet and turboprop aircraft engines. It is a relatively low freezing point distillate of the kerosene type.

Naphtha-type jet fuel: A fuel in the heavy naphtha boiling range with an average gravity of 52.8 degrees API and 10\% to $90 \%$ distillation temperatures of 117 to 233 degrees centigrade used for turbojet and turboprop aircraft engines, primarily by the military. Excludes ramjet and petroleum. 
Kerosene - A petroleum distillate in the 300 to 500 degrees Fahrenheit boiling range and generally having a flash point higher than 100 degrees Fahrenheit by the American Society of Testing and Material (ASTM) Method D56, a gravity range from 40 to 46 degrees API, and a burning point in the range of 150 to 175 degrees Fahrenheit. It is a clean-burning product suitable for use as an illuminant when burned in wick lamps. Includes grades of kerosene called range oil having properties similar to Number 1 fuel oil, but with a gravity of about 43 degrees API and an end point of 625 degrees Fahrenheit. Used in space heaters, cooking stoves, and water heaters.

Kerosene-type jet fuel - See Jet fuel.

Large car - See Automobile size classifications.

Lease Condensate - A liquid recovered from natural gas at the well or at small gas/oil separators in the field. Consists primarily of pentanes and heavier hydrocarbons (also called field condensate).

Light duty vehicles - Automobiles and light trucks combined.

Light truck - Unless otherwise noted, light trucks are defined in this publication as two-axle, four-tire trucks. The U.S. Bureau of Census classifies all trucks with a gross vehicle weight less than 10,000 pounds as light trucks (See Truck size classifications).

Light-heavy truck - See Truck size classifications.

Liquified petroleum gas (lpg) - Consists of propane and butane and is usually derived from natural gas. In locations where there is no natural gas and the gasoline consumption is low, naphtha is converted to lpg by catalytic reforming.

Load factor - Total passenger miles divided by total vehicle miles.

Low emission vehicle - Any vehicle certified to the low emission standards which are set by the Federal government and/or the state of California.

M85 - 85\% methanol and 15\% gasoline.

M100 - 100\% methanol.

Medium truck - See Truck size classifications.

Methanol $\left(\mathrm{CH}_{3} \mathrm{OH}\right)$ - A colorless highly toxic liquid with essentially no odor and very little taste. It is the simplest alcohol and boils at 64.7 degrees Celsius. In transportation, methanol is used as a vehicle fuel by itself (M100), or blended with gasoline (M85).

Midsize car - See Automobile size classifications.

Minicompact car - See Automobile size classifications.

Model year - In this publication, model year is referring to the "sales" model year, the period from October 1 to the next September 31.

Motor bus - See Bus.

Motor gasoline - A mixture of volatile hydrocarbons suitable for operation of an internal combustion engine whose major components are hydrocarbons with boiling points ranging from 78 to 217 
degrees centigrade and whose source is distillation of petroleum and cracking, polymerization, and other chemical reactions by which the naturally occurring petroleum hydrocarbons are converted into those that have superior fuel properties.

Regular gasoline: Gasoline having an antiknock index, i.e., octane rating, greater than or equal to 85 and less than 88 . Note: Octane requirements may vary by altitude.

Midgrade gasoline: Gasoline having an antiknock index, i.e., octane rating, greater than or equal to 88 and less than or equal to 90 . Note: Octane requirements may vary by altitude.

Premium gasoline: Gasoline having an antiknock index, i.e., octane rating, greater than 90 . Note: Octane requirements may vary by altitude.

Reformulated gasoline: Finished motor gasoline formulated for use in motor vehicles, the composition and properties of which meet the requirements of the reformulated gasoline regulations promulgated by the U.S. Environmental Protection Agency under Section 211(k) of the Clean Air Act. For details on this clean fuel program see http://www.epa.gov/otaq/rfg.htm. Note: This category includes oxygenated fuels program reformulated gasoline (OPRG) but excludes reformulated gasoline blendstock for oxygenate blending (RBOB).

MTBE - Methyl Tertiary Butyl Ether-a colorless, flammable, liquid oxygenated hydrocarbon containing 18.15 percent oxygen.

Naphtha-type jet fuel - See Jet fuel.

National income - See Income.

Nationwide Personal Transportation Survey (NPTS) - A nationwide survey of households that provides information on the characteristics and personal travel patterns of the U.S. population. Surveys were conducted in 1969, 1977, 1983, 1990, and 1995 by the U.S. Bureau of Census for the U.S. Department of Transportation.

Natural gas - A mixture of hydrocarbon compounds and small quantities of various non-hydrocarbons existing in the gaseous phase or in solution with crude oil in natural underground reservoirs at reservoir conditions.

Natural gas, dry: Natural gas which remains after: 1) the liquefiable hydrocarbon portion has been removed from the gas stream; and 2) any volumes of nonhydrocarbon gases have been removed where they occur in sufficient quantity to render the gas unmarketable. Dry natural gas is also known as consumer-grade natural gas. The parameters for measurement are cubic feet at 60 degrees Fahrenheit and 14.73 pounds per square inch absolute.

Natural gas, wet: The volume of natural gas remaining after removal of lease condensate in lease and/or field separation facilities, if any, and after exclusion of nonhydrocarbon gases where they occur in sufficient quantity to render the gas unmarketable. Natural gas liquids may be recovered from volumes of natural gas, wet after lease separation, at natural gas processing plants.

Natural gas plant liquids: Natural gas liquids recovered from natural gas in processing plants and from natural gas field facilities and fractionators. Products obtained include ethane, propane, normal butane, isobutane, pentanes plus, and other products from natural gas processing plants.

Nitrogen oxides $\left(\mathbf{N O}_{\mathbf{x}}\right)$ - A product of combustion of fossil fuels whose production increases with the temperature of the process. It can become an air pollutant if concentrations are excessive. 
Nonattainment area - Any area that does not meet the national primary or secondary ambient air quality standard established by the Environmental Protection Agency for designated pollutants, such as carbon monoxide and ozone.

Oil Stocks - Oil stocks include crude oil (including strategic reserves), unfinished oils, natural gas plant liquids, and refined petroleum products.

\section{Operating cost -}

Fixed operating cost: In reference to passenger car operating cost, refers to those expenditures that are independent of the amount of use of the car, such as insurance costs, fees for license and registration, depreciation and finance charges.

Variable operating cost: In reference to passenger car operating cost, expenditures which are dependent on the amount of use of the car, such as the cost of gas and oil, tires, and other maintenance.

Organization for Economic Cooperation and Development (OECD) - Consists of Australia, Austria, Belgium, Canada, Czech Republic, Denmark, Finland, France, Germany, Greece, Hungary, Iceland, Ireland, Italy, Japan, Luxembourg, Mexico, Netherlands, New Zealand, Norway, Poland, Portugal, South Korea, Spain, Sweden, Switzerland, Turkey, United Kingdom, and United States. Total OECD includes the United States Territories (Guam, Puerto Rico, and the U.S. Virgin Islands). Total OECD excludes data for Czech Republic, Hungary, Mexico, Poland, and South Korea which are not yet available.

OECD Europe: Consists of Austria, Belgium, Czech Republic, Denmark, Finland, France, Germany, Greece, Hungary, Iceland, Ireland, Italy, Luxembourg, Netherlands, Norway, Poland, Portugal, Spain, Sweden, Switzerland, Turkey, and United Kingdom. OECD Europe excludes data for Czech Republic, Hungary, and Poland which are not yet available.

OECD Pacific: Consists of Australia, Japan, and New Zealand.

Organization for Petroleum Exporting Countries (OPEC) - Includes Saudi Arabia, Iran, Venezuela, Libya, Indonesia, United Arab Emirates, Algeria, Nigeria, Ecuador, Gabon, Iraq, Kuwait, and Qatar. Data for Saudi Arabia and Kuwait include their shares from the Partitioned Zone (formerly the Neutral Zone). Angola joined OPEC in December 2006, thus, beginning in 2007, data on OPEC will include Angola.

Arab OPEC - Consists of Algeria, Iraq, Kuwait, Libya, Qatar, Saudi Arabia and the United Arab Emirates.

Other single-unit truck - See Single-unit truck.

Oxygenate - A substance which, when added to gasoline, increases the amount of oxygen in that gasoline blend. Includes fuel ethanol, methanol, and methyl tertiary butyl ether (MTBE).

Particulates - Carbon particles formed by partial oxidation and reduction of the hydrocarbon fuel. Also included are trace quantities of metal oxides and nitrides, originating from engine wear, component degradation, and inorganic fuel additives. In the transportation sector, particulates are emitted mainly from diesel engines.

Passenger-miles traveled (PMT) - One person traveling the distance of one mile. Total passenger-miles traveled, thus, give the total mileage traveled by all persons. 
Passenger rail - See Rail, "Amtrak" and "Transit Railroad".

Persian Gulf countries - Consists of Bahrain, Iran, Iraq, Kuwait, Qatar, Saudi Arabia, and the United Emirates.

Personal Consumption Expenditures (PCE) - As used in the national accounts, the market value of purchases of goods and services by individuals and nonprofit institutions and the value of food, clothing, housing, and financial services received by them as income in kind. It includes the rental value of owner-occupied houses but excludes purchases of dwellings, which are classified as capital goods (investment).

\section{Personal income - See Income.}

Petroleum - A generic term applied to oil and oil products in all forms, such as crude oil, lease condensate, unfinished oil, refined petroleum products, natural gas plant liquids, and nonhydrocarbon compounds blended into finished petroleum products.

Petroleum consumption: A calculated demand for petroleum products obtained by summing domestic production, imports of crude petroleum and natural gas liquids, imports of petroleum products, and the primary stocks at the beginning of the period and then subtracting the exports and the primary stocks at the end of the period.

Petroleum exports: Shipments of petroleum products from the 50 States and the District of Columbia to foreign countries, Puerto Rico, the Virgin Islands, and other U.S. possessions and territories.

Petroleum imports: All imports of crude petroleum, natural gas liquids, and petroleum products from foreign countries and receipts from Guam, Puerto Rico, the Virgin Islands, and the Hawaiian Trade Zone. The commodities included are crude oil, unfinished oils, plant condensate, and refined petroleum products.

Petroleum inventories: The amounts of crude oil, unfinished oil, petroleum products, and natural gas liquids held at refineries, at natural gas processing plants, in pipelines, at bulk terminals operated by refining and pipeline companies, and at independent bulk terminals. Crude oil held in storage on leases is also included; these stocks are know as primary stocks. Secondary stocks-those held by jobbers dealers, service station operators, and consumers-are excluded. Prior to 1975, stock held at independent bulk terminals were classified as secondary stocks.

Petroleum products supplied: For each petroleum product, the amount supplied is calculated by summing production, crude oil burned directly, imports, and net withdrawals from primary stocks and subtracting exports.

Processing Gain - The amount by which the total volume of refinery output is greater than the volume of input for given period of time. The processing gain arises when crude oil and other hydrocarbons are processed into products that are, on average, less dense than the input.

Processing Loss - The amount by which the total volume of refinery output is less than the volume of input for given period of time. The processing loss arises when crude oil and other hydrocarbons are processed into products that are, on average, more dense than the input.

Proved Reserves of Crude Oil - The estimated quantities of all liquids defined as crude oil, which geological and engineering data demonstrate with reasonable certainty to be recoverable in future years from known reservoirs under existing economic and operating conditions. 
Quad - Quadrillion, 105 . In this publication, a Quad refers to Quadrillion Btu.

Rail -

Amtrak (American Railroad Tracks): Operated by the National Railroad Passenger Corporation of Washington, DC. This rail system was created by President Nixon in 1970, and was given the responsibility for the operation of intercity, as distinct from suburban, passenger trains between points designated by the Secretary of Transportation.

Class I freight railroad: Defined by the Interstate Commerce Commission each year based on annual operating revenue. A railroad is dropped from the Class I list if it fails to meet the annual earnings threshold for three consecutive years.

Commuter railroad: Those portions of mainline railroad (not electric railway) transportation operations which encompass urban passenger train service for local travel between a central city and adjacent suburbs. Commuter railroad service-using both locomotive-hauled and selfpropelled railroad passenger cars-is characterized by multi-trip tickets, specific station-to-station fares, and usually only one or two stations in the central business district. Also known as suburban railroad.

Transit railroad: Includes "heavy" and "light" transit rail. Heavy transit rail is characterized by exclusive rights-of-way, multi-car trains, high speed rapid acceleration, sophisticated signaling, and high platform loading. Also known as subway, elevated railway, or metropolitan railway (metro). Light transit rail may be on exclusive or shared rights-of-way, high or low platform loading, multi-car trains or single cars, automated or manually operated. In generic usage, light rail includes streetcars, trolley cars, and tramways.

Reformulated gasoline (RFG) - See Motor gasoline.

RFG area - An ozone nonattainment area designated by the Environmental Protection Agency which requires the use of reformulated gasoline.

Residential sector - An energy consuming sector that consists of living quarters for private households. Excludes institutional living quarters.

Residential Transportation Energy Consumption Survey (RTECS) - This survey was designed by the Energy Information Administration of the Department of Energy to provide information on how energy is used by households for personal vehicles. It has been conducted five times since 1979, the most recent being 1991.

Residual fuel oil - The heavier oils that remain after the distillate fuel oils and lighter hydrocarbons are boiled off in refinery operations. Included are products know as ASTM grade numbers 5 and 6 oil, heavy diesel oil, Navy Special Fuel Oil, Bunker C oil, and acid sludge and pitch used as refinery fuels. Residual fuel oil is used for the production of electric power, for heating, and for various industrial purposes.

Rural - Usually refers to areas with population less than 5,000.

Sales period - October 1 of the previous year to September 30 of the given year. Approximately the same as a model year.

Sales-weighted miles per gallon (mpg) - Calculation of a composite vehicle fuel economy based on the distribution of vehicle sales. 
Scrappage rate - As applied to motor vehicles, it is usually expressed as the percentage of vehicles of a certain type in a given age class that are retired from use (lacking registration) in a given year.

School and other nonrevenue bus - See Bus.

Single-unit truck - Includes two-axle, four-tire trucks and other single-unit trucks.

Two-axle, four-tire truck: A motor vehicle consisting primarily of a single motorized device with two axles and four tires.

Other single-unit truck: A motor vehicle consisting primarily of a single motorized device with more than two axles or more than four tires.

Special fuels - Consist primarily of diesel fuel with small amount of liquified petroleum gas, as defined by the Federal Highway Administration.

Specific acceleration power - Measured in watts per kilogram. Acceleration power divided by the battery system weight. Weight must include the total battery system.

Specific energy - Measured in watt hours per kilogram. The rated energy capacity of the battery divided by the total battery system weight.

Subcompact car - See Automobile size classifications.

Supplemental air carrier - See Air carrier.

Test weight - The weight setting at which a vehicle is tested on a dynomometer by the U.S. Environmental Protection Agency (EPA). This weight is determined by the EPA using the inertia weight of the vehicle.

Ton-mile - The movement of one ton of freight the distance of one mile. Ton-miles are computed by multiplying the weight in tons of each shipment transported by the distance hauled.

Transmission types -

A3 - Automatic three speed

A4 - Automatic four speed

A5 - Automatic five speed

L4 - Automatic lockup four speed

M5 - Manual five speed

Transit bus - See Bus.

Transit railroad - See Rail.

Transportation sector - Consists of both private and public passenger and freight transportation, as well as government transportation, including military operations.

Truck Inventory and Use Survey (TIUS) - Survey designed to collect data on the characteristics and operational use of the nation's truck population. It is conducted every five years by the U.S. Bureau of the Census. Surveys were conducted in 1963, 1967, 1972, 1977, 1982, 1987, and 1992. For the 1997 survey, it was renamed the Vehicle Inventory and Use Survey in anticipation of including additional vehicle types. However, no additional vehicle types were added to the 1997 survey. 
Trolley coach - See Bus.

Truck size classifications - U.S. Bureau of the Census has categorized trucks by gross vehicle weight (gvw) as follows:

Light - Less than 10,000 pounds gvw (Also see Light Truck.)

Medium - 10,001 to 20,000 pounds gvw

Light-heavy - 20,001 to 26,000 pounds gvw

Heavy-heavy - 26,001 pounds gvw or more.

Two-axle, four-tire truck - See Single-unit truck.

Two seater car - See Automobile size classifications.

Ultra-low emission vehicle - Any vehicle certified to the ultra-low emission standards which are set by the Federal government and/or the state of California.

Urban - Usually refers to areas with population of 5,000 or greater.

Vanpool - A transit mode made up of vans and sometimes small buses operating as a ridesharing arrangement to provide transportation to a group of individuals traveling directly between their homes and a regular destination within the same geographical area. Most vanpools are privatelyoperated, are not available to the public, and are not considered public transportation. Vanpool data in this report are for vanpools that are owned, purchased or leased by a public entity and are publicly available.

Variable operating cost - See Operating cost.

Vehicle Inventory and Use Survey - See Truck Inventory and Use Survey.

Vehicle-miles traveled (vmt) - One vehicle traveling the distance of one mile. Total vehicle miles, thus, is the total mileage traveled by all vehicles.

Zero-emission vehicle - Any vehicle certified to the zero emission standards which are set by the Federal government and/or the state of California. These standards apply to the vehicle emissions only. 
G-14

TRANSPORTATION ENERGY DATA BOOK: EDITION 29-2010 


\section{TITLE INDEX}


Acquisitions

Federal Fleet Vehicle Acquisitions by Fuel Type, FY 2002-2009 .............................................. 7-6

Advanced

Alternative Fuel and Advanced Technology Vehicles and Characteristics..................................... 6-1

Federal and State Advanced Technology Incentives, 2010 ......................................................... 10-13

Age

Cars in Operation and Vehicle Travel by Age, 1970 and 2001.................................................. 3-11

Trucks in Operation and Vehicle Travel by Age, 1970 and 2001 ............................................... 3-12

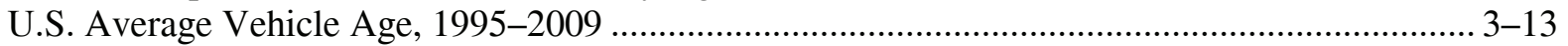

Average Annual Miles per Household Vehicle by Vehicle Age ................................................. 8-12

Share of Vehicles by Annual Miles of Travel and Vehicle Age, 2009 NHTS ................................ 8-15

Daily and Annual Vehicle Miles of Travel and Average Age for Each Vehicle

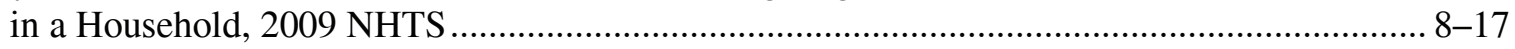

Agency

Federal Government Vehicles by Agency, FY 2009 ................................................................. 7-7

Air

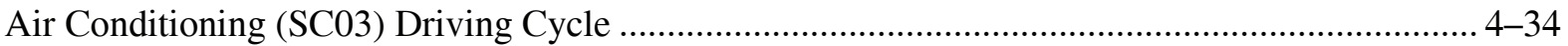

Summary Statistics for U.S. Domestic and International Certificated Route

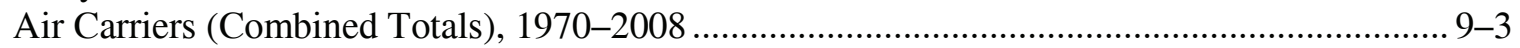

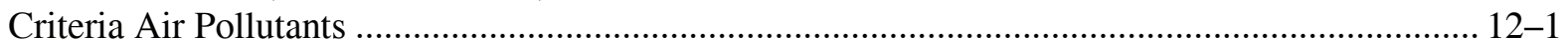

Total National Emissions of the Criteria Air Pollutants by Sector, 2008.................................... 12-2

Alternative

Alternative Fuel and Oxygenate Consumption, 2003-2008 …................................................... 2-5

Alternative Fuel and Advanced Technology Vehicles and Characteristics..................................... 6-1

Estimates of Alternative Fuel Vehicles in Use, 1995-2008 ........................................................ 6-3

Alternative Fuel Vehicles Available by Manufacturer, Model Year 2009...................................... 6-4

Number of Alternative Refuel Sites by State and Fuel Type, 2010 .............................................. 6-6

Properties of Conventional and Alternative Fuels.................................................................... 6 6

Federal and State Alternative Fuel Incentives, 2010 .............................................................. 10-12

Amtrak

Summary Statistics for the National Railroad Passenger Corporation

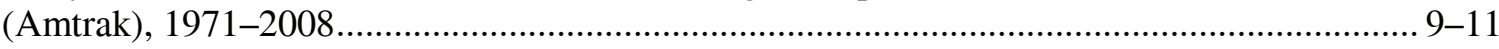

Annual

Average Annual Vehicle-Miles of Travel for Business Fleet Vehicles, 2008................................. 7-3

Average Annual Expenditures of Households by Income, 2008 ................................................ 8-4

Average Annual Vehicle-Miles, Vehicle Trips and Trip Length per

Household 1969, 1977, 1983, 1990, 1995 NPTS and 2001, 2009 NHTS ................................ 8-7

Average Annual Miles per Household Vehicle by Vehicle Age .................................................... 8-12

Self-Reported vs. Odometer Average Annual Miles, 1995 NPTS and

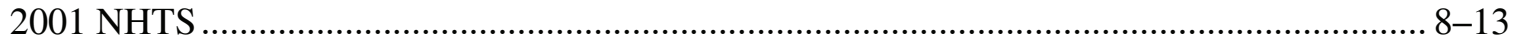

Share of Vehicles by Annual Miles of Travel and Vehicle Age, 2009 NHTS ............................... 8-15

Daily and Annual Vehicle Miles of Travel and Average Age for Each Vehicle

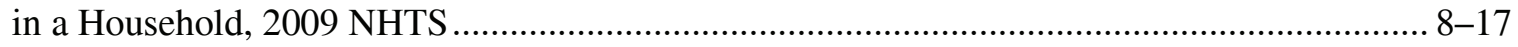

Annual Vehicle Miles of Travel for Each Vehicle in a Household, 2009 NHTS............................ 8-18

Sales-Weighted Annual Carbon Footprint of New Domestic and Import Cars

by Size Class, Model Years 1975-2009

$11-12$

TRANSPORTATION ENERGY DATA BOOK: EDITION 29-2010 
Annual (continued)

Sales-Weighted Annual Carbon Footprint of New Domestic and Import

Light Trucks by Size Class, Model Years 1975-2009 .......................................................... 11-13

Average Annual Carbon Footprint by Vehicle Classification, 1975 and 2009 ............................. 11-14

Appendix

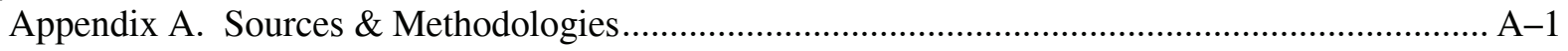

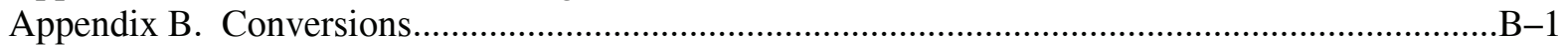

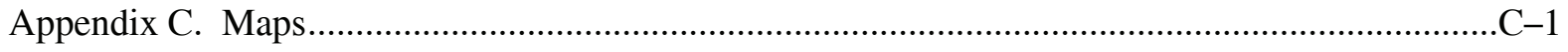

Attributes

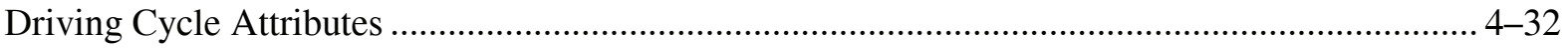

Available

Alternative Fuel Vehicles Available by Manufacturer, Model Year 2009...................................... 6-4

Hybrid Electric Vehicles Available by Manufacturer, Model Year 2009 ........................................ 6-5

Average

U.S. Average Vehicle Age, 1995-2009 ...................................................................................... 3-13

Average Material Consumption for a Light Vehicle, Model Years 1995, 2000,

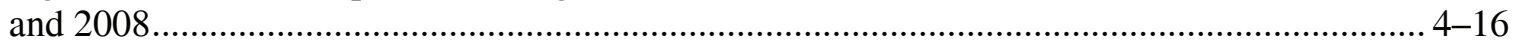

Reformed and Unreformed Light Truck Corporate Average Fuel Economy

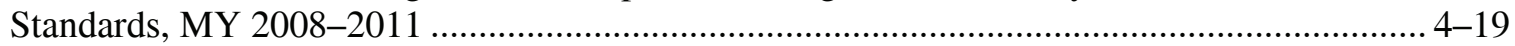

Car Corporate Average Fuel Economy (CAFE) Standards versus Sales-Weighted Fuel Economy Estimates, 1978-2010 ........................................................ 4-22

Light Truck Corporate Average Fuel Economy (CAFE) Standards versus Sales-Weighted Fuel Economy Estimates, 1978-2010 ......................................................... 4-23

Corporate Average Fuel Economy (CAFE) Fines Collected, 1983-2008 ................................... 4-24

Average Length of Time Business Fleet Vehicles are in Service, 2008 ....................................... 7-3

Average Annual Vehicle-Miles of Travel for Business Fleet Vehicles, 2008................................ 7-3

Average Miles per Domestic Federal Vehicle by Vehicle Type, 2009 ......................................... 7-4

Average Annual Expenditures of Households by Income, 2008 ..................................................... 8-4

Average Annual Vehicle-Miles, Vehicle Trips and Trip Length per

Household 1969, 1977, 1983, 1990, 1995 NPTS and 2001, 2009 NHTS ................................ 8-7

Average Number of Vehicles and Vehicle Travel per Household,

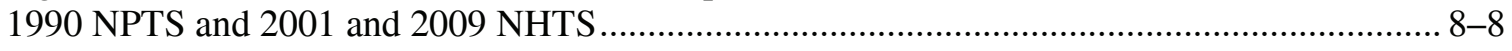

Average Vehicle Occupancy by Vehicle Type, 1995 NPTS and

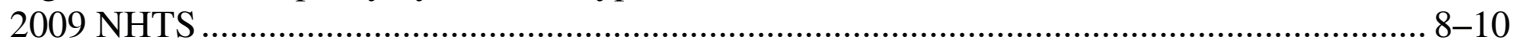

Average Vehicle Occupancy by Trip Purpose, 1977 NPTS and

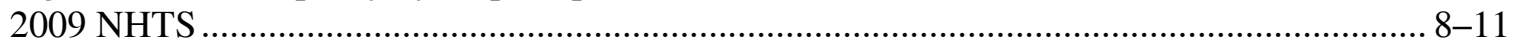

Average Annual Miles per Household Vehicle by Vehicle Age .................................................... 8 8-12

Self-Reported vs. Odometer Average Annual Miles, 1995 NPTS and

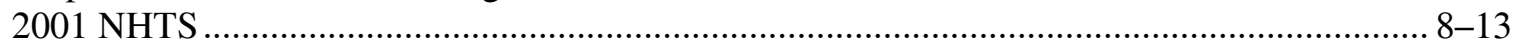

Average Daily Miles Driven (per Driver), 2009 NHTS .............................................................. 8-16

Daily and Annual Vehicle Miles of Travel and Average Age for Each Vehicle

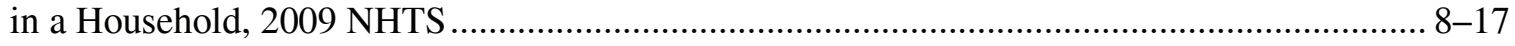

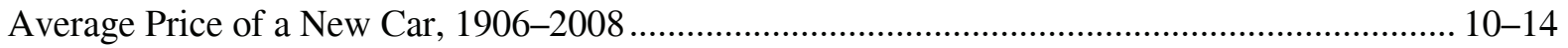

Average Price of a New Car (Domestic and Import), 1970-2008 …........................................ 10-15

Average Annual Carbon Footprint by Vehicle Classification, 1975 and 2009 ............................ 11-14

Aviation

Summary Statistics for General Aviation, 1970-2008 …............................................................ 9-4

Refiner Sales Prices for Aviation Gasoline and Jet Fuel, 1978-2009 ......................................... 10-10 
Axle

Distribution of Five-Axle Tractor-Trailers by On-Road Vehicle Weight, 2008

Barrel

Refinery Yield of Petroleum Products From a Barrel of Crude Oil, 1987-2008 ........................... 1-14

Prices for a Barrel of Crude Oil and a Gallon of Gasoline, 1978-2009. $10-7$

Bicycle

Bicycle Sales, 1981-2008

Bike

Walk and Bike Trips by Trip Purpose, 2009 NHTS.

Boat

Recreational Boat Energy Use, 1970-2008. $9-7$

Bus

Truck and Bus Registrations for Selected Countries, 1960-2008.... 3-3

Buses

Summary Statistics on Transit Buses and Trolleybuses, 1994-2008 $5-24$

Business

Average Length of Time Business Fleet Vehicles are in Service, 2008 $7-3$

Average Annual Vehicle-Miles of Travel for Business Fleet Vehicles, 2008. $7-3$

CAFE

Car Corporate Average Fuel Economy (CAFE) Standards versus

Sales-Weighted Fuel Economy Estimates, 1978-2010

Light Truck Corporate Average Fuel Economy (CAFE) Standards versus

Sales-Weighted Fuel Economy Estimates, 1978-2010

Corporate Average Fuel Economy (CAFE) Fines Collected, 1983-2008

\section{California}

California Cars and Light Trucks Emission Certification Standards

Capita

Vehicles and Vehicle-Miles per Capita, 1950-2008

Car

Car Registrations for Selected Countries, 1960-2008. 3-2

Car Scrappage and Survival Rates, 1970, 1980 and 1990 Model Years

Car and Light Truck Survivability Rates and Lifetime Miles $3-17$

New Retail Car Sales in the United States, 1970-2009 4-5

Car Corporate Average Fuel Economy (CAFE) Standards versus

Sales-Weighted Fuel Economy Estimates, 1978-2010 $4-22$

Average Price of a New Car, 1906-2008 $10-14$

Average Price of a New Car (Domestic and Import), 1970-2008 $10-15$

Car Operating Cost per Mile, 1985-2009 $10-16$

Fixed Car Operating Costs per Year, 1975-2009. $10-17$ 
Carbon

Fuel Economy and Carbon Dioxide Emissions Standards, MY 2012-2016................................ 4-20

Fuel Economy and Carbon Dioxide Targets for Model Year 2016 ........................................... 4-21

World Carbon Dioxide Emissions, 1990 and 2006 .................................................................... 11-2

Numerical Estimates of Global Warming Potentials Compared with Carbon Dioxide .................. 11-3

U.S. Carbon Emissions from Fossil Energy Consumption by End-Use

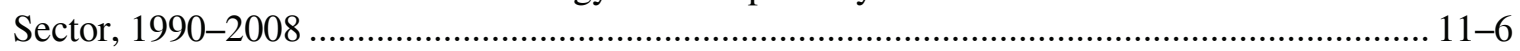

U.S. Carbon Emissions from Energy Use in the Transportation

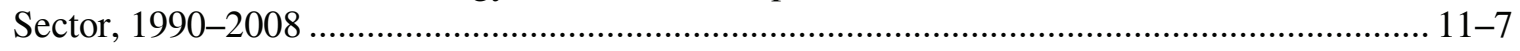

Sales-Weighted Annual Carbon Footprint of New Domestic and Import Cars

by Size Class, Model Years 1975-2009 ............................................................................... 11-12

Sales-Weighted Annual Carbon Footprint of New Domestic and Import

Light Trucks by Size Class, Model Years 1975-2009 ........................................................... 11-13

Average Annual Carbon Footprint by Vehicle Classification, 1975 and 2009 ........................... 11-14

Carbon Dioxide Emissions from a Gallon of Fuel ................................................................ 11-15

Emissions of Carbon Monoxide from Highway Vehicles, 1970-2005 .......................................... 12-4

Carriers

Summary Statistics for U.S. Domestic and International Certificated Route Air Carriers (Combined Totals), 1970-2008 ......................................................................... 9-3

Cars

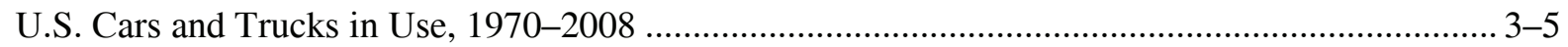

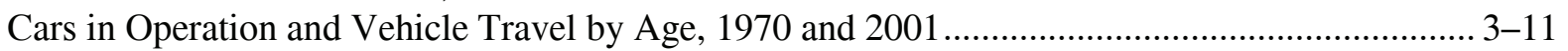

Summary Statistics for Cars, 1970-2008 ............................................................................. 4-2

Period Sales, Market Shares, and Sales-Weighted Fuel Economies of New Domestic and Import Cars, Selected Model Years 1975-2009 ….................................................................... 4-7

Sales-Weighted Curb Weight of New Domestic and Import Cars by Size Class, Model Years 1975-2009...................................................................................................... 4-14

Sales-Weighted Engine Size of New Domestic and Import Cars by Size Class, Model Years 1975-2009.............................................................................................. 4-12

Sales-Weighted Interior Space of New Domestic and Import Cars by Size Class,

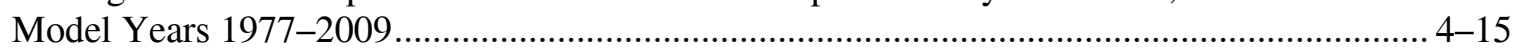

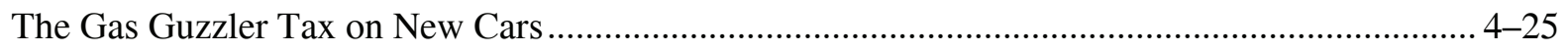

Sales-Weighted Annual Carbon Footprint of New Domestic and Import Cars

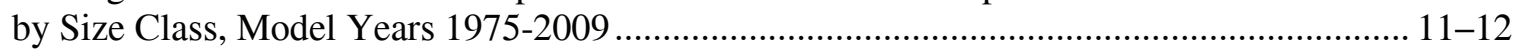

U.S. Tier 2 Emission Standards for Cars and Light Trucks ................................................... 12-13

California Cars and Light Trucks Emission Certification Standards .......................................... 12-15

Census

Household Vehicle Ownership, 1960-2000 Census .................................................................... 8-5

Means of Transportation to Work, 1980, 1990 and 2000 Census .................................................. 8-19

Workers by Commute Time, 1990 and 2000 Census ................................................................... 8-21

Certificated

Summary Statistics for U.S. Domestic and International Certificated Route Air Carriers (Combined Totals), 1970-2008 ……................................................................. 9-3

Certification

California Cars and Light Trucks Emission Certification Standards ......................................... 12-15

Characteristics

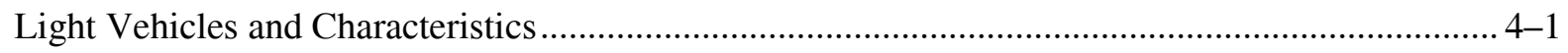


Characteristics (continued)

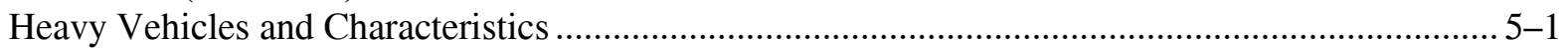

Alternative Fuel and Advanced Technology Vehicles and Characteristics................................... 6-1

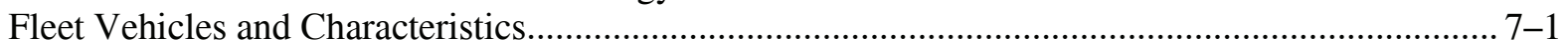

Household Vehicles and Characteristics ............................................................................. 8 8

Characteristics of U.S. Daily per Vehicle Driving vs. Dwelling Unit Type

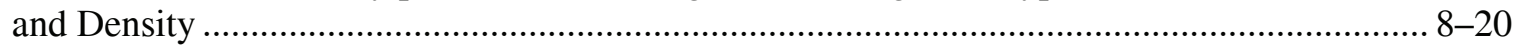

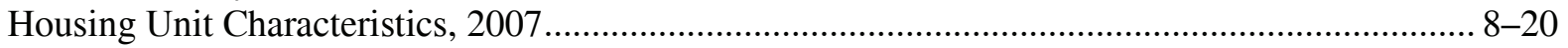

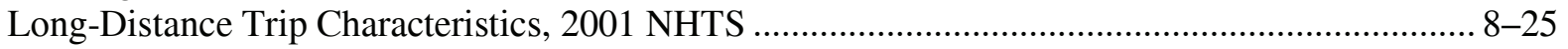

Cities

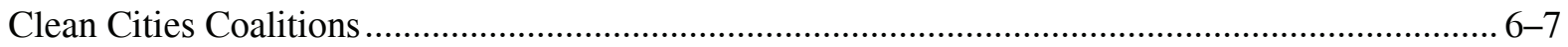

City

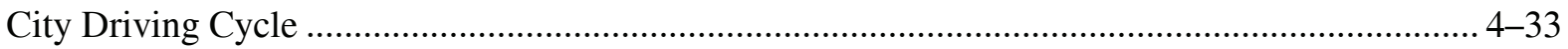

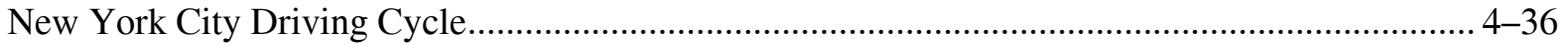

Class

Light Vehicle Market Shares by Size Class, Model Years 1975-2009............................................. 4-10

Sales-Weighted Engine Size of New Domestic and Import Light Trucks by Size Class, Model Years 1975-2009 ................................................................................. 13

Sales-Weighted Curb Weight of New Domestic and Import Cars by Size Class, Model Years 1975-2009 .................................................................................. 4-14

Sales-Weighted Interior Space of New Domestic and Import Cars

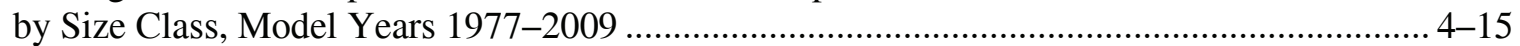

Truck Statistics by Gross Vehicle Weight Class, 2002 …........................................................... 5-6

Truck Harmonic Mean Fuel Economy by Size Class, 1992, 1997, and 2002 .................................. 5-6

Sales-Weighted Annual Carbon Footprint of New Domestic and Import Cars by Size Class, Model Years 1975-2009 ............................................................................... 11-12

Sales-Weighted Annual Carbon Footprint of New Domestic and Import Light Trucks by Size Class, Model Years 1975-2009 ............................................................ 11-13

Class 1

Summary Statistics on Class 1, Class 2a, and Class 2b Light Trucks ........................................... 4-4

Sales Estimates of Class 1, Class 2a, and Class 2b Light Trucks, 1989-1999................................. 4-4

Class I Railroad Freight Systems in the United States Ranked by

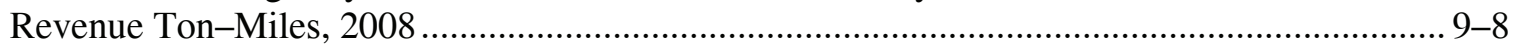

Summary Statistics for Class I Freight Railroads, 1970-2008 ..................................................... 9-9

Class 2a

Summary Statistics on Class 1, Class 2a, and Class 2b Light Trucks ........................................... 4-4

Sales Estimates of Class 1, Class 2a, and Class 2b Light Trucks, 1989-1999................................. 4-4

Class $2 b$

Summary Statistics on Class 1, Class 2a, and Class 2b Light Trucks ............................................ 4-4

Sales Estimates of Class 1, Class 2a, and Class 2b Light Trucks, 1989-1999................................. 4-4

Class 8

Effect of Terrain on Class 8 Truck Fuel Economy...................................................................... 5-14

Fuel Economy for Class 8 Trucks as Function of Speed and Tractor-Trailer

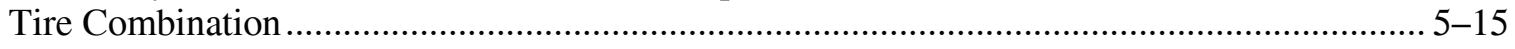


Class 8 (continued)

Class 8 Truck Fuel Economy as a Function of Speed and Tractor-Trailer Tire Combination and Percentage of Total Distance Traveled as a Function

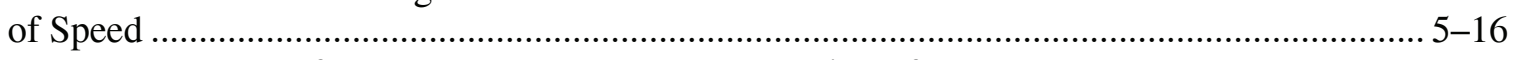

Class 8 Truck Percent of Total Fuel Consumed as a Function of Speed and

Tractor-Trailer Tire Combination ................................................................................... 5-17

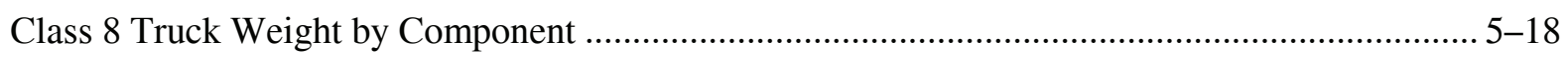

Classification

Average Annual Carbon Footprint by Vehicle Classification, 1975 and 2009 ........................... 11-14

Clean

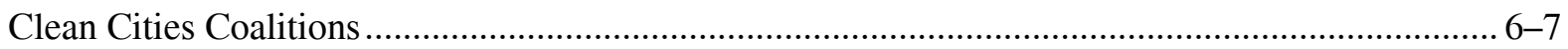

Coalitions

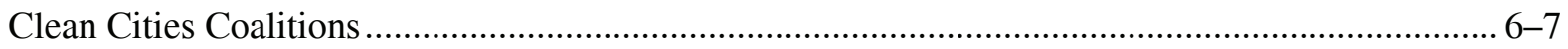

Cold

Cold Temperature (Cold FTP) Driving Cycle ........................................................................ 4-34

Collected

Corporate Average Fuel Economy (CAFE) Fines Collected, 1983-2008 .................................... 4-24

Combination

Summary Statistics for Combination Trucks, 1970-2008............................................................ 5-3

Fuel Economy for Class 8 Trucks as Function of Speed and Tractor-Trailer

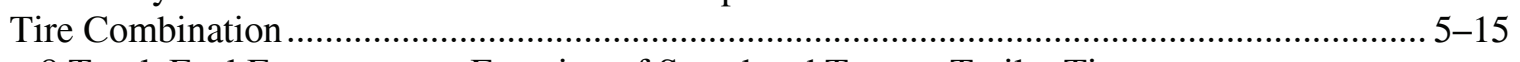

Class 8 Truck Fuel Economy as a Function of Speed and Tractor-Trailer Tire

Combination and Percentage of Total Distance Traveled as a Function

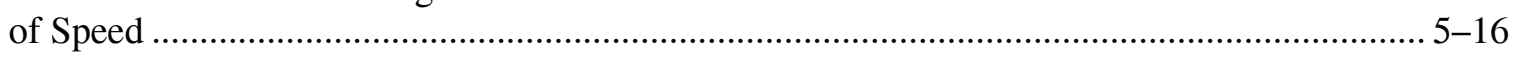

Class 8 Truck Percent of Total Fuel Consumed as a Function of Speed and

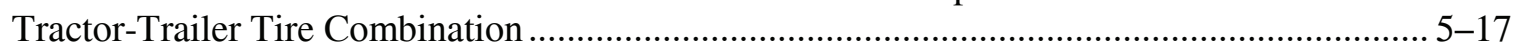

Combined

Summary Statistics for U.S. Domestic and International Certificated Route Air Carriers (Combined Totals), 1970-2008 ............................................................................ 9-3

Commerce

Tonnage Statistics for Domestic and International Waterborne

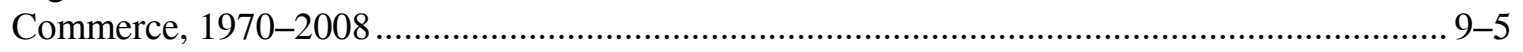

Summary Statistics for Domestic Waterborne Commerce, 1970-2008 _........................................ 9-6

Commodity

Growth of Freight in the United States: Comparison of the 2007, 2002 and 1997 Commodity

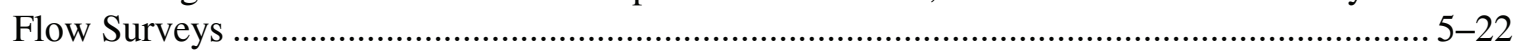

Growth of Freight Miles in the United States: Comparison of the 2007, 2002 and 1997 Commodity Flow Surveys...................................................................................... 5-23

Commute

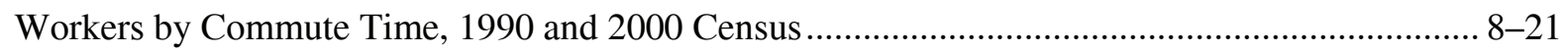


Commuter

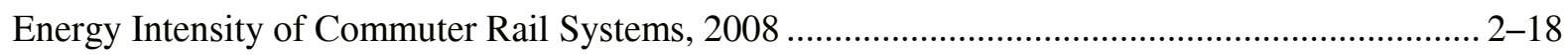

Summary Statistics for Commuter Rail Operations, 1984-2008 .............................................. 9-12

Compared

Vehicles per Thousand People: U.S. (Over Time) Compared to Other

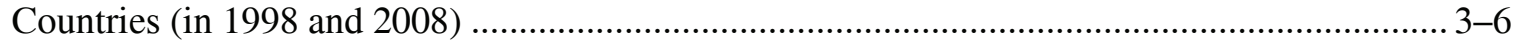

Numerical Estimates of Global Warming Potentials Compared with Carbon Dioxide .................. 11-3

Comparison

Comparison of U.S., European, and Japanese Driving Cycles..................................................... 4-38

Growth of Freight in the United States: Comparison of the 2007, 2002 and 1997 Commodity

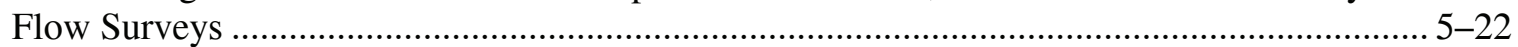

Growth of Freight Miles in the United States: Comparison of the 2007, 2002

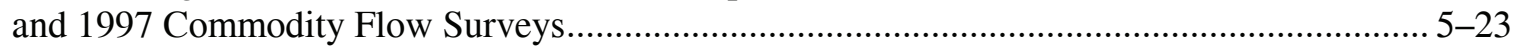

Component

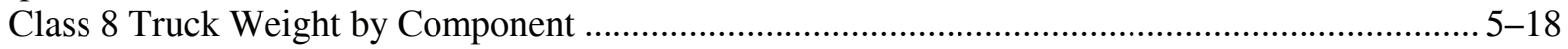

Compounds

Total National Emissions of Volatile Organic Compounds, 1970-2008 ..................................... 12-7

Emissions of Volatile Organic Compounds from Highway Vehicles, 1970-2005 ......................... 12-8

Conditioning

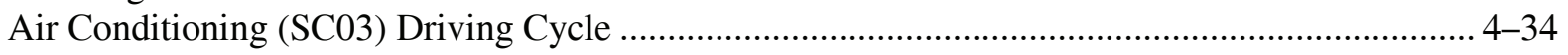

Consumed

Class 8 Truck Percent of Total Fuel Consumed as a Function of Speed and

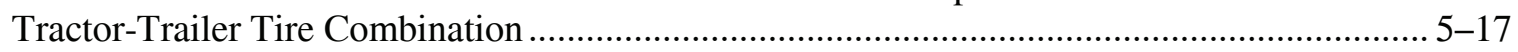

Fuel Consumed by Federal Government Fleets, FY 2000-2009 …........................................... 7-6

Consumer

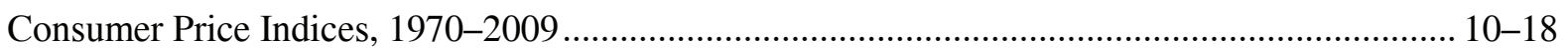

Consumption

World Petroleum Consumption, 1960-2009 …........................................................................... 1-5

World Oil Reserves, Production and Consumption, 2008 ......................................................... 1-6

World Natural Gas Reserves, Production and Consumption, 2008 ................................................ 1-7

Petroleum Production and Consumption and Some Important Percent

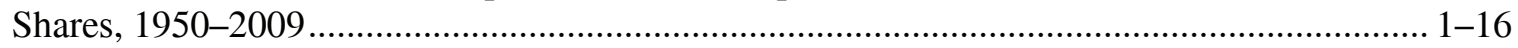

United States Petroleum Production and Consumption - All Sectors,

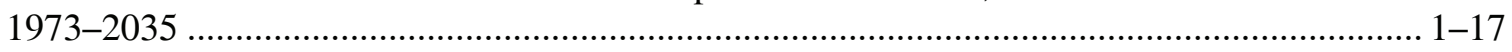

United States Petroleum Production, Transportation and Consumption,

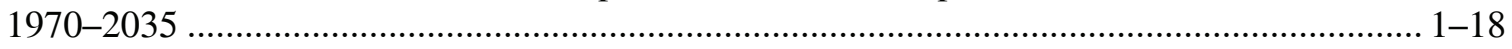

Consumption of Petroleum by End-Use Sector, 1973-2009 ....................................................... 1-19

Highway Transportation Petroleum Consumption by Mode, 1970-2008 ...................................... 1-20

Nonhighway Transportation Petroleum Consumption by Mode, 1970-2008 ............................... 1-21

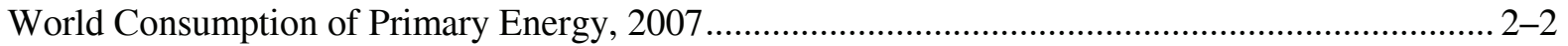

U. S. Consumption of Total Energy by End-Use Sector, 1973-2009 ........................................... 2-3

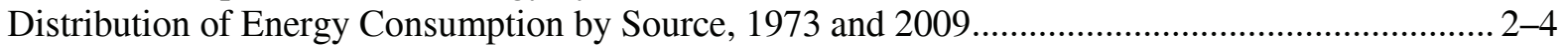

Alternative Fuel and Oxygenate Consumption, 2003-2008 …...................................................... 2-5

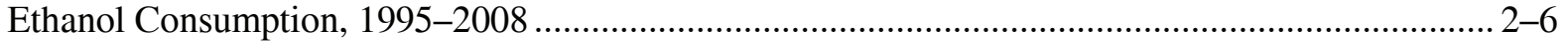


Consumption (continued)

Domestic Consumption of Transportation Energy by Mode and

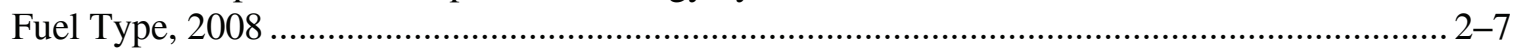

Highway Transportation Energy Consumption by Mode, 1970-2008........................................... 2-9

Nonhighway Transportation Energy Consumption by Mode, 1970-2008.................................... 2-10

Off-highway Transportation-related Fuel Consumption from the NonRoad

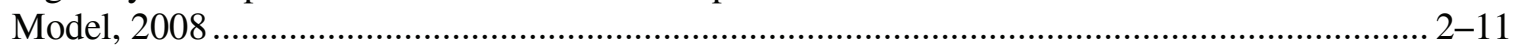

Fuel Consumption from Lawn and Garden Equipment, 2008..................................................... 2-12

Average Material Consumption for a Light Vehicle, Model Years 1995, 2000,

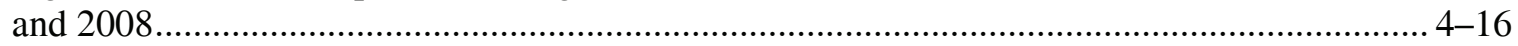

Personal Consumption Expenditures, 1970-2009................................................................... 10-18

U.S. Carbon Emissions from Fossil Energy Consumption by End-Use

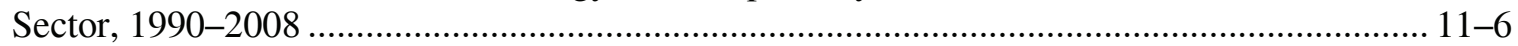

Conventional

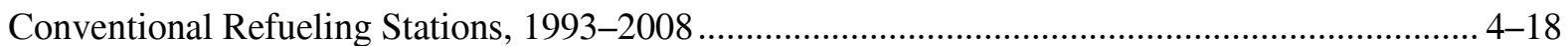

Properties of Conventional and Alternative Fuels......................................................................... 6-9

Conversions

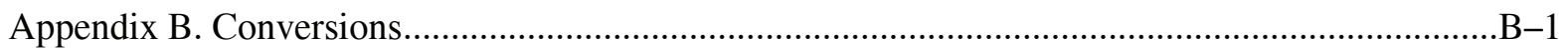

Corporate

Reformed and Unreformed Light Truck Corporate Average Fuel Economy

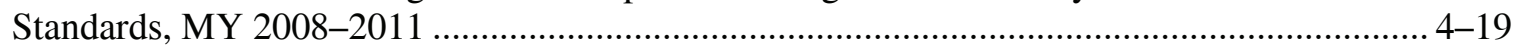

Car Corporate Average Fuel Economy (CAFE) Standards versus

Sales-Weighted Fuel Economy Estimates, 1978-2010 ............................................................ 4-22

Light Truck Corporate Average Fuel Economy (CAFE) Standards versus

Sales-Weighted Fuel Economy Estimates, 1978-2010 ........................................................... 4-23

Corporate Average Fuel Economy (CAFE) Fines Collected, 1983-2008 ................................... 4-24

Corporation

Summary Statistics for the National Railroad Passenger Corporation

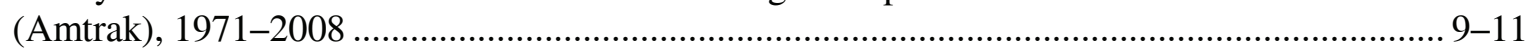

Cost

Car Operating Cost per Mile, 1985-2009 ............................................................................. 10-16

Costs

Costs of Oil Dependence to the U.S. Economy, 1970-2009..................................................... 1-10

Fixed Car Operating Costs per Year, 1975-2009..................................................................... 10-17

Countries

Car Registrations for Selected Countries, 1960-2008............................................................... 3-2

Truck and Bus Registrations for Selected Countries, 1960-2008 .................................................. 3-3

Vehicles per Thousand People: U.S. (Over Time) Compared to Other

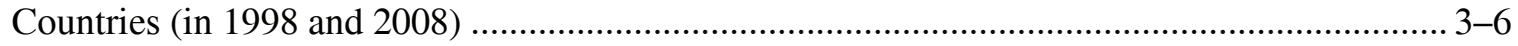

Vehicles per Thousand People in Other Countries, 1998 and 2008 .............................................. 3-8

Gasoline Prices for Selected Countries, 1990-2008 …........................................................... 10-3

Diesel Fuel Prices for Selected Countries, 1998-2008 .............................................................. 10-4

Gasoline Prices for Selected Countries, 1990 and 2008 .......................................................... 10-5

Diesel Prices for Selected Countries, 1990 and 2008 .............................................................. 10-6

Criteria

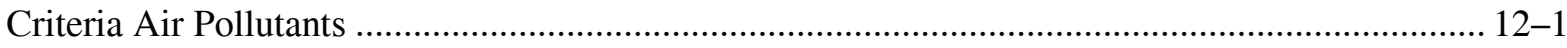


Criteria (continued)

Total National Emissions of the Criteria Air Pollutants by Sector, 2008.

Crude

World Crude Oil Production, 1960-2009 ..................................................................................... 1-3

U.S. Refinery Input of Crude Oil and Petroleum Products, 1987-2008 …................................... 1-13

Refinery Yield of Petroleum Products From a Barrel of Crude Oil, 1987-2008 ........................... 1-14

Prices for a Barrel of Crude Oil and a Gallon of Gasoline, 1978-2009 ........................................ 10-7

Curb

Sales-Weighted Curb Weight of New Domestic and Import Cars

by Size Class, Model Years 1975-2009 ………........................................................................ 4-14

Cycle

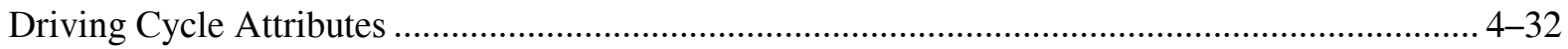

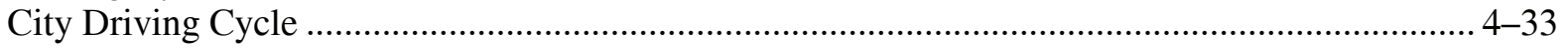

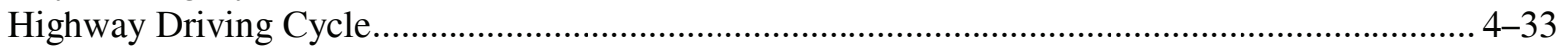

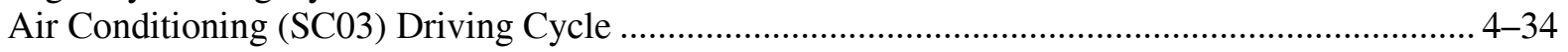

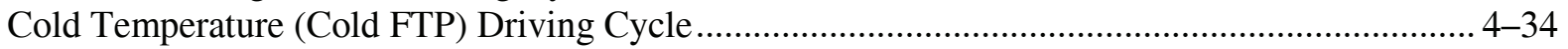

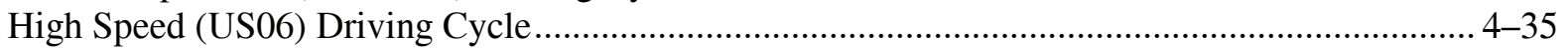

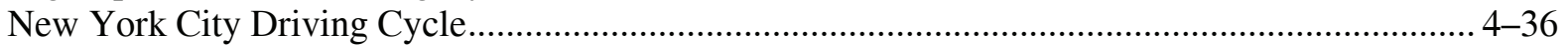

Representative Number Five Driving Cycle ........................................................................... 4-36

Cycles

Projected Fuel Economies from U.S., European, and Japanese Driving Cycles............................. 4-37

Comparison of U.S., European, and Japanese Driving Cycles...................................................... 4-38

Daily

Average Daily Miles Driven (per Driver), 2009 NHTS ..................................................................... 8-16

Daily Vehicle Miles of Travel (per Vehicle) by Number of Vehicles in the

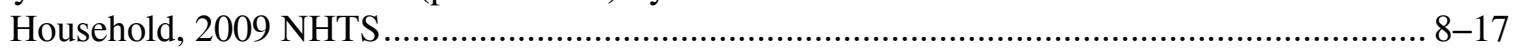

Daily and Annual Vehicle Miles of Travel and Average Age for Each Vehicle

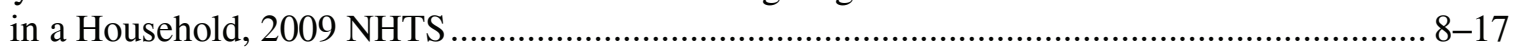

Daily Vehicle Miles of Travel for Each Vehicle in a Household, 2009 NHTS ................................ 8-18

Characteristics of U.S. Daily per Vehicle Driving vs. Dwelling Unit Type and Density

Dealerships

New Light Vehicle Dealerships and Sales, 1970-2008 .............................................................. 4-17

Defending

Summary of Military Expenditures for Defending Oil Supplies from the Middle East..... $1-11$

Definition

Definition of Wagons in Model Year 2009. $4-8$

Demand

Summary Statistics on Demand Response Vehicles, 1994-2008

Demographic

Demographic Statistics from the 1969, 1977, 1983, 1990, 1995 NPTS and 2001, 2009 NHTS 
Density

Characteristics of U.S. Daily per Vehicle Driving vs. Dwelling Unit Type and Density

Dependence

Costs of Oil Dependence to the U.S. Economy, 1970-2009 $1-10$

Diesel

Diesel Fuel Prices for Selected Countries, 1998-2008 $10-4$

Diesel Prices for Selected Countries, 1990 and 2008. $10-6$

Refiner Sales Prices for Propane and No. 2 Diesel, 1978-2009 $10-9$

Dioxide

Fuel Economy and Carbon Dioxide Emissions Standards, MY 2012-2016................................. 4-20

Fuel Economy and Carbon Dioxide Targets for Model Year 2016.......................................... 4-21

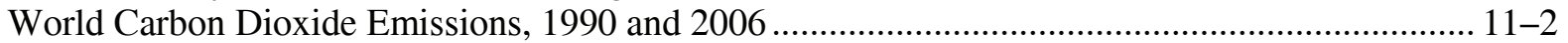

Carbon Dioxide Emissions from a Gallon of Fuel ..................................................................... 11-15

Distance

Class 8 Truck Fuel Economy as a Function of Speed and Tractor-Trailer Tire Combination and Percentage of Total Distance Traveled as a Function of Speed $5-16$

Share of Vehicle Trips by Trip Distance, 2009 NHTS..................................................................... 8

Share of Vehicle Trips to Work by Trip Distance, 2009 NHTS .................................................... 8 8-14

Long-Distance Trip Characteristics, 2001 NHTS ............................................................................... 8-25

Distribution

Distribution of Energy Consumption by Source, 1973 and 2009.................................................. 2-4

Distribution of Trucks over 26,000 lbs. Less than Two Years Old by Vehicle-

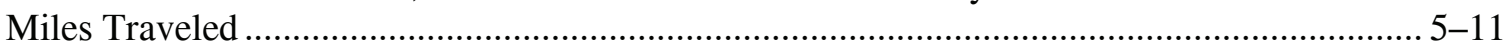

Distribution of Five-Axle Tractor-Trailers by On-Road Vehicle Weight, 2008 ............................ 5-20

Domestic

Domestic Consumption of Transportation Energy by Mode and Fuel Type, 2008

Period Sales, Market Shares, and Sales-Weighted Fuel Economies of New

Domestic and Import Cars, Selected Model Years 1975-2009

Period Sales, Market Shares, and Sales-Weighted Fuel Economies of New

Domestic and Import Light Trucks, Model Years 1975-2009

Sales-Weighted Engine Size of New Domestic and Import Cars

by Size Class, Model Years 1975-2009

Sales-Weighted Engine Size of New Domestic and Import Light Trucks

by Size Class, Model Years 1975-2009

Sales-Weighted Curb Weight of New Domestic and Import Cars

by Size Class, Model Years 1975-2009

Sales-Weighted Interior Space of New Domestic and Import Cars

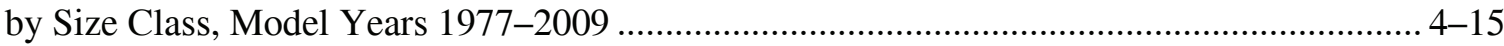

Average Miles per Domestic Federal Vehicle by Vehicle Type, 2009 …........................................ 7-4

Summary Statistics for U.S. Domestic and International Certificated Route Air Carriers (Combined Totals), 1970-2008

Tonnage Statistics for Domestic and International Waterborne Commerce, 1970-2008

Summary Statistics for Domestic Waterborne Commerce, 1970-2008. 
Domestic (continued)

Average Price of a New Car (Domestic and Import), 1970-2008

Sales-Weighted Annual Carbon Footprint of New Domestic and Import Cars by Size Class, Model Years 1975-2009

Sales-Weighted Annual Carbon Footprint of New Domestic and Import

Light Trucks by Size Class, Model Years 1975-2009 $11-13$

Driven

Average Daily Miles Driven (per Driver), 2009 NHTS $8-16$

Driver

Average Daily Miles Driven (per Driver), 2009 NHTS $8-16$

Driving

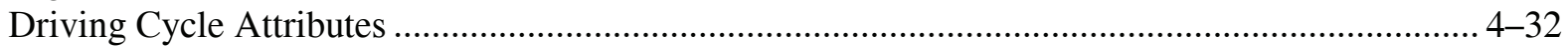

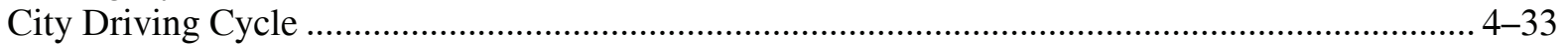

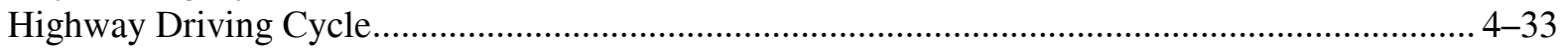

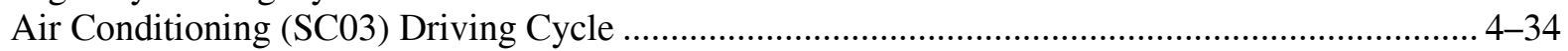

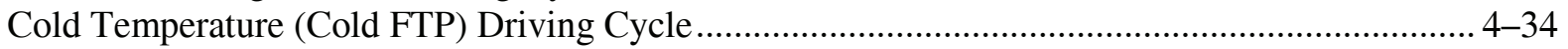

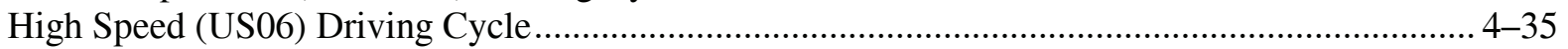

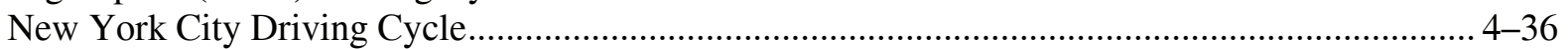

Representative Number Five Driving Cycle ........................................................................... 4-36

Projected Fuel Economies from U.S., European, and Japanese Driving Cycles............................. 4-37

Comparison of U.S., European, and Japanese Driving Cycles...................................................... 4-38

Characteristics of U.S. Daily per Vehicle Driving vs. Dwelling Unit Type

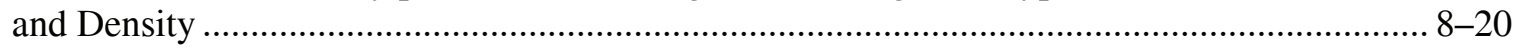

Dwelling

Characteristics of U.S. Daily per Vehicle Driving vs. Dwelling Unit Type

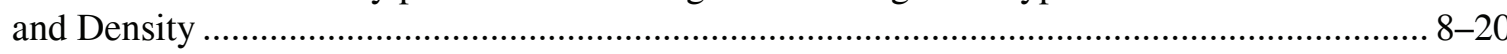

East

Summary of Military Expenditures for Defending Oil Supplies from the

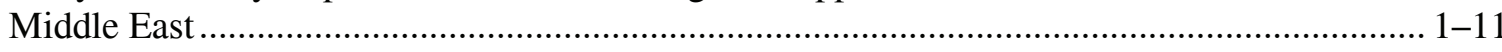

Economic

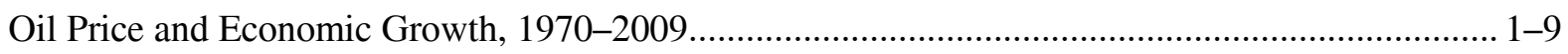

Economies

Period Sales, Market Shares, and Sales-Weighted Fuel Economies of New Domestic and Import Cars, Selected Model Years 1975-2009 ................................................. 4-7

Period Sales, Market Shares, and Sales-Weighted Fuel Economies of New Domestic and Import Light Trucks, Model Years 1975-2009 …............................................ 4-9

Projected Fuel Economies from U.S., European, and Japanese Driving Cycles............................. 4-37

Economy

Costs of Oil Dependence to the U.S. Economy, 1970-2009...................................................... 1-10

Reformed and Unreformed Light Truck Corporate Average Fuel Economy

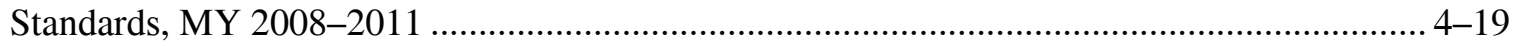

Fuel Economy and Carbon Dioxide Emissions Standards, MY 2012-2016 ................................. 4-20

Fuel Economy and Carbon Dioxide Targets for Model Year 2016.............................................. 4-21

Car Corporate Average Fuel Economy (CAFE) Standards versus Sales-Weighted Fuel Economy Estimates, 1978-2010 
Economy (continued)

Light Truck Corporate Average Fuel Economy (CAFE) Standards versus

Sales-Weighted Fuel Economy Estimates, 1978-2010 ....................................................... 4-23

Corporate Average Fuel Economy (CAFE) Fines Collected, 1983-2008 ..................................... 4-24

Fuel Economy by Speed, PSAT Model Results ...................................................................... 4-27

Fuel Economy by Speed, 1973, 1984, and 1997 Studies ........................................................ 4-28

Steady Speed Fuel Economy for Vehicles Tested in the 1997 Study ............................................ 4-31

Truck Harmonic Mean Fuel Economy by Size Class, 1992, 1997, and 2002 ................................. 5-6

Effect of Terrain on Class 8 Truck Fuel Economy..................................................................... 5-14

Fuel Economy for Class 8 Trucks as Function of Speed and Tractor-Trailer

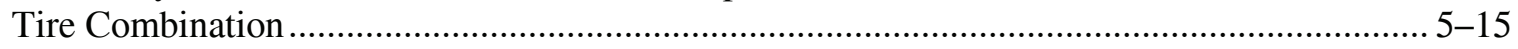

Class 8 Truck Fuel Economy as a Function of Speed and Tractor-Trailer Tire

Combination and Percentage of Total Distance Traveled as a Function

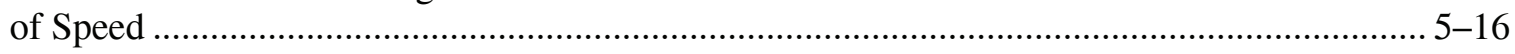

Transportation and the Economy ..................................................................................... 10

Effect

Effect of Terrain on Class 8 Truck Fuel Economy ........................................................................ 5-14

Light Vehicle Exhaust Emission Standards in Effect in 2009 when

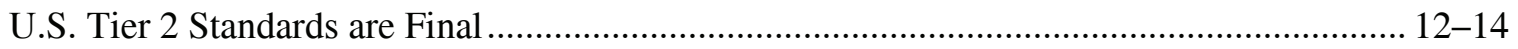

Electric

Hybrid Electric Vehicles Available by Manufacturer, Model Year 2009 ....................................... 6-5

Electronic

Share of Heavy Trucks with Selected Electronic Features, 2002 …........................................... 5-12

Emission

Light Vehicle Exhaust Emission Standards in Effect in 2009 when

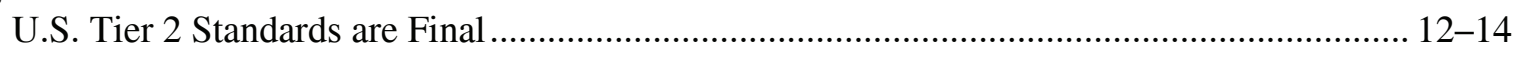

California Cars and Light Trucks Emission Certification Standards ......................................... 12-15

Emissions

Fuel Economy and Carbon Dioxide Emissions Standards, MY 2012-2016................................. 4-20

Greenhouse Gas Emissions ............................................................................................... 11-1

World Carbon Dioxide Emissions, 1990 and 2006 .................................................................. 11-2

U.S. Emissions of Greenhouse Gases, based on Global Warming Potential,

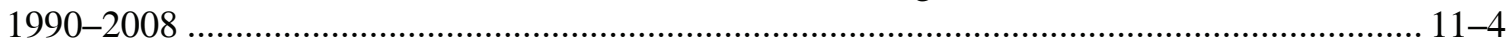

Total U.S. Greenhouse Emissions by End-Use Sector, 2008 ….................................................. 11-5

U.S. Carbon Emissions from Fossil Energy Consumption by End-Use

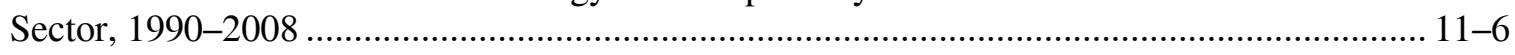

U.S. Carbon Emissions from Energy Use in the Transportation

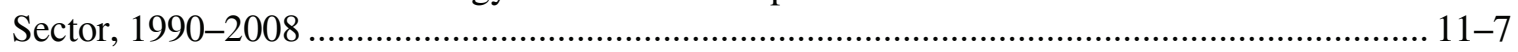

Carbon Dioxide Emissions from a Gallon of Fuel ................................................................. 11-15

Total National Emissions of the Criteria Air Pollutants by Sector, 2008...................................... 12-2

Total National Emissions of Carbon Monoxide, 1970-2008 …................................................... 12-3

Emissions of Carbon Monoxide from Highway Vehicles, 1970-2005 …..................................... 12-4

Total National Emissions of Nitrogen Oxides, 1970-2008 ....................................................... 12-5

Emissions of Nitrogen Oxides from Highway Vehicles, 1970-2005 ......................................... 12-6

Total National Emissions of Volatile Organic Compounds, 1970-2008 ...................................... 12-7

Emissions of Volatile Organic Compounds from Highway Vehicles, 1970-2005 ........................ 12-8

Total National Emissions of Particulate Matter (PM-10), 1970-2008 .......................................... 12-9

Emissions of Particulate Matter (PM-10) from Highway Vehicles, 1970-2005 .......................... 12-10 
Emissions (continued)

Total National Emissions of Particulate Matter (PM-2.5), 1990-2008 ….................................... 12-11

Emissions of Particulate Matter (PM-2.5) from Highway Vehicles, 1990-2005......................... 12-12

U.S. Tier 2 Emission Standards for Cars and Light Trucks .................................................. 12-13

Employment

Transportation-related Employment, 1999 and 2009 …............................................................ 10-19

U.S. Employment for Motor Vehicles and Motor Vehicle Parts

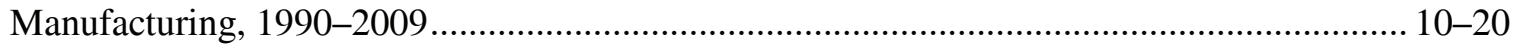

Empty

Gross Vehicle Weight vs. Empty Vehicle Weight ......................................................................... 5-19

End-Use

Consumption of Petroleum by End-Use Sector, 1973-2009.................................................... 1-19

U. S. Consumption of Total Energy by End-Use Sector, 1973-2009 ............................................. 2-3

Total U.S. Greenhouse Emissions by End-Use Sector, 2008 ….................................................. 11-5

U.S. Carbon Emissions from Fossil Energy Consumption by End-Use

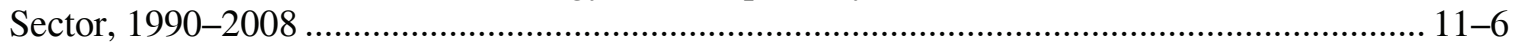

Energy

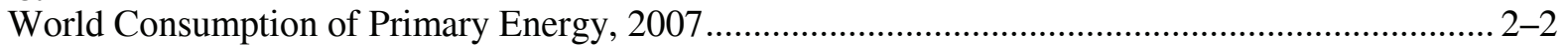

U. S. Consumption of Total Energy by End-Use Sector, 1973-2009 ............................................ 2-3

Distribution of Energy Consumption by Source, 1973 and 2009.................................................... 2-4

Domestic Consumption of Transportation Energy by Mode and

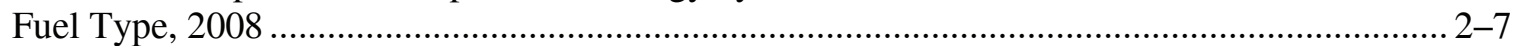

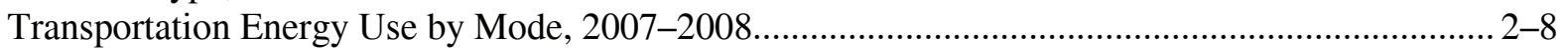

Highway Transportation Energy Consumption by Mode, 1970-2008_.........................................2-9

Nonhighway Transportation Energy Consumption by Mode, 1970-2008.................................... 2-10

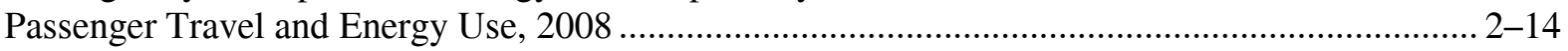

Energy Intensities of Highway Passenger Modes, 1970-2008 ................................................ 2-15

Energy Intensities of Nonhighway Passenger Modes, 1970-2008 ................................................. 2-16

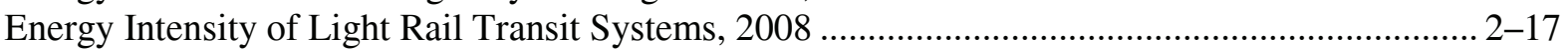

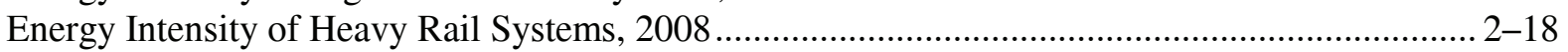

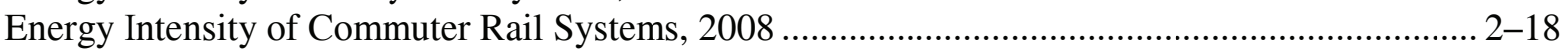

Intercity Freight Movement and Energy Use in the United States, 2007 and 2008 ....................... 2-19

Energy Intensities of Freight Modes, 1970-2008 …......................................................................2-20

Nonhighway Energy Use Shares, 1970-2008 …......................................................................... 9-2

Recreational Boat Energy Use, 1970-2008 …................................................................................. 9-7

U.S. Carbon Emissions from Fossil Energy Consumption by End-Use Sector, 1990-2008 ........... 11-6

U.S. Carbon Emissions from Energy Use in the Transportation Sector, 1990-2008 ...................... 11-7

Engine

Sales-Weighted Engine Size of New Domestic and Import Cars

by Size Class, Model Years 1975-2009 .................................................................................. 12

Sales-Weighted Engine Size of New Domestic and Import Light Trucks

by Size Class, Model Years 1975-2009 ………........................................................................ 4-13

Equipment

Fuel Consumption from Lawn and Garden Equipment, 2008................................................... 2-12

Estimates

Sales Estimates of Class 1, Class 2a, and Class 2b Light Trucks, 1989-1999................................. 4-4 
Estimates (continued)

Car Corporate Average Fuel Economy (CAFE) Standards versus

Sales-Weighted Fuel Economy Estimates, 1978-2010

Light Truck Corporate Average Fuel Economy (CAFE) Standards versus

Sales-Weighted Fuel Economy Estimates, 1978-2010

Estimates of Alternative Fuel Vehicles in Use, 1995-2008

Ethanol

Ethanol Consumption, 1995-2008

European

Projected Fuel Economies from U.S., European, and Japanese Driving Cycles............................ 4-37

Comparison of U.S., European, and Japanese Driving Cycles. 4-38

Excise

Federal Excise Taxes on Motor Fuels, 2008

$10-11$

Exemptions

State Tax Exemptions for Gasohol, 2008

$10-11$

Exhaust

Light Vehicle Exhaust Emission Standards in Effect in 2009 when

U.S. Tier 2 Standards are Final

Expenditures

Summary of Military Expenditures for Defending Oil Supplies from the

Middle East $1-11$

Average Annual Expenditures of Households by Income, 2008 ................................................... 8-4

Personal Consumption Expenditures, 1970-2009

Exports

United States Petroleum Production, Imports and Exports, 1950-2009 ...................................... 1-15

Facility

Percentage of Trucks by Fleet Size and Primary Fueling Facility, 2002 ...................................... 5-9

Share of Trucks by Major Use and Primary Fueling Facility, 2002 ............................................ 5-10

Features

Share of Heavy Trucks with Selected Electronic Features, 2002

Federal

Average Miles per Domestic Federal Vehicle by Vehicle Type, 2009 …..................................... 7-4

Federal Government Vehicles, 2001-2009 ..................................................................................... 7-5

Federal Fleet Vehicle Acquisitions by Fuel Type, FY 2002- 2009 …........................................... 7-6

Fuel Consumed by Federal Government Fleets, FY 2000-2009 ….............................................. 7-6

Federal Government Vehicles by Agency, FY 2009 .............................................................. 7-7

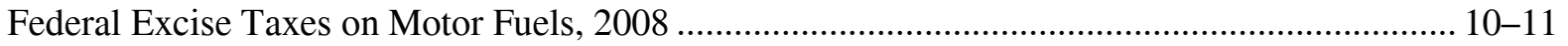

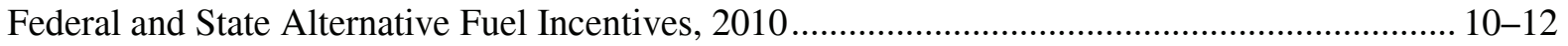

Federal and State Advanced Technology Incentives, 2010 ..................................................... 10-13

Feedstocks

GREET Model Feedstocks and Fuels.

$11-10$ 
Final

Light Vehicle Exhaust Emission Standards in Effect in 2009 when

U.S. Tier 2 Standards are Final

Fines

Corporate Average Fuel Economy (CAFE) Fines Collected, 1983-2008

Fiscal

Federal Fleet Vehicle Acquisitions by Fuel Type, FY 2002-2009 ..................................7-6

Fuel Consumed by Federal Government Fleets, FY 2000-2009 ...................................7-6

Federal Government Vehicles by Agency, FY 2009................................................................... 7-7

Five

Representative Number Five Driving Cycle ......................................................................... 4-36

Distribution of Five-Axle Tractor-Trailers by On-Road Vehicle Weight, 2008 ............................ 5-20

Fixed

Fixed Car Operating Costs per Year, 1975-2009...................................................................... 10-17

Fleet

Percentage of Trucks by Fleet Size and Primary Fueling Facility, 2002 ...................................... 5-9

Fleet Vehicles and Characteristics.................................................................................................

Fleet Vehicles in Service as of January 1, 2009 ...................................................................... 7-2

New Light Fleet Vehicle Registrations by Vehicle Type, Model Year 2008 ................................. 7-3

Average Length of Time Business Fleet Vehicles are in Service, 2008 ....................................... 7-3

Average Annual Vehicle-Miles of Travel for Business Fleet Vehicles, 2008................................. 7-3

Federal Fleet Vehicle Acquisitions by Fuel Type, FY 2002- 2009 …........................................... 7-6

Fleets

Fuel Consumed by Federal Government Fleets, FY 2000-2009 …............................................... 7-6

Flow

Growth of Freight in the United States: Comparison of the 2007, 2002 and 1997 Commodity

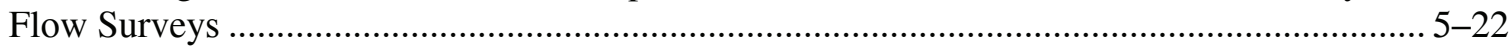

Growth of Freight Miles in the United States: Comparison of the 2007, 2002

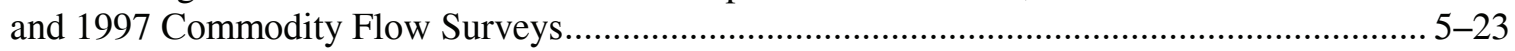

Footprint

Sales-Weighted Annual Carbon Footprint of New Domestic and Import Cars

by Size Class, Model Years 1975-2009 .................................................................................. 11-12

Sales-Weighted Annual Carbon Footprint of New Domestic and Import

Light Trucks by Size Class, Model Years 1975-2009 ........................................................... 11-13

Average Annual Carbon Footprint by Vehicle Classification, 1975 and 2009 ............................ 11-14

Fossil

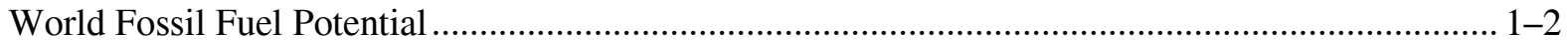

U.S. Carbon Emissions from Fossil Energy Consumption by End-Use

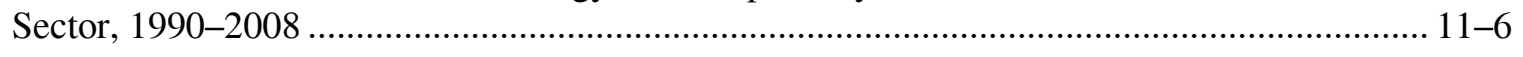

Four-Tire

Summary Statistics for Two-Axle, Four-Tire Trucks, 1970-2008 4-3 
Freight

Intercity Freight Movement and Energy Use in the United States, 2007 and 2008 ...................... 2-19

Energy Intensities of Freight Modes, 1970-2008 .................................................................. 2-20

Growth of Freight in the United States: Comparison of the 2007, 2002 and 1997 Commodity

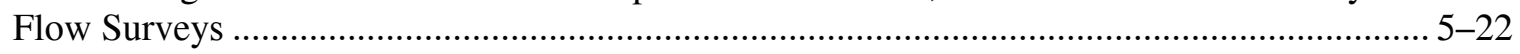

Growth of Freight Miles in the United States: Comparison of the 2007, 2002

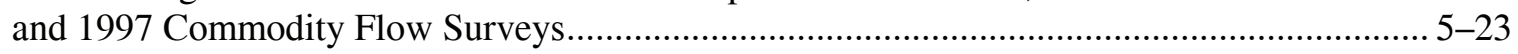

Class I Railroad Freight Systems in the United States Ranked by

Revenue Ton-Miles, 2008 ......................................................................................... 9-8

Summary Statistics for Class I Freight Railroads, 1970-2008 ................................................... 9-9

FTP

Cold Temperature (Cold FTP) Driving Cycle .............................................................................. 4-34

Fuel

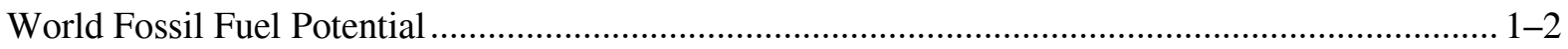

Alternative Fuel and Oxygenate Consumption, 2003-2008 ....................................................... 2-5

Domestic Consumption of Transportation Energy by Mode and

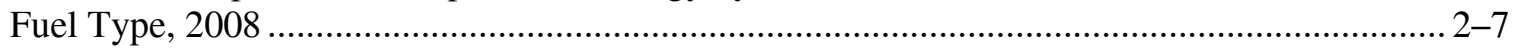

Off-highway Transportation-related Fuel Consumption from the NonRoad

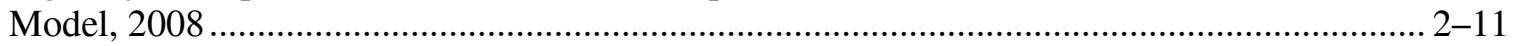

Fuel Consumption from Lawn and Garden Equipment, 2008..................................................... 2-12

Period Sales, Market Shares, and Sales-Weighted Fuel Economies of New Domestic and Import Cars, Selected Model Years 1975-2009 ................................................... 4-7

Period Sales, Market Shares, and Sales-Weighted Fuel Economies of New Domestic and Import Light Trucks, Model Years 1975-2009 .................................................. 4-9

Reformed and Unreformed Light Truck Corporate Average Fuel Economy

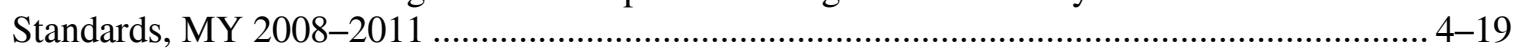

Fuel Economy and Carbon Dioxide Emissions Standards, MY 2012-2016................................ 4-20

Fuel Economy and Carbon Dioxide Targets for Model Year 2016.............................................. 4-21

Car Corporate Average Fuel Economy (CAFE) Standards versus

Sales-Weighted Fuel Economy Estimates, 1978-2010 ......................................................... 4-22

Light Truck Corporate Average Fuel Economy (CAFE) Standards versus

Sales-Weighted Fuel Economy Estimates, 1978-2010 ....................................................... 4-23

Corporate Average Fuel Economy (CAFE) Fines Collected, 1983-2008 .................................... 4-24

Fuel Economy by Speed, PSAT Model Results ............................................................... 4-27

Fuel Economy by Speed, 1973, 1984, and 1997 Studies ......................................................... 4-28

Steady Speed Fuel Economy for Vehicles Tested in the 1997 Study........................................... 4-31

Projected Fuel Economies from U.S., European, and Japanese Driving Cycles............................ 4-37

Truck Harmonic Mean Fuel Economy by Size Class, 1992, 1997, and 2002 ................................ 5-6

Effect of Terrain on Class 8 Truck Fuel Economy.................................................................... 5-14

Fuel Economy for Class 8 Trucks as Function of Speed and Tractor-Trailer

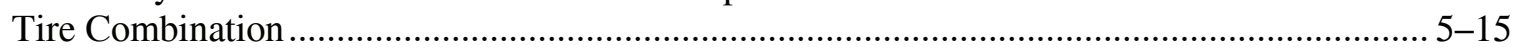

Class 8 Truck Fuel Economy as a Function of Speed and Tractor-Trailer Tire

Combination and Percentage of Total Distance Traveled as a Function

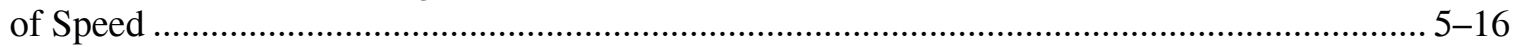

Class 8 Truck Percent of Total Fuel Consumed as a Function of Speed and

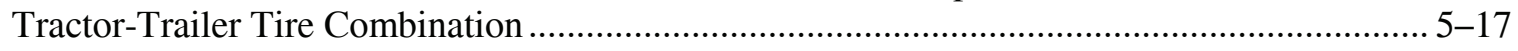

Alternative Fuel and Advanced Technology Vehicles and Characteristics..................................... 6-1

Estimates of Alternative Fuel Vehicles in Use, 1995-2008 ........................................................ 6-3

Alternative Fuel Vehicles Available by Manufacturer, Model Year 2009....................................... 6-4

Number of Alternative Refuel Sites by State and Fuel Type, 2010 ................................................ 6-6

Federal Fleet Vehicle Acquisitions by Fuel Type, FY 2002- 2009 ............................................. 7-6 
Fuel (continued)

Fuel Consumed by Federal Government Fleets, FY 2000-2009 …............................................ 7-6

Diesel Fuel Prices for Selected Countries, 1998-2008 ......................................................... 10-4

Retail Prices for Motor Fuel, 1978-2009 ............................................................................. 10-8

Refiner Sales Prices for Aviation Gasoline and Jet Fuel, 1978-2009 ....................................... 10-10

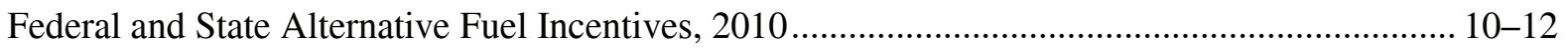

Carbon Dioxide Emissions from a Gallon of Fuel .................................................................. 11-15

Fueling

Percentage of Trucks by Fleet Size and Primary Fueling Facility, 2002 ..................................... 5-9

Share of Trucks by Major Use and Primary Fueling Facility, 2002 ........................................... 5-10

Fuels

Highway Usage of Gasoline and Special Fuels, 1973-2008 ...................................................... 2-13

Properties of Conventional and Alternative Fuels........................................................................... 6-9

Federal Excise Taxes on Motor Fuels, 2008 .............................................................................. 10

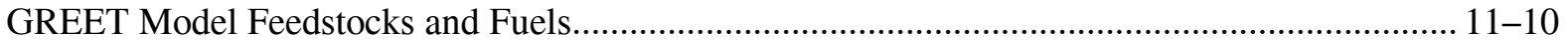

Function

Fuel Economy for Class 8 Trucks as Function of Speed and Tractor-Trailer

Tire Combination

Class 8 Truck Fuel Economy as a Function of Speed and Tractor-Trailer Tire

Combination and Percentage of Total Distance Traveled as a Function

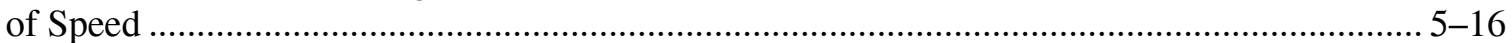

Class 8 Truck Percent of Total Fuel Consumed as a Function of Speed and Tractor-Trailer Tire Combination .........................................................................................

Gallon

Prices for a Barrel of Crude Oil and a Gallon of Gasoline, 1978-2009 ....................................... 10-7

Carbon Dioxide Emissions from a Gallon of Fuel .................................................................. 11-15

Garden

Fuel Consumption from Lawn and Garden Equipment, 2008........................................................ 2-12

Gas

World Natural Gas Reserves, Production and Consumption, 2008 ............................................ 1-7

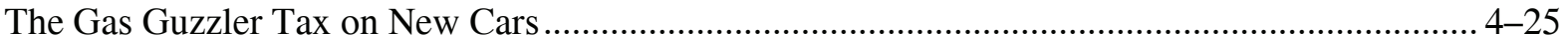

Tax Receipts from the Sale of Gas Guzzlers, 1980-2008 ............................................................. 4-26

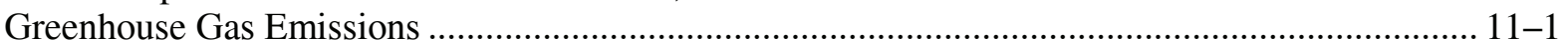

Transportation Greenhouse Gas Emissions by Mode, 1990 and 2008 ...................................... 11-8

Gases

U.S. Emissions of Greenhouse Gases, based on Global Warming Potential,

1990-2008 $11-4$

Gasohol

State Tax Exemptions for Gasohol, 2008 .............................................................................. 10-11

Gasoline

Highway Usage of Gasoline and Special Fuels, 1973-2008 ....................................................... 2-13

Gasoline Prices for Selected Countries, 1990-2008 …............................................................ 10-3

Gasoline Prices for Selected Countries, 1990 and 2008.......................................................... 10-5

Prices for a Barrel of Crude Oil and a Gallon of Gasoline, 1978-2009 ........................................ 10-7 
Gasoline (continued)

Refiner Sales Prices for Aviation Gasoline and Jet Fuel, 1978-2009 $10-10$

General

Summary Statistics for General Aviation, 1970-2008 $9-4$

Global

Numerical Estimates of Global Warming Potentials Compared with Carbon Dioxide $11-3$

U.S. Emissions of Greenhouse Gases, based on Global Warming Potential, 1990-2008. $11-4$

Glossary

Glossary G-1

Government

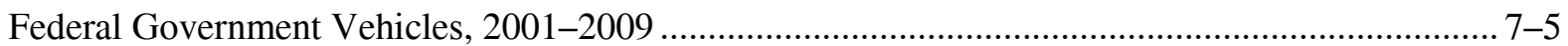

Fuel Consumed by Federal Government Fleets, FY 2000-2009 ….............................................. 7-6

Federal Government Vehicles by Agency, FY 2009............................................................... 7-7

Greenhouse

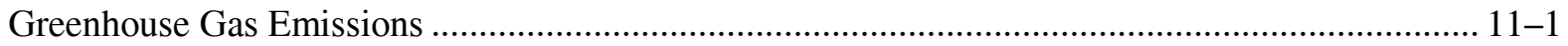

U.S. Emissions of Greenhouse Gases, based on Global Warming Potential,

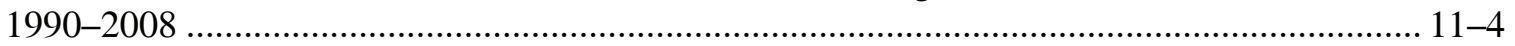

Total U.S. Greenhouse Emissions by End-Use Sector, 2008 …................................................. 11-5

Transportation Greenhouse Gas Emissions by Mode, 1990 and 2008 ........................................... 11-8

\section{GREET}

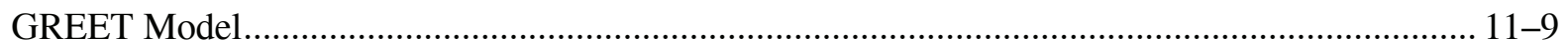

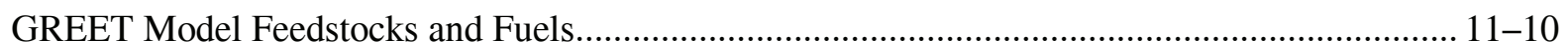

Gross

Refinery Gross Output by World Region, 1999 and 2009 .......................................................... 1-12

New Retail Sales of Trucks 10,000 Pounds GVW and Less in the

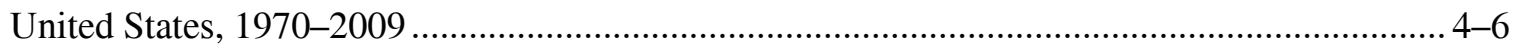

New Retail Truck Sales by Gross Vehicle Weight, 1970-2009....................................................... 5-4

Truck Statistics by Gross Vehicle Weight Class, 2002 ...................................................................5-6

Gross Vehicle Weight vs. Empty Vehicle Weight ....................................................................... 5-19

Growth

Oil Price and Economic Growth, 1970-2009............................................................................ 1-9

Growth of Freight in the United States: Comparison of the 2007, 2002 and 1997 Commodity

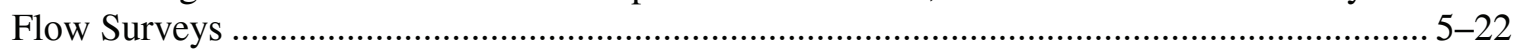

Growth of Freight Miles in the United States: Comparison of the 2007, 2002

and 1997 Commodity Flow Surveys

Guzzler

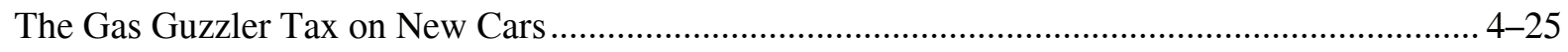

Guzzlers

Tax Receipts from the Sale of Gas Guzzlers, 1980-2008 ….................................................... 4-26

Harmonic

Truck Harmonic Mean Fuel Economy by Size Class, 1992, 1997, and 2002 _............................... 5-6 
Heavy

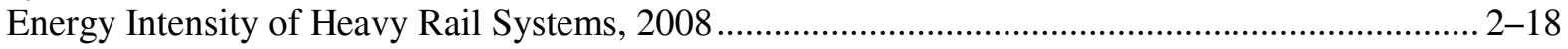

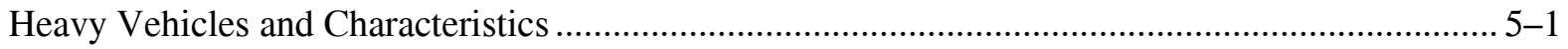

Summary Statistics for Heavy Single-Unit Trucks, 1970-2008 ................................................... 5-2

Share of Heavy Trucks with Selected Electronic Features, 2002 …............................................ 5-12

High

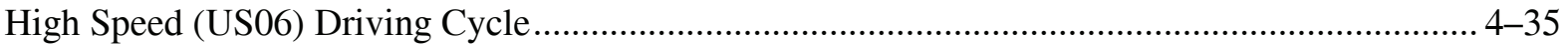

Highway

Highway Transportation Petroleum Consumption by Mode, 1970-2008.................................... 1-20

Highway Transportation Energy Consumption by Mode, 1970-2008 .......................................... 2-9

Highway Usage of Gasoline and Special Fuels, 1973-2008 ....................................................... 2-13

Energy Intensities of Highway Passenger Modes, 1970-2008 .................................................... 2-15

Shares of Highway Vehicle-Miles Traveled by Vehicle Type, 1970-2008 .................................. 3-10

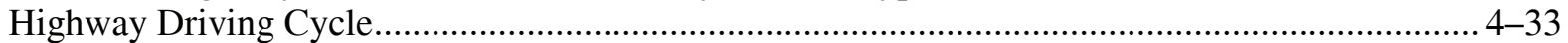

Emissions of Carbon Monoxide from Highway Vehicles, 1970-2005 …....................................... 12-4

Emissions of Nitrogen Oxides from Highway Vehicles, 1970-2005.......................................... 12-6

Emissions of Volatile Organic Compounds from Highway Vehicles, 1970-2005 ......................... 12-8

Emissions of Particulate Matter (PM-10) from Highway Vehicles, 1970-2005 .......................... 12-10

Emissions of Particulate Matter (PM-2.5) from Highway Vehicles, 1990-2005.......................... 12-12

Household

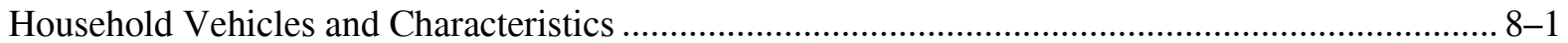

Household Vehicle Ownership, 1960-2000 Census ............................................................................... 8-5

Average Annual Vehicle-Miles, Vehicle Trips and Trip Length per

Household 1969, 1977, 1983, 1990, 1995 NPTS and 2001, 2009 NHTS ................................. 8-7

Average Number of Vehicles and Vehicle Travel per Household,

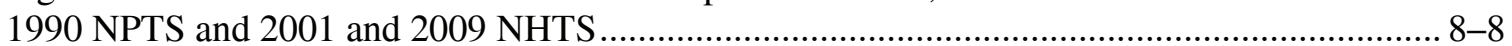

Average Annual Miles per Household Vehicle by Vehicle Age ............................................. 8-12

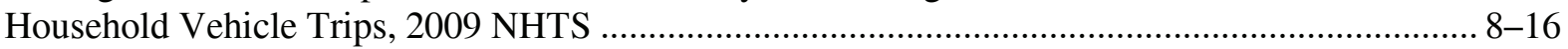

Daily Vehicle Miles of Travel (per Vehicle) by Number of Vehicles in the

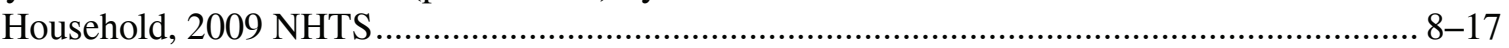

Daily and Annual Vehicle Miles of Travel and Average Age for Each Vehicle

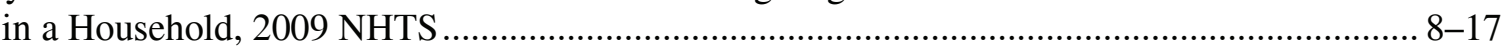

Daily Vehicle Miles of Travel for Each Vehicle in a Household, 2009 NHTS .............................. 8-18

Annual Vehicle Miles of Travel for Each Vehicle in a Household, 2009 NHTS........................... 8-18

Households

Average Annual Expenditures of Households by Income, 2008 ................................................ 8-4

Housing

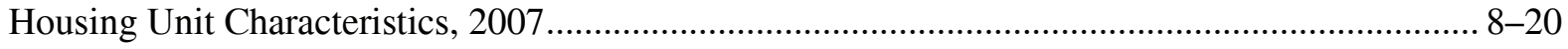

Hybrid

Hybrid Electric Vehicles Available by Manufacturer, Model Year 2009 ....................................... 6-5

Import

Period Sales, Market Shares, and Sales-Weighted Fuel Economies of New

Domestic and Import Cars, Selected Model Years 1975-2009 ................................................. 4-7

Period Sales, Market Shares, and Sales-Weighted Fuel Economies of New

Domestic and Import Light Trucks, Model Years 1975-2009 
Import (continued)

Sales-Weighted Engine Size of New Domestic and Import Cars

by Size Class, Model Years 1975-2009 ............................................................................... 4-12

Sales-Weighted Engine Size of New Domestic and Import Light Trucks

by Size Class, Model Years 1975-2009 ............................................................................ 4-13

Sales-Weighted Curb Weight of New Domestic and Import Cars

by Size Class, Model Years 1975-2009 ............................................................................ 4-14

Sales-Weighted Interior Space of New Domestic and Import Cars

by Size Class, Model Years 1977-2009 ................................................................................ 4-15

Average Price of a New Car (Domestic and Import), 1970-2008 ….......................................... 10-15

Sales-Weighted Annual Carbon Footprint of New Domestic and Import Cars

by Size Class, Model Years 1975-2009 ................................................................................ 11-12

Sales-Weighted Annual Carbon Footprint of New Domestic and Import

Light Trucks by Size Class, Model Years 1975-2009 ......................................................... 11-13

Important

Petroleum Production and Consumption and Some Important Percent

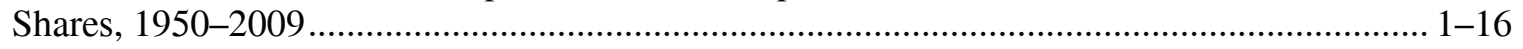

Imports

U.S. Petroleum Imports by World Region of Origin, 1960-2009 .............................................. 1-8

United States Petroleum Production, Imports and Exports, 1950-2009 ........................................ 1-15

Incentives

Federal and State Alternative Fuel Incentives, 2010

$10-12$

Federal and State Advanced Technology Incentives, 2010.................................................. 10-13

Income

Average Annual Expenditures of Households by Income, 2008 .................................................... 8 8

Index

Transportation Services Index, January 1990-January 2010 .................................................... 10-2

Indices

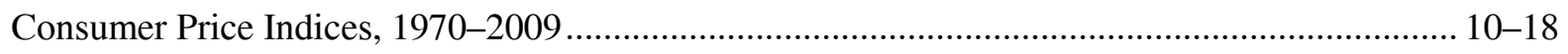

Input

U.S. Refinery Input of Crude Oil and Petroleum Products, 1987-2008 ....................................... 1-13

Intensities

Energy Intensities of Highway Passenger Modes, 1970-2008 …................................................ 2-15

Energy Intensities of Nonhighway Passenger Modes, 1970-2008 .............................................. 2-16

Energy Intensities of Freight Modes, 1970-2008 ...................................................................... 2-20

Intensity

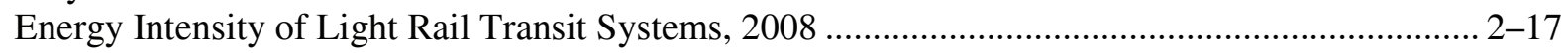

Energy Intensity of Heavy Rail Systems, 2008 ..................................................................... 2-18

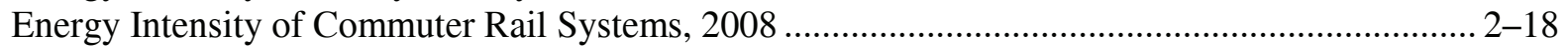

Intercity

Intercity Freight Movement and Energy Use in the United States, 2007 and 2008 ...................... 2-19 
Interior

Sales-Weighted Interior Space of New Domestic and Import Cars

by Size Class, Model Years 1977-2009

Intermodal

Intermodal Rail Traffic, 1965-2008

International

Summary Statistics for U.S. Domestic and International Certificated Route

Air Carriers (Combined Totals), 1970-2008

Tonnage Statistics for Domestic and International Waterborne

Commerce, 1970-2008

Japanese

Projected Fuel Economies from U.S., European, and Japanese Driving Cycles............................ 4-37

Comparison of U.S., European, and Japanese Driving Cycles. 4-38

Jet

Refiner Sales Prices for Aviation Gasoline and Jet Fuel, 1978-2009 $10-10$

Lawn

Fuel Consumption from Lawn and Garden Equipment, 2008 $2-12$

Length

Average Length of Time Business Fleet Vehicles are in Service, 2008 7-3

Average Annual Vehicle-Miles, Vehicle Trips and Trip Length per

Household 1969, 1977, 1983, 1990, 1995 NPTS and 2001, 2009 NHTS

Lifetime

Car and Light Truck Survivability Rates and Lifetime Miles

Light

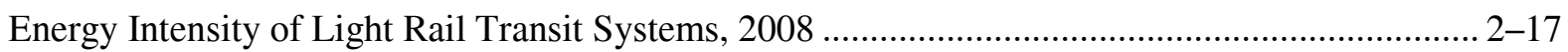

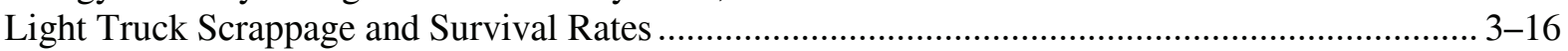

Car and Light Truck Survivability Rates and Lifetime Miles ................................................. 3-17

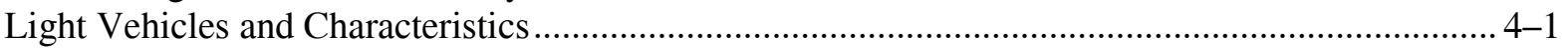

Summary Statistics on Class 1, Class 2a, and Class 2b Light Trucks ............................................ 4-4

Sales Estimates of Class 1, Class 2a, and Class 2b Light Trucks, 1989-1999.................................. 4-4

Period Sales, Market Shares, and Sales-Weighted Fuel Economies of New

Domestic and Import Light Trucks, Model Years 1975-2009 ............................................... 4-9

Light Vehicle Market Shares by Size Class, Model Years 1975-2009......................................... 4-10

Light Vehicle Market Shares, Model Years 1975-2009 .............................................................. 4-11

Sales-Weighted Engine Size of New Domestic and Import Light Trucks

by Size Class, Model Years 1975-2009 …............................................................................. 4-13

Average Material Consumption for a Light Vehicle, Model Years 1995, 2000, and 2008

New Light Vehicle Dealerships and Sales, 1970-2008

Reformed and Unreformed Light Truck Corporate Average Fuel Economy

Standards, MY 2008-2011

Light Truck Corporate Average Fuel Economy (CAFE) Standards versus

Sales-Weighted Fuel Economy Estimates, 1978-2010

New Light Fleet Vehicle Registrations by Vehicle Type, Model Year 2008 
Light (continued)

Sales-Weighted Annual Carbon Footprint of New Domestic and Import

Light Trucks by Size Class, Model Years 1975-2009 .......................................................11-13

U.S. Tier 2 Emission Standards for Cars and Light Trucks ..................................................... 12-13

Light Vehicle Exhaust Emission Standards in Effect in 2009 when

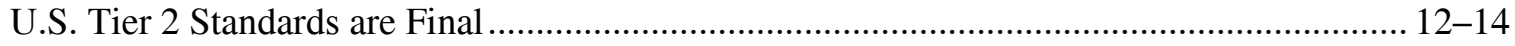

California Cars and Light Trucks Emission Certification Standards ........................................ 12-15

Long-Distance

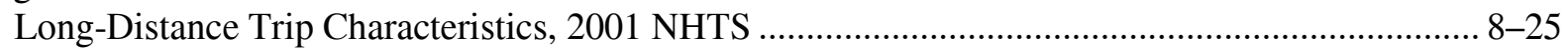

Manufacturer

Alternative Fuel Vehicles Available by Manufacturer, Model Year 2009....................................... 6-4

Hybrid Electric Vehicles Available by Manufacturer, Model Year 2009 ........................................ 6-5

Manufacturing

U.S. Employment for Motor Vehicles and Motor Vehicle Parts

Manufacturing, 1990-2009.

$10-20$

Major

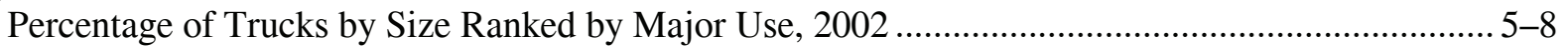

Share of Trucks by Major Use and Primary Fueling Facility, 2002 ............................................ 5-10

Maps

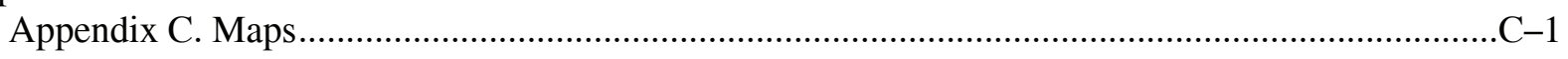

Market

Period Sales, Market Shares, and Sales-Weighted Fuel Economies of New

Domestic and Import Cars, Selected Model Years 1975-2009 ................................................. 4-7

Period Sales, Market Shares, and Sales-Weighted Fuel Economies of New

Domestic and Import Light Trucks, Model Years 1975-2009 ................................................ 4-9

Light Vehicle Market Shares by Size Class, Model Years 1975-2009......................................... 4-10

Light Vehicle Market Shares, Model Years 1975-2009 ................................................................ 4-11

Material

Average Material Consumption for a Light Vehicle, Model Years 1995, 2000,

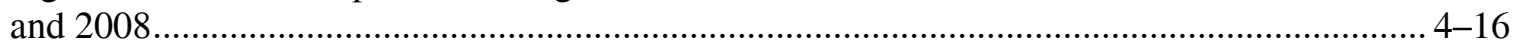

Matter

Total National Emissions of Particulate Matter (PM-10), 1970-2008 ......................................... 12-9

Emissions of Particulate Matter (PM-10) from Highway Vehicles, 1970-2005 ......................... 12-10

Total National Emissions of Particulate Matter (PM-2.5), 1990-2008....................................... 12-11

Emissions of Particulate Matter (PM-2.5) from Highway Vehicles, 1990-2005......................... 12-12

Mean

Truck Harmonic Mean Fuel Economy by Size Class, 1992, 1997, and 2002 ................................ 5-6

Means

Means of Transportation to Work, 1980, 1990 and 2000 Census ................................................ 8-19

Methodologies

Appendix A. Sources \& Methodologies ................................................................................... A-1 
Middle

Summary of Military Expenditures for Defending Oil Supplies from the

Middle East $1-11$

Mile

Car Operating Cost per Mile, 1985-2009 $10-16$

Miles

Car and Light Truck Survivability Rates and Lifetime Miles $3-17$

Distribution of Trucks over 26,000 lbs. Less than Two Years Old by Vehicle-

Miles Traveled $5-11$

Growth of Freight Miles in the United States: Comparison of the 2007, 2002

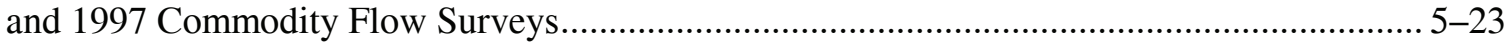

Average Annual Vehicle-Miles of Travel for Business Fleet Vehicles, 2008................................ 7-3

Average Miles per Domestic Federal Vehicle by Vehicle Type, 2009 …....................................... 7-4

Average Annual Vehicle-Miles, Vehicle Trips and Trip Length per Household 1969, 1977, 1983, 1990, 1995 NPTS and 2001, 2009 NHTS ................................. 8-7

Average Annual Miles per Household Vehicle by Vehicle Age ................................................. 8-12

Self-Reported vs. Odometer Average Annual Miles, 1995 NPTS and 2001 NHTS

Share of Vehicles by Annual Miles of Travel and Vehicle Age, 2009 NHTS ............................... 8-15

Average Daily Miles Driven (per Driver), 2009 NHTS ................................................................ 8-16

Daily Vehicle Miles of Travel (per Vehicle) by Number of Vehicles in the Household, 2009 NHTS. $8-17$

Daily and Annual Vehicle Miles of Travel and Average Age for Each Vehicle

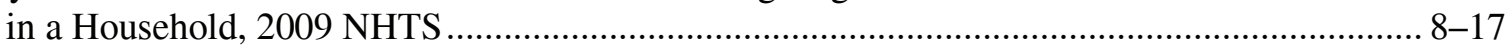

Daily Vehicle Miles of Travel for Each Vehicle in a Household, 2009 NHTS ............................. 8-18

Annual Vehicle Miles of Travel for Each Vehicle in a Household, 2009 NHTS........................... 8-18

Military

Summary of Military Expenditures for Defending Oil Supplies from the Middle East $1-11$

Mode

Highway Transportation Petroleum Consumption by Mode, 1970-2008....................................... 1-20

Nonhighway Transportation Petroleum Consumption by Mode, 1970-2008 ............................... 1-21

Transportation Petroleum Use by Mode, 2007-2008 ................................................................... 1-22

Domestic Consumption of Transportation Energy by Mode and

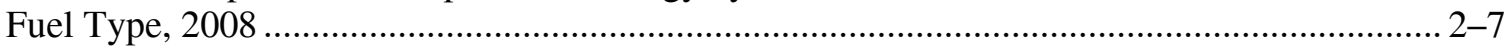

Transportation Energy Use by Mode, 2007-2008................................................................... 2-8

Highway Transportation Energy Consumption by Mode, 1970-2008............................................ 2-9

Nonhighway Transportation Energy Consumption by Mode, 1970-2008 ..................................... 2-10

Transportation Greenhouse Gas Emissions by Mode, 1990 and 2008 ....................................... 11-8

Model

Off-highway Transportation-related Fuel Consumption from the NonRoad

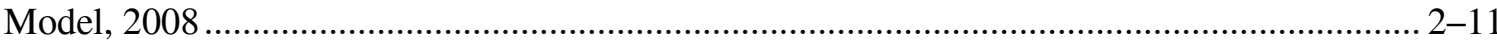

Car Scrappage and Survival Rates, 1970, 1980 and 1990 Model Years ........................................ 3-15

Period Sales, Market Shares, and Sales-Weighted Fuel Economies of New

Domestic and Import Cars, Selected Model Years 1975-2009 _............................................. 4-7

Definition of Wagons in Model Year 2009 .................................................................................... 4-8

Period Sales, Market Shares, and Sales-Weighted Fuel Economies of New

Domestic and Import Light Trucks, Model Years 1975-2009 
Model (continued)

Light Vehicle Market Shares by Size Class, Model Years 1975-2009........................................ 4-10

Light Vehicle Market Shares, Model Years 1975-2009 ................................................................ 4-11

Sales-Weighted Engine Size of New Domestic and Import Cars

by Size Class, Model Years 1975-2009 ..................................................................................... 4-12

Sales-Weighted Engine Size of New Domestic and Import Light Trucks

by Size Class, Model Years 1975-2009 ................................................................................. 4 -13

Sales-Weighted Curb Weight of New Domestic and Import Cars by Size Class, Model Years 1975-2009 ................................................................................ 4-14

Sales-Weighted Interior Space of New Domestic and Import Cars by Size Class, Model Years 1977-2009 ............................................................................... 4-15

Average Material Consumption for a Light Vehicle, Model Years 1995, 2000,

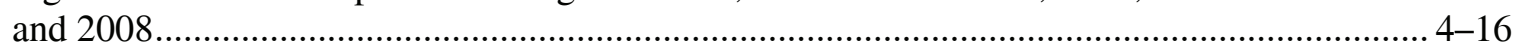

Fuel Economy and Carbon Dioxide Targets for Model Year 2016 ........................................... 4-21

Fuel Economy by Speed, PSAT Model Results ........................................................................... 4-27

Alternative Fuel Vehicles Available by Manufacturer, Model Year 2009....................................... 6-4

Hybrid Electric Vehicles Available by Manufacturer, Model Year 2009 ........................................ 6-5

New Light Fleet Vehicle Registrations by Vehicle Type, Model Year 2008................................... 7-3

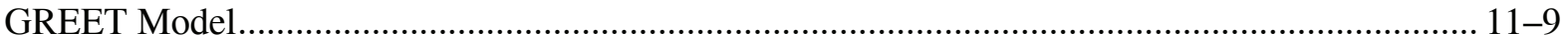

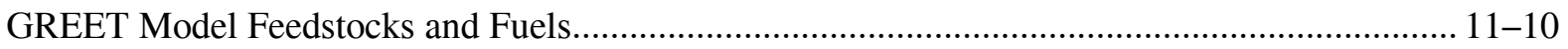

Sales-Weighted Annual Carbon Footprint of New Domestic and Import Cars by Size Class, Model Years 1975-2009 ................................................................................... 11-12

Sales-Weighted Annual Carbon Footprint of New Domestic and Import Light Trucks by Size Class, Model Years 1975-2009 ......................................................... 11-13

Modes

Energy Intensities of Highway Passenger Modes, 1970-2008 .................................................... 2-15

Energy Intensities of Nonhighway Passenger Modes, 1970-2008 .............................................. 2-16

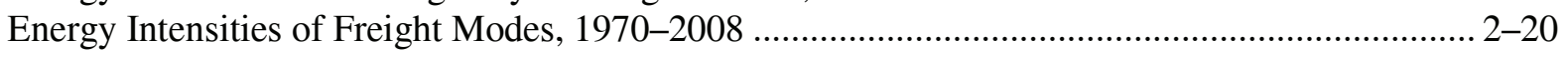

Nonhighway Modes ......................................................................................................

Monoxide

Total National Emissions of Carbon Monoxide, 1970-2008 …................................................ 12-3

Emissions of Carbon Monoxide from Highway Vehicles, 1970-2005 .......................................... 12-4

Motor

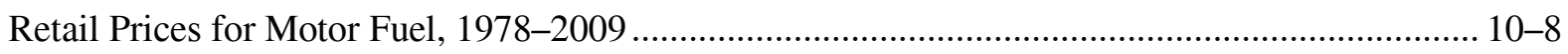

Federal Excise Taxes on Motor Fuels, 2008 ............................................................................... 10-11

U.S. Employment for Motor Vehicles and Motor Vehicle Parts

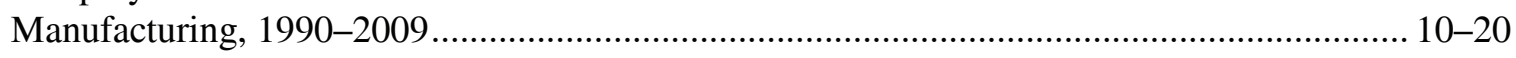

Movement

Intercity Freight Movement and Energy Use in the United States, 2007 and 2008 ....................... 2-19

National

Summary Statistics for the National Railroad Passenger Corporation

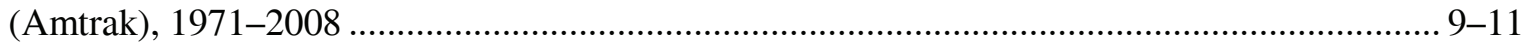

Total National Emissions of the Criteria Air Pollutants by Sector, 2008...................................... 12-2

Total National Emissions of Carbon Monoxide, 1970-2008 ….................................................. 12-3

Total National Emissions of Nitrogen Oxides, 1970-2008..................................................... 12-5

Total National Emissions of Volatile Organic Compounds, 1970-2008 …................................... 12-7

Total National Emissions of Particulate Matter (PM-10), 1970-2008 …...................................... 12-9

Total National Emissions of Particulate Matter (PM-2.5), 1990-2008 ........................................ 12-11 
Natural

World Natural Gas Reserves, Production and Consumption, 2008 $1-7$

New

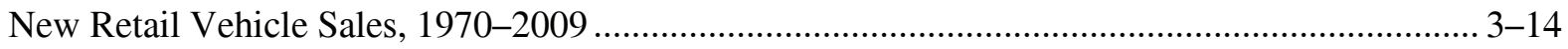

New Retail Car Sales in the United States, 1970-2009 ............................................................... 4-5

Period Sales, Market Shares, and Sales-Weighted Fuel Economies of New

Domestic and Import Cars, Selected Model Years 1975-2009 .................................................. 4-7

Period Sales, Market Shares, and Sales-Weighted Fuel Economies of New

Domestic and Import Light Trucks, Model Years 1975-2009 .................................................. 4-9

Sales-Weighted Engine Size of New Domestic and Import Cars

by Size Class, Model Years 1975-2009 ……............................................................................. 4-12

Sales-Weighted Engine Size of New Domestic and Import Light Trucks

by Size Class, Model Years 1975-2009 …............................................................................... 4-13

Sales-Weighted Curb Weight of New Domestic and Import Cars

by Size Class, Model Years 1975-2009 ..................................................................................... 4-14

Sales-Weighted Interior Space of New Domestic and Import Cars

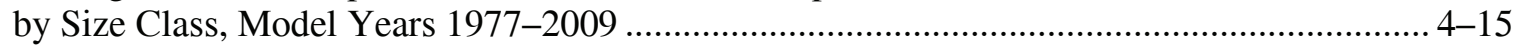

New Light Vehicle Dealerships and Sales, 1970-2008 ................................................................

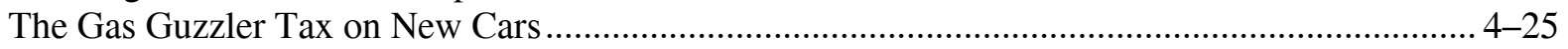

New Retail Truck Sales by Gross Vehicle Weight, 1970-2009.................................................... 5-4

New Light Fleet Vehicle Registrations by Vehicle Type, Model Year 2008 .................................. 7-3

Average Price of a New Car, 1906-2008 ................................................................................. 10-14

Average Price of a New Car (Domestic and Import), 1970-2008 …........................................... 10-15

Sales-Weighted Annual Carbon Footprint of New Domestic and Import Cars by Size Class, Model Years 1975-2009 ................................................................................. 11-12

Sales-Weighted Annual Carbon Footprint of New Domestic and Import Light Trucks by Size Class, Model Years 1975-2009 .......................................................... 11-13

New York

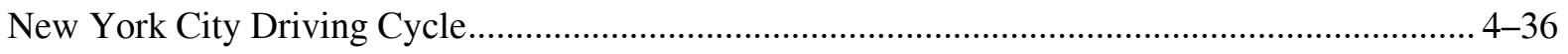

NHTS

Demographic Statistics from the 1969, 1977, 1983, 1990, 1995 NPTS

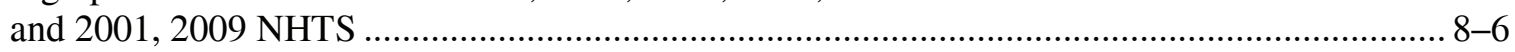

Average Annual Vehicle-Miles, Vehicle Trips and Trip Length per Household 1969, 1977, 1983, 1990, 1995 NPTS and 2001, 2009 NHTS ................................. 8-7

Average Number of Vehicles and Vehicle Travel per Household,

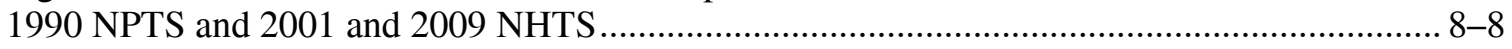

Trip Statistics by Trip Purpose, 2001 and 2009 NHTS ................................................................... 8

Average Vehicle Occupancy by Vehicle Type, 1995 NPTS and

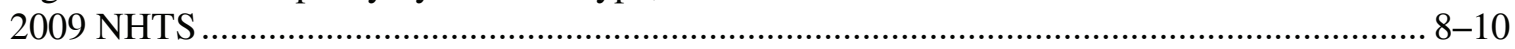

Average Vehicle Occupancy by Trip Purpose, 1977 NPTS and

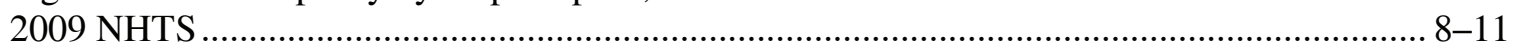

Self-Reported vs. Odometer Average Annual Miles, 1995 NPTS and

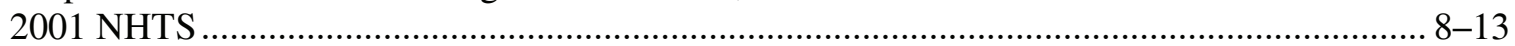

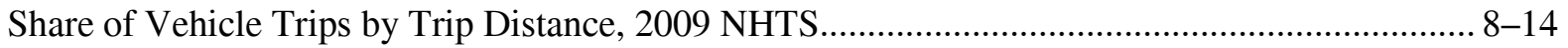

Share of Vehicle Trips to Work by Trip Distance, 2009 NHTS .................................................. 8-14

Share of Vehicles by Annual Miles of Travel and Vehicle Age, 2009 NHTS ............................... 8-15

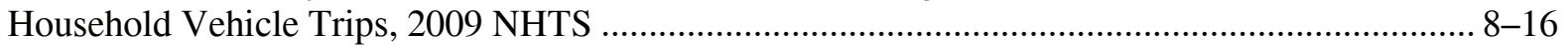

Average Daily Miles Driven (per Driver), 2009 NHTS ................................................................... 8-16

Daily Vehicle Miles of Travel (per Vehicle) by Number of Vehicles in the

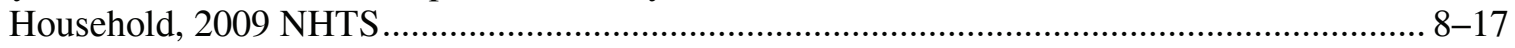


NHTS (continued)

Daily and Annual Vehicle Miles of Travel and Average Age for Each Vehicle

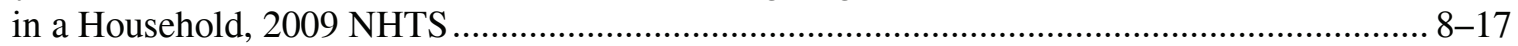

Daily Vehicle Miles of Travel for Each Vehicle in a Household, 2009 NHTS ............................. 8-18

Annual Vehicle Miles of Travel for Each Vehicle in a Household, 2009 NHTS........................... 8-18

Walk and Bike Trips by Trip Purpose, 2009 NHTS................................................................. 8-23

Long-Distance Trip Characteristics, 2001 NHTS ............................................................................... 8-25

Nitrogen

Total National Emissions of Nitrogen Oxides, 1970-2008 .................................................... 12-5

Emissions of Nitrogen Oxides from Highway Vehicles, 1970-2005.......................................... 12-6

Nonhighway

Nonhighway Transportation Petroleum Consumption by Mode, 1970-2008............................... 1-21

Nonhighway Transportation Energy Consumption by Mode, 1970-2008.................................... 2-10

Energy Intensities of Nonhighway Passenger Modes, 1970-2008 .............................................. 2-16

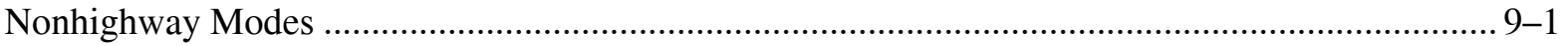

Nonhighway Energy Use Shares, 1970-2008 …….......................................................................... 9-2

NonRoad

Off-highway Transportation-related Fuel Consumption from the NonRoad

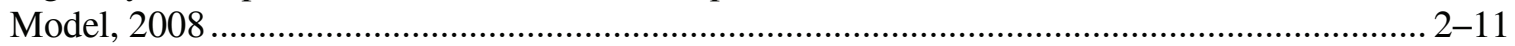

NPTS

Demographic Statistics from the 1969, 1977, 1983, 1990, 1995 NPTS

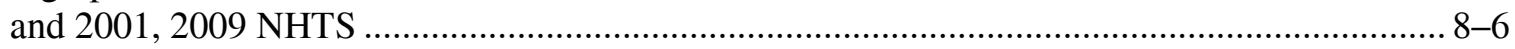

Average Annual Vehicle-Miles, Vehicle Trips and Trip Length per Household 1969, 1977, 1983, 1990, 1995 NPTS and 2001, 2009 NHTS ................................. 8-7

Average Number of Vehicles and Vehicle Travel per Household,

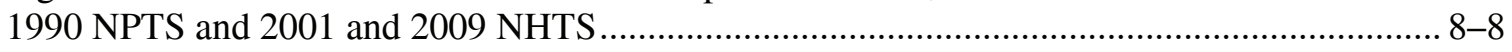

Average Vehicle Occupancy by Vehicle Type, 1995 NPTS and

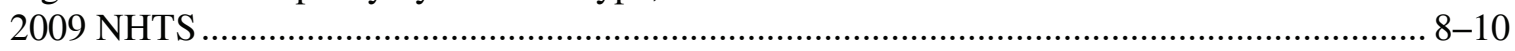

Average Vehicle Occupancy by Trip Purpose, 1977 NPTS and

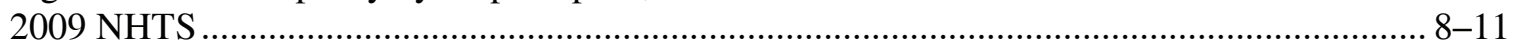

Self-Reported vs. Odometer Average Annual Miles, 1995 NPTS and

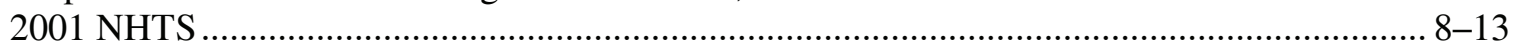

Number

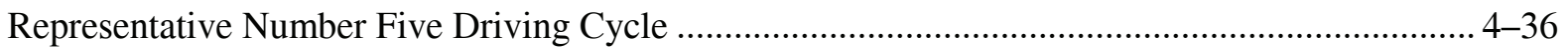

Number of Alternative Refuel Sites by State and Fuel Type, 2010 ............................................ 6-6

Average Number of Vehicles and Vehicle Travel per Household,

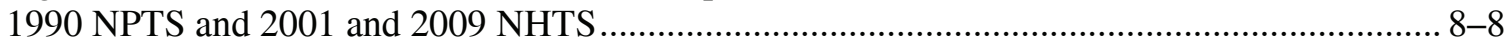

Daily Vehicle Miles of Travel (per Vehicle) by Number of Vehicles in the

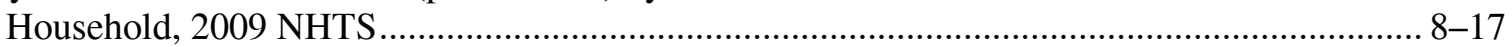

Refiner Sales Prices for Propane and No. 2 Diesel, 1978-2009 ................................................... 10-9

Numerical

Numerical Estimates of Global Warming Potentials Compared with Carbon Dioxide ................... 11-3

Occupancy

Average Vehicle Occupancy by Vehicle Type, 1995 NPTS and 2009 NHTS 
Occupancy (continued)

Average Vehicle Occupancy by Trip Purpose, 1977 NPTS and

2009 NHTS

Odometer

Self-Reported vs. Odometer Average Annual Miles, 1995 NPTS and 2001 NHTS

Off-highway

Off-highway Transportation-related Fuel Consumption from the NonRoad

Model, 2008

Oil

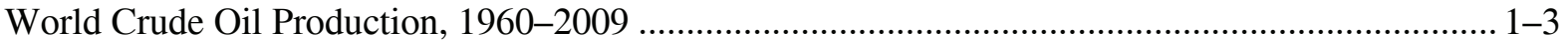

World Oil Reserves, Production and Consumption, 2008 ................................................................ 1-6

Oil Price and Economic Growth, 1970-2009................................................................................. 1-9

Summary of Military Expenditures for Defending Oil Supplies from the

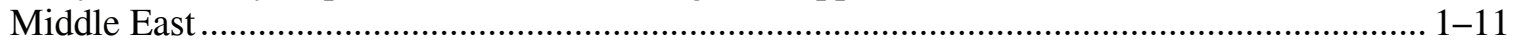

Refinery Yield of Petroleum Products From a Barrel of Crude Oil, 1987-2008 ........................... 1-14

Prices for a Barrel of Crude Oil and a Gallon of Gasoline, 1978-2009 ........................................ 10-7

On-Road

Distribution of Five-Axle Tractor-Trailers by On-Road Vehicle Weight, 2008 ............................. 5-20

Organic

Total National Emissions of Volatile Organic Compounds, 1970-2008 ...................................... 12-7

Emissions of Volatile Organic Compounds from Highway Vehicles, 1970-2005 ......................... 12-8

Origin

U.S. Petroleum Imports by World Region of Origin, 1960-2009............................................... 1-8

Operating

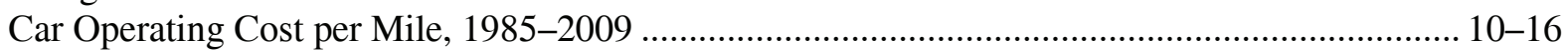

Fixed Car Operating Costs per Year, 1975-2009................................................................. 10-17

Operation

Cars in Operation and Vehicle Travel by Age, 1970 and 2001 ................................................... 3-11

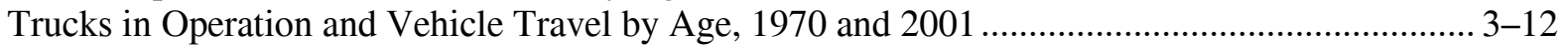

Operations

Summary Statistics for Commuter Rail Operations, 1984-2008 …................................................ 9-12

Summary Statistics for Rail Transit Operations, 1970-2008 ....................................................... 9-13

Other

Vehicles per Thousand People: U.S. (Over Time) Compared to Other

Countries (in 1998 and 2008)

Output

Refinery Gross Output by World Region, 1999 and 2009 $1-12$

Ownership

Household Vehicle Ownership, 1960-2000 Census 
Oxides

Total National Emissions of Nitrogen Oxides, 1970-2008...................................................... 12-5

Emissions of Nitrogen Oxides from Highway Vehicles, 1970-2005......................................... 12-6

Oxygenate

Alternative Fuel and Oxygenate Consumption, 2003-2008 …..................................................... 2-5

Particulate

Total National Emissions of Particulate Matter (PM-10), 1970-2008 …...................................... 12-9

Emissions of Particulate Matter (PM-10) from Highway Vehicles, 1970-2005 .......................... 12-10

Total National Emissions of Particulate Matter (PM-2.5), 1990-2008........................................ 12-11

Emissions of Particulate Matter (PM-2.5) from Highway Vehicles, 1990-2005.......................... 12-12

Parts

U.S. Employment for Motor Vehicles and Motor Vehicle Parts

Manufacturing, 1990-2009.

$10-20$

Passenger

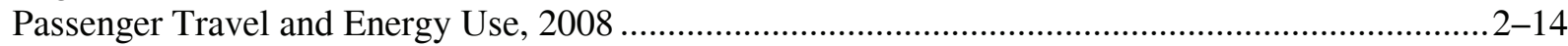

Energy Intensities of Highway Passenger Modes, 1970-2008 ................................................ 2-15

Energy Intensities of Nonhighway Passenger Modes, 1970-2008 ............................................. 2-16

Summary Statistics for the National Railroad Passenger Corporation

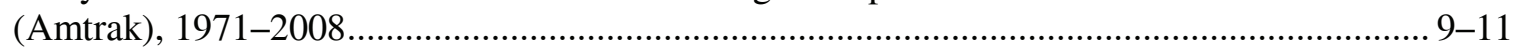

People

Vehicles per Thousand People: U.S. (Over Time) Compared to Other

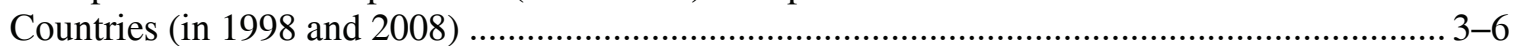

Vehicles per Thousand People in Other Countries, 1998 and 2008 ............................................... 3-8

Vehicles per Thousand People in the United States, 1990-2008 …................................................ 3-9

Percent

Petroleum Production and Consumption and Some Important Percent

Shares, 1950-2009................................................................................................................ 1-16

Class 8 Truck Percent of Total Fuel Consumed as a Function of Speed and

Tractor-Trailer Tire Combination ....................................................................................... $5-17$

Percentage

Percentage of Trucks by Size Ranked by Major Use, 2002 ........................................................ 5-8

Percentage of Trucks by Fleet Size and Primary Fueling Facility, 2002 ......................................... 5-9

Class 8 Truck Fuel Economy as a Function of Speed and Tractor-Trailer Tire

Combination and Percentage of Total Distance Traveled as a Function

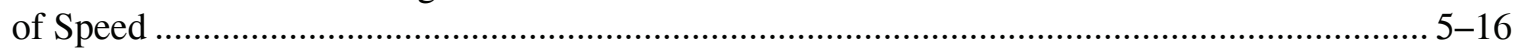

Period

Period Sales, Market Shares, and Sales-Weighted Fuel Economies of New

Domestic and Import Cars, Selected Model Years 1975-2009 .................................................. 4-7

Period Sales, Market Shares, and Sales-Weighted Fuel Economies of New

Domestic and Import Light Trucks, Model Years 1975-2009 ................................................... 4-9

Personal

Personal Consumption Expenditures, 1970-2009.................................................................. 10-18 
Petroleum

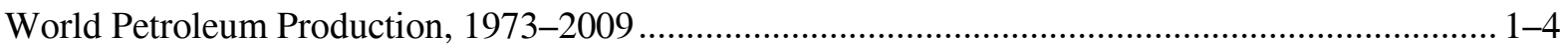

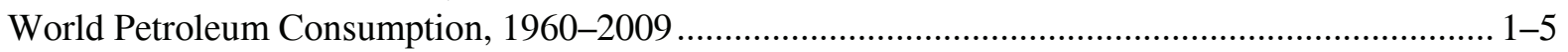

U.S. Petroleum Imports by World Region of Origin, 1960-2009 .............................................. 1-8

U.S. Refinery Input of Crude Oil and Petroleum Products, 1987-2008 …................................. 1-13

Refinery Yield of Petroleum Products From a Barrel of Crude Oil, 1987-2008 …........................ 1-14

United States Petroleum Production, Imports and Exports, 1950-2009 ......................................... 1-15

Petroleum Production and Consumption and Some Important Percent

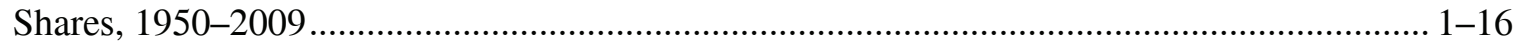

United States Petroleum Production and Consumption - All Sectors,

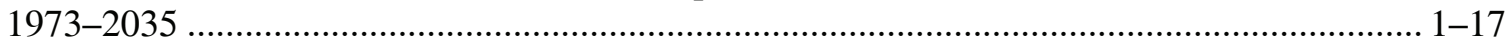

United States Petroleum Production, Transportation and Consumption,

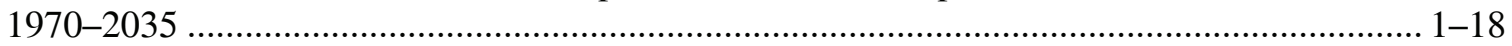

Consumption of Petroleum by End-Use Sector, 1973-2009.......................................................... 1-19

Highway Transportation Petroleum Consumption by Mode, 1970-2008.................................... 1-20

Nonhighway Transportation Petroleum Consumption by Mode, 1970-2008 ................................ 1-21

Transportation Petroleum Use by Mode, 2007-2008 ................................................................... 1-22

PM-10

Total National Emissions of Particulate Matter (PM-10), 1970-2008 ….................................... 12-9

Emissions of Particulate Matter (PM-10) from Highway Vehicles, 1970-2005 ......................... 12-10

\section{PM-2.5}

Total National Emissions of Particulate Matter (PM-2.5), 1990-2008.......................................... 12-11

Emissions of Particulate Matter (PM-2.5) from Highway Vehicles, 1990-2005.......................... 12-12

Pollutants

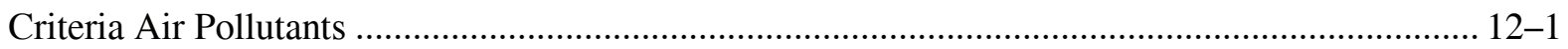

Total National Emissions of the Criteria Air Pollutants by Sector, 2008....................................... 12-2

Population

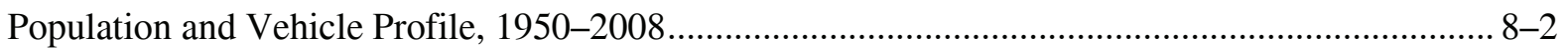

Potential

World Fossil Fuel Potential ..................................................................................................... 1-2

U.S. Emissions of Greenhouse Gases, based on Global Warming Potential,

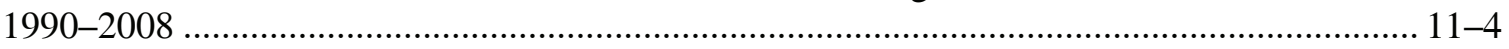

Potentials

Numerical Estimates of Global Warming Potentials Compared with Carbon Dioxide .................. 11-3

Pounds

New Retail Sales of Trucks 10,000 Pounds GVW and Less in the

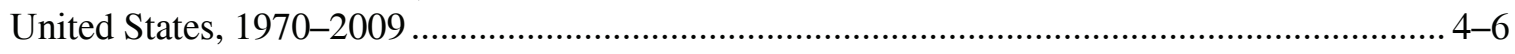

Distribution of Trucks over 26,000 lbs. Less than Two Years Old by Vehicle-

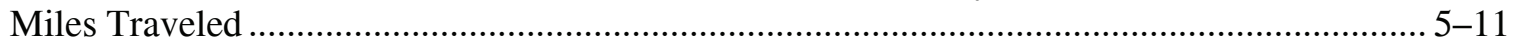

Price

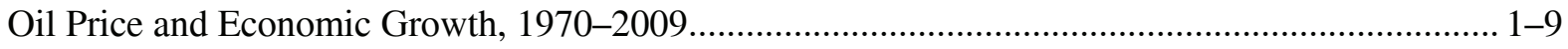

Average Price of a New Car (Domestic and Import), 1970-2008 …........................................ 10-15

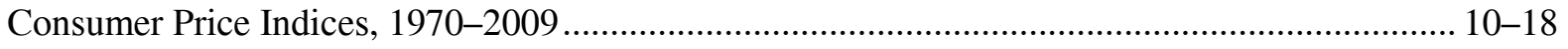

Prices

Gasoline Prices for Selected Countries, 1990-2008 .................................................................. 10-3 
Prices (continued)

Diesel Fuel Prices for Selected Countries, 1998-2008 ........................................................... 10-4

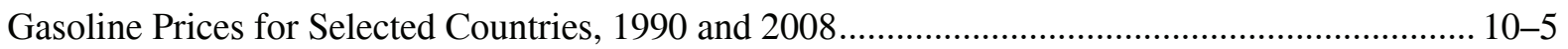

Diesel Prices for Selected Countries, 1990 and 2008............................................................... 10-6

Prices for a Barrel of Crude Oil and a Gallon of Gasoline, 1978-2009 ......................................... 10-7

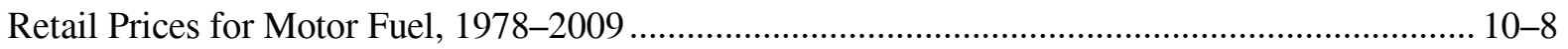

Refiner Sales Prices for Propane and No. 2 Diesel, 1978-2009 ............................................... 10-9

Refiner Sales Prices for Aviation Gasoline and Jet Fuel, 1978-2009 .......................................... 10-10

Average Price of a New Car, 1906-2008 …….......................................................................... 10-14

Primary

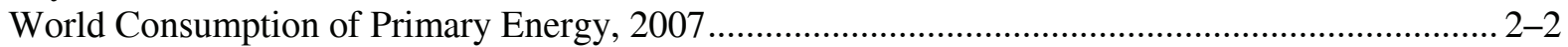

Percentage of Trucks by Fleet Size and Primary Fueling Facility, 2002 ........................................ 5-9

Share of Trucks by Major Use and Primary Fueling Facility, 2002 ........................................... 5-10

Production

World Crude Oil Production, 1960-2009 ..................................................................................... 1-3

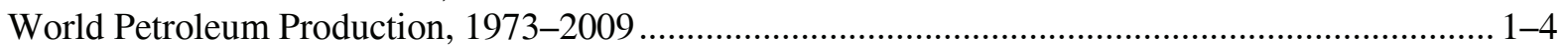

World Oil Reserves, Production and Consumption, 2008 .......................................................... 1-6

World Natural Gas Reserves, Production and Consumption, 2008 ........................................... 1-7

United States Petroleum Production, Imports and Exports, 1950-2009 ……................................ 1-15

Petroleum Production and Consumption and Some Important Percent

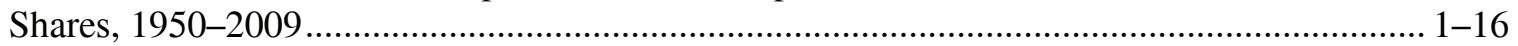

United States Petroleum Production and Consumption - All Sectors,

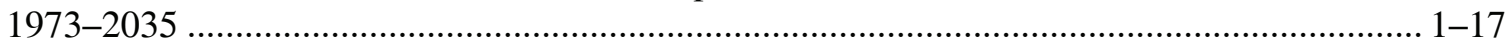

United States Petroleum Production, Transportation and Consumption,

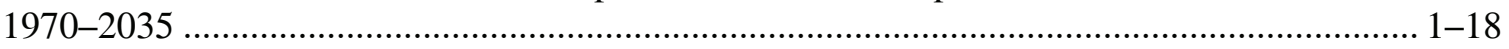

Products

U.S. Refinery Input of Crude Oil and Petroleum Products, 1987-2008 ...................................... 1-13

Refinery Yield of Petroleum Products From a Barrel of Crude Oil, 1987-2008 ............................ 1-14

Profile

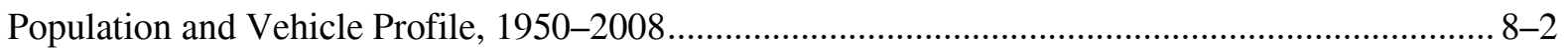

Projected

Projected Fuel Economies from U.S., European, and Japanese Driving Cycles............................ 4-37

Properties

Properties of Conventional and Alternative Fuels.....................................................................

Propane

Refiner Sales Prices for Propane and No. 2 Diesel, 1978-2009 ................................................. 10-9

PSAT

Fuel Economy by Speed, PSAT Model Results ......................................................................... 4-27

Purpose

Trip Statistics by Trip Purpose, 2001 and 2009 NHTS ................................................................. 8 8

Average Vehicle Occupancy by Trip Purpose, 1977 NPTS and

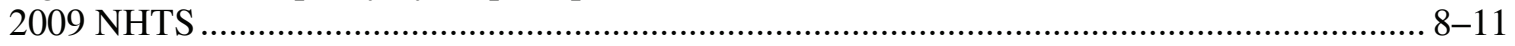

Walk and Bike Trips by Trip Purpose, 2009 NHTS.................................................................... 8-23 
Rail

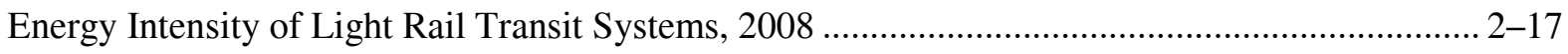

Energy Intensity of Heavy Rail Systems, 2008 …................................................................... 2-18

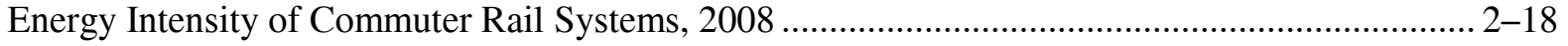

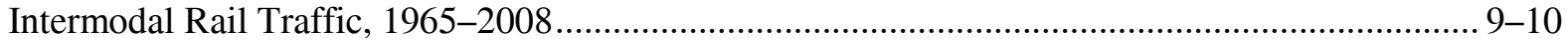

Summary Statistics for Commuter Rail Operations, 1984-2008 …............................................ 9-12

Summary Statistics for Rail Transit Operations, 1970-2008 .................................................... 9-13

Railroad

Class I Railroad Freight Systems in the United States Ranked by

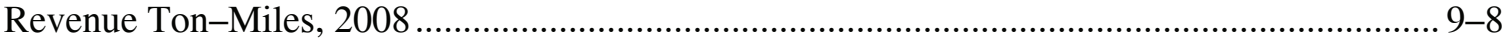

Summary Statistics for the National Railroad Passenger Corporation

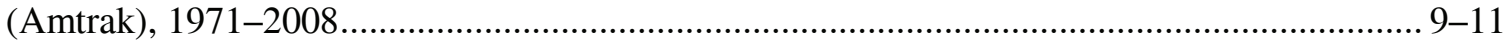

Railroads

Summary Statistics for Class I Freight Railroads, 1970-2008 ...................................................... 9-9

Ranked

Percentage of Trucks by Size Ranked by Major Use, 2002 .......................................................... 5-8

Class I Railroad Freight Systems in the United States Ranked by

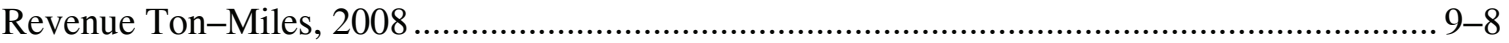

Rates

Car Scrappage and Survival Rates, 1970, 1980 and 1990 Model Years ....................................... 3-15

Light Truck Scrappage and Survival Rates .................................................................................... 3-16

Car and Light Truck Survivability Rates and Lifetime Miles ................................................... 3-17

Receipts

Tax Receipts from the Sale of Gas Guzzlers, 1980-2008 ........................................................... 4-26

Recreational

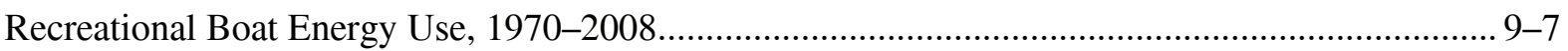

Refiner

Refiner Sales Prices for Propane and No. 2 Diesel, 1978-2009 ................................................ 10-9

Refiner Sales Prices for Aviation Gasoline and Jet Fuel, 1978-2009 ......................................... 10-10

Refinery

Refinery Gross Output by World Region, 1999 and 2009 ......................................................... 1-12

U.S. Refinery Input of Crude Oil and Petroleum Products, 1987-2008 …................................... 1-13

Refinery Yield of Petroleum Products From a Barrel of Crude Oil, 1987-2008 ............................ 1-14

Reformed

Reformed and Unreformed Light Truck Corporate Average Fuel Economy

Standards, MY 2008-2011 ….................................................................................................. 4-19

Refuel

Number of Alternative Refuel Sites by State and Fuel Type, 2010 .............................................. 6-6

Refueling

Conventional Refueling Stations, 1993-2008 …....................................................................... 4-18 
Region

U.S. Petroleum Imports by World Region of Origin, 1960-2009 .............................................. 1-8

Refinery Gross Output by World Region, 1999 and 2009 .......................................................... 1-12

Registrations

Car Registrations for Selected Countries, 1960-2008.............................................................. 3-2

Truck and Bus Registrations for Selected Countries, 1960-2008 ................................................... 3-3

New Light Fleet Vehicle Registrations by Vehicle Type, Model Year 2008 .................................... 7-3

Representative

Representative Number Five Driving Cycle .......................................................................... 4-36

Reserves

World Oil Reserves, Production and Consumption, 2008........................................................... 1-6

World Natural Gas Reserves, Production and Consumption, 2008 .............................................. 1-7

Response

Summary Statistics on Demand Response Vehicles, 1994-2008 ................................................ 4-39

Results

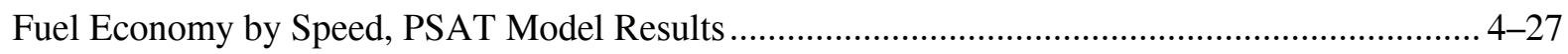

Retail

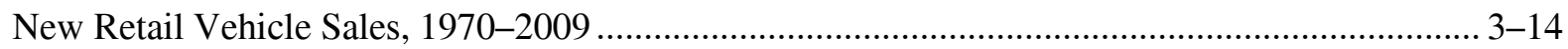

New Retail Car Sales in the United States, 1970-2009 ................................................................ 4-5

New Retail Truck Sales by Gross Vehicle Weight, 1970-2009.................................................. 5-4

Retail Prices for Motor Fuel, 1978-2009 ............................................................................... 10-8

Revenue

Class I Railroad Freight Systems in the United States Ranked by

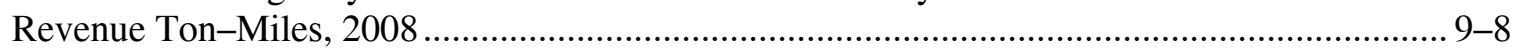

Route

Summary Statistics for U.S. Domestic and International Certificated Route

Air Carriers (Combined Totals), 1970-2008 ….................................................................... 9-3

Sale

Tax Receipts from the Sale of Gas Guzzlers, 1980-2008 …....................................................... 4-26

Sales

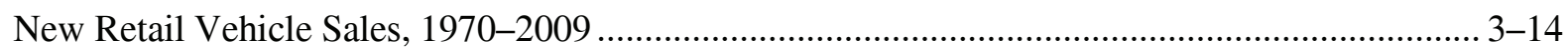

Sales Estimates of Class 1, Class 2a, and Class 2b Light Trucks, 1989-1999................................. 4-4

New Retail Car Sales in the United States, 1970-2009 ................................................................ 4-5

Period Sales, Market Shares, and Sales-Weighted Fuel Economies of New

Domestic and Import Cars, Selected Model Years 1975-2009 ................................................. 4-7

Period Sales, Market Shares, and Sales-Weighted Fuel Economies of New

Domestic and Import Light Trucks, Model Years 1975-2009 .................................................... 4-9

Sales-Weighted Engine Size of New Domestic and Import Cars

by Size Class, Model Years 1975-2009 ................................................................................. 4-12

Sales-Weighted Engine Size of New Domestic and Import Light Trucks

by Size Class, Model Years 1975-2009 ............................................................................. 4-13

Sales-Weighted Curb Weight of New Domestic and Import Cars

by Size Class, Model Years 1975-2009 ................................................................................ 4-14 
Sales (continued)

Sales-Weighted Interior Space of New Domestic and Import Cars

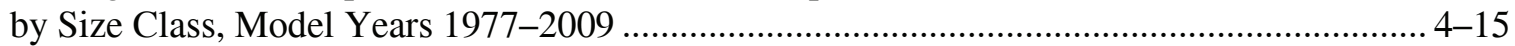

New Light Vehicle Dealerships and Sales, 1970-2008 ............................................................. 4-17

Car Corporate Average Fuel Economy (CAFE) Standards versus

Sales-Weighted Fuel Economy Estimates, 1978-2010 ............................................................ 4-22

Light Truck Corporate Average Fuel Economy (CAFE) Standards versus

Sales-Weighted Fuel Economy Estimates, 1978-2010 ......................................................... 4-23

New Retail Truck Sales by Gross Vehicle Weight, 1970-2009 ................................................. 5-4

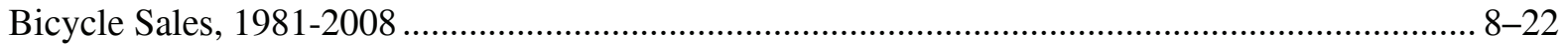

Refiner Sales Prices for Propane and No. 2 Diesel, 1978-2009 .............................................. 10-9

Refiner Sales Prices for Aviation Gasoline and Jet Fuel, 1978-2009 ......................................... 10-10

Sales-Weighted Annual Carbon Footprint of New Domestic and Import Cars

by Size Class, Model Years 1975-2009 ............................................................................... 11-12

Sales-Weighted Annual Carbon Footprint of New Domestic and Import

Light Trucks by Size Class, Model Years 1975-2009 ........................................................... 11-13

Scrappage

Car Scrappage and Survival Rates, 1970, 1980 and 1990 Model Years ....................................... 3-15

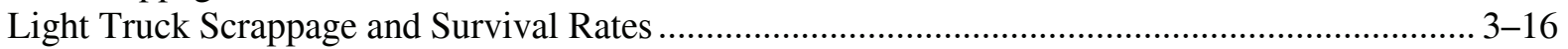

$\mathrm{SC} 03$

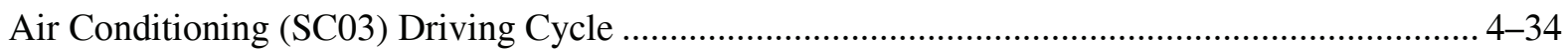

Sector

Consumption of Petroleum by End-Use Sector, 1973-2009................................................... 1-19

U. S. Consumption of Total Energy by End-Use Sector, 1973-2009 …........................................ 2-3

Total U.S. Greenhouse Emissions by End-Use Sector, 2008 ..................................................... 11-5

U.S. Carbon Emissions from Fossil Energy Consumption by End-Use

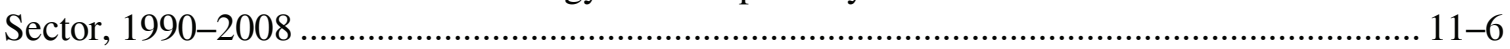

U.S. Carbon Emissions from Energy Use in the Transportation Sector, 1990-2008 ..................... 11-7

Total National Emissions of the Criteria Air Pollutants by Sector, 2008...................................... 12-2

Sectors

United States Petroleum Production and Consumption - All Sectors,

1973-2035

Selected

Car Registrations for Selected Countries, 1960-2008.................................................................. 3-2

Truck and Bus Registrations for Selected Countries, 1960-2008 ................................................... 3-3

Period Sales, Market Shares, and Sales-Weighted Fuel Economies of New

Domestic and Import Cars, Selected Model Years 1975-2009 …............................................... 4-7

Share of Heavy Trucks with Selected Electronic Features, 2002 ............................................... 5-12

Gasoline Prices for Selected Countries, 1990-2008 …................................................................. 10-3

Diesel Fuel Prices for Selected Countries, 1998-2008 ................................................................. 10-4

Gasoline Prices for Selected Countries, 1990 and 2008 ............................................................. 10-5

Diesel Prices for Selected Countries, 1990 and 2008................................................................ 10-6

Self-Reported

Self-Reported vs. Odometer Average Annual Miles, 1995 NPTS and

2001 NHTS 
Service

Fleet Vehicles in Service as of January 1, 2009 …................................................................ 7-2

Average Length of Time Business Fleet Vehicles are in Service, 2008 ....................................... 7-3

Services

Transportation Services Index, January 1990-January 2010 .................................................... 10-2

Share

Share of Trucks by Major Use and Primary Fueling Facility, 2002 …....................................... 5-10

Share of Heavy Trucks with Selected Electronic Features, 2002 ............................................ 5-12

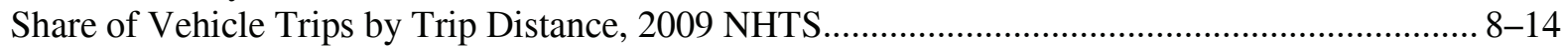

Share of Vehicle Trips to Work by Trip Distance, 2009 NHTS ................................................. 8-14

Share of Vehicles by Annual Miles of Travel and Vehicle Age, 2009 NHTS ................................ 8-15

Shares

Petroleum Production and Consumption and Some Important Percent

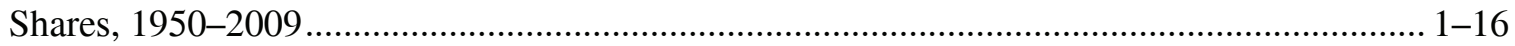

Shares of Highway Vehicle-Miles Traveled by Vehicle Type, 1970-2008 ................................... 3-10

Period Sales, Market Shares, and Sales-Weighted Fuel Economies of New

Domestic and Import Cars, Selected Model Years 1975-2009 .................................................. 4-7

Period Sales, Market Shares, and Sales-Weighted Fuel Economies of New

Domestic and Import Light Trucks, Model Years 1975-2009 ................................................ 4-9

Light Vehicle Market Shares by Size Class, Model Years 1975-2009......................................... 4-10

Light Vehicle Market Shares, Model Years 1975-2009 ........................................................... 4-11

Nonhighway Energy Use Shares, 1970-2008 ......................................................................... 9-2

Single-Unit

Summary Statistics for Heavy Single-Unit Trucks, 1970-2008 …............................................... 5-2

Sites

Number of Alternative Refuel Sites by State and Fuel Type, 2010 ............................................. 6-6

Size

Light Vehicle Market Shares by Size Class, Model Years 1975-2009.......................................... 4-10

Sales-Weighted Engine Size of New Domestic and Import Cars

by Size Class, Model Years 1975-2009 ........................................................................... 4-12

Sales-Weighted Engine Size of New Domestic and Import Light Trucks

by Size Class, Model Years 1975-2009 ............................................................................... 4-13

Sales-Weighted Curb Weight of New Domestic and Import Cars

by Size Class, Model Years 1975-2009 ........................................................................... 4-14

Sales-Weighted Interior Space of New Domestic and Import Cars

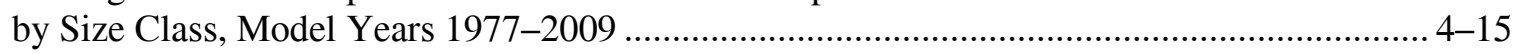

Truck Harmonic Mean Fuel Economy by Size Class, 1992, 1997, and 2002 ................................ 5-6

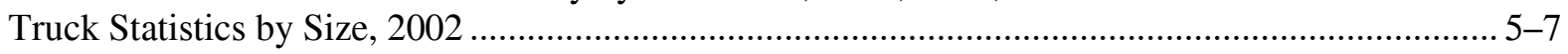

Percentage of Trucks by Size Ranked by Major Use, 2002 ...................................................... 5-8

Percentage of Trucks by Fleet Size and Primary Fueling Facility, 2002 ........................................ 5-9

Sales-Weighted Annual Carbon Footprint of New Domestic and Import Cars by Size Class, Model Years 1975-2009 ................................................................................... 11-12

Sales-Weighted Annual Carbon Footprint of New Domestic and Import Light Trucks by Size Class, Model Years 1975-2009 ........................................................ 11-13

Source

Distribution of Energy Consumption by Source, 1973 and 2009................................................ 2-4 
Sources

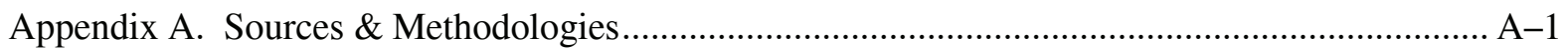

Space

Sales-Weighted Interior Space of New Domestic and Import Cars

by Size Class, Model Years 1977-2009

Special

Highway Usage of Gasoline and Special Fuels, 1973-2008 ........................................................ 2-13

Specifications

Vehicle Specifications for Vehicles Tested in the 1997 Study...................................................... 4-30

Speed

Fuel Economy by Speed, PSAT Model Results ........................................................................... 4-27

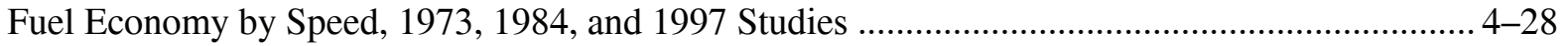

Steady Speed Fuel Economy for Vehicles Tested in the 1997 Study............................................ 4-31

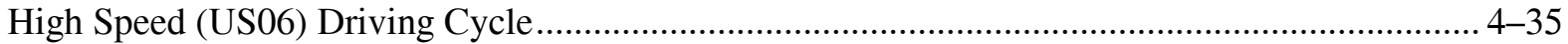

Fuel Economy for Class 8 Trucks as Function of Speed and Tractor-Trailer

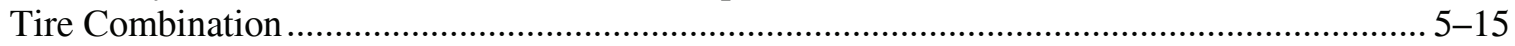

Class 8 Truck Fuel Economy as a Function of Speed and Tractor-Trailer Tire

Combination and Percentage of Total Distance Traveled as a Function

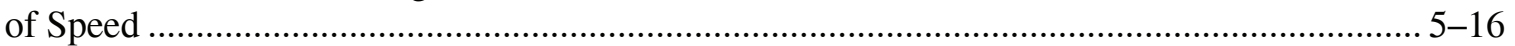

Class 8 Truck Percent of Total Fuel Consumed as a Function of Speed and

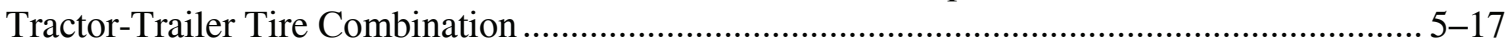

Standards

Reformed and Unreformed Light Truck Corporate Average Fuel Economy

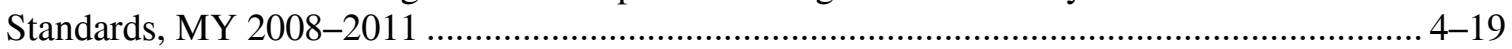

Fuel Economy and Carbon Dioxide Emissions Standards, MY 2012-2016................................ 4-20

Car Corporate Average Fuel Economy (CAFE) Standards versus

Sales-Weighted Fuel Economy Estimates, 1978-2010 .......................................................... 4-22

Light Truck Corporate Average Fuel Economy (CAFE) Standards versus

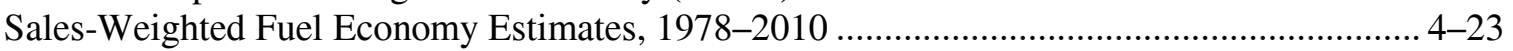

U.S. Tier 2 Emission Standards for Cars and Light Trucks ................................................... 12-13

Light Vehicle Exhaust Emission Standards in Effect in 2009 when

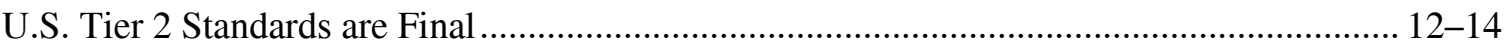

California Cars and Light Trucks Emission Certification Standards ........................................... 12-15

State

Number of Alternative Refuel Sites by State and Fuel Type, 2010 ............................................6-6

State Tax Exemptions for Gasohol, 2008 ............................................................................. 10-11

Federal and State Alternative Fuel Incentives, 2010 ...............................................................10-12

Federal and State Advanced Technology Incentives, 2010 ........................................................ 10-13

States

U.S. Petroleum Imports by World Region of Origin, 1960-2009 ................................................ 1-8

Costs of Oil Dependence to the U.S. Economy, 1970-2009..................................................... 1-10

U.S. Refinery Input of Crude Oil and Petroleum Products, 1987-2008 …................................... 1-13

United States Petroleum Production, Imports and Exports, 1950-2009 ….................................... 1-15

United States Petroleum Production and Consumption - All Sectors, $1973-2035$ 
States (continued)

United States Petroleum Production, Transportation and Consumption, 1970-2035. $1-18$

U. S. Consumption of Total Energy by End-Use Sector, 1973-2009 ............................................ 2-3

Intercity Freight Movement and Energy Use in the United States, 2007 and 2008 ....................... 2-19

Vehicles per Thousand People: U.S. (Over Time) Compared to Other

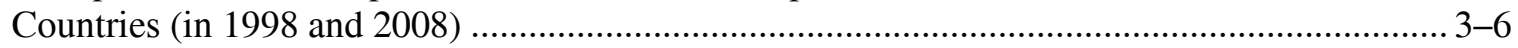

Vehicles per Thousand People in the United States, 1990-2008 …................................................ 3-9

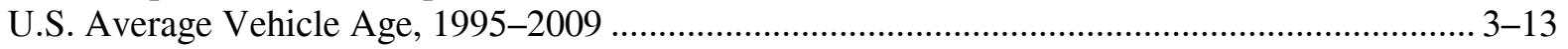

New Retail Car Sales in the United States, 1970-2009 .............................................................. 4-5

New Retail Sales of Trucks 10,000 Pounds GVW and Less in the

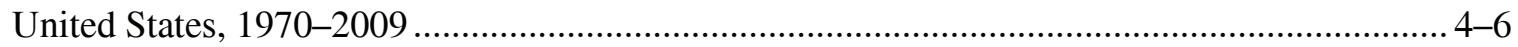

Projected Fuel Economies from U.S., European, and Japanese Driving Cycles............................. 4-37

Comparison of U.S., European, and Japanese Driving Cycles...................................................... 4-38

Growth of Freight in the United States: Comparison of the 2007, 2002 and 1997 Commodity

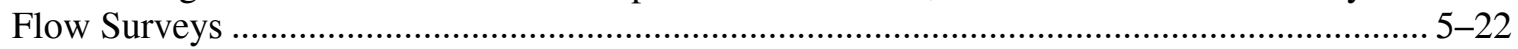

Growth of Freight Miles in the United States: Comparison of the 2007, 2002

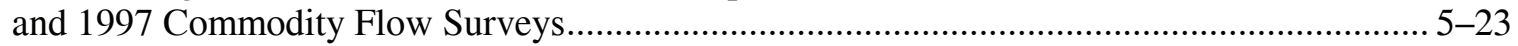

Characteristics of U.S. Daily per Vehicle Driving vs. Dwelling Unit Type

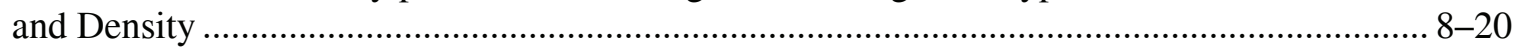

Summary Statistics for U.S. Domestic and International Certificated Route Air Carriers (Combined Totals), 1970-2008 ............................................................................. 9-3

Class I Railroad Freight Systems in the United States Ranked by

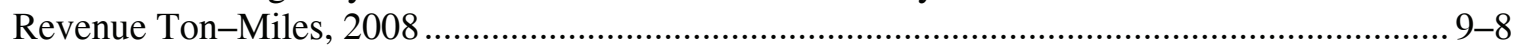

U.S. Employment for Motor Vehicles and Motor Vehicle Parts Manufacturing, 1990-2009. $10-20$

U.S. Emissions of Greenhouse Gases, based on Global Warming Potential,

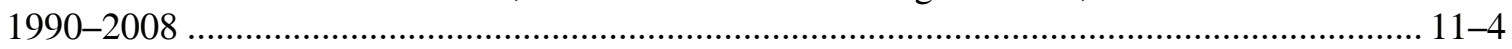

Total U.S. Greenhouse Emissions by End-Use Sector, 2008 ................................................... 11-5

U.S. Carbon Emissions from Fossil Energy Consumption by End-Use

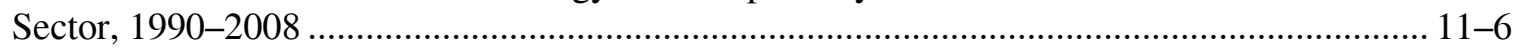

U.S. Carbon Emissions from Energy Use in the Transportation Sector, 1990-2008 ...................... 11-7

U.S. Tier 2 Emission Standards for Cars and Light Trucks ...................................................... 12-13

Light Vehicle Exhaust Emission Standards in Effect in 2009 when

U.S. Tier 2 Standards are Final ..................................................................................... 12-14

Stations

Conventional Refueling Stations, 1993-2008 …...................................................................... 4-18

Statistics

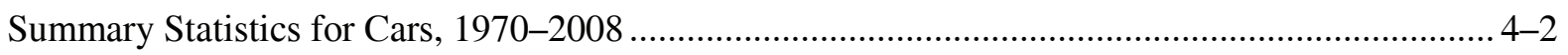

Summary Statistics for Two-Axle, Four-Tire Trucks, 1970-2008................................................ 4-3

Summary Statistics on Class 1, Class 2a, and Class 2b Light Trucks ........................................... 4-4

Summary Statistics on Demand Response Vehicles, 1994-2008 ................................................ 4-39

Summary Statistics for Heavy Single-Unit Trucks, 1970-2008 …................................................ 5-2

Summary Statistics for Combination Trucks, 1970-2008 .............................................................. 5-3

Truck Statistics by Gross Vehicle Weight Class, 2002 ............................................................... 5-6

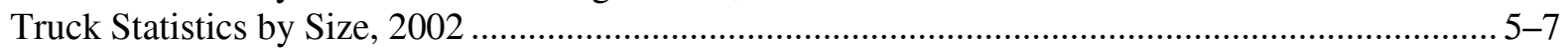

Summary Statistics on Transit Buses and Trolleybuses, 1994-2008 ............................................ 5-24

Demographic Statistics from the 1969, 1977, 1983, 1990, 1995 NPTS

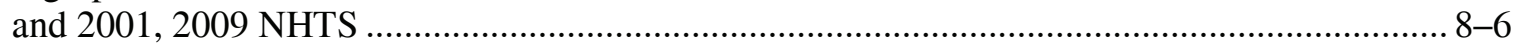

Trip Statistics by Trip Purpose, 2001 and 2009 NHTS .................................................................... 8 
Statistics (continued)

Summary Statistics for U.S. Domestic and International Certificated Route

Air Carriers (Combined Totals), 1970-2008 …........................................................................ 9-3

Summary Statistics for General Aviation, 1970-2008 ................................................................ 9-4

Tonnage Statistics for Domestic and International Waterborne

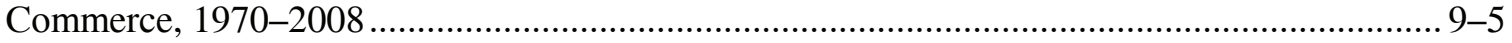

Summary Statistics for Domestic Waterborne Commerce, 1970-2008 .......................................... 9-6

Summary Statistics for Class I Freight Railroads, 1970-2008 ..................................................... 9-9

Summary Statistics for the National Railroad Passenger Corporation

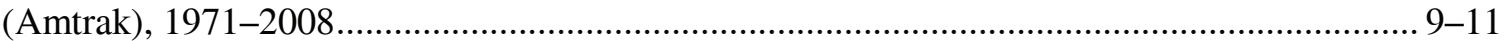

Summary Statistics for Commuter Rail Operations, 1984-2008 ….......................................... 9-12

Summary Statistics for Rail Transit Operations, 1970-2008 …................................................ 9-13

Steady

Steady Speed Fuel Economy for Vehicles Tested in the 1997 Study........................................... 4-31

Studies

Fuel Economy by Speed, 1973, 1984, and 1997 Studies .......................................................... 4-28

Study

Vehicle Specifications for Vehicles Tested in the 1997 Study...................................................... 4-30

Steady Speed Fuel Economy for Vehicles Tested in the 1997 Study............................................. 4-31

Summary

Summary of Military Expenditures for Defending Oil Supplies from the

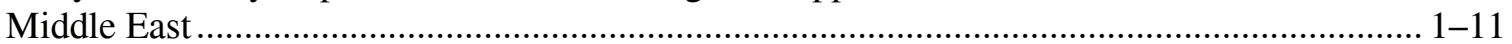

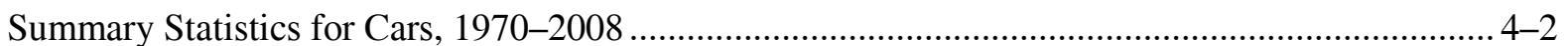

Summary Statistics for Two-Axle, Four-Tire Trucks, 1970-2008................................................ 4-3

Summary Statistics on Class 1, Class 2a, and Class 2b Light Trucks .............................................. 4-4

Summary Statistics on Demand Response Vehicles, 1994-2008 .................................................. 4-39

Summary Statistics for Heavy Single-Unit Trucks, 1970-2008 ................................................. 5-2

Summary Statistics for Combination Trucks, 1970-2008 ........................................................... 5-3

Summary Statistics on Transit Buses and Trolleybuses, 1994-2008 ............................................ 5-24

Summary Statistics for U.S. Domestic and International Certificated Route

Air Carriers (Combined Totals), 1970-2008 …...................................................................... 9-3

Summary Statistics for General Aviation, 1970-2008 ................................................................. 9-4

Summary Statistics for Domestic Waterborne Commerce, 1970-2008 .......................................... 9-6

Summary Statistics for Class I Freight Railroads, 1970-2008 ......................................................... 9-9

Summary Statistics for the National Railroad Passenger Corporation

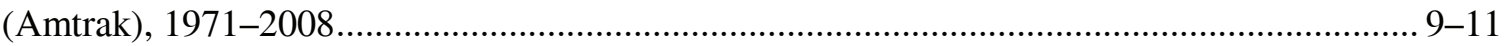

Summary Statistics for Commuter Rail Operations, 1984-2008 …............................................. 9-12

Summary Statistics for Rail Transit Operations, 1970-2008 ..................................................... 9-13

Supplies

Summary of Military Expenditures for Defending Oil Supplies from the

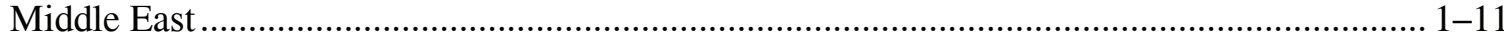

Surveys

Growth of Freight in the United States: Comparison of the 2007, 2002 and 1997 Commodity

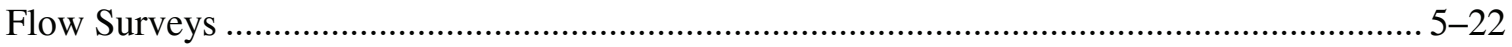

Growth of Freight Miles in the United States: Comparison of the 2007, 2002

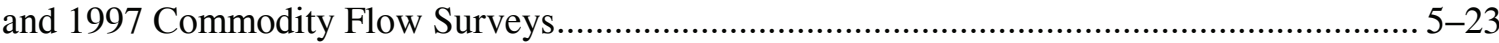


Survivability

Car and Light Truck Survivability Rates and Lifetime Miles

Survival

Car Scrappage and Survival Rates, 1970, 1980 and 1990 Model Years ....................................... 3-15

Light Truck Scrappage and Survival Rates

Systems

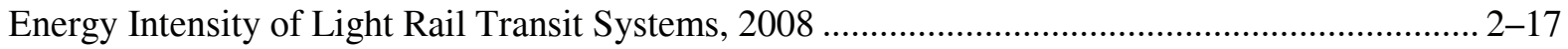

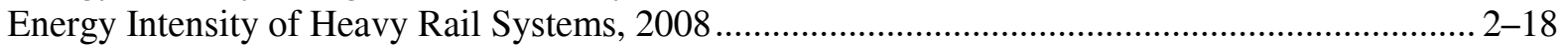

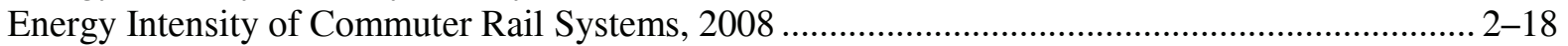

Class I Railroad Freight Systems in the United States Ranked by

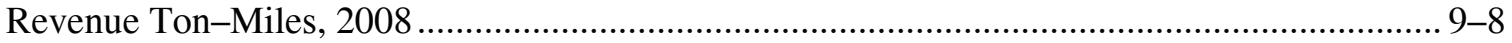

Targets

Fuel Economy and Carbon Dioxide Targets for Model Year 2016.............................................. 4-21

$\operatorname{Tax}$

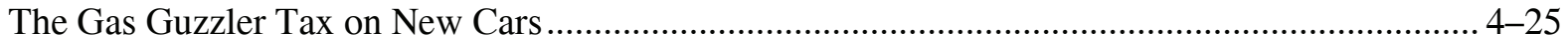

Tax Receipts from the Sale of Gas Guzzlers, 1980-2008 …......................................................26

State Tax Exemptions for Gasohol, 2008 .............................................................................. 10-11

Taxes

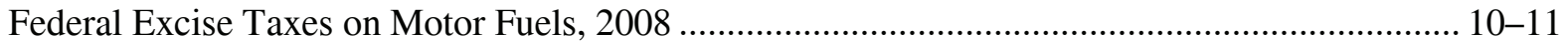

Technology

Alternative Fuel and Advanced Technology Vehicles and Characteristics..................................... 6-1

Federal and State Advanced Technology Incentives, 2010 ..................................................... 10-13

Temperature

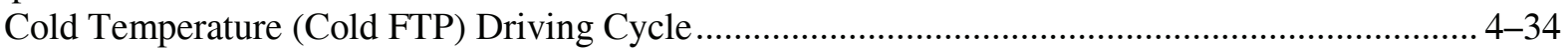

Terrain

Effect of Terrain on Class 8 Truck Fuel Economy......................................................................... 5-14

Tested

Vehicle Specifications for Vehicles Tested in the 1997 Study...................................................... 4-30

Steady Speed Fuel Economy for Vehicles Tested in the 1997 Study.......................................... 4-31

Tier 2

U.S. Tier 2 Emission Standards for Cars and Light Trucks ...................................................... 12-13

Light Vehicle Exhaust Emission Standards in Effect in 2009 when

U.S. Tier 2 Standards are Final

Time

Average Length of Time Business Fleet Vehicles are in Service, 2008 ........................................ 7-3

Workers by Commute Time, 1990 and 2000 Census .................................................................. 8-21

Tire

Fuel Economy for Class 8 Trucks as Function of Speed and Tractor-Trailer

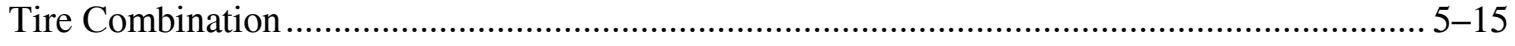


Tire (continued)

Class 8 Truck Fuel Economy as a Function of Speed and Tractor-Trailer Tire Combination and Percentage of Total Distance Traveled as a Function

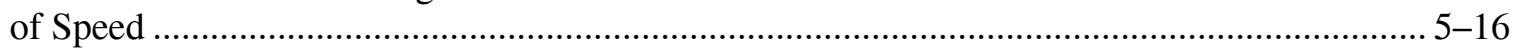

Class 8 Truck Percent of Total Fuel Consumed as a Function of Speed and Tractor-Trailer Tire Combination

Ton-Miles

Class I Railroad Freight Systems in the United States Ranked by

Revenue Ton-Miles, 2008 9-8

Tonnage

Tonnage Statistics for Domestic and International Waterborne Commerce, 1970-2008 $9-5$

Total

U. S. Consumption of Total Energy by End-Use Sector, 1973-2009 $2-3$

Class 8 Truck Fuel Economy as a Function of Speed and Tractor-Trailer Tire Combination and Percentage of Total Distance Traveled as a Function of Speed $5-16$

Class 8 Truck Percent of Total Fuel Consumed as a Function of Speed and

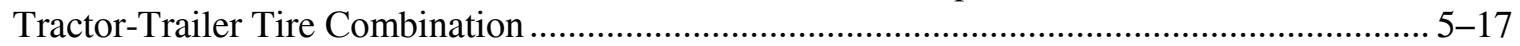

Total U.S. Greenhouse Emissions by End-Use Sector, 2008 …................................................... 11-5

Total National Emissions of the Criteria Air Pollutants by Sector, 2008...................................... 12-2

Total National Emissions of Carbon Monoxide, 1970-2008 ........................................................... 12-3

Total National Emissions of Nitrogen Oxides, 1970-2008 .......................................................... 12-5

Total National Emissions of Volatile Organic Compounds, 1970-2008 ...................................... 12-7

Total National Emissions of Particulate Matter (PM-10), 1970-2008 …...................................... 12-9

Total National Emissions of Particulate Matter (PM-2.5), 1990-2008 ........................................ 12-11

Totals

Summary Statistics for U.S. Domestic and International Certificated Route Air Carriers (Combined Totals), 1970-2008 …........................................................................ 9-3

Tractor-Trailer

Fuel Economy for Class 8 Trucks as Function of Speed and Tractor-Trailer

Tire Combination.

Class 8 Truck Fuel Economy as a Function of Speed and Tractor-Trailer Tire

Combination and Percentage of Total Distance Traveled as a Function

of Speed

Class 8 Truck Percent of Total Fuel Consumed as a Function of Speed and

Tractor-Trailer Tire Combination

Distribution of Five-Axle Tractor-Trailers by On-Road Vehicle Weight, 2008

Traffic

Intermodal Rail Traffic, 1965-2008 $9-10$

Transit

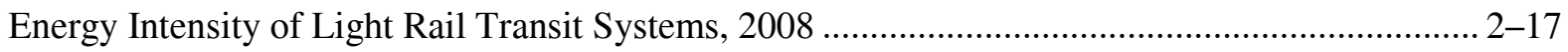

Summary Statistics on Transit Buses and Trolleybuses, 1994-2008 .......................................... 5-24

Summary Statistics for Rail Transit Operations, 1970-2008. 
Transportation

United States Petroleum Production, Transportation and Consumption, 1970-2035

Highway Transportation Petroleum Consumption by Mode, 1970-2008..................................... 1-20

Nonhighway Transportation Petroleum Consumption by Mode, 1970-2008 ................................ 1-21

Transportation Petroleum Use by Mode, 2007-2008 ...................................................................... 1-22

Domestic Consumption of Transportation Energy by Mode and

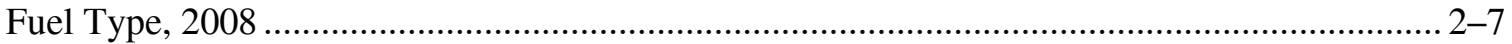

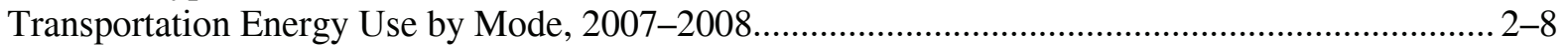

Highway Transportation Energy Consumption by Mode, 1970-2008..........................................2-9

Nonhighway Transportation Energy Consumption by Mode, 1970-2008_................................... 2-10

Off-highway Transportation-related Fuel Consumption from the NonRoad Model, 2008 ............. 2-11

Means of Transportation to Work, 1980, 1990 and 2000 Census ................................................... 8-19

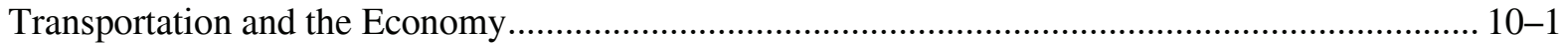

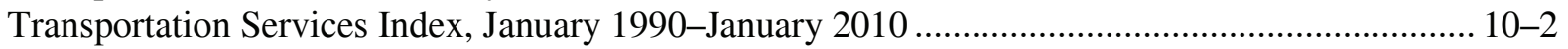

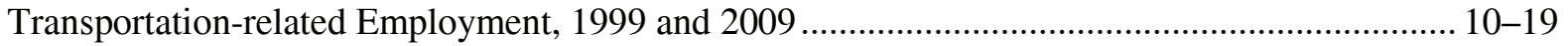

U.S. Carbon Emissions from Energy Use in the Transportation Sector, 1990-2008 ...................... 11-7

Transportation Greenhouse Gas Emissions by Mode, 1990 and 2008 ......................................... 11-8

Travel

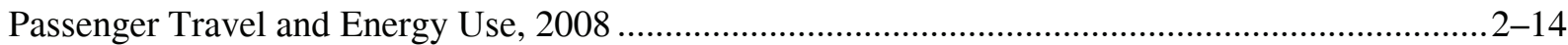

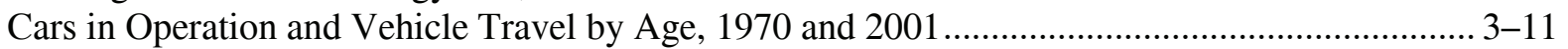

Trucks in Operation and Vehicle Travel by Age, 1970 and 2001 ................................................ 3-12

Average Annual Vehicle-Miles of Travel for Business Fleet Vehicles, 2008................................. 7-3

Average Number of Vehicles and Vehicle Travel per Household,

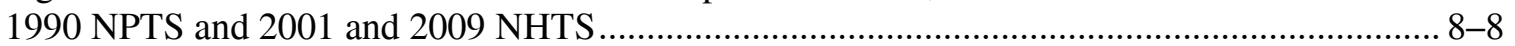

Share of Vehicles by Annual Miles of Travel and Vehicle Age, 2009 NHTS ................................ 8-15

Daily Vehicle Miles of Travel (per Vehicle) by Number of Vehicles in the

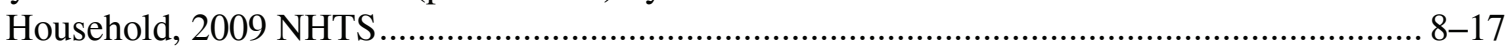

Daily and Annual Vehicle Miles of Travel and Average Age for Each Vehicle in a Household, 2009 NHTS ................................................................................................... 8 8-17

Daily Vehicle Miles of Travel for Each Vehicle in a Household, 2009 NHTS ............................. 8-18

Annual Vehicle Miles of Travel for Each Vehicle in a Household, 2009 NHTS............................ 8-18

Traveled

Shares of Highway Vehicle-Miles Traveled by Vehicle Type, 1970-2008 .................................. 3-10

Distribution of Trucks over 26,000 lbs. Less than Two Years Old by Vehicle-

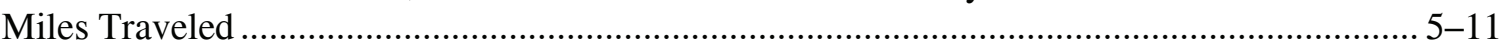

Class 8 Truck Fuel Economy as a Function of Speed and Tractor-Trailer Tire Combination and Percentage of Total Distance Traveled as a Function

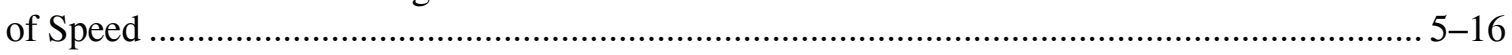

Trip

Average Annual Vehicle-Miles, Vehicle Trips and Trip Length per

Household 1969, 1977, 1983, 1990, 1995 NPTS and 2001, 2009 NHTS ................................. 8-7

Trip Statistics by Trip Purpose, 2001 and 2009 NHTS .................................................................... 8-9

Average Vehicle Occupancy by Trip Purpose, 1977 NPTS and

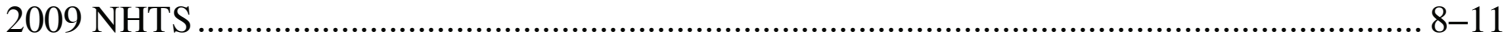

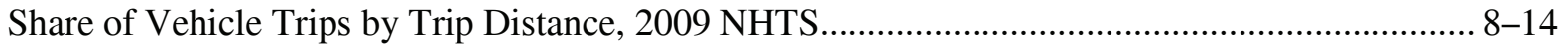

Share of Vehicle Trips to Work by Trip Distance, 2009 NHTS ................................................ 8-14

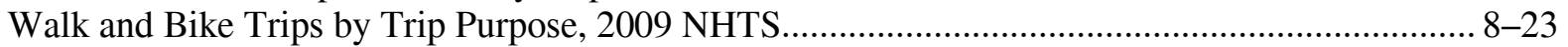

Long-Distance Trip Characteristics, 2001 NHTS ........................................................................... 8-25 
Trips

Average Annual Vehicle-Miles, Vehicle Trips and Trip Length per

Household 1969, 1977, 1983, 1990, 1995 NPTS and 2001, 2009 NHTS ….............................. 8-7

Share of Vehicle Trips by Trip Distance, 2009 NHTS................................................................. 8-14

Share of Vehicle Trips to Work by Trip Distance, 2009 NHTS .................................................... 8-14

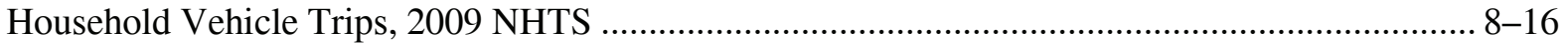

Walk and Bike Trips by Trip Purpose, 2009 NHTS....................................................................... 8-23

Trolleybuses

Summary Statistics on Transit Buses and Trolleybuses, 1994-2008 ............................................ 5-24

Truck

Truck and Bus Registrations for Selected Countries, 1960-2008 .................................................. 3-3

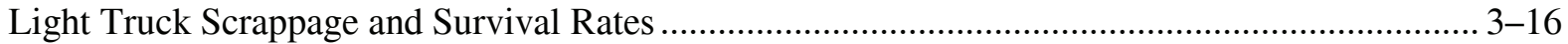

Car and Light Truck Survivability Rates and Lifetime Miles ....................................................... 3-17

Reformed and Unreformed Light Truck Corporate Average Fuel Economy

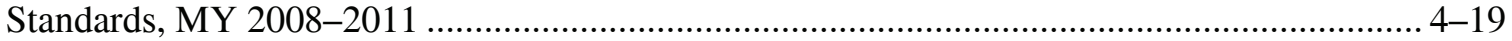

Light Truck Corporate Average Fuel Economy (CAFE) Standards versus

Sales-Weighted Fuel Economy Estimates, 1978-2010 ....................................................... 4-23

Truck Statistics by Gross Vehicle Weight Class, 2002 …......................................................... 5-6

Truck Harmonic Mean Fuel Economy by Size Class, 1992, 1997, and 2002 ................................ 5-6

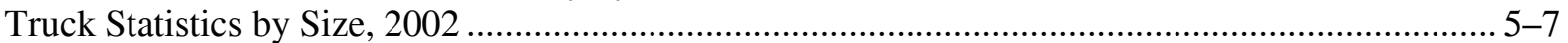

Effect of Terrain on Class 8 Truck Fuel Economy......................................................................... 5-14

Class 8 Truck Fuel Economy as a Function of Speed and Tractor-Trailer Tire

Combination and Percentage of Total Distance Traveled as a Function

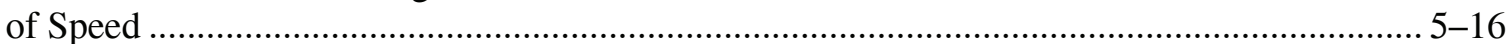

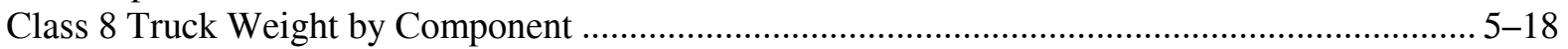

Trucks

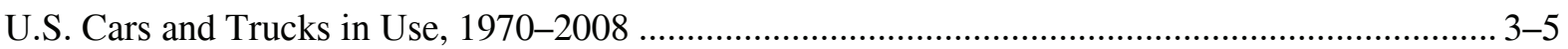

Trucks in Operation and Vehicle Travel by Age, 1970 and 2001 ............................................. 3-12

Summary Statistics for Two-Axle, Four-Tire Trucks, 1970-2008............................................... 4-3

Summary Statistics on Class 1, Class 2a, and Class 2b Light Trucks ............................................ 4-4

Sales Estimates of Class 1, Class 2a, and Class 2b Light Trucks, 1989-1999................................. 4-4

New Retail Sales of Trucks 10,000 Pounds GVW and Less in the

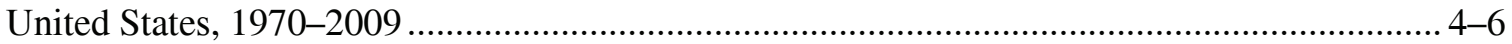

Period Sales, Market Shares, and Sales-Weighted Fuel Economies of New

Domestic and Import Light Trucks, Model Years 1975-2009 ................................................... 4-9

Sales-Weighted Engine Size of New Domestic and Import Light Trucks

by Size Class, Model Years 1975-2009 ……........................................................................... 4-13

Summary Statistics for Heavy Single-Unit Trucks, 1970-2008 …................................................. 5-2

Summary Statistics for Combination Trucks, 1970-2008 ............................................................ 5-3

Percentage of Trucks by Size Ranked by Major Use, 2002 ........................................................ 5-8

Percentage of Trucks by Fleet Size and Primary Fueling Facility, 2002 ........................................ 5-9

Share of Trucks by Major Use and Primary Fueling Facility, 2002 ............................................. 5-10

Distribution of Trucks over 26,000 lbs. Less than Two Years Old by Vehicle-

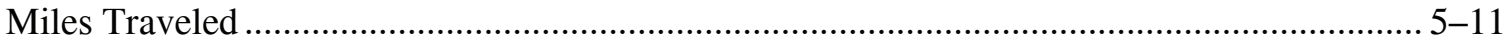

Sales-Weighted Annual Carbon Footprint of New Domestic and Import

Light Trucks by Size Class, Model Years 1975-2009 ........................................................ 11-13

U.S. Tier 2 Emission Standards for Cars and Light Trucks .................................................... 12-13

California Cars and Light Trucks Emission Certification Standards .......................................... 12-15 
Two-Axle

Summary Statistics for Two-Axle, Four-Tire Trucks, 1970-2008 $4-3$

Type

Domestic Consumption of Transportation Energy by Mode and

Fuel Type, 2008 $2-7$

Shares of Highway Vehicle-Miles Traveled by Vehicle Type, 1970-2008.

New Light Fleet Vehicle Registrations by Vehicle Type, Model Year 2008

Average Miles per Domestic Federal Vehicle by Vehicle Type, 2009.

Federal Fleet Vehicle Acquisitions by Fuel Type, FY 2002- 2009 .7-6

Average Vehicle Occupancy by Vehicle Type, 1995 NPTS and 2009 NHTS

Characteristics of U.S. Daily per Vehicle Driving vs. Dwelling Unit Type and Density

Unit

Summary Statistics for Heavy Single-Unit Trucks, 1970-2008

Characteristics of U.S. Daily per Vehicle Driving vs. Dwelling Unit Type and Density

Housing Unit Characteristics, 2007. $8-20$

United

U.S. Petroleum Imports by World Region of Origin, 1960-2009 ................................................... 1-8

Costs of Oil Dependence to the U.S. Economy, 1970-2009 ......................................................... 1-10

U.S. Refinery Input of Crude Oil and Petroleum Products, 1987-2008 …....................................... 1-13

United States Petroleum Production, Imports and Exports, 1950-2009 ….................................... 1-15

United States Petroleum Production and Consumption - All Sectors,

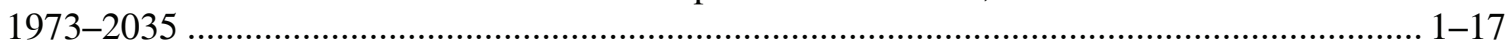

United States Petroleum Production, Transportation and Consumption, 1970-2035 $1-18$

U. S. Consumption of Total Energy by End-Use Sector, 1973-2009 ........................................... 2-3

Intercity Freight Movement and Energy Use in the United States, 2007 and 2008 ....................... 2-19

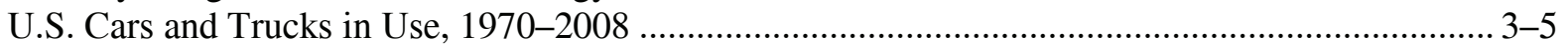

Vehicles per Thousand People in the United States, 1990-2008 ..................................................3-9

U.S. Average Vehicle Age, 1995-2009 .................................................................................... 3-13

New Retail Car Sales in the United States, 1970-2009 ............................................................ 4-5

New Retail Sales of Trucks 10,000 Pounds GVW and Less in the United States, 1970-2009......... 4-6

Projected Fuel Economies from U.S., European, and Japanese Driving Cycles............................. 4-37

Comparison of U.S., European, and Japanese Driving Cycles.................................................. 4-38

Growth of Freight in the United States: Comparison of the 2007, 2002 and 1997 Commodity

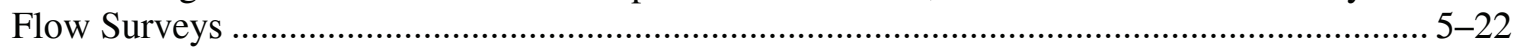

Growth of Freight Miles in the United States: Comparison of the 2007, 2002

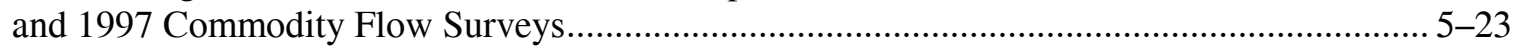

Characteristics of U.S. Daily per Vehicle Driving vs. Dwelling Unit Type and Density

Summary Statistics for U.S. Domestic and International Certificated Route Air Carriers (Combined Totals), 1970-2008 …......................................................................... 9-3

Class I Railroad Freight Systems in the United States Ranked by

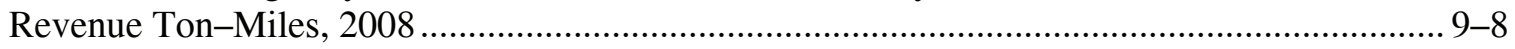

U.S. Employment for Motor Vehicles and Motor Vehicle Parts Manufacturing, 1990-2009. $10-20$

U.S. Emissions of Greenhouse Gases, based on Global Warming Potential, 1990-2008 
United (continued)

Total U.S. Greenhouse Emissions by End-Use Sector, 2008 ..................................................... 11-5

U.S. Carbon Emissions from Fossil Energy Consumption by End-Use

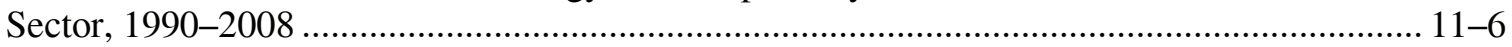

U.S. Carbon Emissions from Energy Use in the Transportation

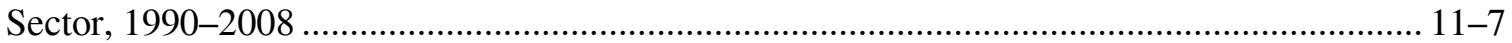

U.S. Tier 2 Emission Standards for Cars and Light Trucks ...................................................... 12-13

Unreformed

Reformed and Unreformed Light Truck Corporate Average Fuel Economy

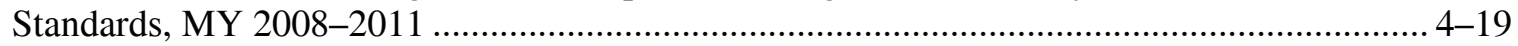

Usage

Highway Usage of Gasoline and Special Fuels, 1973-2008 ........................................................ 2-13

Use

Transportation Petroleum Use by Mode, 2007-2008 .................................................................. 1-22

U. S. Consumption of Total Energy by End-Use Sector, 1973-2009 ........................................... 2-3

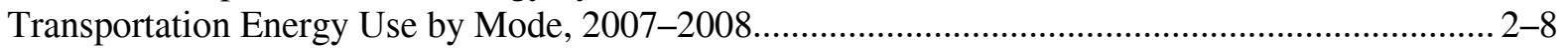

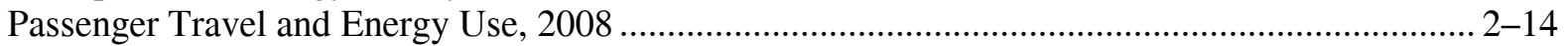

Intercity Freight Movement and Energy Use in the United States, 2007 and 2008 ....................... 2-19

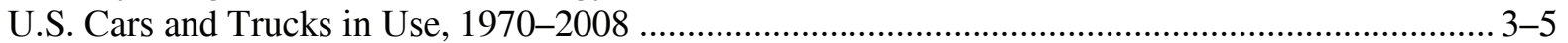

Percentage of Trucks by Size Ranked by Major Use, 2002 .......................................................... 5-8

Share of Trucks by Major Use and Primary Fueling Facility, 2002 .......................................... 5-10

Estimates of Alternative Fuel Vehicles in Use, 1995-2008 _.............................................................. 6-3

Nonhighway Energy Use Shares, 1970-2008 …................................................................. 9-2

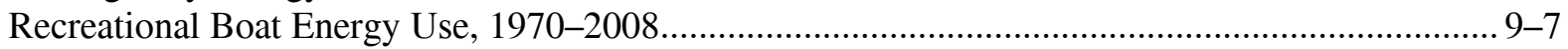

U.S. Carbon Emissions from Energy Use in the Transportation Sector, 1990-2008 ...................... 11-7

US06

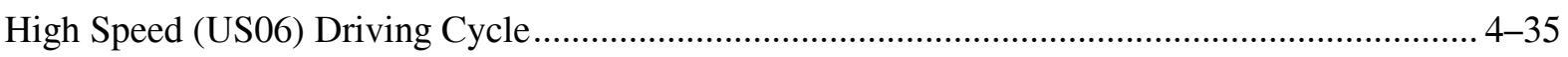

Vehicle

Shares of Highway Vehicle-Miles Traveled by Vehicle Type, 1970-2008 ................................... 3-10

Cars in Operation and Vehicle Travel by Age, 1970 and 2001 ..................................................... 3-11

Trucks in Operation and Vehicle Travel by Age, 1970 and 2001 ................................................. 3-12

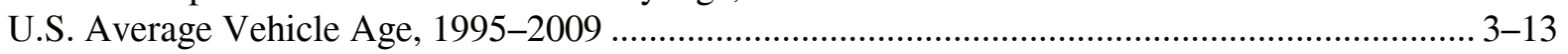

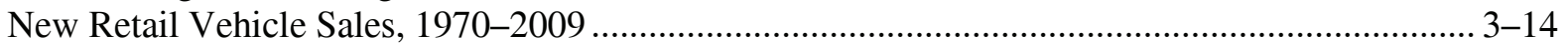

New Retail Sales of Trucks 10,000 Pounds GVW and Less in the

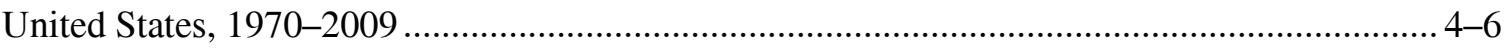

Light Vehicle Market Shares by Size Class, Model Years 1975-2009.......................................... 4-10

Light Vehicle Market Shares, Model Years 1975-2009 ............................................................. 4-11

Average Material Consumption for a Light Vehicle, Model Years 1995, 2000,

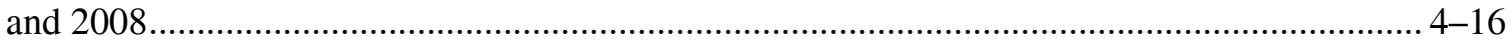

New Light Vehicle Dealerships and Sales, 1970-2008 ............................................................. 4-17

New Retail Truck Sales by Gross Vehicle Weight, 1970-2009 ..................................................... 5-4

Truck Statistics by Gross Vehicle Weight Class, 2002 ............................................................... 5-6

Gross Vehicle Weight vs. Empty Vehicle Weight ..................................................................... 5-19

Distribution of Five-Axle Tractor-Trailers by On-Road Vehicle Weight, 2008 ............................ 5-20

New Light Fleet Vehicle Registrations by Vehicle Type, Model Year 2008 ................................. 7-3

Average Annual Vehicle-Miles of Travel for Business Fleet Vehicles, 2008................................. 7-3

Average Miles per Domestic Federal Vehicle by Vehicle Type, 2009 …....................................... 7-4

Federal Fleet Vehicle Acquisitions by Fuel Type, FY 2002-2009 ............................................... 7-6 
Vehicle (continued)

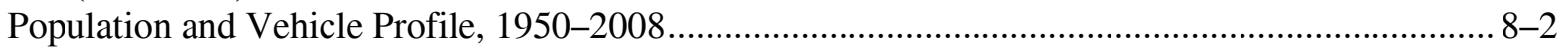

Household Vehicle Ownership, 1960-2000 Census ................................................................... 8-5

Average Vehicle Occupancy by Vehicle Type, 1995 NPTS and

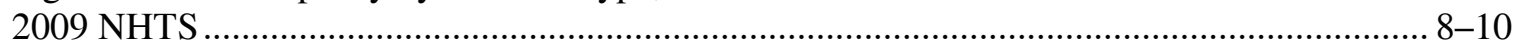

Average Annual Miles per Household Vehicle by Vehicle Age .................................................. 8-12

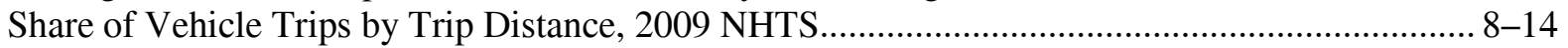

Share of Vehicle Trips to Work by Trip Distance, 2009 NHTS .................................................. 8-14

Share of Vehicles by Annual Miles of Travel and Vehicle Age, 2009 NHTS ................................ 8-15

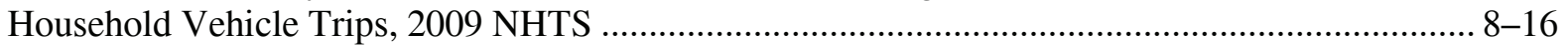

Daily Vehicle Miles of Travel (per Vehicle) by Number of Vehicles in the

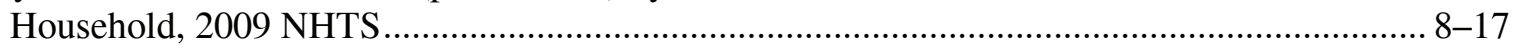

Daily and Annual Vehicle Miles of Travel and Average Age for Each Vehicle

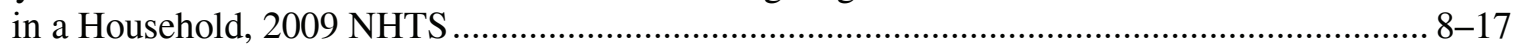

Daily Vehicle Miles of Travel for Each Vehicle in a Household, 2009 NHTS ............................. 8-18

Annual Vehicle Miles of Travel for Each Vehicle in a Household, 2009 NHTS.......................... 8-18

Characteristics of U.S. Daily per Vehicle Driving vs. Dwelling Unit Type

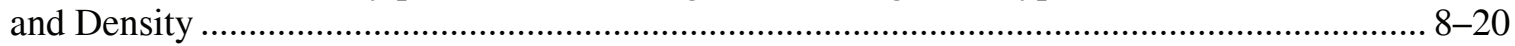

U.S. Employment for Motor Vehicles and Motor Vehicle Parts

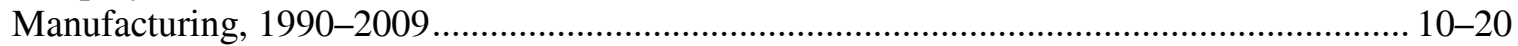

Average Annual Carbon Footprint by Vehicle Classification, 1975 and 2009 ............................ 11-14

Light Vehicle Exhaust Emission Standards in Effect in 2009 when

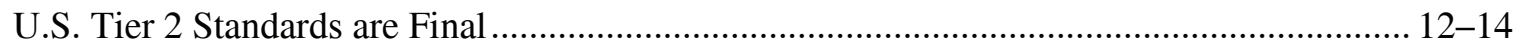

Vehicle-Miles

Shares of Highway Vehicle-Miles Traveled by Vehicle Type, 1970-2008 ................................... 3-10

Distribution of Trucks over 26,000 lbs. Less than Two Years Old by Vehicle-

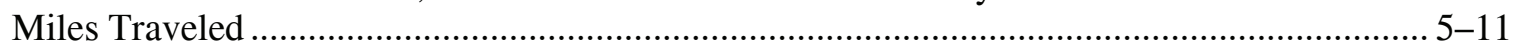

Average Annual Vehicle-Miles of Travel for Business Fleet Vehicles, 2008................................ 7-3

Vehicles and Vehicle-Miles per Capita, 1950-2008 ..................................................................... 8-3

Average Annual Vehicle-Miles, Vehicle Trips and Trip Length per

Household 1969, 1977, 1983, 1990, 1995 NPTS and 2001, 2009 NHTS ................................ 8-7

Vehicles

Vehicles per Thousand People: U.S. (Over Time) Compared to Other

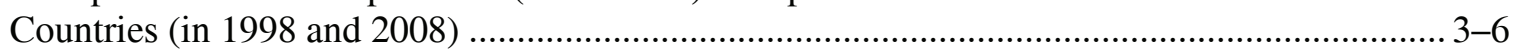

Vehicles per Thousand People in Other Countries, 1998 and 2008 …............................................ 3-8

Vehicles per Thousand People in the United States, 1990-2008 …............................................... 3-9

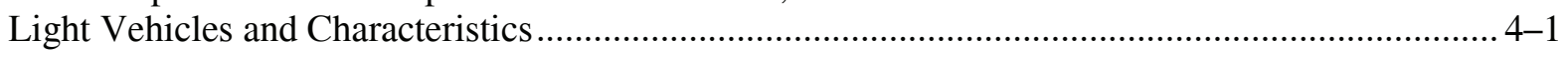

Vehicle Specifications for Vehicles Tested in the 1997 Study................................................... 4-30

Steady Speed Fuel Economy for Vehicles Tested in the 1997 Study........................................... 4-31

Summary Statistics on Demand Response Vehicles, 1994-2008 .............................................. 4-39

Heavy Vehicles and Characteristics ............................................................................................ 5-1

Alternative Fuel and Advanced Technology Vehicles and Characteristics....................................... 6-1

Estimates of Alternative Fuel Vehicles in Use, 1995-2008 ........................................................... 6-3

Alternative Fuel Vehicles Available by Manufacturer, Model Year 2009....................................... 6-4

Hybrid Electric Vehicles Available by Manufacturer, Model Year 2009 ........................................ 6-5

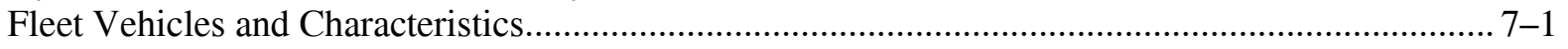

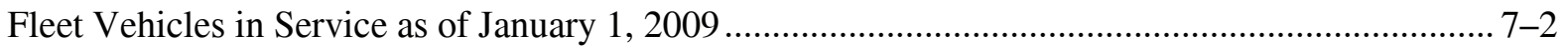

Average Length of Time Business Fleet Vehicles are in Service, 2008 ....................................... 7-3

Average Annual Vehicle-Miles of Travel for Business Fleet Vehicles, 2008................................. 7-3

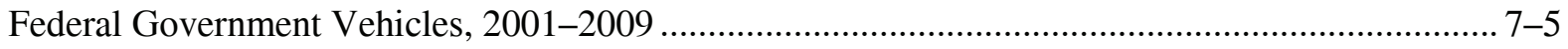

Federal Government Vehicles by Agency, FY 2009 ................................................................... 7-7 
Vehicles (continued)

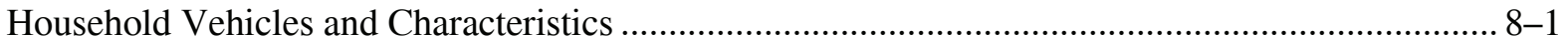

Vehicles and Vehicle-Miles per Capita, 1950-2008 ........................................................................ 8-3

Average Number of Vehicles and Vehicle Travel per Household,

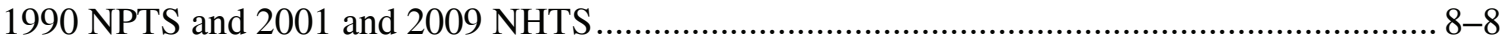

Share of Vehicles by Annual Miles of Travel and Vehicle Age, 2009 NHTS ................................ 8-15

Daily Vehicle Miles of Travel (per Vehicle) by Number of Vehicles in the Household, 2009 NHTS.

U.S. Employment for Motor Vehicles and Motor Vehicle Parts

Manufacturing, 1990-2009.

Emissions of Carbon Monoxide from Highway Vehicles, 1970-2005 ….................................... 12-4

Emissions of Nitrogen Oxides from Highway Vehicles, 1970-2005 .......................................... 12-6

Emissions of Volatile Organic Compounds from Highway Vehicles, 1970-2005 ......................... 12-8

Emissions of Particulate Matter (PM-10) from Highway Vehicles, 1970-2005 ......................... 12-10

Emissions of Particulate Matter (PM-2.5) from Highway Vehicles, 1990-2005.......................... 12-12

Volatile

Total National Emissions of Volatile Organic Compounds, 1970-2008 …................................. 12-7

Emissions of Volatile Organic Compounds from Highway Vehicles, 1970-2005 ......................... 12-8

Wagons

Definition of Wagons in Model Year 2009 ................................................................................. 4-8

Walk

Walk and Bike Trips by Trip Purpose, 2009 NHTS.

Warming

Numerical Estimates of Global Warming Potentials Compared with Carbon Dioxide

U.S. Emissions of Greenhouse Gases, based on Global Warming Potential, 1990-2008

Waterborne

Tonnage Statistics for Domestic and International Waterborne

Commerce, 1970-2008

Summary Statistics for Domestic Waterborne Commerce, 1970-2008 …....................................... 9-6

Weight

New Retail Sales of Trucks 10,000 Pounds GVW and Less in the

United States, 1970-2009

Sales-Weighted Curb Weight of New Domestic and Import Cars

by Size Class, Model Years 1975-2009 …............................................................................ 4-14

New Retail Truck Sales by Gross Vehicle Weight, 1970-2009..................................................... 5-4

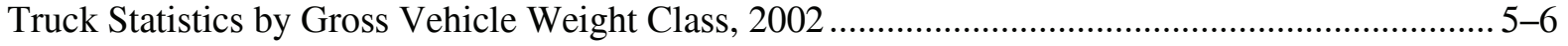

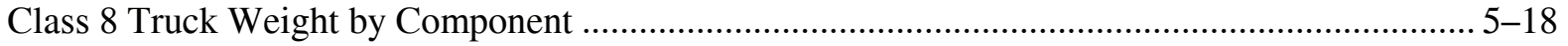

Gross Vehicle Weight vs. Empty Vehicle Weight .......................................................................... 5-19

Distribution of Five-Axle Tractor-Trailers by On-Road Vehicle Weight, 2008 ............................. 5-20

Weighted

Period Sales, Market Shares, and Sales-Weighted Fuel Economies of New

Domestic and Import Cars, Selected Model Years 1975-2009 ................................................. 4-7

Period Sales, Market Shares, and Sales-Weighted Fuel Economies of New

Domestic and Import Light Trucks, Model Years 1975-2009 
Weighted (continued)

Sales-Weighted Engine Size of New Domestic and Import Cars by Size Class, Model Years 1975-2009 ............................................................................... 4-12

Sales-Weighted Engine Size of New Domestic and Import Light Trucks by Size Class, Model Years 1975-2009 .................................................................................... 4-13

Sales-Weighted Curb Weight of New Domestic and Import Cars by Size Class, Model Years 1975-2009 ............................................................................. 4-14

Sales-Weighted Interior Space of New Domestic and Import Cars by Size Class, Model Years 1977-2009 ................................................................................. 4-15

Car Corporate Average Fuel Economy (CAFE) Standards versus Sales-Weighted Fuel Economy Estimates, 1978-2010 ......................................................... 4-22

Light Truck Corporate Average Fuel Economy (CAFE) Standards versus Sales-Weighted Fuel Economy Estimates, 1978-2010 .......................................................... 4-23

Sales-Weighted Annual Carbon Footprint of New Domestic and Import Cars by Size Class, Model Years 1975-2009 .............................................................................. 11-12

Sales-Weighted Annual Carbon Footprint of New Domestic and Import Light Trucks by Size Class, Model Years 1975-2009 ............................................................ 11-13

Work

Share of Vehicle Trips to Work by Trip Distance, 2009 NHTS .................................................. 8-14

Means of Transportation to Work, 1980, 1990 and 2000 Census .................................................. 8-19

Workers

Workers by Commute Time, 1990 and 2000 Census .................................................................. 8-21

World

World Fossil Fuel Potential ...................................................................................................... 1-2

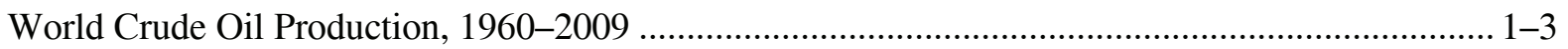

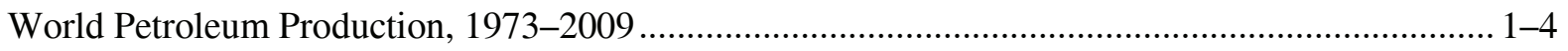

World Oil Reserves, Production and Consumption, 2008 ...................................................... 1-6

World Natural Gas Reserves, Production and Consumption, 2008 ............................................. 1-7

U.S. Petroleum Imports by World Region of Origin, 1960-2009 ................................................ 1-8

Refinery Gross Output by World Region, 1999 and 2009 .......................................................... 1-12

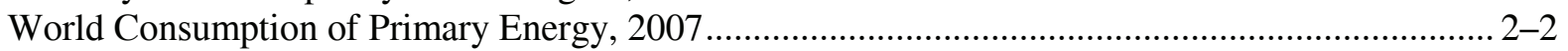

World Carbon Dioxide Emissions, 1990 and 2006 .............................................................. 11-2

Year

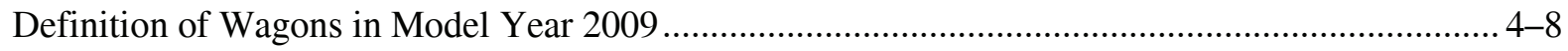

Fuel Economy and Carbon Dioxide Targets for Model Year 2016............................................. 4-21

Hybrid Electric Vehicles Available by Manufacturer, Model Year 2009 ....................................... 6-5

New Light Fleet Vehicle Registrations by Vehicle Type, Model Year 2008 ................................. 7-3

Federal Government Vehicles by Agency, FY 2009............................................................. 7-7

Fixed Car Operating Costs per Year, 1975-2009...................................................................... 10-17

Years

Car Scrappage and Survival Rates, 1970, 1980 and 1990 Model Years ...................................... 3-15

Period Sales, Market Shares, and Sales-Weighted Fuel Economies of New

Domestic and Import Cars, Selected Model Years 1975-2009 .................................................. 4-7

Period Sales, Market Shares, and Sales-Weighted Fuel Economies of New

Domestic and Import Light Trucks, Model Years 1975-2009 ............................................... 4-9

Light Vehicle Market Shares by Size Class, Model Years 1975-2009......................................... 4-10

Light Vehicle Market Shares, Model Years 1975-2009 ................................................................ 4-11 
Years (continued)

Sales-Weighted Engine Size of New Domestic and Import Cars by Size Class, Model Years 1975-2009

Sales-Weighted Engine Size of New Domestic and Import Light Trucks by Size Class, Model Years 1975-2009....

Sales-Weighted Curb Weight of New Domestic and Import Cars by Size Class, Model Years 1975-2009 ……............................................................................. 4-14

Sales-Weighted Interior Space of New Domestic and Import Cars by Size Class, Model Years 1977-2009

Average Material Consumption for a Light Vehicle, Model Years 1995, 2000, and 2008

Distribution of Trucks over 26,000 lbs. Less than Two Years Old by VehicleMiles Traveled

Sales-Weighted Annual Carbon Footprint of New Domestic and Import Cars by Size Class, Model Years 1975-2009

Sales-Weighted Annual Carbon Footprint of New Domestic and Import

Light Trucks by Size Class, Model Years 1975-2009 $11-13$

Yield

Refinery Yield of Petroleum Products From a Barrel of Crude Oil, 1987-2008 $1-14$ 